\title{
Constructivist medicine
}

Citation for published version (APA):

Wackers, G. L. (1994). Constructivist medicine. [Doctoral Thesis, Maastricht University]. Datawyse / Universitaire Pers Maastricht. https://doi.org/10.26481/dis.19941007gw

Document status and date:

Published: 01/01/1994

DOI:

10.26481/dis.19941007gw

Document Version:

Publisher's PDF, also known as Version of record

\section{Please check the document version of this publication:}

- A submitted manuscript is the version of the article upon submission and before peer-review. There can be important differences between the submitted version and the official published version of record.

People interested in the research are advised to contact the author for the final version of the publication, or visit the DOI to the publisher's website.

- The final author version and the galley proof are versions of the publication after peer review.

- The final published version features the final layout of the paper including the volume, issue and page numbers.

Link to publication

\footnotetext{
General rights rights.

- You may freely distribute the URL identifying the publication in the public portal. please follow below link for the End User Agreement:

www.umlib.nl/taverne-license

Take down policy

If you believe that this document breaches copyright please contact us at:

repository@maastrichtuniversity.nl

providing details and we will investigate your claim.
}

Copyright and moral rights for the publications made accessible in the public portal are retained by the authors and/or other copyright owners and it is a condition of accessing publications that users recognise and abide by the legal requirements associated with these

- Users may download and print one copy of any publication from the public portal for the purpose of private study or research.

- You may not further distribute the material or use it for any profit-making activity or commercial gain

If the publication is distributed under the terms of Article $25 \mathrm{fa}$ of the Dutch Copyright Act, indicated by the "Taverne" license above, 


\section{Constructivist Medicine}




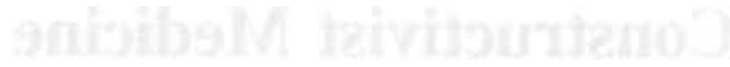




\section{Constructivist Medicine}

\section{Proefschrift}

ter verkrijging van de graad van doctor aan de Rijksuniversiteit Limburg te Maastricht, op gezag van de Rector Magnificus. Prof. dr. H. Philipsen, volgens het besluit van het College van Dekanen, in het openbaar te verdedigen op vrijdag 7 oktober 1994 om 16.00 uur

door

\section{Gerardus Lambertus Wackers}

geboren op 20 januari 1956

te Sittard 
Promotores: Prof. dr. H.A.M.J. ten Have (Katholieke Universiteit Nijmegen) Prof. dr. ir. W.E. Bijker

Beoordelingscommissie: Prof. dr. ir. G. H. de Vries (voorzitter)

Prof. dr. J. W. Arends

Prof. dr. S. S. Blume (Universiteit van Amsterdam)

CIP-GEGEVENS KONINKLIJKE BIBLIOTHEEK, DEN HAAG

Wackers, Gerardus Lambertus

Constructivist medicine / Gerardus Lambertus Wackers. - Maastricht: Universitaire Pers Maastricht. - III.

Proefschrift Maastricht. - Met index, lit. opg. - Met samenvatting in het Nederlands. ISBN 90-5278-152-4

Trefw.: geneeskunde / medische technologie.

(1) 1994, G.L. Wackers, Maastricht

Produktie: Datawyse | Universitaire Pers Maastricht

Omslag: Ruud Leliveld

Druk: Krips Repro, Meppel

Niets uit deze uitgave mag worden verveelvoudigd en/of openbaar gemaakt door middel van druk, microfilm, totokopie of op welke andere wijze ook zonder voorafgaande schriftelijke toestemming van de auteur. 


\section{Contents}

\section{Preface}

1 Into the ways of medicine

1.1 Flashbacks and previews

1.2 Constructivist science and technology studies 14

$\begin{array}{lll}1.3 & \text { Alternative approaches } & 20\end{array}$

$\begin{array}{lll}1.4 \text { Book design } & 21\end{array}$

2 The quest for certainty 27

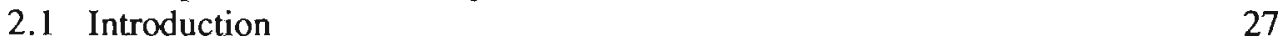

$\begin{array}{ll}2.2 \text { Changing concepts of disease } & 27\end{array}$

2.3 The turn to constructivism: inverting reality 34

2.4 Mute inscriptions and their translators $\quad 40$

2.5 Conclusion 46

3 Existence, presence, identity and performance

Insulin: from hypothesis to standardized treatment $\quad 50$

3.1 Introduction $\quad 50$

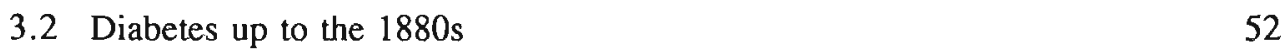

3.3 Minkowski: dogs, pancreases and diabetes $\quad 54$

3.4 Langerhans' islands and diabetes 68

3.5 Moving out of the laboratory: at least attempts to do that 75

3.6 Amplifying the paradox $\quad 79$

3.7 Continuing the search: Georg Ludwig Zuelzer $\quad 82$

3.8 Toronto: the discovery of insulin $\quad 92$

$\begin{array}{ll}3.9 \text { Biological standardization } & 114\end{array}$

$\begin{array}{ll}3.10 \text { Conclusion } & 117\end{array}$ 
4 Theaters of war, truth and competence Intermittent positive pressure respiration during the 1952 polio-epidemic in Copenhagen

4.1 Introduction

4.2 Setting the stage: Blegdamshospital

4.3 Polio in Copenhagen

4.4 Veni, vidi, vici

4.5 Why an anesthetist in the first place?

4.6 Creating identity

4.7 Metabolic alkalosis and respiratory acidosis

4.8 Contingency, rhetorical strength and clinical skill

4.9 Mobilizing hands

4.10 The rhetorics of mortality rate reduction

4.11 Mechanical students

4.12 Acid-base and blood gas analysis

4.13 Kommunehospital's intensive care unit

4.14 Cooled ceilings and big toes

\section{Death in the skull}

Re-negotiating the end of human life

5.1 Introduction

5.2 Dead brains

5.3 Allowing to die? Withdrawal of life-support

5.4 Utility of the (heart-beating) corpse

5.5 On definitions of death

5.6 Definition of total death

5.7 Definition of brain stem death

5.8 Definition of whole brain death

5.9 Definition of neocortical death

5.10 Non-closure

5.11 Partitioning the controversy

$\begin{array}{lll}6.1 \text { Introduction } & 244\end{array}$

6.2 Circumstances of production 246

$\begin{array}{ll}6.3 \text { Programs of action } & 248\end{array}$

6.4 Speaking for the weak 258

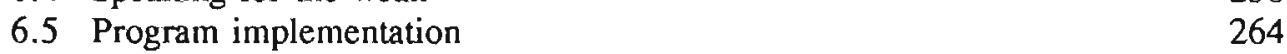

6.6 Conclusion

Summary in Dutch

Glossary

Bibliography

Index 


\section{Preface}

The publication of this study marks the end of a journey through the records and practices of modern medicine. It began some twenty years ago when I decided to become a medical laboratory technician. Since that time I worked in two hospital laboratories, studied medicine, developed a theoretical and historical interest in my own profession and moved into the field of science and technology studies to write this book. Let me briefly outline the various stages of my journey to explain the history of this book and to acknowledge my intellectual and social debts.

Having learned how to grow and identify bacteria from various body fluids and products, I moved to Norway to work in the laboratory of Narvik Sykehus. Being a small hospital with an allround laboratory, Narvik Sykehus provided a most stimulating environment for genuine problem oriënted learning. Two young physicians, Torstein Gundersen and Bjørn Ibsen, deserve special mention for their willingness to discuss special diagnostic problems and to call on our knowledge and skills to contribute to their solution. It was here that I decided to study medicine at the University of Limburg in Maastricht.

In the course of my medical training in Maastricht I was able to probe into the practices of various medical specialties. Early in my studies it was the late professor in medical ethics Paul Sporken, who developed my nascent reflexive attitude. In the group discussions at his house we explored issues of, and attitudes towards, life and death, sexuality, health and physical and mental disability. When his respiratory capacity and vocal cords forced Sporken to abandon teaching. Henk ten Have filled his place. It was in discussions with him that the idea and outlines for a research project emerged. The project found an institutional niche in the Department of Health Ethics and Philosophy of the Faculty of Health Sciences. Physically the department was housed together in one building with the departments of history and philosophy of the Faculty of General Sciences. The people doing work in science and technology studies worked in this building.

In anthropology there always is the risk of 'going native'. That was not my problem. I was a native member of the profession that I wanted to study. My problem was one of doing the work of a philosopher, sociologist and historian. The joint discussions in our profoundly interdisciplinary research group were very helpful in exploring the various disciplinary traditions and vocabularies. The two year curriculum 
of the Netherlands Graduate School in Science and Technology Studies, coördinated by Gerard de Vries and Wiebe Bijker, with regular workshops and three summerschools, provided the inexpendible basic training into various approaches to the study of problems of science, technology and society.

Without the help of my supervisors I would never have been able to complete this book. Being a physician and philosopher, Henk ten Have was a stimulating discussant, both on matters concerning the history of medicine and on issues in the realm of the philosophy of medicine. From the very inception of the research project he provided proper encouragement and direction, while at the same time allowing me to make my own choices as the work proceeded. I am grateful to him for his patience and for his always close reading of my writings.

Wiebe Bijker got involved while the project was well under way. His readingwith-the-text-editor's-red-pen of subsequent drafts of the chapters, and his insistence on the use of plain English whenever possible, did much to improve the readability of the text. But, more than anything else, it was his never ending confidence in my work that stimulated and helped me to see the project through to the completed manuscript. When at the close of 1991 my appointment at the Department of Health Ethics and Philosophy ended, Wiebe Bijker provided the financial resources that allowed me to continue my work at the Department of Philosophy of, what is now called, the Faculty of Cultural Sciences.

I would like to thank professor Uffe Juul Jensen at the Institute of Philosophy, Aarhus University in Denmark, for the provision of facilities, hospitality and friendship during a three month period of research in 1989, and several subsequent visits. With Denmark's best research library in close proximity, and Copenhagen within travel distance, Aarhus provided a perfect setting for the empirical research on the 1952 polio-epidemic in Copenhagen. I would also like to thank Tove Borg and Ulla and Bjørn Runge for their hospitality on several occasions. I am grateful to Lena Olsson for arranging that I could spent a month at the Department of Technology and Social Change, Tema-T, at Linköping University in Sweden. The solitude of Brunby during the weekends provided the proper conditions for the completion of a chapter. Special thanks go to Magnus Johansson for our tour to Sarek. Finally, I would like to thank Wilma van Driel for her search of the brain death literature, Guus van Rooy for drawing the illustrations in figures 3.1 and 3.2, and Jeanet Kalk for translating the German citations of chapter 3 into English.

I do not know how Truus and Helene have endured my recurrent lapses of mental absence and distraction during my spells of writing. If they hoped that these paroxysmal absences would come to an end with the completion of this book, than I have to disappoint them. I vividly remember many occasions on which I pushed the right button by asking Truus about some old laboratory test. She was always able to produce several papers and books from the remotests corners and shelfs of our study. I also remember the many occasions on which her first glance at a text unremittingly disclosed some spelling error. Her close reading of the final manuscript removed several errors I had overseen. Probably Truus suggested some ideas for research that I now think were my own. 


\section{1}

\section{Into the ways of medicine}

\subsection{Flashbacks and previews}

\section{Toronto 1922}

A fourteen year old boy suffered from diabetes mellitus. He had reached the final stage of the disease. He had been on Allen's very strict dietary regime that reduced his calory intake to a minimum, reducing him to skin and bones. It was likely that he would die of starvation, if the disease would not kill him first. On the other side of the road a couple of researchers had been trying to extract from animal pancreases a substance that should be able to restore a diabetic's blood sugar utilization. They had prepared an extract that worked in pancreatectomized animals. With approval of the boy's father and of the patient's physician, the boy was chosen as the first human patient to receive the extract. After having received $5 \mathrm{ml}$ in the morning and $20 \mathrm{ml}$ in the afternoon, the boy's clinical condition improved considerably. He became brighter, more active. He looked better and said that he felt stronger. Laboratory tests showed a decline in blood sugar, and in sugar excretion in the urine. Researchers and clinicians alike felt that this was the first successful clinical test of an anti-diabetic pancreatic extract on a human diabetic patient, a persuasive demonstration that insulin existed. The Torontonian researchers were awarded the 1923 Nobel Prize in medicine and physiology for their achievement.

\section{Copenhagen 1952}

The city was struck by a severe polio-epidemic. A twelve year old girl, rammed by the disease, had been admitted to Blegdamshospital. Legs and arms were paralyzed. She was not able to breathe, swallow or speak. She suffered from a form of the disease called bulbar polio. A young medical student was doing the breathing for her by squeezing a ventilation bag that was connected to a tube in her windpipe. The girl had lost the ability to speak, but not the ability to communicate. Whenever the student slackened at the squeezing she would signal to him with her eyes that he should keep up the pace. Medical students worked in shifts, 24 hours per doy, to ventilate the girl 
and some seventy other bulbar polio-rammed patients, to keep them alive with their own hands. This therapeutic set up, manual intermittent positive pressure ventilation, was new in the treatment of polio. The girl, at the brink of death after admission, had been the first patient to be kept alive by a medical intervention in an epidemic that had killed 27 out of the first 30 patients with bulbar polio, and in which all traditional therapeutic measures failed.

Somewhere, an intensive care unit 1993

A mother was grieving over her twenty year old son. He had been involved in a traffic accident and incurred a severe head injury, less than 24 hours ago. He had been taken to this intensive care unit where they succeeded in stabilizing his condition. They told her that he had difficulty breathing, and that it was therefore that he had been connected to the respirator. Her son's chest moved up and down in tandem with the respirator's wheezing sounds. A monitor, recording his ECG, showed that her son's heart was still beating forcefully. As he did not regain consciousness the intensive care physicians started doing some more neurological tests, some of which were repeated by another neurologist a couple of hours later. After the completion of the tests the physicians told her that her son would never regain consciousness, that he was actually dead, brain dead. Another doctor asked her to give permission for the removal of her son's kidneys, liver and heart for transplantation purposes. In that way her son's death would save other people's lives, giving meaning to an otherwise meaningless premature death of a young, healthy man. Even if she would refuse permission for transplantation, the physicians would stop further treatment, because the patient was dead. Being a stranger in this world, the mother was confused. How could he be dead? His heart was still beating; she could see that on the monitor, and she felt his pulse when she held his hand. His chest was moving in respiration. His skin was warm to her touch. Was he not still alive? Would not the removal of his heart kill him? If she refused the transplantation of his heart, would he not die a second time?

This book is about modern medicine. It investigates changes in medicine's cognitive content, in its technological tool kit and in its moral and social order. This broadness of scope is fostered by genuine excitement and concern simultaneously; genuine excitement about and admiration for impressively well-crafted laboratory experiments and clinical, therapeutic achievements; a recognition also of the thrill and excitement shared by witnesses and many commentators of important achievements in medicine. Especially when life-saving achievements are concerned this thrill is often expressed in superlative terms. Bliss, commenting on the discovery of insulin, wrote that 'those who watched the first starved, sometimes comatose, diabetics receive insulin and return to life saw one of the genuine miracles of modern medicine. They were present at the closest approach to the resurrection of the body that our secular society can achieve, and at the discovery of what has become the elixir of life for millions of human beings around the world'. (Bliss 1982: 11) Mushin, writing about other European countries' responses to the revolutionary new treatment of tracheostomy and manually controlled respiration for bulbar polio, which was first adopted during the 1952 polio-epidemic in Copenhagen, expressed the thrill of the time as follows. 'As 
the winds of alarm at the approaching catastrophe fanned the embers of inventive genius an abundance of new mechanical solutions ... were forged in the flames'. (Mushin 1980: 207)

Thrill and excitement easily translates into a general confidence in a science- and technology-based medicine. Science and technology are credited for medicine's enormously increased potential to cure, and to be effective against previously lethal or invalidating infectious and metabolic diseases. The balance of power between physicians and 'killer' diseases has been reversed in many instances. As the old diseases are repelled and people live longer, new diseases come to the fore: cardiovascular diseases, cancer, degenerative diseases, AIDS. Medical science may have some difficulties in finding effective preventive measures or causal therapies for these diseases, but with an increased effort and enough time medicine will also be able to fight these diseases. In the mean time, medical science and technology have come a long way in redeeming the damage and restore functional losses.

The installation of laboratories in the institutions of medicine in the nineteenth century is seen as the means whereby medicine became scientific. Scientists in these laboratories recognized a truth, the truth that it is the experimental methods of the laboratory that are the most powerful instruments of scientific progress in applied sciences such as medicine. Laboratory education and the pursuit of laboratory-based research turned physicians into scientists and it turned medicine into a scientific discipline capable of sustained progress both in the questions it addressed and in the answers it delivered.'

However, scientific and technological achievements in modern medicine give not only rise to excitement, thrill and admiration. There is also reason for genuine concern; concern about the aggressiveness that has seeped into some areas of medical practice; concern also about medicine bringing more and more aspects of personal life under its dominion. People have become increasingly dependent on medical expertise, in reproduction and child raising, in caring for the sick and dying. Modern medicine has blurred the distinction between life and death. Allowing people to die has become a serious problem. These developments have led critics of medicine to reclaim people's right to live their own life and to die their own death.

Justified concern and criticism easily translate into a general skepticism about a science- and technology based medicine. Medical science and technology have been blamed for the expulsion of the subject. There is more to being a good doctor than knowing and doing what has been established by science and applied in medical technologies. There is more to a human being than can be captured by an experimental science. The argument goes that medicine's limitations are inherent to the cognitiveinstrumental rationality it embodies. Philosophers have tried to conceptualize the wholeness of the human being that was being reduced to its chemical and physical substrate of its body by experimental medical sciences. Medical professionals have tried to develop alternative forms of doing medicine. Forms of medicine that would make up for the reductionism of modern medicine and recognize non-chemical, nonphysical, non-biological (that is: psychological, social and political) determinants of health and disease.

Scientific and technological developments in medicine in recent decades have fostered a good deal of societal skepticism. The costs of health care have risen tre- 
mendously over the past decades. Most western countries have accommodated their national budgets to meet medicine's increasing demands. But there is and must be a limit to the amount of money a country can spend on health care. Furthermore, there is societal skepticism, in a moral sense, about the very desirability and acceptability of the development of scientific knowledge and technological capabilities in certain fields, like, for example, genetic engineering, the complete and permanent replacement of vital organs with artificial substitutes, complete extra-corporeal reproduction, brain transplantation, etc. It seems as if medical technology has run out of control. Society has only very recently and hesitatingly begun to develop means to assess the impact of these technological developments on society and mechanisms to control the introduction and use of new medical technologies in health care.

I recognize and share both the thrill and the concern. Both have been important incentives to embark on a study of modern medicine, and especially of medical technologies. It is the omnipresence of technology in modern medicine that sets it off against traditional forms of medicine. Technology is taken to account for the success of an interventionist medical practice. However, it is also technology that is held responsible for the disruption of traditional value systems, for objectionable moral and social consequences. Any attempt to understand the current shape of modern medicine - its cognitive content, its technologies, the pattern and course of moral disputes should take into account the role that science and technology played in its generation. In other words, an understanding of medicine requires an understanding of science and technology.

However, sharing both the thrill and the excitement suggested me to move away from generalistic critiques of medical technologies and of the reductionist, instrumental rationality that they are supposed to embody. In this study I do not want to defend medicine's virtues, for it has champions enough. Nor do I wish to join the ranks of critics who apply ethical theories or societal value systems to pass judgement about modern medicine's impact on man and society. Neither will I claim that medical science and technology are morally neutral and that it is only their use that allows moral and social value judgments. I will not presuppose any of these claims as a priori assumptions about the nature of modern medicine and technologies. To the contrary, the leading questions throughout this study will be: What kind of achievement are scientific discoveries and technological innovations? In what terms can we most plausibly account for them? How do technological achievements transform the content of medical knowledge, and how do they transform medicine's moral and social order? How can we account for simultaneous cognitive, technological and social changes on the one hand, and for the undeniable durability and obduracy of knowledge, technologies and moral principles on the other hand? How come that a technology that is life-saving in one setting, like the application of manual positive pressure ventilation in the 1952 polio-epidemic in Copenhagen, becomes highly problematic in another setting only a few years later, in respiration centers and intensive care units? Why is it, as Ten Have (1985; Ten Have and Kimsma 1987) observed, that academic medical ethics often operates like a fire brigade, rushing from one (moral) fire to another, always lagging behind scientific and technological developments and primarily dealing with ethical issues arising from the use of medical technologies that have already been introduced? How come that often the moral and political controversy is in the public 
domain, the opponents in the dispute being outsiders? How are new medical practices established despite the fact that the involved technologies still are very controversial?

As the medical-historical work of this project proceeded and as the details of the medical narrative were filled in, it became clear that boundaries between facts and values, the scientific and the social, inside medicine and outside public are from time to time renegotiated and redefined. Constructivist science and technology studies suggests another way into the ways of medicine. Contrary to much of the work in conventional philosophy of medicine and medical ethics, this approach suggested a type of analysis that does not rest on a priori assumptions about the distinctions between science, technology and society and their ontological and epistemological nature.

Consistent with much of the work in constructivist science and technology studies this study sets out to account for the way in which the scientific and technological content of modern medicine and its moral and social order are co-produced by the heterogenous and collective work of a variety of actors. The shape of modern medicine is rooted in these actors' practices. ${ }^{2}$ The argument is that the stabilization of new medical knowledge and new medical technologies requires simultaneous changes in medicine's moral and social order. Vice versa, the establishment of a new moral and social order requires re-constructive work on the cognitive and technological content of medicine. By tracing and maintaining new distinctions and boundaries actors produce and re-produce their own world.

Given the primary aim of this study, that is to provide an account of modern medicine, while drawing on work in constructivist science and technology studies, I hope that this book will of interest to different audiences. Students of science and technology will be familiar with the type of analysis developed in this book. It is not a simple application of an extant theoretical framework, however. On the one hand they may be interested in the way in which some familiar theoretical concepts have been appropriated to fit the details of the case studies. On the other hand scholars in constructivist science and technology studies may be interested in the medical case studies themselves. Medicine offers fascinating opportunities for the pursuit of 'constructivist' research questions.

However, I also hope that this study will be of interest for those readers who have a considerable advance knowledge in medical matters, either by virtue of them being medical or health care professionals, or by virtue of them having dealt with medicine as the subject matter of their academic science (philosophers of medicine, medical historians). Seen from the perspective of philosophy of medicine, this book may be seen as contributing to the literature on various concepts of disease. This study develops what might be called a constructivist concept of disease. But the book also contains a chapter on the issue of brain death, a subject on which philosophers of medicine have written extensively. Historians of medicine may find the chapter on the 1952 polio-epidemic in Copenhagen of particular interest. This is a salient episode in the history of artificial respiration where, as yet, no professional historian has devoted much scholarly time. This latter group of readers may be less, or not at all, familiar with the theoretical positions, problems and stakes in constructivist studies of science, technology and society. I have tried to write each of the chapters relating a particular case study as a complete narrative whole in itself that can be read separately on itself. 
In these empirical chapters I have kept a rather low theoretical profile. Where I introduce theoretical notions I have tried to fit them into the structure of the narrative so as not to disrupt the flow of the story too much. In the other I have also tried not to assume too much advance knowledge about theoretical issues in science and technology studies.

Section 1.2 will provide a brief outline of developments in the field of constructivist science and technology studies. For readers who are not familiar with the work in this field, it will provide the basic tenets of a constructivist approach. It will also explain my own position within this area of research. Section 1.3 will explain why a multi-case study, rather than a single-case study design has been chosen. It will also explain the role of theory in this book. Section 1.4 will conclude this chapter by outlining the design of the book.

\subsection{Constructivist science and technology studies}

Constructivist science and technology studies grew out of several sociological approaches to knowledge-making. In the 1970s British scholars like Barry Barnes, David Bloor, Harry Collins, Alex Dolby, Richard Whitley and Michael Mulkay made the bold claim that the technical content of scientific knowledge was amenable to sociological investigation and explanation. For the first time in the history of sociological thought scholars embarked on a sustained program of empirical research subjecting natural and scientific knowledge to the same scrutiny which had long been brought to bear on other systems of beliefs, such as religious or political thought. With roots in the writings of Marx, Durkheim and Mannheim, the sociological discipline that examined these latter systems of beliefs was known as the sociology of knowledge, broadly defined as a theory of the social and existential conditioning of thought. With the emergence of a sociology of scientific knowledge sociological scrutiny was extended into the realm of the most authoritative and esoteric system of knowledge in modern societies, that of the natural sciences. (Knorr-Cetina and Mulkay 1983)

In this thrust the sociology of scientific knowledge was at variance with the sociology of science as it was pioneered since the 1930s by Robert Merton. which stopped short for the social explanation of scientific content. As Collins (1983) put it. traditional Mertonian sociology of science turned on the elucidation of the set of normative and other institutional arrangements that enable science - the asking and answering of questions about Nature - to exist and function efficiently. A crucial feature of this program consisted in the assumption that the ultimate answers to the questions asked by scientists were Nature's, mankind being only a mediator. Proper institutional arrangements must obviate the effect of mundane disagreements and biases, enabling correct answers to emerge. The new program of inquiry in the sociology of scientific knowledge, however, was, according to Collins (1983), concerned with 'how certain views about the physical and mathematical world come to count as correct within a society, rather than how a society can be arranged so that truth will emerge'. The new program was concerned with the 'social construction' of scientific knowledge. 
Thus, in the 1970s sociologists of scientific knowledge brought the content of science within the scope of thorough-going sociological scrutiny, for which, as Bloor (1976/1991) put it, traditional sociologists of knowledge and science had lacked the nerve and will. In doing so they battled the misguided assumption that there could only be a sociology of error and distortion, of blind alleys and wrong pathways, whereas successful theories and true knowledge would provide their own sufficient explanation. According to Bloor (1976/1991: 9), 'the general structure of these explanations stands out clearly. They all divide behaviour or belief in two types: right and wrong, true and false, rational or irrational. They invoke sociological or psychological causes to explain the negative side of the division. Such causes explain error, limitation and deviation. The positive side of the evaluative divide is quite different. Here logic, rationality and truth appear to be their own explanation. Here psycho-social causes do not need to be invoked'. In response to these asymmetrical explanations of scientific success and failure Bloor formulated two important tenets for his 'strong programme' in the sociology of knowledge: symmetry and impartiality. The analyst should be impartial with respect to truth and falsity, rationality or irrationality, success or failure. Both sides of these dichotomies require symmetrical explanation, that is, the same types of factors should be invoked to explain true and false beliefs, rational and irrational behavior. These two requirements, formulated by Bloor in 1976, stand firm in constructivist science and technology studies today. ${ }^{3}$

The sociology of scientific knowledge was supported by the work of philosophers like Kuhn, Duhem, Quine and Wittgenstein, engendering a relativistic epistemological stance. Kuhn (1962) made a strong case for the conventional nature of scientific knowledge and the theory-loadedness of observation. Observations, the argument goes, are always impregnated with theoretical assumptions and auxiliary assumptions in the form of measurement theories and the like. Furthermore, what counts as relevant evidence is partly determined by the theoretical paradigm informing the experimental designs. As a consequence, it is always open to scientists to doubt observational data by challenging the central theoretical or auxiliary assumptions, or the competence of the experimenter. The bearing observational evidence can have on theory depends on its being accepted as valid information, which in turn depends on the unproblematic acceptance of the background assumptions which are constitutive of the observation. Thus, observations cannot serve as independent arbiters in questions of theory choice. (Knorr-Cetina and Mulkay 1983)

The names of Duhem and Quine are associated with the thesis of the underdetermination of scientific theories by the evidence. Knorr-Cetina and Mulkay (1983) summarize the Duhem-Quine thesis by saying that any theory can be maintained in face of any evidence, provided that we make sufficiently radical adjustments elsewhere in our beliefs. This follows from the fact that no one single theory or theoretical hypothesis can ever be extricated from the ever present web of collateral assumptions so as to be open to conclusive refutation.' A theory whose predictions do not materialize can always in principle be retained by making appropriate adjustments in the auxiliary hypotheses. Conversely, there are in principle always alternative theories which are equally consistent with the evidence and which might reasonably be adopted by scientists. What is reasonable, logical or rational depends, using Wittgenstein's notion, on the specific form of life that scientists share and are part of. ${ }^{4}$ 
This is a philosophical argument about the epistemological, but also about the historical and cultural relativity of science. In this relativism the natural world has a small or non-existent role in the construction of scientific knowledge. Observational data do not determine in any sense the way in which they have to or will be interpreted. Potentially, in science there will always be local interpretative flexibility which prevents observation or experimentation from being decisive. Potentially the debate about interpretations is endless. In practice, however, controversies come to an end. In a series of empirical studies sociologists of scientific knowledge set out to examine and explain how this closure of controversies in science is brought about.

According to Collins (1981), sociologists of scientific knowledge engaged in what has become known as the Empirical Program of Relativism (EPOR), uncovered the equivalent of the philosophical argument made by Duhem-Quine in the day-to-day activity of contemporary laboratory science. Interpretative flexibility was the main message of EPOR's first stage. The second stage of the program goes on to describe and analyze the closure mechanisms which limit interpretative flexibility and thus allow controversies to come to an end. The agenda for the third stage of the empirical program of relativism is to relate the closure and outcome of scientific controversies to the wider social and political structure. This last step is necessary to fulfil EPOR's programmatic goal of explaining the content of scientific knowledge as far as possible in social terms.

The 1970s and the 1980s also saw the emergence of an interest in a new type of technology studies. This emerging interest was partly independent of, and partly closely related to the sociology of scientific knowledge. The explanatory aims are similar. The sociology of scientific knowledge is concerned with, in Collins' (1983) words, 'how certain views about the physical and mathematical world come to count as correct within a society, rather than how a society can be arranged so that truth will emerge'. According to MacKenzie and Wajcman (1985), social scientists writing about technology have tended to concentrate on the 'effects' of technology, on the 'impact' of technological change on society, leaving a prior, and perhaps more important question unasked and therefore unanswered: What has shaped the technology that is having 'effects?' What has caused and is causing the technological changes whose 'impact' we are experiencing? Hence, a new type of technology studies emerged that was concerned with the social factors that shape technological change, with the social shaping of technology. The sociology of scientific knowledge is concerned with the social construction of scientific knowledge, whereas the 'new' sociology of technology is concerned with the social construction of technology.

Scholars engaged in these new sociological approaches to technology-making moved away from the individual inventor or genius as a central explanatory concept, they battled the notion of technological determinism, and they avoided making a priori distinctions between technical, social, economic and political aspects of technological development.

Trevor Pinch and Wiebe Bijker (1984) were inspired by the sociology of scientific knowledge. Their approach, which has become known as the Social Construction of Technology (SCOT). ${ }^{5}$ SCOT was 'mapped' on the three stages of EPOR. Its central claim is that technological artefacts are open to sociological analysis, not just in their usage but especially with respect to their design and technical 
content. Starting from the interpretative flexibility of artefacts, which can be uncovered by tracing the different meanings that 'relevant social groups' attribute to an artefact, SCOT proceeds by describing the mechanisms of closure and stabilization of a technology. In recent work Bijker $(1987 ; 1990 ; 1992 a, b)$ tries to relate these closure mechanisms to the wider socio-cultural milieu.

A second approach stems largely from the work of the historian of technology Thomas Hughes $(1983 ; 1987)$ who treats technology in terms of a systems metaphor. Emphasizing the different but interlocking elements of physical artefacts, institutions and their environment, the systems approach is tailored to understand and explain the evolution of large technological systems such as power networks or rail road systems. ${ }^{6}$ Hughes (1986) introduced the metaphor of a 'seamless web' of science, technology and society to emphasize that the analyst should not make a priori distinctions between these different aspects, but treat them as heterogeneous elements in one, albeit complex system.

A third approach, associated with the work of Michel Callon, Bruno Latour, and John Law, describes the seamless web of science, technology and society in terms of 'actor-networks'. Studies in this line of work describe the work and strategies through which actors (networkbuilders) enroll other actors, human and non-human, in their network.' The methods by which an actor enrolls others are captured under the key notion of 'translation'. These methods involve both, the definition of roles, their distribution, and the delineation of a scenario, as well as the displacement imposed upon others as they are forced to follow the itinerary that has been imposed. (Callon et al. 1986) The thesis defended by Callon (1986a; 1986b;1987) is that the study of technology itself can be transformed into sociological tool of analysis, and that this is not only possible but also desirable. Callon sets out to show that engineers who elaborate a new technology constantly construct hypotheses and forms of argument that pull participants into the field of sociological analysis. By studying the work of these engineer-sociologists one also studies the construction and reconstruction of society. However, this society is no longer made up of humans and inter-human relationships exclusively. Society is made up of human and non-humans in sociotechnical assemblies.

Although avoiding a 'technical' semiotic vocabulary, some of the key concepts of actor network theory, in particular the notion of translation, will be employed in subsequent chapters of this study. These will be introduced in more detail in the second chapter.

The field of constructivist science and technology studies did not only experience a rapid growth and proliferation of different approaches during the past two decades, it also witnessed rapid theoretical developments. It falls outside the scope of this introductory section to review these developments in detail. However, for a proper understanding of the design of and the type of analysis attempted in this study it is important to discuss some of them briefly.

The unit of analysis, the object of study has changed. The early work of scholars in the empirical program of relativism were concerned with the fairly homogeneous content of scientific knowledge. The early studies conducted in the Social Construction of Technology program focussed on artefacts such as bicycles, bakelite and fluorescent lighting, also fairly homogeneous objects of study. In recent work sociologists of 
scientific knowledge have chosen technologies as their object of study. (Collins 1990; Mackenzie 1990) The change in the object of study, however, does not consist only of a shift from scientific knowledge to technology, or from science or technology to technoscience. Most consequential is the inclusion of the social in the object of study. Not in the sense that the object of study now falls into two parts, the technical and the social, but rather, that there is only one, albeit heterogeneous, in Bijker's (1992) words, sociotechnical ensemble. There is no 'technology' external to 'society', nor is there a 'society' external to 'technology'. Actor network theory and Hughes technological systems approach were designed to grasp these heterogeneous assemblies of humans and non-humans, of devices, engineers, entrepreneurs and organizations. Actor-network theorists emphasize that scientists and engineers in elaborating a new technology also do and have to redesign and reconstruct society. This heterogeneity of the object of study has important consequences for what it means to provide a social explanation. According to Latour and Woolgar (1986), 'social' retained meaning when used by Mertonians to define a realm of study which excluded consideration of 'scientific content'. It also had meaning in the strong program's attempts to explain the technical content of science, in contrast with internalist explanations of technical content. 'In all such uses, 'social' was primarily a term of antagonism, one part of a binary opposition. But ... by demonstrating its pervasive applicability, the social study of science has rendered 'social' devoid of any meaning'. (Latour and Woolgar 1986: $281)^{8}$

In other words, having eliminated the social as an exogenous cause for technical change and the technical as an exogenous cause for the social leaves the question of how to explain sociotechnical change. When sociotechnology is the unit of analysis and no exogenous causes are allowed, the changes must result from sources within the sociotechnical system. Scholars in constructivist science and technology studies focus on the work and strategies employed by a variety of actors engaged in controversies, or in overcoming resistances to the implementation of new sociotechnical practices. taking into account the idiosyncracies of particular empirical situations. The specific shape of a sociotechnical practice is considered to be the (temporarily) stabilized result of an ongoing process of change fuelled by actors' unflagging hands-on work to overcome, what Bijker and Law (1992: 9) called, 'conflict, difference or resistance'. In Latour's vocabulary, the verb 'to resist' is inseparable from the verb 'to try'. A 'trial of strength' (or weakness) is Latour's generic term for the kind of frictions Bijker and Law allude to. Furthermore, according to Latour (1988: 158), 'whatever resists trials is real'. Trials of strength may overthrow the old order and establish a new one, which in its turn will be tried. Or the old order resists the trial of strength. The particular shape of a sociotechnical practice has been performed by trials of strength. Only, once a form is stable, it no longer appears to be a trial of strength. These concepts of trials of strength, resistance and reality as that what resists, have informed, as sensitizing notions, the selection of case studies in this study and much of the description and analysis in the following chapters. They are Latour's conceptualization of one of the most basic tenets of a constructivist ${ }^{9}$ approach to science and technology, namely that 'Reality', and related notions like 'Nature', 'Truth' and 'Objectivity', are the upshot of changing practices (scientific, technological, economic, social). These notions are the result of what people do. Because they 
are a result they are what has to be explained (explanandum). Hence, these notions cannot be used to explain (explanans) the cognitive authority of scientific knowledge in general. nor the certainty with which we hold specific scientific representations about the world to be true, nor the working of a technology. They cannot serve as a source for legitimizing or founding cognitive authority and technological superiority. ${ }^{10}$

In a methodological sense 'trials of strength' are the proper, or strategic research sites for a student of scientific and technological change. ${ }^{11}$ They are the right places to look. This also means that there are wrong places to do the research. According to Collins (1975), much of our knowledge seems so 'solid' as to require a justification in terms other than those which describe human actions. This, he explains, is because when we consider the grounds of knowledge. we do it within an environment filled with objects of knowledge which are already established. 'To speak figuratively, it is as though epistemologists are concerned with the characteristics of ships (knowledge) in bottles (validity) while living in a world where all ships are already in bottles with the glue dried and the strings cut. A ship within a bottle is a natural object in this world, and because there is no way to reverse the process, it is not easy to accept that the ship was ever just a bundle of sticks'. (Collins 1975: 205). The proper locus of research for a sociologist of scientific knowledge, Collins (1975: 206) argued, was in places in which 'the figurative 'ships' are still being built by human actors, to be subsequently erected in their bottles by a trick invented and worked by human actors also'. ${ }^{12}$ Latour (1987) phrased the same point in terms of 'science in the making' versus 'ready made science'. It is in these strategic research sites that the processes by which outcomes are brought about through the concrete and situated work of participants can be studied. This concern with the processes through which scientific knowledge and technologies are constructed also reflects what is considered to be the proper job for the analyst: to find the sources and strategies of certainty and obduracy.

To avoid undue misunderstandings a warning is in place here: the job of the analyst is not to demonstrate that scientific knowledge is fiction, rather than fact. Providing a constructivist account of, for example, the discovery of insulin, does not imply that scientific knowledge about insulin is wrong, or that insulin does not 'really' exist. Neither does it mean that all knowledge claims are equally valid. We should avoid what Hernstein Smith (1988) in this respect has called the egalitarian fallacy. Knorr-Cetina and Mulkay (1983) have made the same point by emphasizing the distinction between epistemic and judgmental relativism. Epistemic relativism, which lies at the basis of constructivist science and technology studies, asserts that knowledge is rooted in a particular time and culture. Some knowledge claims are endowed with more certainty than others through the work of participants, thus producing inequality between competing knowledge claims. Judgmental relativism, however, does not only assert that knowledge is rooted in a particular time and culture, in addition it asserts that all forms of knowledge are equally valid, and that we cannot compare different forms of knowledge and discriminate among them. It should be clear, Knorr-Cetina and Mulkay (1983: 6) argue, 'that judgmental relativism manifestly does not follow from epistemic relativism. The belief that scientific knowledge does not merely replicate nature in no way commits the epistemic relativist to the view that therefore all forms of knowledge will be equally successful in solving a practical problem, equally 
adequate in explaining a puzzling phenomenon or, in general, equally acceptable to all participants'. It is precisely the project of constructivist science and technology studies to work out in what sense and to what degree we can speak coherently of knowledge as being rooted in the concrete, hands-on work of participants working in a particular time and culture. This study will attempt to work out the sources and strategies through which new propositions and entities (about the existence of certain substances or about the end of human life, new interpretations of clinical conditions or laboratory results, new technologies, etcetera) are invested with so much certainty that they become a seemingly inexorable part of the world's (cognitive, technological or social) order; at the expense of 'old' truths and ways of seeing or doing things.

\subsection{Alternative approaches}

At the outset the investigation of medical practices might be approached roughly in two different ways, which can conveniently be introduced by way of the two following notions: 'powers of ten' and 'the arctic grail'.

In 1977 Ray and Charles Eaton produced a short film Powers of $\operatorname{Ten}^{13}$ that comprised a series of 41 successive pictures. These pictures provide a visual image of current knowledge about the universe, from the sub-atomic dimensions of a carbon atom in a human DNA-molecule $\left(10^{-16} \mathrm{~m}\right)$ to the expanding emptiness in remote galaxies $\left(10^{25} \mathrm{~m}\right)$. The area covered by each frame was ten times smaller or bigger than the next or preceding one. Moving from the greater to the smaller dimensions in successive steps creates the impression of 'zooming in', of coming closer and seeing more and more detail, each new frame being within the next one.

In a series of successive steps, then, the subject matter of medical practice is progressively narrowed, to the exclusion of other subjects in other fields. If not already in a specific field of research this process of narrowing the subject matter is often accompanied by an eliminative choice for a specific scientific discipline with its own theoretical framework and its own methodological standards. The aim of the study may be on two levels, that is, on the one hand, to develop an understanding of the particular practice or development under study, and, on the other hand, to test, improve on and develop the theoretical framework employed. This approach yields mono-disciplinary single-case studies that tend to be methodologically sound, exhaustive in their description and rich in empirical detail. Although making eliminative choices in the course of the investigation is unavoidable, the powers of ten approach has several disadvantages. The generalizability of the theoretical insights obtained in the particular case study is limited. The study itself does not have a comparative angle that would allow conclusions transcending the spatio-temporal limitations of the case. Furthermore, an early choice for a specific theoretical framework carries with it the disadvantage that it introduces all the a priori choices and distinctions characteristic of that discipline, reinforcing socio-cognitive, disciplinary boundaries.

The Arctic Grail is the title of Pierre Berton's (1988) book on the history of 19th-century arctic exploration. It tells the story of the endeavour to ascertain the practicability of a passage from the Atlantic to the Pacific Ocean along the Northern coast of the North American continent, a quest that had obsessed and frustrated 
English mariners and explorers for centuries. In the ice-clogged northern seas, scattered with islands and peninsulas lining a tangled web of channels that were frozen most of the year, still blanc on the map of the Arctic, explorers set out to find a passage that might not even exist. Consequential choices have to be made all the time. A wide channel opening up promising in front may prove to be a blind alley. Constraining land and ice masses may force you to turn back and try again. Distant clouds over a haze of mist may be misinterpreted as an ice capped mountain range luring you to pass by the only open passage that exists this summer. Prior to the era of aviation and satellite photography this exploration at sea level was the only way to learn more about the shape of water and the land, to understand the intricacies and contingencies of the interplay between land, water, ice and the arctic and sub-arctic climate. Having reached the other side you will know that there was a passage, at least this summer, but you do not know whether is was the only, the easiest or the quickest one. You may want to come back to explore other channels.

I was not prepared to make the eliminative and profound choices associated with the 'powers of ten' approach at an early stage of my research. This study, for which the arctic grail provides a proper metaphor, has been an explorative study, an exploration that proceeded on an empirical (medical) and a theoretical level simultaneously. On the empirical level I worked myself through the details of three medical case studies, three ice-clogged channels to be explored. These case studies constitute the empirical core of this book. Taken together they cover experimental laboratory research, and clinical practice as well debates about medicine in the public domain. They cover scientific discovery, therapeutic or technological innovation and moral controversy. Furthermore, they allow a comparative perspective within the scope of this study. On the theoretical level I explored the tangled web of distinct bodies of literature on medicine, science and technology. In this myriad of ontological and epistemological assumptions and methodological procedures and conventions, 1 had to find and determine my own position and find or adjust some theoretical notions that were fruitful in charting my way through the empirical material of the case studies: a theoretical perspective that would suggest a structure and a plot for the corresponding chapters. On each level the choices that I have made have been influenced and informed by my work on the other level. My reading of the empirical material of the case-studies has been informed by the theoretical notions I adopted. Vice versa, my final settlement on the translation theoretical approach that is employed in this study has been influenced by the problems and intricacies of the empirical material.

\subsection{Book design}

Chapter 2 roughly falls into two parts. In the first part some space is devoted to the most basic, epistemological tenet of a constructivist approach, namely that 'reality' is the stabilized result of concrete work in scientific and technological practices. This claim will be rather counterintuitive to health care practitioners and many philosophers of medicine. It amounts to an inversion of the traditional epistemological position in which reality is a pre-existent entity of which science produces theories and repre- 
sentations. Scientific representations of the world can be more or less correct. The problem, however, is how one can know for certain that one scientific representation is better than another. How, in other words, the certainty with which we hold scientific knowledge to be true can be grounded in reality. The first part of the second chapter discusses essentialist, biological and evolutionary concepts of disease to show how philosophers of medicine failed to solve this problem. It will not be argued that constructivism provides the solution to this long standing philosophical problem, but that a constructivist approach is an interesting programmatic answer to avoid or to get out of the dilemma. The second part of chapter 2 continues to explain how a constructivist approach changes our view of discoveries and of medical imaging. Finally, it introduces some of the constructivist, translation-theoretical notions that are employed in subsequent chapters. They will be introduced, not as a full-blown theoretical framework, but as a set of sensitizing notions that are fruitful in the exploration of the empirical material and the analysis of the various case-studies.

Chapters 3, 4 and 5 constitute the empirical core of this book. To allow for a successive development of the argument through these chapters, they each emphasize different aspects of medical practice. One criterium for their selection was that, taken together, they should cover a wide enough variety of practices, varying from experimental laboratory work to clinical diagnostic and therapeutic work, from the hand work of handling animals, equipment and patients to the hand work of writing and arguing in articles and books. Another criterium for the selection of these three case studies was the occurrence of profound, multi-faceted changes of medical practices, indicating the presence of 'trials of strength'. Situations and periods were selected in which simultaneous changes occurred in scientific knowledge, in diagnostic and therapeutic technologies and capabilities, in moral perceptions and evaluations, in disciplinary and institutional organizations.

Chapter 3 deals with the (pre-)discovery work on insulin between 1889 en 1926. According to the canons of medical history insulin was discovered in 1921 by a Canadian research group in Toronto. The importance of this discovery was widely recognized. The 1923 Nobel Prize in medicine and physiology was awarded to two of the members of the team. Banting and Macleod. For the first time insulin provided physicians with an effective therapy for diabetes mellitus. In its severest forms diabetes mellitus had been a unremediable, lethal disease. However, the recognized discovery of insulin is only the turning point at which a long period of doubt and uncertainty about the very existence and identity of insulin ended. This period had lasted for 32 years since Minkowski's first formulation of a theory about the pancreatic origin of diabetes mellitus. Minkowski (pro)posed the existence of an internal secretion produced in the pancreas. 32 years of research yielded conflicting experimental evidence and failed attempts to capture the internal secretion. In the wake of the discovery not only insulin stabilized as an entity in the world and as a reliable and effective technology, the pancreas and the disease, diabetes mellitus, had changed too. In chapter 3, emphasis will be on the science, on the dilemma of insulin's existence, presence and identity, and the resolution of this dilemma in the 'discovery' of insulin and the establishment of a worldwide network of new industrial organizations and institutions set up for the production, distribution and international standardization of insulin. ${ }^{14}$ 
Chapter 4 analyzes the events during the 1952 polio-epidemic in Copenhagen. Emphasis will be on technological and therapeutic innovation in a Danish hospital specialized in the treatment of infectious diseases, where, in the fall of 1952, the medical staff had to cope with a high mortality epidemic of poliomyelitis. Epidemics often relentlessly expose shortcomings in the health care system and existing therapeutic regimes. They tend to unbalance the political, social and moral order, cast doubt about the truth of accepted knowledge and the effectivity and adequateness of technologies. Mortality among patients who were admitted to the hospital with so called bulbar polio was approximately $90 \%$. The breakthrough in this dramatic situation came not from the hospital's medical staff and specialists, but from an outsider, a free lance working anesthetist who was not even affiliated to the hospital. His intervention, the application of manual positive pressure ventilation. reduced the mortality rate of bulbar polio to $15 \%$ within a couple of weeks. This was the beginning of the end of negative pressure ventilators (iron lungs and cuirass respirators). A new dividing boundary was traced through the 'old' clinical syndrome of bulbar polio. The 'old' irremediable disease was divided in a lethal but remediable 'disease' of carbon dioxide accumulation and a viral infection of motor centers in the spinal cord and brain stem, causing transient paralysis of the muscles involved in swallowing and breathing. Carbon dioxide accumulation was a ventilation problem. The viral infection was no longer lethal when adequate ventilation could be maintained by means of positive pressure ventilation. The anesthetist's successful intervention during this polio-epidemic gave impetus to the re-evaluation of anesthesiology's status as a medical discipline. There was no time for a public controversy to develop. Changes were quick and radical.

Chapter 5 deals with the controversy concerning the definition and criteria of human (brain) death. Unlike the first and second case study, the second and third case study 'connect' to each other in a nose to tail fashion. The positive pressure respirator which received an enormous impetus through its successful deployment in the 1952 polio-epidemic in Copenhagen, became the centerpiece of the respiration centers and intensive care units that where subsequently established. It also became the symbol for modern medicine's 'not-allowing-people-to-die'. The 'plug' in 'pulling the plug' is the respirator's plug and stands for the withdrawal of life-supporting treatment in cases where the patient is considered to be already dead or where the remaining human life and lasting suffering is considered to be non-humane. The development, during the $1950 \mathrm{~s}$ and 1960s, of respiration centers and intensive care units, of closed-chest pulmonary resuscitation, and of techniques to reduce and modulate the immune response that made solid organ transplantation (kidney, heart, liver) feasible, cast serious doubt on the 'old' robustness with which a patient could be said to have died. The old criteria of the irreversible cessation of respiration and circulation did not suffice anymore. Brain related definitions of death were defended, and contested, as well by medical professionals and lawyers as by philosophers. The meaning of brain death, a condition that until the late 1960s was conceived of as the death of the organ brain in an otherwise living patient, shifted and was now to be equated with the death of the human being. It is in this case study that moral and legal issues come to the fore most explicitly, linked to arguments about matters of fact and technological feasibility and reliability. A controversy emerged. People were afraid to be declared 
death prematurely. Neurologists were accused of 'inventing' brain death to facilitate the harvesting of donor organs from patients who, judged by the old criteria, were not yet dead. The various positions in this debate about brain death are far more complex than the simple opposition between traditional heart-lung and new brain related criteria of death. Many of the controversial issues have remained unresolved since the end of the 1960s. Many western countries have adopted some kind of brain related definition of death that can be employed legally in situations where life support systems hinder the diagnosis of death by the traditional criteria, and where consent has been obtained to remove organs for transplantation purposes after the patient's death. No closure of the controversy on brain death has occurred, yet legally endorsed practices of declaring brain dead people dead have been established.

Chapter 6 will provide a resume of the theoretical argument developed in the course of the previous chapters. The notion of a 'program of action' will be proposed as the concept that will allow comparisons to be made between the various case studies. On the basis of these comparisons some generalizations and conclusions will be offered.

\section{Notes to chapter 1}

1. See for example Coleman and Holmes (1988), and Cunningham and Williams (1992).

2. The term practice, as I use it in this study, refers simply to the work people do when they pursue their business. This is in line with the way in which Pickering (1992: 2-3) uses the term practice in conjunction with culture. In Pickering's usage, building on the constructivist insight that doing science is real work and that real work requires resources for its accomplishment, culture denotes the field of resources that scientists draw upon in their work, and practice refers to the acts of making (and unmaking) that they perform in that field. Practice thus has a temporal aspect that culture lacks. Culture encompasses all the resources, many of them humble and mundane, that scientists deploy and transform in their practice. It is not, according to Pickering, a way of gesturing at grand, all encompassing world views.

3. Bloor formulated four tenets defining the strong programme in the sociology of knowledge: causality, impartiality, symmetry and reflexivity. Causality is concerned with the conditions about belief or states of knowledge. Bloor allowed for other types of causes apart from social ones which cooperate in bringing about belief. The notion of reflexivity entails that. in principle, the patterns of explanation that would explain the content of other sciences, would also have to be applicable to sociology itself. Causality and reflexivity have engendered considerable debate within the field of constructivist science and technology studies, various scholars taking different stances. See for example Woolgar (1988b), Ashmore (1989), and the 'epistemological chicken' debate in Pickering (1992).

4. Wittgenstein has strongly influenced the work of scholars like Bloor, Barnes and Collins. Bloor (1973; 1976/1991) in particular is credited for making explicit the relevance of Wittgenstein for the sociology of scientific knowledge. Barnes (1974) showed the relevance of Wirtgenstein for theoretical debates in anthropology, establishing a link between social studies of science and anthropological and ethnomethodological traditions in the social sciences. See also Collins (1975) and (1983), and the debate between David Bloor and Michael Lynch in Pickering (1992).

5. Capital letters are deliberately used here to distinguish the Social Construction of Technology as a specific theory with its own theoretical concepts and methodology from other social constructivist 
approaches to technology. The phrase 'social construction of technology' (in lower case letters), and the acronym SCOT have also been used as a generic term for the wider range of social constructivist approaches to technology gathered in Bijker, Pinch and Hughes (1987). The title of this latter book, The Social Construction of Technological Systems easily translated into the same acronym SCOT. The specific and generic usage of the same acronym has engendered many misunderstandings.

6. See also Mayntz and Hughes (1988) and La Porte (1991).

7. Actor-network theory draws on a French, semiotic philosophical tradition, rather than on the AngloSaxon Wittgensteinian tradition in which EPOR and SCOT scholars work. The latter tradition reserves the notion of an actor for intentionally acting human beings. Parallel to the distinction between human beings and things runs the distinction between action, what is what humans do, and behavior, what is what non-humans or things do. See for example Collins (1990), a book which is based on this distinction. The use of the English words actor and action by actor-network theorists has engendered many misunderstandings and debate. An actor in actor network theory is not a traditional sociological actor, but is a semiotic actant. An actant, Akrich and Latour (1992) explain, is 'whatever acts or shifts actions, action itself being defined by a list of performances through trials; from these performances are deduced a set of competences with which the actant is endowed'. The theoretical concepts employed by actor-network theorists are designed to develop a vocabulary that allows one to treat humans and non-humans symmetrically. For this principle of generalized symmetry see Callon (1986a).

8. This is how Latour and Woolgar explained the difference in the subtitles of the first 1979 edition and the second 1986 edition of their Laboratory Life'. The subtitle of the first edition read The Social Construction of Scientific Facts, whereas the subtitle of the second edition reads The Construction of Scientific Facts.

9. The words constructivism or constructionism cover a variety of meanings and different approaches. They share a recognition of the importance to investigate social influences as co-determinants of the phenomenon under study. See for example Coulter (1979), Harre (1986), Kitzinger (1987), Gusfield (1981), Lorenz (1987); Lock and Gordon (1988). For a recent review of the various uses of the construction metaphor in social studies of science and technology, see Sismondo (1993).

10. Constructivism, as the term is used in this study, considers philosophical attempts to 'ground' the truth and objectivity of scientific knowledge in 'Nature', 'Reality', or 'Rationality', to have failed. To this failure to ground scientific knowledge in 'nature' may be added the failure of moral foundationalism, that is, the attempt to 'ground' the certainty with which we hold certain moral judgments to be better than others, in some kind of transcendental locus of moral authority, be it 'Nature', 'God' or 'Reason'. (Wackers 1992)

11. The phrase strategic research sites was coined by Pinch in Bijker et al. (1987), to describe the various places and domains contributors to the volume had chosen as their locus of research, concerned as they were to show how technology itself can be understond as a social product, or at least as possessing a social dimension. The phrase reflects the general, methodological point stressed here, that it is important not to study 'ready-made' or 'black-boxed' technologies, but rather, to study the (social) processes through which certain technologies come to be considered as effective, safe, in short as 'working'.

12. Collins used the same metaphor in the preface of Collins (1985).

13. The full title of the film was: Powers of Ten. A Film Dealing with the Relative Size of Things in the Universe and the Effect of Adding a Zero. The Office of Charles \& Ray Eames pruduced a first version of the film 1968: A Rough Sketch for a Proposed Film Dealing with the Powers of Ten and the Relative Size of Things in the Universe. The 4I pictures of the 1977 film were used to produce a book. (Morrison et al. 1982)

14. I could also have chosen another route through the empirical material, more in line with a controversy study. The controversy was between proponents of the new internal secretion theory of diabetes mellitus on one hand, and proponents of a neurogenic or neurovascular theory of diabetes mellitus - in the tradition of Claude Bernard - on the other hand. The one claimed the existence of an 
entity produced in and secreted into the blood by the pancreas, the other claimed the existence of a sugar center in the brain stem, a 'twin sister' of our now well recognized respiration center. Insulin came into the world; the sugar center did not make it. The neurogenic theory of diabetes mellitus and the sugar center lost out about a decade before the endocrinologists and insulin won the battle. Chapter 3 touches upon this controversy, but does not explore it to its full extent. For more on Pflüger's dispute with Minkowski see Schlich (1993a, 1993b). 


\section{2 \\ The quest for certainty}

\subsection{Introduction}

The purpose of this chapter is twofold. First, it addresses the basic, epistemological tenet of a constructivist approach: that 'reality' is the stabilized result of hands-on work in scientific and technological practices. Sections 2.2 and 2.3 speak primarily to health care practitioners and philosophers of medicine who find this inversion problematic. Rather than addressing this issue in epistemological terms, section 2.2 will discuss how philosophers of medicine have tried to grasp the notion of a disease in essentialist, biological and evolutionary concepts of disease. On the one hand, philosophers have criticized the 'naive' essentialist concept of disease that underlies the familiar image of medical science uncovering more and more aspects of diseases, their causes and the pathophysiological mechanism through which they work. While recognizing the conventional nature of scientific knowledge about diseases, they fail, however, to provide precise accounts of how this socially produced scientific knowledge relates to unchanging, or even evolving diseases in the world.

The second purpose of this chapter, then, is to suggest that a constructivist approach is an interesting way out of this long standing philosophical quandary. Section 2.3 makes this turn to constructivism by juxtaposing 'traditional' and constructivist views on discoveries and on medical imaging. Although a constructivist approach suggests one to give up attempts to justify the certainty with which we hold knowledge to be true, it will provide a better understanding of how that certainty is produced and maintained. Section 2.4 introduces some sensitizing theoretical notions that are fruitful in the exploration of the empirical material and the analysis of subsequent chapters.

\subsection{Changing concepts of disease}

Medical practitioners are familiar with the idea of changing knowledge about diseases from historical introductory sections in medical textbooks. These sections also convey 
a standard image of scientific progress as the proper way to interpret these changes over time. They provide a chronicle of the discoveries leading up to current, state-ofthe-art textbook knowledge about a disease, displaying earlier conceptualizations to be wrong or incomplete. For the purpose of this section I will borrow an example from Ten Have (1984): thalassemia.

In 1925 Cooley and Lee published a paper in an American pediatric journal describing a series of children with a strange combination of symptoms. All children had chronic anemia which had become progressively worse. They had a characteristic face with high, protruding cheek-bones and a bulging forehead. Spleen and liver were enlarged and microscopic examination of red blood cells revealed several abnormalities. Most of these children did not live into adulthood. Cooley and Lee claimed to have isolated a specific disease entity from the until then poorly differentiated group of anemias. Their claim was accepted and the new disease was enlisted in medical textbooks as 'Cooley's anemia'. A few years later it was discovered that the disease mainly occurs in children living in or coming from the area around the Mediterranean Sea; that is why the disease was also referred to as thalassemia (from the Greek thalassa $=$ sea).

Around 1940 several authors described the occurrence of mild anemia with the same hematological characteristics as in Cooley's anemia in adults of Mediterranean stock. Wintrobe discovered that both parents of children with Cooley's anemia also suffered from the same type of mild anemia. Thalassemia could now be conceptualized as a hereditary disease, the hematological abnormalities in children and adults being expression of one disease. The hereditary nature of the disease could also explain the differences in severity: in homozygote patients, possessing two 'thalassemia genes' on corresponding loci on a pair of chromosomes, the disease would develop its full potential, giving rise to severe anemia and premature death (thalassemia major); in heterozygote patients, with the deviant gene on only one of the chromosomes, the other being normal, the disease would give rise to only mild anemia which could go unnoticed (thalassemia minor). Cooley's anemia was considered to be extremely rare; one hundred cases had been reported in the literature in 1942. Thalassemia, however, was found frequently, in up to $20 \%$ of the population in the northern parts of Italy. Because the disease also occurred in adults it was no longer referred to as a specific pediatric condition.

During the 1940s and early 1950s it became possible to determine the amino acid sequence of peptides. Within the hemoglobin molecule, the oxygen carrying pigment of red blood cells, scientists distinguished four different peptide chains. In normal adults the hemoglobin molecule consists of two alpha and two beta chains. In newborn children so called fetal hemoglobin is found which consists mainly of two alpha and two gamma chains, and to a lesser degree of two alpha and two delta chains. As the child grows older the hemoglobin with gamma or delta chains is gradually, although never completely replaced by normal adult hemoglobin. In 1955 is was reported that the alpha-delta variant of hemoglobin was increased in patients with thalassemia. This initial discovery was followed by more biochemical research into the hemoglobin abnormalities in thalassemia patients. It was concluded from this research that thalassemia was basically a disturbance in the synthesis of alpha or beta chains for the peptide part of the molecule. The deficient production of these chains was com- 
pensated by an increased production of gamma and delta chains. Several thalassemias were distinguished, depending on which chain was deficient and which chain was compensating.

Research in molecular genetics during the following years focussed on the unravelling of the genetic defect responsible for the deficient hemoglobin synthesis. It was discovered that in thalassemia the defect was located in the messenger-RNA containing the information for alpha or beta chain, blocking the synthesis of the chain. At the molecular level more than fifty different defects in the messenger-RNA have been discovered, all causing the clinical symptoms of thalassemia in more or less severe forms. To date, from both a genetic and clinical perspective, the thalassemias are a heterogeneous group of disorders varying widely in prevalence and severity.

At first sight there is no problem here. This is a clear-cut case of scientific progress. Through the employment of research methods from epidemiology, microscopy, biochemical and molecular genetics scientists have unravelled the pathophysiological mechanisms of a group of anemias that was first singled out by Cooley and Lee. Cooley and Lee's assumption that the disease occurred only in children was wrong. Milder forms of the same disease occurred in adults. In these milder cases there was no malformation of the facial bones. High, protruding cheek-bones and a bulging forehead only occurred in the most severe cases, secondary to a compensatory expansion of red blood cell producing tissue in these bones in early life. As diagnostic criteria they were of limited value. Because Cooley and Lee did not recognize the milder cases of the disease they also failed to recognize the hereditary nature of the disorder. Advanced biochemical methods for amino acid sequencing were simply not yet available to Cooley and Lee in 1925 .

An orthodox account of these changes in the concept of thalassemia as an obvious case of scientific progress, and of progress in scientific technology, rests on a distinction between the disease and knowledge about that disease. It rests also on the assumption that scientific technologies, like the amino acid sequencer, enable the scientist to gain access to the material reality of the abnormal chemical processes that make up the disease. The disease itself did not change since 1925; only the knowledge about the disease, the content of the disease concept, changed. The 'new' disease enlisted in medical textbooks following Cooley and Lee's 1925 paper was not actually a new disease. Only the knowledge in the textbook was new, and it was adjusted as better knowledge became available through the employment of new technologies. According to Ten Have (1984), the changes in the clinical picture during the course of time were not interpreted as a transformation, but as an explication of our understanding of the disease: gradually more and more of the real nature of the disease was being discovered. If it would be possible to retrieve samples of blood from the children described by Cooley and Lee and to run the tests currently available we would find the same type of abnormalities. Although nobody knows how old thalassemias are, it makes sense to say that thalassemias existed prior to their discovery.

An account of changing concepts of disease in terms of the standard image of scientific progress necessarily rests on a second distinction, namely the distinction between objective, scientific knowledge and non-scientific beliefs. Our modern, scientific knowledge about diseases differs in an essential way from the beliefs about these 
conditions held by pre-scientific cultures. Contrary to the beliefs held by pre-scientific communities, objective knowledge is not informed by cultural pre-conceptions or tainted by individual or group idiosyncracies; if it was it would not be objective knowledge. ${ }^{1}$ Scientific theories about the pathophysiological mechanisms involved are considered to be true representations or reflections of actual processes going on in the bodies of patients. Objective knowledge about a disease defines its essence, that is, the characteristics that are both necessary and sufficient for its occurrence. Defining a disease's essence makes it possible, in principle, to distinguish between the diseased state and the healthy condition and it provides a rational basis for a specific therapy. The medical community is well aware that this goal has not been achieved in all diseases. ${ }^{2}$ But despite these problems scientific medicine strives for the elucidation of the essence of a disease in terms of causal pathophysiological mechanisms.

Philosophers of medicine articulated (and criticized) the general essentialist concept of disease that has dominated medical thinking. Central to the essentialist concept of disease is the assumption that in reality there exist discrete disease entities, which are assumed to be autonomous, specific and durable. Disease entities are autonomous in the sense that they exist independent of the individuals in which they occur. Diseases can be distinguished from the physical and physiological characleristics of the individual patient, which enables one to say that several individuals suffer from the same disease. Disease entities are specific in the sense that they can be distinguished from each other on the basis of a characteristic set of properties. Disease entities are durable in the sense that they existed prior to their discovery and each of them is identifiable throughout history and in various cultures. (Ten Have 1984) Two examples relevant to the case studies in the following chapters can illustrate this essentialist view on diseases.

In the history of diabetes mellitus, for example, it is commonplace to say that diabetes has been recognized from antiquity. The oldest medical text that is held to allude to diabetes mellitus is the papyrus 'Ebers'. ${ }^{3}$ It contains a prescription for overabundant urine. The first clinical description and the first use of the term diabetes is attributed to Aretaeus of Capadokia in the second century A.D. Aretaeus wrote:

Diabetes is a wonderful affection, not very frequent among men, being a melting
down of the flesh and limbs into urine. The patients never stop making water, but
the flow is incessant, as if the opening of aquaducts. Life is short, disgusting and
painful; thirst unquenchable; excessive drinking, which, however, is disproportio-
nate to the large quantity of urine, for more urine is passed; and one cannot stop
them either from drinking nor making water. Or if for a time they abstain from
drinking, their mouth becomes parched and their body dry; the viscera seems as if
scorched up; they are affected with nausea, restlessness and a burning thirst: and at
no distant term they expire. (Papaspyros 1964: 8)

Archaeologists studying Egyptian art consider the engraving reproduced in figure 2.1 to be the first depiction of a man who fell victim to poliomyelitis (infantile paralysis). ${ }^{4}$ He survived, but the neurological damage produced by the virus infection caused the muscles of his right leg to atrophy, resulting in the thin and shortened leg depicted. 


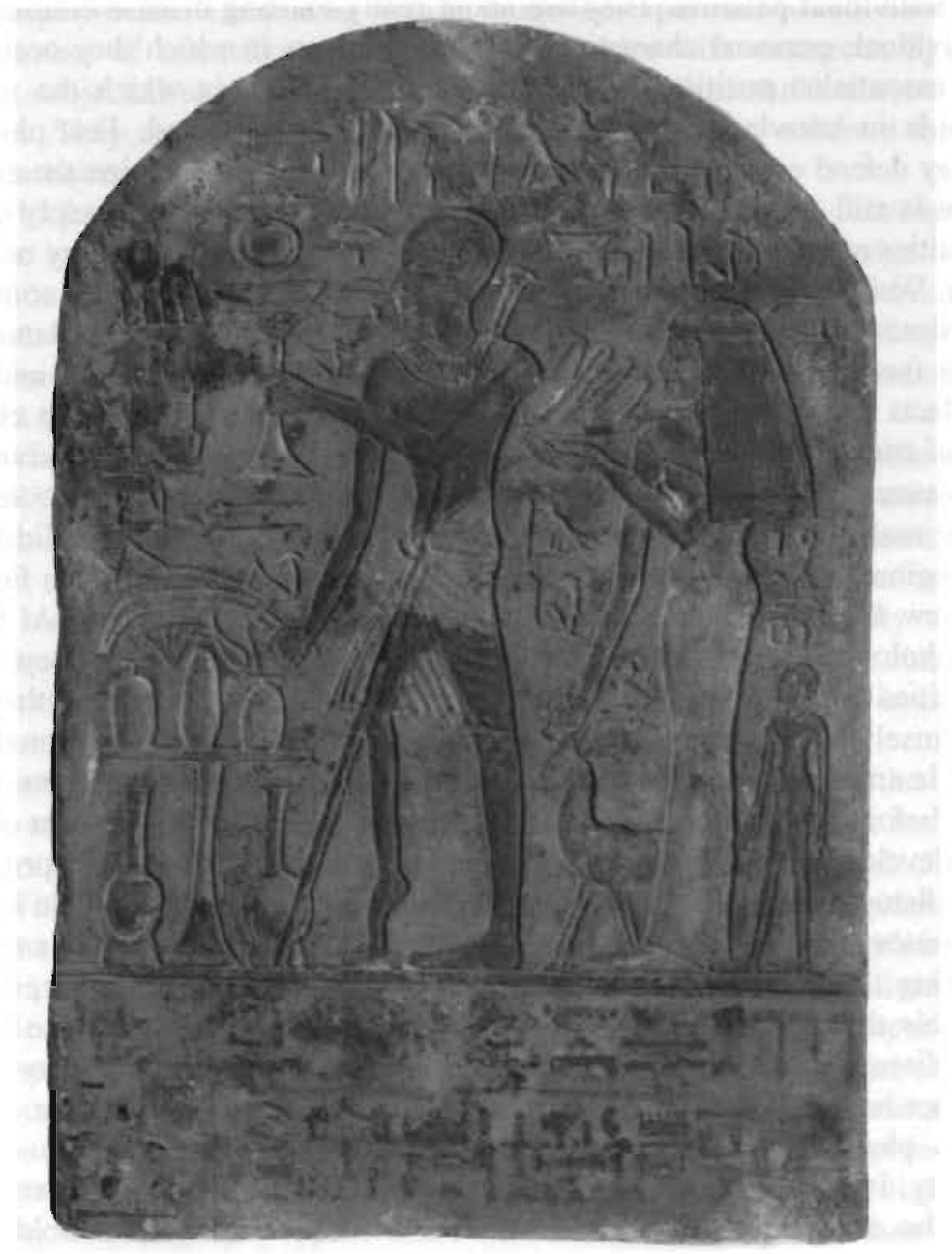

Figure 2.1

Egyptian engraving from approximately 1400 B.C., showing polio-victim and his family offering to the goddess Istar.

Ny Carlsberg Glyptotek, Copenhagen, Denmark

Reproduced with permission

We know that people in antiquity entertained a cosmology that was vastly different from our own. Yet, an essentialist concept of disease allows us to recognize that the patient described by Aretaeus suffered from the same disease diabetes mellitus that we know today, and that the man on the Egyptian engraving suffered from the same virus infection that causes infantile paralysis today, irrespective of the extent or content of the knowledge, beliefs or attributed meanings of Aretaeus, Galen and their contemporaries. Descriptions like Aretaeus', or textbook sections on thalassemias, are 
not about individual patients. They are about really existing disease entities stripped of the individual, personal characteristics of the patients in which they occur.

The essentialist position can be characterized as one in which the role of the world-as-it-is in knowledge production has remained unreflected. Few philosophers would today defend essentialism in its classical sense, but in medicine the essentialist inheritance is still very viable in the strive for exact definitions of sharply delineated disease entities and pathophysiological explanations in terms of necessary or sufficient conditions. Wulff et al. (1986) acknowledge that essentialism underlines correctly that any classification of natural phenomena must reflect the realities of nature. But it ignores the fact that classifications also depend on our choice of criteria and that this choice reflects our practical interests and the extent of our knowledge. In elaborating a biological concept of disease, Wulff and his collaborators provide an account of how science ensures that this transitive knowledge reflects at least some aspects of intransitive reality. During the scientific revolution, which in medicine did not occur until the beginning of the 19 th century, speculative reasoning and realism finally gave way to a new kind of realism which was subject to empirical control. At that time, French pathologists developed an anatomical theory of disease, as they identified disease entities with anatomical lesions, and unlike their predecessors, they did not content themselves with armchair reasoning. They performed meticulous studies at the autopsy table and related their anatomical findings to those clinical observations which they made before the patients died. Later that century, another generation of medical scientists developed a physiological theory as they regarded disease processes as functional disturbances, and they, too, tested their ideas by research in the laboratory. These laboratory-oriented medical scientists were philosophical realists as they aimed at discovering the mechanisms of disease. They tested their hypotheses empirically, molding their theories on reality. The end-result of these efforts was the biological concept of disease which combines all these theories, but excludes all theory formation which cannot be tested empirically. Disease entities are now defined by a mixture of anatomical, physiological, microbiological and other criteria, and this conceptual heterogeneity is the strength rather than the weakness of contemporary medical thinking. The mechanical model constitutes a human construction, molded on the world-as-it-really is. The fact that many of its components can be tested within different conceptual frameworks, under a variety of experimental conditions, helps to ensure that it reflects at least some aspects of reality. ${ }^{5}$

The metaphor of molding knowledge on reality is problematic, though. Pushing the metaphor a little bit further, the process of molding theories on reality might be thought of in two different ways: reality (the mould) as a frame or matrix on which some pliable material (disease classification) is molded into shape, or reality can be thought of as a blacksmith's anvil. In the former the molded material would reflect the shape of the mould; or, in other words, even in the absence of the mould itself the shape of the mould can be inferred from the shape of the finished product. In the latter, the resistance and curves of the anvil are used to mould the black iron into shape by hammering, but in its absence the shape of the anvil can in no way be inferred from the shape of the finished product. Wulff et al. seem to be inclined towards the former, the matrix metaphor. Writing about the modern scientific asthma theory the authors argue that this is the only theory which fits a multitude of carefuily 
executed experiments. Those who wish to probe the experimental will have to look up a large number of scientific articles presenting tables with numerous figures which have been subjected to statistical analysis. These statistical truths are not an end in themselves. They are only a means to the establishment of a coherent theory which the authors believe reflects the reality behind the observations.

The biological concept of disease presumes an intransitive, unchanging natural order. The real-world diseases on which science molds its knowledge and disease classification, are the pre-given, specific, discrete and unchanging entities of the essentialist. They are, as Jensen $(1984 ; 1987)$ puts it, the species of the species of the pre-Darwinian biologist. Evolutionary biology implies a radical rejection of traditional essentialist views of nature and the natural order.

Drawing on evolutionary thinking in biology and in other scientific disciplines, Jensen (1987) develops an alternative concept of disease, replacing static essentialist notions by a dynamic evolutionary approach. In Jensen's view diseases are to be understood as courses and processes, not distinct entities to be defined once and for all. Like biological species, diseases are characterized by variability and change. Diseases are evolving entities, changing under the influence of genetic change, as well changing external circumstances like socio-economic development. Diseases are units of evolution in a changing nature.

The recognition of change and variability as basic features of the world does, according to Jensen (1987), not imply that patterns and laws cannot be found. It is possible to acquire usable knowledge about the world. Variation and change is not chaotic. Within limited geographical areas and periods of time biological species and diseases manifest a number of common features, which make classification, that is taxonomy, possible. Taxonomy is a human and social practice. Classification involves the selection of proto-typical cases that serve as a standard for comparison. Prototypes in medicine consist of a number of descriptions and rules: a list of symptoms, results of complex examination procedures and laboratory tests. These proto-typical standards are of a conventional nature; they are not defined once and for all. Prototypical standards may change under the influence of changes in the disease itself; a disease may normally now appear associated with other symptoms than it did at the beginning of the century. On the other hand, developments within laboratory medicine have furnished the clinic with a number of procedures by which new clinical syndromes are established.

The units in the medical classification of diseases that is thus established, are temporary, standard- and practice-dependent definitions which serve as diagnostic labels in clinical practice. The unit of classification in use in a particular time and place are, in Jensen's words, 'time-slices' of an evolving and changing disease entity, which is the unit of evolution. ${ }^{6}$ However, Jensen is not able to suggest a way in which scientists or physicians could determine whether the diagnostic label used in clinical practice at a particular time fits the corresponding phase of the evolving disease in nature. For practical purposes the socially created order of the disease classification, Jensen agrees, is, despite its historical, cultural and practical relativeness, the natural order. In admitting that, eventually, in practice, the disease-in-nature can not be distinguished from the socially created, practice dependent definition of disease, Jensen follows Ten Have (1984), who conflates the disease and the conceptualization of the 
disease into a single entity. Ten Have argues that disease itself can be considered to be a human construction. Not the issue of the relation between the disease and the disease concept is the most interesting, but the question concerning the functionality and possibility of different kinds of disease constructions. In that case the issue is not whether our understanding of disease today is better than in former days, but whether our disease constructions are satisfactory or adequate. This pertains to the goals with which such constructions have been made and are being handled.

\subsection{The turn to constructivism: inverting reality}

Although reluctant to take the final step, Jensen and Ten Have come close to a constructivist position. Whereas Wulff $e t$ al. argue that with increasing certainty it is warranted to assume that medical knowledge reflects at least some aspects of a preexistent world, Jensen and Ten Have agree that in practice no such distinction can be made. The socially created definition of a disease $i s$ the disease, as far as medical practice is concerned. The final step into a constructivist position is to accept that the entities socially created in various scientific and medical practices make up reality; to accept that reality, in the sense of what we consider to be real, is the outcome of a human, social and practical process of inquiry through which the certainty attached to particular (fallible) beliefs or hypotheses increases beyond doubt. The question then is not whether or to what extent true knowledge reflects the world, but the question is: from where do we derive the certainty with which we hold knowledge to be true, if it is not from the world-as-it-really-is? The task then is to explain how scientific medical knowledge has been made true, real, objective, and not to justify it. In the constructivist account attempted in this study no assumptions will be made about the structure or identity of the world. The theoretical concepts employed allow us to account for changes (and stability) in medicine's cognitive content, in its technologies and in its moral and social order without making any reference to reality-as-it-is. This ontological agnosticism is not a position that denies the existence of a pre-existent world. It only denies that there is any privileged way of acquiring knowledge about that world that would allow us to judge, from a God's eye perspective, whether the knowledge produced in scientific and medical practices is true or not.

While adopting an agnostic position as an analyst I will also assume that, at the outset, the actors in my case studies are in the same predicament and that their ability, at the end of the day, to speak confidently about the world is an accomplishment that has to be explained (and not taken for granted). The shift from justifications of the certainty with which scientific knowledge is held to be true, entails an interest in the conditions of the production of this certainty. This study will stress the situatedness of hands-on practical work, underlining the contingent character of medical practices: that is, the idea that a particular practice is neither predetermined nor logically necessary but the cumulative result of numerous small changes, modifications, negotiations and decisions. Wulff and his collaborators were right in emphasizing the meticulous studies of 19th century French pathologists at the autopsy table. and of 19th century experimental physiologists in their laboratories, but not because they were testing theories. It was through this hands-on practical work in laboratories, clinical wards 
and autopsy rooms that these researchers crafted a new cognitive content and new technologies for medicine.

The adoption of a constructivist approach is consequential for two 'phenomena' on which modern medical practice is highly dependent: discoveries and medical imaging technologies. These 'phenomena' play an important role in the medical narrative in the following three chapters. A discussion of the traditional and constructivist views on discoveries and imaging technologies may contribute to a proper understanding of the analysis attempted in the case-studies, and help to clarify the rather abstract and counterintuitive idea of reality being the result of scientific practices.

New discoveries are generally reckoned to be about objects, events and processes of which we have no previous knowledge. The metaphor of scientific discovery, the idea of dis-covering, is precisely that of uncovering and revealing something which had been there all along. One removes the covers and thereby exposes the thing for what it is; one pulls back the curtain on the facts. The central assumption in discovery is that the discovered object enjoyed an independent existence and identity before the discoverers discovered it. (Woolgar 1988a) In other words, the existence of a newly discovered entity or process is projected back into time, it has always been there. Science only re-presents pre-existing objects and processes in recordings, images and scientific theories. The period prior to the discovery is usually construed as one in which neither the proper knowledge, nor the procedures, technologies or skills were available to make the discovery.

Many historical accounts of scientific discoveries share this traditional vicw on discovery. According to Stanford (1986), historians are concerned with change. Change implies movement through time, a 'before' and an 'after'. Historians are concerned with the past as different from the present and with tracing change as movement over a period of time. The traditional form in which a history is written is that of a narrative, a diachronical narrative that tells the story of change through the verticalities of time from the past to the present. This type of diachronical, with-thebenefit-of-hindsight history writing, is frequently encountered both in introductory sections in medical textbooks listing some of the landmarks in the history of the discipline or subject, and in the work of professional historians of medicine.

Every historical account of a discovery, and of the scientific developments leading up to it, is an after-the-fact reconstruction. The discovery has already been made. Priority disputes rage or have been settled. Credit has been attributed. The discovered entity or process is part of the world the historian lives in. Historians who take the newly established knowledge to be a proper representation of the world, will be inclined to use it both as a search-light and as a standard: a search-light to locate those who anticipated and contributed to current knowledge, and a standard to judge those were right and those who adhered to wrong theories.

'Progress-to-truth' histories have been severely criticized and disqualified as being whiggish ${ }^{7}$, an invective term, carrying a connotation of contemptuous rejection. The label whiggish refers to the idea of history as a tale of progress, permitting to judge past figures by their role in fostering enlightenment as we now understand it. The sin in historical composition is, according to Butterfield, 
to abstract events from their context and set them up in implied comparison with the present day, and then to pretend that by this 'the facts' are being allowed to 'speak for themselves'. It is to imagine that history as such, or historical research however intense, or historical surveys however broad, can give us judgments of value - to assume that this ideal or that person can be proved to have been wrong by the mere lapse of time. (Butterfield 1931: 105-106)

The fallacy of whig history divides behavior and beliefs into two types: right or wrong, true or false, rational or irrational. It invites a-symmetrical types of explanation. The explanation of why a scientist draws the right conclusions from a set of premises appears to reside in the principles of logical inference themselves. An internal, whiggish historical account seems to be self-sufficient. The deviation may be due to a whole variety of external psychological or sociological factors.

A constructivist view on discoveries starts from the question: In what sense can we say that an object exists even though nobody is there to observe it? Our absence from the scene does not prevent us from conjecturing the existence of an object. Our ability to speak as if realities exist independent of our knowing them is a key function of language and representation. But can an object exist independent of our practices of representation? (Woolgar 1988a) Is there a sense in which we can talk about objects prior to their discovery, about processes that have not yet been discovered? Any attempt to talk about the world as it is, including non-discovered objects, would involve the use of words, pictures, models or mathematical formulas that have emerged in (other) attempts to grasp or represent the world; they are not the world as it is. According to Latour and Woolgar (1986) there are severe difficulties in adequately describing the nature of the 'out there-ness' in which objects are said to reside because descriptions of scientific reality frequently comprise a reformulation or restatement of the statement which purports to 'be about' this reality.

Thus, if there is no sense in which an object can be said to have an independent existence from the scientific practices in which it was 'discovered' or 're-presented', one might as well say that the 'discovery' marks the beginning of the object's existence, that its identity is shaped through the scientific instruments and procedures used in its representation. In chapter 6, drawing on examples from the three casestudies I will explain in what sense this can be thought of as a cogent claim. In Latour and Woolgar's (1986) account of the isolation and identification of a new hypothalamic (neuro)hormone. TRF, they point out that 'in the beginning' there is only a controversial statement: a hypothesis about the possible existence of hormone, the biochemical compositions and physical properties of which are not yet known. Members of the laboratory were unable to determine whether statements were true or false, objective or subjective, highly likely or quite probable. While the agonistic process of the controversy was raging, modalities ${ }^{8}$ were constantly added, dropped, inverted, or modified. Once the statement began to stabilize an important change took place. The statement became a split entity. On the one hand, it was a set of words which represented a statement about an object. On the other hand, it corresponded to an object in itself which took on a life of its own. It was as if the original statement had projected a virtual image of itself which existed outside the statement. Previously scientists were dealing with statements. At the point of stabilization, however, there 
appeared to be both objects and statements about these objects. (Latour and Woolgar 1986)

Latour and Woolgar argue that the strength of the correspondence between objects and statements about these objects stems from the splitting and inversion of a statement within the laboratory context. Once this process of stabilization, and of splitting and inversion, is completed, even the most cynical observers and committed relativists will have difficulty in resisting the impression that the real, and pre-existent object has been 'dis-covered'.

The case of 'discovery' is about new knowledge, about hitherto unknown objects and processes. But what about the routinized use of measuring and imaging instruments in science in general, and in medicine in particular? The traditional view on laboratory tests and imaging technologies comes in two versions. Both start from the idea that the outside world is available to us through our senses: sight, hearing, touch, smell and taste are built in instruments through which we can perceive the world in which we live. ${ }^{9}$ However, the natural perceptive range of our unaided senses is limited. Our eyesight is limited to the narrow band of what we call visible light. The range of operation of the auditory system is limited too. The section of the world that is directly accessible to our senses is very limited. The world beyond these limits is inaccessible. Scientists and physicians have learned to overcome the natural limits of their perceptive capacities by putting intermediating instruments between themselves and the world. One version of the traditional view on imaging technologies, argues that, as instruments of perception, these technologies extend or substitute for the

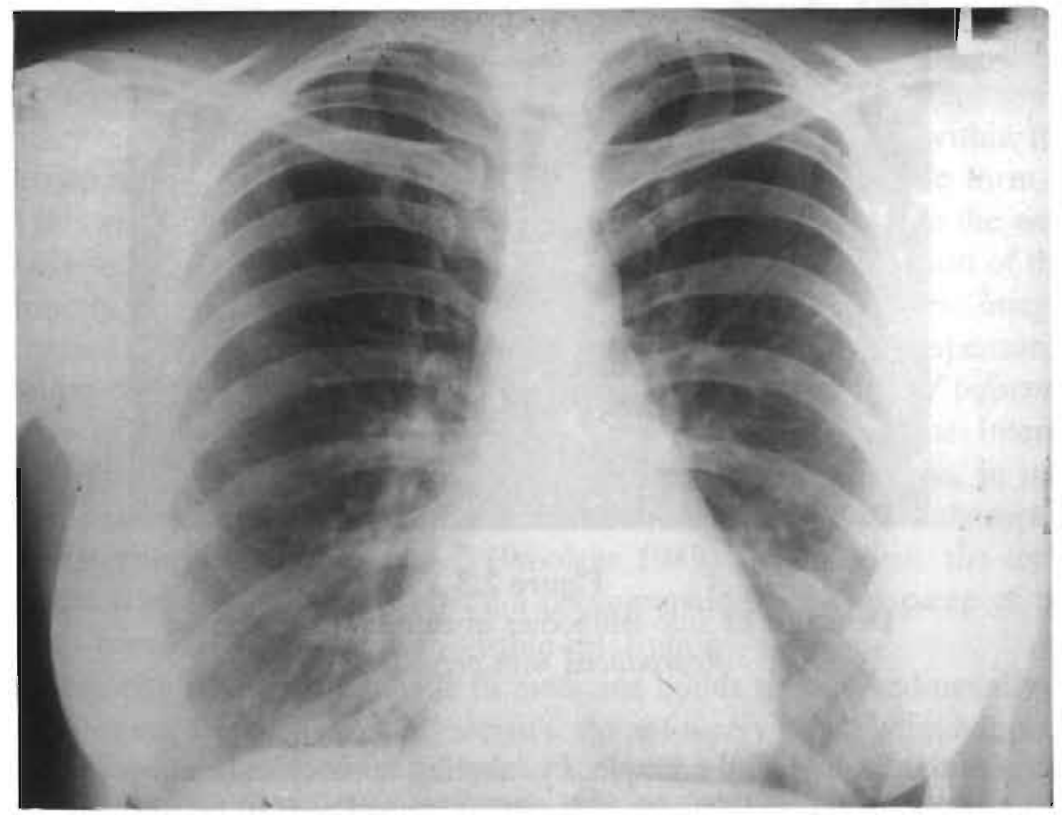

Figure 2.2

$\mathrm{X}$-ray picture of a thorax

Reproduced with permission 
limited natural perceptive capacities of the sense organs of our body, of our sight. hearing and touch. Astronomers use optical and radio-telescopes to look at and listen to the universe. X-ray pictures give access to anatomical structures that otherwise would have remained hidden in the darkness of the living body (see figure 2.2).

Biologists and pathologists use optical and electron microscopes, in combination with appropriate staining techniques, to investigate the cellular and sub-cellular

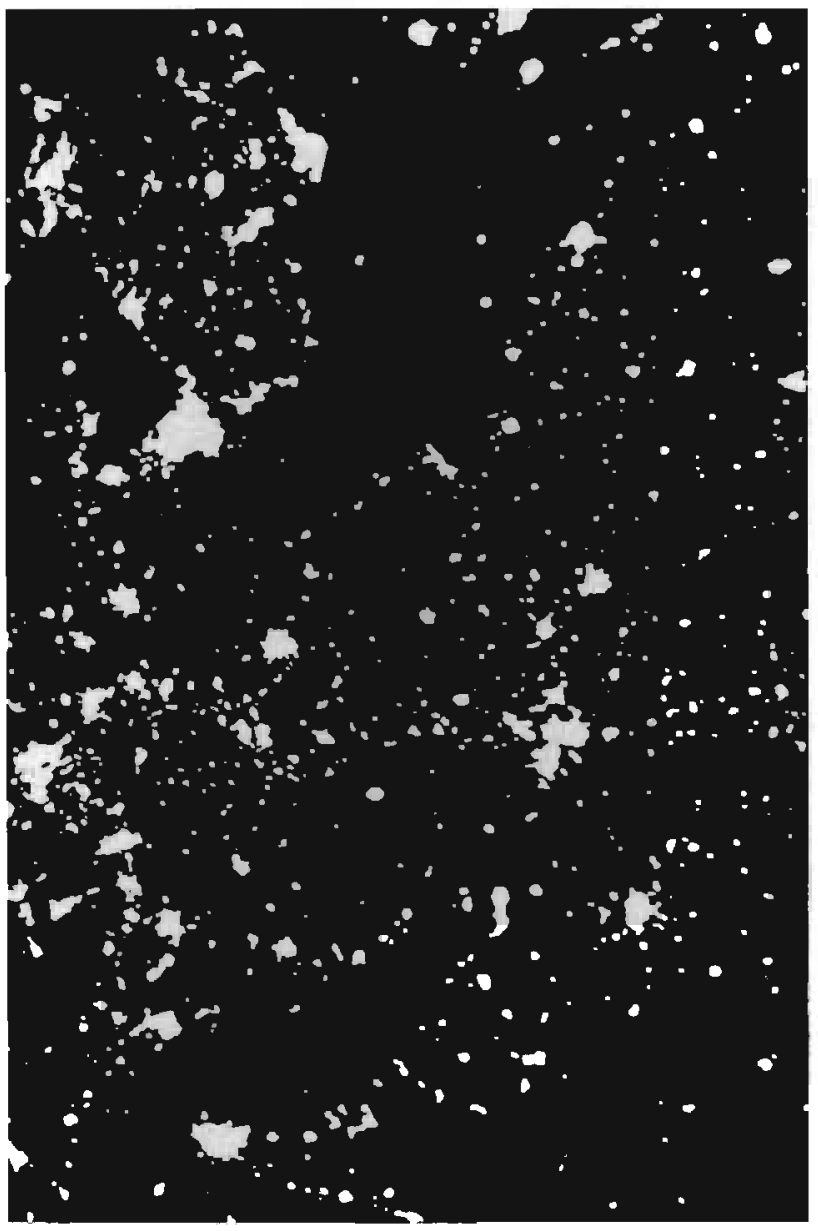

Figure 2.3

Detection of auto-antibodies in auto-immune disease Reproduced with permission

dimensions of the tissues. For example, by labeling monoclonal antibodies against the tale section of other antibodies with a fluorescent marker it is possible to visualize a patient's antibodies against her own tissue antigens in auto-immune disease (see figure 2.3). 


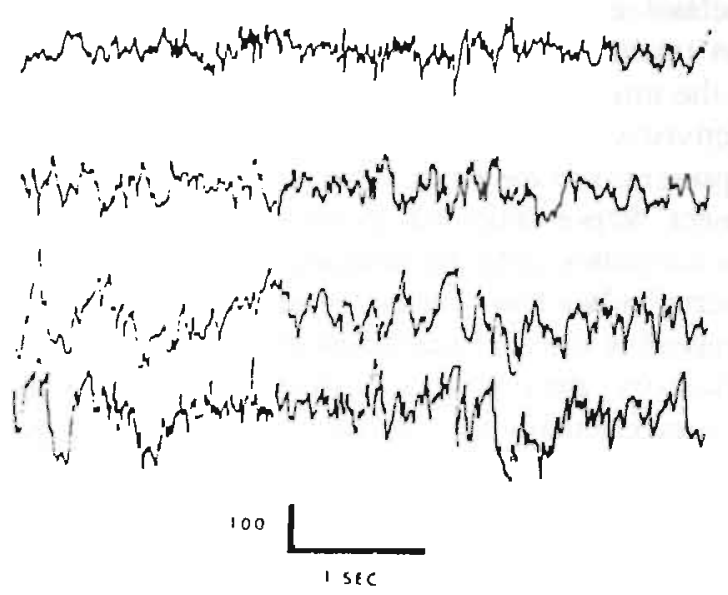

Figure 2.4

ECG-recording showing

the chaos of ventricular fibrillation

Reproduced with permission

ECG and EEG apparatus record the weak electrical currents of the heart and brain on an electrocardiogram and an electroencephalogram respectively; currents that are unaccessible to our unaided senses. During ventricular fibrillation the muscular fibers of the heart contract in a uncoordinated, chaotic way, destroying the familiar pattern of a normal beating heart (see figure 2.4).

In a second version of the traditional view on imaging technologies, these intermediary technologies translate the world in inscriptions that fall within the limits of the perceptive capacities of our unaided senses, usually in a visible form. (Reiser 1978) In this view our bodily perceptive apparatus is not extended into the world, but the world is in a specific sense mobilized and brought within the section of the world that is directly accessible to us. The inscriptions (recordings, pictures, images) produced, contain and convey essential information about the world. Indispensable in the interpretation of these images are the assumptions of conservation of information in the process of producing the images and of the passiveness of the intermediary technology. The role of the technology is assumed to be a passive one, in the sense, that it only enables or facilitates representation without affecting the character of the world or distorting the information. ${ }^{10}$ (Woolgar 1989) In this view, the technology facilitates the world to inscribe itself on a photographic plate or a piece of paper, to transmit and translate information in visible inscriptions. "

The majority of diagnoses made in medicine builds on one and usually more of these technologies. In most cases physician's do not worry about what happens in the translation process and they are confident to accept the images and numbers produced by these technologies as meaningful, information-containing representations of the patient's body. The images provide a window on the world. In the routine application of these technologies the role of the human agent. the interpreter of the images, seems to be a passive one. The human interpreter is only reading the information that is 
revealed by the technology, without affecting it. He is an onlooker watching an event take place without taking part in it. He is only looking into deeper structures and processes beyond the limits of his own natural perceptive capacities.

The constructivist view on laboratory tests and imaging technologies holds that objects are not represented in the images and registrations, but that the representation constitutes the object. Representational technologies do not extend or substitute for limited perceptive capacities allowing sensory access to a larger part of the world. Neither are, according to Woolgar (1988a), representational practices merely adjuncts, passive tools for revealing the character of these things for what they actually are. As in the case of 'discovery' there is no way in which we can know what the objects, structures and processes actually are. We have no independent access to the world as it is or to judge whether information has been conserved, whether it has been translated in visible inscriptions without distortion. The impression that the meaning of inscriptions derives from an intrinsic relationship between the inscription and the object or process it represents, is the result of a stabilization process in line with the splitting and inversion in the case of new knowledge about new objects. In this process the apparatus and the meaning of the inscriptions it produces stabilize simultaneously, the one requiring the other. ${ }^{12}$

The following three chapters contain several examples where unproblematic interpretations of laboratory tests and recordings were challenged; where, in other words. the intrinsic relationship between object, the inscription and the interpretation dissolved and where the inscriptions were exposed as being mute.

\subsection{Mute inscriptions and their translators}

In the account of modern medicine developed in this study, 'things' like laboratory test recordings generated by various kinds of instruments and procedures, physical signs and symptoms, figures, tables and graphical displays, will be of central importance. These 'things' may be captured under the general notion of inscriptions. The notion of inscriptions carries the (restrictive) connotation of artificially produced numbers, lines and images inscribed on sheets of paper or photographic emulsions in a laboratory setting. These inscriptions are considered to be durable records in which aspects of the world have been captured. In this study, inscriptions is used in a broader sense to encompass both transient phenomena or symptoms that are considered to be expressions of an underlying disease, as well as inscriptions on durable media. Hence, the notion of inscriptions encompasses also non-artificially produced 'inscriptions", ranging from petrified footprints and other fossils of pre-historic animals to pathologic changes that can be observed in skeletons found in an archeological quarry and anatomic changes observed in a patient's dead body during autopsy. Inscriptions are the raw material scientists and physicians work with. It is inscriptions they have to make sense of and which they use in communication with others.

Latour (1990) suggests that we should understand inscriptions in terms of persuasion and mobilization. Inscriptions have properties of being mobile but also immutable, presentable, readable and combinable with one another. The mobility of 
inscriptions allows one to mobilize worlds distant in space and bring them home. Islands, geological strata, stars and planets, populations, cannot be moved, but they can be translated into maps, diagrams, pictures, etcetera. A map of the island, or a diagram of a geological formation along with its coordinates can be brought home to the cartographer's or geologist's office. A whole population cannot be mobilized and gathered in one place to assess its health. Morbidity and mortality records can.

Sometimes it is possible to mobilize parts of the world and gather them in another place. Specimens of plants and animals have been collected and gathered in zoos and museums of natural history. Specimens of fossils have been collected in quarries and brought home to the paleontologist's laboratory ${ }^{13}$ In medicine blood and tissue samples are frequently extracted from the patients' bodies and gathered for examination in routine or research laboratories. In every case it is paramount that the collected inscriptions or specimens do not change over time, or as Latour puts it, that they are immutable.

When gathered in one place, immutable mobiles, obtained in different places, at different times, can be combined for comparison, classification, statistical analysis, superimposement, subtraction and graphic presentation. Combination of anatomical changes during autopsy with signs and symptoms documented during the patients' life allowed French pathologists to define diseases as having a locus in the body, characterized by specific patho-anatomical changes. An X-ray picture showing a tumor prior to treatment can be combined with an X-ray picture obtained after a few weeks of treatment to evaluate the effect of the therapy. When the pictures are taken under the same standardized conditions measurement of the diameter of the tumor allows physicians to determine whether the tumor continues to grow, whether the growth has been stopped, or whether tumor size decreased. Comparison of a stained specimen prepared from a tumor, with a similar specimen (retrieved from the storage room) prepared from a tumor removed from the same patient several months ago, allows pathologists to determine whether the current tumor is a new, primary one, or whether it is a metastasis from the old tumor. Similarly, combination of morbidity rates with information about social stratification allows scientists to attribute differences in health to inequalities in socio-economic conditions. Combination of morbidity and mortality rates with geographical data allows scientists to identify healthy and less healthy cities and to make claims about the adequacy of public management by local authorities. (Houwaart 1991)

One of the most important advantages of inscriptions is, according to Latour (1987; 1990), that they can be made part of a written text and reproduced and disseminated at little cost. ${ }^{14}$ Pictures, tables and graphs in a text are not simple 'illustrations' of that text. They are devices employed by the author to make the text strong enough to resist dissent. The author does this by presenting the very thing he wants the readers to believe in the text. These 'illustrations' are produced from the raw material of inscriptions through a complex process of reshuffling. recombination, calculation, cleaning, working up and graphic display. The text carries in it all there is to see, or maybe better, all the authors wants the reader to see. The figure shows what the text says. The world the author writes or speaks about, in the verbal parts of the text, is there, present in the text, where the reader can see for himself. To make sure that the reader sees what the author wants him to see a legend is added explaining 
what there is to be seen or how to read the figure. Belief in the author's word is replaced by the inspection of 'figures'. If there is any doubt about where the picture comes from, the legend will offer a new line of support.

In this view the world is mobilized by a speaker or author, re-presented, and put to work in a text, spoken or written, designed to persuade an audience that the world is as the author claims it to be, that an experimental intervention has the consequences that the author claims it to have, or that a therapy or technology has the effect the author claims it to have. There is a sense in which the text can be seen to be designed to mobilize the reader, that is to say, to make the reader move from some other position to the position advocated by the author, and to act accordingly. Using the word 'mobilization' in the sense of 'marshalling for action', one could say that in a scientific text the world is mobilized to mobilize others.

When we take inscriptions to be the 'stuff' scientists work with, the general notion of translation captures the variety of actions and processes that produce inscriptions, as well as the variety of actions performed by scientists on or with respect to them. Translation covers two important aspects of these actions. It refers both to transformation (change of identity) and to displacement (movement, change of place).

Inscriptions in the form of immutable mobiles do not 'preserve' the world as it really is or was. The process of preservation of the world in immutable mobiles always involves a more or less profound transformation. This is obvious in the case of maps and morbidity statistics. In these cases only some features are preserved, such as the shape of a land mass, the relative proportions of its parts, or the diagnoses established by physicians. Fossilized organisms have been transformed under the influence of specific geophysical conditions and processes of petrifaction. Sometimes only the shape of (part of) an organism has been preserved in the cast of a shell that subsequently dissolved, or in a petrified footprint. The transformation was less profound in the (exceptional) cases of animal and human bodies mummified under exceptional conditions in oxygen-free or permanently frozen marshes in Denmark or Siberia, in Italian glaciers, or in windy, cold but dry Inuit graves on the western coast of Greenland. When taken from their preserving and protective environments these bodies tend to decompose, and wither away, unless they are immediately preserved by artificial means. ${ }^{15}$ In medicine tissue samples are subjected to a complicated series of manipulations prior to their examination under the microscope. Pathologic specimens get soaked in formaldehyde, cast in paraffin, frozen, cut, dehydrated and stained. After completion of this process the specimen can be recorded and kept in the laboratory's storage room for many years and retrieved when necessary. ${ }^{16}$ In electrocardiographic recordings the weak currents picked up by the electrodes on the patient's chest are amplified and filtered before they are translated (transformed) from electrical currents into movements of the pen of a pen-chart recorder.

Concurrent with the transformation there is always a displacement, a movement through space and time. Maps are moved from the place where they have been drawn to the cartographer's office. Fossils are moved from the quarry to the paleontologist's office. Tissue samples are moved from the patient's body to the pathologist's laboratory and onto a glass slide. Electrical currents from the patient's chest are displaced to the ECG-paper, which in turn is moved to the physician's office for 
interpretation, and comparison and combination with other inscriptions sent to his office from other laboratories.

Similarly, all manipulations of individual inscriptions towards more aggregate forms, for example for incorporation in a text, involves transformation and displacement, and can thus also be captured under the notion of translation. Morbidity and mortality rates do not recount every individual patient on which a specific diagnosis or cause of death has been established; they present an aggregated figure representing the number of cases per hundred or thousand (inhabitants, or persons belonging to a specific social stratum, or age group, etc.). Individual inscriptions (on morbidity and mortality registration forms) have been translated (transformed) into a limited number of aggregated figures and displaced to the paper on which these figures are printed, multiplied and distributed.

The most important activity captured under the notion of translation is one associated with the common sense meaning of the word translating: translating for scientists and physicians, is to say or write in their own words what they think the inscriptions are saying. Or, as Callon (1986a) puts it, to translate is to express in one's own language what others say and want, why they act in the way they do and how they associate with each other: it is to establish oneself as a spokesman.

Scientists and physicians act as spokespersons for inscriptions. They translate entities that do not have an articulate language, and as such they act as their representative. Simultaneously scientists speak for the entities that are assumed to be represented by the inscriptions, for the world behind the inscriptions. Scientists and physicians speak for the mute inscriptions they work with, similar to an interpreter who speaks (translates) for her client, an attorney who speaks for his client in court, or a union-negotiator who speaks for the union-members in negotiations with the employer. The central issue, of course, is how scientists and physicians arrive at 'their translation'. This is not only a central question from a science studies perspective. It is also a central problem for scientists and physicians themselves. The following chapters will provide several examples illustrating this point. For now, it is important to get familiar with the notion of a spokesperson in science and medicine.

Whether we are watching ECG-recordings or X-ray pictures that are being explained to us, or whether we are reading a scientific or medical text, a spokesperson is never far away. We are always attending an 'audiovisual' spectacle. There is a visual set of inscriptions produced by the instrument and a verbal commentary uttered by the scientist. Latour describes the actor giving the verbal comment as a spokesman (or spokeswoman, or spokesperson, or mouthpiece). The author behaves, Latour (1987) argues, as if he or she were the mouthpiece of what is inscribed on the window of the instrument.

In scientific and medical texts spokespersons can usually be found in the captions accompanying tables, figures, pictures, etc. Several books and articles about infantile paralysis (polio) use the engraving represented in figure 2.1 as an illustration. The caption usually reads that this Egyptian engraving from the 14th - 13th century B.C. is the first representation of a polio-victim. He survived the disease, but the damage to the nerves to his right leg caused the muscles to atrophy. In the caption it is the archeologist, who tries to make sense of the engraving, who speaks for the inscription in the stone. We will never know whether the man on the engraving really suffered 
from polio. It is possible to think of several other causes for an atrophied leg. However, the interpretation of this engraving as representing a polio-victim goes unchallenged.

Figures 2.2, 2.3 and 2.4 in section 2.3 of this chapter also carry captions in which a spokesperson explains what there is to be seen. The caption of figure 2.2 says that the picture is a X-ray picture of a thorax. The caption of figure 2.3 says that the white spots in the picture represent antibodies against a patient's own cells and tissues. The caption of figure 2.4 says that the represented registration is an electrocardiogram, a registration of electrical currents in the fibrillating heart. The chaotic shape of the trace is due to the rapid and repetitive, but uncoordinated excitation of myocardial fibers without the coordinated contraction of the ventricle. While reading this section you probably accepted the explanation offered in these captions without giving them a second thought.

Please, consider figure 2.5 and its caption and compare it to figure 2.4. You will

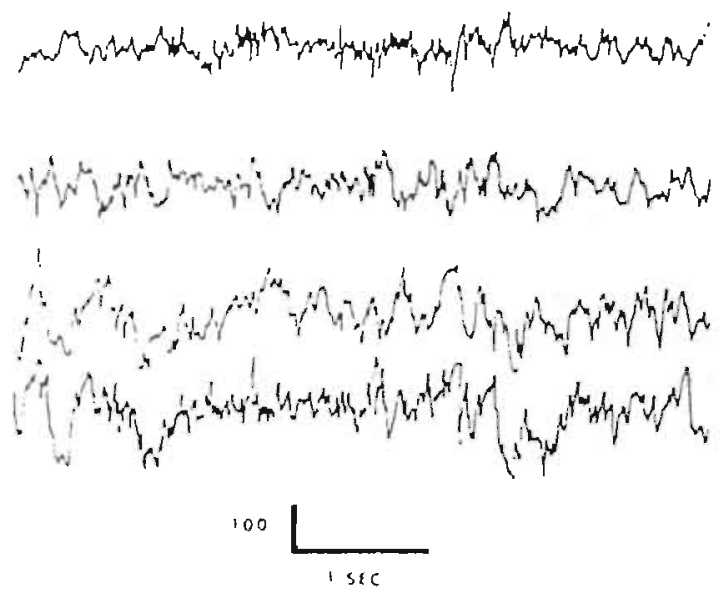

Figure 2.5

Foetal heart rate during labor

notice that it is the same inscription, but the caption is different. The caption in figure 2.5 says that the trace represents the characteristic rapid and irregular changes in the frequency of a baby's heartbeat during labor.

Similarly, the picture in figure 2.3 is the same as in figure 2.6. The spokesperson is different though. The spokesperson is now present in the picture, saying that one of the white dots is Stockholm. Taking a closer look at the distribution of the white spots in the picture we now recognize the shape of the Gulf of Bothnia, with Finland to the right and southern Sweden to the left. We also recognize the shape Denmark with Copenhagen on the island 'touching' Sweden. The caption to the picture in the book where figure 2.5 is taken from, said that the picture was taken from some point in space, a satellite in orbit around the earth, showing Europe's city lighting by night. (Schwarz and Jansma 1988) ${ }^{17}$ 


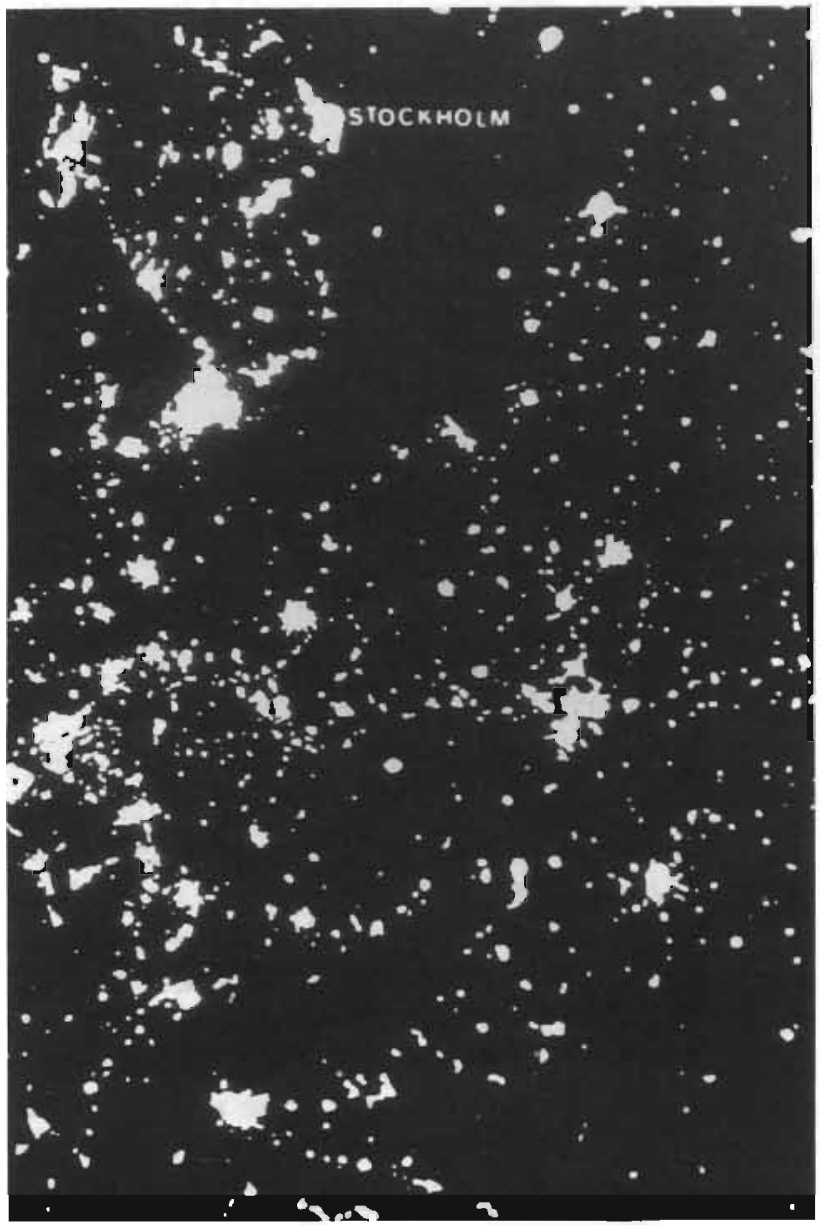

Figure 2.6

The argument here is not only that scientists and physicians speak for the inscriptions they work on, but also that inscriptions do not speak for themselves, that they are mute. Consider figure 2.7 and try to make sense of this picture without a person to speak for it, neither in a caption nor in the text.

The muteness of inscriptions becomes apparent when we are confronted with pictures from a discipline that we are not familiar with. A proper training in that discipline would be a training to act as a spokesperson for the inscriptions the discipline produces.

The muteness of inscriptions also becomes apparent when alternative, competing interpretations (translations) are offered for the same inscription or set of inscriptions. In offering alternative interpretations scientists also challenge their competitors legitimacy as a spokesperson. This is what, according to Callon (1986a), makes a controversy. Controversy is all the manifestations by which the representativity of the spokesman is questioned, discussed, negotiated, rejected, etc. 


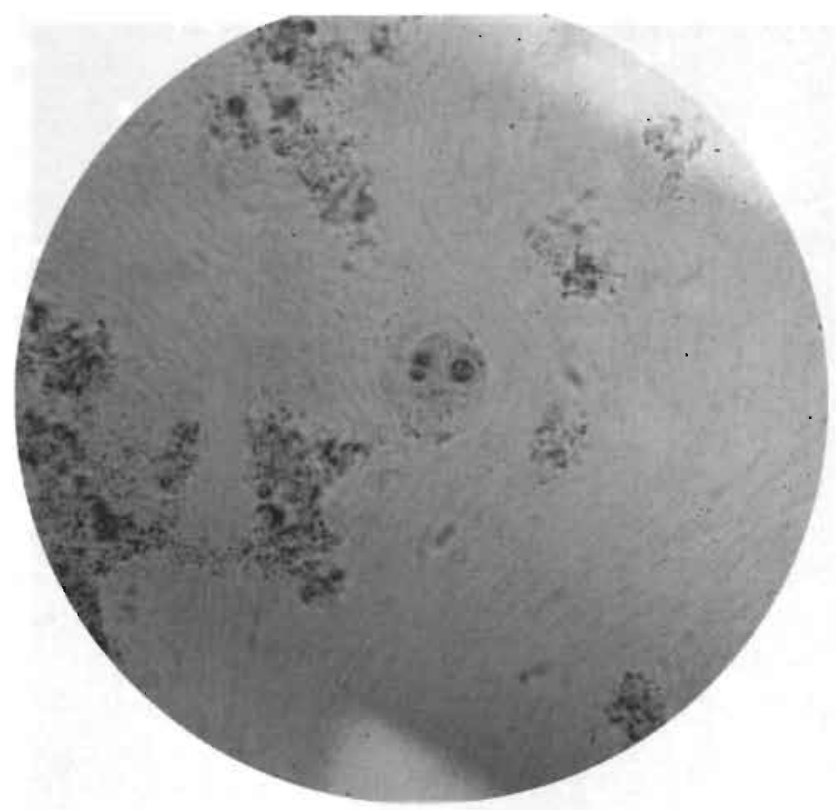

Figure 2.7

Reproduced with permission

The following chapters contain several examples where long standing, taken for granted interpretations of experimental and clinical evidence were challenged and replaced by alternative explanations. With the acceptance of the new way to interpret the data, and the establishment of the legitimacy of the new spokespersons, the world, that was assumed to be represented by the inscriptions, changed too.

A third situation in which the muteness of inscriptions is apparent is in cases where new experimental interventions produce new, and unexpected sets of inscriptions, for which no plausible or authorized interpretation (translation) exists, yet. The next chapter, on the pre-discovery work on insulin, provides a 'telling' example.

\subsection{Conclusion}

The thrust of this chapter is to suggest to give up attempts to justify the certainty with which we hold knowledge to be true by reference to the pre-existent world-as-it-reallyis. This entails giving up the familiar images of scientific progress, discovery and representation (medical imaging). Conceding that the world-as-it-really-is is an inaccessible virtual image of a constructed reality, does not mean that we should give up an interest in certainty (and uncertainty). It puts one in a rather awkward. agnostic. starting position though. Where do we start when we concede that the world does not play any significant role in the production of certain knowledge? Where do physicians and scientists start? How do they make sense of the world? How do they endow their hypotheses with so much certainty that they become facts about the world? How do 
they make technologies work? These are the questions to bear in mind while reading the following chapters. This is the quest for certainty that will be pursued in this study, not by addressing the issue in the abstract, but by working through de details of three different case studies.

The theoretical concepts introduced in section 2.4 provide the sensitizing notions required to get started. The de novo translation of mute inscriptions forms the root of a translation-theoretical account, in which translation is first of all conceived as a problem, and as an endeavour that may fail or succeed. The problem of translating is one of providing a plausible, persuasive interpretation of mute inscriptions. It is also a problem of persuading others to accept the interpretation proposed and to act accordingly. The analysis will focus on all of the process by which something (inscriptions, images, recordings, cognitive or moral propositions, technologies) is invested with so much certainty, hardness, obduracy, that it becomes a seemingly inexorable part of the world's order. It will not only be concerned with discursive forms of persuasion. Since all action and activity is situated, the analysis also comprises unintended, contingent and non-linguistic aspects of persuasive situations, as well as the work that has to be done to develop and maintain the practices providing stability.

Rather than adhering to an (in the end) unjustifiable belief in pre-existent reality as a foundation for scientific knowledge, a constructivist approach and a translationtheoretical account will augment our understanding of how certainty in medicine is produced. It will emphasize the situated and collective work of people to explain the modern medicine, rather than recurring to the operation of an instrumental rationality to explain either the success or the shortcomings of medical science and technology.

The following chapter will account for the hardness and doubtlessness of an entity that has become an inexorable part of the world: insulin. It will describe the work through which insulin became a matter of fact, an entity in the bodies of animals and human beings, and a reliable technological tool in the treatment of a lethal disease. The focus will be on a scientist's problem to make sense of the inscriptions he gathered from pancreatectomized dogs, to find out what these inscriptions were saying about the world from which they were 'extracted'. What were these inscriptions representations of? Or were they just artefacts, produced in the process of producing the inscriptions? The analysis will be concerned with the identification of the resurces and strategies actors use in their attempts to translate the specific inscriptions at hand, how they attempt to increase the level of certainty and the robustness of their account. Having solved this first problem by working out an explanation for their experimental results, postulating the existence of an internal secretion of the pancreas, scientists faced the problem of persuading others. This endeavour was hampered, for approximately three decades, by the accumulation of failing attempts to extract the hypothetical internal secretion from pancreatic tissue. Finally, the account will also be concerned with the material practices that had to be developed to extend the newly established insulin beyond the limits of local laboratories and to turn it into an effective and save therapy in hospitals and in diabetics' homes. 


\section{Notes to chapter 2}

\section{For historical accounts of the shifting uses and meanings of the notion of objectivity see Daston} (1992).

2. Clinical syndromes like systemic lupus erythematosus or rheumatoid arthritis still present considerable problems. There are classical text book cases and lists of symptoms frequently, but not always, associated with the syndrome, and there are conventions as to how many of these symptoms must be present to warrant the diagnosis. However, eventually it is up to the individual clinician to decide whether his patient resembles the typical text book case to such an extent that the diagnosis is warranted. (Wulff et al. 1986)

3. This paper, written about 1550 B.C. was discovered in a tomb at Thebes and translated by the German Egyptologist, Georg Ebers. See for example Poulsen (1982).

4. The Egyptian engraving reproduced in figure 2.1 is in the collections of $\mathrm{Ny}$ Carlsberg Glyptotek, a museum in Copenhagen, Denmark. In the 1950s this engraving was frequently used to illustrate the medical literature on infantile paralysis, either as a picture in the text or on the cover of books and reports.

5. Wulff et al. (1986) accept that we can never be sure that our theories are true, but there are other good reasons to belief in scientific theories, namely the efficacy of modern technology, which at least to some extent is based on scientific theories, proves beyond doubt that we have in some areas reached what Popper calls 'an approximation to the truth'.

6. If we accept Jensen's evolutionary concept of disease it will he hard to maintain that the patient described by Aretaeus and diabetics today suffer from the 'same' disease in the sense that Aretaeus" patients and present day diabetics necessarily share a set of biological characteristics or abnormalities that makes out the essence of the disease. During the course of its evolution the transformation of a disease may have been so extensive that present day cases bear little or no resemblance with earlier cases. Furthermore, when diseases are evolving entities changing over time, it will be equally hard to construe changes in (scientific) knowledge as a progressively better understanding of a pre-existent reality.

7. The notion of whig history is coined by the historian Herbert Butterfield (1931) who criticized the strategy of 19th century English gentleman historians allied with the Whig party who wrote the history of their nation as a progressive approach to their political ideals.

8. In the sense in which Latour and Woolgar use the term, a modality is a sentence about a sentence. The modality usually modifies or qualities the other sentence. Latour (1987) calls positive modalities those sentences that lead a statement away from its conditions of production, making it solid enough to render some other consequences necessary. He calls negative modalities those sentences that lead a statement in the other direction towards its conditions of production and that explain in derail why it is solid or weak instead of using it to render some other consequences more necessary.

9. In medicine, the physical examination of a patient depends on the proper use of the senses. Inspection of a patient's body - anatomical characteristics, color of the skin, etc. - depends on eyesight. Auscultation, usually with the aid of a stethoscope depends on hearing. The discrimination of the sounds elicited in percussion also depends on hearing. Palpation of anatomical structures, of skin texture or temperature depends on touch. Although nowadays smell and taste are less important in the physical examination of the patient, a physician will keep his nose open, since also characteristic smells can give important information about the condition of the patient.

10. Distortion of information can result in false negative and false positive outcomes of tests. Scientists refer to the latter case as an artefact, a symbol unintentionally produced in process that usually represents an aspect of the world, but in this case it represents nothing. 
11. Physicians praised Einthoven's electrocardiograph as so accurate that any observer could duplicate the results upon the same patient, even if they did not use the same machine. Einthoven claimed the form of the electrocardiogram to be independent of the instrument used, and every registration, when and where it may have been recorded, to be immediately comparable with every other registration. Physicians welcomed the electrocardiograph as a way of capturing transient clinical events through visual records. Reiser cites Nash, who wrote, that " $\mathrm{t}] \mathrm{h}$ little strips of paper, imprinted by the disease itself, form permanent and unquestionable testimony of events which have occurred. (Reiser 1978: 109; my emphasis)

12. See for example Pasveer (1992).

13. See for example Rudwick (1985), Stemerding (1991), and Gould (1989).

14. Historians and sociologists of science have drawn attention to the use of visual materials or inscriptions as an important, or the most important, innovation in science and medicine. Writing on the history of geology and paleontology Rudwick $(1976 ; 1985)$ argued that the striking increase in the quantity and quality of illustrations (topographical maps, diagrams of geological strata) in genlogical works, which was in part consequent on the introduction of new ways of producing and reproducing visual materials, reflects the development of a new range of kinds of visual expression, which paralleled and was an essential part of the development of a self-conscious new science. An essential part of this complex historical process was the construction of a visual language that was appropriate to the subject matter of the science, and which could complement verbal descriptions and theories by communicating observations and ideas that could not be expressed in words. The maps and diagrams employed by geologists went beyond the level of 'simple' illustrations or drawings of local topographies. Diagrams of progressively greater degrees of abstraction and formalization incorporated greater theoretical loading into the forms of illustration.

15. In Denmark techniques for the preservations of mummified corpses found in marshes were not available, but had to be invented, when the first body was found near Tollund. During this process of trial and error most of the body was lost and only the head could be preserved, which is now exhibited in a museum in Silkeborg ('Tollund-manden').

16. The transformation of the 'original' object into an 'immutable mobile' is well recognized by scientists and physicians. Nevertheless, despite the severity of the transformation, in natural or artificial processes of preservation, it is assumed that some information has been retained. The extraction of this information involves an understanding, or theory about, natural processes of mummification, fossilization, and artificial preservation.

17. Or are we looking at a picture taken with a telescope, showing a galactic cluster of stars with only a purely coincidental resemblance to Europe's city lighting by night? 


\title{
Existence, presence, identity and performance
}

\author{
Insulin: from hypothesis to standardized treatment
}

\subsection{Introduction}

The discovery of insulin at the University of Toronto in $1921-1922$ was one of the most dramatic events in the history of the treatment of disease. Insulin's impact was so sensational because of the incredible effect it had on diabetic patients. Those who watched the first starved, sometimes comatose, diabetics receive insulin and return to life saw one of the genuine miracles of modern medicine. They were present at the closest approach to the resurrection of the body that our secular society can achieve, and at the discovery of what has become the elixir of life for millions of human beings around the world. (Bliss 1982: 11).

These are the opening lines of Bliss' historical account of the discovery of insulin by Frederick Banting, Charles Best, John J.R. Macleod and James Collip in Toronto. For this major achievement, a breakthrough in the treatment of diabetes mellitus, Banting and Macleod were awarded the 1923 Nobel Prize in physiology and medicine, which they shared with their collaborators Best and Collip. The discovery of insulin is a salient landmark in the history of diabetes. From 1922 on physicians were provided with a powerful weapon to combat a previously lethal disease.

\section{Theoretical discovery and practical isolation}

According to the received canons of narrative medical history on insulin it was Minkowski who in 1889 discovered, in his experimental work on animals, that the pancreas played a major role in the body's carbohydrate metabolism and that the pancreas' failure caused the disease known as diabetes mellitus. In an attempt to understand and explain the pathophysiological mechanisms involved Oskar Minkowski postulated the existence of a substance produced in the pancreatic tissue, secreted into the blood and mediated through the blood vessels to the body's tissues where it exerted its function. This hypothetical substance, which M.E. Laguesse called insulin, was 
actually discovered in 1921 - 1922 by the Canadian research group in Toronto. Following Minkowski's theoretical articulation of the pancreatic etiology of diabetes mellitus the discovery of insulin had only been a matter of time and technical skills in purifying pancreatic extracts. According to Bliss,

\begin{abstract}
the internal secretion of the pancreas had been 'discovered' theoretically back in 1889; its practical isolation and therapeutic use was only a matter of time, determination, ingenuity, technical skill, and resources. Many of Toronto's predecessors ... did have active pancreatic extracts - that is, extracts containing insulin. None of them, however, had been able to purify their extracts sufficiently to eliminate their toxic properties and convince the medical world that the internal secretion had been obtained. (Bliss 1982: 210)
\end{abstract}

\title{
The problem of insulin's existence
}

Like most traditional histories of diabetes and insulin' Bliss' account, although extremely rich in empirical detail, suffers from a common bias arising from unarticulated theoretical assumptions. These histories characterize the experimental evidence as providing convincing and definite proof of insulin's existence. The failures to make effective anti-diabetic pancreatic extracts over a period of 32 years, on the other hand, are characterized as a technical extraction and purification problem. However, it is only with the benefit of hindsight that we can say that the problem of pancreatic extractions was a purification problem for which neither knowledge, nor procedures nor skills were available. Prior to the 'discovery of insulin' in 1921/1922 it was as much an existence problem as a purification problem, although only a few scientists drew the conclusion that insulin did not exist. Most 'extractors' gave up without challenging the existence of insulin, maybe even without publishing their negative results.

It is the existence problem of insulin on which I will focus in this chapter. The term 'existence problem' pertains to the scientific doubt whether the hypothetical internal secretion of the pancreas called insulin existed at all. Scientists who believed in the existence of insulin had to establish its presence in the pancreatic extract and to define its identity. Continuing doubt about one affects the others. Experimental scientists working on anti-diabetic pancreatic extracts had to convince themselve's of the existence of the substance. Secondly, they had to convince others that it existed. Thirdly, they had to make sure the substance performed its existence, presence and identity in other places in the scientists' absence.

What experimental evidence convinced Minkowski and other researchers that there really was a substance, produced and secreted by the pancreas, that played a role in carbohydrate metabolism? Sections 3.3, 3.4 and 3.6 will be concerned with this question. Knowing nothing about its chemical composition and physical behavior (for example solubility) how did scientists know whether they had captured the sought for substance in a pancreatic extract? What should the substance or the extract be able to do in which test systems? What were, in other words, insulin's criteria of existence? These questions will be addressed in sections 3.5, 3.7 and 3.8. Finally, how did physicians and scientists make sure that, once they had a working extract, it worked not only in the laboratory but also in hospital wards and patients' homes, close by and 
at long distances? Focussing on the issue of international biological standardization of insulin, parts of section 3.8 and section 3.9 will be concerned with this issue.

It follows from these questions that the strategic research site to look for answers is the pre-discovery work on insulin, beginning with Minkowski's performance of a total pancreatectomy in a dog. The first problem we have to address, however, is to grasp what diabetes was in the 1880's. As a first step in an attempt to do this it is helpful to review, briefly, some of the landmarks in the history of the disease.

\subsection{Diabetes up to the $1880 \mathrm{~s}$}

\section{Diabetes as a clinical entity}

Towards the end of the 19th century diabetes mellitus was mainly a clinical entity, a disease observed in patients. The list of characteristics defining the disease changed over time. It was extended considerably since the application of chemical methods to investigate the composition of body fluids like urine and blood.

It was long held that diabetes was a kidney affection. Aretaeus, working in the second century A.D., found it not improbable that something pernicious attacks the bladder and kidneys. According to Galen, also in the second century A.D., the disease was caused by the kidney's inability to retain water. Paracelsus in the beginning of the sixteenth century believed that diabetes was caused by a kidney disease in which the kidneys excrete an abnormal amount of salt. When he evaporated the urine of diabetic patients a white powder remained which he assumed to be salt; four ounces of salt in one litre of urine, and that is the salt that causes thirst of the kidneys and brings polyuria.

In European medicine it was the English physician Thomas Willis who in 1674 noticed the sweet taste of diabetic urine, as if it contained sugar or honey. He distinguished between diabetes mellitus (polyuria with sweet tasting urine) and diabetes insipidus (polyuria without sweet tasting urine). Willis explained the sweet taste as being due to different salts and acids. (Poulsen 1982) It was Mathew Dobson who in 1775 found the sweet taste of diabetic urine and, similarly, the sweet taste of diabetic serum, to be due to the presence of (fermentable) sugar in them.

William Cullen, living in the eighteenth century tended to explain every organic phenomenon as originating in the nervous system, and he was led to the conclusion that diabetes was a disturbance of the nervous system. According to Papaspyros (1964: 16), Cullen for the first time used the term mellitus in conjunction with diabetes.

John Rollo at the end of the eighteenth century broke with Galen's doctrine. Rollo assumed diabetes to be an affection of the stomach; in diabetes there is an increased activity of the gastro-intestinal tract producing too much sugar from the carbohydrate-rich food in the stomach. Bouchardat in France accepted Rollo's view and dietary treatment which aimed at reducing the carbohydrate content of the food intake. This diet regularly caused the disappearance of sugar from the urine in mild cases. (Poulsen 1982)

In 1788 Thomas Cawley found diabetes to occur more often when the pancreas had suffered injury or after the formation of calculi in the pancreatic duct. Stones in 
the pancreas in conjunction with diabetes were later, in the nineteenth century, also reported by several other authors. (Papaspyros 1964)

In 1874 the German physician Adolf Kussmaul described the peculiar type of breathing observed in cases of diabetic coma; it still carries his name. He believed that it must be due to a chemical disturbance caused by diabetes. In the beginning of the 1880 's Naunyn, Stadelman and Minkowski identified the acid excreted in the urine of diabetic patients as B-oxybutyric acid. Naunyn introduced the term acidosis to describe the metabolic condition of acid formation in diabetic coma.

\section{Experimental diabetes}

Since the introduction of experimental methods in physiological research, in the first half of the 19th century (Allen 1978; Coleman and Holmes 1988), diabetes also became an artificially induced condition in laboratory animals.

In the middle of the 19th century Claude Bernard, the French experimental physiologist, demonstrated that sugar can be formed in the animal organism - in contradiction to the prevalent belief that all sugar originated from vegetable substances, the organism only being able to burn. By doing perfusion experiments on isolated livers he found the blood leaving the liver to contain more sugar than the blood entering it. For this faculty of the tissues to produce a substance and secrete it into the bloodstream, Bernard coined the term internal secretion. Bernard also demonstrated the presence of glycogen in the liver. Of great importance is Bernard's piquare diabétique, an experiment in which the bottom of the fourth brain ventricle is damaged, resulting in a transitory diabetic state. In Bernard's view the liver, affected by pathogenic influences from the nervous system, was central in the pathophysiology of diabetes.

There were a great number of other experimental interventions that were followed by the excretion of sugar in the urine, all designated as diabetes. Glycosuria following the injection of phloridzin was called Phloridzindiabetes. Glycosuria following the injection of adrenal extracts was called adrenal diabetes (Nebennierendiabetes). The ingestion of strychnine and some other poisons also caused glycosuria. Several surgical interventions, involving the nervous system or, for example, the salivary glands or the thyroid, were accompanied by glycosuria too, although not invariably.

\section{Diabetes in different places}

Thus, in the 1880 s diabetes could be encountered in three different places that were distant in space and time: a) in the living patient, that is. in the patient's home, in the physician's office or in the hospital; b) in the dead patient, that is, in the autopsy room; c) in dogs, frogs, ducks and other experimental animals in the research laboratory of the experimental physiologist. The relevance of what could be observed in the dead body, or in experimental laboratory animals, for the understanding of pathophysiological mechanisms at work in the living patient, was never obvious. In attempts to explain the pathophysiological mechanisms of diabetes mellitus, or in other words, in theories about the cause of the disease, diabetes had been linked to a whole range of organs and processes by different authors. Some of these links were, although speculative, linked to clinical experience, like in Rollo's case, linking diabetes to the 
stomach. Some other links were related to occasional, maybe incidental, findings during abdominal surgery and findings in the autopsy room, like in Cawley's case, linking diabetes to the pancreas. Still others were related to deliberately induced changes and observations in animals under laboratory conditions, like in the case of Bernard, linking diabetes to the liver and the nervous system. None of these links, which were dependent on different types of activities in different places, was very strong however. They provided clues, suggestions for further research or therapeutic approaches.

\section{Glycosuria: transitory and persistent}

Towards the end of the nineteenth century the word diabetes was used to designate any kind of glycosuria, transitory or persistent, experimental or not.

Transitory glycosurias in humans often co-occurred with other ailments: infectious diseases, neurological disorders, traumas, but also psychiatric disorders. (Frerichs 1884) Some of these diseases were assumed to stand in a causal relationship to diabetes. Only a facilitating role was attributed to other diseases. For the greater part, however, the nature of the relationship with diabetes, remained obscure; that is, if there was a relationship.

Severe forms of diabetes resulting in the patient's death were well known. Prognosis was bad. A whole myriad of therapies, based on speculative assumptions about the disease's nature, had been proposed and tried. Without success.

Pathologists were unable to identify specific lesions in the dead bodies of patients that had experienced diabetes during life. Numerous lesions were found, though, but none of them were encountered so regularly and frequently in diabetics as to suggest a relationship with diabetes.

The diversity of experimental interventions that could cause diabetes, but also the diversity of co-occurring diseases and differences in the clinical course in different patients, suggested that diabetes mellitus was not one disease, with one pathophysiological mechanism, but that diabetes rather was a group of diseases which had one thing in common: glycosuria.

\subsection{Minkowski: dogs, pancreases and diabetes}

\section{Total pancreatectomy and diabetes}

It was against this backdrop that in 1889 Oscar Minkowski, working in the laboratory of the Medical Clinic of the University of Strassbourg (headed by Bernard Naunyn) operated on a dog and took out its pancreas (see figure 3.1). The operation was intended to demonstrate the feasibility of total pancreatectomies as an experimental method for studying the digestion of food in the gut in the absence of pancreatic juice. ${ }^{2}$ On the day after the operation Minkowski noticed that the dog passed a lot of urine. ${ }^{3}$ Working in a medical department where diabetes mellitus was the subject of extensive clinical and experimental research ${ }^{4}$ Minkowski associated the polyuria of the newly pancreatectomized $\mathrm{dog}$ in the laboratory with the polyuria of the diabetic patients on the hospital's wards. He collected some of the urine in a pipette, did a Trommer's test and found the urine to contain $12 \%$ sugar. (Minkowski 1929; 


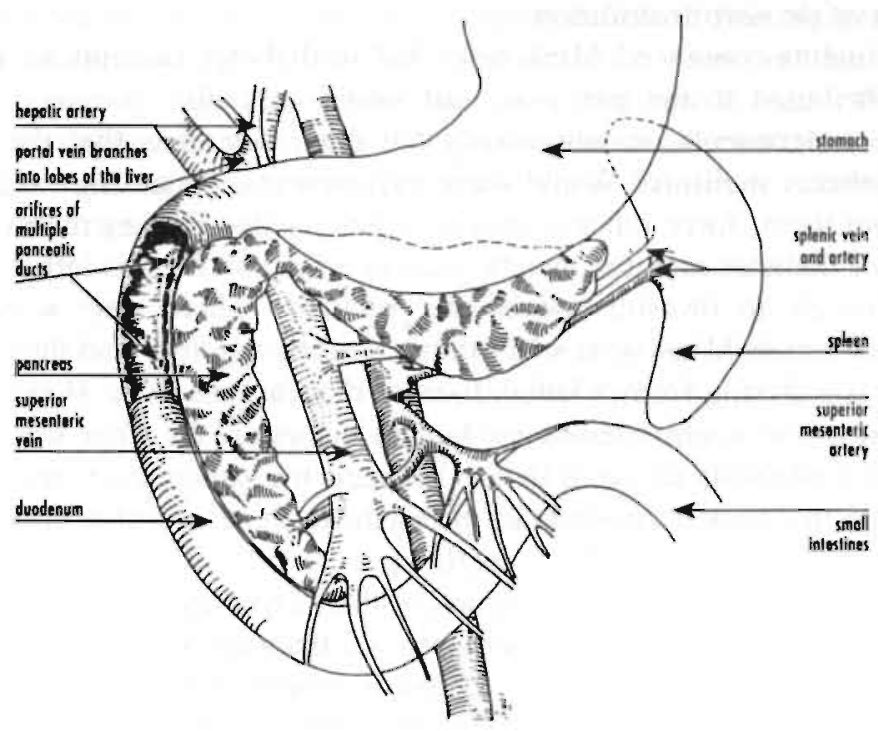

Figure 3.1

Anatomy of the canine pancreas and related structures

Houssay 1954) Minkowski recognized that the dog had some kind of experimental diabetes.

\section{Hesitations}

What would be more obvious than to associate the diabetes of the dog with the organ extirpated the day before, the pancreas, that is, to 'see' the pancreatectomy of the previous day as the cause of the dog's diabetes? Nevertheless, Minkowski hesitated. A possible link between the pancreas and diabetes mellitus, or between the pancreas and carbohydrate metabolism was completely new to him. In 1889 the pancreas played no role in carbohydrate metabolism. Naunyn and Minkowski had been working on the acid poisoning in diabetic coma. In this work the pancreas was of no importance at all. The pancreas secreted digestive enzymes into the gut, but even that function of the organ was much disputed.

There was another reason why Minkowski hesitated to relate the dog's diabetes to the pancreatectomy on the previous day. He did not know whether the dog had sugar in his urine prior to the operation. It was a dog from J. von Mering's laboratory and Von Mering was known for his work on phloridzin-diabetes. The glycosuria might be due to the fact that Von Mering had treated his dog with phloridzin. (Houssay 1952)

Minkowski pancreatectomized three of his own dogs, checking that they had no sugar in their urine prior to the operation. Two of them died within a timespan of two days as a result of necrosis of the duodenum, but both had glycosuria before they died. The third animal survived and displayed a persistent diabetes just like the first dog. (Houssay 1952) 
The problem of de novo translation

These experiments convinced Minkowski that in diabetes mellitus an important role had to be attributed to the pancreas. But would they also convince other people? Would these experiments unambiguously tell other scientists that the pancreas was related to diabetes mellitus? Would these experiments, these dogs and the numbers extracted from them, force other scientists to believe that the key to the elucidation of the mystery of diabetes mellitus, maybe even to a therapy for this lethal disease, could be found through an investigation of the pancreas? There were a couple of dogs without a pancreas in Minkowski's laboratory wetting the floor and there were patients in the hospital suffering from a lethal disease: diabetes mellitus. How could the dogs in the laboratory be made relevant for human diabetics? In other words, Minkowski would have to establish an equivalency between his pancreatectomized dogs in the laboratory and the diabetic patients in the medical clinic in order to be able to relate also the human pancreas to diabetes mellitus.

Other scientists had published reports of their experimental work on the pancreas but none of them reported the emergence of diabetes mellitus in their animals. Minkowski would have to explain the 'negative' results of these scientists.

Furthermore, the pancreas was not the only candidate to be linked up with diabetes mellitus. Other scientists were working on the liver and the nervous system along the lines set out by Claude Bernard. This line of work did not only carry the authority of scientists like Claude Bernard and Eduard Pflüger. The nervous system was the only organ system that was able to influence the function of distant tissues through complex feed back mechanisms, thus playing a central integrating role in the homeostasis of the organism. Glycosuria was known to appear frequently after surgical procedures, also after non-pancreatectomies. Thus, it was more likely that the diabetes was due to the inevitable damage inflicted on the nervous system by the surgery than to the loss of a specific pancreatic function.

Minkowski would have to show that his 'persistent diabetes mellitus' was not identical with the known 'transient post-surgical glycosuria'. He would have to demonstrate that it was the wiping out of the function of the pancreas that caused the appearance of diabetes mellitus and that it was not the surgical procedure as such or the unavoidable damaging of adjacent tissues that was responsible for that. And last but not least, Minkowski would make a better case if he not only would argue that the pancreas was involved in diabetes mellitus, but if he also could provide an answer to the question how the pancreas exerts its influence on carbohydrate metabolism.

Minkowski did not rush to publication with the preliminary results on four dogs, two of which died within two days. Instead he devised a strategy that would strengthen the force of his argument. He searched the literature to find the work that could be used against him and scrutinized it to find a way to render it harmless or to turn it into his advantage. Minkowski devised a series of experiments that strengthened his argument. The first extended paper that Minkowski published about this work was not a straightforward description of a lucky accident with subsequent experiments. (Von Mering and Minkowski 1889) It was a carefully composed article. In the following paragraphs I will discuss this paper in considerable detail in an attempt to display its logic. A second extensive paper, describing a series of experimental pancreatectomies and pancreatic transplantation experiments, was published by Minkowski in 1893 . 
We should not shrink away from the technical details of this experimental work. It is from an understanding of this hands-on practical work in the experimental laboratory that we can begin to understand how Minkowski and other scientists invested the hypothetical, new pancreatic function with enough plausibility to withstand, in the decades to follow, the cumulating failures to extract the active principle from the pancreas.

\section{Why others failed: they did not know their anatomy}

In order to establish a strong link between the pancreas and diabetes mellitus it was necessary for Minkowski to deal with scientific publications that failed to report the emergence of diabetes mellitus after elimination of the function of the pancreas.

The pancreas secreted pancreatic juice to the gut. The ducts through which the juice was secreted were visible to the naked eye and the juice could be collected in the living animal through a catheter placed at or in the outlet of the pancreatic duct. There were two ways to eliminate the function of the pancreas experimentally. One way was through the ligation of the pancreatic duct. Frerichs, Lenz, and Bidder and Schmidt followed this procedure and found no disturbances. But, according to Minkowski these people did not even succeed in keeping the pancreatic juice from the gut, because they did not see that the gland had several draining ducts of which they only ligated a few. They did not know their anatomy. (Von Mering and Minkowski 1889)

\section{The pancreatectomies were incomplete}

The second way of eliminating the function of the pancreas is by extirpation of the pancreas. This was attempted for the first time by the Swiss anatomist John Conrad Brunner in 1673. These operations were performed without proper anesthesia. ${ }^{5}$ The dogs pancreatectomized by Brunner in the 17 th century survived the operation and lived a long time thereafter without displaying any disorders. But, according to Minkowski, Brunner's results could not be of any importance because he left a major (the whole horizontal) portion of the pancreas behind.

A total pancreatectomy was long held to be impracticable. However, progress in surgical procedures and anesthesia made it possible to remove the pancreas of an animal almost completely; and, according to many authors this was good enough to evaluate the function of the pancreas.

In 1888 some reports on experimental pancreatectomies without subsequent diabetes mellitus were published. Senn pancreatectomized several dogs and cats. Most of these animals died within a very short time, either as a consequence of the operation itself, from gangrene of the gut or from peritonitis. These animals did not live long enough to display a persistent diabetes mellitus. One dog survived up to nine days and displayed an undiminished appetite, but lost $2,5 \mathrm{~kg}$ weight in these few days. But, Senn did not examine the urine of the dog. Minkowski suggested that this might well have been a 'positive' result. Hence, 'failing to report diabetes' was not the same as 'failing to produce diabetes'.

Martinotti's pancreatectomized animals recovered completely after the operation and even gained weight. Minkowski pointed out that according to Martinotti's own report small remnants of pancreatic tissue were found at autopsy. Not very big, however. In one dog Martinotti found a piece of pancreatic tissue as big as a chestnut, 
having its own draining duct to the duodenum. In a second dog he found in the same position a smaller remnant, as big as a nut. In a third dog he found an even smaller remnant, as big as a lentil. A fourth dog which displayed considerable digestive problems and loss of weight was killed ten days after the operation. In this dog Martinotti did not find any remnants of pancreatic tissue, but he found an incarceration of the gut which could explain the digestive problems. Thus, in the dogs that lived longest after the operation, in principle long enough to display diabetes, the pancreatectomy had not been total: it was incomplete. Martinotti argued that no physiological importance could be attributed to these small patches of pancreatic tissue. Minkowski, however, argued that these small patches of functional pancreatic tissue might explain why the dogs in Martinotti's experiments did not display diabetes. Furthermore, Martinotti did not examine the urine of his dogs for the presence of sugar either; at least he did not report that he did.

Thus, Minkowski was able to dismiss these 'alleged' negative results because: a) in a trivial sense, the function of the pancreas was not eliminated; b) most pancreatectomies were incomplete, and c) in the cases where there might have been a diabetes they did not look for it.

\section{Creating difference and identity: persistent diabetes in dogs and humans}

Minkowski could not confine himself to explaining why others failed. He had to strengthen the link between the pancreas and diabetes in dogs, and between diabetes in dogs and diabetes in humans in a positive way. In an attempt to achieve this double goal in one move Minkowski argued for a differentiation between persistent experimental diabetes on one hand and experimental transient glucosurias on the other. Only the persistent glucosuria that followed a total pancreatectomy was worthy of the name diabetes mellitus. And only this true and persistent form of experimental pancreatic diabetes resembled the severe forms of diabetes in humans:

...after total extirpation of this organ, the dogs became diabetic. This did not concern transient glycosuria but genuine persistent diabetes mellitus which resembles the severest form of this disease in humans. (Von Mering and Minkowski 1889: 377)

In a first series of pancreatectomies on dogs the 'human-like' diabetes never failed to appear. at least in so far as the animals did not die from the direct consequences of the operation. Only in 3 out of 21 dogs Minkowski was unable to detect any sugar in the urine. But, these three dogs all died within a timespan of 24 hours after the operation without excreting urine in the first place. At autopsy there was found a little urine in the bladder which contained no sugar. But this urine could well have been produced prior to the operation. So these 'negative' results were not really negative results. In all the other cases the diabetes appeared 'ausnahmslos'.

The excretion of sugar began within the course of one day after the pancreatectomy, sometimes already after 4 - 6 hours. The amount of sugar excreted rose to a maximum of $5-11 \%$ within a timespan of $24-48$ hours. Even when the animals were starved during the first week after the operation the sugar did not disappear from 
the urine. Using established laboratory methods for the detection of sugar in urine the sugar excreted by the pancreatectomized dogs could be identified as dextrose:

... fermentative, dextrorotatory dextrose. Comparative saccharometry by means of the polarization apparatus and titration with Fehling's solution showed that urine could on no account contain a noticeable amount of any other kind of sugar. (Von Mering and Minkowski 1889: 377)

Not only the kind of sugar excreted was identified as being the same as in human diabetes mellitus. All the other symptoms that were characteristic of severe forms of human diabetes mellitus were also observed by Minkowski in pancreatectomized dogs.

At first, ... the dogs exhibited an abnormal appetite and an abnormally increased thirst after the pancreatectomy. They rushed on the food offered with an exceptional alacrity, even when they had been sufficiently fed shortly before and they ceaselessly searched for every drop of water they could find. They often devoured their own faeces ... (Von Mering and Minkowski 1889: 377-378)

Concurrent with the increased amount of consumed water the dogs exhibited a considerable polyuria. Despite an abundant amount of fodder the dogs emaciated rapidly and exhibited a severe loss of strength.

The resemblance with human diabetes mellitus did not stop there. Minkowski found that some of the pancreatectomized dogs, besides glucose, also excreted considerable amounts of acetone, acetoacetic acid and B-hydroxybutyric acid. ${ }^{6}$

\begin{abstract}
Sooner or later, larger amounts of acetone, acetoacetic acid and oxybutyric acid could be found in the urine in sporadic instances, besides dextrose and oxybutyric acid, the substances that can often be found in the urine in severe cases of diabetes mellitus. Acetone could usually already be detected during the first days after the operation by means of Lieben's and Legal's reaction. Later on, the amount of that substance increased considerably, and the urine showed an ever-intensifying Gerhard's ferric chloride reaction. The values for the sugar level that were obtained by means of the polarization apparatus came out lower than those obtained by means of titration, which indicated the presence of a laevorotarory substance. In fact, 3 $4 \mathrm{~g}$ of an acid could be produced from the acid ether extract of a dog's 24-hour amount of urine which could be identified as oxybutyric acid both by its specific laevorotation and the fact that it produced crotonic acid when distilled with sulphuric acid. (Von Mering and Minkowski 1889: 378)
\end{abstract}

It was no problem to examine the urine of both humans and animals for the presence of sugar. This was done routinely in the hospital and in the research laboratory. However, it was more cumbersome to determine the presence and concentration of sugar in the blood. A relatively large amount of blood, approximately $20 \mathrm{ml}$, was required to perform one blood sugar estimation. To do frequent blood sugar estimations, either on humans, or on experimental animals, would provoke anemia. It was possible however, to do blood sugar estimations incidentally. When Minkowski 
performed blood sugar estimations on his pancreatectomized dogs he always found the concentration of sugar in the blood increased, as it was in human diabetics.

\section{Post-mortem similarities}

There was not only a strong resemblance between living dogs in a post-total-pancreatectomy-state and living humans suffering from diabetes mellitus; there was also a strong similarity between the two in the post-mortem condition. When Minkowski, during autopsy, examined the liver of the pancreatectomized animal for the presence and amount of glycogen he found that there were only traces (unwägbare Spuren) left. This phenomenon was well known from the diabetes research which centered around the liver. It was found both in forms of experimental diabetes and in autopsy reports on human diabetics. Another salient observation during Minkowski's autopsies was an increased amount of fat in the liver. At autopsy Minkowski never found any pancreatic tissue left in the abdomen of the dogs.

\section{Impaired wound healing}

The severe form of diabetes mellitus which followed the extirpation of the pancreas persisted until the death of the animals. Most of the dogs died within a week after the operation from complicating diseases. Some of the dogs died from necrosis of the duodenum due to impaired circulation of blood, or from peritonitis due to impaired wound healing. (Von Mering and Minkowski 1889) In some cases the abdominal wound burst open again followed by protrusion of the intestines and peritonitis. Only five dogs exhibited a more or less smooth healing of the wound, but nevertheless, none of these dogs lived longer than four weeks.

Minkowski turned this high post-operative complication rate, which easily could have been used against him (bad surgical technique, bad anti-sepsis), into his advantage. It was known from human diabetes mellitus that patients exhibited an impaired wound healing and proneness to complicating infections. The high post-operative complication rate was one more argument that experimental pancreatic diabetes resembled severe forms of diabetes mellitus in humans in many relevant aspects; that it in fact was one and the same disease and that therefore the 'road to the elucidation of the mystery of diabetes led through the pancreas'. Diabetes mellitus, when present, increased the level of difficulty of the operation (which for decades was held to be impracticable) and put high demands on the surgical skill of the operator and on antiseptic procedures during the operation. That Minkowski succeeded in performing a total pancreatectomy and in keeping the animals alive for such a long time after the procedure clearly demonstrated the surgical skill for which Naunyn praised him:

The extirpation of the liver, the extirpation of the pancreas are first-class surgical operations, it took many a year, long after he leamed it, before one learned to perform them in a place other than my laboratory. (Naunyn 1925: 442) ${ }^{7}$

In 1893 Minkowski went even one step further and put doubt on the publications which reported uncomplicated healing after a pancreatectomy. 
And I must confess that according to my experiences, I cannot help but suspect that in the case of all those publications in which extremely favorable healing after the pancreatectomy are reported, a complete extirpation of the organ has not always taken place. Anyhow, it is remarkable that the authors who achieved satisfactory results with their operations are exactly those who frequently observed a course of the diabetes which failed to occur or which was anomalous. (Minkowski 1893: 87)

Minkowski bridged the physical distance between humans suffering from diabetes mellitus in the medical clinic and dogs suffering in the laboratory by systematically juxtaposing (on paper) inscriptions extracted and accumulated from living and dead diabetic patients with inscriptions extracted and accumulated from living and dead pancreatectomized animals. In this way, he created identity between sick people and sick dogs. The creation of this identity made observations in the laboratory relevant for the clinic.

Accompanying injuries: damage to the nervous system?

Although convincing and satisfying to some, Minkowski's results, as presented thus far, would not force any reader into assent, in the sense that they excluded all possible alternative explanations. Eduard Pflüger, one of Germany's leading experimental physiologists, argued that it was possible that the diabetes mellitus following the surgical procedure of total pancreatectomy was not resulting from the total loss of the pancreatic function in the organism of the animal, but that it was the unavoidable, accompanying injury to adjacent tissues and nervous structures in the abdomen that caused the diabetes. Simple as it may seem, Pflüger's alternative explanation of diabetes following pancreatectomy carried the authority of an important neurophysiological tradition in 19th century diabetes research that had articulated a neurogenic theory of diabetes mellitus.

Claude Bernard produced experimental diabetes in laboratory animals by thrusting a specifically designed lancet through the base at the back of a rabbit's skull into the animal's brain stem. The lancet was designed in such a way that, after penetration of the bone of the skull and the meninges, the fibers of the brain stem would be separated rather than severed. It was only when the lancet was pulled back that it cut through the nervous tissue. In this way neurophysiologists could inflict circumscribed damages to an animal's brain stem, the extent of which could be determined exactly in histological sections prepared after the animal's death. Bernard found that when he damaged the floor of the fourth ventricle in the brain stem, this would be followed by excretion of sugar in the urine: diabetes. This was Bernard's famous piqûre diabétique. This glycosuria would last for a couple of hours and then subside. The same pattern of inscriptions could be produced by inflicting damage in other domains of the nervous systems. Experimental neurophysiologists would, for example, expose the large sciatic nerves or branches of the vagus nerve. Simply cutting the nerves already produced the diabetes. Either of the two stumps of the cut nerve could now be stimulated electrically. Electrical stimulation of the lower stump, which had no connections with the brain stem, did not produce diabetes. Electrical stimulation of the upper stump, however, produced a transient diabetes. After the glycosuria had subsided the stimulus could be applied again, and again a transient 
diabetes would develop. The upper stump, which was still in connection with the brain stem, carried the afferent stimulus towards the brain stem where it was translated into an efferent stimulus effecting the release of sugar into the blood which spilled over into the urine. The efferent stimulus could be blocked by cutting the spinal cord above the level where the splanchnic nerves spring from the spinal cord. In these cases electrical stimulation of the upper stump of the cut nerve would not be followed by glycosuria. When the spinal cord was cut below the level where the splanchnic nerves originate the efferent stimulus was unimpaired and glycosuria followed. Exposing and cutting the splanchnic nerves also blocked the efferent stimulus, preventing the appearance of sugar in the urine. In this way the route of the efferent stimulus was traced to the liver, which, as Bernard had shown, was capable of producing and secreting sugar into the blood. These electrical stimuli and responses displayed the same pattern of a reflex arc known from the spinal reflexes. The nervous center in the brain stem where the incoming, afferent stimulus was translated into an outgoing, efferent one effecting a rise in blood sugar, was thought to be located in the floor of the fourth ventricle and was therefore called the sugar center.

In concordance with his commitment to the basic assumption of the intelligibility and rationality of Nature's workings Pflüger sought the articulation of a sufficient cause which would explain the wide variety of phenomena he observed in living organisms, in natural circumstances as well as under experimental conditions. Pflüger developed a comprehensive theory in which he combined the Aristotelian notion of a teleological cause with a radical materialism.

According to Pflüger, only one general point of view, one principle that explains all life functions, could be recognized in the never-ending exchanges of life producing forces. According to this principle only such combinations of causes would arise that would enhance the well-being of the animal and the species. The purpose of Nature is the preservation of life, the promotion of health and the reproduction and upgrading of the species. These observations and reflections prompted Pflüger to the theoretical articulation of the 'teleological mechanicism of living nature'. (Pflüger 1877: 76)

It was the researchers task to reveal the intricate mechanisms, both on a macroscopical level as on the level of molecules and atoms in the living cell, by which this 'law of living nature' was effectuated in each instance. Compensatory left ventricular hypertrophy in aortic stenosis, for example, would constitute an example of the first principle in which an organ, the heart's left ventricle, accommodates itself to the required increase in work with mathematical necessity. Likewise would the compensatory hypertrophy of the remaining kidney when the second has been removed. The full and extended bladder causes, reflexively mediated by the nervous system, its own discharge. Lack of oxygen effects a change in chemical composition which stimulates the respiratory center in the brain, which in its turn acts on the respiratory movements of the chest and lungs providing the oxygen. The one organ which, according to Pflüger, is by far the most important with respect to its role in the satisfaction of other organs' needs is the central nervous system. (Pflüger 1877)

Pflüger's 'teleological mechanicism', including his commitment to the central nervous system as the mediator of the satisfaction of needs, framed his opposition to Minkowski's pancreatogenic theory of diabetes mellitus. In Pflüger's view, lack of sugar in the tissues effects a change in chemical composition which is mediated to the 
brain stem through the nervous system. The brain stem's sugar center translates this incoming signal into an outgoing signal effectuating the release into the blood of sugar by the liver, thus satisfying the tissues sugar requirements. In diabetes mellitus, according to Pflüger, an overstimulation of the brain stem's sugar center occurred due to some abnormal 'irritation' of the nervous system. This abnormal stimulus could originate in any region of the nervous system. (Pflüger 1905) The brain stem's sugar center and the nervous pathways by which the various stimuli were mediated had been discerned and established in extensive experimental, neurophysiological research since Bernard's piqûre diabétique. Support for this view came also from numerous clinical observations concerning the co-occurrence of glycosuria with diseases of the nervous system.

In other words, according to Pflüger, diabetes mellitus was a reflex neurosis, a condition in which an irritating stimulus, mimicking the tissues' need for sugar, reaches the brain stem's sugar center, where it is translated into a signal to the liver to release sugar. Hence, the diabetes following a pancreatectomy would, according to Pflüger not be caused by the abolition of some unknown pancreatic function, but by the injuries afflicted to nervous system during the operation.

A pancreatectomy is a delicate operation to perform. Pancreatic tissue is situated in close proximity to the duodenum from which it has to be isolated. Several important blood vessels, that have to be preserved during the operation to avoid necrosis of the duodenum, traverse the pancreas. It is not at all fictitious that important structures like blood vessels or nerves get damaged during the operation. The plexus coeliacus is of special interest here. In 1869 Klebs and Munk explained the occurrence of diabetes mellitus in cases of diseases of the pancreas as resulting from concurrent lesions of the plexus coeliacus. Minkowski never found such injuries during autopsy. In one case he asked professor Von Recklinghausen to do the autopsy. Von Recklinghausen confirmed that the celiac plexus was undamaged. ${ }^{8}$

\section{Partial pancreatectomies}

Nevertheless, Minkowski decided to do a couple of partial operations. In one dog he cut through the mesentery, leaving the pancreas only in connection with the duodenum. This dog did not become diabetic. In two other dogs he ligated the pancreatic duct and separated the pancreas from the duodenum, leaving it only in connection with its mesentery. These dogs did not become diabetic either. In a series of partial pancreatectomies Minkowski removed always a different part of the pancreas, so that in every dog a different portion of the organ was left behind. The accompanying injuries to adjacent tissues were different in every case. If it was some kind of accompanying injury that caused the occurrence of diabetes mellitus after total pancreatectomy, diabetes should also arise in one or the other of these dogs with partial pancreatectomies. But none of these dogs became diabetic. But, ... these dogs became instantly diabetic when in a second operation the rest of the pancreas was removed. (Von Mering and Minkowski 1889)

\section{A new function, a new substance and blood vessels}

Minkowski's results from total and partial pancreatectomies in dogs provided good reasons to believe in a strong link between the pancreas and diabetes mellitus. Yet, 
these results did not disclose anything concerning the nature of this relationship. They did not provide an answer to the question how the removal of the pancreas caused the diabetes mellitus, or how the pancreas was involved in the regulation of carbohydrate metabolism under normal, physiological circumstances.

Minkowski's experimental results seemed to exclude one important route though. The pancreas did not exert its influence on carbohydrate metabolism through its external secretions. The diabetes mellitus was not the result of the pancreatic secretions not getting to the gut, as authors like Bouchardat, Lanceraux, Popper, Lapierre and Baumel, thought. These authors attributed a causal relationship to the coincidence of pathological changes in the pancreas and diabetes. Minkowski pointed out that these authors assumed

... that it involved a consequence of the pancreas' disturbed secretion, whether it was a non-appearing change the saccharifying nutritive substances had to undergo under the influences of this juice, or an effect this juice could have on the sugar metabolism of the tissues of the organism. (Von Mering and Minkowski 1889: 383)

The partial pancreatectomies in which the portion of the organ connected to the gut by the pancreatic duct was extirpated, and where only a remote portion of the pancreas without any connection to the gut was left in situ, clearly showed that the absence of the external pancreatic secretions had nothing to do with diabetes mellitus. These dogs did not become diabetic."

When the diabetes could not be attributed to the absence of a transformatory influence on the content of the gut, mediated by the external secretions of pancreas, the disturbance produced by the total pancreatectomy had to afflict the intermediary metabolism in the organism's interior.

As to the nature of this disturbance of the intermediary metabolism, Minkowski could think of two possibilities: an accumulation of an abnormal substance or a decline of a normal function:

... either something or other abnormal accumulates in the organism after the pancreatectomy, or some normal function declines after this operation, i.e. that the task of the pancreas is either to produce some, maybe ferment-like or toxin-like substance the retention of which causes the excretion of sugar in the organism, or the function of the pancreas is to bring about the utilization of sugar in the organism, and the loss of this function is the cause of diabetes mellitus. (Von Mering and Minkowski 1889: 383-384)

Minkowski rejected the first possibility immediately. The partial pancreatectomies in which the pancreatic ducts were extirpated, impairing the excretion of any substance, were not followed by diabetes mellitus. The 'accumulation of something abnormal' was not very likely as a causal mechanism in pancreatic diabetes.

After rejecting the possibility of the accumulation of something abnormal Minkowski concluded that:

... the diabetes, as observed after complete extipation of the pancreas, can only be caused by the loss of a function of this organ which is undoubtedly necessary for the 
utilization of sugar in the organism, which shows that we are dealing here with a special, still unknown function of the pancreas. (Von Mering and Minkowski 1889: 384)

Considering all the evidence available to him at that time Minkowski proposed a new function for the pancreas.

The nature of this remarkable function of the pancreas, its significance for the normal conversion of carbohydrates in the organism on the one hand and the development of diabetes mellitus on the other hand, have to be clarified first by means of further research. (Von Mering and Minkowski 1889: 384)

Except for that the new function was probably mediated through the blood, he could say no more at the time. There were only dogs in his laboratory that, following extirpation of the whole or part of the pancreas, did or did not exhibit a number of symptoms that resembled the symptoms in humans who suffered from a disease called diabetes mellitus. They lost weight and strength. They died from pneumonia or peritonitis or from necrosis of the duodenum. Their urine was mixed with solutions of chemical substances and boiled to yield a orange-red (Trommer's test) or red (Fehling's test) deposit in a test tube. Sometimes their liver was taken out to extract and weigh a chemical substance that judged by its performance in chemical reactions was said to be glycogen. It was impossible for Minkowski to know for sure whether this new function of the pancreas existed, and if it did, what its nature was. The only thing he could do was to register carefully what he did and write down what he saw, the behavior of the dogs, the color of the deposits in the test tubes, the readings of the balance and the polarimeter. By juxtaposing numbers in tables and figures he tried to make them speak. He found himself at a road junction. The road to the elucidation of the mystery of diabetes mellitus in humans led through the pancreas of a dog. The route through the known exocrine function of this organ was blocked. According to Minkowski, the only alternative left was to open up a new road, the destination of which was not yet established. The road sign said: 'new function'. This new route was paved with blood and blood vessels carrying an unknown substance to distant tissues where it influenced the utilization of glucose. ${ }^{10}$ It was the beginning of an explanation. ${ }^{11}$

But how could Minkowski say that it were the blood vessels that carricd the unknown substance. In order to understand this we have to go into the surgical details of Minkowski's experiments and how he modified them. An important motive for these experimental modifications was to achieve better survival times of the pancreatectomized dogs (which would allow for proper metabolic research).

\section{Transplantation experiments: two-stage procedures}

Minkowski found that pancreatectomized dogs that lived for a considerable amount of time continued to excrete large quantities of sugar, even when carbohydrates were eliminated from their food. The excretion of sugar continued beyond the time where the glycogen reserves in the liver and muscles were considered to be depleted. Where did the sugar excreted in the urine of these diabetic dogs come from? ${ }^{12}$ 
Proper metabolic research on pancreatectomized dogs required a longer survival time though. Most of the animals did not live long enough. They suffered from complicating diseases like peritonitis or infection of the abdominal wound. Minkowski tried to overcome these difficulties by improving and modifying the surgical procedure.

To avoid or minimize the likelihood of infection of the abdominal wound antiand aseptic measures and procedures should be followed strictly. (Minkowski 1893) By following these procedures Minkowski obtained a smooth and uncomplicated wound healing in (almost) all the animals that did not get diabetic, that is with partial pancreatectomies. ${ }^{13}$ When the pancreatectomy was completed in a second operation, he almost never obtained a healing per primam intentionem of the abdominal wound.

To avoid necrosis of the duodenum the blood supply to this part of the gut had to be secured. Hédon (1891) proposed a two-stage procedure to avoid duodenal necrosis. In a first stage some kind of indifferent substance (melted paraffin, a mixture of carbon and olive-oil, or fat) was injected into the pancreatic duct, as described by Claude Bernard (1855). The resulting obliteration of these ducts initiated a gradually advancing atrophy of the pancreas. According to Hédon, it was rather easy to extirpate the sclerotic and shriveled pancreas a couple of weeks later in a second operation without jeopardizing the blood supply of the duodenum. The risk of leaving small remnants of the pancreas behind was, however, greater than with the removal of the normal pancreas. ${ }^{14}$

Minkowski advocated a two-stage procedure based on his experiences with partial pancreatectomies. The first stage, constituted a partial pancreatectomy in which the most arduous part of the operation, involving the removal of the portions of the pancreas which were closest to the duodenum and its supplying vessels. In this first operation the easier accessible parts of the pancreas were left behind. The second operation was not undertaken until it was clear from the post-operative recovery that the duodenum was not injured. The second stage operation involved an abdominal operation with its accompanying risk for peritonitis in an animal that was going to develop severe diabetes mellitus.

\section{A three-stage procedure}

In an attempt to enhance the survival rate and post-operative condition of pancreatectomized dogs further Minkowski designed a three stage procedure. This procedure further increased the persuasive strength of the experimental set-up.

Encouraged by Eiselberg's (1892) promising results in treating a thyreodectomized cat with a subcutaneous transplant of thyroid tissue Minkowski modified his two stage extirpation procedure, in which pancreatic tissue was either extirpated or left in situ, into an partial transplantation and extirpation procedure. In view of the impaired wound healing and reduced resistance to infectious agents in diabetic dogs, Eiselberg's procedure with pancreatic tissue was likely to fail. A pancreatic transplant devoid of its original blood circulation would not heal sufficiently to restore the function of a removed pancreas. Furthermore, it would probably be destroyed by proteolytic enzymes produced by its own acinar tissue (auto-digestion).

Once again the topographical anatomy of the dog was very favorable for Minkowski's purpose. The distal portion of the descending part of the pancreas in 
dogs is located free in the mesentery. The distal portion of the pancreas was separated from the rest of the gland in such a way that it could be mobilized while attached to a long mesenteric pedicle that contained the blood vessels (see figure 3.2). This piece of pancreatic tissue was then pulled out of the abdominal cavity through an incision in the muscular abdominal wall and fixed in a subcutaneous pouch. The blood supply through the stalk was usually sufficient to obtain smooth healing. The transplants also

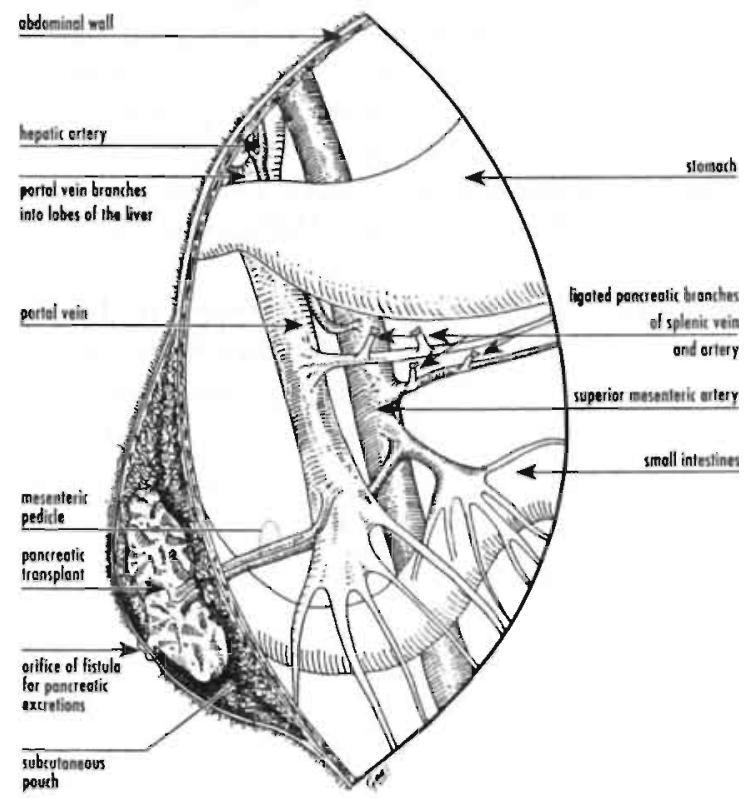

Figure 3.2

Pedicled pancreatic graft in subcutaneous pouch

continued to produce pancreatic juice. To avoid retention of pancreatic excretions (swelling, obstruction of blood supply) the transplant was provided with an outlet, a fistula, to the surface of the skin. This operation constituted the first stage of Minkowski's three stage procedure. These animals did not become diabetic.

The second stage operation was performed after sufficient healing of the subcutaneous transplant. It consisted in the extirpation of the intra-abdominal rest of the pancreas. ${ }^{15} \mathrm{~A}$ well functioning pancreatic transplant fulfilled the function of the whole gland in carbohydrate metabolism. The abdominal part of the operation could thus be completed without the emergence of diabetes. The extensive injuries afflicted in the dog's abdomen were allowed to heal under beneficial conditions. The injuries in the abdomen after this second stage operation were - apart from the mesenteric pedicle - identical with the ones after a total pancreatectomy. If it was not the loss of pancreatic function that caused the diabetes, but rather some kind of accompanying injury to adjacent tissues and structures in the abdomen, the diabetes should have exhibited itself after this second stage operation. But, it did not. ${ }^{16}$ 
That it indeed was the pancreatic transplant in its subcutaneous pouch that held the diabetes off was demonstrated in the third stage operation. This was a comparatively minor operation: the removal of the transplant. This could be done through a simple incision in the skin over the pouch by ligating and cutting the mesenteric pedicle extraperitoneally.

That it was in fact only the subcutaneous intra-abdominal piece of gland that prevented the development of the diabetes in these cases, is shown by the fact that the excretion of sugar immediately occurred with great intensity as soon as the subcutaneous part of the pancreas was removed later. In this way, it is therefore possible, by means of a very simple operation which only takes minutes and can be performed without cutting open the abdomen and in which accompanying injuries are absolutely out of the question, to produce a diabetes of the severest kind which will persist until the death of the animals. (Minkowski 1893: 121)

The mere extraperitoneal ligation of the blood vessels in the pedicle without removal of the transplant - a procedure which Minkowski followed in two dogs, 12 and 17 days after the second stage operation - produced the same result: diabetes. In Minkowski's view there was no doubt that it was the blood in the blood vessels that carried the unknown substance that mediated the pancreatic function in the organism's metabolism.

\section{Distinguishing between external and internal secretion}

The experimental set up of these experiments was also 'revealing' with regard to the relationship between the secretory, or better, between the excretory function of the pancreas and the pancreatic function that mediated the utilization of sugar. The pancreatic juice produced by the subcutaneous transplant drained to the surface of the skin. It could be collected there and examined in the laboratory. When the secretion of pancreatic juice diminished it could be obtained with a catheter through the fistula. Some dogs were not diabetic after the second stage operation, while on the other hand no secretion was obtainable from the fistula of the transplant (neither were there other signs of retention). In some other dogs, reported by Thiroloix (1892), the diabetes ernerged after some time while the excretion of pancreatic juices by the transplant continued unimpaired. Thus, the excretory function of the pancreas and the new function that prevented the appearance of diabetes and mediated the utilization of sugar by the organism, were independent. ${ }^{17}$

\subsection{Langerhans' islands and diabetes}

\section{Ductless glands}

Minkowski's work was well received. It caught the interest of a number of researchers working in different scientific disciplines. Some of them, like Hédon and Thiroloix, started extirpation and transplantation experiments themselves. They tried to evaluate Minkowski's results and claims in their own laboratories and when possible to go beyond them. 
There was another group of researchers who gave their assent to Minkowski's claims without subjecting his work to a test in their own laboratories under similar experimental conditions. They saw no reason to doubt his results. On the contrary, they used it as a starting point or as a heuristic suggestion in their own research. Their work added to the plausibility of Minkowski's claim because they suggested a morphological substrate for the hypothetical new function of the pancreas.

For some anatomists Minkowski's work revived an interest in the groups of cells scattered in the pancreatic tissue. These groups of cells had been described by Paul Langerhans (1869) as distinct histological entities. These anatomists considered it to be unlikely that the glandular (acinar) tissue producing and secreting the pancreatic juice also produced Minkowski's hypothetical substance. Langerhans' islands, lacking a satisfactory explanation in terms of their function ${ }^{18}$, provided a good candidate as the anatomical and morphological substrate for Minkowski's new function of the pancreas.

Laguesse (1893), working on the embryological development of the pancreas and of the islands in different species, seems to have been the first to suggest Langerhans islands as the site of production of the internal secretion of the pancreas ${ }^{19}$. (Papaspyros 1964) Laguesse did not find direct evidence for this assumption in his own research. The only thing he could see under the microscope was that the islands were located in close proximity to a network of capillary blood vessels. Discontent with other explanations concerning the function of the islands he argued that, if the pancreas had some other function in the system besides secreting digestive juice, the islet cells were probably involved.

Schäfer (1895) also associated the internal secretion of the pancreas proposed by Minkowski with this

... peculiar epithelium-like tissue which occurs in isolated patches throughout the organ and which is characterized by its extreme vascularity.(Schäfer 1895: 341)

In a paper On internal secretion, in which he reviewed research on the thyroid and adrenal glands, Schäfer stated that

... [t]he only fact that appears certain in connection with the manner in which the pancreas prevents excessive production of sugar within the body is that this effect must be produced by the formation of some material excreted internally by the gland, and probably by the interstitial vascular islets, and that this internally secreted material profoundly modifies the carbohydrate metabolism of the tissues. (Schäfer 1895: 342)

Schäfer, an experimental physiologist working on internal secretions generally, a subject that he found to be of far-reaching interest, the full importance of which had only lately been recognized, supported Minkowski's explanation of pancreatic diabetes in terms of (the removal by extirpation of an internal secretion. Schäfer suggested a number of other reasons why Langerhans' islands might very well play the role of the production site.

Schäfer emphasized the morphological analogy or similarity between the extremely vascular islands in the pancreas and the thyroid and the adrenal glands, 
which also were very vascular. Work on the thyroid and the adrenal glands had yielded biologically active tissue extracts. The internal secretion of these ductless glands had recently been established. Stressing the high vascularity of the islands Schäfer argued that these cell groups in the pancreas might constitute a ductless gland of their own: a gland secreting its product to the interior of the body (into the blood stream), contained within a gland known to secrete its digestive juice to the exterior surface of the body, that is into the duodenum. If the islands indeed were ductless glands then they were also very likely to produce an internal secretion.

\section{Langerhans' islands and pancreatic atrophy}

There was another argument in Schäfer's 1895-paper. He referred to Minkowski's, Thiroloix' and Hédon's duct-ligation and transplantation experiments. These experiments were designed to show that the pancreas' new function was independent of the hitherto known excretory function of the pancreas. Both ligation of the pancreatic ducts and transplantation of a piece of pancreatic tissue under the skin - while the rest of the pancreas was removed from the abdomen - prevented the passage of pancreatic juice to the gut. Neither of these researchers reported data on the islands in the remaining degenerated gland or in the transplant. Schäfer was not aware whether the gland, which had been caused to atrophy by duct-ligation, still showed the interstitial vascular epithelium-like tissue. Nevertheless, he was almost certain that they did not participate in the atrophy of the secreting part of the gland, and therefore he assumed that

... the pancreas mainly owes its function as an internally secreting organ, at least as far as regards the prevention of diabetes, to this tissue ... (Schäfer 1895: 342)

Schäfer suggested that a strong argument in favor of the internal secretion theory of the pancreas would be provided by the demonstration that the island tissue was the only tissue that remained healthy and intact in an atrophied gland, the subsequent removal of which caused severe diabetes. A couple of years later Schulze (1900) and Ssobolew (1902) set out to do just that.

Instead of ligating the pancreatic ducts, Schulze ligated part of the pancreas itself, allowing the rest of the gland to deliver its juice to the duodenum, thus preventing the digestive problems associated with total blockage of the pancreatic ducts. The ligated part of the pancreas would atrophy, however. He performed this procedure in a series of animals at the same time, and killed them after different time-intervals. Schulze found

... a rapid degeneration of the ligated part of the pancreas with the exception of the islands, which remained completely unchanged .... a gradual progressive atrophy of the pancreas, in which the islands of Langerhans did not participate: they did not display the slightest change and eighty days after the ligature they offered the same view as before. (Schulze 1900: 499-500) 
Schulze partitioned the island tissue off from the excretory digestive system of the pancreas and concluded that they constituted an organ sui generis. an organ in its own right, a gland within a gland, carrying a distinct function.

Ssobolew ligated the pancreatic ducts in a series of rabbits, dogs and cats. These animals were killed at different time intervals after the duct-ligation, varying from 1 to 400 days. In this way Ssobolew was able to study the whole degeneration process in considerable detail. He found that

$\ldots$ in the gland the system that is subject to an atrophy is the one which is closely connected to the excretory passages and apparently only serves the digestion, these are the glandular ducts, whereas another system is preserved, the islands of Langerhans. (Ssobolew 1902: 97)

The island tissue survived all the other tissue elements in the degenerating pancreas and this was sufficient to prevent the emergence of diabetes. Only subsequent removal of the gland would cause diabetes. According to Ssobolew the results confirmed the study's hypothesis that Langerhans' islands carried a second function of the pancreas concerned with the metabolism of carbohydrates in the organism.

In a second series of experiments Ssobolew imitated the first stage of Minkowski's three-stage procedure. He made a subcutaneous pancreatic transplant while leaving the rest of the gland in the abdomen. During the following five or six weeks the transplants continued to excrete pancreatic juice through a fistula in the skin. Then the secretion ceased indicating degeneration of the acinar tissue. Examining the transplants several weeks after cessation of the external secretion Ssobolew found that

... in case of a transplantation under the skin the gland can atrophy in toto, while the islands of Langerhans prove to be the most resistant elements and also prevent the development of a diabetes. (Ssobolew 1902: 107)

\section{Increased sugar load and cellular granules}

Ssobolew set out to provide another strong argument in favor of the hypothesis that Langerhans' islands were the site of production of the internal secretion of the pancreas. He induced different levels of activity of the island's cells, either by starving the animal or by increasing the carbohydrate content of its food. He further increased the sugar load of the organism by subjecting the animal to a partial pancreatectomy with subsequent intravenous sugar injections. He killed the animals a couple of hours later and examined the island tissue of their pancreases. Ssobolew paid special attention to the number of granules in the cells. Ssobolew found the greatest number of granules to be present in island cells of animals that had been starved for 2 - 3 days. The number of granules decreased proportional to the increase in relative sugar load. In a partially pancreatectomized dog which had been given carbohydrate rich fodder and intravenous sugar injections Ssobolew observed a reduction of the volume of the islands' cells, the protoplasm of which contained almost no granules at all. He concluded from these experiments that 
... in the cells of the islands of Langerhans some substance in the form of granules $\ldots$ is produced. The biggest granules are situated against the cell's periphery. The number ... which is the greatest in case of a short period of starvation, decreases considerably when the organism is in one way or the other forced to use a certain quantum of carbohydrates, i.e. either to store them in the form of glycogen in the tissues, or to transform them into other substances, e.g. fat, or eventually to simply bum them. (Ssobolew 1902: 108)

It is important to understand what Ssobolew saw under his microscope and what he did not see. Ssobolew examined different slides with pancreatic tissue. In each distinct slide he found a certain number of red-colored granules in the cells of Langerhans' islands; the number of granules were different in each slide. He could relate each slide to the metabolic condition of the animal from which it was taken. Ssobolew could not see the actual secretion process. If the pancreas produced an internal secretion it might well be produced in the cells of Langerhans' islands, and if it was produced in these cells then the red granules in the cells protoplasm might well contain it. This is how Ssobolew interpreted his results, as arguments in favor of an internal secretion theory for the pancreas and for diabetes.

\section{Working with autopsy materials from human diabetics}

For pathologists working with autopsy materials from human patients matters were more complicated. 'Experimentalists', like Schulze and Ssobolew, worked with healthy animals, They caused the pancreas' excretory system to degenerate and showed that morphologically unaltered, healthy looking islands were sufficient to prevent diabetes.

The starting-point for a non-experimental pathologist was different. He worked with the dead bodies of human patients who might, or who might not have displayed symptoms of a more or less severe form of diabetes mellitus while they were alive. All kinds of different ailments which a patient might have experienced throughout his life, of which only a probably incomplete record was available to the pathologist, might have left traces (inscriptions) in the patients body. Some of these, scars, structural changes in the tissues of the body, might have been visible by the naked eye. Others might only be visible by the use of appropriate preparation and staining techniques and the microscope. It was not always possible to relate specific pathological changes in the dead body to diseases the patient experienced during life.

The same holds for the post mortem examination of diabetics. Often the pathologist found multiple morphological changes in different organ systems, in the brain or in the peripheral nervous system, in the kidneys and sometimes in the pancreas. ${ }^{20}$

Minkowski's work on experimental pancreatic diabetes increased the relevance of pathological changes in the pancreases of dead diabetic patients. When in experimental animals healthy looking islands, in an otherwise degenerated pancreas, prevented diabetes, while their removal induced diabetes, then one would expect to find pathologically affected islands in humans suffering from a diabetic condition. But this was not the case.

According to Carl von Noorden (1893), gross anatomic changes were rarely found in the pancreas of diabetics. In most cases of diabetes mellitus in humans a macroscopically and microscopically healthy pancreas was found. Proponents of a 
pancreatogenic etiology of diabetes had to resort to the hypothesis that morphologically invisible, pure functional deviations in the pancreas' function caused such profound changes in carbohydrate metabolism. (Heiberg 1910)

As a result of the highlight experimental pancreatic diabetes put on the pancreas, directing the pathologist's attention to this organ when doing an autopsy on patients known to have suffered from diabetes during life, more and more anatomical changes were observed and reported. The nature of these changes and their grade of development were wide ranging though. No conclusions could be drawn from them, except the very unsatisfactory conclusion that anatomic changes were more frequent than previously assumed. Despite the registration of more structural deviations found in the pancreases of diabetics, reports of cases of severe and lethal diabetes mellitus were still being published in which neither macro- nor microscopical changes could be demonstrated. With regard to this paradox in the medical literature Ssobolew wrote that

... this contradiction ... confused the authors and made them often think of the enunciation by Goethe's Faust after going through the literature: "There I stand, I poor Thor, and I am as clever, as I was before". (Ssobolew 1902: 119)

\section{Improving pathological observation}

More work had to be done in this field of non-experimental 'human' pathological anatomy. Reviews of the pathological literature concerning anatomical changes of the pancreas in relation to diabetes revealed a number of faults in the older work. The most obvious shortcoming of older pathological work was that most of it was based on macroscopical observations only. Without the proper employment of staining techniques and the microscope relevant but microscopic anatomic changes in the pancreas of diabetics would inevitably be missed. A second shortcoming was in the lack of knowledge concerning Langerhans' islands on the side of the pathologists. Ssobolew complained as late as 1902 that only one of the available textbooks for pathological anatomy contained an adequate description and functional specification of the islands.

A number of pathologists undertook systematic studies of the microstructure of pathological changes in the pancreas. Opie, for example, set out to

... observe what changes [the islands of Langerhans] undergo under pathological conditions, and if possible, to bring such alterations into correlation with concomitant pathological phenomena. From such a study, heretofore little pursued, we may hope for suggestions of the function or anatomical relationship of these obscure structures. (Opie 1900a: 399)

Since the islands of Langerhans did not always show alterations corresponding to those which occurred in the tissues of the acini about them - being little implicated in some varieties of chronic inflammation while markedly affected in others - it was interesting to study the relation of the islands to various forms of chronic pancreatitis that were distinguishable. However, according to Opie, the histological details of chronic pancreatitis had been little studied and only slight attention had been given to the 
classification of various types. In the light of the experimental demonstration of a relation between the pancreas and carbohydrate metabolism the research goal for pathologists was to distinguish a type of chronic pancreatitis constantly associated with diabetes mellitus. ${ }^{21}$ (Opie 1900a)

While studying the relationship between pathological changes in the pancreas' islands of Langerhans and the occurrence of diabetes Opie came across a case in which during the last months of the patient's life diabetes mellitus accompanied pulmonary tuberculosis - which was the cause of death. Post mortem microscopic examination of the pancreas revealed remarkable degenerative changes of the islands of Langerhans only. Opie found deposits of hyaline material in almost every island. The acinar, glandular tissue of the pancreas, on the other hand, was well preserved and morphologically normal. Non-experimental pathological processes had yielded in this patient a state of affairs that scientists had been unable to accomplish in experiments.

Structures so embedded as are these islands in the substance of the gland cannot by any means now at our disposal be subjected to experimental alterations without injury to the surrounding alveoli. In the pancreas described ... a lesion of obscure etiology has destroyed the cells of the islands of Langerhans, while those of the secreting acini, as well as those of other organs, are unaffected. The most successful experiment could no more accurately have selected these bodies. (Opie 1900b: 539)

Admitting that no alteration of the pancreas was demonstrable in many instances of diabetes, and that diabetes was present in only a limited number of cases where pancreatic disease had existed, Opie concluded that where diabetes was caused by a lesion of the pancreas, the lesion was of such a character as to destroy or injure the islands of Langerhans, and where, though the organ was diseased, diabetes was absent, the interacinar islands were relatively unaffected. In the association of diabetes mellitus with the specific destruction of the islands of Langerhans in that one case Opie found convincing proof that

$\ldots$ it is those structures which influence carbohydrate metabolism. What has been learned conceming the relationship of the pancreas to diabetes is the relation of the islands of Langerhans to this disease. (Opie 1900b: 540)

\section{Getting stronger}

Ssobolew, Schulze and Opie did not put Minkowski's work to the test. They assented to Minkowski's central claims and carried them further. Where Minkowski tried to establish a strong link between diabetes mellitus and the pancreas, pathologists and anatomists forged a link between Langerhans' islands and diabetes on one hand, and between the islands and Minkowski's hypothetical internal secretion of the pancreas on the other.

These links seemed to be getting stronger as more work was done. In 1909 Cecil found that 87 per cent of the ninety cases of diabetes he studied post mortem showed either sclerotic or hyaline degenerations of the islets. Thirteen per cent appeared normal both macro- and microscopically. The notion of functional disturbances of the 
islands' cells, which could not be visualized with the available histological techniques, covered for the rest. (Cecil 1909)

In 1920 Barron was confident enough to write that

... the universally admitted facts, that the islets of Langerhans are involved in at least a majority of the cases of diabetes and that no case of alteration of the islets is encountered without an accompanying diabetes, makes it evident that the islets secrete some substance or hormone into the blood which is necessary for the utilization of sugar by the tissues. (Barron 1920: 444) ${ }^{22}$

\subsection{Moving out of the laboratory: at least attempts to do that}

\section{Substitution therapy}

One might be impressed by the subtlety and elegance of Minkowski's extirpation and transplantation experiments. However, one of the most appealing aspects of the internal secretion theory for the explanation of pancreatic diabetes was that it suggested a program of action for the successful treatment of diabetes mellitus in humans. This drew and fixed the interest of a great number of scientists and physicians. They assumed that, if Minkowksi was right, it should be possible to use the internal secretion in fresh pancreatic tissue of animals to substitute the deficiency in human diabetics. The pancreases of animals provided an exogenic source of pancreatic internal secretion that could be utilized to replenish the diminished or lost endogenic production in diabetic patients. The hypothetical character of Minkowski's claim about the pancreas' internal secretion did not hinder them in pursuing its implications. The use of animal tissues in the treatment of diseases had a long tradition called organotherapy or opotherapy. ${ }^{23}$ (Koopman 1926; Rolleston 1936)

In 1891 Murray published a Note on the treatment of myxoedema by hypodermic injections of an extract of the thyroid gland of a sheep, which according to Medvei (1982) became the first generally recognized success of organotherapy. A number of scientists followed the same approach in the case of the pancreas. The analogy between pathophysiological mechanisms in the thyroid and the pancreas was explicitly drawn in an editorial comment in the British Medical Journal of december 31, 1892:

Minkowski has continued his important work on pancreatic diabetes, and Hédon has confirmed most of his results. They find that glycosuria which follows extirpation of the pancreas can be stopped by transplantation of a portion of a living pancreas into the abdominal wall. This, taken in conjunction with the beneficial result that follows injection of the thyroid juice in myxedema shows us that glands have actions in general metabolic processes which were before unknown but the modus vivendi has yet to be discovered. ${ }^{24}$

Although the modus operandi of these tissue extracts was still a sealed book, and the composition of the internal secretion was still unknown, it was presumed that an extract of the fresh organ must contain the active substance in the conglomeration of bodies which are extracted by glycerine, salt solutions, or whatever the solvent used 
may be. For Brown-Sequard this constituted the rationale for the 'organotherapeutic' method.

When a morbid state, as myxedema, or a series of symptoms such as we see in cases of deficiency to the internal secretion of any gland, exists, it is very easy to understand how the cure is obtained when glandular liquid extracts are used: we simply give to the blood the principle or principles missing in it ... (Brown-Séquard 1893: 1213)

The principle was easy to understand. The practice, however, was obstinate. Minkowski's experiments did not allow any conclusion concerning the chemical nature of the hypothetical internal secretion, or concerning its physical properties. Furthermore, there was the question what had to count as success. No biological assay was available yet. ${ }^{25}$ When was the administration of pancreatic tissue (extracts) considered to be effective? The obvious goal of the treatment was the alleviation of diabetic symptoms, that is, reduction of the glycosuria, improvement of the general condition, recovery from diabetic coma etc. But, how soon should the effect be visible: momentarily, within a couple of hours, the next day, the next week? And, how long should it last: a couple of hours, a day, a week or for ever? Some items for the list containing the active principle's criteria of existence could be indicated, but as yet these were rather unspecific.

Successful treatment of experimental diabetes mellitus by means of a pancreatic extract would provide another powerful argument for the existence of an internal secretion of the pancreas. Until its effect could be practically demonstrated the internal secretion was, despite the experimental evidence no more than a good looking hypothesis.

\section{Alternative routes of administration}

Pancreatic tissue extracts, which possibly contained the hypothetical active principle, could be administered in various ways. The first route went through one of the openings of the alimentary canal. Minkowski mixed fresh pancreas with the food for his pancreatectomized dogs. He could not detect a notable decrease in the amount of sugar excreted in the urine. Minkowski was not surprised by the negative result of this procedure:

... because it does indeed not involve an effect of the pancreatic juice in the intestine, but a function of the pancreas in the intermediary metabolism. (Minkowski 1890: 169)

Sandmeyer (1895). Goldscheider (1894), and, later, Pflüger (1905c) administered the pancreatic tissue or a pancreatic glycerin extract as an enema or clyster in the rectum of the animals, without success.

The only alternative to the enteral route for the administration of the hypothetical active principle was the parenteral injection of pancreatic extracts. Subcutaneous injection avoided the ruining influence of digestive juices in the gut. 


\section{Plain negative and harmful results}

Experiences with pancreatic extracts, as they were published in subsequent years by a great number of authors, were extremely disappointing. Most of them were plain negative or even harmful in view of toxic reactions causing fever and necrosis of the injection site. Others were at best ambiguous, leaving room for alternative explanations.

Battistini reported (1893) that he observed a gradual decline in glycosuria and improvement of the general condition in two (human) diabetics whom he treated with successive injections with pancreatic extracts. He obtained these preparations through the extraction of fresh pancreas with saline in one case, and with glycerin in the other. However, simultaneous with the beginning of the injections he prescribed a strict diet to these patients. According to Battistini, the improvement of the patients' condition might well have resulted from this dietary regime. ${ }^{26}$ Goldscheider's (1894) and Fürbringer's (1894) experiments with pancreatic glycerin extracts were also negative. Blumentahl (1898) obtained a pancreatic juice by pressing the tissue in a hydraulic press. He treated the pancreatic juice thus obtained with alcohol. Subcutaneous injections with this extract were followed by necrosis of the injection site, which made him stop the experiments.

In one case, for 2 days sugar metabolism increased by $40 \%$. The success did not last. A subcutaneous use of the extract however conflicts with the fact that it is very toxic ... (Blumentahl 1898: 257)

\section{Trial and error}

Despite the discouragement the search for an effective extract continued. The negative results did not necessarily lead to the conclusion that Minkowski's hypothesis about an internal secretion of the pancreas was false. It was argued that the active principle might be destroyed by proteolytic enzymes, produced by the acinar tissue, during the maceration and extraction process. Several strategies to avoid a possible destruction of the hypothetical internal secretion in the extirpated organ were designed. It was known from earlier work that ligation of the pancreatic duct caused the glandular tissue of the pancreas to atrophy. Langerhans' islands did not suffer from the ligation. This led several scientists to follow a two stage procedure in which they first ligated the pancreatic duct and allowed the organ to atrophy. After a couple of weeks the organ was extirpated and submitted to the extraction procedure. But, the extracts thus obtained did not do better.

Instead of using pancreases of adult animals, Laguesse (1893) suggested the use of extracts made from foetal pancreases instead. During intra-uterine growth and gestation Langerhans' islands develop well before the acinar tissue. But again, the extracts thus obtained did not do any better than the whole, adult pancreas.

Rennie and Fraser (1907), two Scottish researchers in Aberdeen, tried to utilize the specific anatomical conditions in the pancreas of certain species of fish. In fish the island-tissue is not dispersed in small groups of cells. It is arranged in larger united areas, distinct from the acinar tissue, from which it can be easily isolated. Rennie and Fraser prepared an extract from these fish islands and supplied it to four diabetic patients via the enteral route. They observed a gradual reduction of glycosuria after 
two months. These patients were also put on a strict diet. In one patient the authors tried a subcutaneous injection with the extract, which resulted in a toxic reaction. After these discouraging results they gave up. (Rennie and Fraser 1907)

\section{Different conclusions}

The list of publications and authors reporting negative results grew. Who knows how many failing attempts were never published because they were negative? It was a very paradoxical situation. On one hand, there were convincing results from experimental pancreatic transplantation experiments saying that the pancreas produced an internal secretion, an active anti-diabetic principle. On the other hand, there was cumulating evidence saying that no such thing could be found. Those who went to look for the internal secretion were disappointed because they found nothing.

In 1913 two authoritative diabetologists, John J. R. Macleod and Frederick Allen, summed up a generation's attempts to capture the still hypothetical internal secretion of the pancreas in an extract. After due deliberation Macleod articulated the paradoxical conclusion that there might exist an internal secretion of the pancreas, but. in an attempt to explain the failures to abolish glycosuria in pancreatectomized animals by injecting extracts of pancreas, he simultaneously suggested several reasons why it might never be captured in an extract. According to Macleod,

$\ldots$ in a pancreatic extract, besides any substance which may have an action on metabolism, are also several very powerful ferments which may quickly destroy that substance; and, moreover, there may be no ready-made internal secretion in the pancreatic cells themselves: it may be produced in small quantities at a time, according as it is required, or it may exist in the gland as a precursor, and become activated only after is has been secreted into the blood. (Macleod 1913: 91)

According to Allen all authorities agreed upon the failure of pancreatic opotherapy in diabetes. Attempts were continued because of the strong theoretical inducements. (Allen 1913: 813) Allen came to the conclusion that

... injections of pancreatic preparations proved both useless and harmful. The failure began with Minkowski, and has continued to the present without an exception. (Allen 1913: 815)

A few more or less favorable claims had been published. Some authors reported a slight or transient reduction in glycosuria. But these findings could, according to Allen, be doubtless explained by renal or other non-specific changes. For human diabetic patients a genuine benefit was never obtained. According to Allen,

[t] ]he negative reports were numerous and trustworthy. (Allen 1913: 816)

Despite the strong theoretical inducements derived from the transplantation studies one particular conclusion seemed to be unavoidable. This conclusion was expressed by Leschke (1910) who finished an extensive study in which he injected pancreatic saline extracts into pancreatectomized dogs. His results were also negative; in many cases 
administration of pancreatic extract was followed by an increase in the excretion of sugar. Leschke expressed serious doubts about the very existence of an anti-diabetic substance in or produced by the pancreas:

However, the existence of this hypothetical, anti-diabetic substance seems very questionable after all these experiments; and we probably can add the results of these experiments to the numerous arguments that plead against the theory of the internal secretion ... of the pancreatic diabetes. (Leschke 1910: 435; my emphasis)

Pursuing an organotherapy for diabetes mellitus any further seemed pointless to Leschke.

The practical significance of these experiments is that the foundation of the pancreatic therapy of the diabetes is abolished. ... in subcutaneous, intra-peritoneal and intravenous administration of pancreatic extract, the excretion of sugar is increased and a considerable deterioration of the general state of health involving ague, fever, lack of appetite, necrosis of the tissue and possibly suppuration of the injection site is furthermore achieved, so that one does not only not aid but directly harms. (Leschke 1910: 435)

\subsection{Amplifying the paradox}

\section{Parabiosis}

While discouraging negative results concerning the use of pancreatic extracts accumulated on one hand, new experiments in new experimental designs suggested that, nevertheless, the pancreas produced an internal secretion. ${ }^{27}$

Forschbach, working in de medical clinic in Greifswald - which at that time was headed by Minkowski -, realized that parabiosis, as described by Sauerbruch and Heyde (1908), provided a suitable experimental design for the investigation of the pathogenesis of pancreatic diabetes. Sauerbruch and Heyde succeeded in joining a couple young rabbits, from the same sex and from the same birth, in a lasting organic unification, called parabiosis. Either the skin and muscular layers of corresponding areas on opposite sides of the animals were incised, leaving the peritoneum closed, or the depth of the incision was extended through the peritoneum, opening the abdominal cavity. Corresponding wound margins were then sutured together; the wound of one animal was sutured to the wound of the other animal. Then the wound was allowed to heal.

Forschbach (1908a, 1908b) succeeded in doing the same with dogs, both puppies and older dogs (see figure 3.3). Healing was normal in many cases, and as part of this process newly formed blood vessels proliferated into the wound, from one animal to the other. The vascular system of one animal was thus connected with the vascular system of the other, allowing for an exchange of blood and of everything that the blood carried with it. The exchange of blood between the two animals was shown by the injection of iodine in one animal followed by it's excretion in the urine of the 
other. Likewise it could be demonstrated that lethal poisons like strychnine and bacteria found their way from one animal to the other. (Forschbach 1908b)

As a next step in the experiments the pancreas of one of the animals living in parabiosis was extirpated - lege artis, by Minkowski. In these animals diabetes did not appear or did not appear with the same vehemence as in pancreatectomized single dogs. In one of the experiments surgical separation of the animals one week after the

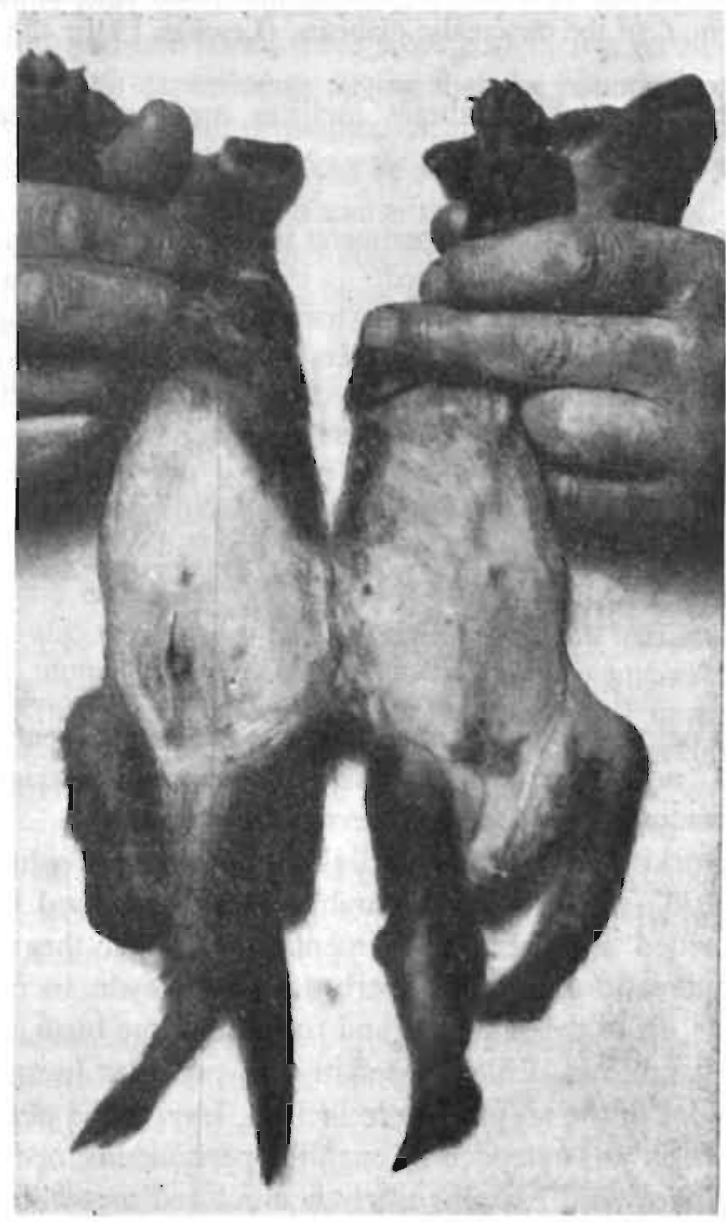

Figure 3.3

Parabiosis in dogs

Reproduced from Forschbach (1908b: 139)

pancreatectomy resulted in the immediate onset of severe diabetes in the pancreatectomized animal, while the other animal, still in possession of its pancreas, survived the operation.

After a small series of experiments of this kind Forschbach concluded that 
[i]f the pancreas of one of the dogs living in parabiosis is completely extirpated, the glycosuria in the other dog does not only stop temporarily, respectively drop, but the behavior of the animal, which hardly differs from that of the healthy dog, the smooth healing of the wound, the absence of cachexia, also lead to the conclusion that the diabetic metabolic disorder is attacked at its root. (Forschbach 1908b: 146)

Forschbach argued that only the internal secretion theory provided a satisfactory explanation for these results. The internal secretion of the remaining pancreas (in one animal) was transferred by the blood through the communicating vessels to the other pancreatectomized animal where it exhibited its anti-diabetic function. ${ }^{28}$ The only possible route of transfer of the anti-diabetic influence or principle was by way of blood or lymph through the parabiotic communication.

\section{Pancreatectomy and pregnancy}

Another paper supporting the internal secretion theory for pancreatic diabetes was published in 1911 in the United States by Carlson and Drennan. During a pancreatectomy on a female dog Carlson and Drennan found that the dog was pregnant. They noticed that after the operation the rise of the sugar content of the urine was slower and that the top of the curve was lower than in non-pregnant pancreatectomized dogs. These incidental observations, together with Forschbach's work on parabiosis and pancreatectomy, led them to believe that the pancreas of the fetus might furnish sufficient internal secretion to influence if not prevent the diabetes in the mother after pancreatectomy. (Carlson and Drennan 1911).

Carlson and Drennan conducted several pancreatectomies in pregnant dogs, at different stages of the pregnancy. A pregnant dog nearing full term of pregnancy recovered well from the pancreatectomy. She continued in good condition and was observed for five days without showing a trace of sugar in the urine. Polyuria was not marked and the wound in the abdomen did not ulcerate as much as was the case in non-pregnant dogs after pancreatectomy. Despite good recovery the dog appeared to grow weak, so that it was doubtful whether she would be capable of giving birth to the pups if allowed to run to full term. A cesarean section was then performed. Recovery was good allowing for the examination of the urinary excretion of sugar during the following days. The dog displayed the sugar excretion curve typical for pancreatic diabetes. From these experiments Carlson and Drennan concluded that

\footnotetext{
... in a normally developed fetus nearing full term the pancreas is so developed, qualitatively and quantitatively, as regards the internal secretion necessary for normal sugar metabolism that the fetal pancreas acts as a residual pancreds of the mother on complete pancreatectomy of the latter. The internal secretion of the fetal pancreas passes through the uterine membranes in sufficient quantity to prevent diabetes in the mother. (Carlson and Drennan 1911: 393) ${ }^{.9}$
}

In reviewing these observations Macleod (1913) argued that it seemed justifiable to conclude that the pancreas owes its influence over carbohydrate metabolism to an internal secretion. This conclusion was greatly strengthened, according to Macleod, by Knowlton and Starling's experimental observations that sugar consumption in an isolated, perfused heart decreased when the heart was perfused with blood from a 
diabetic animal. These results could, according to Macleod, most simply be interpreted as indicating that some hormone necessary for the direct utilization of sugar by the tissues is absent in the diabetic blood. (Macleod 1913)

\subsection{Continuing the search: Georg Ludwig Zuelzer}

\section{Asymmetrical explanations for failure and success}

Despite accumulating discouraging results of attempts to extract the internal secretion, the existence of which was proclaimed by so many experimental physiologists, the search for the internal secretion was not abandoned. Many scientists and physicians gave up. But, there were always new ones who were excited by the idea of extracting an anti-diabetic principle from the pancreas for the benefit of thousands of diabetic patients. Besides the elegance and persuasiveness of a variety of experimental designs suggesting the existence of a pancreatic internal secretion, the promise of such a consequential future program of action for the treatment of diabetes was a strong incentive for many scientists who tried to capture the active principle in a pancreatic extract. In this respect the internal secretion theory stood in contrast with Pflüger's neurogenic theory of diabetes. Explaining the neurological reflex mechanism involved in carbohydrate metabolism, allowing diabetes mellitus to be caused by any abnormal stimulation of nervous tissue in any region of the nervous system, Pflüger admitted that from the perspective of the diabetic patient one might regret such an arrangement, considering the large number of nerves and the extent and distribution of the nervous system in the body. However,

... the physiologist can not agree. He inquires only for the use of the arrangement.

(Pflüger 1905: 403 - 404)

Thus, contrary to Minkowski's internal secretion theory, the neurogenic theory did not suggest a single therapy that, in the future, would save the lives of thousands. As a scientist, Pflüger' interest was in the functionality and fitness of neurological reflex mechanisms in meeting the tissues' oxygen and sugar requirements as part of the intricate and complex physiological processes through which homeostasis is maintained.

However, pursuing the realization of the therapeutic program of action contained in the internal secretion theory of diabetes, required that a belief in the existence of the pancreatic internal secretion be maintained, and that ambiguous, failing and negative results of extraction experiments could be explained. Those who continued the search for the active principle, despite cumulating failures, used asymmetrical, that is, different types of explanations for failure and success. The sought for anti-diabetic effect would have been explained by referring to the activity of the really existing internal secretion, while negative results could be explained by referring to something (everything) else, except the non-existence of the internal secretion. This 'mechanism' can best be illustrated in the work of Georg Ludwig Zuelzer. ${ }^{30}$ Furthermore, Zuelzer's work also illustrates how this problem of explaining failures and success is linked and mixed up with the problem how to know whether the sought for substance is present 
in the extract, when nothing is known about its chemical and physical properties, or about its physiological functions. What should the extract be able to do? Which of the observed effects could be attributed to the activity of the active principle?

\section{Adrenal diabetes}

Zuelzer's interest in work with pancreatic extracts was related to Blum's (1901) report on adrenal diabetes. (Zuelzer 1901) Injection of extracts of adrenal tissue was followed by the excretion of sugar in the urine. This glycosuria lasted for 48 - 72 hours. Blum also observed a rise in the concentration of sugar in the blood.

Zuelzer noticed the resemblance between this experimental adrenal diabetes and human diabetes mellitus, but he also saw the differences:

[Adrenal diabetes] is only distinguished from [human diabetes mellitus] by the duration of its existence and neither does it display a tendency towards progression, i.e. never could it display the final stage of the usually severe human diabetes... (Zuelzer 1907a: 474)

Although many critics considered adrenal diabetes to be no more than a kind of toxic glycosuria, to be distinguished from diabetes mellitus proper, Zuelzer found the analogy with human diabetes strong enough to do more work on it. He drew a different boundary between 'real' diabetes and 'mere' glycosuria and tried to reestablish the relevance of Blum's experimental adrenal diabetes in animals for the understanding of human diabetes mellitus. He rejected the idea that adrenal diabetes constituted an unphysiological toxic reaction of an organism to the injection of adrenal extract.

It did not seem very plausible to me that a body, which appears unchanged, in the way it normally produces and is supplied with the flow of juices from the organism, that such a quasi-physiological body could generate a completely non-physiological reaction. (Zuelzer 1907a: 474)

Zuelzer did not have a laboratory of his own. However, he was allowed to conduct a number of experiments in the laboratory of the physiological institute of the University of Berlin. ${ }^{31}$

\section{Adrenal-pancreatic antagonism}

In his research Zuelzer focussed on the organ that was 'in charge' of sugar regulation in the first place: the liver. Zuelzer perfused the liver of freshly killed dogs with defibrinated blood. He measured the concentration of sugar in pre- and in post-hepatic blood. The dogs were either healthy (prior to the experiment), or pancreatectomized (diabetic), or at the top of an adrenal diabetes. In healthy dogs he found an increase in blood sugar concentration between 8,5 and $15 \%$. In pancreatectomized dogs he found an increase of 27,31 and $66 \%$. In the adrenal diabetic animals he found an increase of 50 and $113 \%$ respectively. Hence, pancreatectomy and subcutaneous injection of adrenal extract had the same kind of effect on the liver. Zuelzer argued that the mechanism was analogous in both cases. In the case of adrenal diabetes there 
was an increased adrenal juice activity caused by the administration of exogenic adrenal extract. In pancreatic diabetes there was also an artificial increase of adrenal juice activity, but this time it was endogenic. The pancreatectomy provided either a stimulus to the adrenal glands to secrete more juice, or, which seemed more plausible to Zuelzer, a normal amount of adrenal juice could effectuate its activity on the liver because pancreatic secretions, which in physiological circumstances neutralize the adrenal juices, were absent.

In other words, I assumed that the secretion of the adrenal glands is usually neutralized by the secretion of the pancreas, so that as a consequence, the pancreatic diabetes discovered by Minkowski represents an adrenal diabetes. (Zuelzer 1907a: 475)

Experimental diabetes following pancreatectomy was in fact, according to Zuelzer (1908) a negative pancreatic diabetes and a positive adrenal diabetes. It followed from this hypothesis about a adrenal-pancreatic antagonism that simultaneous injections of adrenal and pancreatic extracts would neutralize each other, that is. glycosuria would not occur. Zuelzer followed this procedure and injected rabbits simultaneously with pancreatic and adrenal extracts. According to Zuelzer, glycosuria was never found. ${ }^{32}$

It also followed from Zuelzer's hypothesis that simultaneous elimination of endogenic sources of pancreatic and adrenal secretions would have the same effect, that is, no glycosuria. Pancreatic secretions could be eliminated by pancreatectomy according to Minkowski's procedures. No animal, however, survived extirpation of the adrenal glands. However, the ligation of the adrenal veins provided an alternative to adrenal extirpation because it also prevented the adrenal secretions to get into the circulation. A number of these operations failed (death within 24 hours). Eventually Zuelzer was able to report the successful conduct of two such operations on dogs. One of them lived 24 hours after the operation. The other survived for 36 hours. Apart from a transient glycosuria in the first urine portion, which was also observed when the adrenal veins were ligated alone, no glycosuria was found in these animals. Zuelzer performed over a hundred simple pancreatectomies in dogs and always found severe glycosuria emerging within a time span of six to eight hours following the operation. (Zuelzer 1908)

\section{The same therapeutic implications}

These results enhanced Zuelzer's belief in the heuristic utility of his hypothesis. Although both spokesmen, Minkowski and Zuelzer, offered different translations of the results obtained in extirpation experiments, they shared the conclusion that the pancreas produced an internal secretion that exerted an anti-diabetic function in the organism. Concerning the mechanism by which this internal secretion prevented diabetes they disagreed. Minkowski argued that the internal secretion facilitated the utilization of sugar. In diabetes this facilitating capacity was reduced, and as a consequence, nothing being wrong with the production of sugar: the concentration of sugar in the blood rose and spilled over in the urine, producing glycosuria. According to Zuelzer insulin neutralized adrenalin. In diabetes this neutralizing capacity was reduced. As a consequence adrenalin could mobilize more sugar from the liver thus 
giving rise to an increase in blood sugar concentration and glycosuria. The therapeutic implication of both theories was the same, despite their differences in content: there was an internal secretion produced by the pancreas, and if it could be extracted it might be used therapeutically in human diabetes mellitus. ${ }^{33}$ Theory, in Zuelzer's view, served a heuristic function.

$[\mathrm{H}]$ as this theory ... fulfilled its task to serve us as a bridge into therapeutic terra incognita? If the terra incognita does not prove to be a land of fantasy, the theoretical bridges may therefore be pulled down; there will be more than enough roads that lead back to the mainland of proved truth. (Zuelzer et al. 1908: 1383)

Zuelzer tried to prepare a pancreatic extract that could be administered to human diabetics without side affects.

\section{Sources of resources and of internal secretion}

First there was the problem of the raw material to be used. To conduct an extensive series of experiments he would need a rather large amount of fresh pancreases. The richest source of pancreases was the slaughterhouse. Pancreases were not used for human consumption and disposed with the rest of non-utilizable viscera. To obtain these pancreases, however, Zuelzer needed the cooperation of the butchers. They had to cut the organs out and set them aside. But the butchers were not very cooperative. Zuelzer obtained pancreases from the slaughterhouse only now and then. Moreover. the extracts prepared from these pancreases were rather weak, that is, he needed 10 $\mathrm{g}$ of pancreatic extract to neutralize $1 \mathrm{mg}$ of adrenalin.

In search for another and above all richer source Zuelzer turned to larger dogs. He tried to enrich the (hypothetical) insulin content of their pancreases by ligating the pancreatic veins, causing congestion, and subsequent extirpation at the height of the digestive process after a meal. Following this procedure the extract of $0.2 \mathrm{~g}$ of pancreas neutralized $1 \mathrm{mg}$ of adrenalin. (Zuelzer et al. 1908)

Apart from the problem of the raw material to be used for extract preparation, there was another problem. For the further conduct of this line of work he needed additional financial support and co-workers. Zuelzer tried to interest several pharmaceutical companies. He made an effort to convince these companies that the successful fabrication of an effective anti-diabetic preparation would have beneficial consequences for the firm's economy. In most cases he got no for an answer. Eventually, however, the Chemische Fabrik auf Actien (now called Schering) was interested and provided Zuelzer with material and financial support. Moreover, they placed two assistants, Max Dohrn and Anton Marxer, at his disposal. (Mellinghoff 1972)

The extraction procedure was patented in Germany, England and the United States. Patentee of the German 1907 patent was Chemische Fabrik auf Actien. The American patent application was issued on Zuelzer's own name. Zuelzer was never very clear about his extraction procedures, neither in his publications, nor in the patent application. ${ }^{34}$ Zuelzer probably modified the procedure as he went along without publishing them. 


\section{What should the extract be able to do?}

Thinking in terms of an adrenal-pancreatic antagonism Zuelzer argued that the pancreatic extract, in order to be effective, should be able to neutralize the glycosuric effects of an injection with adrenal extract. Zuelzer was convinced that an animal, particularly a rabbit, with adrenal diabetes (Adrenalintier) provided excellent experimental conditions to test pancreatic extracts and gradually improve their effectivity. (Zuelzer et al. 1908) In other words, he believed it could, potentially, be developed into a useful biological assay for the pancreatic internal secretion. The neutralization of adrenal extracts was the first item on Zuelzer's list of criteria for the active principle's existence, the fulfillment of which allowed the conclusion that insulin had been captured and was present in that particular batch of extract.

To test the extracts for anti-diabetic activity Zuelzer also used pancreatectomized dogs, since experimental pancreatic diabetes by most authors, following Minkowski, was considered to be the only 'true' diabetes. Furthermore, Pflüger, who was the most authoritative critic of the internal secretion theory, had stated that

... he would no sooner believe in the internal secretion of the pancreas, than if it was proved that, by administration of the product of the internal secretion of the pancreas, the pancreatic diabetes in the pancreatectomized dog can be influenced in an explicit manner. (Zuelzer 1908: 307-308)

Thus, amelioration or neutralization of the diabetic condition in pancreatectomized dogs was the second item on Zuelzer's list of what the pancreatic extract should be able to do.

\section{Animal experiments}

In 1908 Zuelzer reported two experiments to which he attributed a certain conclusive force. The first experiment was on a $8 \mathrm{~kg}$ male dog. Zuelzer did not follow Minkowski's three-stage procedure for total pancreatectomy, but pancreatectomized the dog in one operation. The dog became diabetic and excreted $4.5-8 \%$ sugar in every portion of urine. On the fourth day after the operation the dog received two subcutaneous injections of $5 \mathrm{~g}$ pancreatic extract each, at $13.00 \mathrm{~h}$ and at $18.00 \mathrm{~h}$. In the two portions of urine following the injections Zuelzer found no more than 1.1 and $2.2 \%$ sugar, a dramatic effect. Then the abdominal wound ruptured, the intestines protruding from the wound and the dog died from severe blood loss. According to Zuelzer the sudden death of the dog was not caused by the injection of pancreatic extract. ${ }^{35}$

The second experiment was conducted on a pancreatectomized dog which Zuelzer borrowed from Dr. Mohr who used the dog in metabolic studies. The dog excreted more than $20 \mathrm{~g}$ of sugar per day. $1 \mathrm{~g}$ of pancreatic extract per day was injected directly into the blood on four subsequent days. The amount of sugar excreted in the urine decreased with one third. In the following days the glycosuria rose to pre-experimental levels. No more extract was available. Zuelzer could not repeat the experiment. No blood sugar estimations were made, nor any other chemical analysis of the urine. 


\section{Moving on to human diabetics}

In view of the substantial support Zuelzer received from Schering these results were disappointing. Nevertheless he decided to move on.

I have thus not yet furnished the experimental proof and I was kept from this because I was accidentally offered the opportunity to test the harmlessness of the preparation and its effect on humans. (Zuelzer et al. 1908: 1384)

Zuelzer moved out of the laboratory into the hospital ward with real patients suffering from diabetes mellitus. To provide a treatment for these patients had been the goal of his work from the outset. Simultaneously, the amelioration of the diabetic condition in humans was the third item on Zuelzer's list of criteria for the existence and presence of the internal secretion in the extract.

Because I had the opportunity to test the harmlessness of the preparation on humans in a comatose and doubtless moribund human, and because I could already determine a certain effect regarding the diabetic process in this case, the animal experiments retreated naturally into the background, without denying the theoretical significance of these animal experiments. (Zuelzer 1908: 309)

The first patient to whom Zuelzer administered his pancreatic extract was a fifty year old diabetic man. One week after the amputation of part of his leg (gangrene) the patient was severely ill, excreting both sugar, acetone and acetoacetic acid in his urine. Zuelzer made an effort to inject the pancreatic tissue intravenously, but failed because of the bad peripheral circulation of blood in the patient's arm. Therefore $3 \mathrm{~g}$ of pancreatic extract was injected subcutaneously. On the next day another $5 \mathrm{~g}$ of extract was injected subcutaneously. The patient was incontinent for urine. Therefore, on the days following the injections the urine was not examined. The general condition of the patient improved. So did his appetite. ${ }^{36}$ However, no more pancreatic extract was available. Five days later the patient went into coma again and died. From this case history Zuelzer drew the following conclusion.

This proved the harmlessness of the preparation. No abscess had been formed. Patient had recovered without doubt. I could now begin to continue the research in appropriate cases. The first case in the Medical Clinic II had a certain evidential value. (Zuelzer et al. 1908: 1384)

New patients of different age and sex were placed at Zuelzer's disposal in a number of Berlin's hospitals. The effect of his pancreatic extracts in these patients varied. Sometimes he noticed no change in the patient's condition or urine at all. Nevertheless, Zuelzer was able to report encouraging results in eight patients. In a 27 year old diabetic the glycosuria diminished almost completely following one injection of 2 $\mathrm{g}$ pancreatic extract, while acetone and acetoacetic acid disappeared completely from the urine. The glycosuria increased again four days later. A second injection in the same patient was without any effect. In one patient Zuelzer claimed that the pancreatic extract facilitated wound healing in a diabetic patient whose leg had been amputated. 
In most cases the injection of pancreatic extract was followed by more or less severe toxic reactions such as high fever.

Most of Zuelzer's observations and experiments were made in very complex clinical situations which complicated the attribution of changes in the patient's (diabetic) condition to the injections of pancreatic extracts. Despite these varying and sometimes ambiguous results Zuelzer was confident that he was on the right track. $\mathrm{He}$ concluded:

The results which were reported in the case histories mentioned above only justify the conclusion that it is possible to diminish the excretion of sugar in a diabetic just as the excretion of acetone and acetoacetic acid without change of diet by means of injection of a pancreatic extract. (Zuelzer 1908: 316)

More, and more specific, items are added to the list of what a pancreatic extract containing insulin should be able to do: a decrease in the excretion of sugar in urine, and a decrease in the excretion of acetone and acetoacetic acid, that is, the disappearance of urinary acidosis.

\section{Forschbach's work on Zuelzer's extracts}

A few months after concluding the clinical experiments Zuelzer and Schering sent some samples of pancreatic extract to Dr. J. Forschbach who worked in the medical university clinic in Breslau. This medical clinic was at the time headed by Minkowski who was appointed there as a professor. Forschbach was allowed to do some experiments using Zuelzer's pancreatic extracts.

Forschbach conducted three experiments on pancreatectomized dogs. The urine of the dogs was collected by means of a catheter in portions comprising the urine production of 12 hour intervals. In all three dogs the injection of Zuelzer's pancreatic extracts, a different sample in each case, produced a marked reduction of the absolute and relative amount of sugar excreted. In one dog the injection was also followed by fever.

Forschbach was encouraged by this what he considered to be an 'unmistakable effect' to move from the experimental animal to the human diabetic. He used Zuelzer's pancreatic extract on two human diabetics.

The first patient was a 20 year old man who even with the most restricting diet excreted $200-300 \mathrm{~g}$ sugar per day. He also excreted acetone and acetoacetic acid and continuously tended to slide away into coma; daily injection of $40 \mathrm{~g}$ sodium bicarbonate prevented this. Injection of Zuelzer's pancreatic extract, which at that moment was 16 days old, had no effect on the patient's condition, nor on the intensity of his glycosuria. But, there was also no rise in body temperature, no toxic reaction.

In the second patient, who also had a severe form of diabetes mellitus, Forschbach decided to collect the urine in portions of 4 hour intervals and to measure the body temperature every second hour. The pancreatic extract used on this patient was freshly prepared. The result was ambiguous though.

Following injection of a freshly fabricated preparation, the secretion of urine stops completely in the next eight hours. The subsequently excreted urine contains, in 
accordance with its low specific weight, already less sugar than the urine of the previous period. The decrease in the relative and absolute amount of excreted sugar lasts 48 hours, so that its daily amount comes to approximately one fifth of the amount of the previous day. The temperature rises to 40.4 several hours after the injection; the rise of the body temperature ... lasts approximately 40 hours. Coinciding with the decrease of the fever at the first day after the injection (after 40 hours), there is an increase in the relative and absolute amount of sugar excreted: on the second day after the injection the excretion of sugar has reached its earlier level. (Forschbach 1909: 2054)

Following the injection the patient became severe ill, with a high pulse rate, vomiting and anuria. These toxic reactions in this patient forced Foschbach to withdraw from further experimental administration of Zuelzer's extracts to humans.

\section{Co-incidence and co-absence}

Forschbach admitted that Zuelzer had, for the first time, fabricated from the pancreas a preparation that reduced the excretion of sugar in the urine also in cases where the composition of the food intake remained unchanged. Forschbach could not think of any reason not to explain this effect as the activity of the unknown sought for substance in the extract, if it were not for the serious toxic side effects that interfered with getting a clearer view of what exactly happened. The injection of pancreatic extract unmistakably produced a toxic reaction. It was hard to imagine that such a severe reaction would have no influence on metabolism. In the medical literature of the time there were a number of publications reporting the reduction of glycosuria and the disappearance of acetone and acetoacetic acid during fever attacks, both in pancreatectomized animals as in human diabetics. Probably it was not the fever as such that caused the decrease in glycosuria and acid excretion. Forschbach's focus was more on the specific activity of toxic substances producing a toxic syndrome of which the fever was just one element. A toxic influence on metabolism might be present in the absence of fever. This would also explain a prolonged effect after decline of the fever. The case history of the patient in which Zuelzer reported an positive effect without an increase in body temperature was, according to Forschbach not very conclusive, since this patient already was feverish prior to the injection.

The record of Forschbach's first patient was important too:

... after injection of an extract that probably had become ineffective due to prolonged standing, both the toxic effects (hyperthermia etc.) and glycosuria reducing effect were absent. (Forschbach 1909: 2055)

The co-incidence and co-absence of 'anti-diabetic' effect and toxic reaction was crucial. Was the 'anti-diabetic effect' produced by a pancreatic internal secretion, while the 'side effects' were produced by distinct contaminating toxic substances? Or were both effects, anti-diabetic and toxic, produced by one substance? According to Forschbach, it might be that it was wrong to make a distinction between anti-diabetic and toxic effects, that both were part of one toxic syndrome. If that was the case, then there was not even an anti-diabetic effect in these cases. Although being a proponent of the internal secretion theory of diabetes mellitus, Forschbach was in doubt about 
insulin's criteria of existence and presence. He had doubts about the correctness of Zuelzer's list. Some of the items on Zuelzer's list for insulin might well belong to another list that defined a toxic syndrome.

\section{Mute inscriptions}

It was possible, on the basis of theoretical considerations, to draw up a list of actions that an effective anti-diabetic pancreatic extract containing the active principle should be able to perform: recovery from coma, decrease of sugar, acetone and acetoacetic acid excretion in urine, etc. But some of these effects had also been described in concurrence with fever, the fever being pointed out as their cause. If fever accompanied the decrease in urinary sugar, acetone and acetoacetic acid excretion, how could one tell whether the sought for effects were attributable to the presence of insulin in the extract or to the fever? Was the fever an alternative cause for the wanted effects, or was it itself an effect? Were there effects and side-effects attributable to different causes or was there one substance causing both the wanted and the unwanted effects?

There were two sick people, and two records with numbers and tables and other inscriptions extracted from the patients during their illness. It is clear that the inscriptions produced by Zuelzer and Forschbach in their research did not speak for themselves. They needed someone to speak for them. But the spokespersons were uncertain as to what these inscriptions were saying. They could only say what they thought the numbers and inscriptions were or were not saying. The resulting translations and conclusions differed. Whereas Zuelzer held on to the belief that the anti-diabetic effect was caused by the pancreatic internal secretion which had been captured, while the toxic effects were caused by some contaminating substance, Forschbach inclined to the conclusion that there was only one toxic syndrome with a singular cause. Concerning the utility of Zuelzer's pancreatic extracts Forschbach concluded:

For the practical usefulness, the discussed side-effects naturally prove to be an insurmountable barrier. The fear of worse results after injection of the pancreatic preparation kept me from performing further experiments on patients. After all, for the time being, it factually involves an operation which, besides a passing useful effect, involves damage to the organism ... It will be difficult to convince a patient who becomes seriously ill after a single injection, of the fact that it is accompanied by a essential healing power for his diabetes. (Forschbach 1909: 2055)

Judgment was passed. There was no future in Zuelzer's extract.

\section{The breakdown of Zuelier's network}

Forschbach's evaluation of Zuelzer's pancreatic extracts, coming from a medical clinic and research laboratory headed by one of the most authoritative figures in contemporary diabetes research, that is Minkowski, referred Zuelzer back to his laboratory. But Zuelzer had no laboratory no more. In 1908 Schering lost interest and withdrew its support to Zuelzer. ${ }^{37}$ For Zuelzer this meant: no money, no co-workers, 
no equipment, no laboratory. But, he did not give up, although, he had to start from scratch again.

In the summer of 1908 Zuelzer submitted a grant application to the medical faculty of Berlin's Humboldt-Universität for a grant of DM 500,- from the Gräfin Bose-Stiftung. The grant would be used to do laboratory research during six weeks in a zoological research laboratory in Italy. Zuelzer intended to investigate whether a non-toxic but effective anti-diabetic preparation could be fabricated from the insular tissue of fish, in which the islands of Langerhans are arranged in larger distinct areas. But the money was not granted to Zuelzer. ${ }^{38}$

Zuelzer gave up the idea of extracting fish-islands. In 1908 he was appointed as director of Berlin's Hasenheide-hospital. The amount of extracting work he could do was limited by the amount of his own investments he could afford. To obtain pancreases Zuelzer had to bribe slaughterhouse workers. ${ }^{39}$

It was clear for Zuelzer that there was no future in this muddling on. He kept on hawking from one drug company to another. In 1910 he tried to interest Farbwerken Hoechst for his 'old' procedure. Although Hoechst was interested at first. a cooperation between Zuelzer and Hoechst was never established.

\section{Back in business, and out again}

Eventually Hoffman-La Roche allowed him to establish a new laboratory in his own hospital Hasenheide in Berlin, and to appoint a co-worker of his own choice. Zuelzer chose the 25 year old chemist Camille Reuter as his assistant. They started working in their laboratory during a couple of years. ${ }^{40}$ During those years they evidently made so much progress that Hoffmann-La Roche permitted them to extract $100 \mathrm{~kg}$ of pancreas in the company's laboratory in Grenzach.

Zuelzer injected a sample of the first batch of pancreatic extract in pancreatectomized animals. The injection was unexpectedly followed by severe convulsions. Zuelzer had never seen a reaction like that before, neither in experimental animals nor in the human diabetics. Zuelzer attributed these convulsions to some kind of poison in the extract. In his view these convulsions were not a property of the sought for pancreatic internal secretion. Neither did he consider the possibility that the internal secretion he was looking for did not exist. He learned that his assistant Reuter had modified the extraction process under the influence of the (older) head of the laboratory in Grenzach. Furthermore, in Grenzach copper containers were used. According to Reuter these might be the source of the toxic material. The convulsions were a serious draw back. The first step to scaling up failed and Zuelzer and Reuter were referred back to the laboratory bench. But, at least the laboratory was still there. Hoffman-La Roche did not withdraw their support.

The next round of experiments failed because of the absence of any effect. But once again Zuelzer did not consider the possibility of the non-existence of insulin. He explained the failure to capture the active principle in this batch of extract with reference to the horses of Ostpreußen. It were their pancreases he had used. Zuelzer attributed the ineffectiveness of the extract to the circumstance that most of the horses from which he got the pancreases came from Ostpreußen, from where they arrived after a three to four days transport during which they were starved before they were slaughtered in Berlin. 
Zuelzer claimed that he had an effective anti-diabetic pancreatic extract a few months later, but this time it was the war that put an end to his extraction work. Zuelzer was called to the front. Hasenheide-hospital was turned over to the military. The war did not put an end to Zuelzer, however. After the war he made an effort to resume his work. But, the post-war economical situation was bad. Zuelzer started hawking again. Hoffman-La Roche was no longer interested. While Zuelzer negotiated with some other company the attention of the world was in 1921 directed to Toronto in Canada where Banting, Best, Macleod and Collip 'discovered' insulin.

\subsection{Toronto: the discovery of insulin ${ }^{41}$}

\section{Frederick Grant Banting: assembling a laboratory}

Banting's medical education was interrupted by World War $\mathrm{I}$, and as a result of that it remained very deficient. ${ }^{42}$ He served in the army during the war as a surgeon, was wounded and returned to Toronto in 1919, where he was appointed as a resident in surgery at the Hospital for Sick Children in Toronto. He did not succeed to obtain a permanent position on the hospital's staff. Instead, he set up a private practice in a ncarby town, London, Ontario.

Banting's London private practice did not run well. He had to supply his income with a temporary teaching position at London's Western University. Soon he began assisting Western University's professor in physiology, F.R. Miller, in experiments on cerebral and cerebellar localization.

On Sunday, October 30, 1920, Banting spent several hours preparing a lecture he had to give on carbohydrate metabolism. Neither carbohydrate metabolism nor diabetes had his particular interest. He had only seen one diabetic patient at Toronto General Hospital during medical training. He wrote later that

[t]here was no such thing as a diabetic in any ward in my surgical experience ... (Bliss 1982: 48)

Banting had just received the new November issue of Surgery, Gynecology and Obstetrics. With the lecture on carbohydrate metabolism and diabetes ahead, he read Barron's (1920) article on The Relation of the Islets of Langerhans to Diabetes with Special Reference to Cases of Pancreatic Lithiasis. Barron presented some of his own observations concerning morphological changes in the pancreas in cases where stones occluded the pancreatic duct. He also reviewed the literature on experimental ligation of the pancreatic ducts. He described how the acinar tissue of the pancreas degenerated, while the islands of Langerhans were not affected. Barron also mentioned, with much confidence, that many researchers held the view that the islands produced the hypothetical internal secretion of the pancreas. Experiments conducted by pathologists like Schulze and Ssobolew had shown that the health of the islands was the key variable in the genesis of (experimental) diabetes. (Barron 1920)

It was from reading Barron's paper that Banting derived the idea for the basic design of a research plan. Ligation of the pancreatic ducts would cause the acinar tissue to atrophy. The islands would be unaffected. The pancreas' internal secretion 
could be obtained from such a degenerated gland, untroubled by the destructive influence of the external secretions of the pancreas. ${ }^{+3}$ Late at night he wrote the following words down in his notebook:

\section{Diabetus}

Ligate pancreatic ducts of dog. Keep dogs alive till acini degenerate Islets

Try to insolate the internal secretion of these to relieve glycosuria (Bliss 1982: 50)

Banting discussed his idea with Miller. Miller, however, knew little about metabolic research. Furthermore, Western University would not be able to support such an investigation. Miller advised Banting to go and talk to professor Macleod at the University of Toronto. ${ }^{44}$ Macleod was a leading scientist in carbohydrate metabolism research.

Macleod was not very impressed.

Macleod was confronted with a young surgeon who had walked in virtually off the street, had no significant experience in physiological research, and was talking haltingly, about a topic he knew about only from standard textbooks and one article.

(Bliss 1982: 52)

Nevertheless, Macleod was struck by something in Banting's proposal. Macleod, who was very cognizant on the subject of pancreatic extracts, insulin and experimental diabetes, knew of no one who had ever used degenerated pancreas for extraction or grafting purposes. From a theoretical point of view, one could imagine, that if there was an internal secretion, and if it was produced by the islands of Langerhans. and if it was the pancreatic juice that destroyed the internal secretion during extraction, than a procedure as suggested by Banting could provide some interesting results. And being a scientist sui generis Macleod thought that negative results would be valuable too.

Furthermore. laboratory methods for measuring the concentration of sugar in blood had recently been improved. Bang $(1913,1916)$ developed micro methods for blood sugar estimations. Instead of the $20 \mathrm{ml}$ of blood that was necessary for one blood sugar estimation, now $0,20 \mathrm{ml}$ were sufficient. Bang's micromethod had been modified by others and was now widely available. This might be another reason for a new attempt to isolate the internal secretion. Earlier research might have been inconclusive by lack of a reliable, easy and quick blood sugar method that allowed repeated measurements at short time intervals.

A third reason why Macleod decided to give Banting a chance might be in Banting's experience as a surgeon. The research plan involved delicate operations and Banting's skills as a surgeon might come in handy.

Macleod warned Banting that many eminent scientists had been working on the problem of the internal secretion of the pancreas for years, in well equipped laboratories. They had not even proven conclusively that there was an internal secretion there. So, there was a considerable risk that his research would lead to nothing. But, if Banting nevertheless was willing to invest the time it would take, then he, Macleod, 
would allow him to work in his laboratory at the University of Toronto. Banting decided to go to Toronto to begin with the research in april 1921 .

Macleod helped Banting to get started. They worked out a plan of investigation. First of all Banting had to learn how to render dogs diabetic by extirpating their pancreas and how to ligate the pancreatic duct to induce atrophy. Macleod provided Banting with two student assistants. Charles Best and Clark Noble. They were fourthyear students in physiology and biochemistry earning some extra money as demonstrators and research assistants for Macleod. Now Macleod assigned them to Banting to run the laboratory tests on urine and blood. As it turned out, only Charles Best would be working with Banting. ${ }^{45}$ Macleod made a little room available under the roof in the physiology department where Banting and Best could run their experiments and tests. Banting had his own surgical instruments with him. Towels for the operation had to be borrowed. The University of Toronto supplied the animals, but no salary. The arrangements were made for the summer, until the end of september, $1921 .{ }^{46}$

\section{Acquiring the skills: trial and mostly error ${ }^{A 7}$}

On Tuesday May 17, 1921, Banting had assembled a laboratory and was ready to go. Macleod assisted and instructed Banting in performing the first pancreatectomy on a dog. A two-stage procedure was chosen. After most of the pancreas had been removed, a remnant with its blood supply intact was pulled up through the abdominal wall and inserted under the dog's skin. The abdominal wound would be allowed to heal and about a week later the pedicle could be easily resected, involving only a minor superficial operation. The first-stage operation on the first dog was successful.

The next day Banting and Best began work on their own, without the assistance of Macleod. The first dog they tried to pancreatectomize on their own died from an overdose of anesthetic. The second dog survived the anesthetic but Banting had great difficulty ligating the blood vessels supplying the lower end of the pancreas. He found himself working in a pool of blood and the dog died after the operation. A third dog was operated on the next day. It survived the operation but died two days later. Meanwhile, the dog they operated under the guidance of Macleod was not healing properly neither. The dog died two days after the operation. They had operated on four dogs that week and all were dead.

On saturday Banting operated on a fifth dog, more careful this time in avoiding interference with the major blood vessels. The pedicle was sutured directly to the surface of the skin to allow easy drainage. The dog survived and was in a reasonable good condition on the next day.

Then they started ligating the pancreatic ducts of a number of other dogs. Banting found it difficult to locate and ligate the ducts properly. The first dog in whom they tried to ligate the ducts died three days later from infection. The second dog survived, but Banting was not sure that he had actually found and ligated the ducts; he might just have ligated a piece of pancreatic tissue. The third 'ligated' dog survived for two days but died from general infection. By the end of the second week seven out of ten dogs Banting and Best had operated on were dead.

The university's animal lab's supply apparently could not keep up with the pace in which Banting and Best used dogs. Banting and Best had to go out and buy dogs on the streets of Toronto for a couple of dollars each. ${ }^{4 s}$ 
In the next week there was gradual progress. One dog survived the first stage and the second stage of the operation, allowing for the first biochemical tests to be run on its blood and urine. These indicated the presence of a diabetic state after completion of the pancreatectomy in the second stage. The dog died three days later.

By the end of the fourth week, while Macleod left to spend his summer holidays in his native country Scotland, Banting had two partially pancreatectomized and seven ligated dogs under way. It was only a matter of time until the pancreatectomies could be completed to induce a diabetic state. Then the atrophied pancreases could be removed for use in the critical experiments.

But things did not work out that well. It was difficult enough to induce a proper diabetic state in pancreatectomized dogs. They healed well enough, but while one would still not become diabetic after removal of the pedicle, the other seemed to become diabetic after the first stage. When, however, five weeks after ligating the pancreatic ducts of seven dogs, Banting opened up one of them he found its pancreas to be quite normal. The duct ligation had not worked at all. Opening up the other six dogs revealed that five of seven had normal pancreases. Banting had failed to isolate and ligate all of their pancreatic ducts. The five dogs with non-atrophied pancreases were done over again, now using silk ligatures instead of catgut. According to Bliss (1982: 67), at this point, 'the whole research program was not far from total failure'. Banting and Best had experimented on nineteen dogs. Fourteen had died, no more than two of them according to the research plan. There were five duct-tied dogs left, and only two of them had gone according to plan.

Towards the end of July 1921 Banting and Best obtained their first positive, encouraging results. ${ }^{49}$ They chose to make an extract of the atrophied pancreas of one of the duct-ligated dogs. They ground it up at low temperatures in Ringer's solution. Injection of the filtered extract in one of the diabetic dogs, whose pedicle had been removed a few days earlier, was followed by a decrease in blood sugar levels of about $40 \%$ within an hour. Repeated injections were not able to bring the blood sugar further down. Despite the injections it rose again. The dog died the next day in coma.

Now there was only one diabetic pancreatectomized dog left. This dog was in coma, on the brink of death. Banting and Best obtained pancreatic extract from a second duct-ligated dog. Again a decrease in blood sugar levels was obtained and the dog came out of his coma, and, according to the laboratory notebook, it was able to stand and walk. But the dog lapsed back into coma and died; a second injection could no prevent this.

\section{Practical problems and shifts in procedure}

Banting and Best were faced with a serious problem. While having a number of ductligated dogs at hand, they had run out of pancreatectomized animals. It would take at least a weak to get a new one ready if a two-stage procedure was followed. Banting decided to try and do the total pancreatectomy at once. He found this procedure to be easier and abandoned the two-stage procedure. They could continue their experiments with extracts the next day. Measuring blood sugar levels they found that repeated injections of pancreatic extract reduced the amount of sugar in the blood. Tests on the dog's urine yielded inconclusive and puzzling results. The general condition of the dog, however, seemed to improve. Boiled extracts remained without effect. So did 
extracts made from liver and spleen. On the third day after the pancreatectomy they mixed the rests of their extracts from two dogs together and injected $25 \mathrm{ml}$ at once. According to the notebook, the dog suddenly became lifeless and appeared to be dying. Attempts to revive the dog with Ringer's solution were not successful, the dog died a few hours later. Autopsy showed widespread infection. This was considered to be the cause of death.

Two new dogs were pancreatectomized. One of them was going to be treated with pancreatic extract, the other was going to serve as a control-dog. The control-dog died four days later. The test-dog, a collie, was kept alive in a reasonable good condition on repeated extract injections. With this dog at hand they tried some variations in extract preparation. An acid extract seemed to be effective, an alkaline one not. Extract incubated with trypsin prior to injection was potent, that is, it reduced blood sugar levels in the collie. ${ }^{50}$

Then another practical problem came up. They ran out of duct-ligated dogs. Without extract injections the collie would die within a couple of days. It was not only unfortunate for this particular dog. The situation revealed a more general supply problem. If they were not able to keep one dog alive on extracts made from atrophied pancreas, which took five to seven weeks to degenerate, then they would surely not be able to treat more animals at the same time, let alone treat human diabetics. Ductligation seemed to work in a small scale laboratory situation, for treatment of one animal for a couple of days, but it certainly would be an impracticable way of procuring effective anti-diabetic extracts in large scale production. An easier and most of all quicker way of preparing the pancreas for extraction had to be found.

For reasons that are not clear, Banting decided to try and make an extract of a whole pancreas of a dog. Injection of this extract in the collie drove its blood sugar level down from $0,30 \mathrm{~g} \%$ to $0,19 \mathrm{~g} \%$ half an hour later, and $0,17 \mathrm{~g} \%$ another 30 minutes later. The reduction in blood sugar levels was as large as they were with extract made from degenerated pancreas. But, for some reason, Banting and Best did not notice this. Probably they were biassed by their working hypothesis concerning the destructive action of the pancreas' external secretion. In the published paper reporting this work they wrote that it was obvious that the whole gland extract was much weaker than that from the degenerated gland. (Banting and Best 1922)

Banting was not prepared to abandon this working hypothesis, and the whole gland extract should not be seen as an attempt to test this hypothesis. Banting and Best still thought that it was necessary to get rid of the destructive external secretion before the pancreas could be ground up.

The acute shortage of degenerated pancreas made Banting try another, quicker way, namely by exhausting the pancreas through stimulation of the external secretion with secretin: a hormone, produced in the duodenal mucosa, that stimulates the external secretion of the pancreas. The plan was to stimulate the pancreas with secretin until it was exhausted and could produce no more external secretion. Then the pancreas could be extirpated and subjected to extraction. It was a complicated procedure, involving resection of a dog's duodenum to obtain the crude secretin, the insertion of a cannula in the pancreatic duct to measure the flow of external secretion, the slow injection of secretin for almost four hours until the production of pancreatic juice stopped, and finally the preparation of the extract. (Bliss 1982) Despite the 
complexity of the procedure it was much faster than duct-ligation and the extract thus prepared worked, preventing the very sick collie to lapse into coma.

A second attempt to exhaust the pancreas of a cat by the secretin-stimulation method failed; the cat died on the operating table. Banting nevertheless made an extract of the cat's pancreas and gave it to the collie. The dog was thrown into profound shock. It died a couple of days later.

New dogs were pancreatectomized for another series of trial and error experiments. According to Bliss, it was not clear what Banting and Best were trying to achieve. The secretin-stimulation method did not work very well. The next attempt to exhaust the pancreas of a dog by the secretin-stimulation method failed. The duodenal extract did not stimulate the pancreas' external secretion. They ground it up anyway and used it as if it were extract of exhausted pancreas. The new extract worked well when injected intravenously. Rectal administration of the extract had no effect. Again the extract was mixed with trypsin. The mixture had no effect. New batches of "more or less' exhausted pancreas were prepared. An intracardial injection of extract caused a moderate decline in blood sugar, shock and death of the test-dog. ${ }^{5 i}$ They also tried subcutaneous injections without success. Blood sugar levels did not change and ulcerations developed at the injection site, causing pain, bleeding and infection.

\section{Professorial caution and persuasive strength}

With these last experiments Banting and Best finished the summer's work. The work was characterized by a 'trial and error' style of working, with a number of ad-hoc and 'What the hell?'-type of experiments. Despite the technical difficulties and practical problems they encountered, which made them depart from Macleod's parting instructions, Banting and Best felt that they had obtained good results, warranting continuation of the project.

Macleod had been on a holiday in Scotland during the summer. In August Banting and Best reported the preliminary results of their research to Macleod in a letter. In his response Macleod noted that the results seemed certainly very encouraging, definitely positive. But this was not good enough for Macleod. Any possibility of mistake had to be ruled out prior to publication. Bliss (1982: 79) speaks of 'professorial caution' here, but a quotation from Macleod's letter reveals that Macleod's prudence is an expression of his awareness of the importance of persuasive strength in scientific work and writing:

You know that if you can prove to the satisfaction of everyone that such extracts really have the power to reduce blood sugar in pancreatic diabetes, you will have achieved a very great deal. Kleiner \& others who have published somewhat similar results have not convinced others because their proofs were not adequate. Its very easy often in science to satisfy ones own self about some point but its very hard to build up a stronghold of proof which others cannot pull down. (Bliss 1982: 79)

Macleod hoped Banting and Best had data on the behavior of the blood sugar of other pancreatectomized dogs so they could prove that their results were not merely normal diumal variations. But Banting and Best had no such data. Macleod hoped that Banting and Best had data to prove that a drop in blood sugar was not a simple dilution 
phenomenon caused by the large volume of fluid injected. But Banting and Best could not provide such data. He advised them to set aside a small tissue-fragment for histological examination from each pancreas used for extraction to prove that it was islet tissue that was causing the effect. Apparently sections were taken, but they never obtained data from them.

When Macleod returned by the end of September 1921 he advised Banting and Best to continue their line of work, to repeat the experiments, get more and better results and wrap the problem concerning the internal secretion of the pancreas up to everyone's satisfaction. ${ }^{52}$

\section{Shifis in the criteria of existence}

In October and November there was not much progress in Banting's and Best's work. The secretin-stimulation method to exhaust the pancreas' external secretion had not at all worked well. They had to fall back on the more time consuming duct ligation method and wait for the pancreas to atrophy. But, impatient as they were, the time lapse between the ligation of the pancreatic ducts and the moment of extirpation and extraction became shorter and shorter. In one instance it was as short as eighteen days. The degenerated pancreases used for extraction were 'more or less' atrophied. The pancreatectomized test-dogs did not do well either. One of them vomited half an hour after the injection of pancreatic extract, followed by anuria and high fever. Neither the first nor a second injection of pancreatic extract had any effect on the blood sugar levels. The dog died a few days later. Autopsy showed a ruptured duodenal ulcer. A second test-dog seemed to respond well to pancreatic extract, but died six days after the pancreatectomy, autopsy showing again a duodenal ulcer and extensive internal bleeding.

Banting and Best used spare time to search the literature more or less systematically. They read scientific articles relevant for their problem in search for ideas and suggestions on how to get beyond this stage of the research on one hand, and as background material for their first article on the other.

Banting had jotted down some notes on an index card concerning alternative explanations for a decrease in blood sugar levels following an injection of pancreatic extract. (Bliss 1982) Impressive as the blood sugar lowering effect of pancreatic extract in some of their experiments might have been, it still did not prove that an internal secretion had been captured. Other substances and conditions, like shock, moribund states, the injection of foreign proteins, might lower blood sugar levels and reduce the excretion without necessarily permitting the diabetic organism to metabolize its food. These factors might have interfered with their own experiments. Kleiner and Meltzer $(1915 \mathrm{a} ; 1915 \mathrm{~b})$ already proposed a shift in the criteria of existence, and as a consequence, in the identity of the internal secretion. Reduction of glycosuria should, according to Kleiner (1919a: 1919b), no longer be regarded as a reliable sign of the active principle's presence and activity: only a decline in blood sugar levels is. In Kleiner's view reduction of glucosuria as such did not represent insulin. For the same reasons Murlin and Kramer (1913) had suggested to use the restoration of the respiratory quotient as a measure for the effect of an anti-diabetic pancreatic extract, since this would indicate that the organism burns food again. Now Banting had come 
to the conclusion that reduction of blood sugar levels as such does not represent insulin either.

\section{Longevity experiments with foetal pancreases}

At November 14, 1921, Banting and Best were invited to give an informal preliminary report to interested colleagues and students on a meeting of the Physiological Journal Club. In the discussion after the presentation N.B. Taylor, a member of the Department of Physiology, suggested to them that a convincing demonstration of the extract's effect would be to show that regular administration of it could prolong the life of diabetic dogs. (Macleod 1978) Another item was added here to the changing list of things that the hypothetical internal secretion of the pancreas, if it existed, should be able to do. If it would be possible to keep a diabetic dog alive over a longer period of time, months maybe, perhaps a year, then this would, in Taylor's words. not only be a convincing demonstration of the extract's effect; it would also be a persuasive demonstration of the very existence of insulin. Was it not possible to prolong the life of diabetic dog then the existence of the internal secretion would still be doubtful. Maybe no internal secretion existed after all; or it might exist, but was not yet captured in the extract. Long term survival in good condition of a pancreatectomized dog on a normal diet and regular injections with pancreatic extract would demonstrate the organism's ability to utilize the nutrients of its food. The injections would then imitate the action of the pancreas still in vascular connection with the body.

Banting, who at several places, in letters, notebooks and desk-calendars, had expressed an interest in the possibility of using the extract of humans, as a cure for diabetes, must also have been struck by the similarity of a longevity experiment on an experimental dog and the necessarily long term clinical treatment of human diabetics. Therefore Banting, Best and Macleod gratefully accepted Taylor's suggestion to do a longevity experiment.

The execution of a longevity experiment once again brought the problem concerning the supply of raw material for the extraction to the fore. Avoiding the destructive action of the external secretion was still, in their eyes, a prerequisite for the preparation of an effective anti-diabetic extract. The secretin-stimulation method had not worked well, and the duct-ligation took too much time. Furthermore, when they decided to try a longevity experiment there was only one duct-ligated dog at hand, yielding barely enough extract for one or two days.

Banting's reading of the literature had suggested another possibility to him. Laguesse had found that in embryonic and foetal life the islands of Langerhans develop and mature earlier and prior to the pancreatic acinar tissue, which later in life produces the external secretion. Banting found additional support for this approach in Carlson's and Drennan's (1911) article in which the authors reported to have found that a pancreatectomized pregnant dog did not develop its diabetic condition until after delivery of the pups; the foetal pancreases supplied, according to Carlson and Drennan, the deficiency of the mother. Digestion, and hence stimulation of the external secretion of the pancreas, does not begin before birth either. So, foetal pancreas would possibly provide a good source of raw material for extraction purposes. 
A swift idea of obtaining foetal pancreases by breeding and subsequently aborting dogs was abandoned in favor of William Davies Company's abattoir in Toronto. Being a farmer's son Banting realized that stock-breeders often bred their cattle just before slaughter to make them better feeders and fatter. There would be plenty of foetal calf pancreases to obtain in slaughterhouses.

Banting and Best went to the slaughterhouse themselves to extirpate the foetal pancreases. They prepared extract from them, using Ringer's solution, and injected it in a diabetic dog. It reduced blood sugar levels and abolished glycosuria. Foetal pancreas extracts worked, that is, they did the same things as degenerated pancreas extracts did. The longevity experiment could proceed, the abattoir providing the foetal pancreases.

While the experiment proceeded Banting and Best tried to improve extraction and administration methods. The extracts were subjected to a final filtration through a Ber-

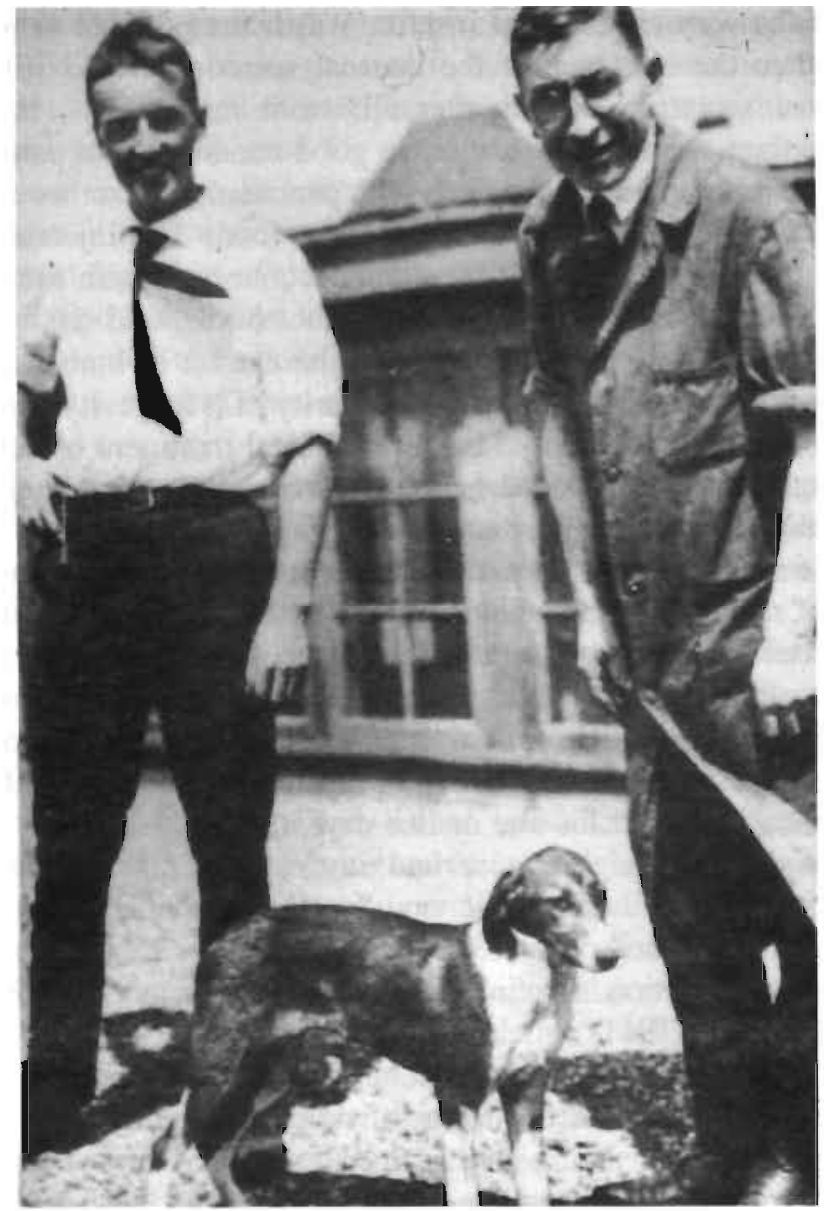

Figure 3.4

Banting and Best on the roof of the Medical Building Reproduced from Bliss (1982) 
kefeld filter, removing particles and clumps and assuring sterility. They also tried to improve stability of the extract by addition of preservatives.

The longevity experiment ran smoothly for about three weeks. Then, suddenly, four hours after an injection the dog developed convulsive twitching, retraction of the head and unconsciousness for several hours. The symptoms recurred following the next injection. The dog did not recover and died. According to Banting and Best the death of the animal was caused by an anaphylactic-like reaction. As Bliss (1982) noted, instead of prolonging the dog's live, the extract had killed it.

Disappointed by the abrupt end and failure of this experiment, but encouraged by its course prior to the dog's death, a new longevity experiment was started. This dog, Marjorie, had been pancreatectomized on November 18, 1921, and was kept alive for about ten weeks, well into $1922 .{ }^{53}$

\section{Alcoholic extracts}

The trouble with extracts made in Ringer's solution was that it was so cumbersome to prepare more concentrated extracts. Boiling away the excess of water destroyed the extract's potency. In the beginning of December, 1921, they decided to try and use alcohol for extraction, as both Zuelzer and Scott had done before them. Alcohol evaporated at much lower temperatures than water, a process that could be accelerated by using a current of warm air flowing over porcelain dishes containing the crude extract. After redissolving the residue in saline it was injected and seemed to work well.

Scott (1911) had explicitly used alcohol to destroy or inactivate the digestive enzymes of the external secretion of the pancreas while preserving the internal secretion. Scott's results led Banting and Best to try and see if, when using alcohol, whole pancreas could be used instead of foetal, degenerated or exhausted pancreas. A dog was pancreatectomized and an extract using alcohol of its own pancreas was prepared and given to it. The blood sugar fell from $0.38 \mathrm{~g} / \mathrm{ml}$ to $0.18 \mathrm{~g} / \mathrm{ml}$ within four hours. Alcoholic whole pancreas extracts worked too. From now on whole pancreas could be used, which was cheap and easily obtainable. ${ }^{54}$

\section{From pancreatectomized dogs to normal rabbits}

In the beginning of December 1921 Macleod conceded to adding James Bertram Collip to the team. Collip was an experienced medical researcher. He was trained in physiology and biochemistry in Toronto, where he took his B.A. in 1912, his M.A. in 1913 and his Ph.D. in 1916. In 1915 he was appointed as a lecturer in physiology at the University of Alberta in Edmonton. In 1920 he was promoted to full professor in charge of a new Department of Biochemistry. Collip was on a sabbatical leave. Supported by the Rockefeller Foundation and a temporary appointment as assistant professor he was spending a year's work in Toronto. ${ }^{55}$

Collip made important contributions to the research. ${ }^{56}$ Following up a suggestion of Macleod Collip began using rabbits as test-animals instead of dogs. Macleod's motive behind his suggestion was economic. There were several ways to induce a diabetic state in rabbits all of which were cheaper and easier than depancreatizing dogs. Macleod's suggestion pertained to rabbits made diabetic experimentally. But Collip found that normal rabbits did as well. His pancreatic extract lowered the blood 
sugars of rabbits from normal to below, just like it lowered the blood sugars in pancreatectomized diabetic dogs or rabbits from above normal to normal. The normal rabbit provided an easy method to test the potency of each batch of extract by measuring its effect of the blood sugar of a normal rabbit. Blood samples could quickly and easily be obtained from a vein in the rabbits ear.

\section{Potency}

The blood sugar lowering effect of an extract in a normal rabbit was not providing further evidence concerning the question whether the pancreas' internal secretion had been captured in the extract. As Banting had noted, reduction of blood sugar levels as such could not confidently be taken to represent the presence and activity of the sought for internal secretion. The researchers were working with extracts that did a number of things that they were supposed to do. The extracts lowered the blood sugar levels in dogs suffering from experimental pancreatic diabetes, they abolished glycosuria, prevented death, and, as the first longevity experiment had shown, kept a diabetic dog alive over a longer period of time. For all these reasons the researchers were convinced that the internal secretion existed and that they had captured it. What they did not know was how much of it was present in every new batch of extract and the normal rabbit seemed to provide an easy way of establishing this. The difference with Zuelzer's use of rabbits as a biological assay to test the effectivity of pancreatic extracts was that Zuelzer did not use normal rabbits. but rabbits with adrenal diabetes (due to the injection of adrenal extract).

\section{Restoration of glycogen formation}

As a next step Collip undertook to investigate whether the pancreatic extracts they were preparing would enable a diabetic dog to convert glucose into glycogen and store this in the liver. The conversion of glucose in glycogen was known to be impaired in both human as in experimental pancreatic diabetes, resulting in a low glycogen content of the liver in these conditions. The restoration of his function in diabetic animals would be an important demonstration of the anti-diabetic potency of their extract.

Banting and Best pancreatectomized a dog for Collip, who took it to his own laboratory where he prepared and tinkered with his own extract. In stead of using a warm air current to evaporate the alcohol from the crude extract he used a vacuum still. He did not evaporate all the liquid in the extract but reduced the volume to about one-fifth of its original volume. This was filtered and injected. It reduced blood sugar in the diabetic dog. Then Collip decided to prepare a solution of the residue of the crude extract and he found this to be even more potent. Assuming that they had the internal secretion in their crude extract Collip found that most of it was not in the filtrate but in the residue.

Collip did not only measure the dog blood sugar levels but he also examined the urine for the presence of sugar and ketone bodies. Both disappeared from the urine following the injection of his pancreatic extracts. According to Bliss (1982) this was the first measured demonstration in Toronto that the extract could abolish ketosis.

Eight days after the dog had been pancreatectomized Collip killed it and took out its liver to examine it for the presence of glycogen. An untreated diabetic dog's liver would contain almost no glycogen, $1.5 \%$ at the most. This liver contained no less 
than $25 \%$ glycogen. This was, in Collip's words, definite proof of the restoration of a function which was definitely known to be lacking in the diabetic state.

\section{Taking shape!?}

In one experiment Collip added two items to the list of things that the extract could do: restoring glycogen formation and storage in diabetic animals, and abolishing ketonuria. These items were derived from what was known at the time about human and experimental pancreatic diabetes. The extract was subjected to tests to see whether it could restore blood sugar levels, abolish glycosuria, prevent death, sustain live. Now the extract was subjected to two new tests to see whether it could restore glycogen formation and storage and whether it made ketone bodies disappear from the urine. And at least in this case, the extract passed the test. By delineating in this way the anti-diabetic properties of their pancreatic extract the Toronto researchers were also successively delineating the properties of the sought for internal secretion of the pancreas. Insulin's identity was gradually taking shape. It was no longer a good looking hypothesis but is was actually there in the extract in a Torontonian laboratory. But it was still fragile. Collip's success in one laboratory was paralleled with failure and disappointment in another. Banting and Best set out to make the largest batch of extract yet. But, as they tested it, it had no potency at all. Maybe they had added to much acid too the alcohol they used for extraction. So they tried again using just alcohol. But also this new batch had no effect. And also a third attempt failed. It only made the dog vomit.

\section{Convincing evidence? The scientific community}

On December 30, 1921, the American Physiological Society held a conference at Yale University in New Haven. Banting, Best, Macleod and Collip were going to present a paper there on The Beneficial Influences of Certain Pancreatic Extracts on Pancreatic Diabetes. Macleod chaired the session, while Banting presented the paper. They were all there: Allen, Joslin, Kleiner, Scott, Carlson; an audience that was highly cognizant of the subject and its problems and preceding failures.

Banting was very nervous. He had never spoken to an audience of this kind before. He spoke very haltingly. According to Macleod,
Banting being nervous, it was evident that he had not succeeded in convincing all of his audience that the results obtained proved the presence of an internal secretion in the pancreas ... any more definitely than had those of previous investigators. (Macleod 1978: 305)

A number of these previous investigators were in the audience and they all commented on the work. They were not as confident as the Torontonians that the work they reported went beyond their own. They asked inconvenient and embarrassing questions uncovering the weak spots in the research. What about toxic effects, for example? Did the extracts cause fever? Banting and Best never kept temperature records of their dogs. They had only one temperature reading ... showing fever. They had to admit that they lost a number of dogs because of noxious reactions to the extracts. The experiment that had to be their trump-card, the first longevity experiment, had ended 
abruptly because of the dog's severe reaction to the extract. The second longevity experiment with Marjory went well, however, showing no toxic side effects. Yes, but why was Marjory doing well, Carlson asked. Was it their extract that kept here alive, or was it pancreatic remnants that did? Had their pancreatectomies been total? Were routine autopsies on the animals sufficient to prove that? Banting and Best had not systematically verified the completeness of the operation by microscopic examinations. They had not kept good records on the experimental dogs' $\mathrm{D}$ (extrose)/ $\mathrm{N}$ (itrogen)ratios, which, in the view of their opponents, were a useful indication for the completeness of the pancreatectomy and an indispensable parameter for the demonstration of an extract's anti-diabetic effect. What about their working hypothesis concerning the deleterious effect of digestive enzymes? How atrophied were the atrophied pancreases they used? How exhausted were the exhausted pancreases employed ? $^{57}$

Macleod, although being the chairman of the session, actively engaged in the discussion. He kept drawing attention to the frequency of a direct relationship between the injections and the lowering of blood sugar and on the prolongation of life of two treated animals. (Macleod 1978: 306) And of course, just before Christmas, Collip had come up with exciting findings about the abolition of ketonuria and restored glycogen formation.

The Torontonians received little praise from the experts in the field. The scientists were looking forward to hearing more in the future.

Not everyone was so reluctant to assent to the Canadians' claims. George Clowes, the research director of Eli Lilly and Company, an American pharmaceutical manufacturing company, was at the meeting too. He had heard about the work in progress in Toronto from one of the people who had attended the meeting of the local Physiological Journal Club in Toronto at the end of November. He had come to New Haven to hear the presentation of the work. Clowes was not as skeptical as the 'experts'. He thought the evidence was convincing. ${ }^{58}$ Clowes got together with Macleod after the meeting and proposed a collaboration between Eli Lilly and the Torontonian team in the subsequent development of the pancreatic extracts into a commercially available product produced on a large scale. But Macleod held him off arguing that the work was not sufficiently advanced for commercial production. Clowes' suggestion would be born in mind.

\section{The hypoglycemic reaction}

In the first days after New Year Collip ran into problems. Occasionally the rabbits on which he tested the extracts he was working on went into convulsive seizures. Their heads snapped back, eyeballs protruding. limbs rigid. They violently tossed themselves from side to side and collapsed into coma, lying still on their sides and breathing rapidly. The slightest stimulation would set them off again. Sometimes the rabbits' limbs moved rapidly, as in running, while lying on their side. In most cases the rabbits died. The extracts must have had toxic properties to cause such a reaction. Collip thought of another possibility. He took a blood sample, dissolved glucose in water, shook it and injected the solution into the comatose rabbit. The rabbit recovered quickly. Subsequent examination of the blood sample revealed the virtual absence of sugar in the blood. (Bliss 1982: 109; Banting et al. 1922b) Collip added a new item 
to the list of actions that were progressively delineating the identity of the pancreas' internal secretion: the hypoglycemic reaction or insulin shock. Blood sugar levels measured in blood samples taken prior to the injection of extract, in coma and after injection of glucose, in conjunction with the immediate recovery after the administration of glucose, a procedure that could easily be repeated, convincingly established the hypoglycemic reaction. The extract could lower the blood sugar to too low a level, inducing a lethal condition. This was an observation of great importance, especially in view of the possible use of the extract in the treatment of human diabetics.

Collip spoke not only for the convulsions in his own rabbits. He offered simultaneously a new explanation (translation) of phenomena that had been interpreted as the effect of toxic substances. In his after-the-fact discussion of the convulsions that Zuelzer attributed to the poisonous copper from the extraction containers, Mellinghoff (1971) argued that these convulsions really were a hypoglycemic reaction. ${ }^{59}$ Were the widespread infections really the cause of death in Banting's dog that suddenly became lifeless and appeared to be dying following a rather large amount of extract? Or was that a hypoglycemic reaction too? And the abrupt end of the first longevity experiment? Was it really an anaphylactic reaction? Or was it an insulin shock that killed the dog? Phenomena that formerly represented a toxic reaction should now be considered to result from the activity of the internal secretion and thus indicate the presence, existence and an aspect of the identity of insulin. Historians who follow Collip and project his newly discovered phenomenon back into time are led astray. By arguing, as Bliss did, that many of Toronto's predecessors had active pancreatic extracts but that none of them had been able to purify their extracts sufficiently to eliminate toxic properties. fail to recognize the considerable doubt concerning insulin's very existence, and if it existed, when it could be said to be present in an extract in what quantity.

\section{Precipitating insulin}

Collip continued tinkering on the details of the extraction and purification procedures in an attempt to prepare extracts that were suitable for treatment of human diabetics. He was tinkering with the concentration of the alcohol used in the extraction process. By steadily increasing the concentration of the alcohol he found that the extract remained potent at higher concentrations where proteins precipitated out. But there was a limit. At an alcohol concentration a little over $90 \%$ the potency disappeared from the extract, but was now to be found in the precipitate. Bliss (1982) summarized the event as follows:

There it was. You cold 'trap' the active principle ... by first producing the concentration of alcohol in which it was soluble but most of its protein contaminants were not, and then moving to the concentration that would precipitate it. (Bliss 1982: 117)

Collip was thrilled. As he put it later: 
I experienced then and there all alone in the top story of the old Pathology Building perhaps the greatest thrill which has ever been given me to realize. (Bliss 1982:

117)

It was as if Collip actually saw insulin. As far as he was concerned, at that moment he did.

The extracts produced in this way were purer than any previous extract. Its potency was tested on rabbits. The animals were carefully checked for side effects and abscesses at the injection site. At January 231922 they were ready to move out; out of the laboratory to the clinic.

As far as insulin was 'materialized' during this period it only existed in a particular laboratory in a particular place, in the form of rather crude pancreatic extracts that irregularly displayed biological activities that were supposed to be antidiabetic. And it was still very fragile. It could disrupt any moment. Insulin did at that moment not yet exist outside the Torontonian laboratory. It did not exist in the lives of the numerous human diabetic patients.

\section{Crossing the Rubicon}

Banting was very eager to start clinical testing. On December 21,1921 , frustrated by the break down of their ability to make potent extracts in three subsequent attempts, Banting decided to give some extract they knew to be potent, prepared prior to the breakdown, to a human diabetic, Joe Gilchrist. Gilchrist, one of Banting's classmates and a physician himself, was deteriorating rapidly, progressing to the final stage of the disease. However, it was too risky to inject the extract intravenously or subcutaneously. Banting and Best decided to give him the extract by mouth, on an empty stomach. No beneficial result was recorded. (Bliss 1982)

After returning from the New Haven conference Banting made a new attempt to start clinical testing on human diabetics. Being the only physician on the team, he was determined to participate in the first clinical trial. The problem was to get access to diabetic patients. Banting had known Gilchrist personally. That was an off the record attempt for which he only needed Gilchrist's consent. Banting had no private practice anymore. The diabetic patients to which he had to gain access for clinical testing were on the other side of the street, College Street, in Toronto General Hospital's diabetic clinic; opposite to the university's Medical Building in which their research laboratories were housed. But Banting had no standing there. He approached Duncan Graham, Eaton Professor of Medicine and head of the Department of Medicine, and applied for a temporary appointment in order to make it possible for him to test his pancreatic extract on humans. Graham was, as Bliss (1982: 111) puts it, 'a tough cookie' at all times, but particularly so when it came to protecting patients in the hospital wards under his control for premature experiments or investigations. Graham asked embarrassing questions. What right did he, as a surgeon who was currently not in practice, have to treat diabetic patients? How many of them had Banting ever treated? Not one. Graham decided that Banting was not qualified to experiment on his patients.

Persistent as Banting was, he urged Macleod to persuade Graham to give them a chance to try some of the pancreatic extract Banting and Best were making on one 
of the patients in Toronto General Hospital. Although reluctantly, Macleod agreed, talked to Graham and got permission for a trial.

Leonard Thompson, a fourteen year old diabetic boy, was chosen as the first patient to receive the extract. Allen's dietary therapy had reduced the boy to skin and bones. ${ }^{60}$ He had reached the final stage of the disease, sliding away into death if nothing else could be done. The boy's father agreed to let them try the extract on Leonard.

Banting and Best prepared the extract, sterilized it by putting it through a Berkefeld filter and tested its potency on a dog. They advised Campbell, head of the diabetic clinic, on the timing for taking blood and urine samples. Banting and Best had to wait in the corridor while a member of the clinic's house staff, Ed Jeffery, injected the extract. The result was somewhat disappointing. There was only a very moderate reduction of blood sugar levels and sugar excretion in the urine. The laboratory test for ketones in urine continued to be strongly positive. Furthermore the injection had no beneficial effect on the boy's clinical condition. A sterile abscess developed at the injection site. (Banting et al. 1922c) The clinicians decided that the extract's moderate effect did not outweigh the adverse reaction it caused. They decided not to give the boy any more of Banting's and Best's extract. This extract was useless for continued administration. The laboratory experimenters were referred back to their laboratory. Their first attempt to cross the street had failed.

About a fortnight later another attempt was made to go back to the clinic. Collip had found that he could improve the extract by stepwise increasing the concentration of the alcohol used in the extraction procedure to a level where the active principle precipitated out. By redissolving the precipitate a purer extract was obtained, which was potent in rabbits and caused no abscesses at the injection site.

On January 23, 1922, Campbell resumed treatment of Leonard Thompson, who was still alive. The boy received $5 \mathrm{ml}$ of Collip's extract in the morning, and $20 \mathrm{ml}$ in the afternoon. The next day he received two injections of $10 \mathrm{ml}$ each. Blood and urine sample were taken regularly. Examinations of these samples was not only important for the scientific record. For lack of a measure of the potency of the extract they ran a risk of inducing a hypoglycemic reaction. On the 23rd Leonard's blood sugar had been $0.520 \mathrm{~g} / 1$. On the 24 th it dropped to $0.120 \mathrm{~g} / 1$. Glycosuria almost disappeared. Ketonuria disappeared completely. The boy's clinical condition improved too. He became brighter, more active. He looked better and said that he felt stronger. No extract was available on the 25th and 26th. On the 27th the treatment was resumed. (Bliss 1982; Banting et al. 1922c). They all felt that this was the first successful clinical test of an anti-diabetic pancreatic extract on a human diabetic patient. It worked. Now they had a new weapon in the combat of a hitherto lethal disease.

The Rubicon, which in the geography of insulin was Toronto's College Street. running between the hospital and the university, had been crossed, meaning that from the clinical side there could be no real withdrawal. (Bliss 1982) It might be a pity and a waste to see a pancreatectomized dog die for lack of extract. But they could not let that happen to a human patient. Once a therapy with pancreatic extracts was instituted in a diabetic patient they were morally obliged to continue it as long as necessary, that is during the rest of the patient's life. If continuation of the treatment could not be 
guaranteed it was better not to start treatment at all. A gradual increase in the number of patients treated would have to be parallelled by an increase in the production of extract.

A few more severely diabetic patients were treated with the pancreatic extract that could be produced in the small scale laboratory setting. In the meantime large(r) scale production facilities were under development, a process in which the scientists took an active part.

\section{The insulin famine}

The Connaught Anti-Toxin Laboratories was financing the assemblage of production facilities in the basement of the medical building. Collip was going to be in charge of the scaling up of the production of pancreatic extract for clinical use.

By the end of February, 1922, everything was set to go. But, to everyone's surprise and dismay Collip failed. He was unable to make potent extracts when using the machinery that was assembled down there. Furthermore, he also failed to do it in his own lab, failing to reproduce the procedures that had been successful in January and February. Insulin was still so fragile that it could disrupt any moment, as it did now.

Collip's failure did not shake the Torontonian's belief in the existence of the pancreas' internal secretion. Leonard Thompson had been successfully treated with extracts they had made. Collip had virtually seen the active principle precipitating in his laboratory tubes. They had no idea what caused the failure. Were the methods too crude? Was the equipment unreliable? The failure underscored that they did not know anything about the chemical composition of the substance they were trying to produce.

Collip's failure resulted in an insulin famine in Toronto during March and April and part of May, 1922. This was the price that had to be paid for crossing the Rubicon. The treatment of diabetic patients with extract could not be continued. Fortunately there were not many of them. Those who had regained enough weight and strength to carry on starving for a few months were put back on a dietary regime. While struggling to regain the capability of making effective extracts Collip sometimes came up with weak ones. These batches were given to those patients who needed them most. Massive doses of weak extract were given to a girl who gradually slipped into coma. The injections brought her back to consciousness, the first recovery from coma at Toronto. But, since no more extract was available for days she slipped back into coma and died. She was the first patient in Toronto who had been treated with the extract and died for lack of it. (Bliss 1982) It took them until mid-May to re-acquire the skill of making effective pancreatic extracts again.

Banting had taken little part in the treatment of patients at Toronto General Hospital; Graham had denied him access. Banting felt that he, as the discoverer of insulin, had a legitimate claim to its application in clinical practice. As things were now he ran the risk of being outmaneuvered. To get back in business again Banting established an office in the city and began a private practice of medicine. This instantly gave him the right to use the facilities of Toronto General Hospital's private patients pavilion. In April, 1922, a few weeks after an interview with the Director of Medical Services for the Canadian Department of Soldiers Civil Re-Establishment, Banting was appointed as head of a new diabetes clinic at Toronto's Christie Street Military Hospital. Now he had all the facilities he needed. An agreement was reached 
about the distribution of the extracts for clinical use produced by the Connaught Laboratories. One-third of the production was to go to Banting for his private practice, one-third to Banting for his clinic in the Military Hospital and one-third would be available for clinical use in Toronto General Hospital and the Hospital for Sick Children. (Bliss 1982) This put Banting back into the center of the headlight concerning the clinical use of insulin.

\title{
Patents and licenses
}

In May, 1922, the (North-American) world already knew that Toronto had been successful. At a very early stage in January a reporter from a local newspaper had gotten wind of what was going on. Macleod had urged him not to publish anything, afraid as he was that premature publicity might awaken false hopes, especially in those diabetics that were desperate for treatment. They themselves had published reports in medical and other scientific journals. Inquiries concerning progress of the work and about the expected time at which the preparation would be available on a larger scale were coming from physicians and scientists in both Canada and the United States. The increasing demands for insulin from Banting's private practice and hospital clinics, and from outside Toronto added to the pressure resulting from their own failure to produce effective extracts and the insulin famine.

What if someone else set to work and succeeded in making effective extracts? Macleod felt that they ran a risk of loosing the discovery to someone who might take out a patent on it for commercial exploitation.

Clowes, himself being a representative of Eli Lilly, expressed the same worries to Macleod. That is why he had offered Lilly's cooperation in Toronto's work. In March Clowes wrote to Macleod:

\begin{abstract}
Public interest in this work will naturally be very great and the demand for the product will be such as to lead to attempts on the part of unprincipled individuals to victimize the public unless some steps are taken to arrange for the manufacture of the product by the procedures recommended by Dr. Collip and the control of the product by means of such tests as you and your associates would consider necessary. (Bliss 1982: 132)
\end{abstract}

Working with Lilly at this moment would not solve the problem of potential commercial monopolization of insulin. The only way to prevent that was by taking out a patent. A patent, usually associated with monopoly of production and profit, could be used to stop anyone else from producing insulin. But, and that was the point, it could also be used to prevent someone else from gaining a monopoly position. In April, 1922, Toronto took out a Canadian patent on the extraction process on the names of the two lay members of the group, Best and Collip, and immediately assigned the patent to the Board of Governors of the University of Toronto. In May they also applied for a patent in the United States.

Large scale production: Eli Lilly \& Co.

When Collip, by mid-May, succeeded in making potent pancreatic extracts again treatment of human diabetics could also be resumed on a small scale. By this time, 
however, the demand for the extract was by far exceeding the limited production. It gradually became clear that the limited Connaught production facilities in the basement of the medical building could never fulfill the demand for insulin that was mounting. Further experimentation in large scale production procedures would be impossible without the collaboration with a well equipped and well staffed commercial firm, prepared to invest company's money in the development of the product. Since the New Haven conference on December 30, 1921, Macleod had been reluctant to enter into a collaboration with Eli Lilly \& Company. But, in May 1922 Lilly was invited to come to Toronto to discuss and work out the details of such a collaboration. In these negotiations the Torontonians were concerned to secure the possibility for other companies to manufacture insulin too. No commercial firm should be allowed to monopolize insulin production, neither Eli Lilly nor any other firm. This had been the leading motive in the decision to take out a 'security patent' in April, and it was a leading motive in the design of an agreement with Eli Lilly. ${ }^{61}$

The meeting resulted in a contract, an 'indenture' dated May 30,1922, between the Board of Governors of the University of Toronto and Eli Lilly and Company. Eli Lilly wanted an exclusive license for an experimental period of one year. During this year there would be complete pooling of knowledge and improvements made in the manufacturing process. Lilly was not allowed to spread information concerning the manufacturing process to other parties. The Toronto researchers, however, were allowed to distribute information about the extraction process. To forestall anycriticism as to handling unethical or unfair, to protect themselves from charges of unethical secrecy the research team published their method, in order to make sure others would know how to make insulin when Lilly's exclusive license expired. (Bliss 1982; Collip 1923) Lilly would supply extracts free of charge for the initial stages of clinical trials in Toronto and in the United States, involving a select group of physicians and institutions. For later stages in the clinical trials Lilly would be selling it at cost. At the end of the experimental year, Lilly wanted a license to manufacture insulin on the same terms as other licensees. The agreement limited Lilly's territorial rights to the United States, Central and South America.

The Board of Governors of the University of Toronto appointed a special committee to do the actual work concerning the licensing resulting from the assignment of patent rights to the university and from the agreement with Eli Lilly and Company. This committee evolved into Toronto's Insulin Committee.

On June 2 and 3, 1922, Best and Collip went to Indianapolis to instruct Eli Lilly's research chemists on the extraction procedures and to help them to prepare a first batch. Pouring in resources it did not last long for Lilly to establish a regular supply of pancreatic extract for clinical testing. During the summer of 1922 the number of American clinicians taking part in the clinical trials gradually increased. During the autumn of the same year insulin was also made available for clinical testing in a number of other Canadian clinics outside Toronto. By February 1923 Clowes estimated that more than one thousand diabetic patients were receiving Lilly's insulin from more than two hundred and fifty physicians in sixty clinics in the United States and Canada. ${ }^{62}$ (Bliss 1982) These clinical trials produced several sets of persuasive 'before and after pictures', some of which were published in medical journals (see 

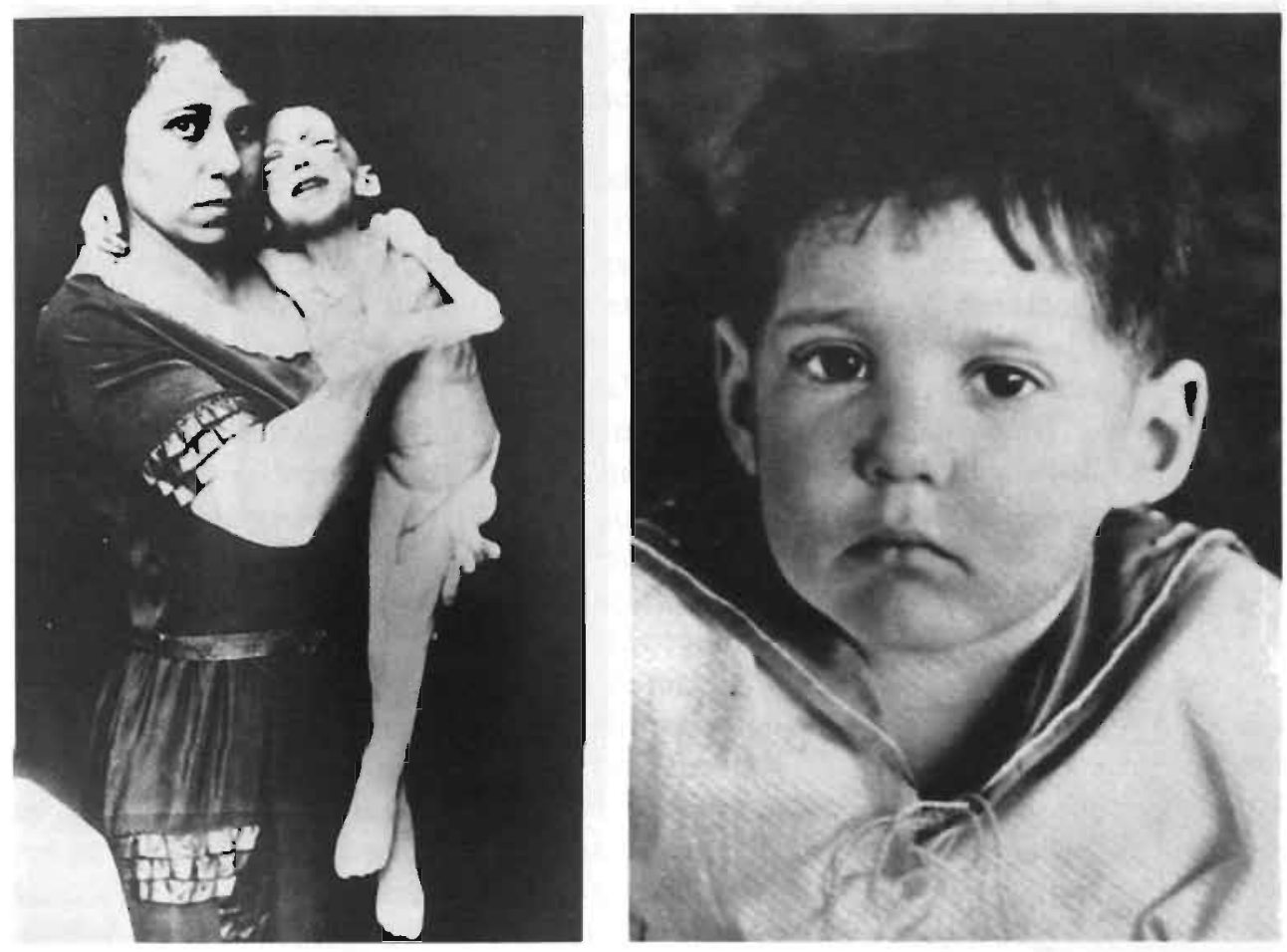

Figure 3.5a

Before and after pictures

I

Before insulin.

Age 3 years, weight 15 lbs.

December 15, 1922

These spectacular pictures appeared in the issue of the Journal of the American

Medical Association that introduced insulin to the profession.

Reproduced from Bliss (1982)

figures 3.5a and 3.5b). Insulin worked, not only in Toronto, but also in other places, and everybody could see insulin's effect with their his or her own eyes.

\section{Extending the network: Great Britain and Denmark}

The patent and license system provided Toronto's Insulin Committee with a legal instrument to exert long distance control over the development of insulin production facilities in other countries. With regard to Britain and the rest of the commonwealth Toronto's Insulin Committee decided, in June 1922, to offer the patent rights to the British Medical Research Council. 

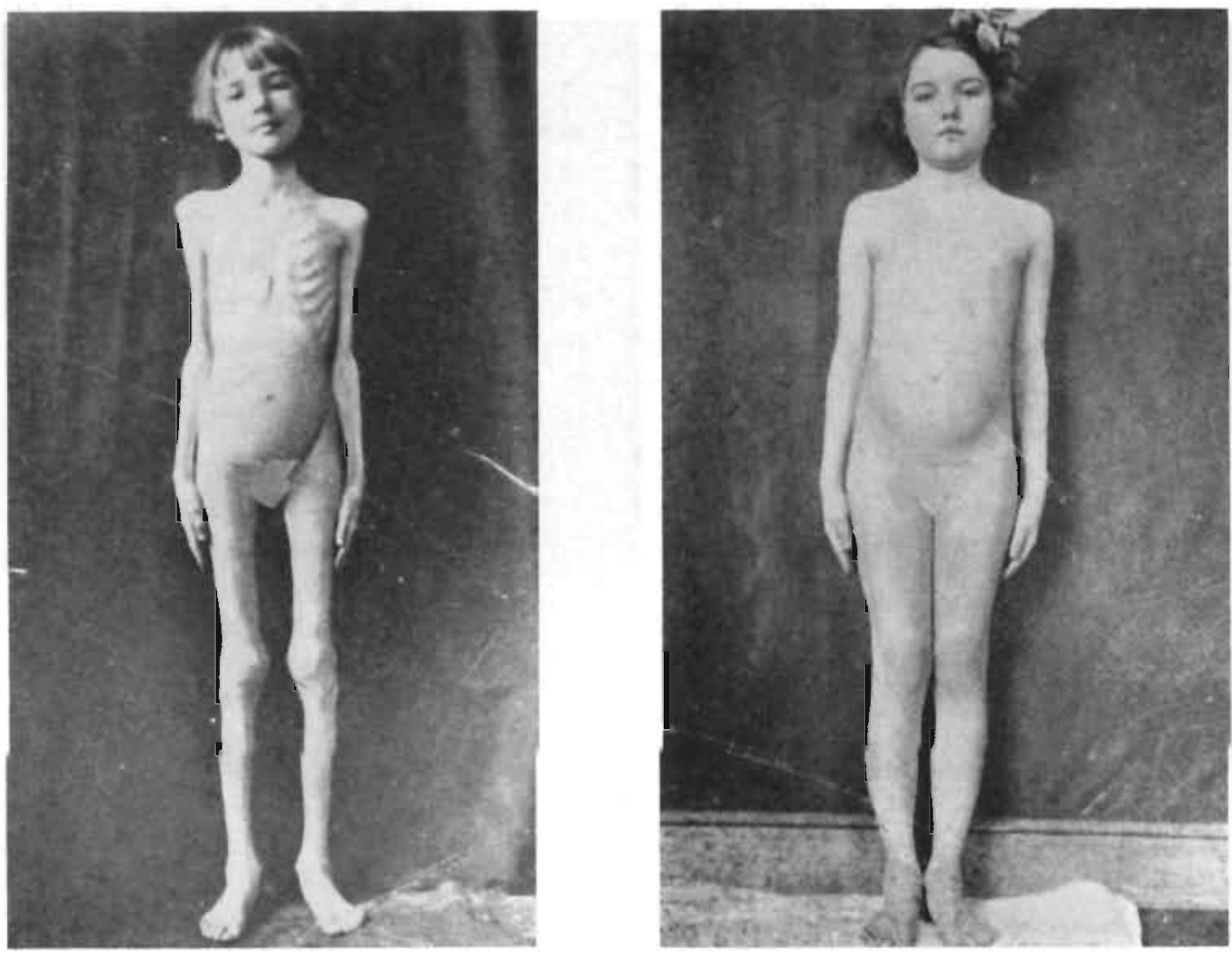

Figure 3.5b

III

Before and after pictures of a 1922 patient of Dr. H. Rawle Geyelin, thought to be too indelicate for lay viewing in the 1920s.

Reproduced from Bliss (1982)

Henry Hallet Dale, director of the biochemistry and pharmacology department of the council's National Institute for Medical Research, together with a colleague, biochemist Harold Dudley, visited Toronto, Eli Lilly in Indianapolis, and several clinics in the United States. Dale and Dudley were very impressed by what they saw in North America. not in the least by some striking cases of diabetic patients treated successfully with insulin. Just like Macleod and Banting. Dale disliked the prospect of being associated with patented drugs. Nevertheless, they admitted that patenting was necessary and advised the British Medical Research Council (MRC) to accept control of the British patent. In their report to the council Dale and Dudley wrote that holding the patent would at least enable the council to

... exercise a moral control over the manufacturers, and would induce the latter to submit to a system of supervision, as regards this product, which the law does not enable the Council at present to enforce. (Bliss 1982: 166) 
The MRC adopted a two stream program that involved, on one hand, the experimental manufacture and clinical testing of insulin, and, on the other hand, efforts were made to develop large-scale commercial production facilities involving a selected number of manufacturers.

Production facilities were established in other European countries too. ${ }^{63}$ In October and November 1922, August Krogh, professor in physiology at the University of Copenhagen, visited Toronto. ${ }^{64}$ Impressed by what he saw there, Krogh decided to take the initiative for the establishment of insulin production facilities for the Scandinavian countries.

Krogh joined forces with Denmark's leading diabetologist, Hagedorn, and with a pharmacist, Kongsted, who privately owned Loven Kemiske Fabrik and Loveapoteket. Krogh and Hagedorn directed and participated in the laboratory research. Hagedorn supervised the clinical testing of batches of extract, while Kongsted financed the laboratory research and set up large scale production facilities as a special division of his firm. In 1924 they were able to supply insulin for Denmark, Norway and Sweden, and even export insulin to other countries.

Kongsted had agreed to refrain from making profit on the production of insulin. Within the Scandinavian countries insulin was supplied at cost. A profit of $35 \%$ was added to the price for insulin sold in non-Scandinavian countries by the - to that end established - International Distributing Company for Danish Insulin Leo. The financial return for insulin sales to non-Scandinavian countries made it possible to separate the insulin production facilities from the rest of Lovens Kemiske Fabrik and establish a new non-profit company: Nordisk Insulinlaboratorium. It was arranged that any profits made by Nordisk on the production of insulin could not be used for anything else than the financial support of scientific research and facilities for diabetic patients in Scandinavia. For the administration of this money Hagedorn and Krogh, established, in cooperation with several other physiologists and physicians from Norway and Sweden, the Nordisk Insulinfund. In addition to the administration of its financial resources the Insulinfund also acted as a Scandinavian insulin committee, comparable to Toronto's Insulin Committee and the British Medical Research Council. (Krogh 1924)

These cursory descriptions of the development of insulin production facilities in Britain and in the Scandinavian countries, illustrates how within a couple of years after the successful treatment of a human diabetic patient a production and distribution network evolved that supplied thousands of physicians and the diabetic patients in their care with insulin. Scientists were actively engaged in the development of this network. They engaged in this organizational work in the interest of individual diabetic patients and of the public in general. They exerted their influence in such a way as to prevent commercial monopolization and, in their view, inadmissible exploitation of insulin production. The shape of the network as it evolved bore an imprint of these scientists' moral conviction concerning the question who should benefit from achievements in medicine and science.

Neither Dale nor Krogh awaited the resolution of tricky questions that could be asked from a strictly scientific point of view. What they saw in terms of persuasive beneficial effects of a treatment with pancreatic extracts was sufficient for them to 
engage. It was first of all the establishment of a therapeutic practice that supported the existence of insulin outside the laboratory.

\subsection{Biological standardization}

\section{Potency testing: disparate rabbit doses}

It is important to grasp what insulin was in these days. What did the word insulin refer to? Insulin was a set of biological activities exerted or performed by a pancreatic extract. It was first of all a pancreatic extract that had a beneficial therapeutic effect on diabetic patients, first on one, Leonard Thompson, later on many. That this biological activity could be precipitated out, dried and redissolved made it obvious that the active principle that exerted the biological activity and therapeutic effect was material in character, a substance. However, the precipitate itself, or the white powder that remained after drying, could not be considered to be that substance. It could be considered to contain the substance, as well as a lot of other substances. Since the precipitate gave a positive biuret reaction, the extract could be considered to contain proteins. But, whether the active principle also was a protein or not was not known.

Little was known about the physico-chemical properties of the active principle. But. that was no real problem. A practical way to capture the active principle and to administer it to diabetic patients had been found. The real problem, both in the development of large scale production facilities as in the treatment of patients, was to estimate the potency, not the amount of substance, of different batches of pancreatic extract.

Collip. when he commenced work on the Torontonian research team, found the normal, healthy rabbit to provide an easy method to test the potency of each batch of extract. However, by measuring the extract's effect on the blood sugar of a normal rabbit he was able to demonstrate that it was potent, but not exactly how' potent it was. A reliable method for the assessment of how potent a batch was, was indispensable, both to evaluate the yield of variations in extraction procedures and to improve the safety of the therapy. ${ }^{\text {is }}$

Nevertheless, Collip's rabbit blood sugar method helped to standardize injections in the first clinical trials in Toronto General Hospital in January and February 1922. Toronto expressed the potency of their batches of pancreatic extract in terms of rabbit doses. One rabbit dose was defined as the amount of extract necessary to lower the blood sugar of a normal rabbit by $50 \%$, to the point where the animal became convulsive, in one to three hours. (Banting et al. 1922b)

In May 1922 Toronto resumed extract production in the Connaught facilities in the basement of the medical building. In June Lilly started production in Indianapolis. The first batches of extract produced by Lilly were sent to Toronto for potency testing and for use in Toronto's clinical trials. Later Lilly tested the potency of their extracts themselves before sending them out, using Toronto's rabbit blood sugar method. In August 1922 physicians participating in the clinical trials were complaining about the lack of potency of Lilly's insulin. Apparently one Lilly rabbit dose was not the same as one Toronto rabbit dose. When looking into this problem they found that Toronto 
and Indianapolis were using 'different' rabbit tests. Lilly used fasting one-kilogram rabbits, while Toronto used a well-fed two kilogram rabbit.

It was relatively easy to adjust the methodological disparities between Lilly and Toronto. The problem became more salient however when more insulin production sites were established throughout the world, in Britain and Denmark for example.

\section{Danish mouse units}

When Krogh and his collaborators in Denmark, in December 1922, started work on insulin they used Toronto's rabbit blood sugar method to test their first batches for potency. When they found their extract to be effective in the rabbit, they injected it into a mouse. Krogh investigated the possibility of using much cheaper mice, instead of rabbits, as a biological assay for insulin. He found that mice exhibit a specific hypoglycemic syndrome, involving first paralysis of the limbs followed by severe convulsions prior to death. Using mice, the Danes developed a new biological insulin assay. They expressed the potency of their batches in terms of the percentage of mice that after injection with a specific amount of extract died or exhibited convulsions. (Krogh 1924)

In March 1923 they received pancreatic extract from Toronto, the potency of which had been tested and expressed in rabbit units. Krogh compared Toronto's rabbit unit with their mouse unit and found 1 rabbit unit to correspond to 600 mouse units.

\section{Comparative testing}

Krogh found that as a rule the mouse convulsion method worked well. It produced reproducible results. But sometimes there were exceptions to the rule. There were so many variables that could influence the outcome of the assay: individual variations between mice, variations in body temperature and room temperature, variations in feeding conditions, etc. Most of these conditions could be controlled. Some could not. When a particular run of the assay produced a negative or unexpected low result, how could one know whether the fault was in the production proces of that particular batch of extract or in the assay itself.

One way of solving this problem was comparative testing; a so called cross over design. (Stewart 1974) The risk of not discovering a failed run of a biological assay could be reduced by simultaneous and comparative testing of each new batch of extract with the previous one. In this way the potency of a new batch could always be related to the previous one. In the treatment of patients, this minimized the risk of inducing hypoglycemic reactions resulting from unexpected potency differences between subsequent batches.

Krogh, however, did not use the previous batch for comparative testing with a new batch. In the autumn of 1923 Hagedorn had prepared a rather large batch of potent pancreatic extract. Krogh used this one batch as a standard in comparative testing. In every run of the mouse convulsion assay for insulin two extracts were used: the standard and the new batch. (Krogh 1924) The stability of a pancreatic extract was a problem though. Deterioration of extracts, the decline of their potency over time, was a problem for therapeutic purposes, but is was even more salient when a liquid extract was intended to serve as a standard over a longer period of time. 


\section{International standardization: the groundwork}

Dale was, more than Macleod or Krogh, engaged in the general problem of biological standardization. In Britain, the National Institute for Medical Research was responsible for the custody, control and supply of biological standards. Dale was the Medical Research Council's chief scientific advisor on the subject. He was largely responsible for initiating policy in this field, including international aspects. He directed the research on biological standardization at the National Institute. (Feldberg 1970)

The ground for international biological standardization had been prepared by Ehrlich with his work on a (stable) standard for diphtheria anti-toxin. Ehrlich proposed to define a unit as the specific activity of an exact weight of that standard. Ehrlich's standard was worldwide accepted. It was kept in Frankfurt. National institutions responsible for the work concerning international biological standards were established in several countries: the National Institute for Medical Research in Britain, the Connaught Anti-Toxin Laboratories in Canada, the State Serum Institute in Denmark.

After World War I, in 1921, an international conference on biological standardization was held in London to endeavour new international agreements and to reverify the correspondence between national diphtheria anti-toxin standards and the international standard in Frankfurt. This 1921 meeting in London was the starting point for an international organization under the League of Nations by which standards were going to be accepted and units of activity defined. International standards for immune sera were going to be kept at the State Serum Institute in Copenhagen.

\section{Crustalline insulin}

When Dale and Dudley in 1922 advised the Medical Research Council to accept the patent rights for insulin for Britain, they were actively engaged in the problem of standardizing pancreatic extracts. In the laboratories of the National Institute Dudley not only succeeded in making potent pancreatic extracts, but also in obtaining insulin in a dry and stable form by precipitating it with picrate. Dale took a vial containing this dry, stable crystalline insulin with him to a 1923 conference in Edinburgh, also attended by Macleod and Krogh. They discussed the standardization of insulin. In 1960 Dale wrote about this meeting:

We had practically a whole day's discussion, largely futile, on the rival claims for a unit defined as the dose of insulin which would throw 2 out of 3 rabbits into convulsions, as put forward by Macleod, who tried to dominate the situation on behalf of the Toronto team, and Krogh's insistence that the proper base of the unit would be to determine the dose which would produce the hypoglycemic convulsions in 6 out of 12 mice. I listened to this wrangle with growing impatience during most of the day, and intervened when I thought that they were getting tired of the controversy without any prospect of a solution. My intervention took the form of insisting that it was complete nonsense to try to define any unit of any remedy in absolute terms of reactions in limited numbers of animals; and that, from the international point of view, the only sensible thing was to obtain the remedy in perfectly stable form, and define the unit in terms of an absolute quantity of such a standard sample ... Macleod replied that he had no doubt that such a policy would be ideal, but that he had no reason to believe that the preparation of a sample of insulin, in a dry and indefinitely stable form, was a practical possibility. At that 
point I was glad to be able to take from my waistcoat pocket a small tube of the preparation, which was to become the first international standard, and roll it across the table to Macleod with the statement: 'Well, there it is'. (Feldberg 1970: 122) ${ }^{66}$

\section{An international standard for insulin}

At the 1923 conference in Edinburgh it was decided to organize an international cooperative effort to investigate the suitability of dry, stabilized standards for posterior pituitary extract, for digitalis and for insulin. This international effort resulted in the adoption at an international conference, organized by the League of Nations Health Organization in Geneva in 1925 and chaired by Dale, of the first international standard for insulin. It was agreed that this standard for insulin, and other international standards for hormones, vitamins and drugs as well, were confided to Dale's National Institute for Medical Research in Hampstead, Britain. (League of Nations 1926)

\subsection{Conclusion}

There it was, insulin, in its most ostensible form: a powder in a tube in a laboratory of the National Institute for Medical Research's special department for Biological Standards in Hampstead, Britain. The powder still contained impurities that were precipitated together with insulin. (Stewart 1974) Insulin's chemical composition was still unknown. But, at least, now there was a basis for further inquiries into insulin's chemical and physical properties. It was widely used in physiological research, as a research tool this time.

Between the 23rd of January 1922 and 1926 insulin's existence became indisputable. As a result of the great persuasive strength of the experiences in the treatment of nearly dead, emaciated diabetic patients, scientific doubts about the internal secretion theory of pancreatic diabetes faded, as the clinical effectiveness of the pancreatic extract in the treatment of diabetes mellitus in humans was acknowledged by leading diabetologists. Supported by as yet small scale laboratory and clinical practices insulin materialized as a set of biological activities exerted by a pancreatic extract. The reduction of doubt through the persuasive success of the treatment of emaciated human diabetics, pertained simultaneously to the existence of insulin as an entity in the world and to the scientific knowledge about this entity. The emergence of insulin as an entity is, however, indistinguishable from insulin as a medical technology.

In 1926 insulin materialized in the form of a powder in a tube, the first international standard for insulin. Verbal statements about its existence, which had been hypothetical and troubled by accumulating counter-evidence. became hard as rock. The successful administration of pancreatic extracts in the treatment of diabetic patients, the resurrection of their bodies, was one of the most persuasive achievements of all, resolving the longstanding paradox of insulin's existence. The establishment in subsequent years of a worldwide network of production facilities and of international standardization procedures further shaped and stabilized insulin's identity as defined by the list of biological and chemical actions performed by pancreatic extracts under a set of variable experimental and clinical conditions. 
In the wake of this process, links that in previous decades had been forged between the then hypothetical internal secretion of the pancreas and other entities stabilized too. The identity of the pancreas had been redefined. Prior to 1889 the pancreas had only one excretory function. Now the pancreas has two functions: one secreting digestive juices to the gut, the other secreting insulin into the blood stream. Langerhans islands, left without a definite function since their establishment as a histological entity in 1869 , were finally equipped with one in 1922 . The disease, diabetes mellitus, was redefined into a endocrine metabolic disorder. Diabetes mellitus in its severest form is no longer a lethal disease. The balance of power between the disease on one hand and the physician and the patient on the other is inverted, thereby profoundly changing the management of diabetic patients in clinical practice.

No scientific knowledge was 'applied' in the development of insulin as a technology. The search for insulin was motivated by the prospect of a possible future therapeutic program of action that could save the lives of thousands of diabetic patients. This program of action, which was contained in the pancreatic internal secretion theory of diabetes mellitus, mobilized many scientists to attempt the extraction of the active principle. In that sense this program of action contributed to the persuasive strength of Minkowski's work. The search for insulin was also motivated by Minkowski's extirpation and transplantation experiments, by the heuristic force of the successtul treatment of myxedema with thyroid gland extracts, and by the morphological analogy between Langerhans' islands and other vascular ductless glands.

Is it possible to talk about insulin as a biological entity, about knowledge about that entity as distinct from the biological entity itself, and about insulin as a technology that can be purchased from Eli Lilly or Nordisk as a commodity? These three faces are sides of one triangular coin. Their distinctiveness is only fictional. Insulin, performs its existence, its presence, and its identity, in the chemical, physical, biological and clinical tests to which it is put. It is supported by the maintenance of these practices, in laboratories, in international standardization agreements, in hospitals. Without these practices it makes no sense to talk about insulin at all.

Now that insulin has stabilized and is part of our world as biological entity, as scientific knowledge about that entity and as a therapeutic technology in the treatment of diabetes mellitus, it is hard to imagine a world in which it, in a sense, did not yet exist. A constructivist account of insulin does not prove the knowledge to be wrong. or the entity to be non-existing, or the technology to be ineffective. However, by making an effort to understand the details of the practices scientists and physicians were engaged in and to describe the events as seen through their eyes, a constructivist approach provides a better feel for what it is that scientist do, how they in their attempt to discover and produce knowledge restructure the world we live in; a restructured world that is subsequently used by scientists and historians to judge the previous, obscuring the doubts and uncertainties their predecessors faced. 


\section{Notes to chapter 3}

1. See for example Engelhardt (1989), Murray (1969), Papaspyros (1964), Poulsen (1982), Pratt (1954), Wrenshall, Hetenyi and Feasby (1962).

2. One day in April 1889 Minkowski went over to the Hoppe-Seyler's Institute to consult some chemical periodicals in its library which were not available in the Medical Clinic. In the library Minkowski met J. von Mering. They discussed the possibility of total pancreatectomy as a experimental design in fat research. Unaware of the fact that Claude Bernard had stated that animals could not be kept alive after complete removal of the pancreas, Minkowski assumed that it could not be more difficult than a hepatectomy, which he had performed successfully several times. Von Mering had a dog available and they decided to give it a try. (Minkowski 1929)

3. Minkowski intended to return the dog to von Mering, who the day after the operation urgently had to go to his father in law who was seriously ill from pneumonia. Because there was no suitable cage available the dog was simply kept tied up in one part of the laboratory. The dog was house-trained and a servant was available to let it out. But even though the dog was housebroken and regularly taken out it kept urinating on the floor. Minkowski at first scolded the servant for not letting it out frequently enough, but the latter replied that the animal was queer; as soon as it came back it passed water again even if it has just done so outside.

4. Minkowski worked as an assistant to Bernhard Naunyn (1839-1925), who was considered to be the most outstanding German diabetologist of that time. Naunyn studied medicine at the University of Berlin where he also received his training in internal medicine at the Charite under Friedrich Theodor von Frerichs, one of Germany's leading diabetologists. Naunyn inherited Frerich's interest in diabetes and devoted his life to it. After working seven years with Frerichs in Berlin Naunyn was nominated professor of clinical medicine at the Imperial Russian University in Dorpat in 1864 . He transferred to Bern in 1871 and to Köningsberg in 1872. Here, in Köningsberg he demanded the establishment of a laboratory as a condition for accepting the post. Oscar Minkowski became Naunyn's assistant in 1882. Minkowski demonstrated that the acid excreted in large quantities during diabetic coma was B-hydroxybutyric acid. When Adolph Kussmaul in 1888 retired from his professorship at the University of Strassbourg the faculty of medicine in Strassbourg unanimously nominated Bernhard Naunyn for the chair of medicine after Adolph Kussmaul. Considering that the University of Strassbourg was one of the prominent universities in Germany this was an indication that Naunyn really was considered to be an authority in German clinical medicine. In Strassbourg Naunyn was heading a medical department where diabetes mellitus was the subject of extensive clinical and experimental research and where experimental scientists like Oscar Minkowski, being a practising physician as well, had easy access to diabetic patients exhibiting all the classical symptoms of the disease. See also Astrup (1985).

5. In 1682 Brunner (1653 - 1895) published a written account of seven experiments in his book Experimenta Nova Circa Pancrease. For a discussion of Brunner's work see Busnardo (1983) and Zimmermann (1989).

6. Minkowski drew on his experience and skills from working on the subject of acid poisoning in human diabetes mellitus, which he gained while working in Königsberg. (Minkowski 1884)

7. To underscore Minkowski's surgical skills Naunyn related the following story: 'Als ich nach Straßbourg gekommen war, stellte sich mir ein Herr vor bei dem ich einen kleinen Polypen genau in der vorderen Kommissur des Kehlkopfs fand. Solche kleine Geschwülste sind an dieser Stelle schwer genug zu sehen, geschweige denn zu operieren. Da damals in Straßbourg niemand war, der sich an den Fall gewagt hätte, so bat ich Minkowski, er möge das machen. Minkowski, der noch nie an eine Kehlkopfoperation gedacht hatte, lachte und wollte nicht. Schließlich aber entschloß er sich, übte sich einige Tage, und nach etwa 14 Tagen konnte er mir melden, daß er den Polypen vollständig und glatt in einer Sitzung entfernt habe'. (Naunyn 1925: 442) 
8. In a note Minkowski added that according to Lustig extirpation of the coeliac plexus is followed by acetonuria and transient glycosuria, not by diabetes.

9. Minkowski also argued that diabetes also developed when the animals were starved for several days after total pancreatectomy, as a result of which the gut was completely empty.

10. At this stage of the research Minkowski did not use Bernard's term internal secretion. In Bernard's definition the term pertained to any substance produced in a tissue and secreted into the blood stream. The liver, for example, was able to produce glucose and secrete it into the blood of the hepatic veins. Hence, glucose was an internal secretion.

11. For the publication of the manuscript Minkowski had easy access to the Archiv fur experimentelle Pathologie und Pharmakologie. Bernard Naunyn, who held Minkowski in high esteem, was editor of this seientific journal.

12. Using Kjeldahl's procedure Minkowski ran nitrogen tests on the urine of pancreatectomized dogs in the first series of experiments. The quantity of nitrogen excreted in the urine was considered to be a measure for the turn over of protein in the organism. By juxtaposing the results of the sugar and the nitrogen determinations Minkowski calculated a sugar-nitrogen ratio: $\mathrm{D} / \mathrm{N}$. In persistent diabetes this $\mathrm{D} / \mathrm{N}$ ratio seemed to be rather constant: $\mathrm{D}: \mathrm{N}=3: 2$.

13. In some of the animals in which only a small portion of the pancreas was left behind a less severe form of diabetes developed. Wound healing in these animals was, despite the rigorous antiseptic procedures, less favorable.

14. Minkowski used double ligations of the blood vessels and cut them in between. In this way he kept the operation field free of blood and procured a clear view on all anatomical structures. He learned which blood vessels were indispensable to warrant a sufficient circulation of blood in the duodenum, and which vessels could be missed. Minkowski succeeded in performing a total pancreatectomy in one session without duodenal necrosis.

15. To prevent necrosis of the duodenum after removal of the intra-abdominal rest of the pancreas Minkowski ligated some of the hlood vessels that ran from the wall of the duodenum to the pancreas during the first stage operation to stimulate the formation of a collateral blood circulation.

16. In two cases a severe diabetes appeared after removal of the intra-abdominal rest of the pancreas in the second stage operation. In these two cases the transplant was not vital enough because the excretions were not allowed to drain to the skin.

17. Assuming the relevance of these observations on dogs in the laboratory for human diabetics, they also suggested that diabetes mellitus occurred in people without any impairment of the excretory function of the pancreas.

18. Since Langerhans' original descriptions of the islands a number of views had been expressed concerning their function in the healthy organism and their pathological significance in disease. None of these explanation gained significant support. Langerhans himself had considered the possibility that the islands were nervous elements, that they belonged to the nervous system. Others thought they were lymph follicles belonging to the lymphatic system. The islands were also considered to be embryonic remnants, to produce raw materials for the production of ferments by the acinar tissue, or to constitute exhausted, regressive or degenerative acinar tissue, dead points in an otherwise healthy and active gland. (Schulze 1900; Ssobolew 1902)

19. Laguesse in 1893 was the first to name the islands after their discoverer. He coined the expression Iles de Langerhans.

20. Links between diseases during life and pathological changes in the tissues and organs of the dead body did not present themselves to the examiner's eye, they could not be found in the corpse. They had to be established in an active process by the pathologist. 
21. Opie (1900a) distinguished two types of chronic interstitial inflammation. In interlobular pancreatitis the inflammatory process was localized chiefly at the periphery of pancreatic lobules. The islands of Langerhans were only implicated when the sclerotic process had reached a very advanced grade. In interacinar pancreatitis the process was more diffuse, invading the lobules and the islands of Langerhans. In only one of eleven cases of interlobular pancreatitis diabetes of mild intensity occurred. The sclerosis was in this case far advanced and affected the islands of Langerhans. Diabetes was present in two of three cases of interacinar pancreatitis, in which the islands were more often affected.

22. It was Barron's article that inspired Banting to attempt to isolate the internal secretion from ductligated and subsequently degenerated pancreas. Barron presented examples of typical changes in islets found in cases of true diabetes together with a detailed study of the histopathology found in a case of pancreatic lithiasis with special reference to the islets, correlated with findings recorded in the literature as obtained in experimental ligation of the pancreatic ducts in animals.

23. During most of its history the rationale for this kind of therapy was not to administer some kind of internal secretion or active principle present in the tissue or in its extract. Animal tissues were applied to transfer its spirit or potency to the patient. Since 1889 physicians in France and in other countries had become very interested in the therapeutic potential of extracts of animal tissues. In june 1889 Charles Edouard Brown-Séquard, the French neurologist and physiologist, at the age of 72 , published his experiences with the subcutaneous injection (on himself) of a liquid obtained by the maceration on a mortar of the testicle of a dog or of a guinea pig to which a little water was added. He suggested to the Society of Biology in Paris that the testes contained an active, dynamogenic, invigorating substance, which he thought he could obtain from animals and inject into men and rejuvenate them. According to Borell (1976a; 1976b) Brown-Séquard's experiments provoked a worldwide response. Within weeks, testicular extract was being given to patients with every kind of illness. Within two years, many physicians thought that not only the testes, but every organ of the body possessed some active principle which might be of immediate therapeutic value. Experiences with Brown-Séquard's testicular extract were ambiguous. While the interest in Brown-Sequard's testicular therapy waned positive results were published regarding the use of adrenal and thyroid extracts. Schäfer, professor of physiology at University College in Britain, holding the chair after Oliver Sharpey, interpreted the data on the pancreas by reference to a hypothetical internal secretion: 'The only fact that appears certain in connection with the manner in which the pancreas prevents excessive production of sugar within the body is that this effect must be produced by the formation of some material, secreted internally by the gland and probably by the interstitial vascular islets, and that this internally secreted material profoundly modifies the carbohydrate metabolism of the tissues.' (Schäfer 1895: 322)

24. The heuristic function of the analogy was also expressed by Mansell-Jones (1893). See also Mackenzie (1893), Wood (1893), Hale White (1893) and an editorial comment in the British Medical Journal. (BMJ 1893)

25. In the following sections this 'assay problem' will recur several times. It will be argued that the establishment of a biological assay follows the resolution of the existence problem. The biological activity of an extract is then going to define the identity of the internal secretion, and not vice versa.

26. See also Mellinghoff (1971).

27. Martina transplanted pancreatic fragments in a dog in highly vascular tissues like the spleen, and removed the rest of the pancreas. In such sanguineous environments these fragments, devoid of any connection to their original blood vessels and nerves, continued to function. Although the dog excreted a certain amount of sugar in his urine the diabetes did not develop its full strength. The most important result was that this 'pancreatectomized' dog with only a small transplanted fragment left in his spleen lived for a number of months after the operation. Cited in Forschbach (1908a).

28. Forschbach also considered the possibility that only the excess of sugar was transferred from the pancreatectomized animal to the healthy animal where it was burned in the tissues (not excreted). $\mathrm{He}$ 
rejected this possibility, since he judged the extent of the vascular communication too small to account, on its own, for the prohibition of diabetes. To support this assumption Forschbach injected lactose in one animal and found that the majority of the injected amount of lactose was excreted by the animal in which it was injected. (Forschbach 1908b)

29. Carlson and Drennan were troubled by the discrepancy between their experimental results and clinical experience regarding the effects of pregnancy on the course of diabetes in humans. Pregnancy augments the diabetic symptoms, especially towards the end of gestation. This would indicate a primary difference in the etiology of diabetes in man and of experimental pancreatic diabetes in other mammals. Carlson and Drennan tried to rescue the internal secretion theory of diabetes by arguing that the difference might only be apparent. If the diabetes in the mother was caused by the depression of the pancreas by some substance in the blood. these substances would in all probability act in the same way on the fetal pancreas or pancreatic secretion, thus giving the usual clinical findings. (Carlson and Drennan 1911)

30. Zuelzer, born in 1870 in Berlin, hegan his medical training in the year in which Minkowski performed his first pancreatectomy: 1889. He graduated from medical school in 1893 and worked as an assistant to, among others, Raphael Lepine and Carl von Noorden. In 1900 he started working as specialist in internal medicine in Berlin. (Mellinghoff 1971)

31. Zuelzer was supported by professors I. Munk and P. Schulz. Financial support was granted by the Grätin Bose-Stittung.

32. Contained Zuelzer's pancreatic extract insulin? At this place I would only like to refer to the problem of what effect had to be regarded as proof for the existence and presence of insulin: reduction of glycosuria, recovery from diabetic coma? Zuelzer argued that the neutralization of adrenal extracts should be on the list too.

33. Zuelzer was aware of the cumulating failing attempts to prepare an effective pancreatic extract containing the hypothetical internal secretion of the pancreas, including the pessimistic conclusions of some authors as to the achievability of such an endeavour. However, apart from his own experiments on rabbits, Zuelzer was encouraged by Starling's theoretical and experimental work on hormones. (Zuelzer et al. 1908)

34. The American patent application contained the most detailed, but nevertheless rather vague, description of the extraction procedure. For details see Mellinghoff (1971). For comments on extraction procedures, especially concerning the alleged destructive action of proteolytic enzymes, see Pratt (1954), Feasby (1958), Mellinghoff (1971) and Bliss (1982).

35. According to Zuelzer the rupture of the wound was a coincidence. 'Der Hund war im vollstem Wohlsein plötzlich gestorben.' (Zuelzer et al. 1908: 1383)

36. Zuelzer's original publications were not very clear on the point whether the patient was actually in coma at the moment of injection.

37. No information could be retrieved concerning the reasons why Schering withdrew their support. Schering's pre-war archives were lost in the war and post-war period. Mellinghoff (1971) only mentioned that Schering lost their interest because of the "hohen Fabrikationskosten des einzelner Präparates von 50,- Mark'.

38. See Mellinghoff (1971).

39. He found an inside-ally in '... eine auf dem Schlachthof quasi beheimatete Frau Huwe, die in resoluter Weise für Zuelzer's Anliegen eintrat und sich im Laufe der Zeit auf die Gewinnung und Frischerhaltung der Drüsen im größten Maßstabe spezialisiert hat.' (Mellinghoff 1971: 45)

40. Nothing was ever published about what they did there.

41. Where, in this and the following sections, the events in Toronto are concerned I rely much on Bliss' excellent historical account of the discovery of insulin in Toronto. Bliss, working as a historian 
in Toronto, had access to valuable sources, including laboratory notebooks, desk calendars, interview material, archives of Toronto University, Eli Lilly and Company, Karolinska Institute, correspondence, letters etc. Bliss' is the most detailed written account available at this moment.

42. Frederick Grant Banting grew up on a farm in small town called London in Ontario, Canada. He went to higher education at the University of Toronto. Toronto's medical faculty was one of the largest in North America, well equipped and well staffed. Toronto General Hospital, the faculty's teaching hospital, had recently been rebuilt. Banting enrolled in Medicine in 1912. Banting was an average student, but studious. Bliss (1982) characterized his studiousness as a kind of dogged determination to get through, but it never won him more than average grades. For biographical details see Stevenson (1947).

43. Banting was not the first North American researcher who tried to extract the hypothetical active principle from the pancreas. Scott $(1911 ; 1912 ; 1913)$ and KJeiner $(1919 a)$ had been working on the subject too. They both got very encouraging results. They both believed that they were on the right track, but none of them continued the work. After tinishing his thesis Scott drifted into specific problems of determining the concentration of sugar in the blood. Kleiner left the problem for personal reasons. He accepted an appointment at another university which had no laboratory facilities and resources available to continue the work on pancreatic extracts.

44. Before going to Toronto Banting visited Western's library to search the literature for publications, but did not find anything. This contributed to his excitement, that he was on to something, maybe even worth dropping his private practice for.

45. Because Banting intended to work through the summer one of them would have to split his summer holidays. They tossed a coin to see who would work first. This happened to be Best. They agreed to change later, but when time came they decided that it was better that Best continued working with Banting. So Noble never got in to the project. (Noble's letter to Guy's Hospital Gazette, 28-8-71, cited in Medvei 1982: 468)

46. The arrangements resulted in a private working place for Banting, a place for trial and error, but also a place that sheltered him from the outside world. Although most Torontonians had little to do with research going on at the university, there was an active anti-vivisectionist movement in Toronto at that time, opposing to the exploitation of animals in medical research. Anti-vivisectionist criticism caused Macleod and other medical researchers a fair amount of trouble. Macleod set up the university's Surgical Research Committee as a line of defence to head off the anti-vivisectionists. (Bliss 1982) When Banting later that summer, dissatisfied with the little room in the physiology building, used the better operating facilities Starr had offered to him, Macleod advised him to be careful. No one should see him transporting operated animals from one building to another on campus.

47. The most comprehensive reconstruction of what happened in the laboratory, compiled from Banting's and Best's original laboratory notebooks can be found in Bliss (1982). In comparing thest' notebooks with the first article reporting this work Bliss found considerable discrepancies.

48. Bliss pleads Banting and Best free from dog-napping: 'If any dog-napping was done to supply the university's animal labs, as Torontonians occasionally feared, and the anti-vivisectionists in the city ... regularly charged, at least technically it was not by Banting and Best. (Bliss 1982: 61) But they did not bother asking how their suppliers procured the dogs.

49. Plans for transplants or grafts were apparently abandoned in favor of the easier and quicker method of extracting. Banting's notes from June and Macleod's parting instructions from June 14. included plans for both intra-peritoneal and subcutaneous transplants of atrophied pancreas in pancreatectomized dogs. (Bliss 1982)

50. In retrospect, this is a very curious result. Trypsin, being a proteolytic enzyme, should have destroyed insulin, which we now know is a protein. The extract should have been ineffective. So something curious was going on there. It might also be argued that this result cast doubt on the 
correctness of Banting's working hypothesis concerning the destructive action of the external secretion on insulin, because trypsin is an active ingredient of the external secretion of the pancreas. It should be remembered, however, that at that time nothing was known concerning the nature of the internal secretion, whether it was a protein or not. That in vilro incubation with trypsin did not destroy the extracts action did not falsify the working hypothesis. During the extraction process it could be one of the other constituents of the external secretion that destroyed the internal secretion.

51. The cause of death seemed to be poorly ground particles in the extract damaging the dog's veins.

52. Banting had not received any salary from the University of Toronto during the summer. Convinced that he was on the right track in the beginning of September he had decided to sell his London-house. Banting would not be able to continue his research without some kind of financial support. This problem was solved by Velyien Henderson, professor of pharmacology at the University of Toronto. Banting had met Henderson in September and told him about his precarious financial situation. Henderson offered Banting a job as special assistant in pharmacology from October 1, 1921. Macleod arranged for retroactive salary for Banting and Best, for the work they had been doing in the summer.

53. Later Banting and Best found that Marjorie was their best evidence that they could make potent and non-toxic anti-diabetic extracts. They regretted, however, that they kept so poor records of her.

54. In December 1922 Roberts (1922) criticized Banting's and Best's work by stating that the production of insulin originated in a wrongly conceived, wrongly conducted and wrongly interpreted series of experiments. According to Roberts, it was in 1922 one of the best established facts in physiology that the proteolytic enzyme exists in the pancreas in an inactive form, trypsinogen, which is activated normally only on contact with another ferment. enterokinase, secreted by the small intestine. It can also be activated on autolysis of the pancreas whole or macerated at body temperature, but only slowly. Roberts critique of Banting's and Best's work remained an important issue in all critical historical accounts of the discovery of insulin. See also Pratt (1954).

55. Banting had met Collip during one of his first meetings with Macleod. Collip, with his expertise in biochemistry and interest in tissue extracts, had offered to help when he came to Toronto. He had actually been eager to help at an earlier stage, and Banting had urged Macleod to bring Collip in. But Macleod found that this was Banting's and Best's research and that they had to wrap up the first stage of their work. Then if results were good, he himself and Collip would come in to develop the project further.

56. His first attempt to make a potent pancreatic extract, using alcohol and fresh whole gland, failed. He didn't get the same results as Banting and Best did. It turned out that he instructed his lab boy to go to the abattoir for sweetbreads. The boy was bringing back thymus, which Collip took to be pancreas. (Bliss 1982)

57. According to Bliss, no record was kept on the discussion following Banting's presentation of the paper. Some of the issues discussed could be traced, however, through the recollections of a number of people who attended the meeting. Other issues were likely to be discussed considering the work of people like Kleiner, Scott and Carlson who were in the audience. Bliss was cautious: 'Few of these questions, if they were asked, could be answered satisfactorily by the Toronto team'. (Bliss 1982: 105)

58. Later in 1948 Clowes wrote that anyone who was at all cognizant with the subject must have realized that a great discovery had been made and provided the work could be brought to fruition there was every prospect that an important means of treating diabetes would be developed. (Clowes 1948)

59. Mellinghoff (1971) regretted that Zuelzer did not perform blood sugar estimations on these dogs. Mellinghoff granted that Zuelzer could not be blamed for that. In Zuelzer's view only adrenalin could mobilize sugar from the liver. The pancreatic internal secretion only neutralized adrenalin. Insulin had no sugar-mobilizing capacity. An overdose of insulin, that is, more than was required to neutralize the adrenal secretion, would not cause hypoglycemia. 
60 . By the end of the second decade of the 20th century no curative treatment for diabetes mellitus had been found. The prognosis for severe cases of diabetes mellitus was as bad as it had ever been before. The 'treatment' most widely used was Allen's diet. In Allen's dietary regulation of diabetes food and caloric intake was reduced to a minimum. Patients were literally starved to death. They gained maybe a couple of month, maybe a year. But caloric intake was so low that finally they would die from starvation, if not the diabetes or some complicating disease killed them first.

61. Eli Lilly and Company, a U.S. firm seated in Indianapolis, had been in the business of manufacturing pharmaceuticals since 1876. It was, as Bliss (1982: 138) ealled it, an 'ethical' drug company, that is, they produced no patented medicines, they made no extravagant claims and advertisements and sales were focussed on physicians and pharmacists only. Lilly's research director, Clowes, had also considered it appropriate for insulin licensees to pay Toronto royalties on all the insulin sold. This money could in turn be used to finance further diabetes research. Eli Lilly and Company was one of the first pharmaceutical industries that had decided to develop, as an important element in the company's policy, links with the scientific community. Lilly had hired Clowes in 1919 as a research chemist. Clowes was appointed director of research in 1920. Lilly had developed respectable research facilities by May 1922. As, Clowes pointed out at the meeting in Toronto, Lilly had a good team of themists ready to work on insulin.

62. See for example Allen and Sherrill (1922a; 1922b), and Williams (1922).

63. Ernst Laqueur, professor in pharmacology at the University of Amsterdam, visited North America and Toronto in the summer of 1922 . He obtained a license for the manufacture of insulin in the Netherlands. Laqueur persuaded Saal von Swanenberg, director of Zwanenberg's Slaughterhouses and Factories, to establish a new pharmaceutical company: Organon. Laqueur moved basic equipment from his laboratory in Amsterdam to Oss and was appointed head of Organon's research laboratory. Insulin was the first drug produced by Organon. (Tausk 1978)

64. Krogh had been awarded the 1920 Nobel Prize in physiology and medicine for his work on capillary action during exercise. He had been invited to the United States to lecture on his capillary work. While he was there, he heard the news about the Toronto achievements.

65. Human diabetics soon experienced the hazards of this new therapy that had to save their lives. Dr. Williams in Rochester threw one of the first patients he treated with the pancreatic extract into a profound insulin shock. He was so lifeless that the chief of the surgical staff pronounced him dead. Fortunately, Williams was able to 'restore the patient to life' by the injection of some glucose. (Bliss 1982)

66. See also Murnaghan and Talalay (1992). 


\section{Theaters of war, truth and competence Intermittent positive pressure respiration during the 1952 polio-epidemic in Copenhagen}

\subsection{Introduction}

During the last couple of decades social historians have recognized the role of epidemics as catalysts for political and social change. By their disturbing and upsetting effects epidemics, like wars ${ }^{1}$, force the display of strengths and weaknesses in the political order of the infested societies. Social historians apprehended epidemics as strategic research sites for the investigation of the dynamics of social change. Briggs (1961) wrote that whenever cholera threatened European countries, it quickened social apprehensions. Whenever it appeared, it tested the efficiency and resilience of local administrative structures. It exposed relentlessly political, social and moral shortcomings.

According to Rosenberg an epidemic, if sufficiently severe, necessarily evokes responses in every sector of society. Values and attitudes, especially in the areas of religion, of traditionalism and innovation are, for example, inevitably displayed during an epidemic. An epidemic, Rosenberg argues (1992), provides a convenient and effective sampling device for studying in their structural relationships some of the fundamental components of social change.

But epidemics do not only provide a fruitful sampling device for studying social change, they also provide an interesting research site for studying the dynamics of technological innovation and scientific change. This chapter relates the story of a major breakthrough in the treatment of lethal cases of infantile paralysis (poliomyelitis anterior acuta) during the 1952 polio-epidemic in Copenhagen. The, in Mushin's (1980: 207) words, 'revolutionary new treatment' of tracheostomy and manually controlled respiration did not only reduce mortality rates of bulbar poliomyelitis, it also marked a significant episode in the development of positive pressure respirators and their use ${ }^{2}$. In chapter 3 emphasis was on the science, on the onto-epistemological dilemma of insulin's existence, presence and identity, and the resolution of this dilemma in the 'discovering' establishment of insulin as a biological entity. But the 
account in chapter 3 also touched upon the development of a worldwide network of new industrial organizations and institutions set up for the production, distribution and international standardization of insulin, securing its availability, reliability and stability as a medical technology. In this chapter emphasis will be on technological and therapeutic innovation, situated in a Danish hospital specialized in the treatment of infectious diseases, where, in the fall of 1952, the medical staff had to cope with a high mortality epidemic of poliomyelitis, manifesting a high incidence of a specific form of the disease, bulbar polio, characterized by paralysis of the muscles involved in swallowing and breathing. But this chapter will also touch upon associated cognitive changes. A dividing boundary was traced through the old irremediable clinical syndrome of lethal bulbar polio, splitting it into a lethal but remediable condition of carbon dioxide accumulation on the one hand, and a viral infection of the spinal cord and brain stem which was no longer lethal when the adequate ventilation could be maintained by means of positive pressure ventilation. The success of positive pressure ventilation, which was proposed by and applied under the direction of anesthesiologists, gave impetus to the re-evaluation of anesthesiology's status as a medical discipline, the establishment of anesthesiological departments in academic and general hospitals, and to new chairs in anesthesiology.

The trial or test to which the 1952 polio-epidemic put Copenhagen's medical system reshuffled prevalent knowledge, technologies and relations between medical professions. The battle took place in Copenhagen's hospital for communicable diseases: Blegdamshospital.

\subsection{Setting the stage: Blegdamshospital}

Blegdamshospital itself was a sequel of a cholera epidemic which infested the city of Copenhagen in $1853 .{ }^{3}$ Being one of the most densely populated capital cities in Europe, Copenhagen counted approximately 140.000 inhabitants within its system of fortifications. Hygienic conditions were very poor. Three and four story houses were in-

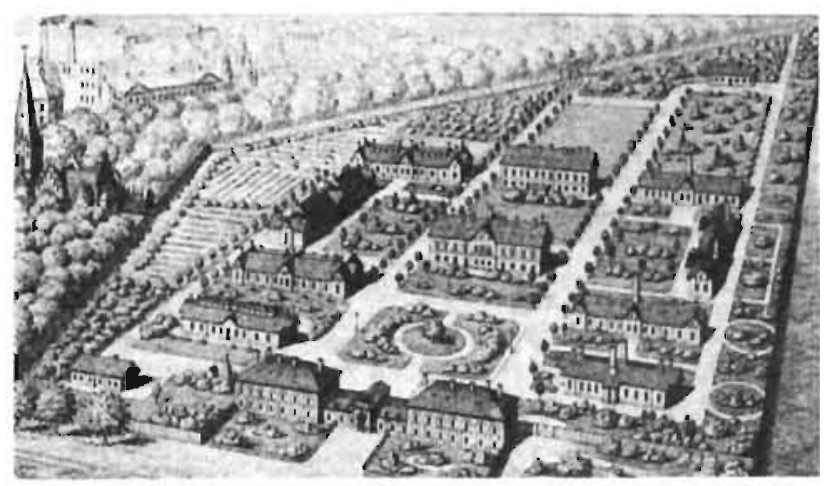

Figure 4.1

Blegdamshospital 1884

Reproduced from Faber et al. (1979: 16) 
habited from the attic to the cellar. Sewage accumulated in cesspits. Once or twice a year the sewage was collected and transferred to a location outside the city by horse and wagon. Where sanitary conditions were poorest sewage ran directly into the open system of gutters in the streets. Unrefined surface water was led into the city through an open system of wooded channels and gutters which were easily polluted by sewage

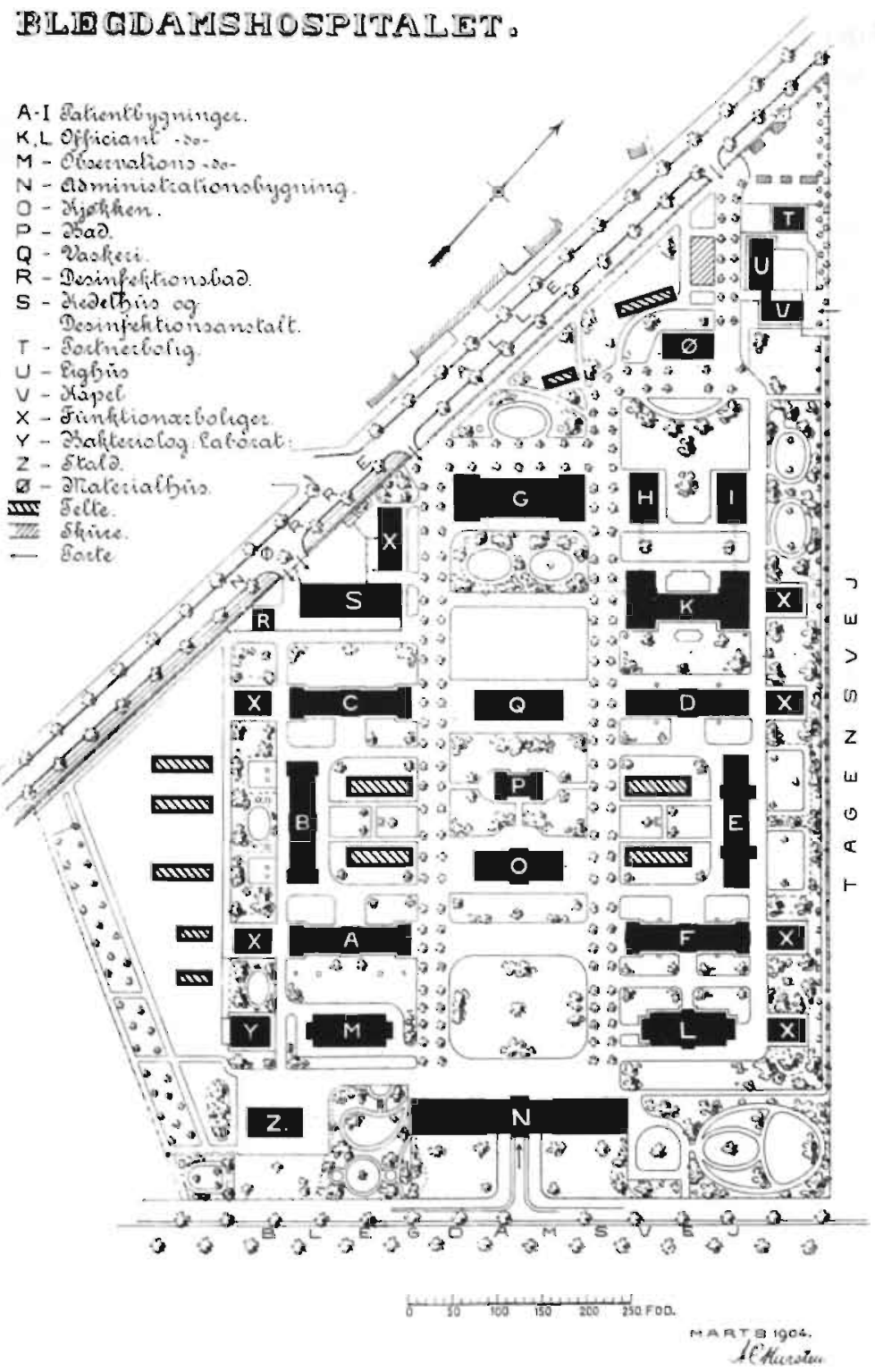

Figure 4.2

Reproduced from Kjobenhavns Magistrat (1904: 28) 
(Hertel 1980). Leading hygienists called for radical sanitary reforms and improvements in Copenhagen. While cholera held sway in surrounding countries quarantine regulations on cholera were lifted in 1852 in concordance with prevailing miasmatic views concerning the cause of the disease. ${ }^{4}$ One year later the 1853 cholera epidemic had free reign in Copenhagen. (Drachmann 1943; Hertel 1980) ${ }^{5}$ Morbidity and mortality statistics, itemized per quarter, confirmed that the epidemic raged most violently in the city's poorest and most populous quarters and hospitals, where hygienists had predicted that it would. The devastating experiences with the cholera epidemic supported the hygienists' demands for sanitary reforms. Reform programs for the improvement of housing, sewerage, water supply and lighting were instigated.

To make up for Copenhagen's hospitals' inability to meet the challenge of epidemic diseases a new hospital, Kommunehospital, was built and opened in 1863 . Kommunehospital was equipped with a separate isolation building which housed the ward for epidemic, communicable diseases, such as smallpox and cholera. With 20 beds, however, its capacity was rather limited. Within a few months after it coming into operation the number of patients admitted outnumbered the available space in the isolation building. Patients with dangerous infectious diseases had to be admitted to other, regular wards too. With some makeshift contrivances the Kommunehospital managed for several years, but it was clear that in the long run an adequate solution had to be found. (Kjøbenhavns Magistrat 1904)

In 1871 Copenhagen's administrative authorities decided to instigate the building of a new hospital with 170 beds for a selected number of epidemic and communicable diseases only: Blegdamshospital ${ }^{6}$ (see figure 4.1 and 4.2).

Three principles underlay the design and construction of the hospital. The hospital should allow for the isolation of affected patients, and thus the protection of non-affected people. It should provide a clean, well ventilated, sunny and healthy environment for affected patients to recover from their disease. And, ready-at-hand facilities should be adjusted to routine handling of patients under non-epidemic conditions, while on the other hand facilities should be available for a rapid increase of the hospital's capacity in the case of epidemics (from 170 to 300 beds). (Kjøbenhavns Magistrat 1904; Faber et al. 1979) Blegdamshospital came in operation in 1879.

Over the next decades treatment grew increasingly important in addition to Blegdamshospital's primary isolation and care functions. Blegdarnshospital developed into a center of medical and epidemiological expertise with regard to infectious, communicable diseases (smallpox, dysenteria, scarlet fever, diphtheria, poliomyelitis).

\subsection{Polio in Copenhagen}

Although paleo-epidemiologists assert the occurrence of poliomyelitis in ancient times. as indicated by the atrophic leg depicted in the Egyptian engraving represented in figure 2.1 , it was not defined as a distinct, clinical disease entity before the nineteenth century. The German physician Jacob von Heine in 1840 described the neurological sequelae and muscular deformations remaining after the acute onset of the disease. The first epidemics of poliomyelitis occurred in the Scandinavian countries during the nineteenth century. These epidemics were described in 1897 by the Swedish physician 
Oscar Medin?. Although paralytic cases of Heine-Medin disease, as poliomyelitis was called for some time, were registered in Denmark and Copenhagen since 1905, major epidemics did not occur until the thirties of the twentieth century (see figure 4.3). ${ }^{8}$ (Lassen 1939)

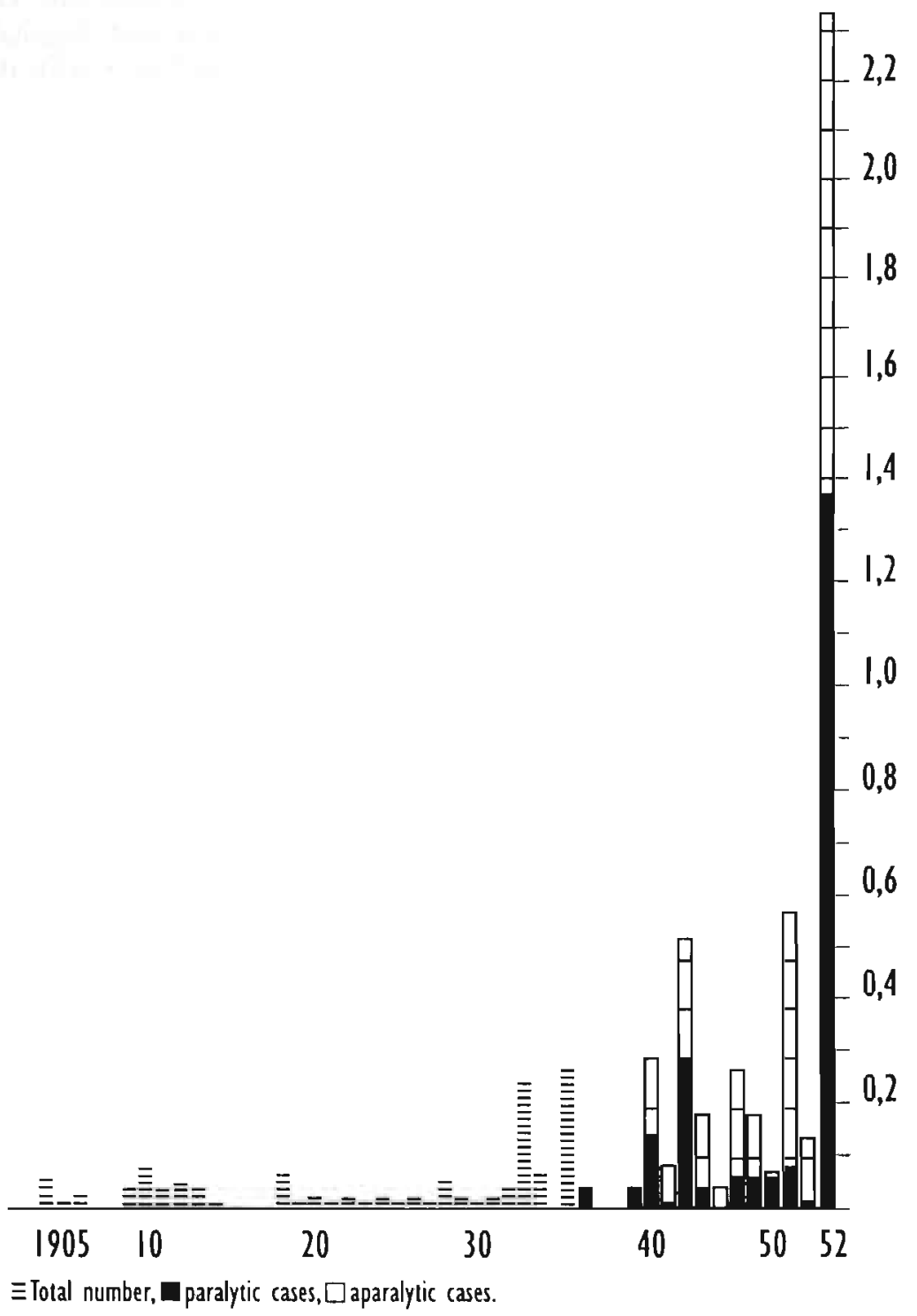

Figure 4.3

Poliomyelitis in Copenhagen

Notified cases per 1,000 inhabitants, $1905-1952$

Modified from Ammundsen (1953: 20) 
The 1952 polio-epidemic's severity

The severity of the 1952 polio-epidemic in Copenhagen was unprecedented in the history of the city. It was the severest ever for several reasons. The number of cases of poliomyelitis, in which the diagnosis was verified, requiring admission to hospital and treatment, was higher than ever (see figure 4.3). With a total number of cases of 2,899 (see table 4.1 ) on a population of 1.2 million in the metropolitan area of Copenhagen, the attack rate was 238 per 100,000 inhabitants:

POLIOMYELITIS IN DENMAKK, 1952

\begin{tabular}{|c|c|c|c|c|c|}
\hline \multirow{2}{*}{$\begin{array}{l} \\
\text { Entire country } \\
\text { Copenhagen }\end{array}$} & \multirow{2}{*}{$\begin{array}{c}\begin{array}{c}\text { Total number } \\
\text { of cases }\end{array} \\
5.676 \\
2,899\end{array}$} & \multicolumn{2}{|c|}{$\begin{array}{l}\text { Paralytic } \\
\text { No. per } 100,000\end{array}$} & \multicolumn{2}{|c|}{$\begin{array}{l}\text { Non-paralytic } \\
\text { No. per } 100,000\end{array}$} \\
\hline & & $\begin{array}{l}2,450 \\
1,280\end{array}$ & $\begin{array}{r}56 \\
105\end{array}$ & $\begin{array}{l}3.226 \\
1.619\end{array}$ & $\begin{array}{r}52 \\
133\end{array}$ \\
\hline
\end{tabular}

Table 4.1

Reproduced from Lassen (1956: 1)

... a figure far exceeding the figures for the three big epidemics in New York in 1916, 1931 and 1944, and the severe epidemics in the United States of America in 1952 and in Sweden in 1953. (Lassen 1956: 1)

Although the majority of patients was under the age of fifteen, the 1952 epidemic distinguished itself from previous epidemics through the relatively high attack rate among adults. Among the female population of Copenhagen for example the attack rate was 118 per 100,000 in the age group from 25 - 29 years (see table 4.2). (Lassen 1956) Furthermore, the incidence of involvement of the upper part of the central nervous system surpassed all previous experience (see figure 4.3). Blegdamshospital

Paralytic poliomeletts in Relation to Age and Sex.

(RATES PER 100,000 INHAAITANTS)

(HAMTOFT, 1953)

\begin{tabular}{|c|c|c|c|c|c|c|}
\hline \multirow[t]{2}{*}{ Age } & \multicolumn{2}{|c|}{ Entire country } & \multicolumn{2}{|c|}{ Copenhagen } & \multicolumn{2}{|c|}{$\begin{array}{l}\text { Entire country } \\
\text { Outside } \\
\text { Copenhagen }\end{array}$} \\
\hline & Male & Female. & Male & Female & Male & Female \\
\hline Under 1 year & 99. & 106 & 248 & 255 & 49 & 58 \\
\hline 1 to 4 years & 277 & 226 & 592 & 445 & 157 & 149 \\
\hline 5 to $9 "$ & 169 & 121 & 324 & 251 & 118 & 77 \\
\hline 10 to $14=$ & 55 & 40 & 114 & 78 & 38 & 29 \\
\hline 15 to $19 \% "$ & 38 & 62 & 65 & 134 & 31 & 40 \\
\hline 20 to 24 & 37 & 54 & 56 & 86 & 30 & 40 \\
\hline 25 to 29 & 46 & 66 & 84 & 118 & 30 & 42 \\
\hline 30 to 44 . & 27 & 28 & 47 & 50 & 18 & 16 \\
\hline over & 3 & 2 & 3 & 2 & 3 & 2 \\
\hline
\end{tabular}

Table 4.2

Reproduced from Lassen (1956: 2) 
served as a the polio-center for the metropolitan area of Copenhagen and the eastern parts of Denmark.

\section{Fighting a lost battle}

During the first four months of the epidemic the hospital treated three times as many cases of poliomyelitis with respiratory insufficiency, paralysis of the ninth, tenth and twelfth cranial nerves, and involvement of the bulbar respiratory and vasomotor centers as in the preceding ten years.

From the beginning of August [1952] to the end of the year nearly 3,000 patients with a diagnosis of poliomyelitis were admitted to the Blegdam Hospital and in 2,241 the diagnosis was verified. Of a total of about 1,250 cases with paralysis no less than 345 had insufficiency of respiration or impairment of swallowing or both, and required special treatment. (Lassen 1956: ix)

Patients with bulbar and respiratory poliomyelitis had been treated in Blegdamshospital prior to the 1952 epidemic, but invariably with unsatisfactory results, the mortality rate never being under $80 \% .15$ out of 76 patients with respiratory and bulbar affections had survived in the previous 10 years. (Nielsen 1946) The treatment of bulbar poliomyelitis was considered to be one of the most complex therapeutic problems in medicine. It was thought that

... patients with bulbar and respiratory poliomyelitis died because of overwhelming, intractable 'bulbar' virus infection, i.e., infection of the medulla oblongata, pons, and midbrain. This caused an understandable therapeutic defeatism. (Lassen 1955: 157)

In the introductory remarks to the scientific report and evaluation of the 1952 polioepidemic in Copenhagen Henry Cai Alexander Lassen (1900 - 1974), chief physician and epidemiologist of Blegdamshospital since 1939, admitted that

... although we thought we knew something about the management of bulbar and respiratory poliomyelitis, it soon became clear that only very little of what we did know at the beginning of the epidemic was really worth knowing. (Lassen 1956: ix)

During the first three weeks of the epidemic 31 patients with respiratory or bulbar poliomyelitis had been admitted to Blegdamshospital and treated in negative pressure respirators (one 'iron lung' and six 'cuirass respirators'). 27 of them died; 19 within three days of admission. That is a mortality rate of $90 \%$. (Lassen 1953; Ibsen 1956)

Just being the beginning of what seemed to develop into a major epidemic with patients with bulbar and respiratory poliomyelitis pouring in (see figure 4.4) the hospital's medical staff faced a catastrophe they certainly were inadequately prepared to cope with. 


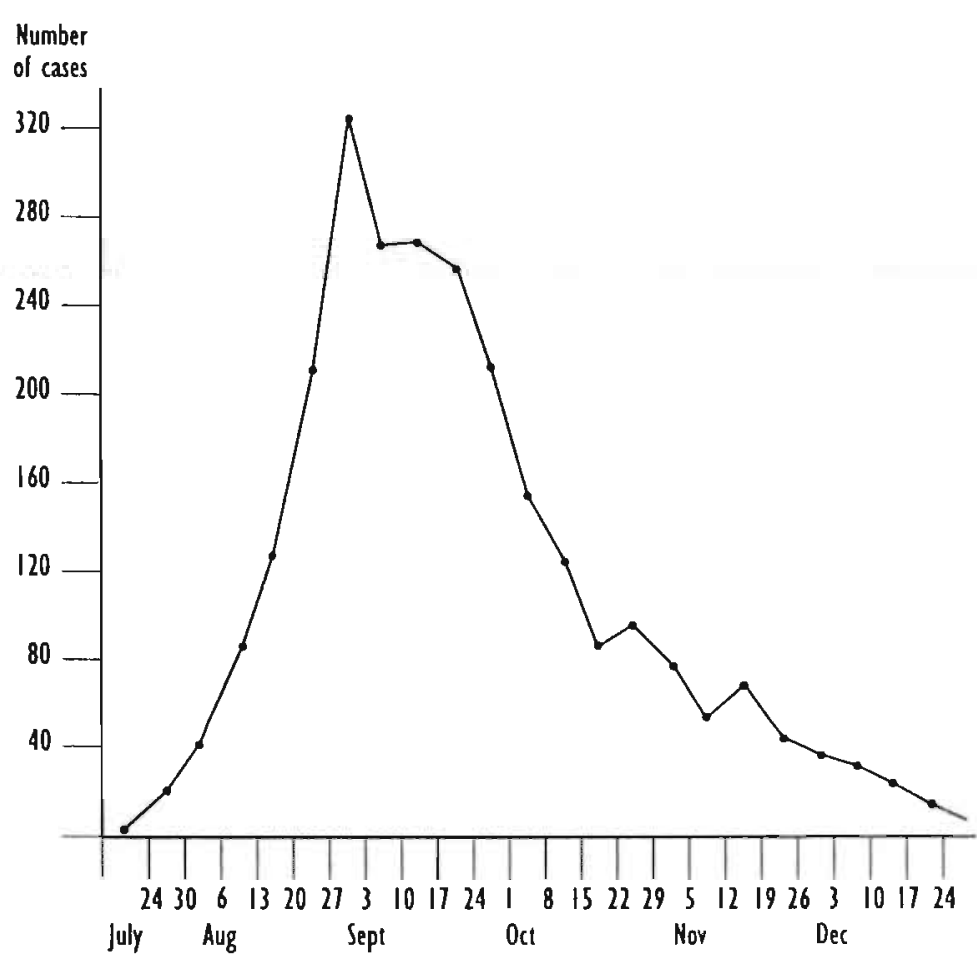

Figure 4.4

Weekly admissions to Blegdamshospital of cases of poliomyelitis in 1952

Modified from Lassen (1956: 3)

With one 'iron lung' (Emerson) and six 'cuirass respirators' at their disposal they were not nearly adequately equipped to meet an emergency of such vast proportions. They were soon faced with the dilemma of having to choose which patients to treat in the few respirators at hand, and which not. The need for improvisation became imperative. They were fighting a lost battle.

\section{Modern anesthesiological principles}

What happened next? In several formal, scientific accounts chief physician H.C.A. Lassen $(1952,1955,1956)$ related, in more or less the same wording, that eventually, they coped with the situation through the application of modern anesthesiological principles.

[A]s it was felt that application of modern anaesthesiologic principles to the problem of airway obstruction and muscular respiratory insufficiency in our desperately ill patients might be of value, anaesthetists were invited to join our staff, and from 27 August an emergency method was introduced which, under the circumstances, saved many lives. The mortality rate dropped from over $80 \%$ in August to $25 \%$ in 
December, and this in spite of the constant severity of the cases throughout the whole epidemic period. ... The emergency method consisted in: high tracheotomy with introduction of an inflatable rubber cuff-tube in order to seal off the air-passage from the hypopharynx, postural drainage with frequent aspiration of the airway, and manual ventilation with a mixture of about $50 \%$ oxygen from a rubber bag. (Lassen 1955: 158)

Lassen's writings, however, give little hint concerning the question of how this remarkable accomplishment had been achieved. There are reasons to assume that, at the time, it was by no means obvious that an anesthetist, or modern anesthesiological principles, could improve Blegdamshospital's results in the treatment of life-threatening, bulbar and respiratory poliomyelitis. Lassen's vague statement that 'it was felt that application of modern anaesthesiologic principles ... might be of value' conceals more than it reveals. Who brought the anesthetists in? How were prevalent interpretations of pathophysiological mechanisms overthrown? How was the shift brought about in the definition of what kind of problem bulbar poliomyelitis constituted? In other words, how was bulbar poliomyelitis redefined from an irremediable neurological destruction problem, resulting from an 'overwhelming, intractable "bulbar" virus infection', into, what Lassen (1955: 157) later called, 'the newer concept of bulbar and respiratory poliomyelitis as chiefly a problem of maintaining an unobstructed airway'? A problem for which the modern anesthesiological principles to the problem of airway obstruction and muscular respiratory insufficiency held the solution.

In the next section I will tell the story of events in Blegdamshospital in the last week of August and the first weeks of September 1952 in a rather condensed format. As a result of this condensed format the story acquires a 'veni-vidi-vici' character. In subsequent sections I will strip this account of its hagiographic overtones through a more detailed description and analysis of an anesthesiologist's management of polio patients which not only reduced mortality rates, but also came to have, in Ibsen's words. importance for the future development of the specialty in Copenhagen, since it initiated the formation of intensive therapy units, later run by anesthesiologists in their own hospital departments and, as a matter of fact, changed the approach to the technique of artificial respiration all over the world.

\subsection{Veni, vidi, vici}

\section{Consultations}

Three weeks after the beginning of the 1952 polio-epidemic in Copenhagen, three weeks in which 27 out of 31 patients admitted to Blegdamshospital with paralytic polio and treated in respirators died, Mogens Bjørneboe, member of the hospital's medical staff, urged Lassen to consult Bjørn Ibsen, a free lance ${ }^{9}$ working anesthetist at Copenhagen's university hospital. At the outset Lassen was very skeptical about the contribution which Ibsen as an anesthetist could make. In 1952 anesthesia in Denmark was an emerging but low status medical discipline. It had acquired the official status of medical specialty in 1950. (Møller 1958) Only a few anesthetists were employed by hospitals in permanent jobs. Most anesthetists worked on a free lance basis. Their 
range of action and acknowledged competence was restricted to the operating theater where they delivered anesthesia to patients. Formally however, anesthesia, including the choice of anesthetic, remained the surgeon's responsibility. ${ }^{10}$

With established treatment regimes failing, Blegdamshospital's medical staff felt as being in a state of war almost empty-handed. (Lassen 1953) Having been chief physician for 13 years in Denmark's polio-center and center of expertise in epidemic diseases, Lassen had to overcome a certain degree of professional pride in soliciting 'outside' help. He was supposed to be the expert. But the mortality rate and the recognition that this was only the beginning forced him to consult others, at first of course those whom he expected to be able to make valuable contributions. Lassen invited, for example, the well known physiologist Einar Lundsgaard to come to Blegdamshospital. Lundsgaard assessed the situation but could not come up with a solution. Still empty-handed Lassen agreed to consult Bjørn Ibsen.

\section{Terminal bulbar polio}

Ibsen came on August 25, armed with textbook-knowledge about polio at most, having no practical experience in its treatment whatsoever. A meeting was held to discuss the problem. Besides Lassen and Bjørneboe, this meeting was also attended by a senior physician, Frits Neukirch, and by the head of the clinical laboratory, Poul Astrup.

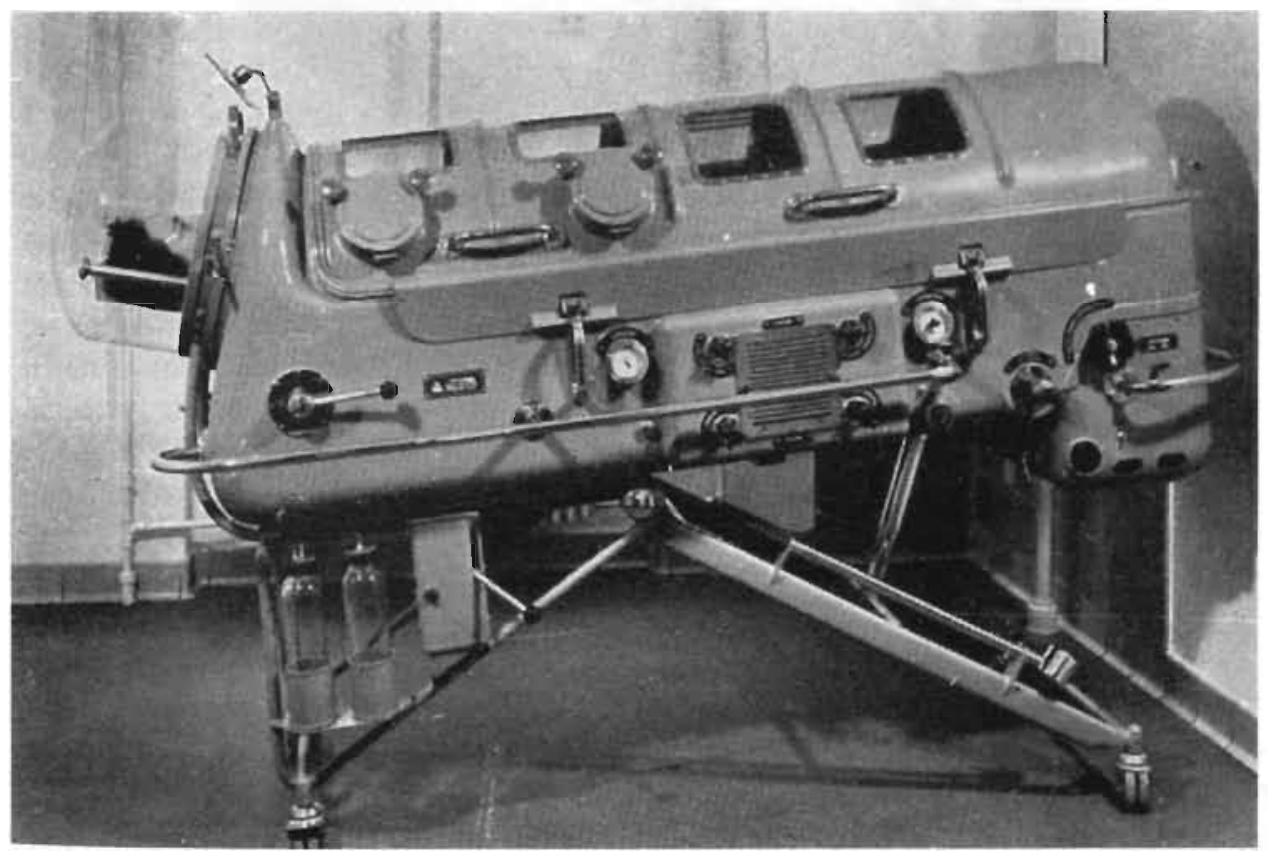

Figure 4.5

'Iron lung' or negative pressure tank-respirator Reproduced from I.assen (1956: 55) 
Astrup was appointed head of the clinical laboratory at Blegdamshospital on the first of May, 1952. He had been on holiday since the first of August, a few days prior to the outbreak of the epidemic, and was called in by Lassen to attend the meeting on August 25.

Ibsen examined some polio patients on the hospital's wards, he studied patients'

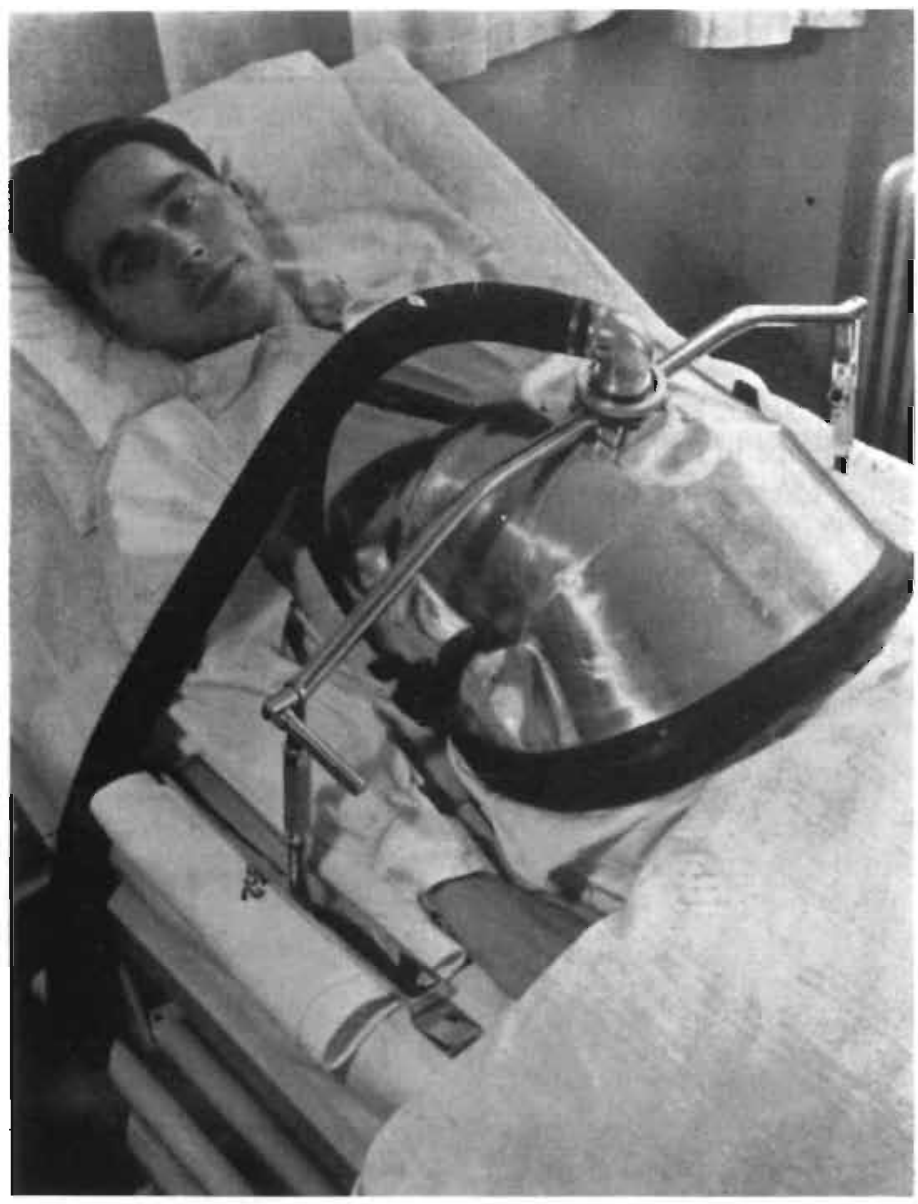

Figure 4.6

Kifa cuirass respirator

Reproduced from Lassen (1956: 58)

records and examined four patients at the autopsy room who had succumbed to the disruptive consequences of bulbar poliomyelitis. Patients presented with paralysis of the muscles of arms and legs, with paralysis of the diaphragm and other respiratory muscles in the trunk (respiratory polio), and with paralysis of the (swallowing) muscles of the throat (bulbar polio), in different combinations. Patients with respiratory and/or bulbar polio felt as if they were suffocating from lack of air and drowning in their own secretions which they could not swallow down. The lack of oxygen 
which resulted from the respiratory insufficiency produced the foul blue skin color known as cyanosis. In addition to these symptoms the final stage of the disease was characterized by high fever, sometimes more than $40^{\circ} \mathrm{C}$, a clammy skin. high blood pressure, and finally, a high total $\mathrm{CO}_{2}$ content in plasma. Blegdamshospital's physicians considered the high total $\mathrm{CO}_{2}$ content to indicate a metabolic alkalosis. The lungs of the patients in the autopsy room showed some degree of atelectasis, but, in Ibsen's view, not sufficient to make adequate ventilation impossible. (Ibsen 1975) Patients with severe respiratory insufficiency were treated in negative pressure respirators - one 'iron lung' (see figure 4.5) and six cuirass respirators (see figure 4.6) - to support their breathing. Oxygen was given to counteract cyanosis and restore skin color to normal. These patients were well oxygenated due to the oxygen supply. Yet, they became unconscious and died.

\section{Remarkable conclusions}

On the basis of his findings Ibsen drew some remarkable conclusions which were in disagreement with local clinical interpretations, prevalent in Blegdamshospital. Most of the symptoms and abnormal laboratory results characterizing the final stage of bulbar polio were usually considered to be specific consequences of the disease process, that is of the neurological damage caused by the virus infection in the central nervous system:

Quite a large number ... presented symptoms conventionally thought to be due to involvement of the bulbar centers. (Lassen 1953: 38)

Ibsen attributed the high blood pressure and the high total $\mathrm{CO}_{2}$ content in plasma to an accumulation of carbon dioxide in the patients' blood. The high total $\mathrm{CO}_{2}$ content was, according to Ibsen, not an expression of a metabolic alkalosis but it indicated. quite to the contrary, a respiratory acidosis: which in physiological terms is exactly the opposite. Blegdamshospital's physicians had focused on the blood's oxygenation by assisting or taking over the patients' breathing and by supplying additional oxygen when cyanosis occurred, restoring skin color to normal. They had not recognized, however, the role of $\mathrm{CO}_{2}$ accumulation in the production of the symptoms, and as a result of that $\mathrm{CO}_{2}$ accumulation had not been treated. According to Ibsen, patients did not die from immediate and specific consequences of the virus infection. They died from the consequences of inadequate gas exchange in the lungs. It was, according to Ibsen, a ventilation problem.

\section{A new treatment}

Ibsen proposed a new treatment (see figures 4.7 and 4.8). He proposed to make a tracheotomy in the patients' throat under local anesthesia. Applying a suction tube through the tracheotomy would then allow them to remove obstructions deep down in the trachea and branches of the lungs' bronchial tubes.

In terms of a clear airway this procedure would produce far better results than the hitherto used methods of postural drainage and aspiration from the pharynx could ever accomplish. As a next step a cuffed, rubber tube should be inserted through the tracheotomy and positioned in the trachea above the junction into a left and right 


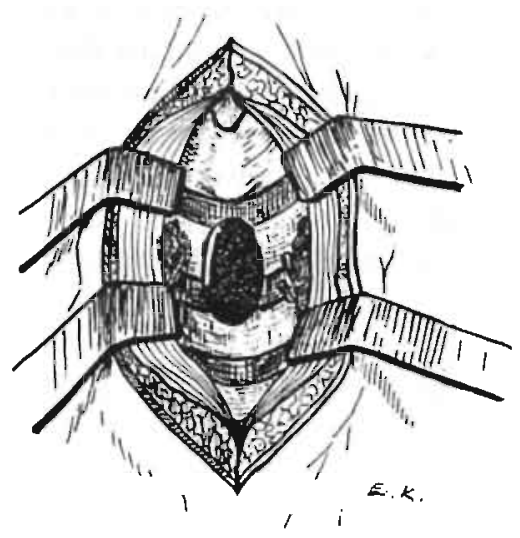

Figure 4.7

Tracheotomy

Reproduced from Lassen (1956: 47)

bronchus. When inflated the balloon at the tracheal end would close the trachea completely, thus preventing aspiration of stomach contents and of saliva and from the throat. A bag attached to the side of the other end of the tube would allow for manual positive pressure ventilation. In stead of creating a negative pressure outside the patient's body, allowing the atmospheric pressure to inflate the thorax and produce an inspiration movement - the principle of operation of negative pressure respirators -, the air in the bag was to be squeezed into the patient's lungs applying, by manual

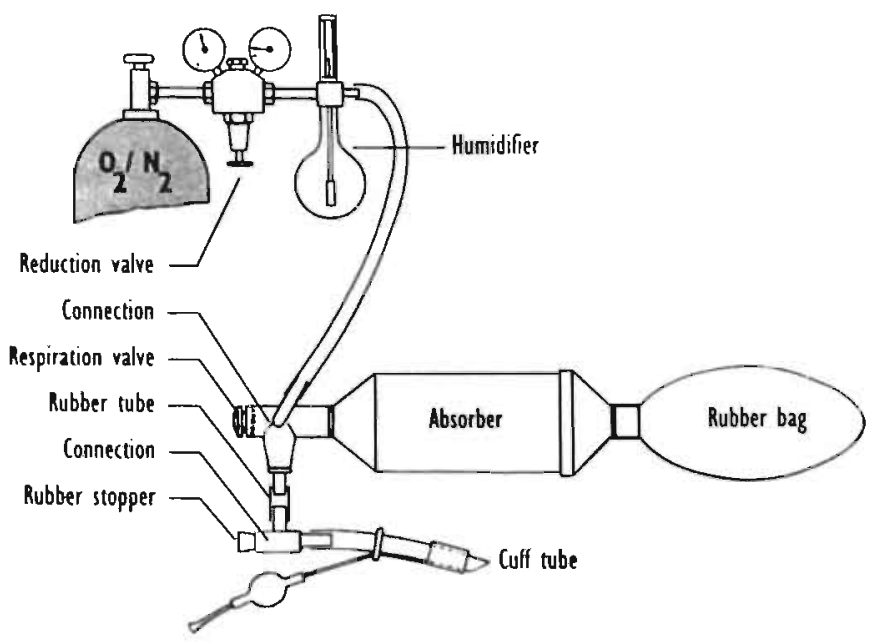

Figure 4.8

Schematic representation of manual bag ventilation

Reproduced from Lassen (1956: 61) 
force, a pressure higher than the pressure in the lungs: hence, manual positive pressure ventilation. Using a valve system the bag could be linked up with a cylinder containing equal parts of compressed oxygen and nitrogen $\left(\mathrm{O}_{2} / \mathrm{N}_{2}\right)$ and with a canister containing granulated soda lime to absorb carbon dioxide from expired air, known as a Water's absorber. A rubber stopper closed the tube, the removal of which would allow for efficient endotracheal suction during treatment.

The bottom line of Ibsen's proposal was to replace the expensive, failing and cumbersome, mechanical negative pressure respirators by cheap and simple, manual positive pressure ventilation which would not only allow for sufficient ventilation but which would also make it a lot easier for nurses to care for the patients. Ibsen found it

... rather easy to suggest possibilities for improvements in therapy at Blegdam Hospital, but not necessarily always easy to get the suggestions accepted. (Ibsen, 1975: 22)

\section{Benefit of the doubt}

Neither Ibsen's dissenting interpretation of the high total $\mathrm{CO}_{2}$ content running against prevailing clinical practice, nor his unusual diagnosis of $\mathrm{CO}_{2}$ accumulation being largely responsible for the patients' death, nor his proposal for a new therapy was immediately accepted. Lassen pointed out that tracheotomy had been tried in Blegdamshospital in 1948, but without success.

In 1948 we started using tracheostomy in all cases where it proved impossible to maintain an open airway because of pooling of secretions and aspiration into the lungs ... all patients treated by tracheostomy and with respirators died, whereas treatment with respirators alone had a somewhat better prognosis ... (Lassen 1953: 37)

Positive pressure ventilation had never been applied in Blegdamshospital, but Lassen had no reason to disqualify the negative pressure respirators as insufficient. In most cases of poliomyelitis with respiratory insufficiency the iron lung and cuirass respirators worked well. In patients with spinal polio the damage to the nervous system was limited and reversible. In other words, the virus could impair a nerve cell's function temporarily without destroying it. In other cases, notably in bulbar poliomyelitis, the involvement of nervous tissue in vital portions of the central nervous system (upper spinal cord, medulla oblongata, pons and midbrain) would lead to its irreversible destruction. Destruction of motor centers located in the 'bulbar' portion of the medulla oblongata, innervating the muscles of the throat, resulted in swallowing paralysis. Widespread lesions in these parts of the central nervous system, where also other vital regulatory centers are located, had been demonstrated in post-mortem studies. Nervous tissue was known to have no regenerative potential once it was destroyed. Sure, these patients died in the negative pressure respirators. But they did not die as a result of the respirator's failure. Their death was a result of the extent and location of neurological damage inflicted by the virus. 
[I]t was generally assumed that the ventilation [with body-type respirators] was sufficient. The polio-encephalitis in itself was mainly considered as the cause of the fatal outcome. (Engström 1963: 7)

It was not obvious at all, maybe even unlikely, that the method of positive pressure ventilation proposed by lbsen could save the lives of patients with such extensive lesions in their brain stem.

Ibsen recalled, and had also taken with him to the meeting at August 25, a copy of a paper published in 1950 by Bower (1950) in the United States in the Annals of Western Medicine and Surgery. Bower claimed to have reduced the overall mortality for cases of bulbar poliomyelitis from $90 \%$ to approximately $20 \%$ over a period of four years. Bower combined total-body-type negative pressure respirators with positive pressure ventilation through an endotracheal tube inserted through the mouth. Blegdamshospital's physicians were not impressed.

It was argued that the reason Bower's results were so good was that many patients were put in respirators too early - patients who never should have been there. In Copenhagen those patients would never have been in respirators, thus accounting for the high percentage of success in Bower's material. (Ibsen 1975: 22)

Although not convinced. Lassen gave Ibsen the benefit of the doubt. Since no one else could offer a solution, arrangements were made for Ibsen to apply his proposed treatment to one of the patients and demonstrate whether he could accomplish something or not. On August 27, Lassen picked a twelve year old girl who was

... in a very bad condition, with paralysis of all four extremities. She ... was gasping for breath and drowning in her own secretions. Her temperature was 40,2 ${ }^{\circ}$ C. She was cyanotic and sweating. (Ibsen 1975: 23; see also Lassen 1956: 14)

\section{Between failure and success}

Ibsen asked an E.N.T.-surgeon to make a tracheotomy under local anesthesia. Ibsen introduced a cuffed tube and a ventilation bag. During this procedure the girl became unconscious. Despite good endotracheal suction Ibsen was not able to inflate the girl's lung properly which he thought partly to be due to secretions obstructing airways and partly to bronchospasm resulting from the manipulations during the operation and subsequent suction. The girl was dying in his hands. With new polio patients coming in, in addition to the patients who already were in the hospital, some of the other physicians, who had been watching, had to leave and attend other pressing duties.

In this desperate situation Ibsen decided to give her $100 \mathrm{mg}$ of Pentothal intravenously to make her stop struggling. The girl collapsed immediately. Her own respiration stopped. Now he was able to ventilate the girl properly. The removal of the excess $\mathrm{CO}_{2}$ from the blood contributed to the drop in blood pressure, which was treated with plasma replacing infusions. Ibsen succeeded in stabilizing the girl's condition. When the other physicians returned a few minutes later the girl's skin color had returned to normal - without the supply of extra oxygen. Blood pressure was restored to normal 
too. This twelve year old girl was the first patient during this polio epidemic who survived as a result of a medical intervention. (Ibsen 1956; 1975)

Following Ibsen's demonstration of the effectiveness and superiority of manual positive pressure ventilation in the treatment of bulbar poliomyelitis Lassen decided immediately to apply this 'emergency' treatment to all patients with respiratory insufficiency and swallowing impairment. In the week from August 28 to September 3, 1952, 335 polio patients were admitted to Blegdamshospital - approximately 50 per day. More successful treatments of patients with bulbar polio followed. To avoid complications small adjustments were made in the therapeutic set up. Within a few weeks mortality rates dropped from $90 \%$ to approximately $15 \%$. In the context of this epidemic no complex statistical calculations were required to demonstrate that this new treatment made a significant difference.

\section{Little human force}

Let us be careful to avoid the pitfall of hagiographic history writing, that is telling stories about Great Men who, against all odds, unselfishly and with perseverance, put science on the right track and save hundreds or thousands of human lives. According to Latour (1988: 62), hagiographers attribute powers that Great Men 'could not have possessed but omit to credit them with the only thing they did with their little human force'.

Bjørn Ibsen did not invent a new technology at the spot. The history of manual positive pressure (bag) ventilation can be traced back to the sixteenth century and seventeenth century as a solution for the pneumothorax problem in experimental physiology, that is for the problem of maintaining life while the chest was open. The lungs would collapse as soon as the surgeon opened the chest, respiration would become labored and the mediastinum would begin to flap violently. making unhurried surgical work inside the thorax impossible. Inflation of the lungs by means of intermittent positive pressure ventilation solved this problems. The introduction of the laryngoscope, allowing for proper intubation of the pharynx in humans, and of antiseptic techniques in the second half of the nineteenth century made extensive thoracic surgery in humans, using positive pressure ventilation, feasible from around the turn of the century. Positive pressure ventilation was also used to administer inhalation anesthetics during anesthesia. (Mushin 1980)

By introducing the notions of respiratory acidosis and $\mathrm{CO}_{2}$ accumulation Ibsen did not invent or discover new concepts in the pathophysiology of respiration either. Denmark had a longstanding tradition in respiratory and acid-base physiology. August Krogh, the Nobel laureate featuring in the previous chapter concerning his work in the production and standardization of insulin, did important work in respiratory physiology demonstrating that gas exchange in the lungs took place by passive diffusion and was not an active secretion process. (Krogh 1910a) Krogh also recognized both the blood's $\mathrm{CO}_{2}$ content's influence on blood pressure and positive pressure ventilation's influence on the carbon dioxide content of the blood. (Krogh 1910b; 1910c; 1910d; see also Møllgaard 1910a; 1910b; and Engström 1950) In 1917 another Danish physiologist, Karl Albert Hasselbalch, revised Lawrence Joseph Henderson's mathematical representation of the buffering capacity of blood, yielding what is known as the Henderson- 
Hasselbalch equation, defining acid-base status of the blood, possible disturbances and compensatory mechanisms. (Astrup and Severinghaus 1985)

If Ibsen can not be credited for the discovery of new pathophysiological mechanisms or for the invention of a new technology, what is it then that Ibsen can be credited for? What did he accomplish with his little human force? To answer this question we will have to consider the details of the events, and their background, in Blegdamshospital during the 1952 polio-epidemic.

\subsection{Why an anesthetist in the first place?}

In Lassen's eyes anesthesia was not the most obvious place to look for a solution. As an epidemiologist and specialist in internal medicine Lassen was not very familiar with modern anesthesiological principles. Blegdamshospital did not count an anesthetist among its ranks. In general, anesthetists worked exclusively in the operating room, mostly on a free lance basis. Furthermore, in 1952 the social structure in the Danish health care system had not changed very much since the pre-war period. As Ibsen explained later,

[t]he organization of the medical personnel in the different departments in Danish hospitals before World War II resembled the old-fashioned Prussian system, with the chief the commanding general at the top of the pyramid formed by the personnel on different levels. (Ibsen, 1975: 10)

The chief physician's view was usually decisive. Bjørn Ibsen, a free lance working anesthetist, member of a low status discipline on a lower rank in the hierarchy, having no practical experience in the treatment of poliomyelitis, was not the expert that could provide the 'outside help' Lassen needed. It was only because other consultants had not been able to offer substantial help that Lassen agreed to Mogens Bjørneboe's proposal to invite Ibsen. ${ }^{11}$ Why then did Bjørneboe believe that Ibsen could accomplish something with respect to the treatment of life-threatening bulbar poliomyelitis?

Bjorneboe's and Ibsen's 'collaboration' was the result of an accidental acquaintance. Ibsen was, in fact, the only anesthetist that Bjørneboe knew. ${ }^{12}$ In June 1952, while Lassen was on a holiday, but with the approval of Blegdamshospital's senior physician Frits Neukirch, Bjørneboe invited Ibsen to come and help in the treatment of a newborn child with tetanus neonatorum.

\section{Tetanus neonatorum}

Tetanus occurs in man and animals when a wound - in newborns the umbilical cord is often the port d'entree - is infected with Clostridium tetani under conditions that allow the bacteria to multiply and produce a neurotoxic substance, the tetanus (exo)toxin. ${ }^{13}$ The toxin causes hyperexcitability of the voluntary musculature. The clinical picture is characterized by increased muscle tonus and exaggerated muscular responses to trivial stimuli. Sudden bursts of contractions of various muscle groups may develop into more or less widespread convulsions and persistent spasms. Persistent spasms of the muscles of the jaw (lockjaw) are often a early symptom of the 
disease in man. Spasms of the facial muscles produce a fixed sardonic grin, known as risus sardonicus. As time passes the spasms become more severe, powerful and exhausting. The patient is completely conscious during the course of the disease and experiences intense pain. Spasms of the muscles of the throat and of respiratory muscles may produce respiratory obstruction, resulting in cyanosis and asphyxia.

At the end of the forties and the beginning of the fifties medical treatment of tetanus aimed at the neutralization of the neurotoxin by means of injection of tetanus anti-toxin, an anti-serum produced in a horse containing pre-formed antibodies against tetanus toxin. Sedatives were given to reduce muscular excitability. Tracheotomy was sometimes performed in an attempt to maintain a free airway.

\section{Curare}

At the end of the forties, from 1947, sporadic publications appeared in American medical literature recommending the use of the neuromuscular blocking agent curare to reduce spasms in tetanus. The administration of curare was intended to control spasms and convulsions while sparing respiration, that is without inducing complete respiratory paralysis. Patients in whom, despite this intention, respiratory paralysis did occur, were treated for a short period of time in negative pressure respirators or with positive pressure respiration, until enough curare had been metabolized to restore respiratory functions.

93 patients with tetanus had been treated in Blegdamshospital between 1940 en 1952; 28 of them, that is $30 \%$, had died. Between May 1950 and 1952 five out 26 severely ill tetanus patients had been treated with tracheotomy combined with sedatives, but no curare. All five tracheotomized patients had died. When invited in June 1952 to assist in the treatment of a newborn with tetanus neonatorum Ibsen was the first physician in Blegdamshospital who used curare in the treatment of tetanus. The curare induced complete respiratory paralysis in the child, which Ibsen treated with manual positive pressure ventilation through a tracheotomy. Eventually however they were not able to keep the child alive. (Bjørneboe et al. 1953)

\section{Similarities}

Despite the eventual death of the child, in June 1952 Bjørneboe had witnessed, that is he had seen with his own eyes that Ibsen, an anesthetist, kept a completely curarized child alive through the application of manual positive pressure ventilation. Bjørneboe recognized the similarities between completely curarized tetanus patients and patients with respiratory and bulbar polio. In both there was paralysis of the respiratory muscles. In tetanus patients the respiratory paralysis was induced by the administration of curare. In poliomyelitis patients the paralysis resulted from neurological impairment or destruction of the motor centers in the upper spinal cord and brain stem.

Despite the eventual death of the tetanus patient in June 1952, Bjørneboe considered it to be worth consulting Ibsen when Blegdamshospital, in August of that same year, faced the high mortality rates of a polio epidemic developing such vast proportions. 


\subsection{Creating identity}

The similarity between the polio patient with respiratory paralysis and the completely curarized tetanus patient extends to the patients under general anesthesia in the operating room. The use of curarizing agents still being very controversial, and the choice of anesthetic being the surgeon's, anesthesia was usually induced through inhalation of a mixture of nitrous oxide and oxygen $\left(\mathrm{N}_{2} \mathrm{O} / \mathrm{O}_{2}\right)$ combined with ether. During anesthesia respiration was assisted with intermittent positive pressure ventilation using a bag. By overventilation a cessation of spontaneous breathing, apnea, could be induced, after which the patient's breathing was completely controlled by the anesthetist.

Ibsen learned the induction of anesthesia during his medical training. After the war he received further training in anesthesia in the United States, where he worked for one year as assistant resident at Massachusetts General Hospital in Boston. It was here, giving anesthesia to a tracheotomized child, that he learned to appreciate the convenience of a tracheotomy for securing free airways, which in turn made it easier to administer an inhalation anesthetic. (Ibsen 1975) Back in Copenhagen Ibsen participated in the international training course in anesthesia, which was held under the auspices of the World Health Organization in six of Copenhagen's largest hospitals between 1950 and 1973, first as a trainee, later as instructor. The anesthetist's main task in the operating room was to induce in the patient a condition that allowed the surgeon to perform his surgery, while maintaining a sufficient circulation (of blood) and respiration and treating circulatory and respiratory complications. That is what Ibser had learned to do.

Curare allowed Ibsen to induce in the newborn tetanus patient a condition identical to the ones he had learned to handle. In the twelve year old polio patient the virus had gone a long way to do the same. Pentothal, a barbiturate having a general depressing effect, did the rest. Knowing virtually nothing about the established treatment of polio Ibsen did what he had learned to do in the operating room, but this time he did it in a place where anesthetists were not considered to have any competence.

\subsection{Metabolic alkalosis and respiratory acidosis}

The next question that requires our attention is: How could Ibsen attribute the characteristic symptoms of terminal stage bulbar polio, that is high fever, clammy skin, high blood pressure and high total $\mathrm{CO}_{2}$ content in plasma, to an accumulation of carbon dioxide in the patients' blood? How could he, contrary to prevalent clinical experts' opinion in Blegdamshospital, interpret the high total $\mathrm{CO}_{2}$ content as an expression of a respiratory acidosis? Total $\mathrm{CO}_{2}$ content in plasma, using Van Slyke's method, was the only laboratory parameter for a patient's acid-base status that was clinically available at the time. ${ }^{14}$ The numbers did not change, but, apparently they did not speak for themselves. Ibsen disagreed with Lassen and the other physicians about what they were saying, about what kind of pathophysiological condition or mechanism these numbers were an expression of. ${ }^{15}$ How come? 


\section{Brinkman's Carbovisor}

In 1951 a Danish surgeon, H.C. Engell, supplemented his training in thoracic surgery with cardiology in professor Erik Warburg's Department of Medicine at Rigshospital in Copenhagen. On the instigation of Warburg, Engell was send to the Postgraduate Course in Fundamentals of Thoracic Surgery and Intrathoracic Diagnosis held in Groningen, in the Netherlands, from March 20

until May 12, 1951. The course, covering important issues in respiratory physiology, was organized by professor $\mathbf{R}$. Brinkman. At the time Brinkman was working on an apparatus to measure carbon dioxide in expired air continuously. Brinkman called this instrument a Carbovisor. The principle of the procedure was that expired air was led through an indicator solution. By means of a membrane pump operated by a synchronous motor the air, which was intended to be analyzed, was blown through an absorption cell containing a $0.005 \%$ bromthymol blue solution, at a rate of 140 $\mathrm{ml} / \mathrm{min}$. Equilibrium between the $\mathrm{CO}_{2}$-tension of the air and of the liquid soon took place. The color in the bromthymol blue indicator varied according to the $\mathrm{CO}_{2}$ concentration of the air that passed through it, being of a pale yellow with $\mathrm{pH} 7.6$. The variations of the color of the indicator were recorded by a photo-electric cell connected to a galvanometer of suitable sensitivity to read the results. (Engell and Ibsen 1952)

On condition that gas exchange in the lungs - across the tissue barrier between the alveolar space and the blood in the capillary blood vessels - was unimpaired, changes in $\mathrm{CO}_{2}$-concentrations in alveolar and expired air were considered to reflect changes in $\mathrm{PCO}_{2}$ in the blood.

Brinkman promised Engell that he would be the first to apply the Carbovisor in clinical research in the operating theater during surgery. Returning home in May 1951, Engell was able to carry a prototype of the Carbovisor with him back to Copenhagen. ${ }^{16}$ Back in Copenhagen Engell was allowed to work the Carbovisor whenever he wanted. Starting in May 1951 Engell, together with Bjørn Ibsen, commenced research in Copenhagen's university hospital applying Brinkman's Carbovisor to measure continually carbon dioxide in the patient's expired air during thoracic surgery. ${ }^{17}$ (Engell and Ibsen 1952) While Engell ran the Carbovisor, Ibsen administered anesthesia and assisted the patient's respiration by manual, intermittent positive pressure ventilation.

Ibsen saw how some of the surgeon's manipulations, compromising the lungs' ventilatory capacity, gave rise to an accumulation of $\mathrm{CO}_{2}$ in the blood as indicated by the changing color of the bromine thymol blue solution in the Carbovisor and the resulting deflection of needle of the galvanometer. He saw, for example, how the surgeon's compression of a lung to get access to the operation site, thereby temporarily reducing the alveolar surface area available for gas exchange, was followed by $\mathrm{CO}_{2}$-retention in the blood. A rise of $\mathrm{CO}_{2}$ in expired air during or after an operation could also be caused by a developing complication, the accumulation of secretions in the lungs or bronchospasms. In one patient, on whom pneumonectomy was performed for cancer of the lung, $\mathrm{CO}_{2}$ - concentration varied from 1.5 to $3 \%$ during the operation, until the thoracic wall was closed. During the surgical closure of the thoracic wall the Carbovisor recorded a rise to $16 \%$ in the course of 30 minutes, despite energetic ventilation. After aspiration of $400 \mathrm{ml}$ of air from the left 
thoracic cavity the $\mathrm{CO}_{2}$-concentration fell rapidly. During the closure of the thorax air had been drawn into the cavity by a sort of valve action, creating a high intra-thoracic pressure, against which it had been impossible to ventilate the patient sufficiently. (Engell and Ibsen 1952)

Monitoring these patient's general conditions and vital functions, Ibsen was in the best position to observe changes that coincided with the changes indicated by the Carbovisor, for example, changes in skin humidity and blood pressure.

Ibsen was not only in the best position to observe the effect of the surgeon's manipulations in the patient's thorax on the $\mathrm{CO}_{2}$-content of the expired air, he could also observe how changes in the frequency and force with which he himself compressed the balloon in applying manual positive pressure ventilation affected the Carbovisor's coloring in immediate spatial en temporal association with other changes in the patient's condition. During these months of Carbovisor research in the operating theater Ibsen learned which symptoms to attribute to $\mathrm{CO}_{2}$-accumulation and which not. In other words, he learned to discern the $\mathrm{CO}_{2}$-retention syndrome from other cooccurring symptoms attributable to other diseases or conditions. ${ }^{18}$

\section{Inadequate ventilation as a cause of death}

When on August 25, 1952, Ibsen examined patients suffering from bulbar polio at Blegdamshospital's wards and when he examined the records of the patients who had died in the previous weeks, he was confronted with the same set of symptoms which he, during the previous year of Carbovisor research in the operating theater, had learned to attribute to $\mathrm{CO}_{2}$-retention. Furthermore, examining the lungs of four patients at the autopsy room, he judged that despite some degree of atelectasis their ventilatory capacity at the moment of their death had not been compromised to make adequate ventilation impossible. Considering the high blood pressure and the high total $\mathrm{CO}_{2}$ in plasma at the moment of their death they must have suffered from $\mathrm{CO}_{2}$ accumulation. A glance at Henderson-Hasselbalch's equation then would suffice to infer that an increase in $\mathrm{PCO}_{2}$ would result in a decrease in $\mathrm{pH}$, that is in acidosis; more specifically, an acidosis following respiratory insufficiency: respiratory acidosis.

Since most vital, physicochemical body functions require a specific $\mathrm{pH}$, which may vary only within a very narrow range, the decrease in blood $\mathrm{pH}$ must have had a devastating. deranging, indeed a lethal effect on the patients' condition. In Ibsen's view it was the $\mathrm{CO}_{2}$-accumulation that killed them. And considering the only moderately compromised ventilatory capacity of the patients he examined in the autopsy room, that is, the area of alveolar surface available for gas exchange, these patients could have been kept alive when adequate ventilation had been applied artificially. Apparently the ventilatory support offered by the negative pressure respirators, the iron lung and the cuirass respirators, had not been sufficient. Maybe manual positive pressure ventilation would, especially when combined with tracheotomy, which Ibsen had learned to appreciate both as a convenient way to administer inhalation anesthetics and as an efficient way to keep a free airway.

\section{Demonstrating $\mathrm{CO}_{2}$-accumulation}

After successfully stabilizing the twelve year old girl Ibsen and Engell, who was present too, connected Brinkman's Carbovisor to her. In addition to the Carbovisor 
they applied a Millikan Oximeter to measure (percutaneously) the oxygen saturation of the girl's blood. Millikan's Oximeter is a photo-electric device developed during the second world war to monitor the oxygen saturation in the blood of airplane pilots during combat in a non-invasive way to facilitate the automatic adjustment of oxygen supply through their masks. The device utilizes the differences in infrared light absorbing capacities of oxygen rich and oxygen poor blood and is applied to the highly vascular earlobe. (Severinghaus and Astrup 1987)

With Brinkman's Carbovisor and Millikan's Oximeter in place Ibsen was able to show Blegdamshospital's physicians, who had returned, how the carbon dioxide content of the expired air varied with the frequency and applied force of balloon compression in manual positive pressure ventilation. He showed them how the $\mathrm{CO}_{2}-$ content as indicated by the Carbovisor rose when the frequency of balloon compression was reduced and how simultaneously blood pressure rose and the patient's skin became clammy and sweating. He could make the symptoms attributable to $\mathrm{CO}_{2}-$ retention return and go away, thereby separating them from other symptoms, like muscular paralysis, assigning those to the poliomyelitis. Assisted by the Millikan Oximeter and the Carbovisor, he was also able to show that cyanosis could be successfully abolished through the administration of oxygen, while on the other hand a life threatening $\mathrm{CO}_{2}$-retention endured, as indicated by the Carbovisor.

The twelve year old girl was now transferred to a negative pressure respirator. When the negative pressure respirator worked alone, the Carbovisor demonstrated the gradual development of $\mathrm{CO}_{2}$-retention, accompanied by a worsening in the patient's condition, which in previous weeks had given rise to patients' death in the respirators. When the respiration in the negative pressure respirator was assisted by intermittent squeezing of the bag connected with the tracheotomy tube, the child's condition improved again. (Ibsen 1975) Blood samples taken by Astrup after the institution of the positive pressure ventilation showed a reduction to normal of the values for 'total $\mathrm{CO}_{2}$ in plasma'.

In a very immediate, almost tangible, way Ibsen displayed the insufficiency of the iron lung and cuirass respirators, an insufficiency for which Lassen, prior to Ibsen's demonstration, had no reason to assume or assent to that it existed. ${ }^{19}$

Ibsen's success in persuading Blegdamshospital's physicians regarding his account of the pathophysiological mechanisms leading to death in terminal stage bulbar polio depended on one condition: that his audience, especially chief physician Lassen, accepted that Brinkman's Carbovisor really measured carbon dioxide in alveolar air, which under the circumstances they did.

\subsection{Contingency, rhetorical strength and clinical skill}

In the previous sections I addressed the questions forwarded in section 3 , that is: Who brought the anesthetist in? How were prevalent interpretations of pathophysiological mechanisms overthrown? How was bulbar poliomyelitis redefined from an incurable neurological destruction problem, resulting from an overwhelming, intractable bulbar virus infection, into the newer concept of bulbar and respiratory poliomyelitis as chiefly a problem of maintaining an unobstructed airway? At this point in the analysis 
we are in better position to answer the question posed at the end of section 4: If Ibsen can not be credited for the discovery of new pathophysiological mechanisms or for the invention of a new technology, what is it then that Ibsen can be credited for? What did he, in Latour's words, accomplish with his little human force?

\section{A rhetorical accomplishment}

Ibsen's accomplishment is rhetorical. He persuaded Blegdamshospital's medical staff and chief physician that he, as an anesthetist, could make a difference, that anesthetists' knowledge and manual skills were not only of value in the operating theatre, but also in other domains of clinical work. He persuaded them that the therapeutical approach he proposed was not only effective, but that it also was superior to the treatment regimes that hitherto had been used. He persuaded them that his account of the pathophysiological mechanism causing the patients' death was the best offered thus far, if not simply true. In other words, Ibsen challenged the legitimacy of Lassen's spokesmanship and established himself as the legitimate spokesperson for blood pressure readings, for the numbers produced in the measurement of total $\mathrm{CO}_{2}$ in plasma and for the Carbovisor's recordings, inscriptions that did not speak for themselves.

Similar to Minkowski's framing of the problem of diabetes mellitus in terms of the impaired release of an internal secretion by the pancreas into the blood, suggesting that it should be possible to extract such an internal secretion from animal pancreases and usc it as an exogenous source in the treatment of human diabetes, Ibsen's anesthesiological way of framing the problem of lethal bulbar poliomyelitis as a ventilation problem offered a program of action for the future. Framing the problem in terms of an overwhelming viral infection of vital portions of the medulla oblongata, pons, and midbrain causing extended neurological damage, had, according to Lassen, fostered therapeutic defeatism. (Lassen 1955) The neurological damage being irreversible, the only hope could be in the prevention of the disease or in a causal therapy that could kill the virus in the patient's body. Neither of them was available in 1952. Framing the problem as a ventilation problem, as Ibsen did, suggested that many more patients could be saved, thus extending its persuasive appeal far beyond the individual case of the twelve year old girl. The majority of patients affected by bulbar polio were children and young adults who were not likely to present with pre-existent, chronic pulmonary diseases compromising their ventilatory capacity. In tracheotomy allowing intratracheal and intrabronchial suction, physical therapy, postural drainage and antibiotic treatment of secondary pulmonary infection sufficient means were available to maintain a maximum of ventilatory capacity, so that there was no reason to believe that the mortality rate of bulbar polio could not be significantly reduced.

\section{Perceptive immediacy in a theater of truth}

Arguing that Ibsen's accomplishment was rhetorical begs the question of what constituted Ibsen's persuasiveness. What constituted rhetorical power or rhetorical strength in this case?

The rhetorical strength was not in Ibsen's rational, verbal line of argument. His initial account of bulbar polio as a ventilation problem was countered with another coherent line of argument which made reference to previous experience in the 
treatment of the disease and to extended neurological damage found in autopsy reports. The rhetorical strength was neither in the mobilization of Bower through Ibsen's 'production' of a paper that said that mortality rates in bulbar polio had been reduced through a combination of negative pressure respiration with endotracheal positive pressure ventilation. Bower was criticized for putting patients in the respirator too early, patients who would also have survived without respirator treatment.

The rhetorical strength was in the demonstration, in the display of competence and skill. The rhetorical strength was in the immediate perceptibility, both visual and tangible, of the surviving twelve year old girl on the one hand, and the Carbovisor display on the other. Recognizing the centrality of, what I would like to call, perceptive immediacy, in an analysis of rhetorical power, Bruno Latour aptly introduced the metaphor of a theater of proof. Latour used this metaphor with reference to Pasteur's 1881 staged demonstration of the effectiveness of his anthrax vaccine at Pouilly-leFort, leaving the vaccinated and visibly marked sheep alive, invulnerable to fatal inoculation with the anthrax bacillus, surrounded by the corpses of unvaccinated sheep. (Latour 1988). Contrary to Pasteur's demonstration at Pouilly-le-Fort, Ibsen did not 'stage' the 1952 polio epidemic in Copenhagen. In this respect the epidemic was contingent. But the contingency of the situation did not reduce its rhetorical characteristics. What had been a theater of war for Blegdamshospital's medical staff during the first three weeks of the epidemic served for Ibsen as a theater of proof, competence and effectiveness. He proved the plausibility of his pathophysiological account. He displayed his own competence and skill as an anesthetist, and representing the discipline, the competence and skill that anesthesiologists in general could offer outside their traditional domain of the operating theater. Ibsen also displayed the effectiveness and superiority of intermittent positive pressure ventilation over negative pressure ventilation.

Ibsen was a competent clinician who successfully applied his knowledge and skills, which he had learned in the previous years in the operating theater, on a crucial moment; an arduous task in trying circumstances. What if the twelve year old girl had died, despite Ibsen's efforts? Would not the whole situation have turned into a theater of incompetence, of failure? Would he have been given a second chance?

The polio-epidemic, the surviving, manually ventilated twelve year old girl and the Carbovisor, constituted a turbulent trial displaying the weaknesses in the 'old' cognitive, technical and social order at Blegdamshospital; a catalytic rapid in which old truths were severed, developments that had already started were accelerated and in which new lines of research were initiated.

\subsection{Mobilizing hands}

Despite his initial skepticism Lassen energetically dedicated himself to the implementation of the new, emergency therapy for all patients with respiratory insufficiency and swallowing impairment. The treatment required no special equipment. Balloons, cuffed tubes, oxygen cylinders, etc. could be sufficiently supplied. Teams of internists, otolaryngologists and anesthetists were established. Depending on the extent and location of the paralysis patients were selected for different forms of treatment. Patients with 
paralysis of the extremities only, that is without respiratory insufficiency, were transferred to other wards and hospitals. Patients with pooling secretions in the upper airways and swallowing impairment, but without respiratory paralysis, were tracheotomized to allow for good suction and free airways, after which the patients could maintain sufficient ventilation themselves. Patients with moderate respiratory insufficiency, but without secretion problems, so called $d r y$ cases, were treated in negative pressure respirators to support their breathing. This treatment could be combined with tracheotomy in patients whose hypopharynx was not entirely free from secretions, the tracheotomy allowing for proper suction of the upper airways. Manual positive pressure ventilation was not applied in these patients. Patients with respiratory insufficiency, pooling secretions and swallowing impairment, so called wet cases, were subjected to early tracheotomy followed by manual positive pressure ventilation. (Lassen 1953)

\section{Medical students working shifts}

The consequence of large scale application of manual intermittent positive pressure ventilation was that every patient who was kept alive should be ventilated 24 hours per day, until their own spontaneous respiration had recovered sufficiently to allow the artificial respiration to be gradually phased out. Most patients reached this point within three months. With new patients with bulbar paralysis coming in every day, during the first weeks following the introduction of the new treatment there was a rapid increase in the number of patients to be manually ventilated continually. Manual ventilation 24 hours per day meant that 24 hours per day somebody had to sit at the patient's bedside to squeeze the balloon. At the height of the epidemic 70 patients had to be manually ventilated simultaneously. It was clear that the physicians could not do this themselves. They were occupied with examining new patients, establishing the diagnosis, assessing the severity of the disease and selecting them for different forms of treatment, depending on the location and extent of paralysis and the presence or absence of secretory problems. The nursing staff too was occupied with the intensive care these patients needed.

In want of first and foremost hands Lassen called on the students of the university's medical faculty. With the help of Medicinske Studenterradet Lassen made an appeal to the medical students to come and assist in the emergency situation at Blegdamshospital. (Bjørneboe 1953b) Lassen urged the dean of the medical faculty to give students who volunteered dispensation from attending obligatory courses in the medical curriculum.

The students were deployed in shift work, four shifts of six hours each. After a few days the task of recruiting new students and organizing and scheduling the shift work was assigned to and taken over by students themselves, first by the chairman of Studenterrådet, Arne M. Boiesen, and later by Villy Frølund Thomsen and Uffe Kirk. (Frolund Thomsen 1953) Copenhagen's municipal authorities agreed to a moderate financial allowance for the students, which was also administrated by the students themselves. (Hansen 1953)

The students were briefly instructed in important aspects concerning the equipment and clinical features of the disease and the patients they were assigned to. They were not only supposed to squeeze the bag but also to monitor the functioning 
of the equipment and the clinical condition of the patients, in order to call in help when technical or medical complications arose.

Working at Blegdamshospital during the polio-epidemic was for most of the students an emotional and impressive experience, especially at night. They were literally keeping the patients to whom they had been assigned alive with their hands.

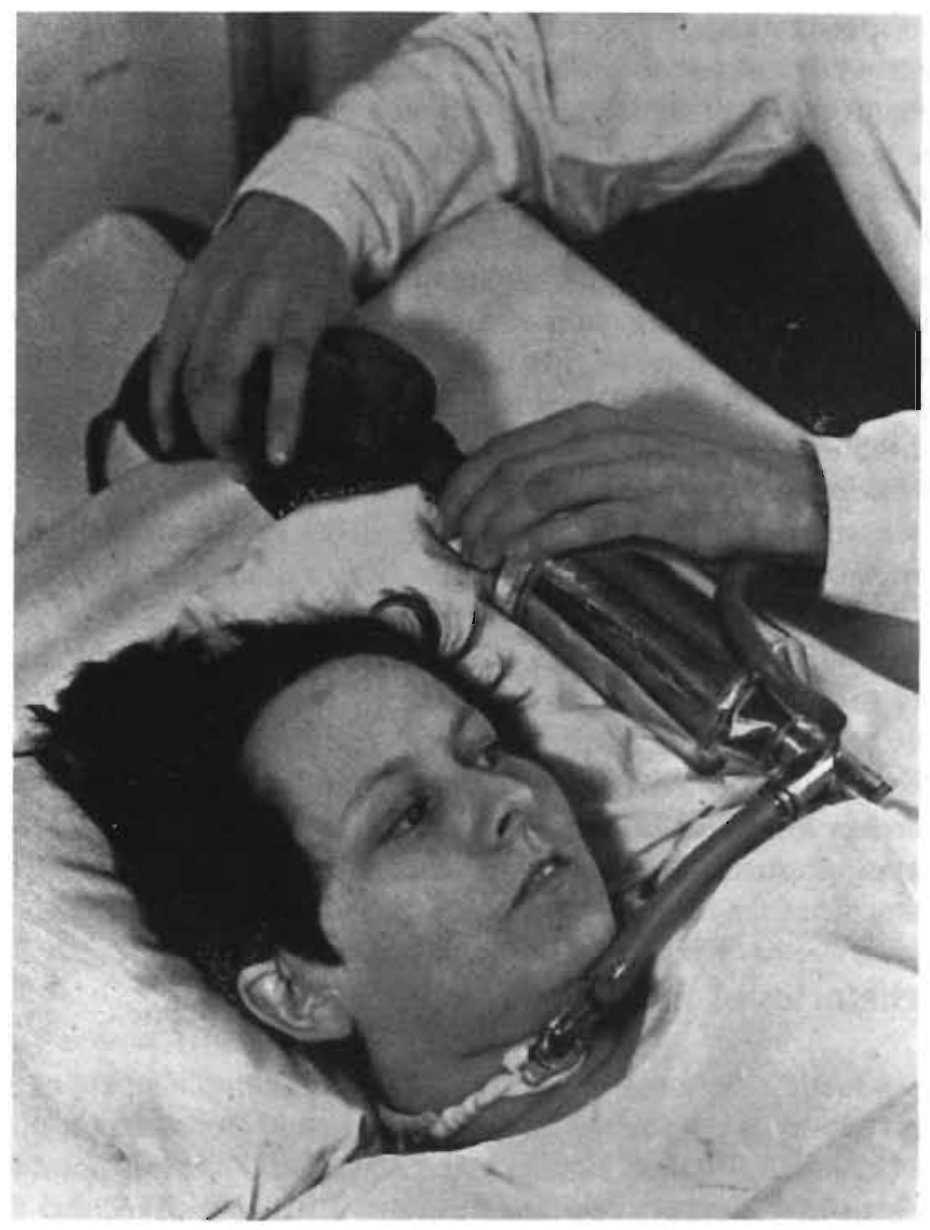

Figure 4.9

Manual bag ventilation (with absorber) by student Reproduced from Lassen (1956: 62)

Six hours at a row, with only one or two ten minute breaks, they were sitting at the patients' side compressing the bag (see figure 4.9). Every slack in attention and frequency of balloon compression had immediate consequences for the patient, inducing a sensation of suffocation. As a result of the respiratory and swallowing paralysis, and as a result of the tracheotomy, patients, the majority of which were 
children, were not able to speak. New forms of communication were invented on the spot.

\section{Recruiting dental students}

The recruitment of new students was a continuous proces. During the first weeks following the introduction of the new treatment the number of hands needed rose as the number of patients requiring continuous manual ventilation increased. In addition to the students who worked the shifts it was necessary to have some students standing by in case one of the students scheduled fell sick or cancelled for whatever reason.

Besides acute reasons of not being able to continue working at Blegdamshospital many students decided to stop after a few weeks. The reasons for quitting were manifold. Although in hindsight, after the epidemic, none of the hospital's staff, neither physicians, nor nurses, nor students, acquired poliomyelitis ${ }^{20}$, during the epidemic the risk of being contaminated and infected was strongly felt. Little was known about the virus' way of spreading. ${ }^{21}$ The confrontation with severely ill children and young adults struggling to survive, perfectly healthy prior to the outbreak of polio, and the fear of falling victim to the disease themselves, added to the physical burden of working in shifts and could well lead to near emotional exhaustion. In addition to the emotional burden that working in Blegdamshospital imposed on the students there was the problem of delay in medical training that made many students decide to return to their studies after a number of weeks.

On November 5, 1952 a number of lectures were given for medical students at the university to encourage them to volunteer. (Medicinske Studenterradet 1952) In november 1952 the 'supply' of medical students ran short, which made Lassen decide to recruit dental students. As Lassen put his request forward, the school's director used the intercom system to call upon his students to respond to Lassen's appeal for support. In total, approximately 1.500 students put in 165,000 hours. (Hansen 1953)

\subsection{The rhetorics of mortality rate reduction}

In the previous section the focus shifted from Ibsen's accomplishment as an individual to the collective level of the successful deployment and 'orchestration', by Lassen, of what Bijker (1992a) aptly called a sociotechnical ensemble. At this point the rhetorical analysis can also be extended. For those working in Blegdamshospital the reduction in mortality rate had the same quality of perceptive immediacy as Ibsen's demonstration of the effectiveness of intermittent positive pressure ventilation and the visualization of the $\mathrm{CO}_{2}$-retention syndrome by means of Brinkman's Carbovisor. No mathematical calculations of statistical significance were required to see that the mortality rate was down. Two features contributing to this perceptive immediacy of mortality rate reduction should be highlighted here.

\section{Reduction of spatial and temporal distance}

The establishment of Blegdamshospital in 1879, and its subsequent development and concentration of medical expertise in diagnosis and treatment of infectious diseases, prepared the ground for the hospital serving as a polio-center for the metropolitan area 
of Copenhagen in 1952. Hence, paralytic polio patients dispersed over quite a large geographical area were all admitted to Blegdamshospital and thus concentrated. This reduction in spatial distance between polio patients was paralleled by a similar reduction in temporal distance brought about by the epidemic character of the outbreak. A relatively large number of patients with bulbar polio were admitted and treated within a short span of time. In four months the hospital treated three times as many cases of poliomyelitis with respiratory insufficiency, paralysis of the ninth, tenth and twelfth cranial nerves, and involvement of the bulbar respiratory and vasomotor centers as in the preceding ten years. When individual cases are seen spread over a long period of time, or when small clusters of cases are treated with large time intervals, any reduction in mortality rates must be extracted from aggregated case reviews and statistical analysis. In the 1952 polio epidemic both distances in space and time were considerably reduced, contributing to the perceptive immediacy of mortality rate reduction.

Bringing down the mortality rate of bulbar poliomyelitis was a collective effort. The students' hands, and all the organizational work to deploy them, were as indispensable as the bags, cuffed tubes, absorbers, humidifiers and oxygen cylinders. As we will see in the next section, the human share in this work would soon be delegated to non-humans, to mechanical positive pressure respirators.

\subsection{Mechanical students}

By 1952 manual intermittent positive pressure ventilation had been used for several decades in operating rooms. Without positive pressure ventilation open thorax surgery would not have been feasible at all. Long before the 1952 polio-epidemic in Copenhagen mechanical positive pressure respirators had been build. At the end of the 1930s for example, Anderson, Crafoord and Frencker (1940) developed a machine which they called the 'Spiropulsator', a pressure regulated positive pressure respirator.

\section{Impairment of venous return}

The applicability of these early respirators was limited however, especially in time. In other words, intermittent positive pressure ventilators could be used for only short periods of time, for example, the few hours it took to perform a thoracic operation. By 1950 the disadvantageous, injurious effects of these respirators on the patients' circulation were still a big problem, hampering their prolonged use.

In mechanical respirators not only the application of pressure or force to compress the bag was mechanized, they also had to contain a mechanism to determine the end of the inspiration and begin the expiration phase. As the name indicates, in pressure regulated respirators a pressure gauge was used for this purpose. The inspiration phase was ended when a pre-selected pressure had been reached, whereafter the weight and elasticity of the thorax took care of expiration. It was this pre-selected pressure that determined the intra-thoracic pressure at the end of the inspiration phase, that is, the pressure encircling the main venous blood vessels travelling through the thoracic cavity on their way to the heart (vena cave inferior and superior). The venous return to the heart is influenced by the difference between the venous blood pressure 
on the one hand, and the intra-thoracic pressure on the other. Selecting too high a pressure on the respirator would cause the intra-thoracic pressure at the end of inspiration to approximate, or even equal or exceed, the venous blood pressure in the inferior and superior vena cava, resulting in a reduced amount of blood returning from the greater circulation to the heart. This in turn would result in a reduced amount of blood entering the pulmonary circulation and in a disadvantageous, decreasing effect on the blood pressure in the greater circulation. (Werkö 1947) However, when the pre-selected pressure on the respirator was chosen too low, to prevent circulatory effects, this threshold value would be reached before a sufficient volume of gas was blown into the patient's lungs, resulting in less than adequate ventilation and, consequently, in the gradual accumulation of $\mathrm{CO}_{2}$ in the patient's blood. This problem could also arise when the pre-selected respirator pressure was set at a higher level. For example, when during thorax surgery the lungs were compressed, preventing normal expansion of the lungs, the respirator's threshold value would be reached before a minimally required volume of gas had been administered. ${ }^{22}$

\section{Engström's respirator}

These circulatory and ventilatory complications and risks prevented prolonged use of mechanical respirators. In the operating theater the duration of mechanized positive pressure ventilation was never longer than a few hours. According to the Swedish physician Engström,

... no successful treatment of intermittent positive or positive-negative ventilation continuously given for longer periods had been reported by 1950 . No devices which could implement the use of intermittent positive-pressure breathing for more than a few hours ... had been constructed, and no methods for the control of the ventilatory function suitable for routine clinical use were available. (Engström 1963: 8)

In 1950 Carl-Gunnar Engström designed and built a prototype of a new respirator type in which the switch from inspiration to expiration was determined by a pre-selected volume. In Engström's respirator the inspiration phase continued even when, as a result of intra-pulmonal resistance, the maximum pressure had been reached at a very early stage. The inspiration continued until a pre-selected volume of gas had been administered, thus warranting, according to Engström, adequate ventilation. In the expiration phase Engström's machine 'sucked' a negative pressure to enhance venous return, thus compensating for any impairment of venous return in the inspiration phase.

In 1951 Engström's prototype was successfully used in the treatment of a patient with chronic poliomyelitis where the negative pressure respirator seemed to be insufficient.

In the fall of 1952, Engström's volume-regulated respirator was brought to Copenhagen to use it in the treatment of bulbar polio patients in Blegdamshospital, where it was considered to perform well (see figure 4.10). 


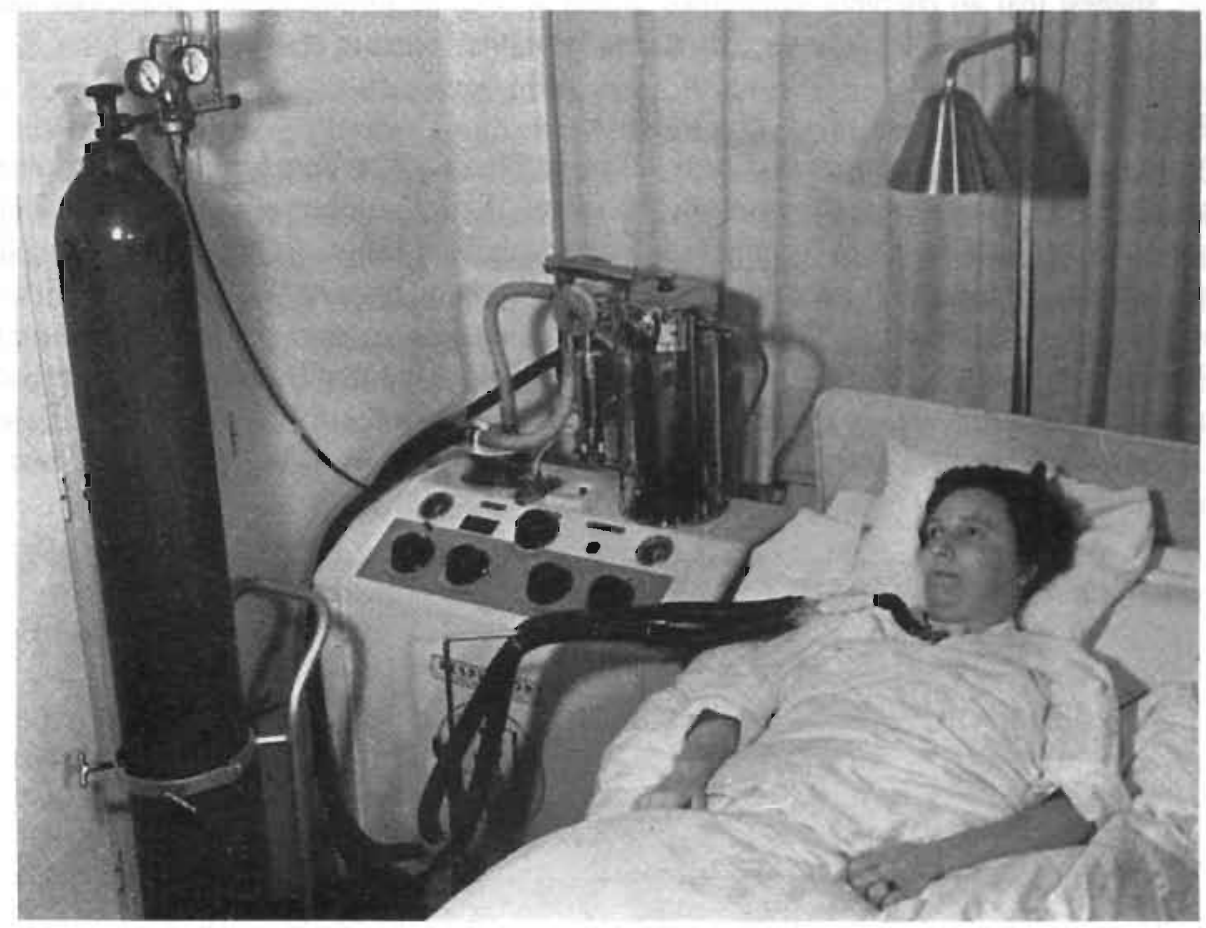

Figure 4.10

The Engström respirator

Reproduced from Lassen (1956: 66)

\section{Building 'mechanical students'}

Struck by the extent and severity of the 1952 polio-epidemic in Copenhagen Swedish health care authorities wondered whether they could cope with a similar epidemic. (Gordh and Gordh 1952; Haglund 1952) The encouraging experiences with Engström's respirator at Blegdamshospital made them decide to take Engström's respirator in production. As Stockholm was confronted with a polio epidemic of similar magnitude one year later, in the fall of 1953, all patients with bulbar polio could be treated with mechanical Engström-respirators. (Ström 1956) Stockholm did not have to call on her medical students to lend a hand.

As the word spread that a breakthrough in the treatment of bulbar polio had been accomplished in Copenhagen, physicians from abroad visited Blegdamshospital. Impressed by what they witnessed there, these physicians called upon their own government to develop and produce mechanical students.

The possibility of producing a 'mechanical student' to squeeze the bag must also be tackled ... We feel ... that this problem must be tackled on a national scale. The central supply of cylinders and pumps, and the production of a 'mechanical student' ... are all matters which cannot be economically dealt with on a local basis ... We 
suggest that an energetic committee should at once be formed so that plans can be perfected before the poliomyelitis season is on us. (Christie and Esplen 1953)

\section{Bang's respirator}

As the epidemic raged initiative was taken in Denmark to develop and build mechanical positive pressure respirators. In the fall of 1952 a number of patients with bulbar polio from Jylland were treated in the hospital in Skive. One of the hospital's physicians, Claus Bang, designed a positive pressure respirators. Together with two employees from Bang \& Olufsen in Struer, an engineer and an instrument maker, Bang built a prototype and used this machine in the treatment of a patient with bulbar polio in Skive hospital in january 1953. (Bang 1954) In the following months Bang \& Olufsen built several respirators more. In march 1953 six B\&O-respirators were in operation. $^{23}$

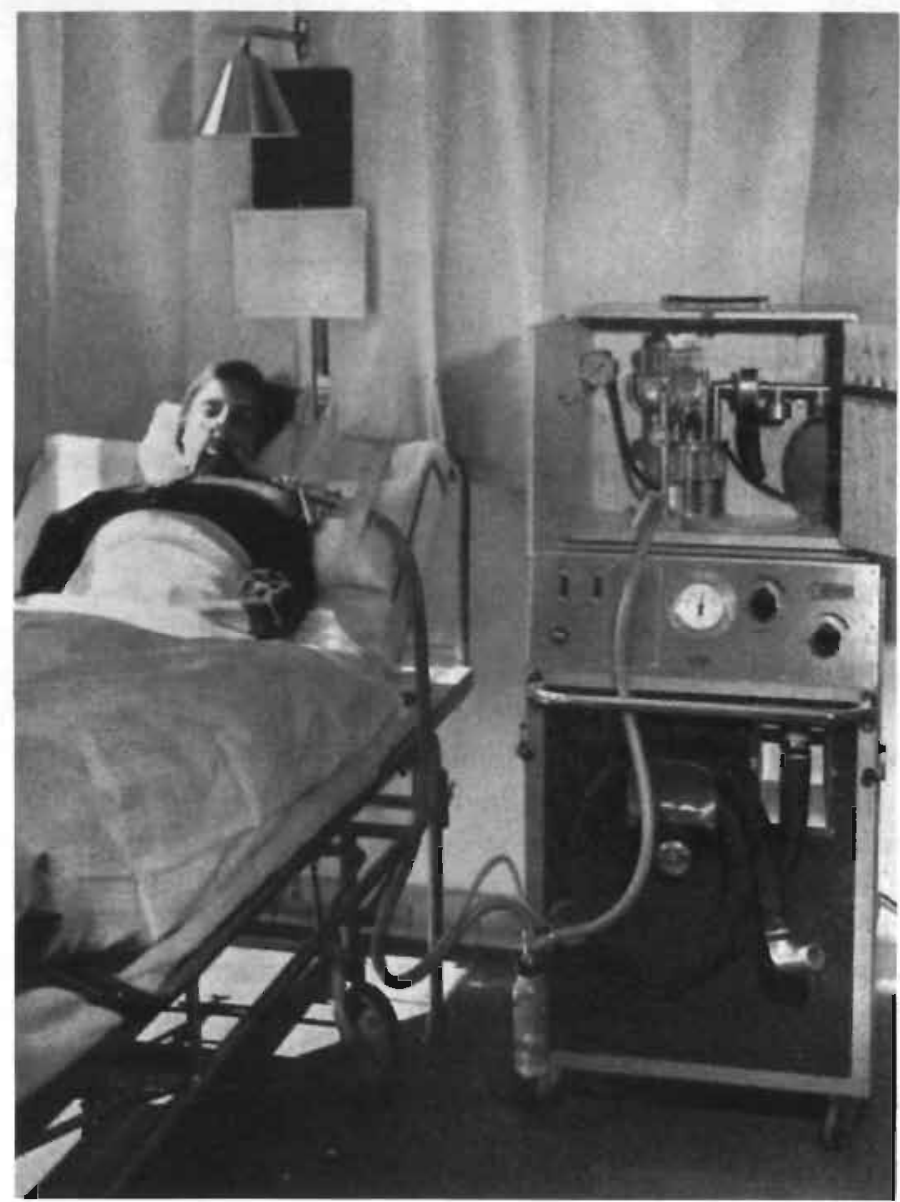

Figure 4.11

The Gullberg-Kifa respirator

Reproduced from Lassen (1956: 67) 


\section{More respirators}

In Scandinavia, as well as in England and Germany, respirators were designed, produced and released with an incredible speed. ${ }^{24}$ Already in 1953 hospitals looking for positive pressure respirators could choose from a great number of different models and trade-marks. These respirators differed in the devices that were used to switch the machine from inspiration to expiration, as well as in the particular solutions constructed to compensate for venous return impairment, thus preventing injurious circulatory effects in prolonged use. Whereas Engström's respirator combined the positive pressured inspiration phase with an intra-tracheal applied negative pressure in expiration, the Swedish Gullberg-Kifa-respirator combined a positive pressure respirator with a pre-existent cuirass respirator, that is with an extra-thoracic applied negative pressure (see figure 4.11).

\section{Obsolescence for negative pressure respirators}

In Europe the negative pressure respirators (iron lungs and cuirass respirators) were soon to become obsolete. According to Mushin,

[a]ll branches of the medical profession, neurologists, anesthetists, E.N.T.-surgeons, and epidemiologists studied the lessons of the Danish epidemic. In the space of three or four years the accepted methods of treatment for life-threatening spino-bulbar poliomyelitis in western Europe were transformed so that, while patients in U.S. epidemics in the middle 1950s were still being treated in 'tank respirators', British and European thought had already largely consigned the tank or body respirator to the limbo of obsolescence. (Mushin 1980: 209 - 211)

In Blegdamshospital negative pressure respirators were used until the end of the 1950s in the treatment of a small number of patients with 'dry' respiratory insufficiency, that is without secretion problems in the airways. (Faber et al. 1979) Preference was given to positive pressure ventilation. Although some physicians, among whom lbsen, preferred manual positive pressure ventilation - the crudeness of mechanical respirators did not match the subtlety in volume and pressure regulation manual ventilation allowed in skilled hands ${ }^{25}$ - mechanical devices for intermittent positive pressure respiration (IPPR) gained wide acceptance and use, not only in the treatment of poliomyelitis.

\section{Prolonged IPPR for respiratory insufficiency of other etiology}

The new volume-regulated respirators, which had proven their applicability in the prolonged artificial maintenance of a patient's breathing, fed back into the operating theater allowing surgery to be performed on patients with a severely compromised pulmonary capacity.

It was then [1954] shown that it was possible to carry through patients postoperatively with acute complications in the only remaining lung, and also to accept patients with only minimal lung function for thoracic surgery through the application of early tracheotomy and controlled ventilation ... (Engström 1963: 10) ${ }^{26}$ 
But also patients with acute or chronic respiratory insufficiency resulting from other causes, such as drug intoxications, skull trauma, brain tumors, etc., were soon successfully treated with intermittent positive pressure respirators.

It soon became evident that the method had a wider field of application than only poliomyelitis patients. Through a complete taking over of the respiratory work it might be successfully used for patients with respiratory insufficiency - either manifest or impending - with other aetiology. Thus in the beginning of 1953 some patients with severe respiratory insufficiency caused by skull trauma, brain tumor and barbiturate intoxication were treated. (Engström 1963: 9)

The recurring impending threat of poliomyelitis epidemics was eliminated in the years following the extensive pilot study with Salk's polio vaccine in the United States in 1954. In Denmark, Statens Seruminstitut had been building up vaccine production capacity while waiting on the results of the US pilot study. As the word about the vaccine's effectiveness came, the institute was able to respond rapidly and organize and coordinate campaigns for the vaccination of Denmark's population. By then however, the mechanical positive pressure respirator did no longer depend on the treatment of poliomyelitis, but had shifted towards other diseases and medical conditions providing it with supporting, stabilizing niches.

An important change in artificial ventilation practices that occurred during the 1952 polio-epidemic in Copenhagen's Blegdamshospital was the prolongation of the time during which either manual or mechanical positive pressure ventilation was given. As indicated by Engström (1963), in the operating theater positive pressure ventilation was never given for periods exceeding a few hours. During the polioepidemic in Copenhagen positive pressure ventilation was given for days, weeks, months and even years without interruption. 26 of the surviving polio patients never regained spontaneous breathing completely and were committed to the respirator for the rest of their lives. (Stig Christensen et al. 1975; Astrup et al. 1989) To avoid long term injurious effects of such prolonged artificial respiration methods to monitor the patient's acid-base status were indispensable.

\subsection{Acid-base and blood gas analysis}

As exemplified by Krogh and Hasselbalch, Denmark had a long tradition in scientific research concerning gas exchange in the lungs and the physiology and pathophysiology of these respiratory gases in the blood. For research purposes methods had been developed to assess acid-base status. both in terms of technological devices for the measurement of $\mathrm{pH}$ and blood gas analysis as in terms of equations and models. However, the application and use of these technologies was largely confined to the research laboratory. They were too complicated and required a too large amount of blood and time to be appropriate for frequent routine examinations in a hospital's laboratory, serviceable primarily to patient care. Although Blegdamshospital' laboratory was equipped with a pH meter, in 1952 'total $\mathrm{CO}_{2}$ in plasma' was the only laboratory parameter used for the assessment of acid-base status in patients. 
Total $\mathrm{CO}_{2}$ in plasma

Total $\mathrm{CO}_{2}$ in plasma was measured according to the method developed by Donald van Slyke in the 1920s in New York. This method measured the total amount (volume) of gaseous carbon dioxide which could be extracted or expelled in a vacuum from a certain amount of blood through the addition of strong acid. Van Slyke's method comprised a chemical mechanism, the addition of sulfuric acid, and a physical mechanism, the application of a vacuum to the sample, for the extraction of $\mathrm{CO}_{2}$. Carbon dioxide was considered to be present in blood or plasma in two different states: chemically bound and physically dissolved. To be able to measure the total $\mathrm{CO}_{2}$ content of blood the chemically bound carbon dioxide had to be transferred into a physically dissolved state, after which it could attain its gaseous form when a different (lower) $\mathrm{CO}_{2}$ pressure was present or applied. Henderson-Hassselbalch's equation was considered to represent chemically bound $\mathrm{CO}_{2}$ in blood:

$$
\mathrm{H}^{+}+\mathrm{HCO}_{3} \rightleftarrows \mathrm{H}_{2} \mathrm{CO}_{3} \rightleftarrows \mathrm{H}_{2} \mathrm{O}+\mathrm{CO}_{2}
$$

The addition of acid amounted to the addition of hydrogen ions $\left(\mathrm{H}^{+}\right)$on the left side of the equation, shifting the equilibrium to the right, releasing $\mathrm{CO}_{2}$ from its chemical shackles on the right side of the equation, allowing it to enter a physically dissolved and subsequent gaseous state.

\section{Metabolic disturbances}

In accordance with John Esben Kirk's ${ }^{27}$ influential 1942 textbook Acidosens klinik og behandling, in Blegdamshospital's clinical practice low values of total $\mathrm{CO}_{2}$ in plasma were usually interpreted as a metabolic acidosis: a rise in hydrogen ion $\left(\mathrm{H}^{+}\right)$ concentration resulting from an accumulation of non-volatile acids deriving from metabolism in the body's tissues; that is, on the left side of the equation. The rise in $\mathrm{H}^{+}$-ion concentration shifted the equilibrium to the right, expelling $\mathrm{CO}_{2}$, which was then subsequently removed in the lungs through breathing. In metabolic acidosis then, less $\mathrm{CO}_{2}$ would be chemically bound, resulting in a low value of total $\mathrm{CO}_{2}$ in plasma when a blood sample was subjected to Van Slyke's method. Metabolic acidosis was the condition which was most often encountered in the hospital.

Less frequent high values of total $\mathrm{CO}_{2}$ in plasma were found. These were usually interpreted as a metabolic alkalosis: a reduction of $\mathrm{H}^{+}$-ion concentration, deriving from some pathophysiological, metabolic mechanism in the bodies tissues, on the left side of the equation. This reduction would pull the equilibrium to the left, resulting in a larger amount of $\mathrm{CO}_{2}$ bound in $\mathrm{HCO}_{3}$ and $\mathrm{H}_{2} \mathrm{CO}_{3}$. A blood sample subjected to Van Slyke's method would thus show a high value of total $\mathrm{CO}_{2}$ in plasma.

The respective shifts in equilibrium described here constitute mechanisms to compensate the impending fall or rise in $\mathrm{pH}$ by limiting the increase or decrease of free hydrogen ions.

\section{Respiratory disturbances}

In research laboratories respiratory disturbances of the acid-base equilibrium were well known. In hospitals and hospital's laboratories they were almost never encountered. Diseases of the lungs causing significant, no longer compensated, disturbances in the 
patient's acid-base equilibrium in the long run, were often complicated by pulmonary infections causing death at an earlier stage. (Astrup et al. 1989) Results from 'total $\mathrm{CO}_{2}$ in plasma' measurements according to Van Slyke's method, which would be ambiguous in the setting of research laboratory if not supplemented with a direct measurement of $\mathrm{pH}$, received a more limited, but for clinical purposes sufficiently robust interpretation in clinical settings.

This theoretical framework for the clinical interpretation of total $\mathrm{CO}_{2}$ in plasma values, combined with a clinical experience in which long term respiratory disturbances of acid-base status were virtually absent, formed the background for the interpretation of the high total $\mathrm{CO}_{2}$ in plasma values found in terminal bulbar polio as a metabolic alkalosis. According to this interpretation, the increased amount of $\mathrm{CO}_{2}$ would be chemically bound. Metabolic alkalosis would not yield an increased amount of physically dissolved $\mathrm{CO}_{2}$, and hence, would not result in an increased concentration of $\mathrm{CO}_{2}$ in expired air.

Applying Brinkman's Carbovisor on the twelve year old girl Ibsen showed that she had an increased concentration of $\mathrm{CO}_{2}$ in expired air. Hence, she must have had an increased amount of physically dissolved $\mathrm{CO}_{2}$ in her blood. In terms of the equation for chemically bound $\mathrm{CO}_{2}$ in blood mentioned above: the physically dissolved $\mathrm{CO}_{2}$ on the right side of the equation could not get out because the pulmonary $\mathrm{CO}_{2}$-disposal mechanism, ventilation, was impaired by secretions blocking the airways. As a result of this, $\mathrm{CO}_{2}$ was accumulating, pushing the equilibrium to the left, giving rise to an increase of free $\mathrm{H}^{+}$-ions, which, if not completely compensated, would result in a decrease in pH: a respiratory acidosis. Ibsen was confident that a direct measurement of the $\mathrm{pH}$ in the blood of a patient in the terminal stage of bulbar polio would reveal a lowered $\mathrm{pH}$.

\section{Measuring $\mathrm{pH}$ in blood}

Poul Astrup, head of Blegdamshospital's laboratory, called on Radiometer A/S in Copenhagen, a firm producing laboratory instruments for chemical industries, to supply a pH electrode that had recently been developed by Radiometer for a laboratory interested in biological $\mathrm{pH}$ measurement. This electrode was furnished with a cap surrounding the electrode and a suction device (see figure 4.12). The liquid to be examined would spread between the inside surface of the cap and the outside surface

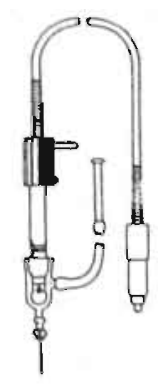

Figure 4.12

Radiometer's capped pH electrode 
of the electrode, allowing $\mathrm{pH}$ measurement on a small sample of blood. One day after Ibsen's treatment of the twelve year old girl Astrup was able to measure $\mathrm{pH}$ in blood directly using Radiometer's new electrode. The pH measurements confirmed that patients with terminal stage bulbar polio had acidosis, and not alkalosis.

While providing additional support to Ibsen's account of the pathophysiological mechanisms leading to the patients' death, Astrup's pH measurements simultaneously displayed the inadequacy of 'total $\mathrm{CO}_{2}$ in plasma' as a reliable parameter in the clinical assessment of acid-base status. The figure which, until then, had indicated metabolic alkalosis with sufficient practical robustness, was no longer unambiguous, not even in a clinical setting. One and the same figure could now represent a metabolic alkalosis and a respiratory acidosis. The numbers themselves were mute, allowing multiple interpretations. 'Total $\mathrm{CO}_{2}$ in plasma' would at least have to be supplemented with a direct $\mathrm{pH}$ measurement.

\section{Monitoring prolonged artificial ventilation}

Ibsen's exposure of the $\mathrm{CO}_{2}$-retention syndrome as the 'killer' in bulbar polio emphasized the amount of physically dissolved $\mathrm{CO}_{2}$ in the patients blood $\left(\mathrm{PCO}_{2}\right)$ as the appropriate parameter to monitor the adequacy of the prolonged manual positive pressure ventilation. Direct $\mathrm{pH}$ measurements and 'total $\mathrm{CO}_{2}$ in plasma' estimations provided the variables which could be inserted in Henderson-Hasselbalch's equation to calculate the actual $\mathrm{PCO}_{2}$ in the patients' blood. During the epidemic regular arterial blood samples were taken from manually ventilated patients for measurement of $\mathrm{pH}$ at $37^{\circ} \mathrm{C}$ and 'total $\mathrm{CO}_{2}$ in plasma'. (Astrup 1956a) The $\mathrm{PCO}_{2}$ was then calculated and correlated with the patient's condition. If necessary the ventilating students were given instructions about frequency and intensity of the manual ventilation they were applying.

Astrup's research interests focused, during and in the years after the polioepidemic, on acid-base and blood gas physiology. (Astrup 1954) Together with Svend Schrøder, brother to one of the founders of Radiometer A/S, Astrup designed and built an apparatus for blood gas analysis based on a new principle. (Astrup and Schrøder 1956; Astrup 1956b) In the clinically relevant range blood exhibited a linear relationship between $\mathrm{pH}$ and $\mathrm{PCO}_{2}$. In his new method, Astrup first measured the actual $\mathrm{pH}$ of a blood sample. Subsequently he perfused the same blood sample two times with gas from two cylinders, containing $\mathrm{CO}_{2}$ in known concentrations, one having a lower and one having higher $\mathrm{CO}_{2}$ concentration respectively. After perfusion the blood sample would have the same $\mathrm{CO}_{2}$ concentration as the gas; the $\mathrm{pH}$, exhibiting a linear relationship with $\mathrm{PCO}_{2}$, would have shifted accordingly. By measuring the $\mathrm{pH}$ of the blood sample after completion of the perfusion process. which Astrup called equilibration, he obtained two points of reference. Plotting these two points in a graph he could draw a straight line between them and locate the actual pH of the blood sample on this line, allowing him to infer the corresponding actual $\mathrm{PCO}_{2}$ of the blood sample. Although still being an indirect way of measuring $\mathrm{PCO}_{2}$ it allowed Astrup to drop the rather cumbersome and time consuming measurement of 


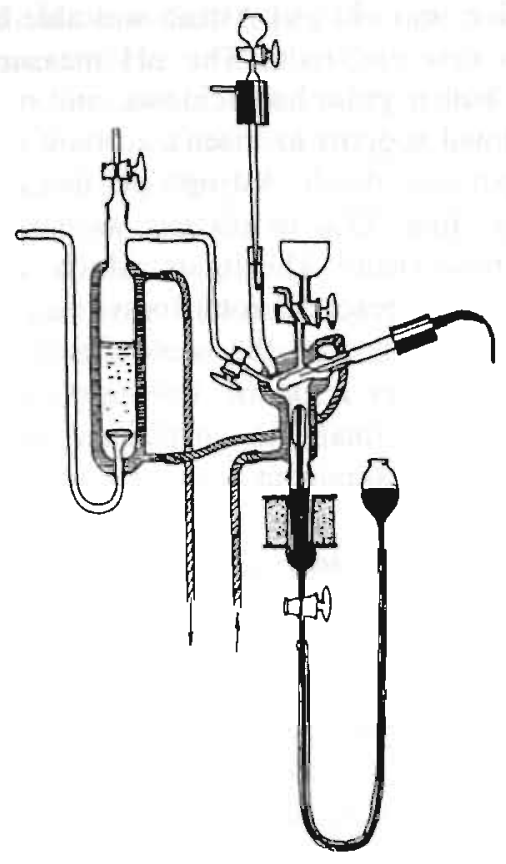

Figure 4.13

The first Astrup apparatus, designed by

Astrup and Schroder

Reproduced from Severinghaus

and Astrup (1987: 42)

'total $\mathrm{CO}_{2}$ in plasma' according to Van Slyke's method. Astrup's 'equilibration' method would be faster and require a smaller blood sample. Radiometer A/S developed the apparatus further and took it in production. Astrup's apparatus gained wide acceptance in hospital laboratories where in for several decades an 'Astrup' was synonymous with blood gas analysis. ${ }^{28}$

Astrup and his collaborators worked on new ways of graphically representing disturbances in the acid-base equilibrium in a clinically relevant manner. (Siggaard Andersen and Engel 1960; Siggaard Andersen 1960) They worked on new parameters like base excess and standard bicarbonate. new concepts which were designed to provide insight in the metabolic and respiratory components in disturbances and compensatory mechanisms of the acid-base equilibrium in blood. (Siggaard Andersen et al. 1960)

This transformation and movement of knowledge and technologies from the physiological research laboratory to the hospital was accompanied by a no less important shift from a rather marginal to a central position for the clinical laboratory in the care for critically ill patients. The monitoring service of the clinical laboratory was indispensable for the development of prolonged, controlled artificial ventilation 
with mechanical positive pressure respirators, and for the development of very intensive care and treatment regimes in intensive care units.

\subsection{Kommunehospital's intensive care unit}

During the 1952 polio-epidemic Ibsen had shown that anesthetists had a task and a competence outside the operating theater. This competence was in the area of the treatment of critically ill patients. An anesthetist's competence was not however, in disease specific treatment, not in the treatment of diseases, but in the maintenance of a patient's vital functions: respiration, circulation, blood pressure and temperature regulation.

The polio-epidemic, and Ibsen's role in it as an anesthetist, provided the beginning professionalization of anesthesiology as a medical specialty with an important impetus. The 1952 polio-epidemic was followed by a marked increase in the pace with which new and permanent jobs were created in Danish hospitals for anesthetists. An increasing number of hospitals established special anesthesiology departments. Copenhagen's university hospital, Rigshospital, appointed Ole Secher on April 1, 1953, on her newly established anesthesiology department. (Jakobsen et al. 1978) Ole Secher was the first anesthesiologist appointed in a senior position in Denmark. In 1964 he was appointed the first professor in anesthesiology in Scandinavia.

Ibsen was appointed in a permanent job at Copenhagen's Kommunehospital's surgical department. Together with Rigshospital, the university hospital, Kommunehospital was one of the most prestigious hospitals in Denmark.

\section{From recovery room to intensive care unit}

At Kommunehospital Ibsen extended the anesthetist's responsibility for the patient beyond the moment the patient left the operating theater to include the post-operative period. Surgeons, Ibsen complained, had neglected post-operative care. Good postoperative care was indispensable for the patient's recovery and prognosis, if it was only for the prevention of post-operative complications. The anesthetist, who had managed the patient during surgery was the best qualified person to attend to the patient while recovering. On July 1, 1953, Ibsen opened a ten beds recovery room at Kommunehospital, which initially was only operational between eight o'clock in the morning until four o'clock in the afternoon. From December 1, 1953, however, the recovery room was operational 24 hours per day.

On April 1, 1954, Kommunehospital too established her own special anesthesiology department. Ibsen was appointed in a senior position to head the new department. In this senior position Ibsen was no longer dependent on the surgeons. $\mathrm{He}$ now had his own patients on his own ward.

Urged by Ibsen, the municipality of Copenhagen agreed to finance the establishment of an intensive care unit in Kommunehospital ${ }^{29}$, the first in Denmark. ${ }^{k 3}$ Ibsen was allowed to design and organize this intensive care according to his own anesthesiological insights. Using the metaphor of an umbrella Ibsen explained that it was the anesthetist's job to hold on to the handle, representing vital respiratory and circulatory functions, as well as temperature regulation. (Ibsen 1969b) If that could 
be done properly assaulting diseases or other pathological conditions would not get the chance to kill the patient. Time was gained then for surgery, for causal therapies to accomplish their effect or, if no causal therapy was available, as in the case of poliomyelitis, to allow the disease the take its natural course and give the body time to recover from the damage. (Ibsen and Dahl Kvittingen 1958; Ibsen 1969)

\section{Credit for survival}

Apparently the rhetorical power of Ibsen's work during the polio-epidemic, and the credibility that followed, were short lived. Similar to light's intensity reduction as a function of the distance from the source, the rhetorical strength of Ibsen's demonstration of the appropriateness of the anesthesiological approach seemed to diminish as a function of the distance from the actual theater of competence in Blegdamshospital, and of the lapse of time. Among Kommunehospital's specialists this recognition, and the acceptance of the anesthesiologist as their equal in the social hierarchy of the hospital, was not self-evident. Ibsen had to win the necessary recognition of the appropriateness of the anesthesiological point of view and the competencies of the anesthesiologist. And in doing this a certain amount of rhetorical sensitivity can not be denied. This time, less was left to contingent situations.

Patients were transferred to Ibsen's intensive care unit from surgical and other departments. Prior to the patient's acceptance Ibsen demanded of the physician who had been treating the patient up to that moment that he make a written statement in the patient's record that he considered the patient to be moribund; that, in other words, with regard to possibilities for further treatment he had nothing more to offer and that the patient was about to die.

[A]t the beginning of my work with intensive therapy I demanded that the chief surgeon made a written statement in the record that the patient was moribund, before I would receive him for I.T. ... I wanted to make sure that if the patient recovered, it would be recognised to be due to our treatment, and that if he did not recover, our treatment would not be blamed. (Ibsen 1975: 33)

\subsection{Cooled ceilings and big toes}

In the intensive care unit Ibsen concentrated and orchestrated the activities of a large number of people, hospital departments (clinical laboratory, X-ray department, blood bank etc.), and diagnostic and therapeutic technologies. Positive pressure ventilation constituted one central element in this sociotechnical ensemble, but the cooled ceiling was no less important.

\section{Distribution of body heat}

Drawing on Johannes Ipsen's research on skin temperature regulation in intensive therapy lbsen accorded central importance, not to the maintenance of body temperature as such, but to the regulation of the distribution of heat in the patient's body. Not only the central, rectally measured, body temperature was important, but the peripheral skin temperature as measured on the patient's thumbs and big toes was equally 
important. When the room temperature surrounding the patient did not change, changes in peripheral skin temperature indicated, according to Ibsen, changes in the distribution of blood in the patient's body. Peripheral vasoconstriction for example would show by a decrease in peripheral skin temperature, vasodilation by a rise in skin temperature. But Ibsen used skin temperature measurements not only for diagnostic purposes, he used them also to monitor the therapeutic effect of vaso-active drugs, blood transfusions and plasma replacing infusions. (Ibsen 1969a; Ibsen and Jensen 1971) To that purpose, however, a controllable room temperature was indispensable. Existing methods to heat and cool the interior of buildings and rooms did not exhibit the required controllability.

\section{Cooled ceilings}

Together with two engineers Ibsen designed a new air conditioning system for intensive care units. Whereas existing systems brought about a decrease of room temperature through the replacement of air, the new air conditioning system conveyed heat to a cooled ceiling through radiation. (Clemmesen et al. 1963; Ibsen 1975)

Through regulation of the ceiling's temperature the room temperature surrounding the patient could be employed not only for diagnostic and monitoring purposes, but also for therapeutic ends. For example, for the treatment of patients who were admitted to the hospital with very low body temperature, room temperature was first lowered to their the level of their low body temperature and then gradually increased to warm them up.

Ibsen had the cooled ceiling installed in his own intensive care unit in Kommunehospital in 1957. On his recommendation a cooled ceiling was also installed in the new intensive care unit of Hvidovrehospital in Copenhagen.

\subsection{Conclusion}

The 1952 polio-epidemic subjected Blegdamshospital's prevalent medical expertise to, what Latour (1988) called, a trial of strength. The epidemic tried the robustness of prevalent pathophysiological knowledge and accounts. It tested the effectiveness of negative pressure respirators in the treatment of severe respiratory insufficiency. It tried the prevalent social organization of the medical profession and the authority of the chief as the most qualified person to tell what exactly was going on and how the problem was to be resolved. Manual positive pressure ventilation was deployed in the battle against a lethal disease, reversing the balance of power between the disease on the one hand and Copenhagen's health care system on the other. The definition of the high mortality rate in bulbar polio as primarily a ventilation problem, and the success of manual positive pressure ventilation in its treatment facilitated the mechanization of the manual technique and its transfer to other medical problem areas in which respiratory insufficiency was of central importance. By the time the vaccination campaign with Salk's polio vaccine took over the mechanical positive pressure respirator was entrenched, not only in the operating theater but also in the new sociotechnical ensemble of the intensive care units. The intensive care unit resulted 
from the changing professional, organizational, technological and architectural constellation surrounding the care and treatment of critically ill patients in the hospital.

In the decades to follow, the intensive care unit would, in effectuating her power to artificially maintain vital biological, bodily functions, constitute a trial of strength for the robustness with which death could be said to have occurred. The deployment of resuscitation and life-supporting technologies drove the moment at which the death process could be said to have begun and the moment at which it could be said to have been completed apart. The tenacity with which the dying were treated detained some of them somewhere in between the beginning and the end. New conceptual, diagnostic, legal and moral problems arose. In 1975 Ibsen captured the quandaries of intensive therapy as follows:

[A]t the beginning of intensive therapy it was a problem to keep the patient alive today is has become a problem to let him die. (Ibsen 1975: 33)

Or have they died already? The conscious, yet respirator dependent survivors of the polio-epidemic certainly have not. We would not consider to let them die. But what about the respirator-driven patients who suffered brain damage to an extent that they have irreversibly lost the capacity for consciousness? Or those who have irreversibly lost the capacity for consciousness but are not respirator dependent? Have they reached the endpoint in their death process, or are they not even dying? Should death be pronounced and the remaining bodily functions be stopped in a convenient way? Or, are they locked between beginning and end and should they be allowed to die?

I will not attempt to provide answers to these questions, yet the doubt that is expressed in these questions will be the subject of the next chapter.

\section{Notes to chapter 4}

1. Military metaphors are often employed to describe the spread and devastating effects of infectious diseases like pest and cholera. In cartoons and drawings the personified death often represents these diseases, while in epidemiological narratives they are often depicted as hostile, invading armies, only to be halted by a 'military cordon sanitaire'. (Evans 1987: 226)

2. Very little was known about the mechanisms by which the virus was transmitted from one person to another. Hence, no effective measures were available to prevent an individual's contamination with the virus. Sera containing preformed antibodies against poliomyelitis virus had been prepared and applied to produce passively acquired immunity in monkeys, but prophylactic and therapeutic experiments in humans were at best inconclusive. In the United States Jonas Salk worked on the preparation of a vaccine using killed poliomyelitis virus, but this would not be available for large scale vaccination programs of the population before 1954 .

3. The tourist map of the city of Copenhagen provided by the Tourist Office in 1989, when I commenced research on the events during the 1952 polio-epidemic, was not a reliable representation of the city's real world situation. Blegdamshospital was on the 1989-map but no longer in the real world. Blegdamshospital's role as a medical center for communicable diseases was taken over by the Rigshospital in 1975. (Faber et al. 1979: 10) Its buildings were torn down and the university's 
Panuminstitute was built in its place. Peter Ludvig Panum was appointed professor in physiology and comparative anatomy at the University of Copenhagen in 1864. In 1850 he investigated a smaller cholera outbreak in Bandholm in Denmark, but he was not able to come to an unambiguous conclusion concerning the contagionist or miasmatic origin of the disease.

4. An epidemic disease is a disease that attacks many people in the same region at the same time, widely diffused and rapidly spreading, which is only occasionally present in a human community. According to middle-of-the-nineteenth-century views an epidemic disease might be caused by a transmittable, infectious agent (contagium), or by foul odors and putrid effluvia emanating from sewage, dead animals, and sick people (miasmata), accumulating in dirty and ill-aired houses in the poor and populous quarters. In some diseases, as in cholera, where the pattern of occurrence and spreading was ambiguous, people allowed for the possibility that a miasmatic disease could be transmitted once it had originated.

5. The official death rate of $4 \%$ gives a distorted impression of the epidemic's severity since a considerable number of Copenhagen's inhabitants, among whom Hans Christian Andersen and Søren Kierkegaard, fled from the city to the countryside.

6. In 1874 it was decided to exclude cholera patients from admission to the new Blegdamshospital and to build a small quarantine hospital near the harbor, Øresundshospital, to accommodate cholera patients from visiting ships. (Kjøbenhavns Magistrat 1904)

7. For an overview of the history of poliomyelitis see the historical summary in International Committee for the Study of Infantile Paralysis (1932).

8. The report published by Copenhagen's authorities in 1904 on the occasion of Blegdamshospital's 25th anniversary did not even mention the admission and treatment of patients suffering from poliomyelitis. (Kjøbenhavns Magistrat 1904) Between 1910 and 1933 only four minor epidemics with no more than 389 paralytic cases (per epidemic) were recorded. (Hertel 1980)

9. The nature of Ibsen's position at the beginning of the 1952 polio-epidemic is often misrepresented in historical surveys. Hilberman (1975: 161) and Reiser (1985: 219), for example, attribute to him the status of 'senior anaesthetist of the [Blegdams]hospital'. See Ibsen (1975).

10. Anesthesia was not always delivered by an anesthetist. Medical students and nurses administered anaesthesia too under the surgeon's responsibility and direction. From 1950 to 1973 Copenhagen hosted an international anesthesiological training center under auspices of the World Health Organization. Leading anesthesiologists from the USA and Britain taught trainees in the operating rooms of six of the largest hospitals in Copenhagen. The WHO-course contributed to the establishment of anesthesiology as a discipline in Denmark, but it was restricted to work in the operating room. (Ibsen 1975)

11. In a historical review of the 1952 polio-epidemic, Copenhagen anesthesiologist Ole Secher expressed Lassen's doubt as follows: 'What good could a young doctor from a discipline he hardly knew, tell old experienced epidemiologists, who had done all that was possible and even more, though they had no explanation of the cause of death? The hyperpyrexia was considered to be due to the cerebral affection per se. (Secher 1989)

12. In january 1950 Ibsen's wife and daughters returned home with a ship from New York while Ibsen stayed in the US to work in Beecher's anesthesia department. On board the same boat was Bjørneboe, thus learning from Ibsen's wife that Ibsen was in Boston to study anesthesia.

13. Conditions which favor the multiplication of the bacteria are, for example, lacerated wounds in which the tissue is deprived of its blood supply, necrotic tissue and effused blood, wounds containing foreign bodies etc.

14. Possibilities to measure $\mathrm{pH}$ of blood in a hospital's laboratory were limited. Methods available in research and industrial chemistry laboratories, using a large $\mathrm{pH}$-electrode, required a large quantity of blood and were of little value in hospital laboratories. Repeated $\mathrm{pH}$ measurements on large 
quantities of blood would render the patient anemic. Although Blegdamshospital was equipped with a pH meter, with 'usual' electrodes requiring a large quantity of blood, it was not routinely used to measure $\mathrm{pH}$ in patients during the first weeks of the polio epidemic in August 1952. See also section 4.12.

15. In the years after the 1952 polio epidemic it was pointed out that, curiously enough, in 1946 one of Blegdamshospital's physicians, Nielsen (1946) had published a paper reviewing Blegdamshospital's experiences with Sahlin's cuirass respirator in the treatment of polio patients, during a ten year period (1934 - 1944). 82 out of 110 patients died. In reviewing terminal stage laboratory (increased total $\mathrm{CO}_{2}$ in plasma) and autopsy findings Nielsen put forward the suggestion that death in these patients could have been due to an increase in blood carbon dioxide, due to hypoventilation, increased dead space and atelectasis, which in turn was due to aspiration due to an impaired swallowing reflex. If it was not the primary cause of death, it was at least a contributory cause. (Secher 1989; Astrup et al. 1989) However, this review did not result in a change in Blegdamshospital's treatment of polio patients. In their 1989 paper Astrup, Bjørneboe and Neukirch write that, in their opinion, most Danish physicians would have interpreted the high values for 'total $\mathrm{CO}_{2}$ in plasma' as a metabolic alkalosis. (Astrup et al. 1989)

16. In March 1952 professor Brinkman was guest lecturer at the WHO Anaesthesia Course in Copenhagen. Engell carried with him a prototype of the Carbovisor. Later, a commercial model was obtainable from Kipp, Delft, the Netherlands.

17. In clinical research with the Carbovisor, the respiratory air to be analyzed was obtained by continuous suction from the main bronchus or from a lower lobe bronchus through a catheter. The sample thus obtained never consisted only of expiratory air; the sample was always a mixture of inspiratory and expiratory air. Experiments on normal suhjects were performed to estimate the ratio of this mixture, and thus, of the ratio of between the $\mathrm{CO}_{2}$-concentration measured and the $\mathrm{CO}_{2}$ concentration of alveolar air.

18. Brinkman's Carbovisor was not the first device ever built to measure carbon dioxide in air. See for example Hesser (1949). Neither were Engell and Ibsen the first to report on the problem of acidosis in thoracic surgery. Priority on these matters is not the issue here.

19. The day after Ibsen's successful treatment of the twelve year old girl further evidence for his rendering of pathophysiological mechanisms was provided by the results of $\mathrm{pH}$ measurements in blood - showing acidosis - performed by laboratory physician Poul Astrup using a new pH-electrode which he had obtained from Radiometer Copenhagen. Measurements of $\mathrm{pH}$ in blood were subsequently used to monitor the adequateness of the manual positive pressure ventilation. See also section 4.12.

20. One of the children of one of the physicians, a six year old girl, living at Blegdamshospital's grounds, contracted what was considered to be incipient polio with high fever and meningismus. Fortunately no paralysis occurred. One nurse, working in the hospital, developed a paresis. which was later diagnosed to be 'hysterical'.

21. Fecal contamination was considered to be one of the possible ways of the virus' spreading. As a public policy measure lavatories on the local trains were closed. They were never opened again. Up to today the local trains in Copenhagen. $S$-tuget, do not have lavatories. Epidemiological research during the epidemic did not come up with a conclusive answer.

22. Of course, manual positive pressure ventilation earried the same risks. The students were instructed to be alert on any intra-pulmonary resistance to the manual ventilation, which could arise from atelectasis or secretions blocking airways (bronchi). Furthermore, blood samples for blood gas analysis and measurement of $\mathrm{pH}$ were taken regularly to monitor the adequacy of the manual ventilation by the students, and, if necessary, give instructions for adjustment. In manual positive pressure ventilation, however, it is the human ventilator who decides when to stop inspiration and allow expiration. 
23. In the corporate history of Bang \& Olufsen in Struer mechanical respirators attained only a marginal position. Being unprofitable Bang \& Olufsen terminated the production of respirators, giving the priority to the development and production of audio-visual equipment. (Rørbæk Madsen 1975)

24. Mushin (1980: 207 - 209) lists a number of ventilators developed 'as the winds of alarm at the approaching catastrophe fanned the embers of inventive genius'. 'In Sweden the Engström ventilator had appeared in 1951 and now production was expedited. In Denmark there appeared the Bang ventilator (1953), the Aga 'Pulmospirator', the Lundia (1955), and the Gullberg (1955). British ventilators designed for this purpose included the Beaver (1953), the 'Clevedon' modification (1953) of the Bang, the Radcliffe (1953), the Smith-Clarke (1955), the Radcliffe positive-negative model (1956), the Blease 'Pulmoflator' (1956) and the 'Barnet' (1958). In Germany the Dräger Company produced the 'Poliomat' (1955).

25. Medical students who had gained some skill in manual positive pressure ventilation where regularly called upon in subsequent years to (manually) ventilate patients in Copenhagen's hospitals.

26. See also Bjørk \& Engström (1955).

27. John Esben Kirk introduced Van Slyke's method in Denmark.

28. For the history of blood gas analysis, including the developments after 1952, see Severinghaus and Astrup (1987).

29. Ibsen had gained some credibility among municipal authorities, yet a large amount of money was not furnished without some floundering. Ibsen billed the city of Copenhagen for all the time he had invested in the manual ventilation of a tetanus patient in 1953 which extended for more than three weeks, a bill exceeding the required money for the intensive care unit by far. Eventually the municipality provided the money.

30. This intensive care was not the first in the world, but, again, priority is not an issue here. For a general history of the development of intensive care units see for example Hilberman (1975). 


\section{Death in the skull}

Re-negotiating the end of human life

\subsection{Introduction}

Today, the procedure of external cardiac massage by intermittent compression of the sternum is well-known and widely distributed, also outside professional medical circles. It appears in every first aid manual. Combined with artificial mouth-to-mouth or mouth-to-nose respiration cardiac massage has become part of the standard procedure in cardiopulmonary resuscitation, taught to medical students and laymen in first aid courses. Also well-known, although not applied by laymen, is the procedure of defibrillation in which, using two electrodes, an electrical current is applied across the patient's chest to restore the normal rhythm of the heart after cardiac arrest. The massive jerk passing through the patient's body when the current is applied is a familiar, though not very comforting, image for most of us. The clause 'closed-chest', to indicate that these procedures are performed with the chest of the patient closed, seems to be entirely superfluous. We should realize, however, that the 'closed-chest' variety of these procedures has been introduced only very recently. Closed-chest defibrillation in a small series of human patients was reported in the middle of the 1950 's by Zoll (1956) and his colleagues, whereas the now commonplace procedure of closed-chest cardiac massage was first described by Kouwenhoven in 1960. Prior to the employment of these techniques in man the only alternative for rapid intervention in emergency situations of cardiac and circulatory arrest was through 'open-chest' procedures requiring thoracotomy. ${ }^{1}$

During the 1950 s hospitals started to organize their cardiac resuscitation facilities. Boxes were assembled containing instruments for emergency thoracotomies, intubation, manual positive pressure ventilation, requisites for defibrillation, drugs and syringes, instructions for direct cardiac massage and for distribution of tasks among physicians, nurses and other personnel. These boxes were placed on every ward and in every department where cardiac arrests could be expected to occur. Residents were 
trained in the required skills. Emergency treatment could thus be initiated while expert help and equipment was called in. (Nauta 1959; Aleman 1962)

In hospitals, procedures and technologies for closed-chest cardiac resuscitation were quickly appropriated in these and other already existing sociotechnical ensembles like respiration centers and intensive care units. ${ }^{2}$ External cardiac massage and mouthto-mouth breathing were propagated by red cross and rescue organizations and incorporated in their training programs and manuals. Since the experimental studies comparing manual and 'expired air' methods, mouth-to-mouth breathing was considered to be the preferable method for 'rescue breathing'. (Elam et al. 1954; Safar 1958; Safar et al. 1958; Gordon et al. 1958; Elam et al. 1958; Elam et al. 1960; Nouhuys 1960)

Due to the uncertainty as to how long cardiac standstill or fibrillation persisted before external cardiac massage and artificial ventilation were initiated, efforts to save a patient's life could succeed in the sense that circulation, breathing and other physiological functions were restored, whereas they failed in restoring consciousness. ${ }^{3}$ These patients survived an episode of cardiac arrest, but they remained severely brain damaged. Dead brains in bodies with hearts still, or again, beating. Heart-beating patients with severe brain damage, secondary to ventricular fibrillation or head trauma, were not unknown in medicine. ${ }^{4}$ But, with the widespread use of external cardiac massage and mouth-to-mouth breathing, supplemented with closed-chest defibrillation and supportive drug therapy, their numbers increased during the 1960s and 1970 s.

In effectuating their power to artificially maintain vital bodily functions, cardiopulmonary resuscitation, artificial respiration and intensive care units constituted a trial of strength challenging the taken for granted character of one of the basic principles in medical work: the humanitarian obligation to save and preserve human life by all means. Respiration and intensive care units challenged the robustness with which death could be said to have occurred.

With regard to severely brain damaged, artificially ventilated and heart-beating patients under treatment in modern intensive care arrangements doubt arose whether life was still present, or whether death had already arrived. Traditional ways of concluding that death had occurred from the permanent cessation of the heart beat and of spontaneous respiration did not suffice anymore in patients in whom the process of dying had been arrested somewhere between its beginning and its completion.

Towards the end of the 1950 s physicians were looking for criteria to determine, in severely brain damaged, artificially ventilated patients, the moment after which further treatment could no longer be expected to be successful; to determine, in other words, the point of no return. In medical journals publications appeared proposing the dead brain, that is, the death of the organ brain, as this point of no return. French physicians coined the term coma dépassé to describe this state. (Mollaret and Goulon 1959; Mollaret et al. 1959; Bertrand et al. 1959) It was argued that when the brain had been irreversibly damaged, life supporting treatment should be withdrawn, after which the death of the patient would soon follow. These issues will be addressed in the first part of this chapter (sections 5.2 and 5.3).

In the course of the $1960 \mathrm{~s}$, following improvements in solid organ transplantation techniques (section 5.4), a shift occurred from brain death, as the death of the organ 
brain in a living (although dying) patient, towards brain death as the death of the patient. The dead brain came to mark the transition from a living human being to a corpse. Most western countries have replaced traditional heart-lung criteria of death with brain related criteria of death to be employed in the special circumstances of the intensive care unit when the 'heart beats in a body with a dead brain'.

In the public debate the shift from traditional heart-lung criteria of death to brain death has received most emphasis. A closer reading of the literature on this subject reveals, however, that in the debate at least three different concepts of brain death, with widely different consequences, have been carved out. A discussion of these different concepts of human death will comprise the major part of this chapter (sections 5.5 - 5.9).

In section 5.10, I will argue that the matter is not closed; the debate is still going on. Yet, in many countries with modern health care systems a practice of solid organ transplantation, involving the use of donor organs from artificially ventilated brain dead patients, has been established; against the backdrop of an ongoing debate. In section 5.11 an important leading question will be how a relatively unproblematic practice of 'declaring patients with dead brains dead' and using their organs for transplantation purposes has been established in a public environment of controversy concerning the end of human life.

As a starting point for the description and analysis let us consider the way in which physicians, at the end of the 1950s and the beginning of the 1960s, accounted (scientifically) for a dead brain in a living body.

\subsection{Dead brains}

\section{Reanimation}

By the end of the 1950s the term 'resuscitation' - 'reanimatie' in Dutch, 'réanimation' in French and 'Wiedererlebung' in German - was used to indicate the whole array of interventions aiming at reversal of medical catastrophes occurring in the course of severe diseases, or as a result of accidents: respiratory arrest, cardiac arrest, occasionally accompanied by severe vegetative or endocrine dysregulations; and, depending on the speed with which the catastrophe were counteracted: the consequences of transient hypoxemia of vital centers. Resuscitation comprised the application of artificial respiration in its widest sense, combined, if necessary with thoracotomy and direct (intra-thoracic) cardiac massage and/or defibrillation; also combined with the regulation of acid-base and water balance, sodium and potassium levels, hormonal substitution and support, if necessary combined with hibernation. The term 'resuscitation' indicated, in short, in various combinations, the most intensive treatment at the highest technical level the medical profession could master. (Kooyman $1959 ; 1960)$

The term 'reanimation' was also used to indicate the therapeutic goal of this intensive treatment. But 'reanimation' was not understood as the (mere) restoration of life in a patient who was at the brink of death or who, measured by traditional standards like the cessation of heartbeat and breathing, had died already. The 'restored' life resuscitation aimed at was often qualified in the sense that the intensive, 
technical medical interventions aimed at the restoration of a conscious natural life. (Kooyman 1959: 441)

Patients subjected to attempts at reanimation were not dead yet. Their prognosis was bad. They were dying. Death was imminent, sure. Death would inevitably follow if nothing was done, if the impending catastrophe could not be reversed. But these patients were not dead yet. They were not brought back from the other side.

Although resuscitation was attempted to restore a conscious natural life this therapeutic goal was not always achieved. An artificially prolonged deathbed might develop, in which suffering was aggravated by the respirator, sucking, infusions and measurements; a prolongation and intensification of suffering not accompanied by an increased chance at recovery. In 1959 Kooyman, neurologist in Rotterdam, put it as follows:

Already countless are the examples of patients with amyotrofic lateral sclerosis or an inoperable cerebral malignancy who become, because of respiratory arrest, connected to a respirator and stay shackled to it, sometimes for years, until no machine can stop death. (Kooyman 1959: 442)

\section{Clinical rounds}

How did they exactly look like, these patients who could not be restored to a conscious natural life? What symptoms did they display? Which signs were interpreted as human life still being present, even if endangered and comprised? How did, according to the physicians. in respiration centers and intensive care units, death display its identity and presence? Let us follow doctor Kramer on a (virtual) 1961 clinical round through his respiration center in Leiden, where he presents the case histories of four patients. (Kramer 1962)

The first patient, patient $\mathrm{A}$, is an eight year old girl, admitted to the hospital nine weeks previously after a street accident. Initially her condition seemed to be very grave being deeply unconscious and having extension spasms. Fifteen hours after the brain damage her respiration became superficial, as a result of which she had be tracheotomized and connected to a Poliomat. She was ventilated artificially during three days in which her consciousness returned. The first two weeks following the accident the patient had symptoms of a left hemiparesis, but no rest symptoms remain now. On the basis of the clinical picture and course, and on the changes in the electroencephalogram, the diagnosis was established as contusion of the brainstem.

The second patient, patient B, is sitting in a chair, an eighteen year old boy. $\mathrm{He}$ breathes spontaneously, but he can not talk and exhibits neurological regression phenomena, such as the palmomental reflex and the sucking reflex. He is severely demented and suffers from compulsive crying and laughing. He is tetraplegic as a result of which he can not walk nor eat without help. This young man suffered from an acute encephalomyelitis, probably caused by a viral infection. This patient would undoubtedly have died without modern reanimation methods, the administration of antibiotics, etc. They succeeded in prolonging his life, but not in making it more agreeable, because they did not succeed in bringing him back to a conscious and natural life. 
The third patient, patient $\mathrm{C}$ in the next room, is still connected to a respirator. $\mathrm{He}$ is unconscious. Left and right cornea reflexes are symmetrically present. The man demonstrates a typical posture with his arms flexed and his legs extended, a posture often seen in 'decorticated' people. This thirty six year old man has been artificially ventilated for the past three months because of a central respiratory arrest caused by skull damage. This patient's life has to be maintained artificially, but reanimation is out of the question, because the pathological brain process, a cerebral contusion, is irreversible.

Patient D is a nine year old, artificially ventilated girl. She is atonic. Her eyes are motionless, her eyelids half-closed. Her pupils are dilated and refractory to light. She is totally unresponsive to stimuli; all reflexes are absent. Tonelli's sign is positive. The skin has a livid color and most vegetative functions are absent. The electroencephalogram is electrically silent, even when electrodes are positioned in the depth of the brain's gray matter. Kramer concludes from the clinical picture and from the electro-encephalogram that, in this stage, the brain has died, while the heart still beats and the circulation of blood and nourishment to the tissues is more or less maintained. Pressure on the carotid sinus or the eyeball does not influence the pulse frequency. Intravenous injection of $1 \mathrm{mg}$ of atropine does not change the rhythm of the heart either, from which Kramer concludes that the heart beats automatically.

How did this girl get into this condition? She was going to be operated on a congenital heart defect. During anesthesia suddenly she stopped breathing and her heart arrested. Artificial mouth-to-mouth ventilation was immediately applied. Her chest was opened by thoracotomy and after four or five minutes direct cardiac massage the heart started beating again. The girl had to be ventilated artificially for several hours, but then spontaneous, although irregular, breathing recommenced. She remained in coma. A few days later breathing got worse. A tracheotomy was performed and a Poliomat connected. She did not regain consciousness. Pulse frequency varied considerably. Body temperature was high at one moment and low at the next. Blood pressure tended to decrease so that high doses noradrenaline had to be given. Thus, a first stage (desequilibration) dominated by the primary affection cardiopulmonary arrest and cerebral hypoxia - was followed by a second stage (disintegration) marked by a decline of brain functions, eventually resulting in a third and end stage which Kramer called: deanimation.

... the condition of the body without the brain working ... the brainless existence. (Kramer 1962: 638)

This deanimation stage can last days or weeks, until eventually the heart stops beating.

There was no doubt in Kramer's account that all four patients presented were alive. The case of the first patient is self evident. Treatment in Leiden's respiration center prolonged the life of the second patient, although he could no longer experience the fullness of a conscious and natural life. To indicate the irreversible clinical condition of the third patient Kramer used the term coma artificiale prolongatum. Coma is a condition, an affection of the living. It is not a condition of the dead. Experience learned, according to Kramer, that recovery in this case was out of the question. 
Such a 'prolongation vitae' ends with death, because in most cases respiration and heartbeat cease simultaneously. One calls this kind of dying 'total death', contrary to conditions in which the brain dies first and the heart ceases to beat later. (Kramer 1962: 638)

In the case of the fourth patient the brain died first, resulting in a central apnea necessitating continuous artificial ventilation, while the heart ceased to beat later. This girl died what Kramer called a dissociated death. The 'normal' close association in time between the cessation of brain function, respiration and heartbeat was artificially dissociated by medical interventions. The girl was not considered to be dead, however, until her heart stopped beating. The stage of deanimation was the last phase of her artificially prolonged agony, the last phase of her life. Kramer intended to arouse his audience's interest in

... the tragedy and the science of this artificially slackened way of dying. (Kramer 1962: 640)

All the way until the end Kramer recognized in these 'bodies' a living, although dying and beyond recovery, human being. In his opinion it was possible that the brain was dead while the patient was still alive.

\section{Intravital autolysis}

By 1961 both macroscopic and microscopic morphological characteristics of postmortal autolysis, that is the structural changes occurring in disintegrating brain tissue following 'total death', had been extensively studied. The application of artificial respiration and other life sustaining technologies in respiration centers and intensive care units confronted neurologists and pathologists with a new condition, a new form of encephalopathy: intravital autolysis of the brain. (Kramer 1962: 639) ${ }^{h}$

Kramer goes on to describe the appearance of the brains of deanimated patients. When taken out of the skull during (postmortal) obductions brains of deanimated patients are often tender and pappy. Sometimes they have the consistency of a soft egg. This 'liquefaction' can even occur while the heart still beats, as Kramer observed

... in two cases where brain tissue flowed from drill holes in the skull. (Kramer, 1962: 638)

The microscopic image of the brains of deanimated patients is, according to Kramer, the result of a combination of two distinct processes. First of all, there is the primary disease process or trauma that caused the clinical condition necessitating intensive therapy. To be distinguished from the primary process is a second chain of changes resulting from the therapy and apparently related to the premature death of the brain. The primary changes differ from case to case and are more or less localized. The secondary changes are similar in all cases and generalized.

When the procedure to prepare a slide for microscopic examination from the pappy brain tissue succeeds the disintegration and necrosis of the tissue is apparent. Blood vessels, however, can be, in view of the deplorable state of the nervous tissue, 
remarkably intact, containing red blood cells. The primary pathological process is often not retraceable in the microscopic slides, as if it has come to an end long before the cessation of the heartbeat, after which it has been superseded and erased by the secondary changes.

In Kramer's material the necrotic, autolytic secondary changes were most prominent in the proximal parts of the brain stem and in the cerebellum. Kramer pointed out that the areas of the brain in which the secondary process occurred did not coincide with the location of the primary process. They did not follow the course of the blood vessels either, where he would have expected them if these secondary changes were caused by lack of oxygen during an anoxemic episode. Kramer eliminated both lack of oxygen and the primary process as explanations for the occurrence and location of the secondary changes. What caused these nerve cells to die while the rest of the body survived?

In reviewing the case histories of the deanimated patients Kramer was struck by the large amount of noradrenalin deanimated patients received in an attempt to keep up the blood pressure. The dose of noradrenalin administered was several million times the physiological level. An important assumption in the rather abundant use of noradrenalin had been that there was a wide range between the therapeutic dose and the toxic level; that, in other words, noradrenalin was not toxic, not even in high concentrations. Kramer had to admit that this assumption was wrong. The therapeutic effect, that is the rise in blood pressure, depended not only on the concentration of noradrenalin in the blood, but also on the - gradually decreasing - reactivity of the blood vessels.

Kramer brought to mind that it was known from neurophysiological research that the hypothalamus and brainstem include structures containing catecholamines (adrenalin and noradrenalin). These substances were assumed to play an important role in the transmission of nerve impulses. In the brainstem noradrenalin is found in the reticular substance in an area known to have an activating effect on the whole cerebral cortex: Moruzzi and Magoun's 'activating system'.? It was also known that catecholamines produced in the adrenal glands and poured into the blood had a direct effect on adrenergic ${ }^{8}$ structures in the brain stem.

Kramer suggested the possibility that adrenergic nerve cells in the brainstem die as a result of an overdose of noradrenaline given during intensive treatment and artificial respiration:

... as a result of a chemical 'knock-out'. (Kramer 1962: 640)

This would, according to Kramer explain why necrosis is most prominent in proximal parts of the brain stem. It would also explain the vegetative atonia, that is the dysregulation of body temperature, blood pressure, etc. A chemical 'knock-out' of adrenergic structures in the hypothalamus would explain the endocrinological dysregulations often encountered. Kramer suggested further that through the hormonal intoxication of the 'activating system' the cerebral cortex would be knocked out too. In combination with other harmful conditions. like metabolic disturbances, the chemical knock-out could cause a more generalized death of nerve cells. The death of the nervous tissue would than be followed by an autolysis very similar to "postmortal 
autolysis', characterized by accumulation of lactic acid and enzymatic breakdown of proteins. The maintenance of some circulation carrying oxygenated blood to the brain, and of a more or less normal body temperature would accelerate the 'intravital autolysis' of the brain as compared to 'postmortal autolysis'.

Despite gradual morphologic differences between the two, eventually 'postmortal' and 'intravital' autolysis of the brain were, according to Kramer, identical. Yet, 'postmortal autolysis' of the brain was a condition of the dead, of the corpse. 'Intravital autolysis' of the brain was on the other hand a condition of the living. The boundary between 'postmortal' and 'intravital', between a 'living though dying patient' and a 'corpse', between 'life' and 'death' was marked by the occurrence of 'total death': in patients where brain functions had ceased at an earlier stage, 'total death' occurred when the heart stopped beating. The selective and 'premature' death of the brain was not equated with the death of the patient.

\subsection{Allowing to die? Withdrawal of life-support}

The human being was not pronounced dead until the heart had stopped to function. The boundary between life and death remained quite robust. ${ }^{9}$ There were no scientific problems in conceptualizing the death of a patient's brain or central nervous system as something distinct from the death of the patient. (Kramer 1962; 1966; Wertheimer et al. 1959) The inscriptions produced by the EEG-machine were mute. They did not say that the patient was dead, neither did the inscriptions produced by the doctor in writing down the results of his neurological examinations, nor the inscriptions produced by Radiometer's Astrup-apparatus in the laboratory. The legitimacy of the physicians as spokespersons for these inscriptions was not seriously challenged. According to most physicians heart-beating brain dead patients were still alive.

Patients with a dead brain whose bodies were maintained by artificial life support systems first and foremost challenged one of the basic principles in medical work: the humanitarian obligation to save and preserve human life by all means. ${ }^{10}$

\section{Questions to the Pope}

In 1957 the Austrian anesthetist Bruno Haid gave voice to the questions and dilemmas he and other physicians working in intensive care arrangements were confronted with. Being a catholic, and hoping for some guidance from the church, he addressed these issues in a letter to Pope Pius XII.

Haid put the following questions, concerning the problems arising in the modern practice of resuscitation, before Pope Pius XII:

First, does one have the right, or is one even under the obligation, to use modern artificial respiration equipment in all cases, even those which, in the doctor's judgement, are completely hopeless?

Second, does one have the right, or is one under the obligation, to remove the artificial respiration apparatus when, after several days, the state of deep unconsciousness does not improve if, when it is removed. blood circulation will stop within a few minutes? What must be done in this case if the family of the patient, who has 
already received the last sacraments, urges the doctor to remove the apparatus? Is Extreme Unction still valid at this time?

Third, must a patient plunged into unconsciousness through central paralysis, but whose life - that is to say, blood circulation - is maintained through artificial respiration, and in whom there is no improvement after several days, be considered "de facto" or even "de jure" dead? Must one not wait for blood circulation to stop, in spite of the artificial respiration, before considering him dead? ${ }^{\text {II }}$ (Pope Pius XII 1958: 395)

In answering these questions Pope Pius XII introduced a distinction between ordinary means and extraordinary means. A physician is not, in the pope's opinion, bound to use modern artificial respiration apparatus in all cases of deep unconsciousness, even in those that are considered to be completely hopeless in the opinion of the competent doctor, even against the will of the family. These forms of treatment go beyond the ordinary means to which he is bound. Hence, it cannot be held that there is an obligation to use them nor, consequently, that one is bound to give the doctor permission to use them. The family is, according to the pope, also bound to the use of only ordinary means. If it appears that the attempt at resuscitation constitutes in reality such a burden for the family that one cannot in all conscience impose it upon them. they can lawfully insist that the doctor should discontinue these attempts, and the doctor can lawfully comply. ${ }^{2}$ (Pope Pius XII 1958: 397)

\section{Four different positions}

Although the papal answers carried some legitimizing authority for catholic physicians, the postions adopted in different centers by various physicians varied considerably, also among catholic physicians. In practice, these postions were expressed in concrete decisions to stop or continue treatment in individual cases. They were also expressed in written form and published in various local, national and international medical journals. The various positions or treatment policies can be summarized as follows.

There were physicians who argued that they themselves, nor other physicians had the right ever to stop treatment. Respiratory arrest should always be treated with artificial respiration and, regardless of the prognosis, every complication should be treated too. As a rule one should ventilate until asystole: until the heart stops beating and attempts at cardiac resuscitation fail. Any compromise to this general rule would give room to the less conscientious and less scrupulous to find the solution of their own dilemma in the death of the patient and not in an improvement of therapy. In some religiously inspired formulations it was argued that it is not up to human beings to decide about life and death, to decide when the patient's cup of bitterness has been filled enough. It is not up to us to interfere in God's will and to meddle with the individual human being. By some, withdrawal of life-supporting treatment was equated with killing, which was prohibited by law.

There were also physicians who argued that as a rule each patient with respiratory paralysis should be connected to a respirator. When after some time and evaluation there was no hope at recovery of the patient, artificial respiration should not be continued for more than a couple of days. In a consultation attended by other physicians and the family it should then be decided to stop treatment and allow the 
patient to die. Diagnosis and prognosis should determine the action to be taken. One could even go one step further and argue that the patient under these circumstances should not only be allowed to die, but that physicians do not have the right to impose a prolonged, hard and hopeless death-bed only because the machines to forestall the arrival of death are available. De Lange, for example, considered the unwarranted and artificial prolongation of the death-bed to be the consequence of a modern technomaniacal attitude which refuses to accept death as the natural end of all things.

[A] perfectly normal and physiological death struggle corrupts into a degrading battle with needles, infusions, tubes, against a power none of us can match. (De Lange 1964: 119)

According to De Lange physicians had forgotten how to leave patients alone in their last moments. Too often the patient is inaccessible for his family in a bed surrounded by infusion bottles. urinals and a respirator.

A third group held that physicians had no right to stop treatment that had been commenced, but that they were not under the obligation to treat every emerging complication when the prognosis was bad. Technological interventions would in these cases be performed at the cost of the humaneness of the treatment. One runs the risk of doing harm by artificially extending the patient's agony in a situation where respect for the patient's right to an undisturbed death-bed would be in place. Kooyman (1959) warned against a one-sided technical approach. In stressing the physician's individual responsibility in these matters and the importance of the physician's own attitude to life and death, he pointed out that resuscitation requires from the physician both a hypertechnical and a deeply humane attitude, integrating both professional dedication and love for one's fellow men.

The fourth position held that the decision to stop treatment was valid when the patient's condition had deteriorated beyond some point of no return, but that this decision should not be taken solely on the basis of clinical judgement or statistical prognosis. In the opinion of Wertheimer et al. (1959), the point of no return, identified as the death of the brain, should be objectified, not only by clinical examinations but also by electro-encephalographic recordings employing electrodes introduced into the brain through drilling holes in the skull. When spontaneous respiration does not return and when the cause of the brain damage, clinical and prognostic signs, etc., point in the same direction, the deep electro-encephalographic recordings should be taken after one or two days.

\section{Arificial maintenance of the mute state}

The details of the pros and cons of the various positions do not have to concern us here. It is important to note, however, as De Lange (1964) pointed out, that in resuscitation physicians were confronted with patients who could no longer express their views on the treatment offered. As a consequence the physician had to act on his own initiative. Taking the patient's interest as a point of departure, the physician had to speak for these mute human beings. What did they want? If the patient could speak, or communicate his wishes in some other way'", would he agree to the treatment 
proposed and implemented, or would he refuse the treatment and prefer to be left alone to die?

It was not only in the context of resuscitation that physicians had to deal with mute patients who could not express their opinions about the treatment. It was not the first time either. Neither the irreversibility of the loss of consciousness nor the duration of the mute state as such were new. New was that with the increased use of artificial respiration, other life-supporting technologies, and intensive care arrangements, during the 1950 s and beginning of the $1960 \mathrm{~s}$, the mute state was artificially maintained in situations where all hope at recovery had gone. When the meaning of continuing therapeutic interventions can not be found in the (possibility of) recovery of the patient, the whole treatment soon turns meaningless. Physicians had the means to institute the treatment. And they had the power to withdraw treatment, as a consequence of which the patient would die: Why not use it? ${ }^{14}$

However much it was difficult to reach a decision and accept its consequences in individual cases, decisions were made, one way or another. None of these four positions or policies, not even the last one insisting that the death of the brain be objectified by deep electro-encephalographic recording ${ }^{15}$, required that the boundary between life and death be moved forward. It was not required that death be pronounced at an earlier stage in the process. The 'de facto' and 'de jure' declaration of death, the latter lifting the protection of the individual by the law, could take place when 'total death' had occurred. The point of no return was not necessarily to be equated with the moment of death itself. At the moment when the decision to abstain from further treatment of complications, or to withdraw life supporting technologies, death was imminent, but not present yet. The respirator was not shut down after but prior to the occurrence of death. This discussion about termination of treatment occurred on the living side of the life/death boundary, and, hence, it was a discussion about euthanasia, passive or active. ${ }^{16}$

\section{A fifth postion: moving the boundary}

In addition to the four positions discussed in the previous paragraphs, De Lange developed a fifth position, which differed from the others in that it moved the life/death boundary forward. In response to the dilemma posed by the artificial but hopeless maintenance of heart-beating but mindless patients De Lange proposed to equate the point of no return with the death of the patient.

It had long been recognized that organs taken out of the body of an organism could be kept alive and functioning for quite a long time. Accepting that organs could have a separate and independent life on their own meant that spontaneous or artificially maintained manifestations of organ functions as such did not necessarily represent the presence of the life of the organism, not even when organs considered to be 'vital', like the heart and the lungs, were concerned. Death might have occurred, death might be present, even when 'vital' organs exhibited 'vital' functions. In other words, the organism as a whole might have died while individual organs or sets of organs continued functioning. The organism as a whole died when the coordinator and integrator of the organs that constitute the whole organism was irreversibly destroyed. This was the line of reasoning De Lange followed when he wrote that: 
[W] hen the cerebrum as the coordinator of the functions of the body can no longer perform its function as a result of too great a damage of whatever nature, one has to regard the body as a collection of separately functioning organs which may, true enough, have mutual contact, but can no longer claim to represent a human. (De Lange 1964: 120)

Thus, in contemplating resuscitation problems one had, according to De Lange, to focus on the brain. Despite the insufficiency of medical knowledge and the fallibility of prognosis, the extent of the damage to the brain and the extent to which the brain can recover from its damages should determine a physician's actions.

There was, however, another reason to grant the brain a central position. This reason had to do with what De Lange considered to be the essential characteristic of human life as distinct from non-human organisms. The origination of an organism in gametes from human parents was in De Lange's view not essential. A more or less integrated functioning human body which originated in the fertilization of a human ovum by a human sperm can not claim to represent human life. The brain is also the seat of a function that qualifies an organism as human: conscious processing of information, that is: thinking. ${ }^{17}$

As long as there is a chance that consciousness will return there is no problem: the doctor has the duty to use all available means to keep the patient and his body alive. However, this obligation expires, according to De Lange, when the chance that consciousness will return is, humanly speaking, absent. Since, in the latter case, the patient is dead, treatment may be stopped. This holds not only for the respirator. It equally holds

... for artificial feeding, liquid administration, and automatic treatment of every infection in the irreversibly comatose patient. In case we are convinced that the coma will remain, let us then have the courage (also towards the nursing staff) to accept the consequences, also in the case of a spontaneous breathing patient, by stopping all artificial administrations. (De Lange 1964: 120)

De Lange's argument was one among several other attempts to find a way out of treatments that had been started on a legitimate basis, but had become meaningless as it became clear that the patient would never regain consciousness. But arguments of this type were bound to meet disagreement both from physicians, lawyers and the general public.

There was disagreement about the matter-of-factual question whether the clinical symptoms and electro-encephalographical tracings, that were interpreted as indicating the brain had died, were also signs of the presence of the death of the patient, of the human being. De Lange argued that, yes, the death of the patient as a human being coincided with the death of the patient's brain, even when the heart still beats spontaneously, the chest moves up and down in (artificially supported) respiration, and blood pressure and body temperature are within normal limits. Opponents argued that, no, despite the severe and irreversible brain damage, despite the death of the brain, the patient was still alive. The death of the patient as a human being could not be said 
to have arrived before the irreversible cessation of respiration and heart beat, that is total death.

\section{Undermining trust in medicine}

De Lange also exposed himself to the moral critique raised against the withdrawal of life-supporting treatment in cases of infaust prognosis. Physicians were obliged to preserve and prolong life wherever this was feasible and meaningful. Physicians were the unconditional protectors of life and the living body, the enemies of death, fighting until the end. They should continue their struggle for the patient's life even in situations were the disease had supremacy, how inconsistent this might seem to be, because this was the basis for public thrust in the medical profession. The moment one would grant physicians the right to refrain from treatment in certain cases, one would immediately be confronted with the question where this would lead to. It could happen that in cases where the patient could no longer express his views on the treatment, the physician would 'consult' relatives, and that, as a consequence, other considerations and interests than the patients own, would be going to determine the outcome.

Furthermore, if the law would grant physicians some latitude in deciding about the continuance of life-supporting treatment, it would probably not be the dutiful, competent and trustworthy medical elite that would make most use of it. It would also give leeway to and provide a legal legitimation for the decisions of less responsible physicians not to fight until the end. This implied the risk that physicians, through their insufficient actions or through their refraining from action, would be guilty of violating one of the most important provisions of professional disciplinary law prohibiting actions undermining public thrust in the medical profession. (Catz 1960)

According to this line of argument, physicians should not be allowed to speak for the patient. They should not be allowed to determine what would be in the patient's own interest. Physicians should be held to their duty, to which they have sworn, to respect, save and protect human life unconditionally. Pronouncing a patient dead prematurely would clearly violate this principle and was intolerable.

\section{At variance with the law}

De Lange's proposal to equate the death of a patient's brain with the death of patient departed from extant Dutch judicial practice in which always the heart and lung criteria had been used to determine the moment of death. Like in many other countries, Dutch criminal law provisions prohibiting murder, assistance to murder, manslaughter, killing on request and assistance to suicide, contained no definitions of notions like 'life', 'dead', 'corpse'. Judicial practice had firmly established the cessation of the heart beat and respiration as the end of human life in the sense of the law. In 1956, against the background of the development of respiration centers and intensive care units, some legal experts cautiously began to question the applicability of criminal law provisions 293 and 294 - penalizing killing on request and assistance to suicide - to refraining from further treatment or to the withdrawal of life-supporting treatment in cases where prognosis was infaust. (Enthoven 1988) It was argued that the progress in medicine and medical technologies necessitated a revision of these 19th century criminal law provisions, devised to protect human life in a time that was incognizant of the possibilities and consequences of future resuscitation technologies 
and intensive care arrangements. In search for a legal argument, Langemeyer, attorney-general of the Dutch State Council, even considered the possibility that a solution could be found in a legal revision of the concept of 'life'.

Maybe one could state that a condition in which the most important functions of life are no longer maintained by the central nervous system, but by mechanical means, is not covered by the concept 'life'. (Langemeyer 1960: 190)

Langemeyer admitted that he had been reluctant to defend such a position, because he was under the impression the medical sciences did call such a condition 'life'. According to Langemeyer, in the judicial system one should avoid to interpret a word in variance with the meaning it had in the science that was first of all competent for it. As he learned, however, that even some members of the medical community were prepared to defend the position that patients who suffered severe and irreversible brain damage should no longer be considered to be alive, Langemeyer indicated that he might be willing to include such an interpretation in a legal argument to the effect that stopping a reanimation effort was not covered by any of the provisions in the criminal law pertaining to criminal offenses against life. (Langemeyer 1960: 190)

\section{Praeter legem}

Despite these careful and cautious explorations by some medical and legal experts the medical and legal community remained divided on these issues. In the late 1950 s and early 1960 s none of the alternative interpretations acquired the status of recognized positive law. Statutory and case law remained unchanged. The cessation of respiration and heartbeat were the legal criteria of death still in force. Physicians withdrawing further treatment from brain damaged heart-beating artificially ventilated patients, in anticipation of their 'total death', ran the risk, in principle, of being prosecuted. There was no legal basis for such actions. The risk of being prosecuted was reduced, however, as the legal authorities adopted a policy of non-prosecution, recognizing the medical profession's own responsibility in dealing with the dilemmas raised by modern resuscitation and intensive care technologies and practices. Medical practice, in this respect, was allowed to develop praeter legem. This provided physicians with enough latitude in practice to stop meaningless medical treatment in irreversibly brain damaged patients in whom vital functions could only be maintained by artificial means for a relatively short period of time. Then, when 'total death' had arrived, physicians could seek consent for post-mortem examination of the corpse, either to establish a diagnosis-post-mortem, to verify the cause of death for scientific purposes, or as a check for the adequacy of medical treatment. In practice, therefore, the answer given to the question whether 'brain death' or 'total death' represented the death of the patient was of little practical consequence. Meaningless medical treatments could be terminated and, after the passage of some time, the death of the patient could be established unambiguously. De Lange's proposal to equate the death of the brain with the death of the patient was an interesting intellectual or philosophical question, but there was no practical need to push the issue very hard. Not until, at first incidentally but later more frequently and in more places, the developing practice of solid organ transplantation in man touched the practices of intensive, life-supporting treatment. 
Where these two practices met the patient with a irreversibly damaged, dead brain but who had not yet reached the point of total death, was transformed into a potential organ donor.

\subsection{Utility of the (heart-beating) corpse}

\section{Cardiac transplantation}

The first South African heart transplantation in man, performed in 1967 by Barnard in the Groote Schuur Hospital in Cape Town, stands out as the moment at which an imminent controversy went public. ${ }^{18}$ The heart transplantation received worldwide media coverage. Barnard appeared in television and live radio interviews and even two gramophone records, with a recorded discussion between Barnard and members of his team, were released and on sale to the public. (McMichael 1968; BMJ 1968) It was an epoch-making event.

This first heart transplantation, in which a heart from a human donor was used, elicited a variety of responses, some of which were at variance with each other. By some the operation was heralded as the beginning of a new epoch, a leap forward in transplantation surgery, a major advance in medicine. Barnard and his team were praised for their courage and technical skill. Others, however, criticized Barnard's first heart transplantation for at best being premature. Although progress had been made in histocompatibility testing ${ }^{19}$ and pharmacological immunomodulation ${ }^{20}$, knowledge in these areas was still incomplete. Important differences might exist between the kidneys and the heart, between graft behavior in animals and in man. It still was questionable whether experiences from human kidney transplantations and from cardiac transplantations in animals warranted the attempt of cardiac transplantation in man. Cardiac transplantation was at best palliative and no proof existed that any given allotransplant would function indefinitely. Such palliation should be weighed against other priorities, especially when account was taken of the very heavy burden that cardiac transplantation imposed on all departments of a hospital, and in view of a shortage of operating theaters and pressing waiting-lists for less controversial treatment. (Dempster et al. 1968) The distinction between proponents and opponents did not follow disciplinary boundaries, in the sense that the medical profession was in favor and that others were against. The opposition cut across professional and lay boundaries.

But the issue that was central in the discussion was the issue of the point of death; a question that transcended the particular case of Barnard's first heart transplantation and had implications for all future potential organ donors, if organ transplantation was going to become common practice.

At what point could or should the patient be considered to be dead? When and to what extent was one allowed to interfere in a patient's death process? At what point should one decide to stop resuscitation, to stop attempts to restore the patient to a normal and conscious life? These questions, that had already been raised by the development of resuscitation procedures and intensive care arrangements, became especially urgent when the patient was a potential donor of a vital organ. ${ }^{21}$ 


\section{The corpse as a useful object}

The alignment of a transplantation practice with resuscitation and intensive care practices transformed the identity, not only of the corpse in the traditional sense as referring to the bodily remains after the occurrence of total death, but also of the severely brain damaged, artificially ventilated, but heart-beating patient. Prior to the feasability of solid organ transplantation in man, medical interest in the corpse had been restricted to the possibility to establish a diagnosis-post-mortem, to verify the cause of death for scientific purposes, or as a check for the adequacy of medical treatment. In addition to this, there was also a need for corpses for anatomical research and teaching. But with the establishment of renal transplantation programs and with the South African demonstration of the feasibility of cardiac transplantation, the corpse had turned into a useful and, for certain new purposes, irreplaceable object. (Van Till 1970) In places where intensive care and transplantation practices met, the body of the severely brain damaged, artificially ventilated, but heart-beating, dying patient was transformed into an organ bank containing valuable and re-usable donor organs that could save, or add some time to the lives of one or several other patients badly in need of a transplantation.

The outcome of the transplantation, the survival and continued functioning of the graft, did not only depend on immunological donor-recipient matching and the availability of a pharmacological and adjuvant immunosuppressive therapy. Every graft, whether kidney, heart or liver, experienced a non-functional episode of less favorable, and potentially damaging, metabolic circumstances. In the case of Barnard's heart transplantation oxygen supply to the heart stopped when the respirator was switched off and the circulation of blood arrested when the heart arrested. This anoxic episode lasted while the heart was removed from the donor's body until the heart was restarted by means of an electrical current from a defibrillator in the recipient's body. In the mean time it was transported from one operating room to the adjacent room where the recipient's sick heart had been removed and vascular anastomoses were made.

The ability of the transplanted graft to resume and continue its function in the new body very much depended on the health of the organ prior to its explantation and on the extent of the damage that the organ contracted during ex- and implantation and during transport. Preservation methods, whether in situ or after removal of the organ, aimed at minimizing the damage incurred by these procedures: perfusion of the organ with oxygenated blood, cardiac resuscitation, a heart-lung machine, reduction of the organ's metabolic activity and oxygen requirements by means of cooling in situ with cold perfusion liquids, or after explantation on ice. (Calne 1963; Greep 1970))

But, everything else being equal, different organs also displayed a difference in tolerance to anoxic episodes. Kidneys tolerated most, hearts and livers less. Reasonable success rates could be achieved in renal transplantation using cadaver kidneys from 'total dead' donors. The usability of kidneys from total dead donors, in combination with the artificial kidney, which allowed the pre-operative 'working up' of the patient to the best achievable clinical condition and the return to dialysis in case of rejection, allowed the development of renal transplantation in a time when people were considered to be dead when their heart beat and respiration had stopped. 
In heart and liver transplantation circumstances were less favorable. The reduced quality of the transplanted organ due to damage incurred by ex- and implantation procedures and transport was considered to be an important factor in the disappointing results of cardiac transplantations enthusiastically attempted in the first couple of years after the first South African heart transplantation. For solid organ transplantation programs other than kidney, it would be paramount to achieve an optimal donor organ quality: organs from previously young healthy people who had not suffered an anoxic episode at all. It should not be necessary to wait for the cardio-pulmonary arrest before making the first incision in the explantation procedure. It should not be necessary to wait for the heart and the respiration to stop and death to be pronounced. It should be possible to remove organs for transplantation while still perfused with fully oxygenated blood, to remove the kidneys, the liver, and other organs if requested, before the heart arrested and artificial respiration was stopped. In the case of cardiac transplantation it should be possible to expose the beating, well perfused and well oxygenated heart: it should be up to the surgeon to decide exactly when and how cardiac arrest should be induced (for example by cooling the heart with ice water, a procedure which not only preserves the organ but which also induces cardiac arrest). ${ }^{22}$ In this way there would be no gap between the cardio-pulmonary arrest and the institution of organ preservation measures, thus yielding optimal quality donor organs.

\section{Re-locating human death}

The improvement of graft survival in the recipient depended on the continuance of life-supporting treatment in the donor: artificial respiration, circulation (by a spontaneously beating heart, by a paced heart, or by a extra-corporeal pump), blood pressure and temperature regulation, etc. But, it should not be life that was supported, because that would mean removing vital, unpaired organs from a living, although dying. human being: and that would amount to murder. Both for physicians who wanted to pursue the further development of solid organ transplantation, and for the community that wanted to benefit from these accomplishments in medicine, it was paramount to be able to pronounce the artificially ventilated, heart beating, irreversibly brain damaged patient lawfully dead. It was important to renegotiate the end of human life and to severe the traditional link between human death and the cessation of respiration and heartbeat. It was paramount to mobilize the fixed point of total death. to move the distinction between human life and death forward and redefine it with reference to the death of the brain.

But, as we will see in the next sections, the new 'location' of a new brain-related definition of death was not readily identifiable. Furthermore, there was also a force counter-acting this shift towards an earlier point of death for the benefit of potential recipients. Several authors expressed their primary concern to be with the potential donor, not with the potential recipient. Faced with the feasibility of solid organ transplantation and recognizing the potential conflict of interest between donor and recipient, they stressed the need for proper protection of the donor. This was to be accomplished through an unambiguous definition of death, through a reaffirmation of a person's right to an undisturbed deathbed and of a person's right to his own corpse. (Van Till 1970; 1974) Furthermore, the redefinition of the boundary between human life and death went, according to numerous commentators, far beyond the sphere of 
competence of the medical profession. In the ensuing debate about brain death it was commonplace to describe the analysis of the concept of death as a philosophical matter, whereas the development of diagnostic criteria, and of empirical tests to see whether the criteria have been met, was considered to be a task for medical expertise. The philosophical analysis should determine what it is that medical diagnostic criteria and tests are supposed to determine. A redefinition of death would have far-reaching and diverse consequences. It touched upon fundamental notions about what constitutes life, more particularly human life and human death. This was a matter of public policy, not of biomedical science alone.

\subsection{On definitions of death}

The development of intensive care arrangements, of cardio-pulmonary resuscitation procedures and of solid organ transplantation programs during the 1950s and 1960s tried traditional and stabilized ways of diagnosing death in human beings. The old way did not suffice anymore and was in need of revision. But as soon as the traditional, fixed boundary between human life and 'total' death destabilized and became mobile, the new problem that arose was where to fix it anew. The different proposals for a new location that were put forward can be grouped into four positions, three of which are brain-related. These different positions do not only propose a link between human death on the one hand, and the cessation of functional activity in specified places in the body on the other. They also try to define or trace boundaries between what is allowable and what is not; when and how somebody should be treated as a living person, and when somebody may be treated as a corpse, now and in the future. ${ }^{2.3}$ Hence, each position invokes different future medical practices. They contain, in other words, different programs of action. A proposal for a particular definition of death links (the destruction of) places in the geography of the body and central nervous system to what can and may be done in medicine and health care. Hence, definitions of death establish heterogenous associations of cognitive, moral, social and technical matters in an attempt to (re)shape medical practices. This is the sense in which the term 'definition' will be used in the following sections, rather than as pertaining to a statement expressing the essential nature of something. A definition comprises both an object definition and a strategy for handling that very object. The definitions and the strategies both imply each other. Neither has priority over the other.

My usage of the term definition, links to the notion of a rhetoric as it is used by Lyne (1990). According to Lyne a rhetoric is a discourse strategy spanning and organizing numerous discourses, and acting as a trajectory for discourses yet unorganized. Rhetorics span and organize discourses past, present and future. Rhetorics are discursive strategies bridging different discursive domains, making it easier to cross from, say, a discourse about biology to a discourse about politics. A rhetoric, according to Lyne, makes facts support judgments and actions. The radical separation of is's and ought's is at best sustained only as a logical point. In practical life, jumping over categories (when we are willing to make the jump) is assisted by rhetoric, which functions to make is's dovetail smoothly with ought's. 


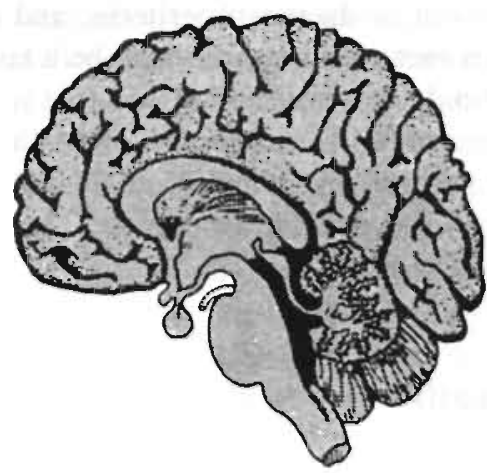

A
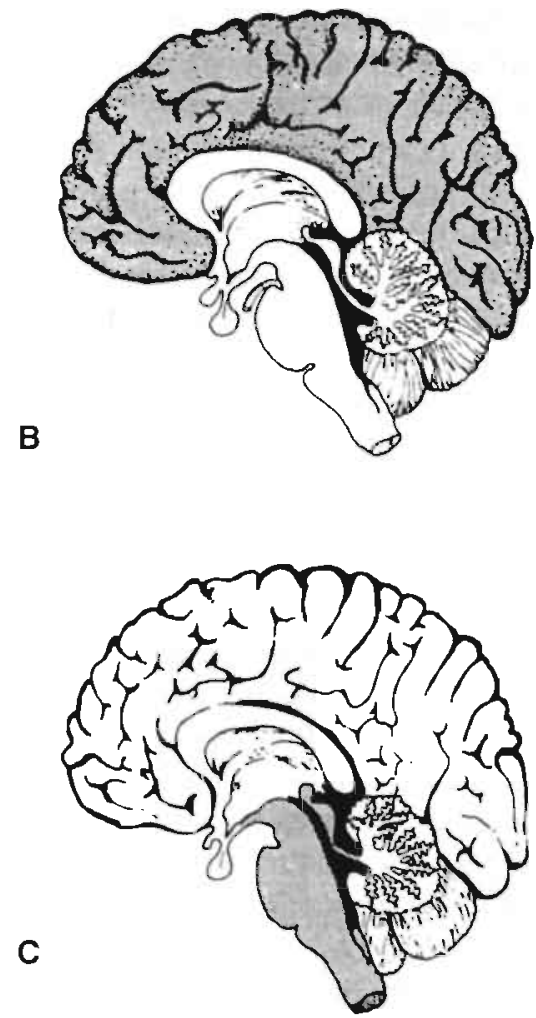

Figure 5.1

The gray area is the portion of the brain that, in the various definitions of brain death, has to be irreversibly destroyed in order to allow the pronouncement of death. A: brain stem death. B: whole brain death. C: neocortical death.

Reproduced from Pallis (1983:3) 
Hence, a definition is, in my understanding, a device drawing on a wide variety of heterogenous resources, encompassing definitions tracing boundaries, distributing ontological and moral status, linking divergent spaces together, spanning and mobilizing past, present and future discourses and programs of actions to gain strength and persuasiveness, in an attempt to shape future practices.

The four proposals for a new location of the boundary between human life and death that emerged in the debate yield as many definitions of death. They may be named after the critical topos in the body. The critical topos is the place in the body the irreversible destruction of which is considered to be sufficient ground to pronounce a person dead. Three out of these four definitions of death locate the critical topos inside the skull, in the brain: in the brainstem, in the whole brain and in the cerebral (neo)cortex respectively (see figure 5.1).

Proponents of these positions have all made the move from a notion of death in which the permanent cessation of cardiac and respiratory functions is central to a brain related notion of human death. They accept that it is conceivable that a brain damaged, artificially ventilated patient with a spontaneously beating heart, warm skin, normal body temperature and blood pressure, nevertheless, is dead. For them the traditional metonymical relationship ${ }^{24}$ between the beating heart and human life is no longer. tenable. However, they differ with respect to what part of the brain should be considered so essential that its irreversible destruction is sufficient to declare death over the remaining body, irrespective of any non-essential rest functions the body, which is now a corpse, might display.

In the following sections I will try to show who speaks for whom or what, how various spokespersons interpret mute inscriptions recorded by machines, what different lines of arguments accomplish, what they exclude or allow, what boundaries they trace, how they distribute ontological and moral status and legal protection, etc. I will try to identify the various and heterogeneous resources they draw on: neurophysiological science, epidemiology and statistics, the establishment of conclusive proof in legal practices, logic, thought experiments, brain imaging techniques. I will, in my text, reproduce examples of pictures, tables and graphs plus legends, used by actors, participants in the debate on the redefinition of death, in their texts in support of their line of argument.

I do not think of these definitions of death and the medical and legal practices they invoke in terms of the traditional opposition between theory and practice. Designing and building a definition is work. Writing and arguing, mobilizing a variety of resources to build a strong and persuasive argument is a kind of practice in itself: a practice of attempts to reshape extant medical and legal practices. Although I will focus in the following sections on the content of the various definitions, it should be borne in mind that most of the actors participating in the debate are also engaged in other kinds of work having to do with re-defining human death, as teachers, as physicians, as lawyers, as members of institutional review or governmental advisory boards, etc. I will return to this kind of work in section 5.11 when I address the question of how, against the backdrop of an ongoing controversy concerning the end of human life, a relatively unproblematic transplantation practice has been established, using donor organs from brain dead people. 
Before turning to the definitions of brain death I will first, briefly, re-describe the 'traditional' definition of 'total death'.

\subsection{Definition of total death}

\section{Integrated functioning}

A definition of total death is a line of argument that holds that death should not be pronounced before the organism as an integrated whole has ceased to exist. ${ }^{25}$ The critical topos is not located in one or other single organ, but it is distributed throughout the whole organism. Integrated functioning of the human being as a biological organism is the key issue here. Through the integrated functioning of a complex system of neural, hormonal and chemical control and feedback mechanism the organism maintains homeostasis, a steady state in its internal environment. This is what human beings share with all living organisms. This is what constitutes life. Death as the absence of life has arrived when integration has been irreversibly lost.

The destruction of one organ or organ system may jeopardize integrated functioning but is in itself not sufficient to be equated with death. Loss of kidney function is lethal when left untreated. However, an artificial kidney or a donor kidney may substitute for the lost kidney function and restore homeostasis. Similarly, the loss of the capacity of the pancreas' Langerhans' islands to produce insulin jeopardizes integrated functioning. Substitution therapy with insulin derived from other animals or produced by genetically manipulated bacteria restores integrated functioning. A pacemaker substitutes for deficiencies in the heart's own impulse generating and conducting tissues. A mechanical respirator replaces the work of the neuro-muscular system that normally controls and performs respiratory movements. A heart-lung machine takes over the functions of the heart and lungs completely.

In all these cases integrated functioning is maintained artificially, sometimes temporarily, sometimes permanently. The artificialness of the support is not essential. In none of these cases patients would be considered to be dead. ${ }^{26}$ Consciousness or, what is generally called, higher brain functions, are important features in human beings. However, the loss of these functions as a result of for example extensive damage to the cerebral cortex does not impede continued integrated functioning when the brain stem is still intact. These are the patients in a persistent vegetative state, who may, when nursed properly, remain (alive) in this condition for decades. ${ }^{27}$

There is no principle reason why an exception should be made for the brainstem. It is true that at present no artificial means to substitute for a loss of brainstem function is available. But it is conceivable. according to Green and Wikler, that at some point in the future an artificial substitute for the brainstem will be feasible.

[The brainstem] is still one among many organs, and, like other organs, could conceivably be replaced by an artificial aid which performed its function. The respirators and other life-supports which maintain body functioning after lower brain death collectively constitute a sort of artificial lower brain, and development of a more perfect mechanical substitute is merely a technological problem. (Green and Wikler 1981: 57) 
Patients with a severely damaged brainstem have a very bad prognosis. They will die very soon. But they are not dead yet, no less than patients on chronic dialysis or on permanent artificial ventilation due to neurological damage caused by a bulbar polioinfection; not as long as some integrated functioning is maintained by means of artificial respiration and other intensive care therapies. ${ }^{28}$ They will suffer from cardiac arrest. It may be possible to restart the heart a few times, but eventually cardiac resuscitation will fail too. Statistics show that this point will be reached within 48 or 72 hours, within a week at most. It is only at this point that integrated functioning of the organism has been lost and that the patient is dead. ${ }^{29}$

Drawing on scientific knowledge from modern biology and physiology a definition of total death argues that the boundary between life and death should remain where it has always been and that it should not be moved forward. Brain damaged, artificially ventilated patients are alive. Their hearts beat, their lungs exchange oxygen and carbon dioxide between alveolar air and circulating blood, their pancreases produce insulin and their tissues metabolize glucose, their body temperatures are within normal ranges and so are their blood pressures. And even if all of these functions are artificially supported, they are part of an organism that still exhibits integrated functioning. Hence, these functions are traces of integrated life still present in the body, and the life supporting technologies do indeed support life. Higher brain functions may be impaired and lost, the EEG may be flat. But impaired brain functions or a flat EEG are no expression of the arrival of death in the organism as a whole.

\section{Protected by the law}

By defining brain damaged, artificially ventilated, heart-beating patients as alive, they are tied to the provisions in a country's statutory law which provides them with the protection the law offers to living people. In principle, actions that bring about death in living people are punishable according to criminal law. This includes medical actions like the extirpation of organs for transplantation purposes.

A definition of total death does not necessarily exclude euthanasia with respect to these patients. It only defines the withdrawal of life supporting treatment as an act of euthanasia, to which a community's provisions and regulations to permit or prohibit euthanasia apply. A brain related definition of death would obscure the real nature of such an intervention in a patient's dying process. A medical redefinition of death, supported by the law, would be to easy a way out of a difficult moral dilemma. The problem is a moral one, and we should deal with it as a moral problem and not redefine it as a medical problem of diagnosing death.

A definition of total death does not preclude the development of a transplantation practice either. However, by defining the artificially ventilated, heart-beating but brain dead organism as being alive it precludes, in principle, the use of these patients as a source of donor organs. If that limits the availability of suitable donor organs for certain kinds of solid organ transplantations, than that is too bad. But, nobody, neither the community nor other patients who need a transplantation, can exert a right to these organs at the cost of the donor.

If a community, nevertheless, would like to create the possibility for organs to be removed for transplantation purposes prior to the irreversible cessation of the 
circulation it would be conceivable that exclusively for these special circumstances appropriate provisions are made in the law. According to the Canadian neurosurgeon Dwight Parkinson, physicians would have to explain to the patient's family

... that everything possible has been done ... that the patient is past the point of no return, being kept alive by artificial means and that they would like to keep life going until the organs can be harvested, after which, the support would be removed, and death would occur and be declared very shortly when the patient's circulation ceased. (Parkinson 1985: 90) ${ }^{30}$

There will, according to Parkinson, always be discussion amongst the medical profession as to just how much of the brain needs to be damaged for the patient to be past the point of no return. Often at autopsy, in patients declared brain death, microscopically large portions of the brain show no signs of earlier death, hence, the term at best would be 'partial' brain death. The creation of a new category of death termed 'brain death' is obfuscating. It all comes down to the inability to keep the individual alive except by artificial means. It is not necessary to redefine death by law. The only change in the law that has to be made is to the effect that it, in special circumstances, permits 'to remove organs earlier if past this point of no return'.

The law should continue to protect the dying, potential organ donor as it protects all dying patients as it protects all living people. Any exceptions to this general rule should be specifically and carefully defined, not by redefining death, but as permissible interventions in the final stage of a patient's life. This would also offer the best protection to the potential organ donor. The acceptance of a brain related definition of death, and its legitimation in the law, would be the first step on a slippery slope to which next patients in a persistent vegetative state, patients suffering from severe dementia and mentally handicapped fall victim.

\subsection{Definition of brainstem death}

\section{The brain as the critical system}

A definition of brainstem death argues that it is legitimate to equate brain death with death, that the necessary and sufficient component of brain death is death of the brain stem, and that a dead brain stem can be reliably diagnosed at the bedside. In contrast to the line of argument in a definition of total death, in a definition of brain death it is argued that death may be equated with the irreversible destruction of the critical system of that organism. The critical system is that system which is irreplaceable by an artifice, be it biological, chemical, or electromechanical. (Korein 1978) In man virtually all organ systems are replaceable, with one exception, however, and that is the brain. The heart can be replaced by a pump, the kidneys by a dialysis unit, endocrine glands by replacement therapy. When, according to Korein, it comes to the neuronal cells that comprise the central nervous system, an individual is born with a fixed number of cells that do not reproduce. A neuron may grow by increasing its dendritic tree and interconnections with other neurons, but if the body of the cell, the 
soma, is destroyed this is a irreversible process. The brain depends on the neurons for its function, and the organism depends on the brain.

If the brain is irreversibly destroyed, the critical system is destroyed, and despite all other systems being maintained by any manner whatsoever, the organism as an individual functioning entity no longer exists. If the critical system, i.e., the brain, in a man is destroyed, the human organism is no longer in a state of minimal entropy production; its state will progressively become more disorganized by spontaneous irreversible fluctuations. Therefore it will never return to its initial state as a sentient human being. The time course may be prolonged by artificial means, depending on the adequacy of functioning subsidiary control, but the outcome of dissolution of the system is just as certain as that resulting from irreversible cardiac arrest. (Korein 1978: 26 - 27)

\section{Vital mimicry}

When the brain is irreversibly destroyed a point (of no return) has been reached where the various subsystems lack neurological integration and, according to Lamb

... their continued (artificial) functioning only mimics integrated life. (Lamb 1985:

37; my emphasis)

The beating heart, the respiratory movements of the chest, the warm skin, etc., of a patient with a dead brain, are no longer to be interpreted as being expressions of human life present in the body. They only mimic life. It is a kind of apparent life. Actually, the patient is already dead.

That structural disintegration of the body follows brain death, is, according to Lamb, not a contingent matter; it is a necessary consequence of the death of the critical system. The death of the brain is the point beyond which other systems cannot survive, with or without mechanical support. The brain's role as integrator, regulator and co-ordinator determines as much its critical centrality in the organism, as does its irreplaceability. (Lamb 1985: 37)

\section{The brainstem as the (doubly) critical system of the brain}

In a definition of brainstem death the critical topos is located even more strictly. in the 'lower brain', or brainstem. It is not the irreversible destruction of the whole brain, but the irreversible destruction of the brain as a whole that is critical. Thus in a brainstem definition of death it is not required that all neurons inside the skull, above the foramen magnum, are destroyed. The critical system of the organism itself has a critical system, the irreversible destruction of which amounts to the death of the brain. This critical system of the critical system, this critical mass of neurons without which the brain cannot function as a whole. is the brainstem.

The centrality of the brainstem derives from two different sources. Extensive neuro- and electrophysiological research has demonstrated the brainstem and adjacent portions in the midbrain and hypothalamus to be essential for the maintenance and integration of many autonomic, vegetative functions. It is essential for spontaneous breathing, for a spontaneous heartbeat, for body temperature regulation and blood pressure control. However. the brainstem also contains Moruzzi and Magoun's 
ascending reticular activating system (ARAS) ${ }^{3 !}$ which is responsible for arousal (as distinct from the content of consciousness). (Moruzzi and Magoun 1949; Moruzzi 1954; Magoun 1954; Magoun 1963) The ARAS is responsible for the activation or 'switching on' of the cerebral hemispheres. Unless there is a functioning brainstem arousing the cerebral cortex one cannot speak of a content of consciousness. The brainstem carries the very capacity for consciousness. ${ }^{32}$

\section{Signals of residual biological activity of the brain}

In so far as the brain cannot function as a whole without a functioning brainstem it follows that, once reliable criteria for loss of brainstem function have been met, the patient can be diagnosed dead. When a patient is pronounced dead according to these criteria it is very well possible that in other areas of the brain there is still some functional nerve tissue left.

An acute, massive, and irreversible brain stem lesion (primary or secondary) prevents meaningful functioning of the 'brain as a whole', even if isolated parts of the brain may, for a short while still emit signals of biological activity. (Pallis 1983:

8)

In other words, transient residual signs of electrical activity in isolated neuronal aggregates in the higher regions of the brain do not indicate persistent functioning of the organism as a whole or even of the brain as a whole. (Lamb 1985) These "signals of biological activity' emitted by isolated parts of the brain, these 'transient residual signs of electrical activity in isolated neuronal aggregates in the higher regions of the brain' may be 'visible' in EEG-recordings. If they do they should not be interpreted as saying that the patient is still living and not yet dead. They are also part of the mimicry of apparent life. EEG-recordings are of little use in diagnosing a dead brain stem, and hence, in diagnosing the death of a human being. If the death of the brainstem is accepted as the death of the patient, electrocerebral silence, that is a flat EEG, cannot be a requirement.

Except for this conceptual argument, electrocerebral silence is not acceptable as a criterion for death for technical reasons.

[A]n intensive care unit is about the most hostile environment imaginable for trying to record 'electrocerebral silence'. Many electroencephalograms in these circumstances show multiple artefacts which may be bizarre and difficult to identify and locate. If the nurse is wearing nylon underwear static electricity may generate false signals. Electromagnetic disturbances from calls on the Tannoy system may also generate confusing information. (Pallis 1983: 30)

When these artefacts are not properly recognized as false signals it might happen that artificial ventilation and intensive treatment is continued in a patient who is already dead. 


\section{Diagnosing a dead brainstem: preconditions and exclusions}

In a definition of brain stem death, the only signs that tell us that death has arrived are the signs that tell us that the brainstem is irreversibly and irremediably destroyed. On the other hand, there are also readily observable signs that say that the patient's brain stem cannot possibly be dead, because they are always associated with retention of one or more brain stem reflexes. According to Pallis,

[A] seizure, generalised or focal, implies the passage of nervous impulses through the brain stem and therefore proves that this part of the nervous system is still viable. Abnormal postures - either 'decorticate' (with flexed forearms and extended legs) or 'decerebrate' (with extended and hyperpronated forearms and extended legs) - likewise imply live neurons in the brain stem. (Pallis 1983: 14)

In these conditions a diagnosis of brain stem death should not even be considered. The diagnosis of brain stem death should not be considered before two essential preconditions have been met and before some necessary exclusions have been made.

The first essential precondition is that the patient should be in apnoeic coma. Coma is used here not in the wide and 'sloppy' sense of 'deeply unconscious', but in the strict sense denoting a state of sleep-like unresponsiveness from which the patient cannot be aroused. Unlike patients in a persistent vegetative state (PVS), patients in a coma do not exhibit spontaneous eye-opening, nor do they have a spontaneous waking-sleeping cycle. PVS-patients have 'arousal', indicating that their ARAS is viable. Just like a seizure or an abnormal posture, 'arousal' should warn the physician that the patient's brainstem cannot possibly be dead, that at least some parts of the brainstem are still viable and that a diagnosis of brain stem death should not be considered. Apnoeic means that the patient should not display spontaneous respiratory movements. Even if they are insufficient, spontaneous respiratory movements indicate that parts of the brainstem are still viable. According to Pallis (1983) it is 'selfevident' that the patient should be on a respirator. When no artificial ventilation has been provided for some time to a patient who is apnoeic, the patient will have proceeded to the stage of 'total death'. His brain stem will still be dead, but making a diagnosis of brain stem death has become meaningless. On the one hand, the body's organs have suffered an anoxic episode which makes them worthless for transplantation purposes. Or, on the other hand, there is no intensive. extraordinary life supporting treatment that could be terminated. A diagnosis of brain stem death is only meaningful prior to the arrival of total death, in situations where the interval between the death of the brain stem and total death is artificially prolonged by the institution of intensive, life supporting treatment.

The second essential precondition that has to be met before a diagnosis of brain stem death should be considered is that the condition is caused by a irremediable structural brain damage due to a disorder which can lead to brain death. In other words, a positive diagnosis concerning the cause of the apnoeic coma has to be made before proceeding with the tests to establish that the brain stem is dead. Drug intoxications, metabolic and endocrine disorders, and hypothermia may cause apnoeic coma, but they are potentially remediable (reversible) and not structural. A sub- or epidural hematoma, that is a collection of blood between the bone of the skull and the 
brain which compresses the brain and results in a herniation though the tentorium and foramen magnum, can cause apnoeic coma, but is remediable. Thus, patients suffering from these conditions do not meet the second essential precondition and a diagnosis of brain stem death should not be considered here. Gross destruction of the brain after severe head trauma, and massive intracerebral bleeding are irremediable and structural. ${ }^{33}$

The necessary exclusions to be made, before proceeding with the tests for brain stem death, pertain to potentially remediable, non-structural conditions that can cause
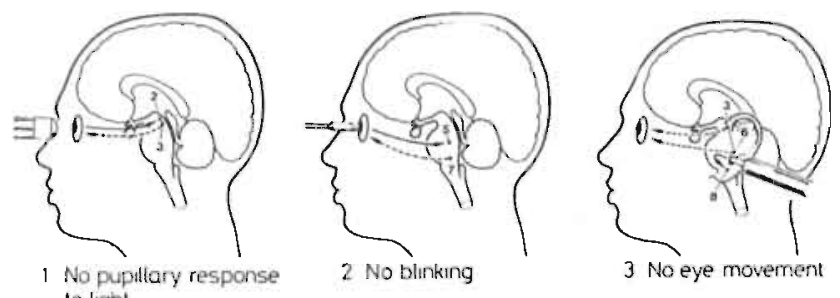

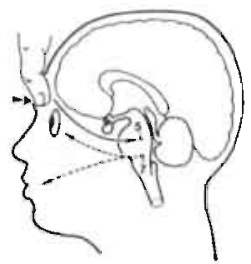

4. No grimacing

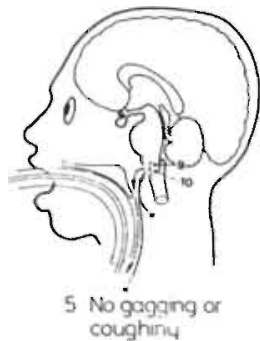

coughiny

Figure 5.2

Testing the five brain stem reflexes.

Reproduced from Pallis (1983: 15)

apnoeic coma in an acute stage and thus mimic irremediable, structural brain stem damage: hypothermia. drug intoxications including alcohol, and metabolic and endocrine disturbance. ${ }^{34}$

\section{Diagnosing a dead brainstem: brainstem reflexes}

Only when the essential preconditions are met and the necessary exclusions have been made is it permissible to proceed with the tests to demonstrate that the brain stem is dead. The anatomical topography of the brain stem allows its viability to be tested in a simple, quick and reliable way, by testing for the presence or absence of a number of brain stem reflexes (see figure 5.2).

Testing the brain stem reflexes enables the functional integrity of the brain stem to be probed in a unique way. ... This is fortunate because the concept of death proposed ... implies that all that is meaningful to human life depends on the 
integrity of these few cubic centimeters of tissue. Tests for brain stem reflexes ... yield vivid and unambiguous results. They look for the presence or absence of responses and not for gradations of function. They are simple to perform and capable of interpretation by any well trained doctor or nurse. ... They do not depend on elaborate machinery, on the vagaries of maintenance, or on the presence - around the clock - of superspecialists. Their basis is easy to convey to both relatives and lay people. Finally, they provide a battery of tests, so that the determination of death does not rely exclusively on a single procedure or on the assessment of a single function. (Pallis 1983: 15)

The five brain stem reflexes that should be systematically tested all involve an 'afferent' stimulus and an 'efferent' response along the cranial nerves (see figure 5.2): 1) contraction of the pupil in response to a bright light in the eye; 2) blinking of the eyelids in response to a strong stimulus to the cornea; 3 ) movement of the eyes in response to injection of cold water into the (clear) external auditory canal; 4) grimacing in response to a painful stimulus (firm supraorbital pressure); and 5) gagging and coughing in response to a irritating stimulus in the throat. Viable tissue at different levels of the brain stem is required to translate the stimulus in a response. These five brain stem reflexes must be absent before the diagnosis brain stem death can be made.

In addition to these five brain stem reflexes the physician may attempt another one. He may test for the presence of 'dolling' (the oculocephalic reflex, or 'doll's

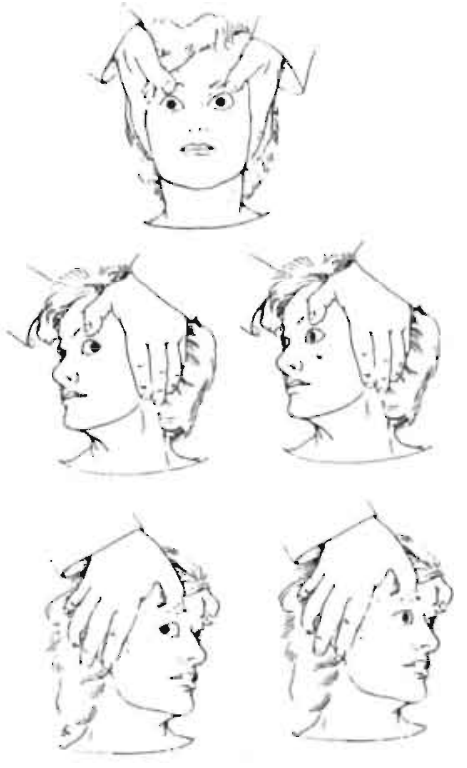

Figure 5.3

Testing for the presence of 'dolling'.

In a fully alert individual the eyes will, within a fraction of a second, orient with the head. In the cadaver the head and eyes will likewise move together.

Reproduced from Pallis (1983: 14) 
head eye phenomenon'; see figure 5.3). Standing at the top of the bed, the physician holds the patient's head between his hand while he raises the eyelids with his thumbs. He then rotates the head first to one side (where it is kept for three or four seconds), and then right over to the other side. In the mean time he keeps a close watch on what happens to the eyes. When 'dolling' is present the patient is not brain stem dead and there is no need to proceed with further tests.

\section{Testing for apnoea}

The final test that must be performed to establish a diagnosis of brain stem death is the test for apnoea, that is to test for the absence of spontaneous respiration - in a patient who is artificially ventilated and in a critical condition. A test for apnoea involves the disconnection of the patient from the respirator for 10 minutes. But disconnection in itself is not sufficient and precautions have to be taken that it does not damage the brain.

It has been established decades ago that it is the lack of oxygen that damages the nervous tissue of the brain, but that it is the rising arterial carbon dioxide tension $\left(\mathrm{PCO}_{2}\right)$ that 'drives' the respiratory center in the lower brain. What is needed during the 'disconnection test' is a high arterial oxygen tension $\left(\mathrm{PO}_{2}\right)$, sufficient to prevent anoxic brain damage during 10 minutes, and a $\mathrm{PCO}_{2}$ level that is adequate to 'drive' the respiratory center, if it is still viable, to respiratory movements.

A high $\mathrm{PO}_{2}$ can be accomplished by 'pre-oxygenating' the patient with $100 \%$ oxygen during 10 minutes prior to the disconnection procedure. This will saturate his tissues with oxygen and build up a $\mathrm{PO}_{2}$ that is sufficient for 10 minutes of apnoea. Furthermore, a sufficient level is maintained by means of 'diffusion oxygenation': pure oxygen that is delivered down the trachea by a catheter.

Patients on a respirator tend to be overventilated, with $\mathrm{PCO}_{2}$ levels below normal. To obtain a sufficient high level of $\mathrm{PCO}_{2}$ within 10 minutes of disconnection the patient should not be hypocapnic at the time of disconnection. To achieve this the rate of the respirator may be reduced, or the patient could be given carbon dioxide in the gas mixture he breathes during the last five minutes prior to disconnection, for example, $5 \% \mathrm{CO}_{2}$ in $95 \% \mathrm{O}_{2}$. (Milhaud et al. 1978; Pallis 1983) If one of these preparatory measures is taken the $\mathrm{PCO}_{2}$ level will rise sufficiently. The actual $\mathrm{PCO}_{2}$ level reached can and should be recorded and documented. When the patient does not respond with an effort to breathe spontaneously the respiratory center can be considered to be dead.

\section{Epidemiological statistics: zero survivors}

Strictly speaking, what these diagnostic procedures establish is the death of the brain stem. A definition of brainstem death argues that the brainstem is the critical mass of neurons in the organism's critical system, and that its irremediable destruction may legitimately be equated with the death of the patient. A definition of brain stem death strongly relies on this conceptual line of argument, but not exclusively. Pallis also draws on epidemiological statistics, not only to provide a persuasive empirical basis for this claim, but also as a defence against (media) reports about alleged survivors of brain stem death. Pallis must claim that no patient who met the UK-criteria for brainstem death ever recovered, that asystole (irreversible cardiac arrest) develops 
Non-drugged parients diagnosed as brain dead by clinical criteria and maintained on ventilator

\begin{tabular}{|c|c|c|c|c|}
\hline Series & Year & $\begin{array}{l}\text { Patients } \\
\text { accepted }\end{array}$ & $\begin{array}{l}\text { Silent EEGs/ } \\
\text { EEGs obtained }\end{array}$ & Survivors \\
\hline Lindgren $e t a l^{7}$ & 1968 & 35 & $27 / 35$ & 0 \\
\hline Becker et al & 1970 & 15 & $6 /$ ?* & 0 \\
\hline Gaches et $a l^{2}$ & 1970 & 69 & $2 / 69$ & 0 \\
\hline Mohandas and Chou ${ }^{3}$ & 1971 & 25 & $3 / 9$ & 0 \\
\hline Ingvar and Widen ${ }^{9}$ & 1972 & 26 & $26 / 26$ & 0 \\
\hline Ouaknine ${ }^{10}$ & 1975 & 42 & $32 / 32$ & 0 \\
\hline Walker and Molinari" & 1975 & 141 & $130 / 141$ & 0 \\
\hline Rappaport $e t a l^{12}$ & 1978 & 3 & $0 / 1$ & 0 \\
\hline Ayim and Clark ${ }^{13}$ & 1979 & 30 & $3 / 10$ & 0 \\
\hline Hicks and Torda's & 1979 & 21 & $18 / 21$ & 0 \\
\hline \multicolumn{5}{|l|}{ Ashwal and } \\
\hline Schneider ${ }^{15}$ & 1979 & 5 & $0 / 5$ & 0 \\
\hline Kaste et $a l^{16}$ & 1979 & 12 & $? / 8^{*}$ & 0 \\
\hline Powner and Fromm ${ }^{17}$ & 1979 & 182 & $164 / 182$ & 0 \\
\hline Jennett et alj & 1981 & 326 & $67 / 70$ & 0 \\
\hline Jofrgensen $^{18}$ & 1981 & 70 & Not stated* & 0 \\
\hline \multirow[t]{2}{*}{ Caronna and Plum ${ }^{1 \theta}$} & 1981 & 34 & $1 / 10$ & 0 \\
\hline & & 1036 & $473 / 611$ & 0 \\
\hline
\end{tabular}

\footnotetext{
*Excluded from totals.

Note. - The number of cases (from each reported series) accepted for analysis in this table is often smaller than the totals mentioned in the series themselves. Despite the development of asystole in all cases some of the patients did not, in my opinion, fulfil adequate clinical criteria of brain death, and some of the series include cases of drug intoxication. An additional 92 cases from two further series 2121 have not been included (although clinically brain stem dead and ventilated to asystole) because it was impossible to ascertain exactly what proportions of these cases were due to structural brain disease. The table deliberately underrepresents the relevant material.
}

\section{Figure 5.4}

Table listing the reports and exclusions in Pallis' calculation of the number of survivors of a dead brain stem. Note also the number of patients without electro-cerebral silence, but who otherwise met Pallis' criteria; none of these survived either.

Reproduced from Pallis (1983: 22)

within a couple of days at most. Numerous reports about the clinical outcome of smaller and larger series of patients diagnosed as brain dead were published since the end of the 1960s. Basically these reports supported Pallis' claim, but unfortunately some of these series were 'contaminated' with cases that did not meet the essential 
preconditions, or with cases in which there still was doubt about the possible role of drug intoxication. In some reports the authors did not provide sufficient information to ascertain the structural nature of the brain disease with certainty. Using from the literature only the cases that met his strict criteria, Pallis calculated the number of survivors of a dead brain stem to be zero (see the right column in Pallis' table in figure 5.4).

\section{Tracing conceptual boundaries}

Building an argument for a specific definition of death always involves an argument against other definitions of death. Any definition of death traces conceptual boundaries vis-à-vis other definitions. Vis-à-vis a definition of total death, a definition of brainstem death grants the brainstem a central and critical position in the organism as a whole. Furthermore, it argues that the artificialness of the support that substitutes for the brainstem's integrating functions does matter; death should be considered to have occurred when a brainstem cannot get the job done on its own, without artificial support. As we will see in the following section on whole brain death, the argument that 'transient residual signs of electrical activity in isolated neuronal aggregates in the higher regions of the brain do not indicate persistent functioning of the organism as a whole or even of the brain as a whole', is simultaneously an argument against a 'whole brain' definition of death arguing that this residual electrical activity might indicate 'residual sentience'.

Pallis, as a proponent of brainstem death, also traces a conceptual boundary visa-vis a definition of neocortical death, that is, against a line of argument that holds that the irreversible destruction of the cerebral cortex is a sufficient condition to pronounce death, irrespective of whether the body displays spontaneous respiration or not. Against a definition of neocortical death Pallis mobilizes a picture of an
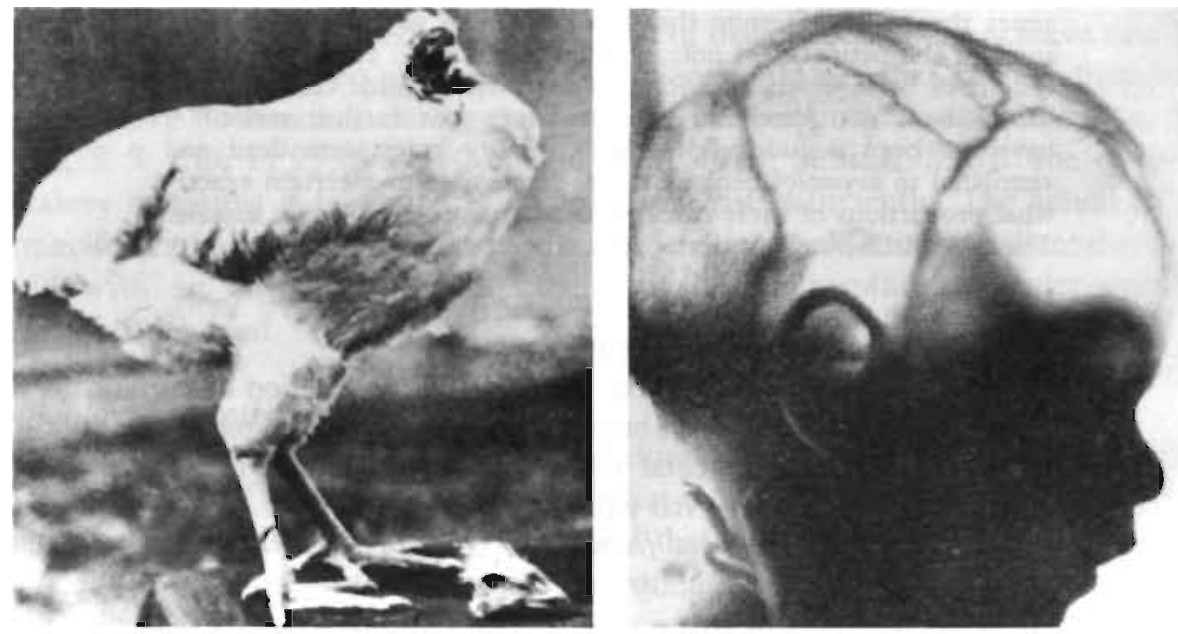

Figure 5.5

Reproduced from Pallis (1983: 3) 
unsuccessfully decapitated chicken (which appeared in a leading magazine) and juxtaposes it with a picture of the transluminated skull of a child with hydranencephaly (see figure 5.5). The forebrain of the chicken has been amputated and lies on the ground, while the brain stem is still in place. The bird, still breathing, was fed with a dropper for several weeks. Was it alive or dead? According to Pallis, the chicken must be considered alive so long as its brainstem is functioning. In a child with hydranencephaly there is a spinal cord, a brain stem, and perhaps some diencephalic structures but certainly no cerebral hemispheres. The cranial cavity is full of cerebrospinal fluid and transluminates when a light is applied to it. The child can breathe spontaneously, swallow, and grimace in response to painful stimuli. Its eyes are open and the heart can beat normally for months. According to Pallis, no culture would declare that hydranencephalic child dead. The condition in the chicken and the hydranencephalic child are similar to the more common condition of the persistent vegetative state to which the neocortical death proponents refer. How can a medical practice that considers hydranencephalic children to be alive, pronounce death over spontaneously breathing and heart beating patients in a persistent vegetative state?

A definition of brainstem death equates the diagnosis of a dead brain stem with the death of the patient. It translates a prognostic statement into a descriptive statement about the presence of death and the absence of life. It turns a dying patient into a dead patient, that is, into a corpse. This translation allows organs to be explanted from a "heart beating body with an irreversibly destroyed brain stem which is maintained on artificial life support systems, because it is no longer life that is supported. ${ }^{35}$ The acceptance of a brainstem definition of death would, according to Pallis,

\section{... lessen human distress, lead to more rational use of our limited intensive care} facilities, and radically alter the life expectancy of thousands of patients with endstage renal failure. (Pallis 1983: 1)

While facilitating the availability of good quality donor organs, both Pallis, the physician, and Lamb, the philosopher, emphasize that the diagnosis of brain stem death has nothing to do with euthanasia. The distinction between diagnosing death and euthanasia runs parallel to the distinction between a dead brain stem and dead hemispheres. According to Lamb, it would be a dangerous mistake to consider a diagnosis of death in patients with dead hemispheres, that is, patients in a persistent vegetative state.

Whilst the vegetative state is often cited as an example of a condition in which the patient may be allowed to die, philosophers who confuse or equate this state with brain death are taking the first step along a problem of factually diagnosing death on to a very dangerous slippery slope that leads to euthanasia. Uncertainty regarding criteria for determining the loss of hemispheric functions - for instance, the limited reliability of the EEG as a positive indicator - suggest that redefinitions of death in this direction run into serious risks of confusing criteria for euthanasia with criteria for diagnosing death. The first is an ethical issue with serious legal implications, the latter a clinical issue to be resolved by clinical expertise. (Lamb, 1985: 112) 
According to Pallis, discussions about 'allowing to die' are different. They seek to answer very difficult questions about how society copes with severely brain-damaged patients who still have some brain stem function (the Karen Quinlan dilemma). There are patients of this kind in long-term care units all over the world. According to Pallis, they present an enormous social problem. Decisions have to be taken about them. But,

... these are social decisions, with important cultural, ethical, religious, and economic implications. There are medical implications too, but doctors should not try to play God: their function is to give an early prognosis (if they can)..... Different societies have taken different attitudes to this problem, which abuts on to the whole subject of euthanasia. But I am not discussing euthanasia. 'The uncomfortable dimensions of the care of the dying' have nothing to do with identifying a dead brain stem. (Pallis 1983: 4)

\subsection{Definition of whole brain death}

\section{All function of all neurones in the whole brain}

A definition of whole brain death shares with a topology of brain stem death the centrality of the brain as the critical topos, the irreversible destruction of which is sufficient to pronounce death. A definition of whole brain death, however, does not single one or other part of the brain out as being more essential than another.

Brain death means irreversible cessation of all brain functions ... The term brain includes the cerebrum, cerebellum, and brainstem with the medulla oblongata. (Jørgensen et al. 1973: 355)

In patients with a dead brain stem or with other types of irreversible coma death is imminent. They are nearly dead and dying. Their brains function poorly. Some portions have even ceased to function. But, a dying patient is still a living patient and should be recognized and protected as such.

The centrality of the brain as the critical topos in whole brain death has to do with the human brain being the structural and functional substrate for human personhood, the faculty that is required to obtain or retain the ticket of legal rights granted to living members of the species Homo sapiens. Hence, a definition of whole brain death agrees that the death of the brain can be equated with the death of the human being. In other words, brain death constitutes the death of the person, but only under the condition that brain death means the irreversible termination of

... all brain function, that is to say all function of all neurones in the whole brain, must have ceased completely and irreversibly before a person may be declared dead. Only this is truly brain death, which is more than irreversible and serious brain dysfunction (Van Till 1975: 134) 


\section{Speaking for the potential donor, against 'civil death'}

Van Till, being a lawyer, is clear whom she speaks for in proposing and defending a whole brain definition of death. The person declared dead loses all his former rights. To all intents and purposes he is 'outlawed' and ceases to exist as a legal subject. He is no longer protected against murder or other forms of maltreatment.

In order to safeguard the citizen against premature loss of rights (such as the right to life and to physical inviolability), whether this has happened through ignorance, carelessness or abuse of power, lawyers, judges and legislators may and must concern themselves with the definition, the diagnosis and the proof of death. (Van Till 1976: 787)

The interests of the dying patient and of the potential organ donor as legal subjects come first. Now, in the era of resuscitation and intensive treatment and of solid organ transplantation, it is necessary to re-establish and safeguard the individual's rights to life, to physical inviolability, to an undisturbed deathbed. This can be accomplished by redefining death, by law, in such a way that it provides maximum certainty for the patient and for physicians and other health care professionals. A definition or criterion of death designed to promote organ transplantation, to avoid legal difficulties associated with the complex problem of euthanasia, or to free hospital beds would be legally unacceptable. These would constitute social or economic rather than medical criteria of death, even if they depend on medical symptoms for their occurrence. A redefinition of death on these grounds would amount to

... the reinstatement of 'civil death' (outlawry) but now pronounced by physicians instead of by a judge. (Van Till 1976: 810)

A legally acceptable definition of death should be the same for all. In ethics and law all people are equal. Biologically all persons function along the same lines. It would be unacceptable to use one definition or criterion for some (for instance, nonEuropeans, handicapped babies, senile elderly or suitable organ donors) and another for others. A legally acceptable definition of death should be exact and unambiguous, giving as much certainty as possible. It should not be quantifiable, that is, it should not be expressed in gradations.

The question of how well or badly the entity functions is left out of consideration, for the question of declaring a person dead is not to be decided on the basis of value judgement but rather on the basis of having or not having certain faculties. (Van Till 1975: 137)

The definition of death should mark the beginning of a state which comes into being by an irreversible and total change which concerns the essence of personal life. The law should be unaccommodating where these requirements are concerned. Otherwise, we will end up with a criterion of death which may be arbitrarily changed and adapted to the needs of the moment or to the private opinion or value judgement of the physician. 


\section{The burden of proof}

Van Till's argument basically proceeds along two lines. First, the burden of proof should be on the physician pronouncing death, and not on the patient. In other words, when the diagnosis of death is concerned, we should not say that, as no sign of life has manifested itself during a certain period, death may be presumed by the doctor in the absence of evidence to the contrary.

[I]n practice [this view] puts the onus of proof on the patient; if within an arbitrary period of time he does not demonstrate that he is alive, he is presumed dead. (Van Till 1975: 139)

Because by declaring a patient dead the physician terminates the legal protection of that patient, we should not equate outwardly perceptible, measurable, or registrable signs of life with life itself, leaving an unknown margin of error open by omitting the immeasurable. ${ }^{36}$ Even in the absence of measurable signs of brain activity we must allow for the possibility that some innate awareness, some residual sentience is left. When residual electrical activity in the brain is being recorded in the EEG, a diagnosis of death should not even be considered.

[E]ven though there is no sign of life the doctor cannot with certainty exclude on these grounds the possibility of the patient being still alive and only apparently dead ... the doctor will have to prove the possibility of the existence of brain function by proving a fact which is incompatible with brain function. (Van Till 1975: 139)

Secondly, a minimum qualification of what constitutes a human person is sufficient. We only need the minimum qualification of a person, because regarding the death criterion we are not interested in knowing how well or how badly a person functions but only in knowing whether there is a person at all.

Hence we need not describe all the faculties which constitute a person; we need only mention the one faculty which gives a biological organism its personality: mind. (Van Till 1976: 811)

To constitute a person there must at least be an organism of the species Homo sapiens with some integrating brain function of its own. However, a person is something more than a merely living biological organism. A person is at least an organism with some inner awareness or perception of itself and its surroundings: a mind body, psychicalphysical or psychosomatic entity.

Mind, neurophysiological research has established that much, is exclusively a function of the brain. This excludes, for example, the spinal cord, which is generally considered to be a part of the central nervous system. Although there is strong resemblance between the brain and the spinal cord from a neuro-anatomical and physiological perspective, there are good reasons to consider the brain and spinal cord as distinct organs. The neural centers in the spinal cord are, for example, further apart from each other than in the brain, probably too far to facilitate the rapid neural communications between neurons that are characteristic of mental activity. The 
number of synapses between neurons in the spinal cord is far less than in the brain. Furthermore, the brain is directly accessible to external stimuli through all five sensory input channels (sensory organs and cranial nerves): sight, hearing, smell, taste and touch. The spinal cord, however, is only directly accessible to tactile stimuli. Furthermore, tactile stimuli entering the spinal cord do not at that level produce awareness of these stimuli. We know from patients with complete transsections of the spinal cord high up in the cervical area that they cannot perceive painful stimuli which are applied below the level of the lesion, neither are they aware of what is happening to their body in general. They may react to painful stimuli with movements. These movements, however, are merely spinal reflexes and are not accompanied by awareness. (Van Till 1976: 818) Hence, as far the diagnosis of death is concerned, a line may be drawn between the spinal cord and the brain, at the level of the foramen magnum, the hole in the skull where the spine attaches and through which the spinal cord passes into the cranial cavity.

When it comes to the brain itself, however, neurologists and neuroscientists disagree as to the precise location of the structures supporting consciousness. Experiments have singled out some areas of the brain (ARAS, cerebral cortex) as being important for normal conscious behavior. However, these experiments do not allow the conclusion that, hence, these functions where located in these areas. That what is variously called mind, consciousness, mental activity or higher brain functions require the functional integrity of areas in the cerebral hemispheres, in the thalamus, in the hypothalamus and in the brain stem.

[I] even the neurologists contradict each other on this point, safety demands that the biggest unit, i.e. the whole brain, should be diagnosed as being dead before the person is declared dead. To content ourselves with partial brain death on uncertain grounds is ethically and legally unacceptable. It is even more unacceptable because there is no positive proof at all that awareness itself, or consciousness, is indeed restricted to the cerebrum. (Van Till 1976: 819)

The next question is: What is minimally required to make a minimal inner awareness the minimal qualification of a person - at least possible? It is not relevant whether this minimal inner awareness expresses itself in outwardly measurable signs like for example EEG-inscriptions. It is only relevant whether the conditions that permit the possibility of inner awareness exist. Scientists maintain that there is a correlation between the function of an organ and its structure. More specifically, neuroscientists maintain that the ultimate structural requirement to make brain function possible is the presence in the brain of functional synapses between mature neurons. Without functional synapses and neurons the introduction and assimilation of stimuli in the brain is impossible.

[O]nce they exist the possibility of brain function can no longer be denied, though the existence of brain function cannot always be demonstrated. (Van Till 1975: 137)

Neurons and synapses are indispensable but not sufficient. They have to be kept alive and functioning. This requires a intracranial circulation of oxygenated blood. 
Consequently, to be certain of the total absence of mental function there must be total destruction or total absence of all synapses and/or neurons in the brain. Altematively the intracranial circulation of oxygenated blood must be completely absent or the oxygenation of the brain or the whole organism must be non-existent. If one of these three conditions exists, we can say for certain that a psychosomatic entity cannot exist. The patient is dead as a person, even if the organism is kept functioning as a robot, by medical manipulation. We also know that brain neurones do not regenerate after birth; consequently the total destruction of all brain neurones is then with certainty irreversible. (Van Till 1976: 816)

In 'normal' cases of death, where there is no interference in the dying process, such as cardiopulmonary resuscitation, permanent cessation of blood circulation or respiration destroys all the neurones and synapses in the brain, eliminating the very possibility of even a minimal inner awareness. After this, the remaining body does not fulfil the minimal requirement for human person anymore and can be considered dead. However, as long as the intracranial circulation could possibly continue as a result of resuscitation treatment, there is also a possibility that a minimal inner awareness exists.

Is it humbug then to think or say that a comatose patient with irremediable structural brain damage, with a flat EEG, without spontaneous respiration, thus respirator dependent, with absent cranial reflexes, etc. is dead? No, of course not, but,

[a]ll these data do no more than give a presumption of death ... the irreversibility of the existing situation ... does not prove the totality of the absence of all brain function ... (Van Till 1976: 804; my emphasis)

Mark the difference, between a presumption of death and conclusive proof of death. Because declaring a patient dead rips the patient of the legal protection every human person is entitled to, death should not be pronounced on presumptuous grounds only. The lasting absence of outwardly perceptible signs of brain function - absent reflexes, a flat EEG, which anyhow only records electrical activity in the cerebral cortex and not in deeper layers of the brain - gives a strong presumption that brain function itself could be absent too. In some cases the presumption that brain death has occurred can be so strong that the physician is personally convinced that his patient is already dead. According to Van Till, however, his opinion is theoretically falsifiable. Consequently he still risks dispute with the next of kin and the law if he labels his warm and oxygen-consuming patient 'dead' on these grounds only. He should also provide the conclusive prove. (Van Till 1976)

Death should only be pronounced when 'conclusive proof' has been provided by the physician declaring death. What counts as 'conclusive proof, hence, the diagnostic procedure for establishing death, is different in cases of 'normal' and 'abnormal' death. Cascs of 'abnormal' death can, according to Van Till, be divided into four groups: 1) cases in which the treatment (resuscitation, heart- lung machine) may mask an already existing death, or, may be maintaining life; 2) cases in which the traditional symptoms of death are present but, because of etiology or other circumstances, need not necessarily truly indicate death (poisoning, anesthesiological accidents, brain stem injury, etc.); 3 ) children, who have been shown to have a longer 'brain revival time', 
a greater tolerance for anoxic episodes; 4) cases in which the physical integrity of the body will be infringed (removal of organs for transplantation), or in which burial or cremation will take place within 24 hours after death has been diagnosed. All other cases, which with certainty are not abnormal, constitute cases of 'normal' death, the vast majority of adult deaths. (Van Till 1975; 1976)

In cases of normal death the traditional practice of using total and permanent cessation of clinically perceptible heartbeat and respiration as criteria for death may be continued. Cardiac and respiratory arrest may be caused by death of the brain, or if left alone, they will inevitably cause the death of brain. The slight inaccuracy of these criteria in cases of normal death is acceptable, because

[i]n normal cases the integrity of the body will, by definition, not be interfered with during at least 24 hours after death has been pronounced, nor will the body be buried or cremated during that time. Hence, even if death was pronounced slightly inaccurately or prematurely, the patient would not incur any risk or harm during the period between permanent cessation of perceptible heart beat and/or respiration on the one hand, and total and permanent cessation of all brain function on the other. (Van Till 1976: 809)

In cases of 'abnormal' death the only conclusive proof that is admissible is the demonstration of the absence of intracranial circulation for at least 15 consecutive minutes by serial angiography of the internal carotid and vertebral arteries.

If bilateral serial angiography of both carotid and vertebral arteries demonstrates a total absence of blood in the brain during at least 15 consecutive minutes, then it is certain that all brain neurones have perished (lost their structure and become dysfunctional), provided that no circumstances or chemicals are present which depress metabolism, and that the patient is not a child. (Van Till 1976: 805)

Because cerebral angiography can in itself be dangerous and cause damage to the brain or give cardiac complications, physicians should make their presumption of death as strong as possible before deciding to proceed with a cerebral angiography. In addition to a thorough clinical, neurological examination and EEG-recordings, other harmless tests (echo-encephalography, scintigraphy, etc.) should be performed first.

Van Till's claim that only serial angiography of the cerebral arteries, demonstrating absence of intracranial circulation for at least 15 consecutive minutes, is admissible as conclusive evidence that death has occurred, draws on a number of arteriographic studies conducted in severely brain damaged patients during the 1950s, 1960s and early 1970s. (Scherzer and Pendl 1973; Bücheler et al. 1973; Ingvar 1973; Jørgensen 1973; Jørgensen et al. 1973)

\section{Cerebral perfusion pressure}

First of all, it has to be recognized that the circulation of blood inside the skull (intracranial) can be absent while nonetheless extracranial circulation of blood persists, artificially maintained or not. Whether blood circulates through the skull depends on the difference in pressure between the arterial blood pressure and the pressure in the skull. The intracranial pressure can be measured by connecting a pressure gauge to 
an open ended catheter in the ventricles of the brain, which are filled with cerebrospinal fluid. (Lundberg 1960) From this ventricular, intracranial pressure and the mean arterial blood pressure ${ }^{37}$ the cerebral perfusion pressure can be calculated as the difference between the mean arterial blood pressure and the mean ventricular pressure. (Jørgensen et al. 1973)

In normal, healthy circumstances the cerebral perfusion pressure is sufficient to permit the circulation of oxygenated blood through the brain. As a result of brain injury, however, the intracranial pressure may rise. equaling or even exceeding the arterial blood pressure. As a result the cerebral perfusion pressure is reduced to zero, and may even become negative. ${ }^{38}$ Intracranial pressure can rise as a result of any space occupying process in the rigid, bony skull: intracranial bleeding, brain tumors, or cerebral edema, which in turn may be caused by a tumor, trauma or by an anoxic

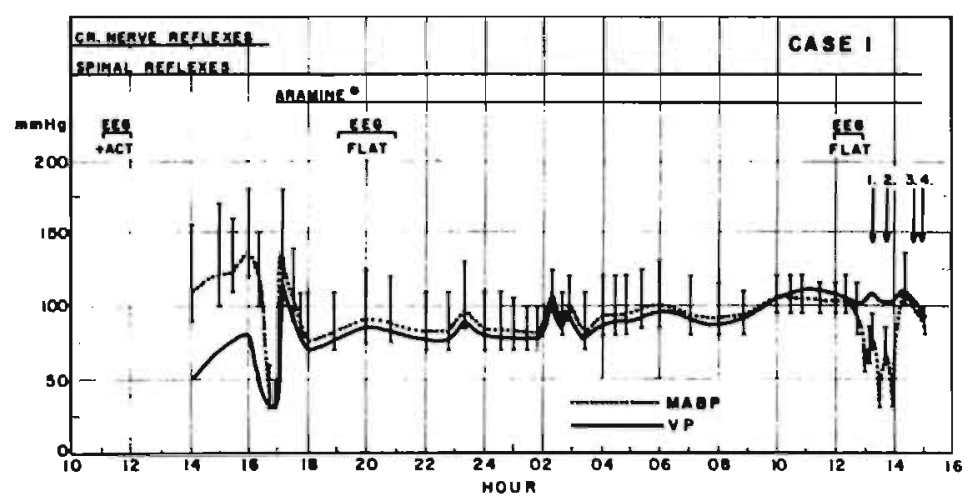

Figure 5.6

MABP (mean arterial blood pressure) and VP (ventricular pressure)

in a 23 year old female with recurrent meningioma in the posterior fossa.

Cerebral perfusion pressure decreased gradually accompanied by disappearance of the cranial nerve reflexes and of cortical activity in the EEG.

Spinal reflexes persisted. Aramine is a vasopressor drug increasing the mean arterial blood pressure.

Reproduced from Jorgensen et al. (1973: 359)

episode following cardiac arrest (see figure 5.6). When the cerebral perfusion pressure decreases below approximately $20 \mathrm{~mm} \mathrm{Hg}$ blood flow in the brain becomes insufficient and may even be obliterated completely. When intracranial blood flow stops cranial nerve reflexes extinguish within a couple of hours and electrocerebral silence follows soon after. ${ }^{34}$

\section{Cerebral angiography}

In cerebral angiography a radiopaque contrast medium (dye) is injected through a catheter into the aortic arch or selectively into the carotid or vertebral arteries. Simultaneously a series of X-ray pictures is taken, or the distribution of the dye in the vascular system, the filling of arteries and veins, is recorded on a video film 
(radiocinematography). Angiographic findings in patients with a low or negative cerebral perfusion pressure can display a variety of filling patterns of cerebral vessels (see figure 5.8 and figure 5.9). When intracranial blood flow is obliterated completely only extracranial vessels in the head (to facial muscles, scalp, etc.) fill, whereas no contrast medium enters into intracranial, cerebral blood vessels, which remain invisible. In figure 5.8, the absence of the intracranial circulation of blood produces the image of an 'empty skull', especially when compared with the normal filling pattern in figure 5.7. Injection of dye under high pressure may result in filling of the initial branches of intracranial arteries but with no, or delayed transit of contrast material from the arterial to the venous circulation (see figure 5.9). Gradual filling of initial branches, but without subsequent venous filling, is also seen in patients in

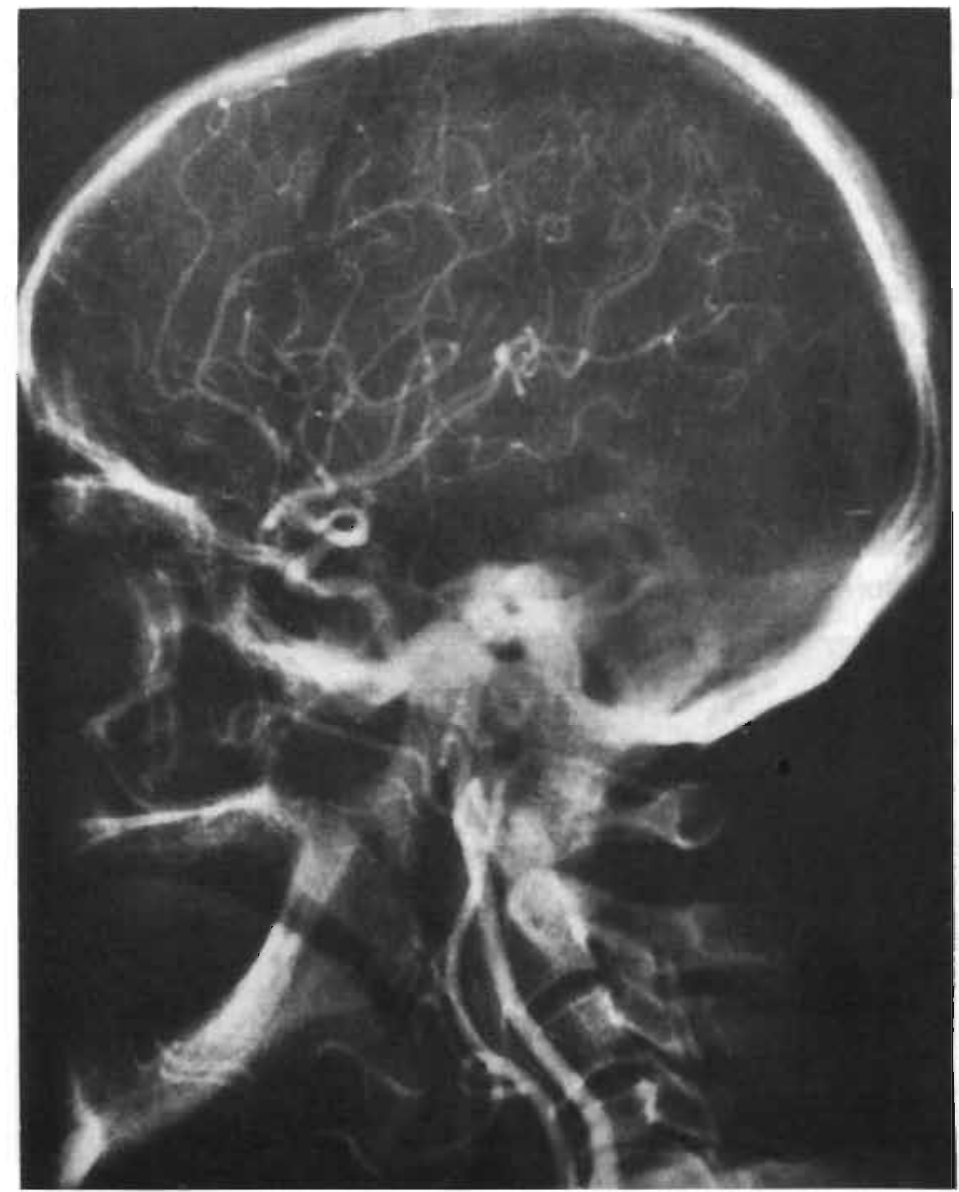

Figure 5.7

Normal filling pattern of intracerebral blood vessels. Reproduced from Mulder en Voorthuizen (1985: plaat 13-6) 

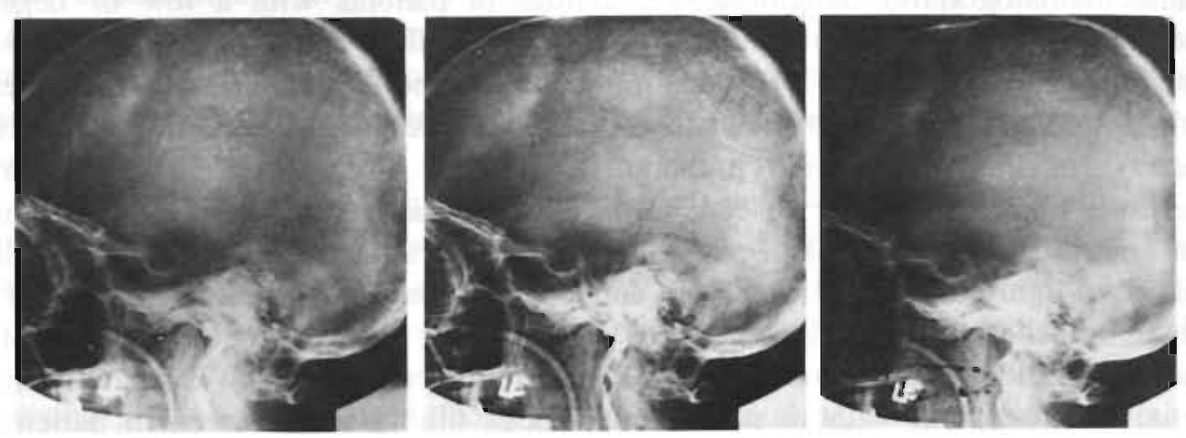

Figure 5.8

Left common carotid angiogram via femoral catheter demonstrating no intracranial filling of cerebral vessels. Lateral projections at 6 seconds (left), 10 seconds (middle) and 29 seconds (right). Reproduced from Kricheff et al. (1978: 172)

whom there is stasis of blood in intracranial blood vessels. When cerebral perfusion pressure is low, but not reduced to zero or negative, intracranial vessels may fill, often slowly and in a thread-like fashion. The contrast material remains too long in the arteries, enters too late into the venous circulation and is, over all, too slowly eliminated from the cerebral circulation. There is flow of blood, but it is prolonged and it will presumably be insufficient to support normal brain functions. There is room for gradations here. (Jørgensen et al. 1973; Kricheff et al. 1978; Walker 1985)

Matters may be even more complicated. In some cases, the rise in intracranial
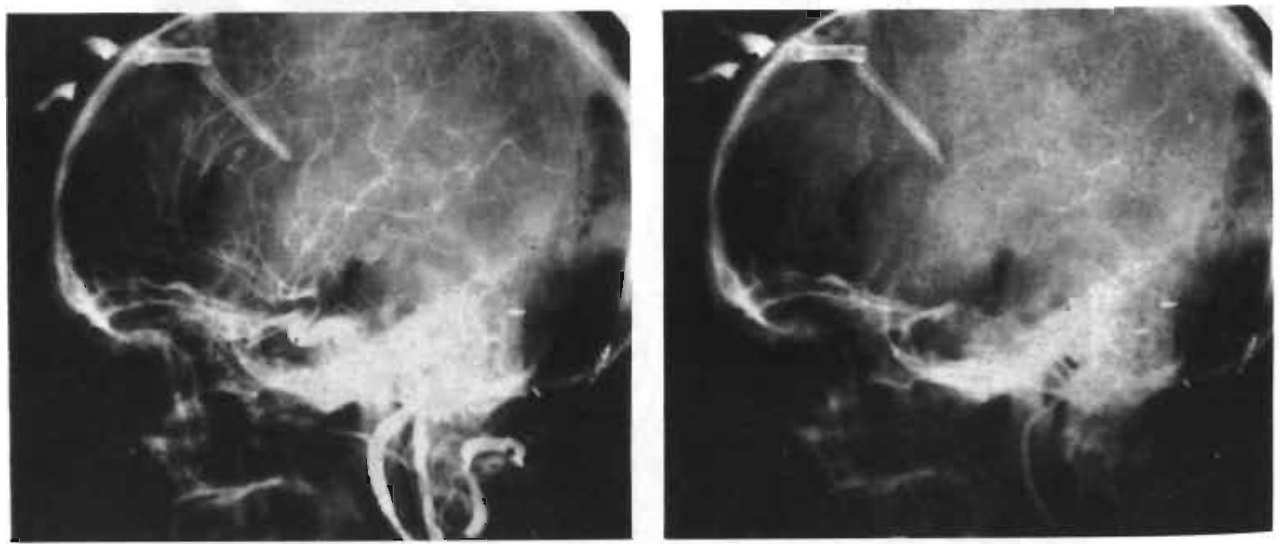

Figure 5.9

Left: Right internal carotid arteriography (frame after 2 seconds). Immediate filling of thread-like anterior and middle cerebral arteries. Right: Right internal carotid arteriography (frame after 54 seconds).

Contrast visible in distal arterial branches and faintly in deep cerebral veins.

Reproduced from Jorgensen er al. (1973: 361) 
pressure is reversible, for example when it was due to cerebral edema, which disappeared again as a result of treatment. The brain may already have suffered irreversible damage when, as a result of an increase in cerebral perfusion pressure, blood flow in the brain recommences. Cerebral angiography then shows intracranial filling of blood vessels. ${ }^{40}$

\section{Organ transplantation}

If angiography shows total absence of intracranial circulation during al least 15 consecutive minutes, this conclusively proves that the patient is dead. Extracranial circulation of blood does not have to be interrupted before removing organs for transplantation.

If, however, the angiogram shows blood in the intracranial vessels, it is not certain whether the patient is dead or not. (Van Till 1976: 820)

The patient may be dead, but this has not been conclusively proven. Death should not yet be pronounced, not until either of the following three conditions are met. First, the cerebral angiography at a later time may demonstrate the required total absence of intracranial circulation during at least 15 consecutive minutes. Secondly, death can be pronounced when cessation of extracranial circulation has persisted for at least 15 consecutive minutes. In that case, confirmation by angiography is no longer necessary. Or, thirdly, death can be pronounced after the passage of at least 15 consecutive minutes after artificial ventilation has been stopped. In the two latter cases, the body's organs, which might have been used for transplantation purposes, have suffered damage from an anoxic episode. That is too bad, but it is no reason to lower our standards of proof or to accept a definition of death that equals a less than total and irreversible loss of all brain function with the dead of the person. ${ }^{41}$

\section{Maintaining trust in medicine}

These diagnostic procedures for establishing the death of the patient and potential organ donor provide maximum (legal) certainty for all involved, a level of certainty that is required to maintain the public's trust in medicine. Patients, and their relatives, can be confident that they will not be declared dead prematurely in the interest of another patient, or because the intensive care ward needs the bed. When the public is confident about the thoroughness of procedures for establishing death, the willingness to consider being an organ donor will increase. Physicians who have accepted the burden to prove conclusively that the patient is dead can be confident that they do not run into legal troubles. Hence, a whole brain definition of death strikes a proper balance between the legal protection every dying patient is entitled to, while, on the other hand, allowing the practice of solid organ transplantation to develop.

If, however, unexpectedly, these procedures for diagnosing death would be to restrictive for the proper development of organ transplantation, then it should be up to the public, and not to physicians, to choose between two possibilities:

1) to give priority to respect for the dying patient's right to physical inviolability until his death has been conclusively proved, for instance by angiography. In that 
case one or more types of organ transplantation may become less successful or impossible;

2) to give priority to successful organ transplantation. (Van Till 1976: 814)

A redefinition of such a fundamental notion as 'death' should be made only by the legislator after extensive public discussion. Physicians should not be allowed to redefine death for practical reasons, and to settle for less than total and irreversible loss of all brain functions. Not in a time when the artificially ventilated, irreversibly comatose patient can be put to other uses. This would be a first step on an extremely slippery slope, a step that is unnecessary and undesirable.

\section{Prohibiting other uses for the 'newly dead'}

The 'newly dead' as a source for donor organs is the utility of the ventilated corpse that has received most attention. This is a purpose that in general is considered to be acceptable when the interests of the dying patient are properly safeguarded. A brainrelated definition of death that accepts a less than total cessation of all brain functions as sufficient to pronounce death would allow for other, in Van Till's view, unacceptable uses of the artificially ventilated, severely brain damaged patient. The ventilated corpse can also be used for other purposes: in the treatment of other patients (not transplantation), in experiments or in teaching. Van Till pointed out that patients pronounced dead on the basis of the criteria proposed in 1968 by the ad hoc committee of the Harvard Medical School, which equate irreversible coma with death, have been used in the treatment of patients with (in principle reversible) hepatic coma.

In want for an 'artificial liver', physicians have tried to find the next best solution by using the in situ livers of monkeys and 'brain dead', irreversibly comatose human donors as substitutes for the patient's own failing liver. This could be accomplished by 'cross circulating' the liver patient and the irreversibly comatose by means of two arteriovenous shunts. One of these shunts carried blood from the patient to the donor, while the other one carried blood back to the patient. This arrangement would last for a couple of days, hopefully enough for the patient's liver to regenerate after surgery and to resume its function. (Summers 1970)

The possibility of the use of irreversibly comatose patients in experiments, like those of White and colleagues (1971), that aim at transplantation of the brain, is, according to Van Till, even more frightening.

The idea that White's experiments on the perfused monkey head could be repeated in man through a premature declaration of death is utterly frightening and repugnant. (Van Till 1975: 140)

White's 'cephalic preparation', an isolated head of a living monkey remained 'alive' as long as it was perfused with oxygenated blood under sufficient pressure (see figure 5.10). It looked, bit, made noises and produced a normal EEG (see figure 5.11). White also succeeded in transplanting the heads of two animals to the other's body and in grafting the head of one animal to the neck of a recipient. 
From 1 to 3 hours after transection, the cephalon awakened and demonstrated a persistence of awareness of its environment during an eight hour period of study.
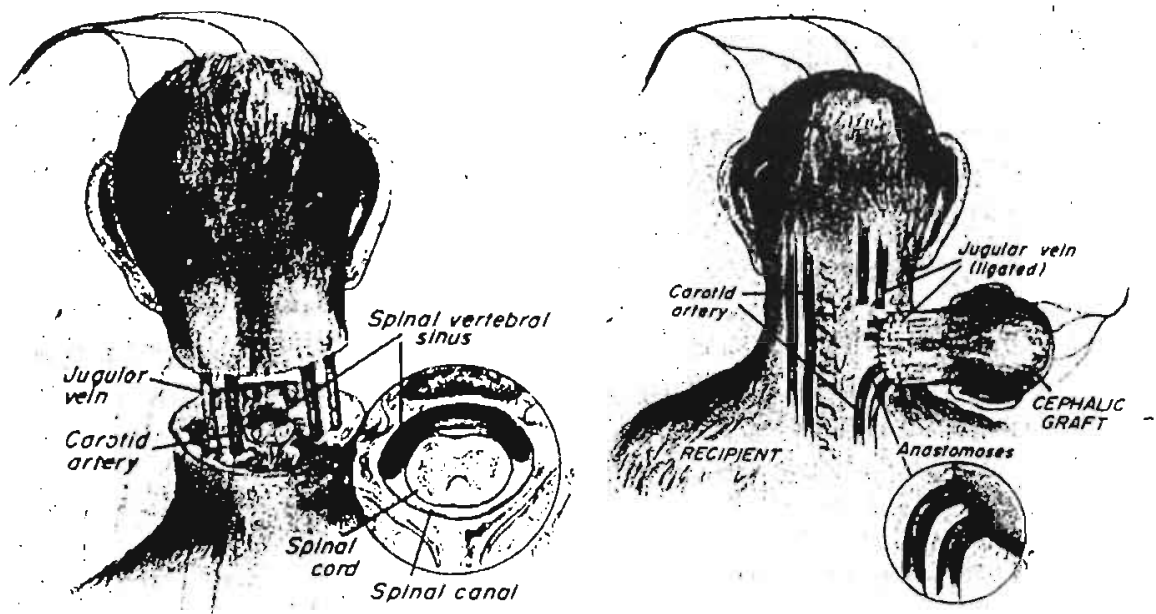

Figure 5.10

Left: Neurologically separated vascularly associated cephalon. All tissues have been separated including the spinal cord, vertebral column, muscular ring and vertebral sinus. Only bilateral carotid-jugular loops are preserved to provide circulatory exchange to brain and head.

Right: Isolated encephalon grafted to the neck of a recipient employing monocarotid-jugular interposition into the cervical circulation. This example illustrates the most advanced model utilizing direct vascular micro-surgical suture anastomosis.

Reproduced from White et al. (1971: 602-603)

These preparations were capable of vocalizing, accepting and chewing food, tracking with their eyes, and biting if orally stimulated. Throughout the period of observation the EEG recorded reflected the established characteristics of an awake pattern ... provided arterial pressure was maintained. (White 1971: 602-603)

Van Till could not find any argument against calling this monkey head, with a functio ning brain, a monkey, though a very incomplete one.

Van Till found evidence that her fear that similar experiments would be conducted on human beings was not totally unwarranted.

American researchers have done similar experiments on eight human foetal heads of 12-17 weeks gestation ... in Helsinki ... These isolated perfused foetal heads consumed nutriments, so presumably they contained a functioning brain: they were psychical-physical entities of the species Homo sapiens. Consequently these heads in fact were persons, though very incomplete ones and not formally registered as such. (Van Till 1976: 818) (2) $^{2}$ 


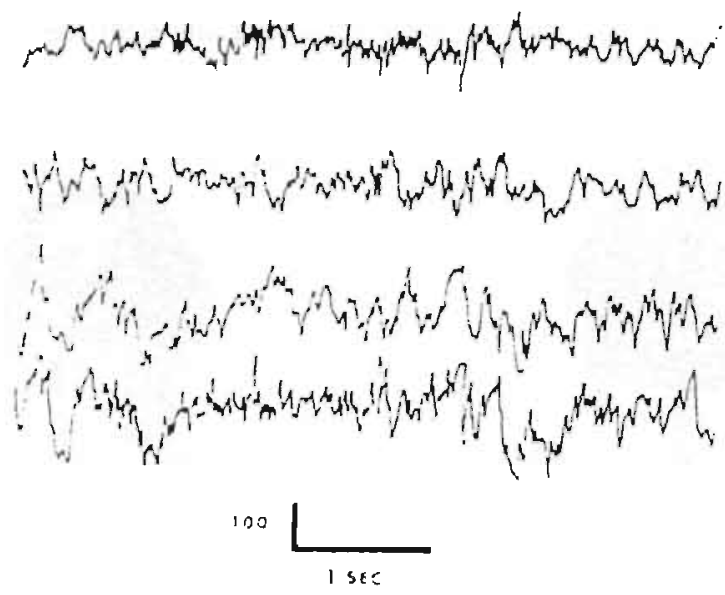

Figure 5.11

Electroencephalographic recording made directly from the cortex of the isolated encephalon three hours and forty-five minutes after vascular transplantation to cervical vessels of a suitable recipient. This recording, conducted during a period when the preparation was alert, reflects an awake EEG.

Reproduced from White et al. (1971: 602-603)

The acceptance of a whole brain definition of death, and its enforcement by the law, would prohibit these and similar kinds of experiments.

\subsection{Definition of neocortical death}

\section{Plaving down the body}

In the three definitions of death discussed in the previous sections the body and bodily integration is of central importance. A definition of total death accepts a dead brain in an organism and considers this organism to be alive as long as the job of integrating various bodily functions is done, even when it is done by artificial substitutes for the brain stem. A definition of brain stem death accepts the death of the cerebral hemispheres or the cerebral cortex, but considers the organism with the dead cerebral cortex to be alive as long the brain stem, being the central integrator and coordinator of bodily functions, is not irremediably destroyed. Both definitions accept that these patients have lost the capacity to return to a natural and conscious life. But this is no reason to declare these patients dead. Bodily integration ranks higher than the capacity to give content to your consciousness and to communicate with your social environment. A definition of whole brain death puts equal emphasis on the capacity for bodily integration (residing in the brain stem) and on the capacity for internal awareness. Only when both capacities have irreversibly been lost a patient may be declared dead. As long as the possibility exists that either one of these capacities persists the patient should be considered to be alive. 
In a definition of neocortical death the importance of the body and of bodily integration is played down significantly. Central importance is attributed to higher brain functions. For these functions a viable, phylogenetically younger, cerebral (neo)cortex has been identified as a necessary, although not sufficient, conditio sine qua non. These are the functions that make up an individual's personality. They constitute what is essential to man's nature, his conscious life. his uniqueness, his capacity for remembering, judging, reasoning, enjoying, worrying, and his capacity to relate to others and to express and perform his essentially social identity and nature. Some authors differentiate between these functions and put more or less emphasis on the capacity for rationality, for consciousness and experiencing or on the capacity for social interaction. (Veatch 1976)

While consciousness is certainly important, man's social nature and embodiment seem to me to be the truly essential characteristics. I therefore belief that death is most appropriately thought of as the irreversible loss of the embodied capacity for social interaction. (Veatch 1976: 42)

When the capacity for higher brain functions has been irreversibly destroyed, then that what is essential for a human being to being alive has been lost. Hence, the person is dead, even if the body which the person occupied prior to his death continues integrated functioning at a biological or physiological level. It makes no sense to treat a body, that does not embody a person, as if it were a living human being. Neither does it make sense to maintain such a body, that does not embody a person, indefinitely by expensive artificial means, at the cost of their relatives or of society. From a moral perspective, treating a dead person as if he were alive is morally as wrong as treating a living person as if he were dead.

\section{Multiple geographies}

These higher functions that make up what is specifically human, are considered to be brain functions, more specifically cerebral functions or functions of the cerebral cortex. Veatch (1988) talks about higher brain functions and a higher brain oriented definition of death. In a definition of neocortical death words like higher and, its opposite, lower brain functions have a double meaning. They define and link together places in different 'geographies': the geography of the brain, the geography of the animal kingdom and the geography of entities that can be objects of moral concern. Higher brain functions refers to (the functions of the) anatomical, electro-physiological and neuro-chemical substrate of the 'higher' provinces of the brain in the cerebral hemispheres and cerebral cortex to which the occurrence of these functions has been attributed. Lower brain functions comprise functions like temperature and blood pressure regulation, respiration and heart beat, etc. These biological functions of homeostasis and bodily integration are attributed to the lower provinces of the brain in the brain stem. But, 'higher brain functions' also refers to the fact that it is through these functions that man rises above the level of other animals. Man shares lower brain functions with other animals, but due to the phylogenetically recent development of the cerebral cortex only man developed the capacity for higher brain functions like consciousness, reasoning, etc. The capacity for higher brain functions is what makes 
an organism human. Irreversible loss of higher brain functions amounts to the loss of humanness. When this occurs the organism transgresses an essential and morally relevant boundary. It becomes an organism at the same level as other animals and is no longer the object of the same moral concern that is granted to human persons.

\section{The neocortex as the critical topos}

For a proponent of brain stem death, like Pallis, whole brain death constitutes death because it partly comprises death of the brain stem. Death of the brain stem is sufficient to pronounce death. Death of the 'upper' brain, that is the cerebral cortex, may have occurred but is not necessary for death to have occurred. For proponents of neocortical death, like Green and Wikler,

[w] hole-brain death is also death for persons, but only because whole-brain death is partly comprised of upper-brain death. Tests for either will be tests for death. (Green and Wikler 1980: 127)

In other words, death of the brain stem may have occurred but is not necessary to call a person dead. It is sufficient that the cerebral neocortex is irreversibly destroyed, and when this has occurred,

[T] he person who entered the hospital has literally ceased to exist. (Green and Wikler 1980: 132)

As a person, a patient with a viable brain stem but with a dead cerebral cortex, is dead.

Neocortical death covers also the class of patients with the clinical condition of persistent vegetative state. ${ }^{43}$ (Jennet and Plum 1972) For the bedside clinician, judging the spontaneous behavior of the patient as well as elicited responses in neurological examination, functions of the cerebral cortex are completely absent. These patients never regain recognizable mental activity. Their bodies, if nursed properly, may survive almost indefinitely. They are not respirator dependent, but breathe spontaneously. Their heart beats spontaneously too. Usually within two or three weeks, due to recovery of the brain stem's ARAS, they develop periods of wakefulness in which their eyes are open and move. At this point they have recovered from coma - in the strict sense of deep, unresponsive sleep-like unconsciousness with permanently closed eyes. But, they remain unresponsive. There is no evidence that they perceive their physical or social environment. There is no evidence of inner awareness, consciousness or intentions. Their wakefulness is empty. They do exhibit a large variety of simple and complex reflex patterns. They respond to strong external stimuli with primitive postural and reflex movements of the limbs. They also exhibit highly complex stereotype patterned behavior like chewing, yawning, grinding or swallowing food. But these primitive patterns of behavior have nothing to do with conscious behavior. They reflect lower brain functions at a vegetative level, not the expression of the presence of an embodied person. There is no sentient individual. It is, in Puccetti's words, a breathing, 'non-apneic corpse'. (Puccetti 1988: 76) 


\section{Diagnosing neocortical death}

In a definition of neocortical death the irreversible loss of higher brain functions is critical in determining whether a person has died. In addition to the clinical judgement that these functions are absent, diagnostic efforts will be concerned with electrophysiological activity in the cerebral cortex. Of course, EEG-recordings are important in this respect. Flat EEG-recordings over longer periods of time and repeated after several hours provide a strong underpinning for the death of the cerebral cortex. Whereas EEG-recordings derived from scalp electrodes are superfluous in brain stem death, and insufficient in whole brain death, the scalp EEG does a good job in neocortical death because it records electrical activity in and between the neurons that are considered to be critical. But, the interpretation of EEG-recordings is a tricky and difficult business. There is always the possibility of error. The diagnostic problem is not completely solved with EEGs alone.

The level of doubt and uncertainty inherent to EEGs can be further reduced with auxiliary diagnostic procedures concerned with blood flow, oxygen consumption and metabolism in the cerebral hemispheres and cortex. According to Puccetti, in recent years,

... with the advent of Positron Emission Tomography, all reasonable grounds for doubt can be removed. In PET scanning, the uptake of oxygen and particularly glucose in selective subregions of cerebral cortex can be measured and displayed in color on a video screen: yellow or green for normal metabolic activity, blue or purple for low or no uptake. Although as of a couple of years ago the PET scan had apparently not been used to confirm a diagnosis of apallic syndrome ${ }^{44}$, nothing could be safer, easier or more certain: the entire surface of the brain would be displayed in blue or purple. (Puccetti 1988: 85 - 86)

It is the loss of functions that is critical in neocortical death. Experimental and clinical studies on brain metabolism have shown that higher brain functions disappear when cerebral blood flow and oxygen supply drop below a critical threshold level or deficit. (Braunstein et al. 1978) Furthermore, Lassen et al. $(1963,1978)^{45}$ reported strong correlations between increases in blood flow to specific regions of the cortex of patients in the waking, conscious state when problem solving, and also a significant overall increase in blood flow to the cerebrum as a whole of about $10 \%$ during such activity. According to Puccetti,

... a repeated finding of diminished cerebral blood flow, to less than $20 \%$ of normal, is itself a powerful evidence of pallial destruction ... it seems exceedingly unlikely that any kind of conscious experience in going on in a brain with use for less than a fifth of normal blood flow. (Puccetti 1988: 85)

Veatch (1988) doesn't require a complete cessation of all cortical activity. Pathological processes causing damage to brain structures rarely follow neatly the anatomical boundaries between brain stem, whole brain and neocortical death drawn by theorists. Often more areas of the brain are affected or selective areas may be spared while other regions are destroyed. It is, thus, possible that the cerebral cortex is irreversibly damaged to the extent that the capacity for consciousness, for awareness, experience, 
sentience, willing, etc., has been lost, but that, on the other hand some groups of neurons in, for example, motor areas of the cortex are still functionally viable. These neurons could even produce a flicker in the EEG, or electrical stimulation of the area might still produce muscular contractions when descending cortico-spinal pathways are intact. But, according to Veatch,

... cellular level function, even at the neocortical level, does not count as anything significantly more than isolated lower brain activity did. Only supercellular or system-level functions count. (Veatch 1988: 171)

Veatch's use of the term 'higher brain oriented' or 'neocortically oriented' concept of death conveys that it is the loss of supercellular functions that are usually associated with the cerebral neocortex that counts, and more specifically the functions that have to do with the capacity to think, feel, be conscious and aware of other people. Some residual motor function, even if it is in the cortex, does not constitute a living person.

I do not want to be confused with my gagging. I would not want society to continue to treat me as if I were alive simply because my body could gag. By the same token, I would not want society to continue to treat me as if I were alive simply because my body retained some isolated perfused motor cortex capable of jerking my arm if stimulated properly. ... I even take it as a kind of insult that I could be confused with any of these trivial bodily capacities. (Veatch 1988: 181)

\section{Ontological clarification}

Proponents of a neocortical definition of death take great care to emphasize that the work of redefining death serves to clarify the real, ontological status of severely brain damaged patients in intensive care arrangements. The distinction between the body that has developed or retained the capacity for higher brain functions (the body that is an embodied person), and the body that has lost this capacity (the body that is a biological remnant after the person has died), is not a value-based or moral divide. Choosing between competing definitions of death is not to decide whether or not we attach any value to the preservation of someone irreversibly comatose, whether we value life even if unconscious, or whether we value life only as a vehicle for consciousness. According to Green and Wikler,

... the value of life often drops well before brain death; years before, in some cases of senility. Indeed, many of us would prefer death over continued existence at the level of some profoundly retarded, institutionalized human beings. For all that, there is no temptation to say that these human beings are dead. When they do die, the change is obvious. A reclassification of healthy, profoundly retarded humans as dead would amount to ontological gerrymandering. (Green and Wikler 1980: 116; my emphasis)

Gervais too, in her plea for a neocortical definition of death, argues that 
[w] must not redefine death to suit any other purpose than that of clarifying, as best of we can, the real status of the patient. If our redefinition is shaped by other purposes, we have failed to fulfill our primary moral obligation to protect the interests of the most vulnerable human, whose resources for self-protection are permanently absent. (Gervais 1986: 60; my emphasis)

A quality of life approach, proposing the patient's own value judgement that life has become valueless for the patient himself as a criterion in choosing between competing definition of death, would, according to Gervais, lead to a simpleminded reductio ad absurdum.

If we allow the disabled and the depressed to speak for themselves, they may decide that life has no value for them. But we cannot connect that decision to a change in the way they are classified. A suicidal person does not differ in status from others because he thinks that life lacks sufficient quality to go on. Considerations of quality of life for someone have no bearing on his status, particularly his life status ... any effort to reclassify persons based on quality of life would lead to 'ontological gerrymandering'. (Gervais 1986: 108)

Adopting a neocortical definition of death is, according to these authors, not the first step on a slippery slope inevitably leading to the 'mercy killing' of the weak, the senile or mentally retarded, of those vulnerable human beings whose resources for self-protection are absent. Green and Wikler stress that their argument requires no moral premises and that it is not sufficient to yield moral conclusions. They hold that

... the brain dead are dead, but it does not follow that brain death is the appropriate moment to turn off ventilators or to remove organs. These acts, and other 'death behavior' might be in order at some earlier time, as circumstances dictate. (Green and Wikler 1980: 128)

Green and Wikler, also argue that is does not follow from their argument that all humans lacking the substrate of consciousness are dead. Anencephalic infants are lacking at birth the cortical material necessary for the development of cognitive functioning and consciousness. Due to the possession of a functioning brain stem, they may have spontaneous breathing and heartbeat, and a good suck.

Accounts which simply identified life with upper brain function would have to classify these infants as dead, which they obviously are not. We, on the other hand, need only point out that the identity criteria for the anencephalic, never-to-be conscious infant do not involve causal substrates for higher level psychological continuity. The conditions for life and existence will be those of human bodies rather than those of persons. (Green and Wikler 1980: 128)

Moral issues concerning the care of patients whose bodies fulfil the conditions of life of human bodies, but not those of persons, are left open. It is an issue of public policy to decide how these patients should be treated. 


\section{Moral relevance of an ontological distinction}

While maintaining that a neocortical definition of death is an ontological position and not a moral one, other authors are less reluctant than Green and Wikler in attributing moral relevance to the ontological distinction between an embodied person and a neocortically dead, breathing, non-apneic corpse. According to Veatch,

[t]o confuse a corpse maintained in irreversible coma with the living human who was once that body is not simply a wasteful luxury, it may be an ethical affront. (Veatch 1976: 257)

According to Puccetti (1988), in his defence for a neocortical brain death definition against a whole brain (encephalon) and brain stem definition, destruction of the neocortical areas necessary for conscious experience brings about a shift in the moral status of the individual.

[E]ither human life is rooted in brain stem function or it is rooted in the capacity for personal experience. If the former, then all vertebrate species are on an equal footing and what counts in medical ethics is just long-term organic functioning independently of our capacity to intervene. If the latter, then there is no ethically relevant difference in the status of encephalically and cerebrally dead people; they have both lost the neocortical basis of an ongoing personal life. The only surprising fact of the apallic syndrome is, or should be, that corpses are really of two kinds: the vast majority that cannot breathe unaided, and a small minority that nevertheless can do this. Apneic or non-apneic, a corpse is still a corpse. (Puccetti 1988: 85)

\section{Disposal of the non-apneic corpse}

The permanent and irreversible loss of consciousness transforms a living human being into a breathing, non-apneic corpse. Further treatment, feeding and nursing of this body will do no good for the deceased. For the same reason, no harm can be done to him or her by stopping his or hers breathing. They are no longer alive; they are dead. And you cannot kill a body that is already dead. That point is missed by critics who accuse neocortical brain death protagonists, advocating active intervention to stop the breathing, of instigating to murder.

You can stab a corpse, but you cannot kill it, for it is already dead whether breathing or not. (Puccetti 1988: 87)

Along the same lines Pucetti argues that it is inappropriate to designate the active intervention to stop breathing, nor the passive non-continuance of treatment, as 'euthanasia', meaning 'mercy killing'.

One cannot be merciful to a cadaver, for cadavers are beyond pain and indeed all further experience of this world. (Puccetti 1988: 87 - 88)

It only makes sense to pronounce death when it, after doing so, is appropriate to treat the body as a corpse, that is to go into mourning, begin a funeral or other ritual, to conduct an autopsy, read the will, remove organs for transplantation. 
Opponents of neocortical death invoke images of 'death behavior' involving Puccetti's breathing, non-apneic, heart-beating corpses to demonstrate the, in their views clearly unacceptable consequences of such a definition and policy of declaring death. Parkinson (1985) argued that no pathologist would start an autopsy on an individual with a beating heart and that no nurse would ever wheel a cadaver with a beating heart covered with a shroud down to the morgue. In the same vain Brody wrote, that

[a] body with a flat EEG but with an intact brain stem may go on breathing indefinitely ... Many of us would find it hard to imagine declaring such a body dead, holding a funeral and lowering it into the grave, still warm and breathing. (Brody 1981: 87)

These are persuasive images of agreeably undesirable futures, but, according to Gervais, they do not change the ontological status of such the patient as being dead. Neither should these images lead us into treating these patients as if they were still alive. It is easy enough to stop bodily rest-functions in an elegant way prior to autopsy or burial.

We recoil at the idea of burying a body that is still spontaneously breathing, but as an argument, this is merely an appeal to the impact of entrenched human sensibilities. When the neocortically dead patient has been declared dead, we would simply stop the remaining bodily functions by an inexpensive, aesthetically tolerable procedure. Those who administer the means for stopping the remaining functions must understand that the person is already dead, and that their action is not killing. Upon the cessation of functions, death practices may be initiated. It is thus unrealistic to raise the specter of a still-breathing body being suffocated by burial. (Gervais 1986: 89 - 90)

A definition of neocortical death moves in quite a different direction than a definition of total death or even brain stem or whole brain death. A definition of neocortical death allows active intervention to stop remaining organic or vegetative functions like respiration and circulation by artificial means, but does not qualify such a procedure as euthanasia nor as murder. Stopping rest-functions is a first step in the now appropriate burial procedure, which is in concordance with the true ontological status of the patient.

When proper consent procedures have been followed a definition of neocortical death also allows the removal of organs for transplantation purposes from patients in a persistent vegetative state, because these patients are dead already; they are nonapneic corpses. On the other hand, every active intervention bringing about the cessation of respiration and heart beat in every other class of not-neocortically dead patients still classifies as active euthanasia, assistance to suicide or murder. Depending on professional standards and provisions developed in the jurisdiction, withdrawal of treatment to not-neocortically dead patients classifies as a case of permissible passive euthanasia or 'letting die', as culpable neglect undermining public trust in the medical profession. or as a criminal offence against life to be prosecuted accordingly. 


\subsection{Non-closure}

\section{Persisting uncertainty}

In the previous sections I have dealt with the various definitions of death. Each of these four definitions of death is at present defensible. Concerning the distinction between human life and death there is still so much doubt, ambiguity and uncertainty that each one of these topologies can be defended without running the risk of making a fool of yourself. You will quickly run into people who do not agree with you, pointing out the philosophical and logical flaws in your reasoning, reminding you that such a course of action has illegal and unethical consequences and that the basis of scientific knowledge you build on is not as solid as you think it is. On the other hand, it will be easy to find people, physicians, scientists, ethicists, lawyers and philosophers in support of your position. Defenses and oppositions, strongholds and flaws have been extensively discussed in almost four decades of literature. Since Haid put his questions before the pope in 1957 - asking whether a patient plunged into unconsciousness through cerebral paralysis but whose life (blood circulation) is maintained through artificial respiration should be considered de facto or even de jure dead - a brain related definition of death has gained considerable strength and has become a respectable, defensible position. Brain related definitions of death are recognized by judicial systems in most western countries as the end of human life in patients in intensive care arrangements. But none of the three brain related definitions of death has gained dominion to the exclusion of the others or to the exclusion of a definition of total death. A variety of spokespersons still speaks with authority for the arrival of death or the (possibility of the) persistence of life. Doubt was raised by Haid and his contemporaries, alternative interpretation prospered, but a new boundary between life and death has never been fixed with the same level of certainty, unambiguity and obduracy as, for example, the existence and identity of insulin has been established.

In a country where there is freedom of speech, one may choose to deny the existence of insulin, but this can not be done without making a fool of oneself. The non-existence of insulin, or the existence of a sugar center in the brain stem, are at present not defensible positions. The existence of insulin is beyond controversy, it is uncontroversial. The controversy on the existence of insulin is closed.

If we take the emerging undeniableness of an entity or fact, its hardness and obduracy. and the demise of competing interpretations, to indicate the closure of a controversy, then, in that sense, in the controversy on the redefinition of death closure has never occurred. The non-closure of the controversy becomes apparent when the public debate flares up again on the occasion of, for example, the adoption of a brain related definition of death in formal statutory law, or when matters concerning medical treatment and care of PVS-patients are brought before a court. Recently, in october and november 1992, a public debate flared up following the announcement of German physicians at the university hospital in Erlangen that they wanted to keep an eighteen year old, brain dead. pregnant woman going on life-support systems until the child she carried would be viable. (Schöne-Seifert 1993) Except for a neocortical definition of brain death this intention would be a contradiction in terms with both a whole brain definition of death. as well as with a brainstem definition of death. As we have seen 
both the latter brain related definitions of death are partly defined by the fact that, in patients with a dead brainstem, a irremediable asystole sets in within three days, a week at most. If it would be possible to keep her body going for another four or five months, then, by definition, she could not have been brain(stem) dead. The public debate covered many issues concerning the definition of brain death, the mother's right to die, and the foetus' right to live. The debate displayed all the doubts and uncertainties characteristic of a controversy in which closure has not been achieved. The foetus aborted and died five weeks later.

\section{The controversy is not everywhere}

A second important point to observe is that, apart from the occasional outburst, intensive care units are far from being the sites of heated controversies and debates about the definition of death or about the occurrence of death in particular patients. There may be diagnostic uncertainty. Among relatives there may be doubt concerning the death of the injured or critically ill patient. They find it hard to accept that their loved one, whose body is warm, whose heart beats and whose chest moves up and down in respiratory movements, that he is dead. There is grief, and anger because the medical staff has surrendered already. But, these eruptions of doubt are manageable and can be kept under control. In these and similar intensive care arrangements the level of doubt has been reduced sufficiently to establish a workable practice in which medical personnel has agreed on and feels comfortable with one definition of death, to the exclusion of others. This choice has subsequently been operationalized in diagnostic and other procedures. Having obtained approval from the hospital's board of directors, from the institutional review board, from legal advisors, the prosecutor or other actors the approval of whom is obligatory, a relatively stable and private domain has been established in which the medical and nursing staff can pursue their business, not at will, but relatively sheltered. In this private domain the negotiability of definitions, diagnostic procedures and interpretation of results, etc., has been minimized in order to be able to provide medical care at all. Possible objections voiced in the public domain have become less relevant in the sense that the medical staff can afford to let the critique pass. They can choose not to let the critique affect what they are doing.

Closure has not occurred in the controversy on the redefinition of death, but something else has happened to the controversy. She is not everywhere anymore. Usually she is not in intensive care units, not in the private domains of medical practice. If anywhere, the controversy is in the public domain of the philosophical, ethical or legal literature or in public media like newspapers and television programs, where she flares up from time to time. What has taken place is a partitioning, an exclusion of controversy, doubt, uncertainty, and limitless negotiability into the public domain. This resulted in the establishment of a relatively stable, 'doubt-free' private space where people can pursue their business. Between the inside and the outside there is a horizon of relevance. ${ }^{47}$

In the following section I will not be concerned with the professional, personal or practical reasons various people might have to adopt one or the other definition of death. What counts is which choices eventually are made in specific documents (health council reports, bills of law, etc.), that had as an effect that an inside/outside or 
private/public boundary was formed: a boundary that partitioned allowable medical practices off from the outside or public domain. This was a dual process in which the inside and the outside were produced simultaneously. In other words, being primarily interested in this process of boundary formation, and partitioning of the controversy, I will be concerned with the effects of these documents, not with the discussions and negotiations that went into the (micro)construction of each of them. I will focus on these legal and semi-legal documents, leaving aside financial and other practical arrangements that were made.

Being the result of and dependent on the collective work of local or national actors, actual choices and resultant medical practices can and do differ from country to country, from jurisdiction to jurisdiction. I will focus on the Netherlands, taking these developments to be exemplary for different but similar developments in other countries.

\subsection{Partitioning the controversy}

\section{Praeter legem}

In the Netherlands the first kidney transplantation, using a kidney from a post-mortal donor, was performed in 1966. Over the following decade the number of kidney transplantations gradually increased to 178 in 1976. (Akveld 1987) Eurotransplant in Leiden was established in 1967. Like in many other countries the development of this kidney transplantation program was initially 'hampered' by the lack of what one could call the 'logistic infrastructure' for such a program, which had to be developed simultaneously (financial reimbursement - who should pay for the donor nephrectomy; development of medical and technical expertise among physicians and nursing staff; informing the public to induce confidence and willingness to donate organs after their death; etc.). Kidney dialysis centers were established two years earlier, in 1964. In 1966, there was already a waiting list of transplantation candidates. During the first decade the availability of donor organs lagged behind the demand. (Akveld 1987; Akveld et al. 1988)

Aside from these logistic problems there were legal problems too. The development of organ transplantation in the Netherlands would depend on the possibilities offered by the law. According to Vink, professor of surgery at the university hospital in Leiden,

[i]t can't be denied that in many countries legal regulations in force hamper the development of organ transplantation. (Vink 1967: 1251)

The law in force was very restrictive. It did not forbid transplantation explicitly, but it was not designed to cover organ transplantation. More specifically, there was no legal basis for the extirpation of healthy organs for transplantation purposes from corpses. It was also recognized that the legally recognized moment of death - although not laid down in statutory law, but 'positivized' in centuries of judicial practice - would constrain the development of a solid organ transplantation program. For postmortal organ donation it would only allow the removal of organs after the definite 
cessation of heart beat and respiration. This in turn would at least reduce the success rate of many transplantations and make some others completely impossible.

The Dutch legislature did not tend to lay standards of medical practice down in legal regulations and statutory law. The law and the legislature were reticent about matters of proper professional medical conduct. The Dutch medical association had published a professional code of conduct. (KNMG 1959) A system of professional disciplinary law was in force. The legislature allowed the medical profession to readjust professional standards to developing and changing views and to new scientific insights and technological achievements. If necessary, legal regulations could be implemented later. In the late 1950 s and early 1960 s a practice of withdrawal of meaningless medical treatment in irreversibly brain damaged patients was allowed to develop. Now, in the second half of the 1960s, a practice of post-mortal kidney transplantation was allowed to develop praeter legem.

Enough latitude was provided to remove healthy organs from corpses for transplantation purposes. However, as a result of the lack of a legal basis for such actions this latitude was imbued with legal uncertainty, both for the potential donor as for the surgeon performing the operation.

In August 1968 the under secretary for Social Affairs and Public Health forwarded a request to the Dutch Health Council to establish a permanent advisory committee on the issue of organ transplantation. The Health Council commissioned three sub-committees to do exploring studies on medical ethics, on brain death criteria and on legal aspects of the transplantation problem. (Gezondheidsraad 1975)

\section{A provisional legal basis by analogy}

Both in medical and legal circles it was argued that, if the Dutch population was to benefit from the great achievements in organ transplantation, the legislature would have to provide a proper legal basis for organ transplantation. This would take years. In the mean time, however, a provisional legal basis had to be found.

By means of an analogy this provisional basis was found in the Dutch Law on the Disposal of the Dead (Wet op de lijkbezorging). This law, which originated as the burial law in 1869 but was repeatedly revised since, defined the legally possible consignments the mortal remains of a deceased or of a stillborn could get. Besides burial, the possibility of cremation had been introduced in the law during the 1950 s. The law also allowed people to consign their body for dissection in anatomical research and teaching. People could donate their corpse to science. The fourth option was that of autopsy. Literally the text of the law spoke of 'opening of the corpse' (lijkopening), or 'partial dissection'. Autopsy or partial dissection allowed physicians to open and examine the body for the cause of death and to remove tissues or organs from the body for further pathological, toxicological or bacteriological examinations. The removal of organs was restricted to diseased tissues and organs. Healthy organs were to be left in situ. (Rang and Rozendal 1971; Sluyters 1970) It was the resemblance between autopsy and partial dissection on the one hand, and the extirpation of healthy organs for transplantation purposes on the other, that provided the legal justification for the latter. Strictly speaking the comparison did not hold water. The resemblance between the two was at best partial. Both procedures are performed on a corpse, and not on a living patient. After removal of the organs, diseased in one case but healthy 
in the other, the rest of the corpse can be cleared for burial or cremation. The analogy failed, however, where the purpose of the two procedures were concerned. Autopsy and partial dissection were performed to verify the diagnosis and cause of death. It was done in the interest of medical science and of future generations who would benefit from what physicians and scientists learned from examining the corpse of a patient. Extirpation of organs for transplantation served no such goal. Organs were extirpated for therapeutic purposes, for the benefit of a particular, individual patient whose further life depended on the availability of suitable donor organ. These discrepancies led Rand and Rozendal to reject the analogy on strictly formal grounds, but to accept it on pragmatic grounds, because it was the only possible solution. (Rang and Rozendal 1971)

The Dutch Law on the Disposal of the Dead also defined the roles of some other actors involved in procedures concerning the handling of dead bodies. Physicians, for example, interested in doing an autopsy on a deceased patient had to obtain the consent of the patient's spouse or close relative. The patient's relatives had the right to deny an autopsy. Other roles were related to the nature of the cause of death.

The law made a distinction between natural and non-natural causes of death. When a patient died as a result of naturally occurring diseases or of old age, the physician had to fill out a standardized death certificate. After having obtained consent from the patient's spouse or closest relative he could proceed with an obduction. The patient could not be buried or cremated however before approval had been obtained from a civil servant at the municipal administration office. In case the patient had consigned his corpse to medical science, approval had to be obtained from the mayor. Physicians suspecting that the patient died from non-natural, usually external causes (sharp or blunt trauma, chemical poisoning, etc.) were held to inform the public prosecutor. In these cases the judicial interest in procuring possible evidence of criminal actions would gain priority. The pathologist was not allowed to begin with an autopsy, or, when the suspicion arose during autopsy, he was not allowed to proceed. In each case of non-natural death the public prosecutor would have to decide whether in this particular case there was a judicial interest, that is, whether a criminal offence, punishable according to criminal law, caused the death of patient. He would commission the coroner to investigate the case, establish the cause of death and report back to him. On the basis of the coroners report he would then clear the body, or claim the body for a judicial autopsy (which could be done without the consent of the patient's relatives).

\section{Non-heart-beating cadaver donors}

The majority of potential kidney donors were brain damaged, non-heart-beating victims from traffic accidents (skull trauma). According to the Law on the Disposal of the Dead, traffic accidents were non-natural causes of death. Thus, the public prosecutor had to be informed and the coroner would have to investigate the case. In comparison with other organs, kidneys tolerate a relatively long anoxic period between cessation of the circulation and the installment of preservative measures. However, due to the obligatory involvement of the public prosecutor and the coroner valuable time and valuable donor organs would be lost. Several solutions to this problem have been proposed or attempted. Some authors have argued for a redefinition of traffic 
accidents, to exclude them from the category of non-natural death and to include them in the category of natural death. In that case extirpation or organs could proceed on the basis from the patient's or relative's consent only. Some hospitals had one of their own physicians, working in the emergency room or at the traumatology department, sworn into the function of coroner, who would then be available in the hospital. This would save a lot of time. However, the law also stated that the physician-coroner who investigated the cause of death in cases in which a non-natural cause of death is suspected should not have been involved in the treatment of the patient. A physiciancoroner working at the hospital's emergency or traumatology department could easily get involved in the treatment of injured victims of traffic accidents, transferred to the hospital while still alive, but dying subsequently in the hospital despite treatment. In these cases the in-house coroner would not be allowed to handle the case. A third option that has been proposed is that, unless there is a strong presumption of a criminal offence, the public prosecutor clears the corpse for extirpation of transplantable organs, prior to the investigation of the coroner. The coroner would then have to do his job, not on the basis of his own first hand investigation, but partly on the basis of second hand information received from the treating physicians. The responsibility to extirpate organs for transplantation purposes would then rest with the physician. ${ }^{48}$

\section{Provisional revision of the law}

The legal justification of the extirpation of healthy organs for transplantation by analogy with autopsy and partial dissection was given a more formal status in a bill submitted to parliament in April 1971. This bill contained a draft of a thorough revision of the Law on the Disposal of the Dead. It was stressed that as a legal basis for organ transplantation the proposed regulations were provisional. A separate law covering the organ transplantation issue was in preparation. The bill left a lot of questions unanswered, but it did two important things. In article 77 the bill states that when, the deceased has consented, the corpse may be submitted to autopsy or organs for transplantation may be removed. Furthermore, when an explicit consent of the deceased for organ donation is not available the patient's spouse, partner or closest relative may speak for him and act as a proxy on his or her behalf.

By submitting the extirpation of organs to the same consent requirements that were applicable to autopsy and partial dissection in the 'old' Law on the Disposal of the Dead, which was still in force, the bill expressed the legislature's principle choice for an explicit consent system. The legislature did not choose for a no-objection system, in which consent would be presumed unless explicit objections had been expressed. The bill pointed out a patient's spouse, partner or close relative as the legitimate spokesperson for the deceased. These spokespersons were not to be silenced or bypassed by physicians giving priority to the recipient's interests despite objections of the donor's surviving relatives.

The bill was mute on the moment and diagnosis of death. This issue was left open. Neither did the bill redefine traffic accidents as natural causes of death. According to many discussants and commentators the bill was not a very good one. It was incomplete and on many issues not specific enough. (Sluyters 1972; De Bijl 1985; Akveld 1987) But, the two basic things it did, allowing the use of cadaver 
organs for transplantation and the choice for a consent system, stood well in following discussion in parliament. Expressing the principle choices of the legislature, which had also been forwarded through the channels of the state health inspectorate (circulars to physicians and coroners), the bill traced a boundary between proponents of the use of cadaver organs for transplantation and possible opponents. It also traced a boundary between proponents of a consent system and proponents of a no-objection system. The latter's critique on the consent system, that it would not yield enough donor organs to meet the growing demand, was rendered less relevant. They too had to obtain either the patient's or the patient's relative's consent, before they could proceed with the removal or organs for transplantation.

As a draft-law the 1971-bill went through several revisions, concerning issues that had nothing to do with organ transplantation. ${ }^{49}$ The bill did not reach the formal status of statutory law before March 1991. Thus, during a quarter of a century of solid organ transplantation in the Netherlands this practice had no formal legal foundation. Yet, the 1971-bill reduced the initial legal uncertainty at the end of the 1960s sufficiently to warrant Rang and Rozendal's view that

... it is not conceivable that a public prosecutor would prosecute when consent has been given in conformity with the regulations laid down for autopsy. (Rang and Rozendal 1971)

The legislator's general attitude towards new developments in health care, and the proposed revision of the Law on the Disposal of the Dead created the initial space in which a transplantation practice could develop. The latitude that allowed the use of non-heart-beating cadaver donors prepared the way for the procurement of donor organs from heart-beating, artificially ventilated, brain dead patients.

\section{Heart-beating donors: converging opinions}

The risk and the consequences of being prosecuted for removing viable and vital organs from the heart-beating, irreversibly brain damaged patient was, at the end of the 1960 s, more serious, however. There was not only no legal basis for employing brain related criteria of death. The extirpation of vital organs from a heart beating body could be conceived as a crime against life (murder, manslaughter or death by fault), covered by the criminal code and punishable accordingly; if such a patient would be considered still to be alive. Prosecution, however, would not inevitably lead to conviction. The law did not define notions like 'life' and 'death'. In 1960. Langemeyer, attorney-general of the Dutch State Council, indicated that in the judicial system one should avoid to interpret a word in variance with the meaning it had in the science that was first of all competent for it (see section 5.3). As Van Agt (1968) pointed out, not every behavior or course of action that violates the law is unlawful. There may be conditions in which it is not. In such exceptional situations a judge may recognize a justification or vindication ground. Exceptional conditions can justify an act, despite the fact that it violates the law, and no penalty will be inflicted." Speculating about possible justification or penalty exemption grounds for the extirpation of vital organs from heart-beating organ donors, Van Agt suggested two possibilities. These would depend on the court's reinterpretation of the word 'life' in 
the penal provisions in the Dutch criminal code. If the court accepted the converging medical opinion that a patient's life ends when his brain dies, it might decide that the patient was already dead when the organs were removed. In that case, no crime would have been committed, on the premise that diagnostic procedures to establish the death of the brain have been performed lege artis. But even if the court did not accept a brain related definition of death, it would be conceivable that the court would accept the removal of a healthy organ from a brain dead but still living patient on the ground that the survival of the recipient justifies the infringement on the remaining vegetative life of the donor. (Van Agt 1968)

In 1960 , there were only some members of the medical community who were prepared to defend a brain related definition of death. Towards the end of the decade there were numerous publications and more or less authoritative reports, national and international, stating that human life ended when the brain had been irreversibly damaged. In 1968, the Ad Hoc Committee of the Harvard Medical School to Examine the Definition of Death published its influential report proposing irreversible coma as a criterion of human death. (Beecher 1968) Also in 1968, the World Health Organization issued a declaration on the determination of the time of death. The WHO stated that clinical interest lies not in the state of preservation of the individual cells but in the fate of a person. The point of death of the different cells and organs is not so important as the certainty that the process has become irreversible by whatever technique of resuscitation that may be employed. The Council for International Organisations of Medical Sciences (CIOMS), at a CIOMS-conference on Heart Transplantation held in june 1968 at the WHO headquarters in Geneva, issued a statement that the cerebral function of the organ donor must have completely and irreversibly ceased. ${ }^{51}$ The Swiss academy for medical sciences (Schweizerische Akademie der medizinische Wissenschaften) published guidelines for the definition and diagnosis of death stating that a patient dies when his brain dies. (Schweizerische Akademie 1969; Van Till 1970)

In 1969, in an editorial in the journal of the Dutch medical association, Medisch Contact, Van Woelderen concluded that concerning the point at which a potential organ donor turns into a real organ donor, opinions and medical criteria to be applied were converging. A consensus was developing that brain death constituted the death of the patient. For it is with the death of the brain that the patient looses the characteristics that distinguishes man from animals: human consciousness, the ability of conscious communication at a rational basis with other members of the species. (Van Woelderen 1969) ${ }^{52}$ Support for a brain-related definition of death was also offered by theologians and ethicists. (Sporken 1968; 1969) Sporken, for example, wrote that

... we accept as human all life that contains ... those elements of the human body, that form the basis for human consciousness ... Although man is more than his brain activity, one can say that the presence of viable brains ... play a very important role in human existence as such. That is why disintegration of the brain will be a very important factor in the whole of criteria for determining death. (Sporken 1969: 244 245) 


\section{Brain death as a boundary object}

The convergence of opinions, the emerging of consensus, did not follow disciplinary boundaries between physicians, ethicists and lawyers. Consensus emerged across disciplinary boundaries. Especially when compared with the fine-grained definitions of death laid out later in the debate, these early statements on brain death were fairly vague. Notions like brain death, 'coma dépassé' and irreversible coma were used almost synonymous. The differential importance of the brainstem or the neocortex with respect to the whole brain was not (yet) recognized. Neither was there a standardized set of procedures by which the irremediable destruction of the brain, and hence, brain death could be determined. Yet, despite its vagueness the notion of brain death seemed to be robust enough to allow cross-disciplinary communication and discussion about this new way of thinking about the end of human life. On the other hand it was imprecise enough to allow adaption to specific disciplinary interpretations and vocabularies. Brain death made sense to the neurophysiologist and biologist in the physician, as well as to the existentialist in the ethicist, as well as to the legislator working on regulations to facilitate organ transplantations. Following Leigh Star and Griesemer (1989), one could say that the notion of brain death served as a boundary object. According to Leigh Star and Griesemer, the analytic notion of boundary objects refers to those abstract (or concrete) objects or concepts which inhabit several intersecting social worlds and satisfy the informational requirements of each of them. Boundary objects are objects which are both plastic enough to adapt to local needs and the constraints of the several parties employing them, yet robust enough to maintain a common identity across sites. They are weakly structured in common use, but become strongly structured in individual-site use. (Leigh Star and Griesemer 1989) Boundary objects play an important role in developing and maintaining coherence across disciplinary worlds, managing both diversity and cooperation. At the end of the 1960s the notion of brain death served this function, facilitating the cross-disciplinary exploration of a medical practice that would allow the procurement of donor organs from brain dead but heart-beating donors.

\section{The Health Council's 1974 report on brain death criteria}

Having received and accepted, in August 1968, the request for advice from the Under Secretary for Social Affairs and Public Health, the Dutch Health Council commissioned a small, but all-medical committee to do an explorative study on brain death criteria. ${ }^{53}$ This committee consulted Van Till about legal matters, but she was not a member of the group. This working party's interim-report, especially the technical part, was largely incorporated in the official Health Council report on brain death criteria prepared by a second, larger and more heterogenous committee established in June 1973. This latter report was published in 1974. (Gezondheidsraad 1974)

By means of this report the Dutch Health Council expressed itself in favor of a whole brain definition of death. The report accepted the brain as being the critical topos, the irreversible destruction of which constituted the death of the human being. 
The human individual as a psycho-somatic unity exists by virtue of the integrating function of the brain. Is this function completely and irreversibly lost, then no human life remains, and the human being has died. (Gezondheidsraad 1974: 2)

In circumstances in which respiration and circulation are maintained by artificial means, the irreversible loss of brain functions can not be diagnosed employing the traditional criteria of cessation of spontaneous respiration and heart beat. In such extraordinary circumstances death should be diagnosed employing the really

... essential criterion: the complete and irreversible loss of brain function. It is the same death that is being diagnosed as in ordinary circumstances, employing, however, a methodology and carefulness that is adapted to the special situation ... Expressed in medical terms, the complete and irreversible loss of the whole encephalon, consisting of the myelencephalon, metencephalon, diencephalon and telencephalon, has to be established. (Gezondheidsraad 1974: 3)

The Health Council distinguished between three principle brain functions: the reception of information, the processing of information and the emission of information to other organs. Science allows only to infer the non-reception of information from failure of brain responses to sensory or nervous stimulation to appear as evoked potentials in EEG-recordings. Strictly speaking, to get an optimal result, the EEG-electrodes used in the recording of evoked potentials should be in immediate contact with various brain structures. This would involve the drilling of burr holes and the insertion of deep electrodes into the tissues of the brain. This consequence, however, is not acceptable for the Health Council on moral grounds. The risk of such a procedure for the patient who would turn out not to have suffered an irreversible loss of brain functions would be unacceptable. The recording of evoked potentials using scalp electrodes would have to suffice, leaving, admittedly, a small margin of uncertainty.

The non-processing of information can also only be inferred from the absence of spontaneous electrical potential variations in the brain, that is: from an iso-electric, flat, EEG. Here too, the Health Council accepts a small margin of uncertainty, because a flat EEG from scalp electrodes only indicates the loss of functions of the cerebral cortex and not of the whole brain.

The non-emission of information to other organs may, according to the Health Council, be concluded from the absence of all spontaneous movements, the absence of all reflectory muscle contractions (except spinal reflexes), the absence of spontaneous respiratory movements, absence of spontaneous regulation of body temperature and blood pressure.

Irreversibility of the loss of brain functions can be inferred from the absence of these measurable brain functions for a period of six hours (precautions have to be taken to avoid the pitfalls of hypothermia, intoxication, etc.). The Health Council recommends to take three EEG-recordings of half an hour each; one at the beginning, one in the middle and one at the end of this period of six hours. (Gezondheidsraad 1974) 
The report was presented to the Dutch government in the beginning of 1974. The report's conclusions and main arguments were published in Medisch Contact soon after its appearance. (MC 1974) In the same year it was adopted by Dutch health care authorities. It was published, by the Ministry of Public Health, in official governmental media like the Staatscourant. It was referred to by the under secretary in his correspondence with parliament concerning the revision of the Law on the Disposal of the Dead.

\section{The Health Council's 1983 report on brain death criteria}

In 1983 the Dutch Health Council published a second report on brain death criteria which was intended to revise and update the 1974 report in the light of the growing body of technoscientific, legal and ethical publications on the subject. The committee that prepared the report largely consisted of physicians from various disciplines, but it also comprised two ethicists, three lawyers and a nurse. Van Till, who had written extensively on the matter, was a member of the committee this time.

This 1983 Health Council report on brain death criteria consolidated a whole brain definition of death. In summary the report stated that the death of a human being is determined by 'brain death'. This was understood to mean that the brain, the brainstem and the medulla oblongata have completely and irreversibly ceased to function. Under normal circumstances it has been found sufficient to employ traditional criteria to establish death (no pulse or respiration for at least ten minutes). These criteria are not reliable or applicable under exceptional circumstances, however. The main aim of diagnosis will then be to establish brain death. The complete cessation of brain function can be established on the basis of the anamnesis and a physical examination of the patient, supplemented by special tests. In the current state of scientific knowledge, the most appropriate supplementary tests are electroencephalography (EEG) and/or cerebral angiography. Anamnesis (and/or heteroanamnesis) and a physical examination are always necessary to establish brain death under exceptional circumstances, and an EEG examination will be required in almost every case as well. Once the doctor has established that the brain has ceased to function according to these criteria, he will have to take the primary causal disease and the patient's condition into account to ascertain whether any additional tests would be useful or necessary to establish that the brain has completely and irreversibly ceased to function. This may involve a second EEG six hours later or cerebral angiography immediately after the first EEG. (Gezondheidsraad 1983)

The committee was not unanimous in its advice. The report contained a majority and a minority position. As a last point in its conclusions the report explicitly stated that

[t]he opinion of a large majority of the commission was that, in the present state of scientific knowledge, cerebral angiography should not be considered obligatory as a means of diagnosing brain death. (Gezondheidsraad 1983:4)

In the minority statement, which was jointly signed by two lawyers, B. Schultz and Van Till, and a neurologist, G.W. Bruyn, it was pointed out that it is false to infer the absence of brain function from the absence of recordable and measurable expression 
of functions. It is false to infer the irreversible cessation of the whole brain from EEG recordings from scalp electrodes that can only register electrical activity in the cerebral cortex. The majority of the committee does not recognize the logical error contained in these inferences and the majority of the committee accepts a margin of uncertainty which is unacceptable on legal grounds and which can be eliminated by means of a cerebral angiography. (Gezondheidsraad 1983) As far as her requirements for conclusive proof for the impossibility of brain function were concerned, Van Till found herself on the outside of the boundary traced, at first by the 1973 report and reaffirmed, by the majority position of the 1983 Health Council report. (Van Till $1983 ; 1984 ; 1986 ; 1989$ )

\section{In-house protocols and transparency of medical practice}

Two hospitals in Enschede ${ }^{54}$ translated the 1983 Health Council's majority position into an in-house protocol containing the whole brain definition of death and guidelines (check-list) for diagnostic procedures when physicians suspect brain death in a patient. This protocol exemplifies how the relatively ill-defined and ill-structured notion of brain death, in its function as a boundary object, becomes less abstract and well structured in the local settings of hospitals. The protocol structures, standardizes and normalizes the inside of the private space. It defines each actor's role and assigns legitimacy of spokesmanship (who can speak for whom or what on which issues). The protocol reflects what Fujimura (1992) refers to as a standardize package. According to Fujimura, a standardized package defines a conceptual and technical work space which is less abstract, more structured, less ambiguous, and more concrete than a boundary object. It combines boundary objects with standardized methods in ways which further restrict and define each object. Such co-definition and co-restriction narrows the range of possible actions and practices. It facilitates fact stabilization. Thus, in the local setting of the Enschede hospitals brain death becomes more of a fact, and less subject and susceptible to the criticism and doubt raised by the ongoing debate in the public domain.

The Enschede protocol defines brain death as the complete and irremediable loss of brain functions, including the brain stem and the medulla oblongata. It specifies the criteria that have to be met. It specifies that brain death has to be established by two different qualified neurologists. When the first neurologist suspects brain death on the basis of a physical, neurological examination of the patient a EEG will be recorded. This EEG-recording has to be iso-electric, both in a spontaneous recording as following sensory stimulation with painful external stimuli. No evoked potentials may show up.

When the EEG is iso-electric, it will be repeated after at least six hours or immediately following the first EEG a 'four-vessel' arteriography will be performed. A second neurologist or neurosurgeon must be consulted now ... Together with clinical signs of coma and absent brain stem reflexes a iso-electric EEG confirms the conditions of brain death. Cerebral angiography is not obligatory as a diagnostic technique. (De Graaff et al. 1984: 591) 
When brain death has been established in this way, when the two neurologists sign the check-list carrying the results of the neurological examinations and additional tests and their conclusion that the patient is brain dead, the official death certificate can be signed and dated.

Such a procedure makes it unambiguously clear for all involved that continuation of the artificial respiration (to facilitate organ donation) does not constitute a prolongation of life. (De Graaff et al. 1984: 592)

Except for their internal function in structuring and standardizing local practices, inhouse protocols also serve an external, what one might call a public relations function. The growth, development and continuation of solid organ transplantation practices depends on people's willingness to donate organs after their death. From the beginning of organ transplantation, using cadaver organs, the public fear of being declared death prematurely had to be negotiated. From the beginning both lawyers and physicians have argued for a strict separation of responsibilities between qualified physicians diagnosing brain death on the one hand, and physicians involved in the extirpation and transplantation of organs on the other hand. The legislature's principle choice for a consent system for organ donation in the 1971 bill on Disposal of the Dead served the same function of negotiating the public's confidence. The public was informed about the possibilities of organ transplantation. It was argued that the donation of organs gives sense and meaning to the unexpected and dramatic death of previously healthy and young people. Medical staff was trained in social skills to make it easier for them to pose the difficult question, asking for permission to extirpate organs from the heart-beating, artificially ventilated patient, to the patient's relatives. When they have been established and published 'in-house' protocols make medical practice transparent: for physicians involved, for the nursing staff, for patients' relatives, for the hospital board, for the public prosecutor, for the public in general.

\section{The 1991 bill on the Donation of Organs}

After the adoption of the Health Council's reports on brain death criteria and organ transplantation by Dutch health care authorities, these reports expressed the legislature's position, intention and policy in these matters. Together with the bill on the Disposal of the Dead, these reports created sufficient legal latitude to allow the declaration of death and the extirpation of healthy organs: in medical places where transplantation and intensive care practices met. It was not until 1991, the year in which the new Law on the Disposal of the Dead obtained its status of statutory law, that the Dutch government submitted a bill on the Donation of Organs to parliament. This bill spoke of brain death as the point at which the person dies. When it was first submitted the bill did not specify any definition of brain death. However, in december 1993 the original text was changed, incorporating a whole brain definition of death in the law. In its revised form the bill states explicitly that brain death is to be defined as the complete and irremediable loss of the functions of the brain, including the brain stem and the medulla oblongata. The bill also states that a diagnosis of brain death is only to be considered in cases of fatal and untreatable brain damage of known cause, after all other causes of unconsciousness and non-responsiveness have been excluded. 
(Derde Nota van Wijziging 1993; Gewijzigd Voorstel van Wet 1994) The bill does not specify the criteria that have to be met nor the diagnostic procedures and procedures that have to be performed to see whether the criteria have been met. The diagnosis of death is considered to be a medical matter. The bill only states that brain death has to be diagnosed in concordance with the standards of medicine. The Health Council will review developments in this field from time to time and revise and re-establish what is to be considered as good medical practice. On the basis of these revisions the Health Council will devise protocols concerning the procedures to be followed in hospitals whenever the diagnosis of brain death is considered in cases where there is an intention to remove organs for transplantation purposes. The bill proposes to make these (in-house) protocols obligatory in transplantation centers, recognizing explicitly their importance in negotiating the public's confidence and willingness to donate organs after their death. (Memorie van Toelichting 1991; Gewijzigd Voorstel van Wet 1994)

\subsection{Conclusion}

In analyzing changing practices in medicine and health care we should be alert not to confuse controversies in which closure has occurred with controversies that have been partitioned into, on the one hand, medical places in which cognitive doubt and moral and legal uncertainty have been reduced to a manageable level, whereas, on the other hand, doubt and debate persists in other places. A horizon of relevance has been established allowing the employment of a brain related definition of death and the extirpation of organs for transplantation purposes. With the establishment of this horizon a boundary was traced between a public domain where the issue is allowed to be controversial, and a private space where for practical purposes the issue is resolved allowing the medical staff to carry on with their work. The opposition against these practices was excluded to the public domain and rendered less relevant. So were proposals to adopt another brain related definition of death. The boundary that was established and has been maintained since, provided legal impunity despite the lack of a formal legal basis, while at the same time allowing medical practice to become transparent.

In the Netherlands the use of a whole brain definition of death is intimately linked up with the practice of solid organ transplantation. This brain death definition will be incorporated in legal regulations facilitating the use of donor organs from heart-beating but brain dead people. It is from this organ transplantation program that the whole brain definition of death in the Netherlands derives its present obduracy. However, it is also this interdependency between the current shape of this practice and the current content of brain death that makes the latter vulnerable and fragile. Future changes in the practice of solid organ transplantation may remove some of the 'latches' from which the Dutch whole brain definition of death derives its current stability. There is no reason to assume that medicine will stop transplanting organs. Nor is there any reason to expect that the development of implantable mechanical devices, like the artificial heart, will to a large extent reduce the need for biological, substitute organs. Consequential changes may result, however, from recent developments in genetic engineering. Scientists try to bridge the genetic and immunological gap between species, not by suppressing the human host's immune respons, but by making genetically manipulated, transgenic animals express human Major Histocompatibility Complex antigens on the surface of their cells and tissues. The large scale availability of 
suitable substitute organs from 'animal donors' would render the use of organs from brain dead patiënts superfluous. It is imaginable that in such circumstances brain death will lose its stability again. Instead of meaning 'the death of the person', the notion of brain death will shift its meaning back to 'the death of the organ brain'. With this other meaning it may still be used to indicate the point at which further medical treatment is useless and the patient may be allowed to die.

\section{Notes to chapter 5}

I. In 1947 Beck, Pritchard and Feil (1947) reported the first successful ventricular defibrillation by electric counter-shock in a human patient, a fourteen year old boy being operated for a 'funnel chest'. The patient recovered completely after a prolonged period of ventricular fibrillation. While direct heart massage was being provided the defibrillator could be brought into position. (Wiggers 1936) Intermittent manual compression of the heart restored a degree of circulation of blood that was sufficient to forestall irreversible anoxic damage to the heart and central nervous system. In 1940 Wiggers reported that with proper technique [cardiac massage followed by countershock] revival now generally occurs even following coronary occlusion and when the fibrillation has continued for five to ten minutes. During thoracic surgery manual compression of the heart could be commenced immediately. In every other situation the chest had to be opened first. Since direct, open-chest cardiac massage required an emergency thoracotumy, its use was limited to operating. recovery and emergency rooms. Open-chest heart massage was rarely employed outside the confines of a hospital. Outside the hospital cardiac arrest was usually fatal. These limitations to the applicability of openchest procedures in cardiac resuscitation had long been recognized and experimental attempts to defibrillate a fibrillating heart in animals through the closed chest were undertaken already during the 1930s. Several laboratories engaged in developing a method for closed-chest cardiac defibrillation in humans, which would not only be effective but which would also be safe for the patient and for the medical staff. (Kouwenhoven et al. 1932; Hooker et al. 1933; Guyton and Satterfield 1951; Kouwenhoven and Harold 1951; Kouwenhoven and Milnor 1954; Kouwenhoven et al. 1957)

2. Combination of capacitor discharge defibrillators with rechargeable batteries made the use of the defibrillator independent of the power line. They could be installed in ambulances and portable units could be constructed. Fibrillating hearts could be defibrillated wherever cars could drive and people could run, in or out of hospital, as long as there was somebody to provide cardiac massage and mouth-to-mouth ventilation to maintain the necessary blood and oxygen supply to the heart and brain. The chances for people to survive an episode of cardiac arrest increased when they were in the vicinity of this mobile sociotechnical ensemble that could provide cardio-pulmonary resuscitation when their heart stopped beating; or when they were in a situation where this network could reach out to them.

3. When the heart stops or starts to fibrillate circulatory arrest ensues. The brain no longer receives blood and oxygen and unconsciousness follows after approximately 10 seconds. Patients with cardiac arrest are often found unconscious. Sometimes the situation in which the patient has been found provides a clue to the question how much time might have lapsed since the heart arrested. But often this is not the case. To the helper, layperson or professional, who has to initiate emergency treatment (or not), the cause of the cardiac arrest, underlying and accompanying diseases are often unknown. In many situations the 'only' option is to start resuscitation. The human body's tissues differ in the extent to which they can tolerate lack of oxygen without suffering irreversible damage. Usually the cerebral cortex is irreparably damaged when anoxia persists for 3 to 5 minutes. After this. reanimation to a normal conscious life has become impossible. This amount of time during which the cerebral cortex can tolerate anoxia is also called the cerebral time. (Aleman 1964) The heart time is 
longer. During the first two minutes following circulatory arrest the chance that normal heart function can be restored to normal is, in Kouwenhoven's work, nine out of ten. But it is not impossible after that. Experience with cardiopulmonary resuscitation in human patients showed that, sometimes, heart function could be restored after circulatory arrest of up to ten minutes.

4. In experimental physiology this problem had already been recognized by Wiggers: 'But even if the heart be revived, we may still be defeated in our race with time. Experimental evidence indicates that the central nervous system can probably not endure the complete anoxia which results from ventricular fibrillation for more than fifteen minutes, and perhaps not that long. Moreover, the possibility of reviving higher centers through elevation of blood pressure by cardiac massage is not as promising as that of reviving the ventricles, and, in the event of resuscitation, the possibility must be kept in mind that, unless revival is prompt, the recovered victim may be left in a state of mental deterioration.' (Wiggers 1940: 420)

5. See also Mollaret and Goulon (1959) and Van der Woude et al. (1956).

6. See also Kramer (1963; 1964; 1966; 1970; 1973) and Kramer and Tuynman (1967). The condition described by Kramer as intravital autolysis of the brain was later called respirator brain. See Moseley et al. (1976).

\section{See also Magnus (1965).}

8. The term 'adrenergic' refers to structures or nerve fibers that are activated by or secrete adrenalin or substances of similar activity.

9. This does not mean that the distinction between life and death always has been robust. For a history of various episodes of doubt on the definition and diagnosis of death see Pernick (1988).

10. The International Code of Medical Ethics adopted by the Third General Assembly of the World Medical Association in London, England, in 1949 articulated the doctor's duties to the sick as follows:

A doctor must always bear in mind the obligation of preserving human life.

A doctor owes to his patient complete loyalty and all the resources of his science. Whenever an examination or treatment is beyond his capacity he should summon another doctor who has the necessary ability.

A doctor must give emergency care as a humanitarian duty unless he is assured that others are willing and able to give such care.

A doctor must observe the principles of "The Declaration of Geneva" approved by The World Medical Association. (Van Till 1970: 144)

11. Although Haid posed the question, whether a deeply unconscious patient whose circulation is maintained through artificial respiration, and in whom there is no improvement after several days, must be considered 'de facto' or even 'de jure' dead, in a rather unambiguous way, Pope Pius XII evaded a direct answer. He rephrased the question as: Has death already occurred after grave trauma of the brain, which has provoked deep unconsciousness and central breathing paralysis, the fatal consequences of which have nevertheless been retarded by artificial respiration? Or does it occur, according to the present opinion of doctors, only when there is complete arrest of circulation despite prolonged artificial respiration? The answer could not, according to Pope Pius XII, be deduced from any religious or moral principle and therefore not fall within the competence of the church. However, considerations of a general nature led him to believe that human life continues for as long as its vital functions - distinguished from the simple life of organs - manifest themselves spontaneously or even with the help of artificial processes. (Pope Pius XII 1958)

12. In answering the question concerning the validity of Extreme Unction administered to deeply unconscious, artificially ventilated patients, Pope Pius XII drew on the doctrine and practice of the church. Sacraments are meant, by virtue of divine institution, for men of this world who are in the course of their earthly life, and, except for baptism itself, presuppose prior baptism of the recipient. He who is not a man, who is not yet a man, or is no longer a man, cannot receive the sacraments. 
If Extreme Unction has not yet been administered to the artificially ventilated patient, one must seek to prolong respiration until this has been done. Concerning the validity of Extreme Unction at the moment when blood circulation stops completely or even after this moment, Pope Pius XII was not able to provide a clear answer because of doubts about the exact moment of separation of the soul from the body. If, according to the pope, in the opinion of doctors, this complete cessation of circulation means a sure separation of the soul from the body, even if particular organs go on functioning, Extreme Unction would certainly not be valid, for the recipient would certainly not be a man anymore. If, on the other hand, doctors are of the opinion that the separation of the soul from the body is doubtful, and that this doubt cannot be solved, the validity of Extreme Unction is also doubtful. In a comment on the pope's answer on this point, De Lange (1964), being a non-religious person, found it curious that the doctor and not the clergy was seen as the expert best capable of determining at which moment the typically metaphysical event of the separation of the soul from the body took place.

13. An entity is mute not only when it lacks the possibilities of verbal language, but when it lacks all capacity to communicate its identity, opinions, etc. A patient with 'locked-in syndrome' who has retained the possibility to voluntarily move his eyes up and down can answer questions put to him with yes and no by moving his eyes up and down respectively. (Cherington et al. 1976) Such a patients is not mute. Similarly, being able to speak does include the use of verbal language as a means of communication, but is not restricted to that.

14. Opponents to withdrawal of life-supporting treatment argued that the termination of treatment caused the patient's death (killing). Proponents argued that it was the completion of a lethal disease process inside the patient's body that caused the death of the patient (letting die).

\section{See also Kramer's position in section 5.2 .}

16. For further reading on the issue of withdrawal of life-supporting treatment see for example Walton (1983).

17. De Lange recalled that Aristotle perceived plants as anima vegetalis, animals as anima sensitiva and humans as anima rationalis. Linnaeus said: plants grow, animals grow and feel, people grow, feel and think. Hence, man as such ceases to exist, according to De Lange, when the potency to think ceases irreversibly. Both patients who have temporarily lost the capability to think - for example during anaesthesia or as a result of cerebral contusion - and people whose 'level of thought' is reduced or who can't communicate their thoughts - as in aphasia - are excluded from De Lange's definition of the death of a human being. These latter categories of people are recognized as human beings and protected as such by the law. (De Lange 1964)

18. Kidneys were the first organs to be transplanted in man. Kolff's artificial kidney, developed during the early 1940 s, provided the possibility to keep patients with fatal kidney failure alive by means of chronic, intermittent dialysis. (Kolff et al. 1943; Kolff and Berk 1944) For these patients an attempted kidney transplantation was an interesting option. When the kidney was rejected they could return to the artificial kidney. The first kidney transplantations were performed between 1950 and 1955 in Boston and Paris. (Greep 1970) However, in the absence of techniques to select the proper donor and to suppress the rejection process, these attempts were bound to fail. Under these circumstances success could only be expected from kidney transplantations between identical twins. This would come as close to auto-transplantation as possible. The first kidney transplantation of this type was performed in Boston in 1954, preceded by a skin graft - that was not rejected. The kidney was donated by the patient's healthy twin brother. The group donor-recipient couples, next to the identical twins, among whom kidney transplantation could be successful, were the non-identical twins. The first kidney transplantation between non-identical twins was successfully performed in 1956 . However, the requirement that a living, healthy twin should be willing to donate one of his or her kidneys to the other half of the twin, severely restricted the number of successful kidney transplantations. In 1963 in the whole world only eleven patients were known to live with a transplanted kidney with a graft survival of more than one year. (Eijswogel 1967) 
19. During the 1950s, while Medawar, Burnett and others performed the experimental and theoretical work that provided the modern conceptual framework for the interpretation of the graft rejection process in terms of a immune response (Medawar 1946; Billingham, Brent and Medawar 1953; Burnett 1959), a method to identify suitable donors developed from work with agglutinating leucocyte antibodies that were found in the blood of people who had received blood transfusions and in the blood of women who had been pregnant. (Dausset 1954; Van Rood, Eernisse and Van Leeuwen 1958) Using a panel of anti-sera containing antibodies against leukocytes researchers began to identify a complex system of leucocyte groups, the Human Leucocyte Antigens (HLA), analogous to the (red) blood groups on erythrocytes. Through HLA-serotyping it was possible to determine the specific antigenic composition of the surface of an individual's white blood cells. By replacing the white blood cells with cells derived from other tissues, like for example fibroblasts from the skin, these other tissues could also be tested for the presence of HLA-antigens. (Engelfriet et al. 1966) HLA-antigens were not found on red blood cells, but they could be demonstrated on many other tissues. When introduced into the body of another individual by means of organ transplantation these antigens could elicit an immune response and graft rejection in the recipient. During the first half of the 1960s HLAtyping of readily accessible leukocytes, which could be isolated from a simple sample of blood, developed into a means to compare and assess the immunological (histo)compatibility of the potential donor and the recipient. (Van Rood et al. 1964; Van Rood et al. 1965; Walford et al. 1965) In other words, HLA-typing allowed for the determination of the degree of foreignness between donor and recipient and thus to improve the outcome of the transplantation procedure.

20. Until the beginning of the 1960 s modification of the rejection process had been attempted through irradiation of the recipient's body with X-rays. It was indeed possible to achieve an increase in the survival time of the graft, but severe side effects limited the utility of this technique. The irradiation did not only suppress the rejection process, it also suppressed the rest of the immune system that was involved in the body's defence against bacteria and viruses, thus leading to an increased and often fatal susceptibility to infections. Towards the end of the 1950 s drugs that were used in the treatment of cancer (leukemias), were also tested for possible immunosuppressive properties. (Schwarz et al. 1959) One of these drugs, 6-mercaptopurine, which was shown to inhibit antibody production after antigenic stimulation, was used in experimental kidney transplantations in dogs, where it prolonged the graft survival time. (Calne 1960) Similar results were obtained when other purine-analogs, notably azathioprine, were used. Due to their structural resemblance with the natural purine base constituents of RNA and DNA (guanine and adenine), these purine-derivatives were considered to compete for the same positions in RNA and DNA synthesis, producing 'fraudulent' molecules interrupting the normal process of protein synthesis and cell replication. The inhibiting effects of 6-mercaptopurine and azathioprine were largest in proliferating cells, like those actively involved in an immune response against a transplanted kidney. Especially azathioprine proved to be useful in kidney transplantation in man. Although azathioprine was not devoid of toxic properties, in clinical practice it was often possible to find a dose that produced the required immunosuppression to halt the rejection process while avoiding serious side effects. Furthermore, when combined with corticosteroids, or other nonspecific immunosuppressive therapies, the azathioprine dose could be lowered while still retaining a sufficient immunosuppressive effect. See also Kriek (1966).

21. In some countries similar questions were raised prior to Barnard's heart transplantation in 1967. In Sweden for example, questions about the time of death were raised publicly concerning a case of kidney transplantation in 1964. See Socialdepartementet (1984). See also Brante and Hallberg (1989; 1991).

22. Induction of cardiac arrest by pouring ice water in the cavities surrounding the exposed heart is used in cardiac surgery when a motionless heart is needed to perform delicate surgery. The heart-lung machine substitutes for the functions of the heart and the lungs. When the surgery is completed the temperature of the heart is allowed to rise to normal again, after which it resumes its function.

23. It is possible to discern a fifth position that defines death as the departure of the soul from the body. As Pope Pius XII pointed out, according to the doctrine of the church the departure of the soul 
from the body, however difficult to establish, has consequences for the validity of the Extreme Unction. He who is not a man, who is not yet a man, or is no longer a man, cannot receive the sacraments. In the following account on the redefinition of death, however, I will leave this line of argument aside.

24. A 'metonymy' is a figure of speech consisting of the use of the name of one thing for that of another of which it is an attribute or with which it is associated (as in 'lands belonging to the crown'). Spontaneous heart beat and respiration have been strongly associated with the presence of human life. Their presence was, in a metonymical sense, synonymous with human life. It is this relationship that has been severed in the debate on brain death. See also Wackers (1993)

25. Death of the organism as a whole should be distinguished from death of the whole organism. It is well known that some organs or cells can survive for some time while at the level of the organism as a whole integrated functioning has been lost. In tissue and cell cultures cells can be kept alive for years. Organs that are transplanted continue to function in their new host long after their former owner died. In a definition of total death the argument is not that death should not be pronounced before all cells of the body are dead.

26. Consider, for example, the case of a patient who is a candidate for a heart transplantation. When a suitable donor organ has heen found, she will be taken to the operating theater. General anaesthesia will be induced and she will be connected to the heart-lung-machine. At some point in the procedure her heart will be removed, while the donor organ has not yet been implanted. At this point it would be possible, by lifting the anesthesia, to let the patient regain consciousness. She is not dead. If something would happen to the donor organ she could be kept alive on artificial life support systems for quite a long time. Death would not occur unless life support systems are withdrawn, as a result of which integrated functioning can no longer be maintained.

27. The case of Karen Ann Quinlann in the US is probably the most well-known example. In the Netherlands the case of Mrs Stinissen has attracted much attention.

28. According to Green and WikJer the source from which integration is maintained and controlled is not essential. The heating system of a home can continue to function even after the thermostat fails, so long as the furnace is turned on and off manually (or by a substitute machine); an airplane continues to fly even after the autopilot fails if human pilots are able to take control. The source of control is not important; what matters is whether the job is done. (Green and Wikler 1981)

29. According to Green and Wikler, the interval during which a brain-dead patient can be maintained by artificial life-support is at present quite limited, but surely it could be extended, perhaps indefinitely. It is difficult to see why the brevity of the interval should have any bearing on the definition of death. There are a host of medical conditions which, given the current power of medicine, also inevitably lead to death of the system as a whole, just as renal failure did only a few years ago. There was and is no temptation to regard the onset of these conditions as the occasion of death, and, failing further argument, this judgement extends naturally to the brain. (Green and Wikler 1981)

30. In a letter to a Swedish colleague in neurosurgery Parkinsun expressed his hope that Sweden would lead the world in passing legislation that would make such a procedure possible. "How wonderful it would be if your country could lead us out of this sticky trend by being the first to pass legislation stating quite plainly that patients are dead when circulation ceases and cannot be started, but that it is permissible to remove organs earlier if past this point of no return. The rest of the world would soon follow your country's legislation like a 'shining beacon' of truth and rationality in the existing fog of semantic distortion'. (Parkinson 1985: 90)

31. On macrosicopic visual inspection of sections of the brainstem more conspicuous fibre bundles and nuclei, consisting predominantly of grey or white matter, can be discerned interspersed with extensive fields of intermingled grey and white matter, collectively termed the brainstem's reticular formation by neuroanatomists. Examined under the microscope with a myelin stain these areas are characterized by an interlacing network of fiber bundles. In this reticular formation fibre bundles interlace in many 
directions and the scattered neurons have diffuse and ili-defined patterns of connectivity. Moruzzi and Magoun, using bipolar electrodes which could be used for both stimulation and pick up from the brainstem, found that it was stimulation of central areas of the interspersed, anatomically ill-defined reticular formation to which the cortical EEG responded with cessation of synchronized high-voltage slow activity and its replacement with low-voltage fast activity. (Moruzzi and Magoun 1949) From the spatial distribution of numerous effective points, combined with the spatial locations of surgical lesions, Moruzzi and Magoun could map an excitable area and project it on the brainstem's anatomy where it included the central core of the brain stem and extended from the bulbar reticular formation forward into portions of the hypothalamus and thalamus in the midbrain. It should be recognized that the ARAS is a functional entity, and not an anatomical one. For further reading on the reticular formation of the brain see Brodal (1957, Cairns (1952), Dell (1963), Hass and Hawkins (1978), Jasper et al. (1958), Magoun (1954: 1963) and Moruzzi (1954, 1972).

32. Neurophysiological research into the role of various parts of the brain aimed at an understanding of the brain mechanisms responsible for consciousness and behavior, both in the healthy animal and human being, and in patients suffering from a bewildering variety of clinical conditions characterized by some form of reduced or disturbed consciousness. Part of the research focussed on the most common of all forms or reduced consciousness, that is: sleep. For early work in this area see Mauthner (1890). For early work on the different EEG patterns produced by the sleeping and waking brain see Berger (1929; 1930), Bremer (1935a; 1935b; 1938) and Adrian and Mathews (1934a; 1934b). For further reading on the subject of brain mechanisms and consciousness see Adrian $e t$ al. (1954), Brain (1958), Cairns (1952), Critchley et al. (1972), Frederiks (1969), Jouvet (1969), Magoun (1963); Moruzzi (1972), Moruzzi et al. (1963), Plum (1972), Plum and Posner (1980), Walshe (1972) and Wolstenholme (1958).

33. Since some remediable conditions may temporarily cause more severe neurological deficits than some irremediable ones, it may be helpful to wait and see how the condition develops before deciding that a disorder is irremediable.

34. Drugs blocking neuromuscular transmissions, for example, impair respiration as well as brain stem reflexes. After the passage of some time during which the patient has to he artificially ventilated, the effect of the drug will wear off and spontaneous respiration and neuromuscular activity will return. Making the necessary exclusions is an active process proceeding on the basis of infurmation obtained from relatives (history taking), on the basis of a physical examination of the patient and, if necessary, additional diagnostic tests. As long as these conditions cannot be pusitively excluded physicians should not consider a diagnosis of brain stem death. Things may sort themselves out after the lapse of some hours or days, one way or the other, resolving doubts.

35. In, what Pallis called, the 'limbo' of the 1960s and early 1970s, decisions were taken to ventilateto-asystole. In those years the plight of the patients' relatives was pitiful. They, the relatives, became emotional hostages of uncomprehending machines. Especially in countries where the next of kin were asked to bear the cost of such care the situation was, according to Pallis, truly appalling. It is bad to ventilate corpses, because of the distress to relatives; because it is bad for the morale of highly trained nursing staff to clean the mouths or treat the pressure areas of patients who are already dead; because it denies limited facilities to those who might benefit from them; and because it is useless in terms of any cost-effectiveness equation. (Pallis 1983)

36. Van Till draws on logic as an 'auxiliary science". The rules of ordinary logic permit the reversal of a true statement, but not all reversals of a true statement are logically correct. The true statement that 'if an animal is a healthy animal then it has a trunk', may be reversed into 'if an animal does not have a trunk then it is not a healthy elephant'. However, it is not correct to argue that 'if an animal has a trunk it is a healthy elephant'; this is incorrect because a tapir also has a trunk but is not an elephant. (Van Till 1975) Consider the true statement: 'if a person is dead he does not breathe spontaneously'. The converse, 'if a person does not breathe spontaneously, he is dead', is not. necessarily true. There are other conditions that cause absence of spontaneous breathing, like, for example, the administration of anaesthetic drugs or an infection with poliomyelitis virus in paralytic 
bulbar polio. The same holds for the following true statement: 'if a patient is dead, then he has an iso-electric EEG, does not breathe spontaneously, does not have a normal spontaneous blood circulation, and cannot be resuscitated'. A reversed version of this statement, namely: 'if a patient has an iso-electric EEG, is not breathing spontaneously, has not spontaneous blood circulation and cannot be resuscitated (to a normal and conscious life), then he is dead', is used in several countries to pronounce death in comatose, respirator dependent patients with a flat EEG. But this reversal is as unreliable as any other logically incorrect reversal in which death is (only) a sufficient cause for the stated consequences, but not a necessary cause. "In theory and in practice each of the early symptoms of normal death, whether occurring separately or together, can have a different cause than death as long as oxygenated blood circulates in the brain'. (Van Till 1976: 804)

37. The mean arterial blood pressure is calculated by adding one third of the recorded systolic blood pressure to the diastolic pressure.

38. This mechanism is very similar to the one occurring in the heart or other muscles when the muscular tonus (the pressure inside the muscle) exceeds the arterial blood pressure, thus obliterating the blood vessels traversing through the muscle and prohibiting the circulation of blood during muscular contractions. This is why the myocardium is provided with oxygenated blood during diastole.

39. It is not possible to overcome an increasing intracranial pressure simply by increasing the arterial blood pressure. In the conditions under consideration here, the rise or fall in blood pressure is often accompanied by an equal rise or fall in ventricular pressure, the cerebral perfusion pressure remaining the same. It is as if in these stages the arterial blood pressure is directly transmitted into the skull because the blood vessels in the brain have lost their capacity to respond with vasodilatation or vasoconstriction. This is sometimes referred to as cerebral vasomotor paralysis.

40. It should also be borne in mind that the cerebral perfusion pressure is a calculated entity, derived from mean arterial blood pressures and mean ventricular pressures. In the brain itself there may be pressure differences and inequal flow of blood in different sections of the cerebral circulation.

41. Strictly speaking a cerebral angiography is not in the interest of the patient. Conclusive evidence of the patient's death can also be obtained by waiting at least 15 minutes after either cessation of the heart beat or respiration, or both. 'To perform angiography is to infringe the right to physical inviolability of the dying or dead patient in the interest of others. (Van Till 1976: 820) In certain conditions such an infringement can nevertheless be ethically and legally acceptable. That is when the patient is in irreversible coma; when previous harmless tests have shown intracranial circulation to be immeasurable minimal or absent; when the patient has given previous consent to be used as an organ donor after his death; and, when his organs will indeed be used for immediate transplantation.

42. Van Till gives no references to published reports on these foetal experiments. However, she is in the possession of documentation bearing witness to the fact that these experiments have been performed, documentation which she showed to the editors of the 24th volume of the Handbook of Clinical Neurology, Vinken and Bruyn, who on Van Till's request agreed to withhold it.

43. Kretschmer's term apallic syndrome, with which he described the simultaneous disturbances of cortical functions, has never gained wide acceptance and was considered to confuse matters more than to clarify the issue. It is used however, in this section, by Pucetti (1988). See Kretschmer (1940).

44. Apallic syndrome is the term coined by Kretschmer in 1940 to describe the simultaneous disturbances of cortical functions resulting from extensive damage to the cortex of the brain's hemispheres. It is taken to refer to the same clinical conditions designated with the now widely used term persistent vegetative state (PVS).

45. N.A. Lassen is son to Henry Cai Lassen, the physician in charge of Blegdamshospital during the 1952 polio-epidemic in Copenhagen.

46. The non-apneic corpse joins the ranks of the animals in similar conditions, since from a moral perspective the main consideration is simply that one can do neither good nor harm to an irreversibly 
comatose being, and that should hold true independently of his species identity. (Puccetti 1988)

47. I borrow this phrase from Kunneman (1986). In Kunneman's Habermasian, communication theoretical vocabulary the notion of a horizon of relevance has to do with the boundary between system and life world, and with problems of (normative) finalization of decisions in system contexts on the basis of norms that have been developed in the life world. I borrow the phrase - not the associated Habermasian system-life world vocabulary - because it conveys the image of an effective boundary between an inside and an outside, that renders outside opposition and criticism less relevant to the inside.

48. For problems concerning the role of the coroner with respect to the extirpation of organs, see Rang and Rozendal (1971), Fassaert (1987), Cremers (1973; 1989).

49. The Law on the Disposal of the Dead also contains regulations concerning graveyards. The growing number of cremations reduced the need for burial space. There was also a significant growth in minority groups having culturally inherited ways of disposing of their dead, that were different from the Dutch. How much latitude should the law provide for these different forms of "death behavior'. Another issue concerned the timespan during which graves were to be maintained and left alone, before being evacuated. Last but not least a parliamentary deregulation committee spotted the bill and proposed some changes. (Van der Putten 1992)

50. A court decision in the field of euthanasia can illustrate this. Until 1973, where statute law was concerned active euthanasia fell under penal provisions 293 and 294 of the Dutch criminal code. It still does at present. In 1971 Bets van Haringa, general practitioner, administered a lethal dose of morphine to her irremediably ill 78 year old mother, on her mother's explicit and repeatedly uttered request. A judicial autopsy was performed on her mother's body and the lethal dose of morphine was discovered. She was prosecuted and accused of violation penal provision 293 of the criminal code, which forbids 'killing on request'. In its verdict the Leeuwarden court accepted, for the first time, several of the justification grounds forwarded by the defence. The court ruled that in certain exceptionable situations an active intervention by a physician, shortening or terminating the patient's life is not unlawful. This is the case when the patient is irremediably ill from disease or accident, when there is unbearable suffering, when the patient acts on his or her own free will, and when the intervention is performed by a physician. Van Haringa should, however, not have administered a lethal dose in one. According to the court, she should have gradually increased the dose of morphine, giving priority to the relief of suffering (pain killing effect of morphine), knowing that the respiration depressing 'side-effect' of the drug would terminate the patient's life. This was the reason why the court inflicted Van Haringa with a conditional sentence of one week imprisonment. This verdict was heralded as a victory by the proponents of voluntary but active euthanasia - during the trial a support group for the accused general practitioner had been formed - and in the same year 1973 the Dutch Society for Voluntary Euthanasia was established. The public prosecutor in the Van Haringa case acquiesced in the verdict and did not appeal. (Enthoven 1988)

51. The cessation of cerebral function could, according to the 24 medical scientists gathered in Geneva, be concluded from: a) loss of all response to the environment; b) complete loss of reflexes and muscle tone; c) absence of spontaneous respiration; d) massive drop in arterial blood pressure when not artificially maintained; and e) an absolutely linear electro-encephalographic tracing recorded under the best technical conditions, even with stimulation of the brain. (Van Till 1970)

52. In the same issue of Medisch Contact Prick argued that, when the brain is dead, the continuance of respiration and circulation by artificial means are organ preservation measures that serve no other purpose than to obtain an optimal donor organ and the best possible chance at a successful transplantation. (Prick 1969)

53. This all-medical committee consisted of $W$. Storm van Leeuwen, clinical neurophysiologist (chairman), J.F. Crul, anesthetist, J.M. Greep, surgenn, A. Kemp, neurologist, S.A. de Lange, neurosurgeon and C.L. van Woelderen (who was editor of Medisch Contact).

54. These hospitals were called Ziekenzorg and De Stadsmaten. 


\section{6}

\section{Programs of action}

\subsection{Introduction}

This study has been an explorative one. It proceeded on the empirical-medical and theoretical level simultaneously. In charting my way through the empirical material of the case studies, making choices for the structure and plot of the various chapters, I passed by several subjects that would have been worthwhile exploring in more detail. My primary interest being in an understanding of modern medicine, I have adopted and appropriated a set of theoretical concepts that I found helpful in developing a theoretical framework that allows me to account of cognitive, technological, moral and social change and obduracy, based on a description and analysis of hands-on, situated practical work. It is now time to compare the case studies and attempt some generalizations and conclusions. Each of the three preceding chapters provided a specific account of a specific episode. Three different maps of three different episodes that can now be juxta- and superimposed in an attempt to develop a more systematic perspective on and understanding of modern medicine. What can we learn, through comparison, from these accounts of different episodes in the history of modern medicine? Is it possible to link the theoretical notions introduced in the second chapter together with concepts added in the course of empirical case-studies in subsequent chapters in order to develop a new way of looking at problems of modern medicine and health care? Will we be able to be more specific about the sources of certainty in times of doubt and change? How is certainty maintained in times of obduracy and stability? In other words, what can we say about the processes by which something is invested with so much certainty that it becomes a seemingly inexorable part of the world?

In this final chapter I will propose the concept of program of action as the specific notion that will allow us to recognize similar patterns in the different case studies. It will allow us to move from one case study to the another, bringing the specific situated developments under a common denominator. Furthermore, it will allow us to link the theoretical notions that have been introduced in the previous chapters together into a more systematic perspective. This systematizing attempt, however, provides only a sketch, an outline. More theoretical work and conceptual 
fine-tuning, remains to be done, but is not the primary aim of this study. The systematizing perspective offered in this chapter is not intended to provide a definite account, neither of the developments described in particular case studies nor of modern medicine in general. To the contrary, I hope that others will find the case studies interesting for other reasons than my own, and apply other analytic perspectives.

I have used the phrase program of action a few times in the previous chapters without giving it much theoretical emphasis. ${ }^{1}$ I will develop the concept in more detail in the following sections, using examples from the case studies. At this point, by way of introduction, I will only sketch some important associations. The term conveys the image of a coherent, detailed plan which has been developed and designed for a particular purpose, for example to provide a particular service or to deal with a particular problem. In this sense, research programs, organ transplantation programs or vaccination programs are instances of programs of action. These programs comprise multiple items which relate in specific ways to each other and to the program as a whole. Individual items and the program as a whole are interdependent. Their identity is determined by their mutual relationships. The notion of a program of action also includes overall program goals to which individual items relate. An example of an explicitly goal-directed program of action is a vaccination program. Individual program items are steps towards ultimate realization of the program's overall goal. In a vaccination program each injection serves to protect the individual, for example against polio. The vaccination of a particular age group like school children serves to protect a generation. But, each new generation has to be vaccinated to attain the overall goal of the program: the eradication of a disease from the population.

Two dimensions of the notion of a program are of central importance. First, that a program can be a plan: a plan of event, jobs and activities that are to take place. including the times at which each thing should happen or be done. In this sense, the program of action is a statement assigning roles to various actors. But it has not been executed (yet). This brings us to the second important dimension of a program: its execution, implementation or performance. It is the implementation of the program that gives presence to what the program tries to establish. Performance realizes, gives existence.

In section 6.2. I will first return to the sensitizing notions that I set out with: mute inscriptions and translator-spokesmen. Using examples from the case studies I will develop the concept of a program of action by emphasizing the circumstances of production of inscriptions. Applying the notion of a program of action, section 6.3 will provide a more fine-grained analysis. In section 6.4 I will explain how the same conceptual framework can account for moral issues. Section 6.5 will address processes and mechanism involved in the implementation of programs of action, resulting in stabilized practices of medicine. Finally, in section 6.6 I will venture, what I think is the most important lesson that can be learned from this study. 


\subsection{Circumstances of production}

In chapter $2 \mathrm{I}$ introduced the notions of mute inscriptions and of translator-spokesmen as two important sensitizing theoretical concepts to start off with. I argued that inscriptions are the raw material scientists work with. It is inscriptions that translatorspokesmen combine and compare, that they aggregate and transform into figures, tables and graphs, and that they have to speak for. What is it the inscriptions say? What is it they represent? The muteness of inscriptions becomes apparent, I argued, when new experimental interventions produce new, and unexpected sets of inscriptions, for which no agreed upon interpretation exists.

When Minkowski, in 1889, successfully performed a total pancreatectomy and kept the dog alive, he produced a new experimental set-up. A new pattern of inscriptions could be observed in pancreatectomized dogs or obtained from their urine, blood and ground up liver. The dogs exhibited polyuria and polydipsia. Doing Trommer's tests Minkowski found their urine to contain sugar which was excreted in large quantities until the animals' deaths. These symptoms signalled a new kind of experimentally inflicted pathological condition. But, what did they represent? The large quantity of urine produced and the sugar in the urine suggested that it was a kind of experimental diabetes. But which pathophysiological mechanisms produced these symptoms?

The notions of mute inscriptions and translator-spokespersons are helpful in grasping the basic, interpretative problem scientists face: the problem of providing a persuasive explanation for experimental results. In an analysis of the way in which Minkowski translated his experimental findings in a hypothesis about an active principle produced by the pancreas and secreted directly into the blood, reference to theory will not be very helpful. There was no pre-existent theoretical framework available to Minkowski that could explain these results. The present-day endocrinological concept of a hormone was not formulated before 1902. In the field of diabetology the dominant theory, in the tradition of Claude Bernard and Eduard Pflüger, explained diabetes mellitus in neurogenic terms, as a condition of the nervous system. This possibility was considered by Minkowski, but it was effectively excluded by the series of partial extirpation experiments inflicting the whole range of surgical damage, however without producing diabetes.

We can begin to understand Minkowski's translation of experimental results into the pancreatic internal secretion hypothesis, not by looking at theories, but by examining the circumstances of production of the various inscriptions, symptoms and biochemical laboratory measurements. An example can illustrate this. Had you been confronted with the picture shown in figures 2.3 and 2.6, but this time without the translation provided by a spokesperson, you would have been asking for the circumstances of its production. How was it produced? Had you learned that the picture was taken with a camera mounted on a microscope, and that the sample under the microscope contained white blood cells mixed with a solution of antibodies labeled with a fluorescent marker, then this would have shaped to a large extent your interpretation of the picture. Apparently the white splashes on the picture indicated the presence of some cellular antigen. Next you would have inquired into the circumstances of production of the antibodies. What substance was injected into the animal that 
produced the antibodies? On the other hand, had you learned that the camera was mounted on a satellite in orbit around the earth, this too would have shaped your interpretation of the picture. Your next inquiries would have been into the alignment of camera relative to the earth. Was it directed towards the earth or was it directed towards space? Similarly, had you been confronted with the inscriptions shown in figures $2.4,2.5$ and 5.10 , without captions, the only possible way that would have allowed a sensible translation of these inscriptions would have been by way of an inquiry into the circumstances of their production. The content of your translation would be different, depending on whether you had learned that the electrodes were on the chest of patient suffering from cardiac arrest in a coronary care unit, on the abdomen of woman during labor or inserted in the cerebral cortex of a monkey whose head has been transplanted to the body of another monkey.

In Minkowski's case the content of his pancreatic internal secretion hypothesis was very much shaped by the design of his series of experiments. Minkowski manipulated the pancreas. He extirpated the whole pancreas, or parts of it, leaving the rest in situ. Or he transplanted a small part of the pancreas on a mesenteric pedicle into a subcutaneous pouch, removing the rest of the pancreas in a second operation (figure 3.2). No diabetes developed as long as the transplant was viable. Next, Minkowski manipulated the arterial and venous blood vessels carrying blood to and from the subcutaneous pancreatic transplant. With all the surgical skill he could muster, Minkowski exposed and ligated the transplant's blood vessels, leaving the rest of pedicle's tissues unimpaired; diabetes developed. ${ }^{2}$ Having effectively destroyed the transplant's connections to the gut, by which route other than through the blood flowing through these blood vessels could the pancreatic transplant exert its influence on sugar metabolism in distant tissues?

Claude Bernard, Eduard Pflüger and several other experimental neurophysiologists also produced a kind of experimental diabetes in laboratory animals. Examining the urine of the animals following an experimental intervention, they also found it to contain sugar. The pattern of the inscriptions they obtained was different from Minkowski's. The glycosuria did not persist until the death of the animal. It was transient. However, the circumstances in which these inscriptions were produced were strikingly different. In Bernard's piquare diabétique the neurophysiologist would thrust a specifically designed lancet through the base at the back of a rabbit's skull into the animal's brain stem. The lancet was designed in such a way that, after penetration of the bone of the skull and the meninges, the fibers of the brain stem would be separated rather than severed. It was only when the lancet was pulled back that it cut through the nervous tissue. In this way Bernard could inflict circumscribed damages to an animal's brain stem, the extent of which could be determined exactly in histological sections prepared after the animal's death. Bernard found that when he damaged the floor of the fourth ventricle in the brain stem, this would be followed by excretion of sugar in the urine: diabetes. This glycosuria would last for a couple of hours and then subside. The same pattern of inscriptions could be produced by inflicting damage in other domains of the nervous system. Experimental neurophysiologists would, for example, expose the large sciatic nerves or branches of the vagus nerve with the same surgical skill and solicitude as Minkowski's in exposing the pancreatic transplant's blood vessels. Simply cutting the nerves already produced the diabetes. Either of the 
two stumps of the cut nerve could now be stimulated electrically. Electrical stimulation of the lower stump, which had no connections with the brain stem, did not produce diabetes. Electrical stimulation of the upper stump, however, produced a transient diabetes. After the glycosuria had subsided the stimulus could be applied again, and again a transient diabetes would develop. The upper stump, which was still in connection with the brain stem, carried the afferent stimulus towards the brain stem where it was translated into an efferent stimulus effecting the release of sugar into the blood which spilled over into the urine. The efferent stimulus could be blocked by cutting the spinal cord above the level where the splanchnic nerves spring from the spinal cord. In these cases electrical stimulation of the upper stump of the cut nerve would not be followed by glycosuria. When the spinal cord was cut below the level where the splanchnic nerves originate the efferent stimulus was unimpaired and glycosuria followed. Exposing and cutting the splanchnic nerves also blocked the efferent stimulus, preventing the appearance of sugar in the urine. In this way the route of the efferent stimulus was traced to the liver, which, as Bernard had shown, was capable of producing and secreting sugar into the blood. These electrical stimuli and responses displayed the same pattern of a reflex arc known from the spinal reflexes. The nervous center in the brain stem where the incoming, afferent stimulus was translated into an outgoing, efferent signal effecting a rise in blood sugar, was thought to be located in the floor of the fourth ventricle and was therefore called the sugar center. Bernard's and Pflüger's experimental manipulation of nerves and the pattern of responses from the animals' bodies shaped to a large extent Pflüger's explanation of these results, and of human diabetes, in terms of a reflex neurosis. In normal circumstances, he thought, this mechanism played an important role in meeting the tissues sugar requirements. Under pathological conditions an abnormal overstimulation of some part of the nervous system could result in an increased release of sugar from the liver causing the symptoms of diabetes mellitus.

Thus, differences in the content of Minkowski's pancreatic internal secretion theory of diabetes mellitus and Pflüger's reflex neurosis theory of diabetes can be traced back to differences in what they were actually doing in the laboratory. The difference in cognitive content can be explained from differences in the experimental circumstances and procedures through which they produced inscriptions. Pflüger's experimental designs featured only nerves, no pancreases, no blood vessels. Minkowski's experiments featured only pancreases and blood vessels, no nerves. Both considered their form of experimental diabetes to be the proper analog to diabetes mellitus in man.

\subsection{Programs of action}

The differences in the circumstances of production of inscriptions between Minkowski and Pflüger are differences in programs of action. A program of action is the list of actions (interventions, measurements) performed by the scientists, and of reactions performed by the bodies under observation. Together these actions are considered to define or constitute a particular entity or condition. It is the performance of a particular agreed upon and stable pattern of actions and reactions that establishes or 
constitutes the identity of an entity. To avoid the reiteration of the whole list or program of action in talking about the entity the program is given a name. Diabetic reflex neurosis and pancreatic diabetes are two different programs of action.

To make full use of the analytical power of the concept of programs of action, it is important to distinguish and emphasize several dimensions or aspects of programs of action. Central to the notion of a program of action is that a program assigns roles and distributes status to those involved in the performance of the program. In an analysis of modern medicine the notion of a program of action will allow us to describe and analyze how a particular program distributes ontological status, cognitive meaning, professional competence, moral value, financial resources. It will allow an analysis of how different programs distribute roles, status, competence and value differently. Furthermore, the notion of a program of action puts emphasis on performance. A program that is not performed, not argued for or implemented, does not accomplish anything. Performance gives presence and establishes existence. Performance makes things happen, it makes things real. Programs of action can be closed, and more or less open-ended. ${ }^{3}$ The former kind of programs does not allow deviations from assigned roles, whereas the latter is not yet able to control the behavior of its actors in a predictable and standardized manner. If we take into account that it takes time to implement a program the notion of a program of action will allow us to describe how a program develops from its inception to its implementation and completion. Thus, we might distinguish between programs that have been realized and programs that have not (yet) been realized.

When Minkowski performed his first total pancreatectomy in 1889 no program of action called pancreatic diabetes existed, whereas the diabetic reflexive neurosis program was several decades old. Following Bernard's piqûre diabétique experimental neurophysiologists had developed the skill, acquired the capacity, to elicit (symptoms characteristic of) diabetes in animals through specific manipulations of the nervous system. This capacity to perform these experimental interventions and to generate this specific pattern of responses from animal bodies required a laboratory, surgical skills, extensive knowledge of species specific nervous anatomy, anesthesia, bladder catheters to collect urine, chemical reagents to do Trommer's test, etcetera. It was this capacity to perform this program of action that backed up Pflüger's rendering of the pathophysiological mechanisms involved in diabetes mellitus in man. The capacity to perform this program of action was an important source of certainty concerning the existence of reflex mechanisms and their involvement in diabetes mellitus: the program made them happen. The program gave them presence. The program made them exist. Without the capacity to perform this program diabetic reflex neurosis would never have existed.

Prior to Minkowski's total pancreatectomy in 1889 pancreatic diabetes did not exist. It took Minkowski several years to develop the program of action that brought pancreatic diabetes into existence. He had to improve on his surgical technique to prevent the dogs from dying too soon after the operation. He had to learn how to prevent duodenal necrosis, how to make a subcutaneous transplant with intact vascularization, until the dogs invariably developed (the pattern of symptoms characteristic of) diabetes when the last remnant of pancreatic tissue was removed from the animal's body. Bliss credits Minkowski for the 'theoretical discovery' of insulin. I 
would say that Minkowski is to be credited for the development of the capacity to perform the program of action called pancreatic diabetes. He is to be credited for the elimination, through the design of the program, of all the possible routes through which the pancreas' influence on sugar metabolism might be mediated, all but one: the blood. In the process of acquiring this capacity Minkowski turned what began as an experiment into a demonstration. It was this capacity to perform the program of action for pancreatic diabetes that Frederick Allen was referring to when, in 1913, he wrote that attempts to capture the pancreas' internal secretion in a pancreatic extract continued because of the strong 'theoretical inducements'.

The basic tenet of a constructivist approach to science and technology implies that what we call 'reality' is the upshot of heterogeneous, temporarily stabilized practices. For insulin, this would mean that it first came into existence after it was discovered by Banting and Best in Toronto and stabilized in worldwide, internationally standardized therapeutic practices. In other words, there is a sense in which insulin could be said not to have existed before its discovery. At this point, we can be more specific about this sense in which insulin did not exist before its discovery. Insulin did not exist because nobody had developed the capacity to perform the stable, repeatable program of action that would bring insulin into existence; a program of action that would give perceptible, visual presence and identity to insulin. Between 1889 and 1921 insulin was an entity without a program of action. That is why it was a hypothesis. Before 1889 it was not even a hypothesis.

All attempts to capture the pancreatic internal secretion in an extract can be seen as a struggle to acquire the capacity to develop and perform a program of action that would unambiguously establish insulin's existence, its presence in an extract and its identity. This was not accomplished before January 1922 when Leonard Thompson was successfully treated with a pancreatic extract Collip had prepared. But even after this date this capacity was temporarily lost by the end of February of the same year resulting in the first insulin famine and the first human diabetic patient dying for lack of insulin. Between 1889 and 1922 we have seen the gradual emergence of a program, but it was still shifting and changing. There was uncertainty about exactly what items, what performances, should be on the list. There was agreement about some items that could be inferred from Minkowski's pancreatic diabetes: the extract should be able to counteract the consequences of a total pancreatectomy. In other words, the extract should be able to abolish glycosuria. In many cases no such response was found, or if it was found that the extract also elicited other responses from the animal's or patient's body, like fever. Often there were other circumstances in the setting that could possibly explain the observed responses, including the disappearance of glucosuria, like for example a change in diet. New program items (longevity. restoration of glycogen formation, blood sugar reduction in normal rabbits) were proposed, others were given lesser significance because they were not very helpful in attributing the observed responses to the sought for substance. But even after the successful treatment of Leonard Thompson insulin's program of action changed. Pancreatectomized dogs were deleted from the program and replaced by rabbits and mice for purposes of testing the potency of new batches of extract and standardization between production facilities in various countries. It is from the capacity to perform this specific, internationally standardized program of action that the certainty derives 
with which we belief in the existence of insulin. Insulin's program is maintained through the work of international and national institutions for biological standardization of hormones and other biologically active substances. Parts of the program are performed everyday; physicians treating diabetic patients and diabetic patients having developed the knowledge and skill to treat themselves. How could we doubt insulin's existence? Insulin exists wherever people have learned how to perform the program. It will continue to exist as long as the program is maintained. Insulin's effectiveness and safety can only be maintained when all the actors involved in this therapeutic program of action play her assigned roles. Pharmaceutical companies producing insulin have to live up to international standardization agreements to make sure that a specific volume of redissolved insulin contains a specified amount of international units. The patient too has to perform her assigned role, balancing her daily carbohydrate intake and exercise pattern to the amount of insulin injected. The patient's willingness to perform her assigned role in a therapeutic program is usually called patient compliance.

Whereas the cases of pancreatic diabetes and insulin are examples of how programs of action have to be developed from scratch, the 1952 polio-epidemic provides an example how different programs of action can exist independently of one another in different places. When at a certain moment in time they come to share the same space they turn out to be relevant to each other.

On the wards of Blegdamshospital, Copenhagen. August 1952, a number of programs of action were in place that could be grouped together because they showed a considerable overlap. One could also consider them to be sub-programs or variants of one program of action called poliomyelitis anterior acuta. When the patient's body temperature was measured it turned out to be increased. When physicians tested the muscles of the arms and legs for strength patients could offer no resistance (paralytic polio). Paralysis of the muscles of the throat, causing difficulties in swallowing and breathing, would identify the patient's condition as bulbar polio. When this condition persisted it developed further: the skin would turn blue (cyanosis), the blood pressure would rise, a measurement of total $\mathrm{CO}_{2}$ content in plasma would yield a high value. This specific pattern of actions and reactions was terminal bulbar polio.

In the months and years before Ibsen was consulted in the 1952 polio-epidemic he had acquired the capacity to perform a program of action designated as $\mathrm{CO}_{2}$ accumulation. The inclusion of Brinkman's Carbovisor into the program had greatly enhanced Ibsen's capacity to visualize the accumulation of $\mathrm{CO}_{2}$ in the blood as a result of compromised ventilation. However, the program of action that constituted and gave presence to $\mathrm{CO}_{2}$-accumulation had mainly been performed in research laboratories working on problems of respiratory physiology, and in operating rooms. Prior to 1952, the program had never been performed in Blegdamshospital's clinical wards. Not with the same persuasive force as Ibsen's demonstration on the 12 year old girl.

During the 1940s Blegdamshospital had treated patients with bulbar polio in its wards, but always with disappointing results, although mortality had never been so high as during the first three weeks of the 1952 polio-epidemic. During the 1944 epidemic Blegdamshospital's physicians had tried to explain why so many of the patients with bulbar polio died while being treated with negative pressure respirators. In 1946 Ellen Margrethe Nielsen (1946) reported that patients were ventilated with a 
frequency of $18-24$ in- and expirations per minute, which is somewhat higher than the normal frequency of $12-14$ expirations per minute. At the normal rate patients felt they were suffocating. At the slightly increased frequency patients felt comfortably. Measurements of oxygen uptake in patients ventilated at this frequency showed normal values. However, Nielsen suspected these patients to be hypoventilated because the increase in frequency made the ventilation 'shallower', that is: the volume of air exchanged with each in- and expiration was smaller. Total $\mathrm{CO}_{2}$ in plasma was found to be increased. Nielsen forwarded $\mathrm{CO}_{2}$-accumulation as a possible cause of death in these patients, suggesting that the increased value of total $\mathrm{CO}_{2}$ in plasma did not represent a (frequently encountered) metabolic alkalosis, but a (rarely encountered) respiratory acidosis. If this was the case the blood's $\mathrm{pH}$ would probably have decreased, she argued. However, measurements of the blood's $\mathrm{pH}$, which would have completed the performance of the program of action for $\mathrm{CO}_{2}$-accumulation in Blegdamshospital's wards, were not included in the investigations conducted during the 1944 polio-epidemic. $\mathrm{CO}_{2}$-accumulation was a 'theoretical' possibility a prudent physician should keep in the back of his head, but which was (almost) never encountered in the clinic. $\mathrm{CO}_{2}$-accumulation had no real existence in Blegdamshospital's wards, not until Ibsen gave it presence, on August 27, 1952, by performing its program of action. One day later Astrup supplied evidence of the acidosis.

Performance brings to mind that the performance of a program of action presupposes that there is an audience. The circumstances of Ibsen's treatment of the 12 year old girl, and his subsequent demonstration of $\mathrm{CO}_{2}$-accumulation, underlines the significance of the physical presence of an audience. Minkowski performed his experiments in the relatively secluded and private space of his research laboratory, to which only a limited and selected number of people had access. The same holds for Banting's and Best's experiments in Toronto, 1921. Their audiences were never physically present; they never came into the laboratory to see the pancreatectomized dogs. They met their audiences on medical and scientific meetings like the informal meeting of Physiological Society Club on November 14, 1921, or the New Haven meeting of the American Physiological Society on December 30, 1921. Or they addressed their audiences in the medical and scientific journals in which they published their results. On these meetings and in these journals Minkowski and Banting and Best represented the pancreatectomized dogs. They spoke for them, but they also gave presence to the dogs, although in another, translated material form: in tables and figures in which the authors had gathered the inscriptions obtained from the animals in the laboratory. The privateness of the laboratory provided them with the space and time that was necessary to give shape to and acquire the capacity to perform the programs of action of pancreatic diabetes and insulin respectively: to master and control the circumstances for the production of a specific pattern of inscriptions. They could proceed by trial and error. Failures, dogs dying, were disappointing for themselves, but they did not immediately hurt the research program as such. As long as the laboratory was available they could continue their work, unlike Zuelzer, who lost his laboratory twice.

There was no private space for Ibsen when he treated the 12 year old girl on August 27, 1952, on Blegdamshospital wards. His audience was physically present. $\mathrm{He}$ had to go public immediately. He had the advantage that he had already acquired 
the required skills and capacity, although he had never done it on a polio patient. The physical presence of the audience made him vulnerable to failure, especially under the trying circumstances of the epidemic. Considering the reservations with which his proposals had been met, it is doubtful whether he would have been given another chance had he failed on the 12 year old girl. But he did not fail. For a life audience, Ibsen provided convincing proof of the effectiveness of positive pressure ventilation, of his competence as an anesthetist and of the truth of the pathophysiological explanation he had offered for the death of bulbar polio patients treated in negative pressure respirators. For Ibsen Blegdamshospital turned out to be a theater of truth and competence. The success of Ibsen's demonstration before a life audience created an unprecedented degree of perceptive immediacy for the realness of the $\mathrm{CO}_{2}$-accumulation and the superiority of positive pressure ventilation. Among the life audience was Henry Cai Lassen, chief physician at Blegdamshospital. Lassen had the authority and power to change treatment policies and begin implementation of the new therapeutic program of action immediately.

The first time when Banting and Best tried to perform their insulin program of action for a life audience, on Leonard Thompson in Toronto General Hospital, they failed to produce convincing results and were referred back to the laboratory. Banting's and Best's extract produced only a very moderate reduction of blood sugar levels. The excretion of ketone bodies was not abolished. There was no beneficial effect on the body's general clinical condition and a sterile abscess developed on the site of injection. A fortnight later Collip was more successful with the extract he had prepared. Blood sugar levels dropped and glycosuria and ketonuria disappeared completely. The boy's general condition improved. Collip's mastery of the program of action was better than Banting's and Best's. The perceptive immediacy that Collip's demonstration produced for a life audience of physicians and diabetologists produced more persuasive force than Banting had been able to generate at the New Haven conference of the American Physiological Society with the combined and aggregated experimental results on dogs.

For each of the four definitions of death discussed in chapter 5 it would be possible to list the diagnostic program of action that would establish the death of a human being. They would be strikingly different though. The program of action that would have to be performed to establish total death would involve some check for the absence or presence of heartbeat and spontaneous respiration, either through visual inspection, palpation (arterial pulse in the neck or groin) or auscultation of the thorax. On lifting the eyelid the pupil should be wide and non-responsive to the light. The resistance offered by the eyeball to a gently applied pressure would be reduced due to a reduction in intraocular pressure. This program of total death shares only the pupillary reflex with the program of action for brain stem death. Heartbeat and respiratory movements, essential items in total death, are of no significance in brain stem death. The core of the program of action that established the death of the brain stem consists of tests for five brain stem reflexes, and a delicate (disconnection) test for apnoea. The test for apnoea requires pre-oxygenation of the patient with $100 \%$ oxygen and an increase in carbon dioxide content of the gas mixture to provide a strong 'drive' for the respiratory center. Whether brain stem reflexes and apnoea are present or absent is of no relevance for the establishment of neocortical death. In 
neocortical death recording an EEG is of central importance, as well as other techniques that allow an estimation and assessment of the circulation of blood to the cerebral hemispheres. Neocortical death does not require the total absence of blood circulation within the confines of the skull. This latter requirement is essential for the establishment of whole brain death.

The diagnostic programs of action that establish total, brain stem, neocortical and whole brain death are intimately related to or are part of other programs at a discursive level. These discursive programs of action provide the verbal and discursive translation of the pattern of inscription produced whenever the diagnostic programs are performed. In the case of various diagnostic programs that establish death, these discursive translations are provided by the definitions of death outlined in chapter 5 . These definitions of death are as many discursive programs of action.

More than in any of the other case studies, a comparison of the various definitions of death reveals how each of these programs of action distributes ontological status and moral value in a specific way. A definition of total death, the 'traditional' definition of death supporting heart-lung criteria, does not recognize the death of (parts of) the brain as the end of human life. The artificially ventilated, heart-beating patient with a dead brain is still a living person and member of our moral community, deserving of the best possible medical care and protection by the law. It is not until the irreversible cessation of heartbeat and respiration that the ontological status of the body changes from a living, although dying, human being into a corpse. The ontological and moral status of a corpse is different from that of a living human being. We can do other things to a corpse than to a living person. The corpse is not without moral status, however. We treat corpses with respect and as much as possible in concordance with the wishes of the deceased. The body or parts of it cannot be claimed by others without the explicit consent of the deceased. Hence, the exact location of the boundary between human life and death carries moral and legal relevance. A definition of total death, opposing the shift towards brain related criteria of death, argues that we should treat future dying patients as we have treated them in the past. We should not, in other words, consider burial, cremation or the extirpation of organs for transplantation purposes at another, earlier moment in the proces of dying, than we would have done before the development of intensive care units and solid organ transplantation.

Each of the brain related definitions of death moves the boundary between human life and death forward: in cases where the process of dying is halted, permanently or temporarily, through medical interventions. An artificially ventilated, heart-beating patient with a dead brain stem, who would be considered to be alive in a program of total death, is considered to be dead in the program of action of brain stem death. Continued intensive treatment of brain stem dead patients in the past, in a number of hospitals throughout the world, was never followed by recovery. None of these patients survived. Electrocerebral silence and irreversible cardiac arrest always followed within hours after the death of the brain stem had been identified. Gathered in epidemiological statistics and represented in tables like the one in figure 5.4, these 'facts' warrant a change in the ontological and moral status of the artificially ventilated, heart-beating brain stem dead patient: from a living patient with a very bad prognosis into a corpse. This redistribution of ontological and moral status is accompanied by a redistribution of human distress, of limited intensive care facilities and 
financial resources, and of life-expectancy for thousands of patients for whom organ transplantation is the only option to survive.

According to the program of action of brain stem death the irreversible destruction of the brain stem is necessary and sufficient to pronounce death. Irreversible destruction of the cerebral hemispheres is not necessary and not sufficient. Patients with irreversibly destroyed hemispheres who have a viable brain stem live. These patients are able to breath spontaneously, to maintain their body temperature and blood pressure, to swallow food. Due to the viability of the brain stem's ARAS, they have periods of wakefulness in which their eyes are open. These patients are in a persistent vegetative state, which is a condition of the living. We consider them to be alive as much as we consider hydranencephalic newborns and anencephalic newborns to be alive. Questions about therapeutic abstinence or active interventions, which would result in the termination of these conditions, are questions about passive or active euthanasia.

Proponents of the program of action of a neocortical definition of death argue that patients in a persistent vegetative state are dead. They argue that their argument is a matter of ontological clarification. When the capacity for higher (neocortical, hemispheric) brain functions has been irreversibly destroyed, then that what is essential for a human being to being alive has been lost. The person is dead. The body does no longer embody a sentient human being. The body is already a corpse that can not be killed or harmed in any other way.

Brain stem death does not require the irreversible cessation of all neurological activity in the cerebral hemispheres. Hence, it does not require a flat EEG in its diagnostic program of action establishing death. When the brain stem is dead, some individual neurons, or groups of neurons, firing in the cerebral cortex, have nothing to do with the persistence of human life, or with the presence of some residual sentience. Furthermore, an intensive care unit with its electronic machinery, beepers and health care personnel wearing nylon clothing or underwear creating a lot of static electricity, is hardly a proper environment for the recording of a silent EEG. Incidental spikes in an otherwise flat EEG might well be artefacts.

Neocortical death does not require the irreversible destruction of the brain stem. Even if the brain stem's ARAS is able to induce a state of arousal, the absence of viable neocortical tissues makes any content of consciousness impossible. Neocortical death attributes no more status to the brain stem reflexes than brain stem death attributes to the spinal reflexes.

A definition of brain stem death draws heavily on several decades of experimental neurophysiological research. This research was spurred by an interest in the problem of consciousness, its anatomical and physiological basis and the bewildering wide variety of clinical conditions of acute, subacute or chronic alterations in consciousness. A definition of neocortical death draws on a variety of theories about personal identity. The resources on which they draw differ as much as the way in which they distribute ontological status differently. They link the boundary between human life and death to the irreversible destruction of different areas of the brain. By doing so they trace the boundary between pronouncing death and euthanasia differently. This boundary is of great importance and consequence for the permissibility of the extirpation of organs for transplantation purposes. 
In the program of action of whole brain death neither a brain stem dead nor a neocortically dead patient would be considered to be dead. Whole brain death does not require the irreversible cessation of heart and lung functions, but it does require the irreversible destruction of all neuronal activity within the confines of the skull (brain stem, midbrain and cerebral hemispheres). Neither brain stem death nor neocortical death excludes beyond all doubt the, maybe extremely remote possibility that their is some residual capacity for consciousness and suffering left. It is exactly because the pronouncement of death changes the ontological and moral status of the patient, that we should demand from physicians that they should take the full burden of proof. The pronouncement of death strips from the patient all the legal and moral rights attributed to a member of our legal and moral community. Physicians pronouncing death should prove beyond all doubt that the patient has lost the capacity for consciousness. The patient should be freed from the burden to prove that she has retained it. The only way for physicians to provide the required proof is to demonstrate the absence of intracranial circulation of oxygenated blood in the brain for more than 15 consecutive minutes. The only way to demonstrate this conclusively in artificially ventilated, heartbeating patients is by means of serial angiography of the blood vessels in the brain. Only when intracranial circulation is absent during 15 consecutive minutes can death be pronounced. At his point, when the extirpation of organs for transplantation purposes is considered, life-support systems change into organ preservation measures.

Minkowski's internal secretion theory of diabetes and Ibsen's redefinition of the terminal bulbar polio as a ventilation problem also share the characteristics of discursive programs of action distributing ontological and epistemological status differently. Minkowksi's internal secretion theory was the discursive translation of the pattern of inscriptions produced in the performance of the program of action realizing experimental pancreatic diabetes in dogs. By juxtaposing and comparing the pattern of symptoms and laboratory results observed in the animals with those found in human diabetics Minkowski linked the experimental research laboratory and the clinic, tracing a boundary between what he considered to be diabetes proper (in which glycosuria persisted until death) on the one hand, and transient glycosurias on the other. Pflüger, drawing on a wide range of neurophysiological experiments and on Aristotelian philosophy concerning teleological causes, thought that a variety of neurological affections could cause diabetes. Minkowski's theory of internal secretion endowed a malfunction of the pancreas with the status of a cause. The brain stem's sugar center, which was of central importance in Pflüger's diabetic reflex neurosis, was a non-entity in Minkowski's world of pancreases and blood vessels. Minkowski's hypothetical internal secretion of the pancreas could have no ontological status, no existence, in Pflüger's world where only the nervous system could influence metabolic functions in distant tissues and organs. Thus, Pflüger's and Minkowksi's respective translations of the pattern of symptoms observed in human diabetes distributed ontological status and causality differently. They also attributed different cognitive relevance to the duration of the glycosuria.

One of the most appealing aspects of Minkowski's theory of internal secretion was that it suggested a future therapeutic program of action for the successful treatment of diabetes mellitus in humans. If Minkowski was right it should be possible to capture an animal's pancreatic internal secretion in an extract, which then could be 
used as an exogenic source to substitute the diminished or lost endogenic production in human diabetics. In a time in which patients suffering from severe forms of diabetes were put on a dietary regime that would starve them to death if the diabetes did not kill them first, this new therapeutic program of action implied in the theory of internal secretion contributed significantly to the theory's persuasive strength. Minkowski had worked out a program of action that established experimental pancreatic diabetes in dogs. However, the therapeutic program that was implied in Minkowski's pancreatic diabetes had still to be developed. The combined force of the design and capacity to perform experimental pancreatic diabetes and the promise of an effective treatment for human diabetes was tested and balanced for several decades by the negative force of cumulating failures to master the program of action that would establish insulin's existence, presence and identity.

Ibsen's discursive translation of the pattern of symptoms and inscriptions observed in the polio victims examined in Blegdamshospital's wards and autopsy room also entailed a therapeutic program of action. Ibsen argued that the terminal stage of polio was brought about by two different pathological conditions: the neurological damage caused by the virus and the accumulation of $\mathrm{CO}_{2}$ in the blood. Arguing that it was the $\mathrm{CO}_{2}$-accumulation that killed them, this definition of the problem suggested the way in which it could be handled. If it was a ventilation problem, proper ventilation should be able to save many patients' lives. Ibsen's discursive program of action challenged the reigning interpretation and definition of the problem of terminal bulbar polio in terms of an overwhelming viral infection destroying vital portions of the brain. In the absence of effective anti-viral agents that could kill the virus in the patient's body, and of an effective polio vaccine that could prevent the spread of the disease, this way of framing the problem had fostered considerable therapeutic defeatism. By demonstrating that it was the $\mathrm{CO}_{2}$-accumulation that caused the high total $\mathrm{CO}_{2}$ values in plasma, the high blood pressure and the shift in the acid-base equilibrium, Ibsen made a case for his redefinition of the epidemic's high mortality as primarily a ventilation problem (for which adequate ventilation by means of manual positive pressure ventilation offered the solution). The positive pressure ventilation was in fact part of the program of action that established the $\mathrm{CO}_{2}$-accumulation. Ibsen was only able to demonstrate the $\mathrm{CO}_{2}$-accumulation after he had successfully applied the manual positive pressure ventilation and stabilized the girl's clinical condition. It was the Pentothal that forced or transformed the struggling girl into the docile body that performed its assigned role in $\mathrm{CO}_{2}$-accumulation's program of action, producing the pattern of symptoms and inscriptions that Ibsen had hoped for. Had Ibsen failed to ventilate the girl properly, he would neither have been able to demonstrate the $\mathrm{CO}_{2}$ accumulation. In his immediately perceptible demonstration of $\mathrm{CO}_{2}$-accumulation on the twelve year old girl, Ibsen also demonstrated with the same perceptive immediacy how proper ventilation could be provided and that he and other anesthesiologists held the capacity to perform this therapeutic program of action. Who could have resisted such persuasive force?

Because the therapeutic program was included in, and prerequisite for, the demonstration of $\mathrm{CO}_{2}$-accumulation, and also because the required equipment was cheap and sufficiently available in Copenhagen's hospitals and from manufacturers and suppliers, they could move so fast towards the implementation of this therapeutic 
program for all the patients with bulbar polio. Under the direction of Lassen, additional equipment and more anesthetists were brought in to play their assigned roles. Medical students were brought in to squeeze the bags. The students had to monitor the patients' clinical condition as well as the proper functioning of the equipment. The students' performance in the program was monitored by the laboratory, taking regular blood samples (from patients) for $\mathrm{pH}$ and total $\mathrm{CO}_{2}$ in plasma measurements. If necessary a student's performance was adjusted. The student's role in the program became well defined and standardized to such a degree that it was possible to replace the student by a machine, the 'mechanical student', the mechanized positive pressure respirator. ${ }^{4}$ The epidemic brought the numbers of bulbar polio patients. Blegdamshospital's function as an epidemic hospital and center of expertise, concentrated these patients in one place, allowing the almost 'physical' experience of a dramatic mortality rate reduction.

\subsection{Speaking for the weak}

In the previous section I focussed on similarities between the three case-studies. But there are apparent differences too. The case studies differ to the extent in which moral issues are explicitly at stake. The difference is not that the debate on the redefinition of human death is a moral issue and that the controversy about the existence and identity of insulin is not. The difference is that the development of intensive care units and solid organ transplantation challenged the existing moral order whereas Minkowksi's internal secretion theory of diabetes did not. The polio-epidemic challenged the cognitive and technical order of medical practice concerning the treatment of polio. Ibsen, a free lance working anesthetist, also challenged the medical social order and disciplinary boundaries between various medical specialties. But he did not challenge a moral order. This does not imply that there was no moral order. It only means that, because moral issues were not explicitly at stake, it is harder to see how a moral order was (re)created or maintained. Yet, on close reading, glimpses of the moral order of turn of the century diabetes research, and of 1952 bulbar polio treatment can be discovered. Pflüger and Minkowski disagreed about many things. They did not disagree about the fact that is was alright to sacrifice animals in research laboratories for the benefit of human diabetics (or for the benefit of human science). Their respective research programs distributed moral status in a specific, but similar way. They agreed upon who should (and could) be the primary beneficiaries and what might be used in instrumental ways to achieve these benefits. ${ }^{5}$ The people who would take issue with the use of animals in experimental research are to be found in anti-vivisectionist movements. This is one of the channels I have not explored in my empirical research, but, to use the same metaphor (of the arctic grail), the entry of the channel opens up there where we find scientists responding to anti-vivisectionist criticism. Claude Bernard, for example, in his An Introduction to the Study of Experimental Medicine (1865) recalls that vivisection in all ages has met with prejudices and detractors. And although he does not intend to answer the arguments of detractors of vivisection, he does consider a few general questions, one of them being the question 
concerning man's right to make experiments on animals and vivisect them. Bernard wrote:

As for me, I think we have this right, wholly and absolutely. It would be strange indeed if we recognized man's right to make use of animals in every walk of life, for domestic service, for food, and then forbade him to make use of them for his own instruction in one of the sciences most useful to humanity. No hesitation is possible; the science of life can be established only through experiment, and we can save living beings from death only after sacrificing others. ... [I]t is essentially moral to make experiments on an animal, even though painful and dangerous to him, if they may be useful to man. (Bernard 1865/1950: 257)

According to Bliss (1982) Toronto also knew an active anti-vivisectionist movement, causing Macleod and other medical researchers a fair amount of trouble. Macleod set up the university's Surgical Research Committee as a line of defence to head off the anti-vivisectionists. When Banting, dissatisfied with the little room in the physiology building, started using better operating facilities in another building, Macleod warned him to be careful. No one should see him transporting operated animals from one building to another on campus.

August Krogh, the Danish Nobel laureate who initiated the production of insulin in Denmark and took part in its international biological standardization, also delended vivisection on animals in experimental physiological research. Vis-à-vis the Danish anti-vivisectionist movement's opposition to a large grand from the Rockefeller Foundation - which in their view, would establish an international center for vivisection in Denmark -, Krogh used the example insulin to emphasize what vivisection was capable of. The discovery of insulin rested on vivisection, from Minkowski's work in 1889 to Banting's and Best's work in 1921 and 1922. According to Krogh (1924), insulin's discovery would have been impossible without vivisection. Physiologists' moral legitimation of vivisection rested on two arguments:

... the truth about an organism's functions cannot be discovered without [vivisection], and ... the suffering of a relatively limited number of animals yields results which alleviate the suffering of a many times larger number of human beings and animals. (Krogh 1924: 32)

These examples, scientists' responses to anti-vivisectionist criticism in the public domain, allow us to recognize the moral order, and document how moral status was distributed in diabetes research. They also indicate the existence of an effective boundary between the inside of the private space of the laboratory and the outside of the public domain. This inside-outside boundary did not immunize the scientists from criticism, but it made the criticism less relevant: a nuisance that takes too much of their time to deal with but that does not stop them from pursuing their research program.

Macleod and Krogh were also actively involved in negotiating the conditions and institutional arrangements under which commercial firms and pharmaceutical industries would be allowed to produce and sell insulin. They tried to make sure that not one firm would get a commercial monopoly on insulin, and that the revenues on the sale 
of insulin would partly flow back to diabetes research. For this latter purpose Krogh set up the Nordisk Insulin Fund. For them it was clear who should benefit from the advances in medical science.

In the case of the 1952-polio epidemic it is even harder to find evidence of the moral order, because it was not challenged. The public in Copenhagen, as well as the municipal and health care authorities supported the work of the physicians in Blegdamshospital without reservations. Nobody raised questions about, for example, the experimental nature of positive pressure ventilation as a new treatment for bulbar polio, or whether informed consent had been obtained. Besides the fact that in Ibsen's view the treatment was not experimental, doing what he had learned to do in the operating theater, it would have been considered immoral even to raise that question. They had no choice. Without Ibsen's treatment of the twelve year old girl, this girl and many others would have died. There was no disagreement about the question whether trying out Ibsen's suggestions was morally responsible. It was. There was a moral order, morally responsible action, but no controversy. It was not until a few months after the epidemic, that some questions arose about the new treatment. By this time it became clear that some patients would be dependent on artificial ventilation for the rest of their lives. Was it a good thing to attempt to save some of the patients' lives if this implied life-long respirator dependency? The surviving patients however, whose ARAS and cerebral hemispheres were vital, could speak for themselves on this matter.

The pre-discovery work on insulin and the 1952 polio-epidemic in Copenhagen were excellent strategic research sites for the study of cognitive and technological change. They were less revealing on moral issues, for which, however, the controversy on brain death made up. Unlike the first two case studies the third one revealed an abundance of inscriptions from which to build an account of the morality of the various programs of action.

How can we explain the difference in the extent to which moral issues were explicitly at stake in the respective case studies? To find an answer we have to look at the way in which the boundaries of our moral and legal community are defined. The human diabetic patients who were to benefit from experimental physiological diabetes research were well within the boundaries of the moral community. The animals that were sacrificed in the laboratories were well outside the boundary. The same holds for the polio victims suffering from bulbar polio, they were well within the confines of the moral community. Severely brain damaged, artificially ventilated, heart-beating but comatose patients were borderline cases. Their numbers increased with the development of respiration centers and intensive care units in the 1950s and 1960s, where they were artificially maintained in this condition for unprecedented lengths of time. A borderline case is very nearly unacceptable as a member of a class or group because it is doubtful whether it has the features or qualities necessary to be included in that group. The brain damaged patients on life-support did not speak for themselves. They were mute, and other people spoke for them, represented and translated them. Other people spoke about their ontological status, whether they were still alive or already dead. They spoke about the significance of one organ over another. They also spoke about the artificial maintenance of vital functions, whether that mattered or not. The representatives spoke about whether these patients could suffer, about 
what would be in the patient's interest, and about the question whether or not they had or could have interests.

Various representatives provided different and competing translations and that is what makes a controversy. According to Callon (1986a: 219), "a controversy is all the manifestations by which the representativity of the spokesman is questioned, discussed, negotiated, rejected, etc.'. The questions now are: Is there a sense in which this controversy is also a moral controversy? What parts of a representative's translation do we think of as being a moral judgement or a moral argument? Is it possible to bring these instances under a common denominator?

The controversy on brain death supports the conclusion that we tend to see the translator-spokesperson in a moral capacity, as a moral agent, when she speaks on behalf of those who cannot speak for themselves, against an infringement of basic interests and legal rights. Thus, we would call an argument against the premature pronouncement of death a moral argument. An argument against an undue delay of the pronouncement of death, depriving patients in need of a transplantation a chance at survival, is also a moral argument.

Elsewhere (Wackers 1988) I have suggested that we can use Jensen's basic moral principle of the responsibility for the weak as an interesting point of departure for an account of moral values and moral action. ${ }^{6}$ Jensen (1987) conceives of values as model activities or paradigmatic examples of responsible action and ethical importance, serving the role of models and paradigms in ordinary human life. We are acting morally responsibly towards other individuals or groups who in a particular context are weaker than us, if we try to act from their point of view, and support them. In other words, speaking for and acting on behalf of the weak is morally responsible when it is pursued from the perspective of the weak and aims at their support. ${ }^{7}$

The weak in the first two case studies were the human diabetics and polio victims suffering from lethal diseases. Minkowski's, Zuelzer's and Banting's experimental research programs aimed at the development of an effective treatment for diabetes mellitus. Maybe you frowned when reading that Zuelzer moved so quickly from animal research to human diabetics, his experimental results being ambiguous at best. Yet, the pancreatic extract Zuelzer had prepared entailed the only chance for these patients to recover and survive. He would not deprive them of that chance. Forschbach, using Zuelzer's extract, also moved to human patients, but he backed off when the transient useful effect of the extract turned out to be closely associated with toxic reactions. Continuing treatment with Zuelzer's extract was not in the interest of these patients. According to Bliss, Duncan Graham, head of the department of Medicine at Toronto General Hospital, protected the patients in the hospital wards under his control for premature experiments and investigations. He wanted to make sure that any experiments would be for the patients' benefit, and not for the benefit of too eager a scientist. The successful treatment of Leonard Thompson with Collip's extract did not only finally give persuasive presence and existence to insulin, it also realized the moral promise entailed in Minkowski's internal secretion theory which had legitimated the suffering and sacrifice of many animals. It is here where medicine demonstrated its potential to cure previously lethal diseases; the fulfillment of a physician's dream in the realization and implementation of an important therapeutic program of action, the morality of which went, and still goes, unchallenged. 
The same holds for the successful application of positive pressure ventilation in the 1952 polio-epidemic in Copenhagen. As part of the new therapeutic program of action positive pressure ventilation was definitely a life-saving technology. The morality of the program's goals and means went unchallenged. The medical community recognized and acknowledged the competence and skills of the physicians who had developed the capacity to treat bulbar polio so effectively in the operating theater. New jobs were created for anesthesiologists. They were given their own departments. Anesthesiologists headed newly established respiration centers and intensive care units. In these units all available means were assembled to save the lives of patients who would otherwise die.

In a paradoxical sense Minkowski's and Ibsen's programs of action, the way in which they defined the problem and the therapeutic programs implied, derived strength from the weakness of patients in the past and in the future. The persuasiveness of Minkowski's internal secretion theory of diabetes mellitus derived not only from its technical implications. Persuasiveness also derived from the weakness of innumerable patients who had suffered and died from diabetes mellitus. By carefully juxtaposing the pattern of symptoms and inscriptions observed in depancreatized dogs with the pattern of symptoms observed in human diabetics, Minkowski gave presence, in his papers describing the experimental work on dogs, to the group of weak people who would have been the primary beneficiaries of the realization of the implied therapeutic program of action, had they not been killed by the disease. The weak that were given presence in Minkowski's papers were the weak of the past and present. The weak of the future were subsequently given presence in the work of those who articulated and explicated the therapeutic implications of the internal secretion theory of diabetes mellitus. The weak of the future would be the real beneficiaries if scientists and physicians succeeded in acquiring the capacity to perform the therapeutic program of action implied in the discursive translation. In other words, the weak of the past were mobilized to mobilize the living (scientists, physicians) to work for and support future's weak. They were mobilized by people who spoke in the name of yesterday's weak on behalf of the weak of the present. (Wackers 1988)

A similar analysis can be made for Ibsen's discursive program of action identifying terminal bulbar polio as being primarily a ventilation problem. Copenhagen had experienced recurrent epidemics for several decades. Mortality among patients suffering from bulbar polio had always been high. Examining four patients in Blegdamshospital's autopsy room on August 25, 1952, Ibsen found that there was nothing in the patients' lungs that would have prevented proper ventilation, implying that these patients would have lived had positive pressure ventilation been applied. Applying positive pressure ventilation to bulbar polio patients who would be admitted to the hospital in the course of the following days and weeks would, in Ibsen's view, save many lives. Thus Ibsen too, mobilized the weak of the past to mobilize the living to save the lives of the future's weak. Yet, in this case, the persuasive force that derived from the patients' weakness was in itself not sufficient to overcome Lassen's skepticism. Even the mobilization of Bower's results in treating polio patients with a combination of negative pressure and endotracheal positive pressure ventilation did not suffice. It took Ibsen demonstration of his capacity to perform the implied therapeutic program of action to overcome skepticism and resistance. 
In many cases intensive care units were, and still are, successful in accomplishing their primary goal, to maintain vital functions in critically ill patients. In other cases, however, they failed. Brain damaged patients were resuscitated, but not reanimated, they did not regain consciousness. Whereas it had been clear what it meant to support a seriously ill patient in the case of diabetes mellitus and bulbar polio, this became very unclear in the deanimated, artificially ventilated patients in intensive care units. Was it still in the best interest of the patient to continue treatment? Or could it be, that, from the perspective of the patient, support would have to take the shape of 'allowing to die', of assisting the patient in the execution of her right at a natural death? As soon as people began raising these questions, and began answering the latter in the affirmative, medicine's moral order was challenged. The same respirator that was life-saving during the polio-epidemics of the 1950s has come to represent and epitomize a reductionist medicine in which technology has run out of control. According to De Lange (1964), modern medicine had adopted a modern technomaniac attitude which refuses to accept death as the natural end of all things, corrupting a perfectly normal and healthy death struggle into a degrading battle with needles, infusions and tubes. Catz (1960) argued strongly against the implications of De Lange's position. Physicians should be the unconditional protectors of life and the living body, enemies of death, fighting until the end. They should continue their struggle for the patient's life even in situations where the disease had supremacy. The moment one would grant physicians the right to refrain from treatment in certain cases, it could happen (in future cases) that the physician would consult relatives, and that, as a consequence, other considerations and interests than the patients' would be going to determine the outcome. The weak given presence by De Lange were those (in the present and in the future) who were not allowed to die their own natural death. The weak given presence by Catz were future patients who would be denied a chance at survival and recovery. Despite these differences, both the De Lange and Catz mobilize the weak and try to derive persuasiveness from their weakness. However, the respective discursive programs of action into which the weak are mobilized move into different directions.

People not only argued for different views on what was in the best interest of severely brain damaged, artificially ventilated patients. They also began to question whether these patients could have interests. For, if they were dead, they would no longer fall into the category of the weak for whom we are morally responsible. Gervais, in her plea for a neocortical definition of death, argued that if our redefinition of death is shaped by other purposes than establishing the real status of the patient, we have failed to fulfill our 'primary moral obligation to protect the interests of the most vulnerable human, whose resources for self-protection are permanently absent'. (Gervais 1986: 60; my emphasis) But, in case they are dead we should consider the interests of others and try, in Pallis' words, to lessen human distress for the patient's relatives, use the limited intensive care facilities for those in need, and if possible. consider organ transplantation for the benefit and support of others who will die when no donor organs are available. Recognizing the legitimate and partly conflicting interests of various groups of patients in need of health care is one thing. However, redefining the boundary between human life and death with the objective to exclude the severely brain damaged, artificially ventilated patient from the moral community 
(by declaring them dead) was unacceptable to Van Till and many others. Van Till did not reject a brain related definition of death, but in her definition of whole brain death she gave priority to the support and interests of the severely brain damaged patients. Others regard even the acceptance of a whole brain definition of death as an unacceptable infringement on patient's basic rights to life and health care. In this view the 'traditional' definition of total death, with its associated heart-lung criteria, still suffices. A shift to brain related definitions of death is unjustified.

Despite the renegotiability of notions like 'support' and 'the weak', Jensen's basic moral principle of responsibility for the weak is useful to talk about the moral dimensions of medicine. We should be prepared, however, to accept that there is nothing in the actions of people exhibiting solidarity with the weak that makes us necessarily perceive these actions as good or morally responsible. Actions have no intrinsic quality that makes them stand out as a model activity, a paradigmatic example of morally responsible action to be used by people as a standard for commenting on and judging past and future actions. Whether certain programs of action stand out as a standard depends on whether the moral order they presuppose, and the morality of their goals and means, goes unchallenged.

\subsection{Program implementation}

In the previous sections I used words like diagnostic, therapeutic and discursive to emphasize one or another aspect of various programs of action. In discussing the various types of programs of action, pointing out how they are related, how one is implied in the other, I provided examples from the case studies. The examples differed in one important aspect, namely, whether the program had been realized or not (yet). In other words, some programs of action may be hypothetical in nature, lacking a corollary in laboratory or clinical practice. In this section I will outline an account for the processes through which hypothetical programs of action are implemented, realized and maintained.

A discursive program of action may still be hypothetical or it may have been realized. It may have been implemented in one place (laboratory, hospital, country) but not in another. The program of action of brain stem death is implemented in England but not in the Netherlands. The Netherlands have implemented a whole brain definition of death, although not to the full extent as it was developed by Van Till. $\mathrm{CO}_{2}$-accumulation had been realized in the operating theater but not yet in the infectious diseases wards of Blegdamshospital. Thus, in case a program of action has been realized, it is important to ask where it has been realized and where not, and to what extent. Programs of action have a history that stretches from their inception to their final implementation and stabilization. The implementation of new programs of action often requires the collaboration of scientists, physicians, engineers, philosophers, lawyers. This implies the coordination of work and the establishment of agreement across disciplinary boundaries.

The history of realized programs of action is characterized by a gradual, sometimes abrupt, reduction of indeterminacy, and an increase of determinacy. Insulin's indeterminacy in 1889 is reflected by the frequent use of expressions like 'active 
principle', a 'substance' produced by the pancreas, or in German: ein gewisses Etwas. In 1926 insulin was determinate. It could be measured in biological assays involving rabbits and mice, and compared with an international standard. The change from indeterminacy to determinacy is a change from doubt and uncertainty to certainty and obduracy, from hypothesis to fact, from promise and implication to effective therapy.

In the beginning there was doubt and indeterminacy. Insulin lacked its own program of action that would give it existence. Yet, several scientists were certain that a pancreatic internal secretion existed. What were the sources of this certainty? I will argue that, despite the lack of its own program of action, insulin (as an hypothesis) was 'latched'8 onto other programs of action. One of these other programs of action was developed by Minkowski. More were subsequently provided by other scientists.

Minkowski's experimental pancreatic diabetes was insulin's home base: its most important latch and primary source of certainty. The point to point comparison of the pattern of symptoms of pancreatic diabetes in dogs and the pattern of symptoms in human diabetics established insulin's relevance for the clinic. However, pancreatic diabetes left insulin indeterminate, an unmeasurable substance. But at least, the experimental design attributed plausibility to Minkowski's claim that something like an internal secretion of the pancreas existed. A second latch was provided by other physiologists, embryologists, anatomists and pathologists working on the pancreas. Laguesse proposed Langerhans' islands in the pancreas as the site of production of the internal secretion. Schäfer emphasized the morphological analogy between the extremely vascular islands of Langerhans in the pancreas and the thyroid and the adrenal glands. The internal secretion of these glands was established in the beginning of the 1890: scientists had developed programs of action, involving the thyroid and adrenal glands. Eiselberg (1892) reported the successful treatment of a thyreodectomized cat with a subcutaneous transplant of thyroid tissue. Murray (1891) succeeded in treating sheep with myxedema by means of subcutaneous injections of an extract of thyroid tissue. In both cases the effects of the transplant or the injection of extract on the animal's overall metabolism could only be explained by assuming that these tissues produced an active principle which was secreted into the blood to exert its function in distant tissues. The morphological analogy between pancreatic island and thyroid tissues allowed scientists to latch Eiselberg's and Murray's programs of action to Minkowski's pancreatic diabetes, bestowing more plausibility on the pancreatic internal secretion.

Pathologists like Schulze and Ssobolew did important experimental work that singled out the pancreatic islands of Langerhans as the most probable site of insulin production. They devised programs of action in which the island tissue was partitioned off from the excretory digestive system of the pancreas, demonstrating that, in preventing the occurrence of pancreatic diabetes, it was the survival of Langerhans' islands that mattered. Also non-experimental pathologists working on autopsy material from human diabetics worked to provide latches for the internal secretion theory of pancreatic diabetes, although in their case this proved to be far more difficult. Structural alterations in Langerhans's islands were only infrequently found, and the notion of functional failure was frequently invoked to save the case.

Further latches were provided in Starling's experimental work and his 1902 articulation of the modern concept of a hormone, but also in Forschbach's parabiosis 
experiments and Carlson and Drennan's experiments on pregnant dogs. These researchers developed their own programs of action. None of them proved the existence of insulin conclusively, but they provided latches for insulin. Insulin was still a hypothesis, an entity lacking its own program of action, but it derived plausibility from other programs of action realized in various laboratories throughout the world. These programs of action were latched together in and by the discursive translations of the researchers who had developed a particular interest in diabetes, or in ductless glands and internal secretion in general. These other programs of action onto which insulin was latched, provided the strength and certainty to resist the cumulating negative evidence emerging from numerous failing attempts to capture the pancreatic internal secretion in a pancreatic extract.

When finally in 1922 the Torontonean researchers successfully performed the program of action that definitely established the existence of insulin, its identity and its presence in the extract, the old latches became dispensable.

Despite its indeterminacy the notion of an internal secretion (of the pancreas) served as a boundary object, facilitating the multiple transactions (latchings) needed to engineer agreements across disciplinary boundaries. As I explained in chapter 5, the notion of boundary objects refers to those abstract (or concrete) objects or concepts which inhabit several intersecting social worlds and satisfy the informational requirements of each of them. Boundary objects are objects which are both plastic enough to adapt to local needs and the constraints of the several parties employing them, yet robust enough to maintain a common identity across sites. They are weakly structured in common use, but become strongly structured in individual-site use. (Leigh Star and Griesemer 1989) Boundary objects play an important role in developing and maintaining coherence across disciplinary worlds, managing both diversity and cooperation. Despite its initial indeterminateness, the notion of internal secretion served this function, facilitating the creation of the emerging field of endocrinology: the field of ductless glands, blood vessels and hormones.

In 1952, the notion of $\mathrm{CO}_{2}$-accumulation and the technique of positive pressure ventilation were far from being indeterminate when compare with insulin prior to its discovery in 1922. But, their determinacy was restricted to particular places and disciplines: the respiration physiological research laboratory, the operating theater, anesthetists. The 1952 polio-epidemic created the kind of circumstances facilitating the world of physicians treating infectious diseases and the world of anesthetists ventilating patients in operating rooms to meet and intersect in one specific and particular place, and at one particular time: Blegdamshospital, August 27, 1952. It was at this point, while sharing the same space, that Ibsen performed the program of action that both established the existence of $\mathrm{CO}_{2}$-accumulation in terminal polio-patients, and positive pressure ventilation as the most effective treatment. In this case it was the 'ensemble' of the 12 year old girl, ventilated by means of a balloon, and attached to Brinkman's Carbovisor, that served the function of a boundary object. The treatment of bulbar polio, at first in Copenhagen, later in Stockholm and other European cities, provided the primary latch for the positive pressure respirator outside the operating theater. The subsequent adoption and implementation of positive pressure ventilation in the treatment of other conditions characterized by severe respiratory insufficiency, firmly latched the respirator into other domains of medical practice. When a few years later 
poliomyelitis was successfully prevented by large scale vaccination programs, the loss of this area of application did not affect the further development of the respirator. It was well entrenched in respiration centers and intensive care units. The negative pressure respirators were relegated to the museum for medical history.

Like the undeterminate concept of internal secretion in the last decade of the 19th century, the notion of brain death, served as a boundary object, facilitating the attainment of agreements among (members of) the worlds of neurologists, transplantation surgeons, of health care authorities, lawyers, philosophers, ethicists. Agreement was reached about the acceptability of the employment of a brain related definition of death in cases of severely brain damaged patients maintained on life support systems in intensive care units. Beyond this, however, opinions split. The rather indeterminate notion of brain death was transformed and adapted into the much more specific definitions of brain stem, whole brain and neocortical death. These programs of action argued for different degrees of constraints to be put on the use of brain damaged patients as resources in solid organ transplantation.

In the Netherlands the implementation of a solid organ transplantation program began in 1966, using kidneys from post-mortal donors. Arrangements were made for financial reimbursement. A provisional legal basis for the extirpation of organs for transplantation purposes was found in the Law on the Disposal of the Dead, on the basis of an analogy with partial dissection for autopsy. The 1971 bill containing a revision of the Law on the Disposal of the Dead gave this analogy a more formal status. The bill also expressed the legislator's principle choice for an explicit consent system, assigning specific rights, roles competencies to patients, relatives, and physicians. It was the 1974 Health Council report on brain death criteria, which, in the Netherlands, provided the first important latch for a whole brain definition of death. Shortly after the publication of the report its recommendations were adopted by Dutch health care authorities and given a semi-legal status by publication in official governmental media (Staatscourant). In this way whole brain death was introduced into the organizational, financial and legal framework through which the solid organ transplantation program in the Netherlands was institutionalized. The health care report defined the whole brain dead patient on artificial ventilation and other life support as a potential source of donor organs. It also defined the diagnostic program of action that had to be performed in order to establish the death of the whole brain. In 1983 the Health Council published a second report on brain death criteria, a revision and update of the 1974 report. In 1991 the Dutch government submitted a bill on the Donation of Organs to parliament. This bill identifies brain death as the point of death at which the person dies.

In the Netherlands whole brain death is firmly latched into the institutionalized solid organ transplantation program. The institutionalization of whole brain death has not silenced the other definitions of brain death, it has not terminated the controversy. The controversy has been partitioned. The various Health Council reports and legal documents have traced a boundary, a horizon of relevance, between the sphere of medical practice where a definition and criteria of whole brain death are employed, and the public domain.

The case studies on insulin and the 1952 polio-epidemic are both characterized by a marked turning point, which is lacking in the case study on the redefinition of 
death. The pattern of developments is different in the latter. In the case of insulin 1922 marks the point at which the doubt concerning the existence of insulin was definitely resolved. The program of action that established insulin as an entity in the bodies of animals and human beings was the program that established insulin as an effective therapy in the treatment of diabetes mellitus. The number of alternative discursive translations for diabetes was reduced, indicating closure of the debate. This closure of the debate coincided with the stabilization of insulin as a technology. The same holds for the second case study. August 27, 1952, Ibsen's persuasive demonstration of terminal bulbar polio as a ventilation problem, reduced the number of discursive translations of bulbar polio, marking the closure of the debate. This closure coincided with the stabilization of manual positive respiration in this new domain of medical practice. In these two cases it seems to make little sense to distinguish between closure and stabilization. Closure and stabilization might as well be used synonymous, or one of the concepts might be abandoned. The third case study, on the re-negotiation of the end of human life, suggests that it may be fruitful not to use them synonymous. In this case, stabilization of a medical practice in which a particular brain related definition of death is employed relatively unproblematically, is not preceded, nor coincidental with, nor followed by a closure of the debate on brain death. However, my usage of the notions of closure and stabilization is somewhat different from the way in which they were originally introduced.

The concept of closure originated in the sociology of scientific knowledge. It is linked to the notion of interpretative flexibility. The availability of multiple interpretations is a characteristic of scientific controversies. The concept of closure pertains to the 'ending', the closure of a scientific controversy, resulting in a reduction of interpretative flexibility. In the process of closure one interpretation becomes dominant. Closure indicates the emergence of consensus among two or more groups. Bijker and Pinch used the concept of closure in an analogous sense in the analysis of technological developments. In the analysis of technology, closure refers the reduction of the interpretative flexibility of an artefact. Closure among the different relevant social groups about the dominant meaning of an artefact emerges and the 'pluralism of artefacts' decreases. As it is used in the sociology of scientific knowledge and in the Social Construction of Technology, the concept of closure is primarily relevant to an inter-group analysis.

The concept of stabilization, on the other hand, is primarily relevant to intragroup analysis: the emergence of consensus within one relevant social group. The concept of stabilization conveys that the production of scientific knowledge and the invention of artefacts are the result of collective work that takes time. Discoveries and invention are not the individual acts of geniuses. In a semiotic analysis increasing and decreasing degrees of stabilization can be traced by looking at the appearance and disappearance of positive or negative modalities. Modalities are statements about statements that affect the latter's factuality. Thus the statements: 'Minkowski claims that the pancreas produces an internal secretion', 'The pancreas produces an internal secretion called insulin, but it has never been captured in an abstract for technical reasons', and 'Insulin exists and can be obtained from Eli Lilly', these statements exhibit progressively greater degrees of stabilization of insulin. Bijker argues that closure and stabilization are two aspects of the same process. 'Closure leads to a 
decrease of interpretative flexibility - to one artifact becoming dominant and others ceasing to exist. As part of the same movement, the dominant artifact will develop an increasing degree of stabilization within one (and possibly) more relevant social groups'. (Bijker 1992a: 96)

The concepts of closure and stabilization outlined above, reflect the 'original' units of analysis: scientific knowledge and technical artefacts. A group in this view is characterized by homogeneity of meaning attributed to a particular (arte)fact. However, as a result of the extension of the unit of analysis from knowledge or technical artefacts to sociotechnology, the notion of a (relevant social) group in this analysis has become highly problematic. This does not mean that we should do away with the concepts of closure and stabilization. They still convey important insights. It only means that they have to be appropriated to fit the new heterogeneous units of analysis.

In my analysis of the implementation of programs of action I use closure to refer to the termination of the debate between two or more competing, but non-implemented, discursive programs of action. Stabilization then, I take to refer to the implementation of a program of action in a routinized, standardized practice in which various actors play their assigned roles. Within this practice debate, doubt and uncertainty are eliminated, whereas the debate might continue in an outside, public domain. Facts and artefacts are elements in this stabilized, heterogeneous practice. The process of the stabilization of these elements may be traced by looking at modalities dropped from or added to particular statements. The stabilization of a practice as a whole can be traced by looking at the standardization of roles of both human actors, bodies and machines. An increase in stabilization is characterized by a decrease in the latitude allowed to actors to deviate from their assigned roles. In other words, the implementation and stabilization of programs of action is characterized by an increasing degree of standardization and routinization through which the range of possible actions and practices is reduced. The various actors in the program have to play their assigned roles. Serious deviations are not allowed.

The performance of the various actors is regulated and monitored. Examples can illustrate this point. The distribution and circulation of samples of the international and national standards of insulin is used to calibrate biological assays used for testing the potency of subsequent batches of pancreatic extract. Through extensive quality control procedures, manufacturers make sure that every milliliter sold contains the same amount of international units of insulin. The syringes used by health care personnel or patients themselves to inject insulin are calibrated and standardized to avoid mistakes. Physicians monitor the patient's body's responses to the insulin, as well as the patient's compliance to therapeutic and dietary guidelines by means of regular measurements of glucose in blood. Equipment and procedures for blood sugar determinations are subject to the same extensive and permanent quality control procedures. International and national institutions for standardization, insulin and insulin syringe manufacturers, physicians, laboratories, patients, but also insulin itself play there assigned roles in the therapeutic program of action that enables diabetic patients to lead a close to normal life. The effectiveness and safety of insulin in hospitals and patients' homes is dependent on the day to day work that goes into the stabilization and maintenance of the mutual relationships between various actors in the program. Intermediaries like samples, control serums, laboratory result sheets. 
prescriptions, dietary instructions, circulate between the actors to monitor performance and to execute corrections of deviations from the norm, whenever necessary.

The same holds for prolonged artificial respiration with positive pressure respirators in intensive care units. In modern hospitals blood samples for blood gas analysis are no longer carried to the laboratory by health care personnel. It is sent directly by pneumatic post to the laboratory where calibrated equipment and personnel provide a 24 hours per day service. Results are sent back the same way, or they are phoned, or dispatched through a computer network. Whatever the route these intermediaries take in a particular hospital, they allow the day to day, and hour to hour monitoring of the respirator's performance, the patient's responses and condition.

In-house protocols to be obeyed when organ donation is requested from a brain dead patient also exemplify the standardization and fine-tuning of roles in an implemented and stabilized program of action. These protocols, latched onto the authorized Health Council reports on brain death criteria, describe rights, obligations and roles assigned to the various actors involved, patients, relatives, various physicians. They describe what diagnostic procedures have to be followed, who has the competence and is allowed to perform them, who is not allowed to be involved in the diagnosis of brain death, whom consent for organ extirpation has to be obtained from. In the hospitals where they have been discussed, implemented and accepted these in-house protocols regulate the performance of everybody involved, facilitating the reduction of doubt and the emergence of agreement. By standardizing roles and regulating various actors' performances, restricting the latitude for deviations from these roles, also restricting the range of alternative opinions to be expressed, in-house protocols support the production of, albeit local, certainty with regard to brain death.

Stabilized practices in medicine are no closed circuits. There is continuous traffic across the inside-outside boundary. Medical practices depend on the influx of patients. On entering medical practice the patient is confronted with the standardized roles for patients laid out. Although medicine tries to educate the public in various ways, the patient's own ideas about her problem, her perspectives on health and disease, may be at variance with the interpretation offered by medicine. The latitude for disagreement may be so restricted that the patient is confronted with a take-it-or-leave-it choice: the patient has to comply, or refrain from the services offered. In many cases patients have no problem with the adoption of the specialist's definition of their health problem. Others feel like strangers in medicine's world: confused like the mother sitting at her brain dead son's bed, unable to accept that he is dead while his body is warm, his heart beats and his chest moves up and down in tandem with the respirator's wheezing sounds (see section 1.1). However, when patients or relatives find medicine's definition of the problem problematic. they may want to seek a second opinion. Eventually, this disagreement may translate into a general distrust in medicine's capability of conceptualizing and dealing adequately with problems. of human health and disease. It is this outsider's unwillingness to accept the interpretations and associated therapeutic strategies of a standardized and routinized medical practice that lie at the root of reductionist critiques of medicine. My analysis suggests that it is possible to explain these 'limitations' of medicine without making reference to a cognitive-instrumental rationality embodied in medicine's science and technologies. My explanation would make reference to the collective work of a multitude of 
actors that defines roles and restrict latitude for deviation, not only for patients, but for all other actors in a particular stabilized practice.

\subsection{Conclusion}

In the previous sections of this final chapter I have tried to link the various concepts together in a more systematic way. In the various case studies many empirical questions came up that I have tried to answer. No doubt many more can be raised that I did not address. I hope that readers will take issue with other aspects of the narratives provided in the case studies and attempt alternative explanations. If there is a general lesson to be learned from this study it must be a strategic one.

A constructivist analysis of medicine emphasizes the contingency of medical practices as they are today. This implies that there is no principle reason why medical practices could not be otherwise. At a microlevel specific changes may be forged by the conscious strategies of one or a small number of actors. The overall shape of a medical practice can, however, hardly be conceived of as being the result of explicitly planned and rationally decided action. There have been many small actors contributing to medicine's present shape and content. Medicine's content and shape, its boundaries with the outside world, are also maintained by the work of many small actors. Hence, a constructivist analysis also emphasizes that it will be hard to change medical practices.

For those who are critical of particular practices in the general domain of modern medicine, it is important to consider their relative position vis-à-vis the inside-outside boundary of that practice. For outsiders, whether they are lay people, policymakers or ethicists, it will be difficult to know what exactly is going on within the private space of, for example, research laboratories. For observers and commentators in the public domain developments will not become visible before they move out of the laboratory. This is an important reason why academic medical ethics often operates like a fire brigade, rushing from one (moral) fire to another. It is why they often lag behind scientific and technological developments, primarily dealing with ethical issues arising from the use of medical technologies that have already been introduced. For insiders of the practice this boundary constitutes a horizon of relevance. Those who want to raise issues about new developments at an early stage must be prepared to find a way to get inside. This will not always be possible, because sometimes the insideoutside boundary is very physical in nature.

Seen from the perspective of a scientist or engineer pursuing a research or technical development program, especially when it is sensitive and controversial, it is of strategic importance to establish an inside-outside boundary, a horizon of relevance, a private space. New programs of action have to be implemented in a field that is shaped by programs of action, practices, that are already in place and extensively latched or institutionalized. In the private spaces of their laboratories scientists and engineers can prepare and develop new technical capabilities. However, in order to develop the program beyond the limited scale of the laboratory they will have to engage established practices. To make a place for themselves it may be necessary to challenge established practice in strategic places, to redefine disease concepts or 
central concepts in physiology, to re-negotiate problem definitions, ontological or moral status. Implementing a new program of action involves the unlatching of the old and relatching of the new. This un- and relatching is not something done lightly and requires a lot of collective work.

Old practices will resist change. The strength of the resistance will depend on whether new programs of action move in different directions on issues that are considered to be very important or essential. These will be the issues where the controversy centers. The debate on brain death is only one such issue, where fundamental legal rights and basic interests of human beings (who can no longer speak for themselves) are at stake. Many more examples can be found in contemporary debates concerning modern medicine, ranging from controversies about (non)biological or genetic causes of homosexuality and criminality to various conflicting scenarios about genetic engineering. Change is fuelled by the hands-on work of actors in these areas of conflict: where two or more programs of action try each others strength. Change will only result when a new program is implemented at the expense of the old one. However, the trial may also result in the reaffirmation of the old practice. The outcome of this process is contingent and in principle reversible.

Although in principle everything can be subjected to trials of strength, not everything is. Glancing over contemporary debates concerning modern medicine, controversy centers around only a limited number of issues. These are the issues that are of strategic importance for the participants in the debate, either to challenge or to defend. In many other areas people are comfortable with the world medical science has produced. Insulin, for example, goes unchallenged. Insulin is obdurate, durable, irreversible. I do not know of anybody who would want to challenge the existence or effectiveness of insulin. I cannot imagine any philosophically or politically interesting reason do so. If I would get diabetes I would probably be a very compliant patient. The situation for brain death is different though. Brain death, or particular versions of brain death are still being challenged. Brain death very much depends for its stability on the institutional framework of the organ transplantation program. Organ transplantation still is very much dependent on brain dead patients as an important source of good quality donor organs. Brain death would lose its stability if, for example, inter-species (xeno-)transplantation would be possible. In other words, if donor organs could be obtained from monkeys and apes - or from genetically manipulated, transgenic pigs ${ }^{10}$-, the necessity to use organs from brain dead patients would be reduced, and so would the necessity to maintain a brain related definition of death. A dead brain would be sufficient to terminate treatment of severely brain damaged patients and allow them to die. The implementation of a transplantation program using routinely animals as organ donors would have to count on considerable resistance from animal rights movements.

For those who are concerned about or want to resist the implementation of new programs of action, whether they are ethicists, philosophers, policy makers or physicians, it is important to be aware of the existence of private spaces in laboratories, R\&D-departments, and hospitals, where developmental work proceeds relatively sheltered from public scrutiny. It is important to penetrate or destabilize the horizon of relevance in order to be able to interfere with that work and challenge it at an early stage. Those who engage in the debate, irrespective of their original training, must be 
prepared to step over disciplinary boundaries and also tackle the scientific and technical issues. In stabilized practices cognitive, technical and moral issues are intimately linked and latched onto each other. Each of them may be challenged to induce a softening of the obduracy of the practice as a whole. The choice of the issue to be challenged will be a strategic choice. Subsequently one must be prepared to do the work to (re)construct a new obduracy and certainty. This will also involve work in, and collaboration with people from other disciplines. Programs of action can never be challenged, nor implemented by a single discipline alone.

\section{Notes to chapter 6}

1. Latour (1992) and Akrich and Latour (1992) use the same phrase for the description of (the history of) any setting, that is, of any assembly of human and non-human actants. Although my usage of the term is not incompatible with theirs, I will not use it in the same technical, semiotic sense. The difference is more in the level of abstraction and the use of a specific vocabulary. The way in which I will use the word action will be in line with the semiotic definition of actant and action, rather than with the traditional sociological definition reserving actor and action for humans. See also chapter 1, note 7 . Thus, I will use the word actions to refer to the actions of humans (experimental scientists, physicians), as well as to the behavior of, for example, insulin in biological assays. I want the notion of a program of action to straddle the actions of humans and the behavior of things, like a lions act in a circus where both the lion's tamer and the lion are performers of the program: take either one of them away and you will luse the act. In realized and stabilized programs of action both human and non-humans must play their assigned roles.

2. Minkowski went one step further (not described in chapter 3 ). When enough time was allowed to pass after the healing of the subcutaneous transplant and the removal of the rest of pancreas in the second operation, blood vessels would grow into the transplant from the surrounding subcutaneous tissues, establishing a second venous drainage route. Blood carried into the pancreatic transplant by the pedicle's artery, could leave the transplant through the pedicle's vein, which would carry it to the portal vein into the liver, or through the newly established microcirculation into the subcutaneous venous blood vessels. When Minkowski exposed and ligated only the pedicle's vein, leaving the artery unimpaired, he found that no diabetes occurred. From this he concluded that the liver was not required for the pancreas to exert its influence on sugar metabolism in the rest of the body. The active principle, was carried to distant tissues through the subcutaneous venous circulation, bypassing the liver. (Minkowski 1893)

3. The words closed and open-endedness refer to the predictability of outcomes. In this perspective an experiment would be an open-ended program of action whereas a demonstration is a stable and closed program of actions.

4. Using Collins vocabulary one could say that this is an example of behavior specific action. See Collins (1990)

5. Linguistic conventions resist a proper 'agnostic' account that tries to avoid a priori distribution of moral and ontological status. We use who to refer to human beings, and what to refer to things. These conventions reflect differences in ontological and moral status, the distinction we make between humans and things. I would like to write that Pflüger and Minkowski agreed upon who should (and could) be the primary beneficiaries, and who might be used in instrumental ways to achieve these benefits; or that Pflüger and Minkowski agreed upon what should (and could) be the primary beneficiaries, and what might be used in instrumental ways to achieve these benefits. But any language. editor would object that it is not correct to use who to refer to animals and that the things (or animals) 
to which what refers cannot be beneficiaries, because things and animals do not have interests.

6. According to Jensen (1987), there are no such things as values in the sense of ideas or ideals of what is right or adequate, the validity of which can be confirmed in abstraction from human communal life. Values are not external, determining the practices of our communal life, but are integral, determining our ways of acting and talking together.

7. According to Jensen (1987), the basis for moral responsibility for other individuals or groups is to be found in the nature of human communal life: human beings living in relationships with others, characterized by dependency and power. It is in the establishment of these relationships that, according to Jensen, the moral responsibility for other individuals or groups, who are weaker than us, is established. I have argued that it is not necessary to make assumptions about the nature or quality of certain activities, about human nature or about the nature of human communal life and relationships. The reason why Jensen's model activities stand out as paradigmatic examples of morally responsible behavior lies in the agency of spokespersons who point them out as such. It is their repeated recommendation in writing, teaching, literature, motion pictures, etc., that contributes to their status as a yardstick for commenting on and judging past and future actions. See also Jensen (1984a) and Wackers (1988).

8. Latching is the word Pirsig uses in his 1991 book Lila, in which Phaedrus explores the question how dynamic, changing patterns become static. Phaedrus' answer is: through latching. To latch (verb) means to catch or get hold, to attach oneself, or to make fast with or as if with a latch. A latch (noun) on for example a gate or a door usually consists of a metal bar which is held in place to lock the door and which can be lifted to open it. Thus, the notion latching conveys an image of attaching something to a fixed point in order to derive stability, plausibility or certainty from it. Latches are sources of certainty. On the other hand it also conveys an image of 'in principle reversibility': a latch can be unlatched.

9. See also Bijker (1994). Bijker refers to take-it-or-leave it choices in the analysis of a sociotechnical ensemble's obduracy. For highly included actors the stabilized ensemble enables and constrains interaction and thinking to a large extent. The ensemble does not guide the interactions and thinking of actors with a low inclusion. To lowly included actors, artefacts that are part of the sociotechnical ensemble present as a take-it-or-leave-it choice: they have no chance of modifying the artefact when they take it, but life can go on quite well when they leave it.

10. In current genetic research attempts are being made to insert human genes, responsible for the expression of socalled Major Histocompatibility Complex antigens, into the DNA of pigs. These MHC-antigens play an important role in the immune system's recognition of self and non-self. The idea is that if pigs can be made to express human MHC-antigens on the external surfaces of their cells. organs transplanted from these genetically manipulated animals to humans would not be reconized as non-self by the recipient's immune system. Immunological rejection would not occur, or it would be manageable by means of pharmacological immunosuppressants. 


\section{Samenvatting}

Dit boek gaat over de cognitieve inhoud van de moderne geneeskunde, haar technologisch instrumentarium en haar sociale en morele orde. Het is de alomtegenwoordigheid van technologie waardoor de moderne geneeskunde zich onderscheidt van traditionele vormen van geneeskunde. Technologie wordt geacht verantwoordelijk te zijn voor het succes van een interveniërende medische praktijk. Echter, technologie wordt ook verantwoordelijk gehouden voor de verstoring van traditionele waardensystemen, voor bezwaarlijke sociale en morele gevolgen. Iedere poging de huidige vorm van de moderne geneeskunde te begrijpen - haar kennis, haar technologieën, het patroon en verloop van morele debatten - zal rekening moeten houden met de rol die wetenschap en technologie hebben gespeeld in haar produktie. Met andere woorden, een begrip van geneeskunde vereist een begrip van wetenschap en technologie.

Deze studie verdedigt niet de deugden van de geneeskunde. De geneeskunde heeft voorsprekers genoeg. Noch voegt zij zich bij de critici die ethische theorieên of maatschappelijke normen en waarden toepassen om een oordeel te vellen over de effecten van de moderne geneeskunde op mens en maatschappij. Geen van deze evaluaties worden hier als a priori vooronderstellingen over de aard van de moderne geneeskunde aanvaard. Begrippen als wetenschappelijke methode en rationaliteit zullen niet gebruikt worden om het succes van de geneeskunde te verklaren. De beschrijving en analyse in deze studie concentreert zich daarentegen op het concrete handwerk van wetenschappers, artsen en een reeks andere actoren bij de produktie van de cognitieve, technologische, morele en sociale orde van de geneeskunde. Daarbij zullen vragen met betrekking tot zowel verandering als stabiliteit aan de orde komen. Leidende vragen in dit onderzoek zijn: Hoe kunnen we in één analyse zowel gelijktijdige cognitieve, technologische en sociale veranderingen als de onmiskenbare duurzaamheid en hardheid van kennis, technologieën en morele principes verklaren? Waardoor wordt een technologie, die levensreddend is in de ene situatie, zeer problematisch in een andere setting, een paar jaar later? Waarom opereert de academische medische ethiek als een brandweerkorps dat van de ene morele brandhaard naar het andere snelt; steeds achter wetenschappelijke en technologische ontwikkelingen aanlopend en zich voornamelijk bezig houdend met ethische vragen voortkomend uit het gebruik van technologieën die al ingevoerd zijn? Hoe komt het dat de morele en politieke controversen zich vaak in het publieke domein 
afspelen, met buitenstaanders als opponenten in het dispuut? Hoe worden nieuwe medische praktijken gevestigd ondanks de controversialiteit van de betrokken technologieën?

Hoofdstuk 1 werkt deze algemene doelstellingen van het boek nader uit. Drie verschillende scenes bieden aan het begin van het hoofdstuk een terugblik in specifieke episoden in de geschiedenis van de moderne geneeskunde. Tegelijkertijd bieden zij een vooruitblik op de drie gevalstudies die de empirische kern van dit onderzoek uitmaken: het werk aan insuline voorafgaande aan haar ontdekking, de polio-epidemie van 1952 in Kopenhagen, en het debat over hersendood. Hootdstuk 1 geeft ook een overzicht van de belangrijkste ontwikkelingen in het constructivistische wetenschaps- en techniekonderzoek. Dit onderzoek vormt een belangrijke inspiratiebron voor de analyse die in de volgende hoofdstukken wordt ontwikkeld.

In de jaren zeventig claimden Britse sociologen voor het eerst dat de technische inhoud van wetenschappelijke kennis toegankelijk was voor sociologische analyse en verklaringen. Voor het eerst in de geschiedenis van de sociologie werd (natuur)wetenschappelijke kennis op systematische wijze aan dezelfde vormen van kritisch, empirisch, sociologisch onderzoek onderworpen die voorheen toegepast werden op religies en politieke denkbeelden. Dit nieuwe, relativistische onderzoeksprogramma in de sociologie van wetenschappelijke kennis ging veeleer over de vraag 'hoe bepaalde ideeën over de fysische en mathematische wereld binnen een samenleving als juist konden gaan gelden', en niet zozeer over de vraag 'hoe de samenleving georganiseerd dient te worden opdat waarheid aan de dag kan treden'. Dit onderzoeksprogramma ging over de 'sociale constructie' van wetenschappelijke kennis. Behalve deze sociologische benaderingen van kennisproduktie, zagen de jaren zeventig en tachtig ook de opkomst van een nieuw type technologieonderzoek met vergelijkbare verklarende doelstellingen. Deze studies hielden zich bezig met de sociale factoren die vorm gaven aan technologische veranderingen, en veel minder met de effecten van technologie op de samenleving. Van de verschillende benaderingen in het constructivistische wetenschapsen techniekonderzoek, ontleent deze studie het meeste aan de door Bruno Latour, Michel Callon en John Law ontwikkelde actor-netwerk- of translatietheorie. Zij hebben met andere constructivisten gemeen dat zij de nadruk leggen op het werk en de strategieën van diverse actoren betrokken bij controversen, of bij het overwinnen van weerstanden tegen de implementatie van nieuwe sociotechnische praktijken. De specifieke vorm van een sociotechnische praktijk wordt gezien als de (tijdelijk) gestabiliseerde uitkomst van een voortdurend proces van veranderingen dat gevoed wordt door het nooit nalatende werk van actoren om conflicten, verschillen en weerstanden te overwinnen. Deze aandacht voor de processen waardoor wetenschappelijke kennis en technologieën geconstrueerd worden weerspiegelt wat als de eigenlijke taak van de analist wordt beschouwd: het vinden van de bronnen en strategieën waarmee zekerheid en hardheid tot stand wordt gebracht. Constructivistisch wetenschaps- en techniekonderzoek probeert aan te geven in welke zin en in welke mate we kunnen spreken over wetenschappelijke kennis als zijnde geworteld in het concrete werk van actoren werkend in een specifieke tijd en cultuur. Het doel van deze studie is te onderzoeken op welke manier propositics en entiteiten (uitspraken over het bestaan van bepaalde biologische actieve stoffen of over het einde van het menselijk leven, nieuwe interpretaties van symptomen of laboratoriumuitslagen, nieuwe technologieën) met zoveel zekerheid toegerust worden dat zij schijnbaar uitwishaar deel gaan uitmaken van onze (cognitieve, technologische of sociale) orde; ten nadele van 'oude' waarheden en 'oude' manieren van doen.

Hoofdstuk 2 bestaat uit twee delen. In het eerste deel wordt aandacht besteed aan het meest basale, epistemologische uitgangspunt van een constructivistische benadering, namelijk dat 'realiteil' de gestabiliseerde uitkomst is van concreet werk in wetenschappelijke en technologische praktijken. Deze contra-intuitieve claim leidt tot een omkering van de traditionele 
epistemologische opvatting waarin de realiteit een pre-existente entiteit is waarover wetenschap theorieën en andere representaties produceert. Volgens deze traditionele opvatting kunnen wetenschappelijke representaties van de wereld meer of minder correct zijn. Het probleem is echter hoe men zeker kan weten dat de ene wetenschappelijke representatie beter is dan een andere. Hoe kan, met andere woorden, de zekerheid waarmee we een wetenschappelijke opvatting voor waar aannemen, in de werkelijkheid worden gefundeerd? Het eerste deel van hoofdstuk 1 richt zich vooral tot artsen en andere werkers in de gezondheidszorg, en tot filosofen van de geneeskunde die deze 'constructivistische omkering' problematisch vinden. Dit probleem wordt echter niet zozeer in epistemologische termen besproken. Hoofdstuk 2 bespreekt de verschillende manieren waarop enkele filosofen van de geneeskunde hebben geprobeerd het idee van een ziekte te vatten in essentialistische, biologische en evolutionaire ziektebegrippen. Enerzijds hebben filosofen het naïeve, essentialistische ziektebegrip bekritiseerd dat ten grondslag ligt aan het vertrouwde beeld van een medische wetenschap die steeds meer aspecten van ziekten ontdekt. Hoewel zij het conventionele karakter van wetenschappelijke kennis erkennen, slagen zij er niet in te verklaren hoe deze sociaal geproduceerde wetenschappelijke kennis zich verhoudt tot niet-veranderende of tot evoluerende ziekten in de wereld. Het argument is dat een constructivistische benadering een interessante uitweg is uit dit eeuwenoude filosofische dilemma. Hoofdstuk 2 maakt deze constructivistische wending door traditionele en constructivistische visies op ontdekkkingen en op medische beeldvormende technieken naast elkaar te plaatsen. Een constructivistische benadering onderneemt geen pogingen meer om de zekerheid waarmee we wetenschappelijke kennis voor waar houden te rechtvaardigen. Een constructivistische benadering geeft daarentegen meer inzicht in de wijze waarop die zekerheid geproduceerd en onderhouden wordt.

In het tweede deel van hoofdstuk 2 worden enkele translatie-theoretische begrippen geïntroduceerd die in de volgende hoofdstukken gebruikt worden. Deze begrippen worden niet als een volledig conceptueel kader gepresenteerd, maar als een set van sensiterende begrippen waarvan vruchtbaar gebruik gemaakt kan worden bij het exploreren van het empirische materiaal en de analyse van de verschillende gevalstudies.

Het eerste van deze sensiterende begrippen is de notie van 'stomme inscripties'. Het begrip 'inscriptie' verwijst naar kunstmatig geproduceerde getallen, lijnen en beelden op vellen papier of fotografische emulsies in een laboratorium setting. Deze inscripties worden beschouwd als duurzame registraties waarin aspecten van de wereld gevangen zijn. In deze studie wordt het begrip inscriptie in een bredere betekenis gebruikt. Het begrip omvat zowel inscripties op duurzame media als voorbijgaande fenomenen en symptomen die als uitdrukking van een onderliggende ziekte beschouwd worden. Het begrip inscriptie omvat dus ook niet-artificieel geproduceerde inscripties zoals anatomische veranderingen die tijdens de obductie in het dode lichaam van de patiënt waargenomen worden. Inscripties zijn het ruwe materiaal waar artsen en wetenschappers mee werken. Wanneer zijn op één plaats bij elkaar gebracht worden, kunnen inscripties die in verschillende plaatsen, en op verschillende tijdstippen, verzameld werden met elkaar gecombineerd worden ter vergelijking, classificatie, statistische analyse, superpositie en grafische presentatie. Inscripties kunnen tot onderdeel van een geschreven tekst gemaakt en vermenigvuldigd worden. Foto's, tabellen en grafieken zijn geen simpele illustraties van die tekst. Het zijn instrumenten die door de auteur gebruikt worden om de tekst sterk genoeg te maken om oppositie te weerstaan. Met andere woorden, het zijn inscripties waar wetenschappers betekenis aan moeten geven en die zij gebruiken in communicatie met anderen. Inscripties zijn echter stom: zij spreken niet voor zichzelf. Zij moeten vertaald worden. Zij hebben iemand nodig die voor hen spreekt. 
Dit is het tweede belangrijke translatie-theoretische begrip dat in hoofdstuk 2 geïntroduceerd wordt: de notie van een woordvoerder die stomme inscripties vertaalt. Wetenschappers en artsen treden op als woordvoerders voor inscripties. Zij vertalen entiteiten die niet over een eigen taal beschikken, en als zodanig treden zij op als hun vertegenwoordigers of representanten. Tegelijkertijd spreken wetenschappers voor de entiteiten die geacht worden door de inscripties te worden gerepresenteerd, voor de wereld achter de inscripties. Het centrale probleem is natuurlijk hoe wetenschappers en artsen tot hun vertaling komen. De de novo vertaling van stomme inscripties vormt het uitgangspunt voor de translatie-theoretische analyse die in deze studie ontwikkeld wordt. Hierbij wordt vertaling of translatie in eerste instantie gezien als een probleem om overtuigende vertalingen van stomme inscripties te maken, en als een onderneming die kan falen of slagen. Om al het werk gesitueerd is, zal de analyse zich ook uitstrekken tot onbedoelde, contingente en niet-talige aspecten van settings, evenals tot al het stabiliteit verlenende werk dat nodig is om specifieke praktijken te ontwikkelen en te onderhouden. De analyse zal zich richten op het gesitueerde en collectieve werk van mensen om zo de vorm van de moderne geneeskunde te verklaren, zonder terug te vallen op de werking van een instrumentele rationaliteit om of het succes, of de tekortkomingen, van medische wetenschap en technologie te verklaren.

De hoofdstukken 3, 4 en 5 vormen de empirische kern van dit boek. Om een geleidelijke ontwikkeling van de argumentatie mogelijk te maken benadrukken deze drie gevalstudies elk verschillende aspecten van de medische praktijk. Eén criterium voor hun selectie was dat zij samen zeer verschillende praktijken moesten bestrijken, variërend van experimenteel laboratoriumwerk tot klinische diagnostiek en therapie, van het handwerk van het omgaan met dieren, apparaten en patiënten tot het handwerk van schrijven en argumenteren in artikelen en boeken. Een tweede criterium voor de selectie van deze drie gevalstudies was het vórkomen van ingrijpende en veelzijdige veranderingen in medische praktijken. Met name die situaties en perioden werden uitgekozen warin gelijktijdige veranderingen plaatsvonden in wetenschappelijke kennis, in diagnostische en therapeutische technologieën en mogelijkheden, in morele percepties en evaluaties, in disciplinaire en institutionele organisaties.

Hoofdstuk 3 gaat vooral over het werk aan insuline dat aan insulines ontdekking vooraf ging, dat wil zeggen over de periode tussen 1889 en 1921. Volgens de canons van de medische geschiedenis werd insuline in 1921 ontdekt door een Canadese onderzoeksgroep in Toronto. Het belang van deze ontdekking werd alom erkend. In 1923 werd de Nobelprijs voor geneeskunde en fysiologie toegekend aan twee van de leden van het onderzoeksteam, Banting en Macleod. Insuline was de eerste effectieve therapie tegen diabetes mellitus waarover artsen konden beschikken. De erkende ontdekking van insuline is echter slechts een keerpunt waarin een lange periode van twijfel en onzekerheid over het bestaan en de identiteit van insuline eindigde. Deze periode duurde 32 jaar, beginnend bij Minkowski's eerste formulering van een theorie over de rol van de alvleesklier (pancreas) in de genese van diabetes mellitus.

Hoofdstuk 3 beschrijft vrij gedetailleerd Minkowski's experimentele werk op honden. In 1889 opereerde Oscar Minkowski, in het laboratorium van de medische kliniek van de universiteit van Strassbourg, een hond en verwijderde zijn pancreas (pancreatectomie). De operatie was bedoeld om de bruikbaarheid aan te tonen van volledige pancreatectomieën als experimentele methode in het onderzoek naar de vertering van voedsel in de darm in afwezigheid van pancreassappen. De dag na de operatie merkte Minkowski dat de hond veel urine produceerde. De urine bevatte $12 \%$ suiker. Minkowski kwam tot de conclusie dat de hond én of andere vorm van experimentele diabetes had. Minkowski ontwierp een serie experimenten die gericht waren op de verheldering van de rol van de pancreas in het 
koolhydraatstofwisseling. Deze experimenten omvatten behalve volledige en partiële pancreatectomieën tevens gecombineerde transplantatie- en extirpatie-experimenten. Minkowski ontwierp deze experimenten om twee redenen: om de post-operatieve overleving van de honden te verbeteren, én om alternatieve verklaringen voor het optreden van de diabetes na de pancreatectomie uit te sluiten. In die tijd werd alleen aan het zenuwstelsel het vermogen toegeschreven om de functie van weefsels op afstand te beïnvloeden. De diabetes na verwijdering van de pancreas zou, in die visie, veroorzaakt kunnen worden door de uitgebreide beschadiging van het zenuwstelsel die het gevolg is van de operatie, en dus niet door de verwijdering van de pancreas.

Minkowski verwijderde de hele pancreas, of slechts delen ervan. Of hij transplanteerde, in een eerste operatie, een klein deel van de pancreas op een vaatsteel naar een onderhuidse zak. De rest van de pancreas werd tijdens een tweede operatie enkele dagen later verwijderd. Zolang als het transplantaat gezond was ontstond geen diabetes. Minkowski isoleerde en ligeerde de bloedvaten van het transplantaat in de vaatsteel, waarna de diabetes zich ontwikkelde. Het transplantaat had geen verbinding meer met de darm. Via welke andere weg dan via deze bloedvaten kon het pancreastransplantaat zijn invloed uitoefenen op de koolhydraatstofwisseling in weefsels op afstand? Het ontwerp van deze experimenten vormden voor een groot deel Minkowski's vertaling van de door hem verzamelde stomme inscripties; dat wil zeggen, zijn hypothese over het bestaan van een intern secreet dat in de pancreas geproduceerd en in het bloed afgescheiden werd.

Minkowski's werk trok de aandacht van een aantal onderzoekers in verschillende wetenschappelijke disciplines. Pathologen en embryologen suggereerden dat dit hypothetische interne secreet geproduceerd zou kunnen worden in de groepjes cellen in de pancreas die in 1869 door Langerhans werden beschreven: de eilandjes van Langerhans. Laguesse stelde voor om dit hypothetische actieve principe insuline te noemen. Fysiologen als Schäfer argumenteerden op grond van de grote vaatrijkdom van de eilandjes van Langerhans dat de pancreas als klier (met afvoergang) een tweede klier (zonder afvoergang) bevatte. Het meest tot de verbeelding sprekende aspect van de theorie van de interne secretie als verklaring voor deze experimentele, pancreatogene diabetes mellitus was dat zij een actieprogramma suggereerde voor de succesvolle behandeling van diabetes mellitus bij mensen: als Minkowski gelijk had zou het mogelijk moeten zijn het interne secreet uit vers pancreas weefsel van dieren te gebruiken om het tekort bij menselijke diabetici aan te vullen (substitutie).

Het principe van substitutietherapie was gemakkelijk te begrijpen. De praktijk was echter weerbarstig. Minkowski's experimenten stonden geen conclusies toe aangaande de chemische en fysische eigenschappen van het hypothetische interne secreet. Bovendien was er de vraag wat als succes gerekend kon worden. Een 'biologisch assay' was (nog) niet beschikbaar. Wanneer kon de toediening van pancreasextract als effectief beschouwd worden? Het voor de hand liggende doel van de behandeling was het verlichten van de symptomen van diabetes mellitus, dat wil zeggen, een vermindering van de glucosurie, verbetering van de algemene conditie en terugkeer uit diabetisch coma. Maar hoe snel zou het eerste effect zichtbaar moeten zijn: meteen, binnen enkele uren, de volgende dag, de volgende week? En hoe lang zou het moeten duren: een paar uur, een dag, een week, voor altijd? In hoofdstuk 3 wordt dit probleem toegelicht aan de hand van het werk van Georg Ludwig Zuelzer in het eerste decennium van de twintigste eeuw. Zuelzer injecteerde in een experiment een pancreasloze hond met pancreasextract. De injectie werd onverwacht gevolgd door emstige convulsies. Zuelzer had een dergelijke reactie nog nooit gezien, noch bij dieren, noch bij menselijke diabetici. Zuelzer schreef deze convulsies toe aan een giftige stof in het extract: een koperverbinding afkomstig van de koperen ketels die in het extractieproces gebruikt 
werden. Volgens Zuelzer waren deze convulsies geen eigenschap van het interne secreet van de pancreas waarnaar hij op zoek was.

In 1921-1922 was het werk van Banting, Best, Macleod en Collip doordrongen van dezelfde onzekerheid aangaande de chemische, fysische en biologische eigenschappen van de gezochte stof. De onderzoekers slaagden erin enkele items toe te voegen aan de lijst van biologische activiteiten die hun pancreasextracten vertoonden: vermindering van boedsuikerconcentraties in gezonde konijnen, het herstel van glycogeenvorming in pancreasloze honden, het opwekken van een door convulsies gekenmerkte hypoglycemische reactie bij konijnen. In de lokale setting van dat onderzoekslaboratorium begon insuline vorm te krijgen. De meest overtuigende demonstratie van insulines bestaan, en haar aanwezigheid in het extract, was de succesvolle behandeling van Leonard Thompson. Dit was een veertienjarige diabetische jongen die als gevolg van Allens dieet vel over been geworden was. Hij had het eindstadium van de ziekte bereikt en zou zonder verdere behandeling in coma raken en sterven. De glucosurie verdween bijna helemaal. De klinische conditie van de jongen verbeterde ook. In de daaropvolgende maanden werden productiefaciliteiten opgezet in Canada, de Verenigde Staten, maar ook in Groot Brittannië en Denemarken. Problemen betreffende verschillen in potentie tussen de verschillende partijen pancreasextract werden opgelost door middel van internationale standaardisatie.

De succesvolle behandeling van experimentele en humane diabetes mellitus door middel van een pancreasextract leverde een sterk argument op voor het bestaan van een intern secreet van de pancreas. Tot dat insulines effect praktisch gedemonstreerd kon worden was het interne secreet, ondanks het experimentele bewijs, niet meer dan een goed ogende hypothese. Wetenschappers die in het bestaan van insuline geloofden moesten haar aanwezigheid in een pancreasextract aantonen en haar identiteit definiëren. Wetenschappers moesten eerst zichzelf overtuigen van het bestaan van de stof. Op de tweede plaats moesten zij anderen overtuigen van het bestaan van insuline. Op de derde plaats moesten zij ervoor zorgen dat insuline ook in hun afwezigheid, op andere plaatsen dan het laboratorium, haar bestaan, aanwezigheid en identiteit uitvoerde. In het kielzog van de 'ontdekking' stabiliseerde niet alleen insuline als een entiteit in de wereld en als een betrouwbare en effectieve therapie. De pancreas en de ziekte, diabetes mellitus, waren ook veranderd. In hoofdstuk 3 ligt de nadruk dus op de wetenschap, op het dilemma van insulines bestaan, aanwezigheid en identiteit, en op de resolutie van dit dilemma in de ontdekking van insuline en de ontwikkeling van een wereldwijd netwerk van nieuwe industriële organisaties en instituten opgezet voor de produktie, distributie en internationale standaardisatie van insuline.

Hoofdstuk 4 analyseert de gebeurtenissen van de poliomyelitis-epidemie van 1952 in Kopenhagen. In hoofdstuk 4 ligt de nadruk op technologische en therapeutische innovatie in een Deens epidemieziekenhuis, Blegdamshospital, waar in de herfst van 1952, de medische staf geconfronteerd werd met een polio-epidemie met een zeer hoog sterftepercentage. De ernst van deze epidemie was om een aantal redenen van ongekende omvang. Het aantal patiënten, waarbij de diagnose bevestigd kon worden en die behandeling in het ziekenhuis nodig hadden, was groter dan ooit. Hoewel het merendeel van de patiënten jonger dan vijftien jaar was, onderscheidde de epidemie van 1952 zich door een relatief hoog aantal volwassenen. Bovendien was het aantal gevallen met aantasting van de hogere delen van het centraal zenuwstelsel (bulbaire polio) uitzonderlijk hoog. Blegdamshospital functioneerde als het poliocentrum voor Kopenhagen en de oostelijk delen van Denemarken.

Epidemieën leggen vaak genadeloos de tekortkomingen van gezondheidszorgsystemen en bestaande behandelpraktijken bloot. Zij zaaien twijfel over de waarheid van geaccepteerde kennis en over de effectieviteit en adekwatheid van technologieën. Het sterftepercentage 
onder patiënten die met bulbaire polio in Blegdamshospital werden opgenomen bedroeg $90 \%$. Met één 'ijzeren long' en zes 'kuras-respiratoren' was het ziekenhuis bij lange na niet voldoende toegerust om een epidemie van een dergelijke omvang aan te kunnen. De doorbraak in deze dramatische situatie kwam niet van de medische staf van het ziekenhuis maar van een buitenstaander, een free lance werkend anesthesist, Bjøm Ibsen, die niet eens bij het ziekenhuis in dienst was. Ibsen onderzocht enkele poliopatiënten op de verpleegafdelingen van het ziekenhuis, hij bestudeerde statussen en onderzocht, in de obductiekamer vier patiënten die aan de gevolgen van de aandoening bezweken waren. Patiënten met respiratoire en/of bulbaire polio hadden het gevoel te stikken en te verdrinken in hun eigen speeksel dat zij niet door konden slikken. Het zuurstoftekort dat het gevolg was van de ademhalingsinsufficiëntie veroorzaakte de vieze, blauwe kleur van de huid die als cyanose bekend staat. Bovendien werd het eindstadium van de ziekte gekenmerkt door hoge koorts, soms meer dan $40^{\circ} \mathrm{C}$, een klamme huid, een hoge bloedddruk, en ten slotte, een hoog 'totaal $\mathrm{CO}_{2}$ in plasma'. De artsen van Blegdamshospital interpreteerden het hoge totaal $\mathrm{CO}_{2}$ in plasma als een metabole alkalose. De longen van de patiënten in de obductiekamer vertoonden een geringe mate van atelectase die, volgens Ibsen, echter niet voldoende was om een adekwate ventilatie onmogelijk te maken. Patiënten met ademhalingsinsufficiëntie werden behandeld in negatieve druk respiratoren. Zuurstof werd gegeven om de cyanose te bestrijden. Deze patiënten waren daardoor goed geoxygeneerd. Toch raakten zij in coma en stierven zij.

Op grond van deze bevindingen trok Ibsen een aantal opzienbarende conclusies die strijdig waren met lokale klinische interpretaties. De meeste symptomen en afwijkende laboratoriumresultaten werden gewoonlijk toegeschreven aan de, door het virus in het centrale zenuwstelsel veroorzaakte, neurologische beschadiging. Ibsen schreef echter de hoge bloeddruk en het hoge totaal $\mathrm{CO}_{2}$ in plasma toe aan een opeenhoping van kooldioxide in het bloed. De hoge totaal $\mathrm{CO}_{2}$ in plasma was, volgens Ibsen, niet een uitdrukking van een metabole alkalose, maar een respiratoire acidose: hetgeen in fysiologische zin precies het tegenovergestelde is. De artsen van Blegdamshospital hadden de rol die de $\mathrm{CO}_{2}$-opeenhoping speelde in de produktie van de symptomen niet onderkend, en daardoor onbehandeld gelaten. Volgens Ibsen overleden de patiënten aan de gevolgen van een inadekwate venrilarie.

Ibsen stelde een nieuwe behandeling voor die bestond uit manuele, positieve druk ventilatie door een tracheostomie. Hoofdstuk 4 beschrijft gedetailleerd hoe dit voorstel met scepsis ontvangen werd, maar dat Ibsen het voordeel van de twijfel kreeg. Ibsen mocht zijn behandeling op een daartoe geselecteerde patiënt toepassen: een twaalfjarig meisje in een zeer slechte conditie, met verlammingen van zowel armen als benen, snakkend naar adem en verdrinkend in haar eigen speeksel. $\mathrm{Zij}$ had $40,2^{\circ} \mathrm{C}$ koorts. $\mathrm{Zij}$ was cyanotisch en zweette. Ondanks goed uitzuigen van de keel kon Ibsen in eerste instantie de longen van het meisje niet goed ventileren. Hij schreef dit voor een deel toe aan slijm lager in de luchtwegen, voor een deel aan bronchospasmen die het gevolg waren van de manipulaties tijdens de operatie en het daarop volgende uitzuigen. In deze wanhopige situatie besloot Ibsen het meisje 100 mg Penthotal intraveneus te geven. Hierdoor raakte zij in shock. Haar eigen ademhaling stopte. Nu lukte het echter wel om haar goed te ventileren. De verwijdering van het teveel aan $\mathrm{CO}_{2}$ door de beademing droeg bij aan de daling van de bloeddruk. Deze werd behandeld met plasmavervangende infusies. Het lukte Ibsen het meisje te stabiliseren. Haar huidskleur werd weer normaal, zonder de toevoeging van extra zuurstof. De bloeddruk werd ook weer normaal. Dit twaalfjarige meisje was de eerste patiënt die tijdens deze polio-epidemie door middel van een medische interventie in leven werd gehouden. $\mathrm{Na}$ Ibsens demonstratie van de effectiviteit en superioriteit van manuele positieve druk ventilatie bij de behandeling van bulbaire polio besloot het hoofd van de medische staf van het ziekenhuis, Lassen, onmidde- 
lijk tot de toepassing van deze noodbehandeling bij alle patiënten met ademhalingsinsufficiëntie en slikproblemen.

De toepassing van manuele positieve druk ventilatie bracht het sterftepercentage van bulbaire polio binnen enkele weken terug van 90 naar $15 \%$. Dit was het begin van het einde van de negatieve druk respiratoren (ijzeren longen en kuras-respiratoren). Er was een nieuwe grenslijn dwars door het 'oude' syndroom van de bulbaire polio getrokken. De 'oude' onbehandelbare ziekte was gesplitst in de dodelijke maar behandelbare $\mathrm{CO}_{2}$-accumulatie en een virale infectie van de motorische centra in ruggemerg en hersenstam, welke verbijgaande verlammingen veroorzaakte van de bij ademhaling en slikken betrokken spieren. Het eerste was een ventilatieprobleem. Het laatste was niet langer dodelijk, mits een adekwate ventilatie verzorgd werd door middel van positieve druk ventilatie. De succesvolle interventie van de anesthesist tijdens deze polio-epidemie gaf een belangrijke impuls aan de herwaardering van de anesthesiologie als medisch specialisme.

Hoofdstuk 4 analyseert de retorische kenmerken van deze snelle en radicale veranderingen in termen van de zintuiglijke onmiddelijkheid van het overlevende, twaalfjarige meisje, ondersteund door de beschikbaarheid van een nieuw apparaat om kooldioxide in uitademingslucht te meten. Hierdoor veranderde Blegdamshospital en de epidemie in een waarheids- en competentietheater waar Ibsen de plausibiliteit van zijn pathofysiologische verklaring bewees, waar hij zijn eigen competentie en vaardigheid als anaesthesist demonstreerde. Als vertegenwoordiger van zijn discipline, demonstreerde hij tevens de competentie en vaardigheden die anesthesiologen in het algemeen te bieden hadden buiten hun traditionele domein van de operatiekamer. Ibsen demonstreerde ook de effectiviteit en superioriteit van intermitterende positieve druk ventilatie ten opzichte van negatieve druk ventilatie. Zowel tijdens als vlak na de epidemie in Kopenhagen werden in Scandinavië, maar ook in Engeland en Duitsland, in snel tempo mechanische, positieve druk respiratoren ontworpen, geproduceerd en op de markt gebracht, de ijzeren long tot vergetelheid verdoemend.

Hoofdstuk 5 gaat over de controverse over de definitie van en criteria voor de humane (hersen)dood. De positieve druk respirator, die zo'n impuls kreeg door zijn succesvolle toepassing tijdens de polio-epidemie van 1952 in Kopenhagen, vormde de kern van de beademingscentra en intensive care units die vervolgens werden opgericht. De respirator werd ook het symbool voor een moderne geneeskunde die haar patiënten niet meer toestaat te sterven. De ontwikkeling, in de jaren vijftig en zestig, van beademingscentra en intensive care units, van cardio-pulmonaire resuscitatietechnieken, en van technieken om bij orgaantransplantaties de afstotingsreactie te onderdrukken, stelden de 'oude' robuustheid op de proef waarmee gezegd kon worden dat een patiënt was overleden. De oude hart-long-criteria voldeden niet meer. Aan de hersenen gerelateerde doodsdefinities werden verdedigd, en bestreden, zowel door medici als door juristen en filosofen. De betekenis van 'hersendood', een toestand die tot de late jaren zestig gezien werd als de dood van het orgaan hersenen in een overigens levende patiënt, verschoof en werd geïdentificeerd met de dood van de mens. In deze gevalstudie komen morele en juridische vragen het meest expliciet aan de orde, in hun nauwe verbondenheid met vragen over biologische feiten en technologische haalbaarheid en betrouwbaarheid. Er ontwikkelde zich een controverse. Mensen waren bang dat zij te vroeg dood verklaard zouden worden. Neurologen werden ervan beschuldigd de hersendood 'uitgevonden' te hebben om bij patiënten die volgens de oude criteria nog niet dood waren te kunnen overgaan tot het oogsten van organen voor transplantatiedoeleinden. De verschillende posities in dit debat over hersendood zijn echter veel complexer dan de simpele oppositie tussen traditionele hart-long-criteria en het nieuwe hersendoodbegrip doet vermoeden. Veel van de controversiële punten zijn vanaf het einde van de jaren zestig tot op 
vandaag onopgelost gebleven. Veel westerse landen hebben echter een vorm van aan de hersenen gerelateerde doodsdefinitie aangenomen. Deze mag gebruikt worden in situaties waar de toepassing van resuscitatie en intensive care technologieën een doodsdiagnose volgens de oude criteria onmogelijk maken en waar toestemming werd verkregen voor de verwijdering van organen voor transplantatiedoeleinden na de dood van de patiënt; dat wil zeggen, op plaatsen waar intensive care en transplantatiepraktijken elkaar ontmoeten. Met andere woorden, sluiting van de controverse heeft niet plaatsgevonden, terwijl toch juridisch gelegitimeerde medische praktijken, waarin patiënten met dode hersenen dood verklaard worden, tot ontwikkeling zijn gekomen.

Hoofdstuk 5 onderzoekt in detail de vier verschillende posities waarin de verschillende voorstellen voor de (her)definiëring van het einde van het menselijk leven gegroepeerd kunnen worden. Drie van deze posities zijn aan de hersenen gerelateerde definties. Naast de traditionele 'totale dood', worden de hersenstamdood, de volledige hersendood en de neocorticale hersendood onderscheiden. Deze doodsdefinities stellen niet alleen maar een bepaald verband tussen de menselijke dood en het stoppen van functionele activiteit in een bepaald deel van het lichaam voor. Zij proberen tevens te definiëren en grenzen te trekken tussen wat, nu en in de toekomst, toegestaan is en wat niet; wanneer en hoe een lichaam behandeld dient te worden als een levende persoon, en wanneer een lichaam als een lijk behandeld dient te worden. De doodsdefinities roepen verschillende toekomstige medische praktijken op en omvatten, met andere woorden, verschillende actieprogramma's. Doodsdefinities brengen verschillende heterogene associaties tussen cognitieve, morele, sociale en technische zaken tot stand in een poging medische praktijken vorm te geven (of om te vormen). In de beschrijving van de verschillende doodsdefinities in hoofdstuk 5 ligt de nadruk op hoe deze definities ontologische, epistemologische, morele en juridische status verschillend verdelen. Dit is de manier waarop de notie van een 'definitie' in dit hoofdstuk gebruikt wordt, en niet als een uitspraak over de essentie van jets.

Hoofdstuk 5 beschrijft vervolgens hoe in Nederland de controverse over de redefiniering van de dood gepartitioneerd werd. De controverse is niet meer overal. Zij bestat gewoonlijk niet meer in de intensive care units, niet meer in het private domein van de medische praktijk. De controverse bestaat wel nog in het publieke domein van de filosofische, ethische en juridische literatuur, of in publieke media zoals dagbladen en televisieprogramma's, waar zij van tijd tot tijd opvlamt. Wat plaats heeft gevonden is een partitionering, een uitsluiting naar het publieke domein van de controverse, van twijfel, onzekerheid en onbegrensde onderhandelbaarheid. Dit resulteerde in een relatief stabiel, 'twijfelarm' privaat domein waar mensen hun werk kunnen doen. Tussen de binnen- en de buitenkant werd een relevantiehorizon gevormd. De beschrijving van dit proces gaat niet over de professionele, persoonlijke of praktische redenen die mensen kunnen hebben om deze of gene doodsdefinitie aan te hangen. Wat telt zijn de keuzes die uiteindelijk gemaakt worden in specifieke documenten (Gezondheidsraadrapporten, wetsvoorstellen, enz.) die tot effect hebben gehad dat een binnen/buiten- of een privaat/publiek-grens werd gevormd. In dit specifieke geval ligt de nadruk op juridische en semi-juridische documenten en wordt geen aandacht besteed aan financiële en praktische regelingen die getroffen werden.

Vroege uitspraken over hersendood, eind jaren vijftig en jaren zestig, waren vrij vraag. Noties als hersendood, 'coma dépassé' en irreversibel coma werden haast als synoniemen gebruikt. Het specifieke en onderscheidende belang van de hersenstam of de neocortex in relatie tot de volledige hersenen werd (nog) niet onderkend. Noch was er een gestandaardiseerde set procedures volgens welke de onherstelbare beschadiging van de hersenen, en dus hersendood, kon worden vastgesteld. Ondanks zijn vaagheid leek de notie van hersendood echter robuust genoeg om communicatie en discussie over deze nieuwe manier van denken 
over het einde van het menselijk leven over disciplinegrenzen heen mogelijk te maken. Anderzijds was de notie van hersendood flexibel genoeg om adaptatie aan verschillende disciplinaire (medische, ethische, juridische) interpretaties en vocabulaires toe te staan. Hersendood betekende zowel iets - hoewel niet hetzelfde - voor de neurofysioloog en biolong in de arts, als voor de existentialist in de filosoof, als voor de wetgever die werkte aan regelgeving die orgaantransplantatie mogelijk moest maken. In die zin functioneerde het hersendoodsbegrip als een grensobject.

Ziekenhuisprotocollen over hersendoodsdiagnostiek die bijna twee decennia later in Nederland gepubliceerd werden bevatten het volledige hersendoodsbegrip en richtlijnen voor diagnostische procedures in die gevallen waar de arts hersendood vermoedt. Deze protocollen laten zien hoe de relatief slecht gedefiniëerde en slecht gestructureerde notie van hersendood minder abstract en beter gestructureerd werd in de lokale settings van ziekenhuizen. Deze protocollen structureren, standaardiseren en normaliseren het private domein van deze praktijken. Zij definiëren zowel de dood als de rol van iedere actor. Zij verdelen de legitimiteit van het woordvoerderschap (wie mag voor wie spreken over welke zaken). Een dergelijke co-definitie en co-restrictie reduceert de ruimte voor alternatieve handelingen en praktijken. Tevens wordt zo de stabilisatie van feiten bevordert. In de locale settings van ziekenhuizen wordt hersendood daardoor harder en feitlijker, en minder gevoelig voor kritiek en twijfel die geuit wordt in het publieke domein.

Hoofdstuk 6 resumeert de theoretische analyse die in de voorafgaande hoofdstukken ontwikkeld werd. De notie van een actieprogramma wordt geintroduceerd als het concept dat het mogelijk maakt de verschillende gevalstudies met elkaar te vergelijken. De term actieprogramma roept het beeld op van een coherent, gedetailleerd plan dat ontwikkeld en ontworpen wordt voor een bepaald doel, bijvoorbeeld om een bepaalde dienst te verlenen of om een bepaald probleem op te lossen. In deze zin zijn onderzoeksprogramma's, orgaantransplantatie- of vaccinatieprogramma's en therapeutische regimes voorbeelden van actieprogramma's. Deze programma's omvatten meerdere items die op een specifieke manier met elkaar en met het programma als geheel samenhangen. Individuele items en het programma als geheel zijn van elkaar afhankelijk. Hun identiteit wordt bepaald door hun onderlinge relaties.

Twee aspecten van de notie van een actieprogramma zijn van groot belang. Ten eerste, een programma kan een plan zijn: een plan van gebeurtenissen, taken en activiteiten die plaats zullen vinden, inclusief de tijden waarop dit zal gebeuren of waarop dit gedaan moet worden. In deze zin is een actieprogramma een lijst met uitspraken die specifieke rollen toewijst aan de verschillende actoren, maar die nog niet uitgevoerd zijn. Het tweede aspect dat van groot belang is bij een actieprogramma is de uitvoering, de implementatie. Het is de implementatie van een actieprogramma die aanwezigheid geeft aan datgene wat het actieprogramma probeert te realiseren. Uitvoering realiseert (het bestaan van een entiteit). Het vermogen om actieprogramma's uit te voeren is een belangrijke bron van de zekerheid waarmee we kennis voor waar houden en waarmee we technologieën effectief vinden.

Een actieprogramma is de lijst van acties (interventies, metingen) die uitgevoerd worden door de wetenschappers, en van reacties die uitgevoerd worden door de entiteiten of lichamen die onderzocht worden. Samen definiëren en constitueren deze acties een bepaalde entiteit of toestand. Het is de uitvoering van een bepaald stabiel patroon van acties en reacties waardoor de identiteit van een entiteit gerealiseerd wordt. Zo ontwikkelde Minkowski de vaardigheid en het vermogen om het actieprogramma dat pancreasdiabetes genoemd werd uit te voeren. Door het ontwerp van dit actieprogramma elimineerde hij op één na, het bloed, alle mogelijke manieren waarop de pancreas de suikerstofwisseling zou kunnen beïnvloeden. 
In de loop van dit proces veranderde wat begon als een experiment in een demonstratie. Insuline bestond in 1889 echter nog niet: omdat nog niemand het vermogen had ontwikkeld om het stabiele, herhaalbare actieprogramma uit te voeren dat aan insuline zijn bestaan zou verlenen; een actieprogramma dat waameembare, zichtbare aanwezigheid en identiteit aan insuline zou verlenen. Tussen 1889 en 1921 was insuline een entiteit zonder actieprogramma; daarom was het een hypothese. Alle pogingen om het interne secreet van de pancreas in een extract te vangen kunnen gezien worden als een poging om een actieprogramma te ontwikkelen en uit te voeren dat ondubbelzinnig insulines bestaan, haar aanwezigheid in een extract en haar identiteit zou realiseren.

In Denemarken werd het actieprogramma dat $\mathrm{CO}_{2}$-accumulatie constitueerde vooral uitgevoerd in onderzoekslaboratoria die aan problemen werkten op het terrein van de ademhalingsfysiologie, en in operatiekamers. Voorafgaande aan de epidemie van 1952 was dit actiepromma nog nooit uitgevoerd op de verpleegafdelingen van Blegdamshospital; niet met dezelfde overtuigingskracht waarmee Ibsen de $\mathrm{CO}_{2}$-accumulatie demonstreerde bij het twaalfjarige meisje.

Voor elk van de vier doodsdefinities die in hoofdstuk 5 werden besproken is het mogelijk het diagnostische actieprogramma op te stellen dat de dood van de patiënt zou realiseren. $\mathrm{Zij}$ zouden echter sterk verschillen. De diagnostische actieprogramma's die totale dood, hersenstamdood, neocorticale dood en volledige hersendood realiseren zijn nauw verbonden met, of maken deel uit van (de als definities geintroduceerde) actieprogramma's op een discursief niveau. Deze discursieve actieprogramma's zorgen voor de verbale en discursieve vertaling van het patroon van inscripties dat geproduceerd wordt iedere ketr als het diagnostische programma wordt uitgevoerd. In deze discursieve actieprogramma's worden, steeds op een andere manier, wetenschappelijke kennis, technologische moge. lijkheden, morele en juridische kwesties met elkaar verbonden. Het is mogelijk over de morele aspecten van deze heterogene actieprogramma's te spreken in termen van Jensens verantwoordelijkheid voor de zwakken. We zullen echter bereid moeten zijn te accepteren dat er niets is in de handelingen van mensen die solidariteit met de zwakken tonen waardoor wij dergelijke handelingen als goed of als moreel verantwoord zouden moeten zien. Handelingen hebben geen intrinsieke kwaliteit waardoor zij als paradigmatische voorbeelden van moreel verantwoord handelen in het oog springen, waardoor anderen deze handelingen als een standaard zouden kunnen gebruiken om handelingen uit verleden of toekomst te kunnen evalueren. Of bepaalde actieprogramma's als standaard fungeren hangt van de vraag of de morele orde die zij veronderstellen ter discussie wordt gesteld of niet.

Discursieve actieprogramma's kunnen nog steeds hypothetisch zijn, maar ze kunnen ook al gerealiseerd zijn. Ze kunnen geïmplementeerd zijn op de ene plaats (laboratorium, ziekenhuis, land) maar niet op een andere. Het actieprogramma van de hersenstamdood is in Engeland geimplementeerd, maar niet in Nederland. Nederland heeft een volledig hersendoodsbegrip aanvaard. $\mathrm{CO}_{2}$-accumulatie was wel gerealiseerd in de operatiekamer maar nog niet op de afdelingen van Blegdamshospital. In het geval dat een actieprogramma gerealiseerd is, is het belang te vragen waar het gerealiseerd is en waar niet, en in welke mate. Actieprogramma's hebben een geschiedenis die zich uitstrekt van het begin tot aan de uiteindelijke implementatie en stabilisatie. De implementatie van nieuwe actieprogramma's in de geneeskunde vereist vaak de samenwerking van wetenschappers, artsen, ingenieurs, filosofen en juristen. Dit betekent dat werk over disciplinaire grenzen heen gecoördineerd moet worden om het ontstaan van consensus mogelijk te maken. De geschiedenis van gerealiseerde actieprogramma's wordt gekenmerkt door een geleidelijke, maar soms abrupte, reductie van onbepaaldheid, en een toename van bepaaldheid. De verandering van onbe- 
paaldheid naar bepaaldheid is een verandering van twijfel en onzekerheid naar zekerheid en hardheid, van hypothese naar feit, van belofte en implicatie naar effectieve therapie.

In de laatste paragraaf van hoofdstuk 6 wordt tenslotte gesteld dat als er een algemene les uit deze studie getrokken kan worden dat het dan een strategische moet zijn. Een constructivistische analyse van de geneeskunde legt de nadruk op de contingentie van medische praktijken zoals zij vandaag zijn. Dit impliceert dat er geen principiële reden is waarom medische praktijken niet anders zouden kunnen zijn. Op het micronivo kunnen veranderingen het resultaat ziijn van bewuste strategieën van ex́n of een klein aantal actoren. De algehele vorm van een medische praktijk kan echter moeilijk gezien worden als het resultaat van expliciet geplande, rationele actie. Er waren vele kleine actoren die in het verleden bijdroegen aan de vorm en inhoud van de huidige moderne geneeskunde. Deze vorm en inhoud van de geneeskunde, haar grenzen met de buitenwereld, worden tevens door vele kleine actoren onderhouden. Een constructivistische analyse benadrukt dus ook dat het moeilijk zal zijn medische praktijken te veranderen. Voor diegenen die kritisch staan tegenover bepaalde praktijken is het belangrijk hun relatieve positie ten opzichte van de binnenbuiten grens van die praktijk te bepalen. Voor buitenstaanders, of het nu leken, beleidsmakers of ethici zijn, zal het moeilijk zijn om precies te achterhalen welke ontwikkelingen gaande zijn in het private domein van bijvoorbeeld onderzoekslaboratoria. Voor commentatoren in het publieke domein worden ontwikkelingen niet zichtbaar voordat zij uit het laboratorium komen. Dit is een belangrijke reden waarom de academische medische ethiek vaak als een brandweerkorps van de ene morele brandhaard naar de andere snelt. Daarom loopt de medische ethiek vaak achter wetenschappelijke en technologische ontwikkelingen aan en houdt zij zich vooral bezig met ethische problemen die te maken met het gebruik van technologieën die al ingevoerd zijn. Voor de actoren in die specifieke praktijk vormt die binnen-buiten-grens een relevantiehorizon. $\mathrm{Zij}$ die nieuwe ontwikkelingen in een vroeg stadium ter discussie willen stellen zullen een weg moeten vinden om deze grens te passeren. Dit zal echter niet altijd mogelijk zijn, want soms is deze binnen-buiten-grens zeer fysiek van aard.

Ethici, filosofen, beleidsmakers of artsen, kortom iedereen die zich zorgen maakt over. of die zich wil verzetten tegen de implementatie van nieuwe actieprogramma's zal zich bewust moeten zijn van het bestaan en het belang van private domeinen in laboratoria, in industriële onderzoeks- en ontwikkelafdelingen, en in ziekenhuizen. Hier kan het werk aan nieuwe ontwikkelingen voortgang vinden in relatieve beslotenheid en beschermd tegen publiek onderzoek. Het zal van groot belang zijn de relevantiehorizon te penetreren en te destabiliseren om zodoende het ontwikkeingswerk dat daar plaatsvindt in een vroeg stadium ter discussie te kunnen stellen. Zij die zich mengen in het debat zullen, ongeacht hun orspronkelijke opleiding, bereid moeten zijn over disciplinaire grenzen heen te stappen en ook wetenschappelijke en technische kwesties aan moeten pakken. In gestabiliseerde praktijken zijn cognitieve, technische en morele kwesties nauw met elkaar verbonden en aan elkaar vast gemaakt. Elk van deze kwesties kan ter discussie gesteld worden om de hardheid en vanzelfsprekendheid van die praktijk weer vloeibaar te maken. De keuze van de kwestie die ter discussie gesteld moet worden is een strategische keuze. Daarna moet men bereid zijn om het al het werk te doen om een hardheid en zekerheid te (re)construeren. Dit zal ook werk in en samenwerking met mensen van andere disciplines omvatten. Actieprogramma's kunnen nooit door één enkele discipline alleen ter discussie worden gesteld, maar ook niet door éćn discipline worden geïmplementeerd. 


\section{Glossary}

This glossary contains a list of medical terms used in this book. Reference to this glossary may help in the understanding of the technical content of the various case studies. The $\rightarrow$ arrows refer to other entries in the glossary.

acid base status - acid base physiology: pertains to the measuring important parameters that give insight into the biochemical mechanisms (buffers) through which the body maintains a normal $\mathrm{pH}$ of around 7.4 .

acidosis: a pathological condition resulting from the accumulation of acid in, or loss from base from, the body. In acidosis $\mathrm{pH}$ is $<7.4$.

acinar tissue - acinar - acini: acinus is a general term used in anatomical nomenclature to designate a small saclike dilatation, particularly one found in various glands. In the pancreas the acinar tissue is associated with the production of the external secretion of pancreatic juice into the duodenum. adjuvant therapy: a therapy that assists, supports or aids the primary therapy.

adrenal extracts: tissue extracts from the adrenal glands. Adrenal means 'situated near the kidneys'. adrenergic structures: nerve fibers that liberate norepinephrine into a synapse when a nerve impuls passes.

afferent: centripetal, towards a center. An afferent nerve carries an afferent stimulus towards the brain (center). See $\rightarrow$ efferent.

allotransplantation: intra-species transplantation. Transplantation of cells, tissues or organs from one individual to another individual of the same species. See $\rightarrow$ graft.

alveolar: alveolus is a general term used in anatomical nomenclature to designate a small saclike dilatation. In the lungs the alveoli are small outpouchings along the walls of the smallest, terminal branches of the bronchial tubes, through the wall of which gas exchange takes place between alveolar air and capillary blood.

amino acid sequence: the sequence of amino acids that build up a protein molecule.

anamnesis - hetero-anamnesis: history taking; the collected data concerning a patient, her family, environment, previous diseases, etcetera. When the patient is unconscious relatives and friends provide this information in a hetero-anamnesis.

anaphylactic reaction: an unusual or exaggerated allergic reaction of an organism to foreign protein or other substances. Use of the term was originally restricted to a condition of sensitation in laboratory animals produced by the injection of foreign matter, such as horse serum (diphteria antitoxin), but has since been extended to human reactions. Such an injection renders the individual hypersusceptible to a subsequent injection.

anemia: a reduction below normal in the number of erythrocytes, in the quantity of hemoglobin, or in the volume of packed cells per $100 \mathrm{ml}$ of blood which occurs when the balance between blood loss and blood production is disturbed.

anencephaly: congenital absence of the cranial vault, with cerebral hemispheres completely missing or reduced to small masses attached to the base of the skull. 
angiography: the roentgenographic visualization of blood vessels following the introduction of contrast material. See $\rightarrow$ arteriography.

anoxia anoxic: absence or lack of oxygen. Reduction of oxygen in body tissues below physiological levels.

anthrax bacillus: bacteria causing an infectious diseas of ruminant animals. Anthrax results from the ingestion of spores of Bacillus anthracis in the soil, often leading to sudden death due to the proliferation of bacteria in the blood.

antigen: any substance which is capable, under appropriate conditions, of inducing in an organism an immune respons, that is, the formation of antibodies, and of reacting specifically in some detectable manner with the antibodies induced.

anuria: absence of the excretion of urine from the body.

aortic stenosis - aortic arch: the aorta is the main arterial trunk, arising from the left ventricle of the heart, from which the systemic arterial system proceeds. From the left ventricle it passes upwards, bends over (aortic arch) and passes downwards through the thorax. In aortic stenosis there is a narrowing or stricture of the lumen of the vessel at the aortic orifice or in the aorta itself, frequently in the aortic arch.

apallic syndrome: pallium is the cerebral cortex as viewed in its entirety: the mantle of gray matter covering both cerebral hemispheres. A-pallic syndrome is the clinical condition characterized by a loss of functions of both cerebral hemispheres. The condition is better known as $\rightarrow$ persistent vegetative state (PVS).

apn(o)ea - apn(o)eic: cessation of breathing.

ARAS: see $\rightarrow$ ascending reticular activating system.

arteriography: angiography of the arteries. See $\rightarrow$ angiography.

arteriovenous shunt: artificial conncection between an artery and a vein.

ascending reticular activating system: system of nerve fibers, traversing from the brain stem to the midbrain through the meshy (reticular) tissue between the nervous nuclei. Responsible for the activation or arousal of the cerebral hemispheres or neo-cortex. More a functional entity than a distinct anatomical structure. Described by Moruzzi and Magoun.

asphyxia: a condition due to lack of oxygen in respired air.

asytole: systole is the phase of the heart cycle in which the heart's ventricles contract and expulse the blood, which can be felt as the heart beat and pulse. A-systole is the absence of heart beat: cardiac standstill or cardiac arrest.

atelectasis: incomplete expansion of the lungs at birth or collapse of (parts of) the adult lung, for example, due to absorbtion of air behind a bronchial occlusion (as by secretions, a foreign body or tumor).

atony - atonic: lack of normal tone or strength. Used for lack of muscle strength, but also for lack of autonomic, vegetative nerve functions or vegetative dysregulation.

atrophy: a wasting away. A dimunition in the size of a cell, tissue, organ or body part.

atropine: alkaloid drug, derived from species of belladonna or produced synthetically. An anticholinergic drug used for the relaxation of smooth muscles in various organs and to increase heart rate by blocking the vagus nerve.

auto-immune disease: condition characterized by a specitic immune response against the constituents of the body's own tissues.

harbiturate: salt or derivative of barbituric acid. A group of substances used as sedatives.

base excess: parameter for the metabolic component in disturbances of the acid-base equilibrium in the blood.

Berkefeld-filter: bacterial filter used to remove bacteria from liquids.

biuret reaction: laboratory test for quantitative determination of protein concentration. Produces a blue color which can be measured in a spectrophotometer.

blood gas analysis: measurement of dissolved gasses (oxygen, carbon dioxide) in the blood and of parameters that determine acid base status: $\rightarrow p H, \mathrm{PO}_{2}, \rightarrow \mathrm{PCO}_{2}$, oxygen saturation, $\rightarrow$ base excess and $\rightarrow$ standard bicarbonate.

brain stem: stemlike portion of the brain connecting the cerebral hemispheres with the spinal cord. 
bronchospasm: narrowing or stricture of the bronchial ducts in the lungs due to spasmodic contraction of the smooth muscle in the wall of the bronchi.

bulbar polio: form of $\rightarrow$ poliomyelitis (infantile paralysis) affecting nerve centers in the (bulbar portion of the) brainstem, leading to an impairment (paralysis) of the muscles of the throat, hence, to impairment of swallowing. Often in combination with paralysis of muscles of the thorax and diaphragm (respiratory insufficiency).

cachexia: a profound and marked state of general ill health and malnutrition.

calculi: stones or abnormal concretions formed in the body, composed of mineral salts.

carbon dioxide: important gas dissolved in the blood as a waste product of metabolic processes in the tissues. When it accumulates in the blood it causes $\rightarrow$ acidosis. Carbon dioxide drives the respiratory center in the brain stem and is thus important in the regulation of breathing.

cardiopulmonary arrest: cessation of breathing and circulation of blood.

cardiopulmonary resuscitation: emergency treatment applied to counteract $\rightarrow$ cardiopulmonary arrest, consisting of $\rightarrow$ artificial ventilation (mouth-to-mouth breathing or bag ventilation) in combination with (usually $\rightarrow$ closed-chest) cardiac massage, and $\rightarrow$ defibrillation .

carotid sinus: the dilated portion of the internal carotid artery, situated above the division of the common carotid artery into its two main branches (internal to the brain, and external to the facial muscles and scalp). The carotid sinus contains in its wall pressure receptors that are stimulated by changes in blood pressure.

catecholamines: group of compounds mimicking the effect of the sympathetic nervous system.

central respiratory arrest: cessation of breathing due to impairment or destruction of the respiration center in the brain stem.

cephalon - cephalic: head, pertaining to the head. To be distinguished from $\rightarrow$ encephalon, $\rightarrow$ cerebrum, and $\rightarrow$ cerebellum.

cerebellum - cerebellar: part of the brain that occupies the posterior cranial fossa behind the brain stem. It is separated from the cerebral hemispheres by the $\rightarrow$ tentorium. The cerebellum plays and important role in the coordination of movement.

cerebral perfusion pressure: the difference in pressure between the systolic blood pressure in the arteries of the brain and the intra-cranial pressure in the surrounding tissues. This is the pressure with which the brain is perfused with blood

cerebral cortex: the gray matter of the cerebrum. The top-layer of the cerebral hemispheres containing the cell bodies of the neurons.

cerebral vasomotor paralysis: loss of the brain's bloodvessel's capacity to dilate or constrict independently of the blood pressure, due to which changes in bloodpressure are transmitted to the surrounding tissues.

cerebrospinal fluid: the colorless liquid surrounding the brain and the spinal cord.

cerebrum - cerebral: (pertaining to) the upper part of the brain consisting of the two hemispheres.

To be distinguished from $\rightarrow$ encephalon and $\rightarrow$ cerebellum.

cervical: pertaining to the neck, or to the neck of any organ or structure.

cesarean section: delivery of the foetus through an incision in the lower uterine segment.

closed-chest cardiopulmonary resuscitation: $\rightarrow$ cardiopulmonary resuscitation with the chest closed.

To be distinguished from the employment of techniques that require an opening of the chest, that is an emergency $\rightarrow$ thoracotomy.

clyster: an injection into the rectum.

coma dépassé: term introduced by Mollaret et al. in 1959 to indicate a state of coma from which there could be no return to a more or less normal conscious life. Also known as irreversible coma. coma: a state of sleeplike unconsciousness from which the patient can not be aroused, not even by very powerful and painful stimulation. To be distinguished from the patient who has lost her capacity for consciousness due to extensive brain damage, but who displays a waking-sleeping cycle (as in $\rightarrow$ persistent vegetative state).

contusion: a bruise. Injury of a body part without a rupture of the skin 
cornea - cornea reflex: the transparant structure covering the white part of the eye. When the cornea is touched the eye blinks through a reflex mechanism (cornea reflex). This is one of the brain stem reflexes to test the viability of the cranial nerves and brain stem.

cranial nerves: twelve pairs of nerves connected directly to the brain, most of them to the brain stem. In contrast to spinal nerves that spring from the spinal cord.

cuirass respirator: type of negative pressure respirator with the form of a shield that is placed over the thorax and abdomen. The negative pressure in the respirator pulls the $\rightarrow$ diaphram down to produce an inspiration movement.

cyanosis - cyanotic: the foul, blue skin color that results from a reduced oxygen saturation in the blood and tissues.

D/N-ratio: dextrose/nitrogen ratio. Parameter indicative of the balance between carbohydrate and protein metabolism.

decerebration: loss of cerebral functions due to a transection at the level of the brain stem.

decortication: loss of cortical functions due to brain damage above the level of the brain stem.

defibrillation - defibrillator: the act of reverting the heart from a state of ventricular $\rightarrow$ fibrillation to normal coordinated contractions through the application of an electrical counter-shock, and the device employed to do so.

defibrinated blood: blood from which the fibrin is removed by allowing it to clot.

dextrorotatory - laevorotatory: the property of a substance to turn the plane of polarization of a bundle of light to the right (dextro) and left (laevo) respectively.

diabetes mellitus: sugar sickness. To be distinguished from diabetes insipidus in which there is an excessive excretion of urine, but without $\rightarrow$ glycosuria.

diaphragm: the muscular partition between the abdomen and the thorax. Plays an important role in respiration.

diencephalon: the midbrain, consisting mainly of the thalamus and the hypothalamus.

direct cardiac massage: cardiac massage applied directly to the heart by manual compression. This requires an opening of the chest. See $\rightarrow$ closed-chest cardiopulmonary resuscitation.

distal: remote. Farther from any point of reference. See $\rightarrow$ proximal.

duodenum: the first, approximately twelf inches long, portion of the small intestine, extending from the stomach. The duodenum shares its blood supply with portions of the $\rightarrow$ pancreas.

ECG: see $\rightarrow$ electrocardiogram.

echo-encephalography: brain imaging technique employing ultrasound.

edema: the presence of an abnormally large amount of fluid in the intercellular spaces of the tissues (outside the vascular bed).

EEG: see $\rightarrow$ electroencephalogram.

efferent: centrifugal, away from a center. An efferent nerve carries an efferent stimulus from the brain towards a target organ or tissue. See $\rightarrow$ afferent.

electrocardiogram: recording of electrical currents arising in the heart through the employment of electrodes on the skin of the chest.

electrocerebral silence: absence of measurable electrical currents arising from the cortex of the cerebral hemispheres, resulting in a flat $\rightarrow$ electroencephalogram.

electroencephalogram: recording of the electrical currents arising in the cerebral cortex through the use of electrodes placed on the skin of the scalp. Scalp electrodes do not pick up currents from deeper brain structures.

electrophysiological research: a kind of physiological research making use of electrical stimulation of nerve endings and of electrodes positioned in the tissues to pick up electrical currents, often combined with selective surgical destruction of structures to map the routes of nerve signals and to ascertain the function of various structures.

encephalomyelitis: inflammation of the brain and spinal cord, caused, for example, by a viral infection.

encephalon: term used to indicate the (whole) brain. To be distinguished from $\rightarrow$ cerebrum, $\rightarrow$ cephalon and $\rightarrow$ cerebellum.

encephalopathy: general term used to indicate a pathological condition of the brain. 
endogenic: originating from within the organism or arising from causes within the organism. Synonymous with endogenous. See $\rightarrow$ exogenic.

endotracheal suction: the application of suction in the $\rightarrow$ trachea, in order to remove secretions and to maintain a free airway.

enema: see $\rightarrow$ clyster.

epidural hematoma: accumulation of blood between the dura mater, the though membrane covering the brain, and the bony skull. See also $\rightarrow$ subdural hematoma.

epithelium: the tissue, consisting of large, flat cells, covering the in- and external surfaces of the body.

etiology aetiology: the study of factors that cause disease and the method of their introduction to the host.

evoked potentials: significant electrical responses recorded in an $\rightarrow$ EEG, produced by powerful stimulation of the senses (for example, by applying sound to the ear or strong light to the eye while the EEG-apparutus is running).

exogenic: originating outside the organism. Synonymous with exogenous. See $\rightarrow$ endogenic.

exotoxin: toxic substance produced and secreted by the organism to the outside environment.

extension spasms: spasmodic extension of the arms and legs, as seen in $\rightarrow$ decerebration.

extraperitoneally: outside the ( $\rightarrow$ peritoneum lining the) abdominal cavity.

Fehling's test - Fehling's solution: a test for dextrose in urine. The urine is mixed with freshly prepared Fehling's solution and boiled. A red precipitate (of cuprous oxide) shows the presence of dextrose. Fehling's solution is a mixture of equal parts of two solutions: a solution of copper sulfate in water, and a solution of crytallized potassium sodium tartrate and sodium hydroxide in water.

ferments: any substance that causes fermentation (decomposition) in other substances with which it comes into contact.

fibrillation: See $\rightarrow$ ventricular fibrillation.

foramen magnum: the large opening in the occipital bone of the skull interconnecting the vertebral canal and the cranial cavity.

forebrain: frontal portion of the brain, that is the cerebral hemispheres.

gangrene: death of tissue, generally associated with loss of vascular supply and followed by by bacterial invasion and putrefaction.

Gerhard's reaction: a test for acetone and acetoacetic acid in urine. A solution of ferric chloride is added to the urine. When acetone or acetoacetic acid are present a red color is produced.

glycogen: the chief carbohydrate storage material in animals and man. It is formed by and largely stored in the liver and to some extent in muscles, being transformed into glucose and liberated as needed.

glycosuria: the presence of an abnormal amuunt of glucose in the urine.

graft: any suspension of cells, tissue, organs or parts of organs, or body parts for transplantation in the same individual (autotransplantation), to an individual of the same species ( $\rightarrow$ allotransplantation) or to an individual of another species ( $\rightarrow$ xenotransplantation).

Heine-Medin disease: see $\rightarrow$ polio poliomyelitis.

hemiparesis: loss of muscular strength on one side of the body.

Henderson-Hasselbalch equation: chemical equation expression the buffering capacity of carbonic acid in the blood.

hepatic - hepar: (pertaining to) the liver.

herniation: an abnormal protrusion of an organ through a defect or natural opening in a covering membrane, muscle or bone.

heterozygote: an individual possessing different alleles in regard to a given character. See $\rightarrow$ homozygote.

hibernation: artificial, pharmacologically induced state of reduced metabolism, muscle relaxation, and a twilight sleep resembling narcosis.

higher brain functions: functions associated to the cerebral hemispheres or neocortex. Intellectual functions, speech, etcetera. 
histocompatibility testing: test to ascertain whether a specific recipient will accept tissues and organs from another individual. Histocompatibility testing for organ transplantation purposes focuses on the $\rightarrow$ Major Histocompatibility Complex of the $\rightarrow H L A$-system.

histological: pertaining to tissues.

HLA: see $\rightarrow$ Human Leucocyte Antigens.

homeostasis: a tendency to stability in the internal environment of the organism, achieved by a system of control mechanism activated by negative feed back.

homozygote: an individual posessing a pair of identical alleles for a given character. See $\rightarrow$ heterozygote.

Human Leucocyte Antigens: system of 'tissue groups' first described on white blood cells and anaolog to the system of blood groups on red blood cells.

hyaline: a transluscent substance. In urine hyaline discharges appear as casts of the tubuli in the kidney.

hydranencephaly: complete or almost complete absence of cerebral hemispheres, the space they normally occupy being filled with cerebrospinal fluid.

hypertrophy: the enlargement of an organ of bodypart due to an increase in the size of its constituent cells. See $\rightarrow$ atrophy.

hypocapnia - hypocapnic: (pertaining to) a reduction of carbon dioxide in the blood due to hyperventilation.

hypoglycemic reaction: abnormal reduction of blood sugar levels, which may leed to tremulousness. cold sweat, hypothermia, headache, accompanied by confusion, hallucinations and ultimately convulsions, coma and death.

hypopharynx: lower portion of the throat.

hypothalamus - hypothalamic hormone: hormone produced in the hypothalamus, which is the portion of the brain responsible for activating, regulating and integrating peripheral autonomic functions, endocrine activity and many somatic functions (metabolism, water balance, body temperature, sleep, food intake, development of secondary sex characteristics).

hypothermia: reduction of body temperature.

hypoxemis: a decrease of oxygen in the blood.

immune respons: the reaction of the organism's humoral and/or cellular defence mechanisms towards foreign (non-self) substances.

incarceration: unnatural retention or confinement of a body part, as in herniations.

incidence: the number of new cases of a specific disease occuring during a certain period.

infantile paralysis: see $\rightarrow$ polio : poliomyelitis.

inhalation anesthetics: the use of gases, like ether, that can be inhaled, for the induction of narcosis. insulin shock: see $\rightarrow$ hypoglycemic reaction.

internal carotid arteries: two of the main arterial branches, springing from the common carotid artery running on both sides of the neck, supplying the brain with blood.

internal secretion: term introduced by Claude Bernard to indicate that a tissue produces a substance and secretes it into the interior of the organism (blood), and not through a duct to the exterior surfaces of the body.

interstitial: pertaining to the interspaces of a tissue.

intra-thoracic cardica massage: see $\rightarrow$ direct cardiac massage.

intracranial circulation: the circulation of blood inside the skull. To be distinguished from the extracranial circulation. One can have extra-cranial circulation without intra-cranial circulation.

intravital autolysis: self-destruction of tissues during life, similar to the self-destruction of tissues after death, as occurs in a patient with extensive brain damage whose body is maintained on lifesupport (respirator brain).

intubation: the introduction of a rube in the throat or trachea, to maintain a free airway, to facilitate $\rightarrow$ endotracheal suction and artificial ventilation.

iron lung: negative pressure respirator of the tank or total-body type. The patient's body, except her head, is enclosed by the tank. The negative pressure in the tank creates a pressure difference with the outside air, allowing the atmospheric pressure to produce an inspiration movement. 
irreversible coma: see $\rightarrow$ coma dépassé.

iso-electric: (pertaining to an) $\rightarrow E E G$-recording without changes in electrical currents, displaying a straight, flat line. Also called electrocerebral silence.

jugular veins: large pair of veins running on both sides of the neck.

ketone bodies - ketosis - ketonuria: group of compounds having a carbonyl group ( $\mathrm{CO}$ ) with hydrocarbons attached to it. Ketosis is a condition characterized by an accumulation of ketone bodies in the tissues and blood. When they occur in the urine it is called ketomuria. Ketosis and ketonuria is a complication of diabetes mellitus and starvation.

Langerhans' islands: the group of cells, first described by Paul Langerhans, dispersed among the $\rightarrow$ acinar tissue of the $\rightarrow$ pancreas.

laryngoscope: instrument for the direct visual examination of the lower regions of the throath, the vocal cords and the upper part of the windpipe. Required for the proper introduction of an endotracheal tube in $\rightarrow$ intubation.

lower brain functions: vegetative, bodily functions associated with the brain stem (regulation of body temperature, blood pressure, respiration, heart beat). See $\rightarrow$ higher brain functions.

macroscopical: visible with the unaided eye or without a microscope.

Major Histocompatibility Complex: the group of $\rightarrow H L A$-antigens responsible for inducing an immune respons in a recipient. Compatibility of $\mathrm{MHC}$-antigens between donor and recipient is necessary for successful organ or tissue transplantation.

matching: selecting the best recipient for an available donor organ on the basis of a comparison of $\rightarrow H L A$ - and $\rightarrow M H C$-groups.

mediastinum: the mass of tissues and organs separating the two lungs.

medulla oblongata: the truncated cone of tissue in the brain stem between the spinal cord and the $\rightarrow$ pons.

meningioma: malignant tumor arising from the membranes covering the brain.

mesentery: a membraneous, $\rightarrow$ peritoneal fold attaching various organs to the body wall.

metabolic alkalosis: a disturbance in which the $\rightarrow$ acid-base status of the body shifts towards the alkaline side because of retention of base or loss of non-carbonic or non-wolatile

acids. See $\rightarrow$ respiratory acidosis.

metencephalon: the portion of the brain comprising pons and cerebellum.

MHC: see $\rightarrow$ Major Histocompatibility Complex.

monoclonal antibodies: antibodies produced by one line of cells, directed against one specific antigen.

morbidity: the sick rate. The ratio of sick to healthy persons in a community. To be distinguished from $\rightarrow$ mortality.

moribund: term used to indicate that a patient is in a dying state.

morphological: pertaining to the form and structure (of cells, tissues, organs).

mortality: the death rate. The percentage of deaths in a population during a certain period of time. motor centers: nervous centers in brain stem and spinal cord innervating the muscles.

mucosa: a mucous membrane.

myelencephalon: the portion of the brain comprising the $\rightarrow$ medulla oblongara and the lower part of the fourth $\rightarrow$ ventricle.

myocard - myocardial: (pertaining to) the heart muscle.

necrosis: death of tissue.

negative pressure respirators: mechanical devices used for $\rightarrow$ negative pressure ventilation. They came in two different types: $\rightarrow$ iron lungs and $\rightarrow$ cuirass respirators.

negative pressure ventilation: a kind of artifical respiration in which the inspiration movement is produced by creating a negative pressure outside the patient's body (in a tank or a $\rightarrow$ cuirass). See $\rightarrow$ negative pressure respirators.

neocortex: outer, and $\rightarrow$ phylogenetically newest or youngest layer of the cerebral hemispheres. It is associated with $\rightarrow$ higher brain functions.

nephrectomy: extirpation of a kidney. 
neurogenic theory: theory that locates the causes of a disease (like diabetes mellitus) in the nervous system.

neurological regression phenomena: the re-appearance of neurological reflexes that normally only occur in the newly born child and early childhood.

neuromuscular blocking agent: drug blocking the transfer of a nervous impuls in the junction between the neuron and the muscular fiber.

neurotoxin - neurotoxic: (pertaining to a) substance that is toxic especially for nervous tissues.

noradrenaline: a hormone produce by nerve cells, acting as a transmitter substance. Also produced by the inner tissues (medulla) of the adrenal glands.

oculocephalic reflex: reflex by which the movements of the eyes and head are directed in the interest of visual attention.

otolaryngology: the branch of medicine specialized in diseases of the ear, nose and throath.

oxygenation: saturation with oxygen. To be distinguished from oxydation.

pacemaker: device designed to stimulate, by electrical impulse, the contraction of the heart at a certain rate.

pallial: pertaining to the cerebral cortex. See $\rightarrow$ apallic syndrome.

palliation - palliative: affording relief, but not cure.

palmomental reflex: also palm-chin reflex, twitching of the chin produced by scratching the palm. pancreas - pancreatitis - pancreatectomy: [Gr. pan all + kreas flesh] elongated gland situated behind the stomach, between the spleen and the $\rightarrow$ duodenum. A pancreatitis is an inflammation of the pancreas. A pancreatectomy is the extirpation of the pancreas.

pancreatogenic etiology: locating the causes of a disease or condition in the $\rightarrow$ pancreas.

parabiosis: the union of two individuals of experimental animals by surgical operation.

paralysis: loss or impairment of motor functions in a body part due to lesion of the muscular or neural mechanisms.

parenteral: any route of administration (of drugs, liquids, etc.) not using the digestive sytem.

paresis: slight or incomplete $\rightarrow$ paralysis.

pathogenesis: the abnormal cellular events and reactions occurring in the development of a disease. pathophysiology: the physiology of disoredered function.

$\mathrm{PCO}_{2}$ : the carbon dioxide tension in blood.

Pentothal: trademark for preparations of thiopental, a short acting $\rightarrow$ barbiturate used to induce narcosis.

peptides: proteins. Large molecules consisting of amino acids.

percutaneously: through the skin.

peritoneum - peritonitis: the thin, smooth membrane lining the inside of the walls of the abdominal cavity and enveloping the inner organs and intestines. Peritonitis is an inflammation of the peritoneum.

persistent vegetative state: clinical condition characterized by loss of higher brain functions due to destruction of the cerebral cortex, but with retainment of brain stem functions. PVS -patients are not respirator dependent. They have spontaneous temperature en blood pressure regulation and they have a spontaneous waking-sleeping rhythm.

pH: the negative logarithm of the $\mathrm{H}^{+}$concentration. $\mathrm{pH}$ expresses the acidity or alkalinity of a liquid. pH 7 is neutral. $>7$ is alkaline. $<7$ is acid.

pharmacological immunomodulation: influencing an organism's immune respons by means of drugs.

pharynx: region between the mouth and esophagus.

phloridzin: [Gr. phloios bark + rhiza root] a bitter glycoside from the root bark of apple, cherry, plum and pear trees, used to induce a form of experimental diabetes mellitus. It causes glycosuria by blocking the reabsorbtion of glucose in the kidneys.

phylogenetically: pertaining to the development of the species.

picrate: salt of picric acid, a bitter substance derived from a digitalis species.

plexus coeliacus - c(o)eliac plexus: nervous plexus situated in front and on the sides of the aorta, at the origins of the celiac (arterial) trunc, the superior mesenteric and renal arteries. 
pneumonectomy: extirpation of a lung.

pneumothorax: the accumulation of air or gas in the space between a lung and the chest wall, resulting in a total or partial collapse of the lung. Pneumothorax may occur spontaneously or as a result of trauma or surgery.

polarimeter - polarization apparatus: device for measuring the rotation of plane polarized light. Used to measure the sugar concentration in urine or to distinguish between various sugars that may occur in urine.

polio - poliomyelitis: polio is shorthand for poliomyelitis anterior acuta, a viral infection of the central nervous system affecting, at multple levels, the clusters of neurons innvervating the muscles.

Poliomat: one of the mechanical positive pressure respirators developed in the 1950s (Dräger). Its name reflects its intended use for the treatment of poliomyelitis.

polyuria: the passage of a large volume of urine in a given period (a characteristic of diabetes).

pons: (L. bridge] the part of the brain bridging the region between the $\rightarrow$ medulla oblongata and the midbrain in front of the $\rightarrow$ cerebellum.

positive pressure ventilation: any kind of artificial respiration employing a pressure higher than the patient's intra-thoracic pressure to bring about inspiration and inflation of the lungs (mouth-to-mouth breathing, manual bag ventilation, mechanical positive pressure respirators).

positive pressure respirators: machines designed to provide artificial $\rightarrow$ positive pressure ventilation. positron emission tomography: computerized brain imaging technique.

postmoral autolysis: self-destruction of tissues following death.

proteolytic enzymes: enzymes destroying proteins.

proximal: nearest. Closer to any point of reference, as opposed to $\rightarrow$ distal.

purine analogs - purine derivatives: group of compounds very similar to the purine bases that make

up the DNA and RNA. When incorporated into DNA they produce non-functional molecules, thus blocking normal DNA-synthesis in and reproduction of the cell.

PVS: see $\rightarrow$ persistent vegetative state.

radiocinematography: the combination of a moving picture camera with an $\mathrm{X}$-ray machine, used in $\rightarrow$ angiography.

reanimation: restoration to a normal, conscious life.

renal: pertaining to the kidneys.

respiration center: neural center in the brain stem involved in the regulation of breathing.

respiratory quotient: the ratio of the volume of expired carbon dioxide to the volume of oxygen absorbed by the lungs per unit of time.

respiratory acidosis: a condition due to the excess retention of carbon dioxide in the body. See $\rightarrow$ metabolic alkalosis.

reticular substance reticular formation: meshy nervous tissue between the distinct nervous nuclei in the brain stem. See $\rightarrow$ ARAS.

Ringer's solution: infusion fluid containing various salts in physiological concentrations.

saccharometry: the measurement of the amount of sugar in a solution, or in urin.

Salk's polio vaccine: vaccine prepared from killed polio virusses of three different types, grown in monkey kidney tissue and used for the active immunization against poliomyelitis.

scintigraphy: imaging technique mapping distribution of gamma rays emitted by a radioisotope injected into the body.

sclerosis - sclerotic: hardening of tissues from inflammation due to proliferation of connective tissue. secretin: a hormone produced and secreted by the mucosa of the duodenum. Carried by the blood it stimulates the secretion of pancreatic juice.

sentience - sentient: the capability to suffer.

splanchnic nerves: nerves innervating the organs in the abdominal cavity.

standard bicarbonate: $\rightarrow$ acid-base status parameter for alkali reserve which pertains to the available buffering capacity of $\mathrm{HCO}_{3}^{-}$(bicarbonate) in blood.

subcutaneous: under the skin.

subdural hematoma: accumulation of blood in the subdural space, that is beneath the dura mater, the though membrane covering the brain. 
supraorbital: situated above the bony cavity that contains the eyeball.

synapse: the region of junction between processes of two adjacent nerve cells, forming the place where a nervous impulse is transmitted from one neuron to another.

telencephalon: portion of the brain from which the cerebral hemispheres derive. Also endbrain.

tentorium: a structure formed by a double sheet of the outermost, toughest and most fibrous (dura mater) of the three membranes (meninges) covering the brain and spinal cord. The tentorium supports the occipital lobes of the two hemispheres and covers the cerebellum. Its internal border is free. Its external border is attached to the skull. With an increase in intracranial pressure brain tissue is pressed onto the tentorium and into the $\rightarrow$ foramen magnum.

tetanus neonatorum: tetanus occurring in the newborn child.

tetraplegia - tetraplegic: paralysis of all four extremities. Also quadriplegia.

thorax - thoracotomy: the thorax is the chest. A thoracotomy is a surgical opening of the chest, performed routinely in operations on the heart and lungs. As an emergency procedure it gives access to the heart for direct, manual cardiac massage.

thyreodectomy: extirpation of the thyroid gland.

Tonelli's sign: the pupil becoming oval when some pressure is exerted on the eye. Occurs when the patient is dead (not during life).

topographical anatomy: description of an anatomical region or body part with emphasis on the mutual relationships between various structures (veins, arteries, tendons, nerves, organs).

total $\mathrm{CO}_{2}$ in plasma: laboratory test measuring buth the physically dissolved and the chemically bound $\mathrm{CO}_{2}$ in plasma.

trachea - tracheotomy - tracheostomy: the trachea is the windpipe. Tracheotomy refers to the act of making (cutting) a hole in the windpipe. Tracheostomy, with an s, refers to the hole itself.

transgenic animals: genetically manipulated animals that are made to produce substances or tissues from another species: They are chimeras: organims whose genetic make up derives from two different species.

Trommer's test: a test for dextrose in urine. To two parts of urine one part of potassium hydroxide, and a very dilute solution of copper sulfate is added, whereafter the whole is boiled. Sugar, if present, causes precipitation of an orange-red deposit.

trypsin: one of the proteolytic enzymes of the pancreatic secretion.

vagus nerve: the tenth $\rightarrow$ cranial nerve, playing an important role in the regulation of $\rightarrow$ vegetative funcrions.

vascular anastomoses: the union of two blood vessels by surgical operation (side-to-side, end-to-end or end-to-side).

vaso-active drugs - vasopressor drugs: drugs affecting the tone of the smooth muscles in the walls of blood vessels, resulting either in $\rightarrow$ vasodilation or $\rightarrow$ vasoconstriction.

vasoconstriction: reduction in the lumen (constriction) of a blood vessel.

vasodilation: incease in the lumen (dilation) of a blood vessel.

vasomotor centers: nervous centers innervation of smooth musculature of blood vessels.

vegetative functions - vegetative dysregulations - vegetative atonia: see $\rightarrow$ lower brain functions and $\rightarrow$ atorny.

venous return: the return of blood from the systemic circulation to the heart through the major veins running through the thoracic cavity: vena cava inferior and superior.

ventilatory capacity: the wolume of the lungs that is effectively available for gas exchange.

ventricle: the ventricles of the heart are the cavities of the heart that propel the blood through the arterial systems in the minor and major circulation. The ventricles of the brain are a system of cavities in the brain filled with $\rightarrow$ cerebrospinal fuid. The fourth ventricle is at the level of the $\rightarrow$ medulla oblongata and $\rightarrow$ pons.

ventricular fibrillation: irregular contractions in the walls of the heart's ventricles. due to rapid repetitive excitation of myocardial fibers without coordinated contraction of the ventricle.

vertebral arteries: a pair of arteries running alongside the vertebral column to the brain. Together with the pair of $\rightarrow$ internal carotid arteries they supply the brain with blood.

xenotransplantation: inter-species transplantation. See $\rightarrow$ graft and $\rightarrow$ allotransplantation. 


\section{Bibliography}

Adrian, E.D., Bremer, F. and Jasper, H.H. (eds.) 1954. Brain mechanisms and consciousness. Oxford: Blackwell Scientific Publications.

Adrian, E.D., and Matthews, B.H.C. 1934a. 'The Berger rhytm: potential changes from the occipital lobes in man'. Brain 57: 355 - 385.

Adrian, E.D. and Matthews, B.H.C. 1934b. 'The interpretation of potential waves in the cortex'. Journal of Physiology 81: 440 - 471.

Agerholm, M. 1953. 'Epidemic poliomyelitis and its prevention'. The Lancet 1953: 287 - 293.

Agt, A.A.M. van. 1968. 'Recht op leven en sterven'. Katholiek Artsenblad 1968: 369 - 379.

Akrich, M. and Latour, B. 1992. 'A summary of a convenient vocabulary for the semiotics of human and non-human assemblies'. In: Bijker, W.E. and Law, J. (eds.) Shaping Technology / Building Society. Studies in Sociotechnical Change. Cambridge, Ma.: The MIT Press, pp. 259 - 264.

Akveld, J.E.M. 1987. Transplantatie en wetgeving. Werkelijkheid en ideaal. Lelystad: Koninklijke Vermande BV.

Akveld, J.E.M., Charro, F.Th. de, Hessing, D.J., Kootstra, G., Roscam Abbing, H.D.C. 1988. Orgaandonatie, een kwestie van bereidheid. Lelystad: Koninklijke Vermande BV.

Aleman, P.J. 1962. 'De organisatie van resuscitatie in het ziekenhuis Dijkzigt te Rotterdam'. Het Reddingwezen 51: $207-211$.

Aleman, P.J., Crul, J.F., Slikke, W. van der. 1964. 'Resuscitatie'. Nederlands Tijdschrift woor Geneeskunde 108: $1265-1270$.

Allen, F.M. 1913. Studies Concerning Glycosuria and Diabetes. Cambridge, Ma.: Harvard University Press.

Allen, F.M. and Sherrill, J.W. 1922a. 'Clinical observations on treatment and progress in diabetes'. Journal of Metubolic Research 1922: 377 - 455.

Allen, F.M. and Sherrill, J.W. 1922b. 'Clinical observations with insulin. 1. The use of insulin in diabetic treatment.'. Journal of Metabolic Research 1922: 804 - 985.

Allen, G. 1978. Life Science in the Twentieth Century. Cambridge: Cambridge University Press.

Ammundsen, E. 1953. Sundhedstilstunden i Kobenhavn. Stadslogens drsberetning 1952. Kabenhavn: Kabenhavn Kommune.

Ashmore, M. 1989. The Reflexive Thesis: Wrighting Sociology of Scientific Knowledge. Chicago: University of Chicago Press.

Aström, A. 1952. 'On the action of combined carbon dioxide excess and oxygen deficiency in the regulation of breathing'. Acta Physiologica Scandinavica 27: supplementum 98.

Astrup, P. 1954. 'Om erkendelsen af forstyrrelser i organismens syre- og basestofskifte. I. Definitioner, teori, beregningsmåde, analysemetodik or normalværdier. II. Laboratorieanalysers værdi for diagnosen. III. Problemer vedrørende behandelingen af forstyrrelser samt organismens forsøg på egenregulation'. Ugeskrift for Lager 116: 758 - 771.

Astrup, P. 1956a. 'Laboratory control of gas exchange'. In: Lassen, H.C.A.(ed.) 1956. Management of life-threatening polionyelitis. Copenhagen, 1952 - 1956. With a survey of autopsy findings in 115 cases. Edinburgh and London: E. \& S. Livingstone Ltd., pp. $111-120$. 
Astrup, P. 1956b. 'A simple electrometric technique for the determination of carbon dioxide tension in blood and plasma, total content of carbon dioxide in plasma, and bicarbonate content in separated plasma at a fixed carbon dioxide tension $(40 \mathrm{~mm} \mathrm{Hg})$. Scandinavian Journal of Clinical and Laboratory Investigation 8: 33 - 43.

Astrup, P. 1989. 'I hundreåret for den første måling af kuldioxid i blodet ved diabetisk coma'. Bibliotek for Lager / Medicinsk Forum 181/42: 17 - 28.

Astrup, P., Bjørneboe, M., Neukirch, F. 1989. 'Polioepidemien i København 1952'. Bibliotek for Lager / Medicinsk Forum 181/42: 79 - 110.

Astrup, P., Schrøder, S. 1956. 'Apparatus for anaerobic determination of the $\mathrm{pH}$ of blood at 38 degrees centigrade'. Scandinavian Journal of Clinical and Laboratory Imestigation 8: 30 - 32 .

Astrup. P. and Severinghaus, J.W. 1985. Blodgassernes, syrernes og basernes historie. Kobenhavn: Munksgaard.

Atkinson, R.S. and Boulton, T.B. (eds.) 1989. The History of Anaesthesia. London and New York: Royal Society of Medicine Services and the Pathenon Publishing Group.

Bang, C. 1954. 'Da en respirator kom til verden'. Arbejder Samariten 23: 18 - 23.

Bang, I. 1913. Der Blutzucker. Wiesbaden: Verlag von J.F. Bergmann.

Bang. I. 1916. Methoden zur Mikrohestimmung einiger Blutbestandteile. Wiesbaden: Verlag von J.F. Bergmann.

Bang, J. 1950. 'Om behandling af bornelammelse'. Tidsskrift for sygeplejersker 50: 627 - 631; 643 649.

Banting, F.G. and Best, C.H. 1922. 'The internal secretion of the pancreas'. Journal of Laboratory and Clinical Medicine 7: $256-271$.

Banting, F.G., Best, C.H., Collip, J.B., Macleod, J.J.R., and Noble, E.C. 1922b. 'The effect of pancreatic extract (Insulin) on normal rabbits'. American Journal of Physiology 62: 162 - 176.

Banting, F.G., Best, C.H., Collip, J.B., Campbell, W.R., and Fletcher, A.A. 1922c. 'Pancreatic extracts in the treatment of diabetes mellitus. Preliminary report'. Canadian Medical Association Journal 2: $141-146$.

Barnes, S.B. 1974. Srientific Knowledge and Sociological Theory. London: Routledge \& Kegan Paul. Barron, M. 1920. 'The relation of the islets of Langerhans to diabetes with special reference to cases of pancreatic lithiasis'. Surgery, Gynecology, and Obstetrics 31: 437 - 448.

Battistini, F. 1893. 'Ueber zwei Fälle von Diabetes Mellitus mit Pankreassaft behandelt'. Therapeutische Monatshefie 7: 494 - 498.

Beck, C.S., Pritchard, W.H. and Feil, S.H. 1947. 'Ventricular fibrillation of long duration abolished by electric shock'. Journal of the American Medical Association 135: 985 - 986.

Beecher, H.K. 1968. 'A definition of irreversible coma. Report of the ad hoc committee of the Harvard Medical School to examine the definition of death'. Journal of the American Medical Association 205: 337 - 340.

Berger, H. 1929. 'Über das Elektroenkephalogramm des Menschen'. Archiv fur Psychiatrie 87: 527 570.

Berger, H. 1930. 'Über das Elektroenkephalogramm des Menschen. II. Journal fur Psychologie und Neurologie 40: 160 - 179.

Bergman, R., Huldt, S. 1948. 'De Svenska resultaten av respiratorbehandling vid poliomyelit'. Nordisk Hygienisk Tidsskrift 1948: 135 - 158.

Bernard, C. 1855. Mémoire sur le pancréas. Paris.

Bernard, C. 1865/1950. An Intmduction to the Study of Experimental Medicine. Translated by Henry Greene; published in 1950 by Abelard-Schuman, Publisher. Partly reprinted as 'Vivisection ' in Reiser, S.J., Dyck, A.J. and Curran, W.J. (eds.) 1977. Eshics in Medicine. Historical Perspectives and Contemporary Concerns. Cambridge, Ma.: The MIT Press, pp. 257 - 259.

Berton, P. 1988. The Arctic Grail. The quest for the north west passage and the north pole, 1818 1909. New York: Viking Penguin.

Bertrand, I., Lhermitte. F., Antoine, B., Ducrot, H. 1959. 'Nécroses massives de système nerveux central dans une survie artificielle'. Revue Neurologique 101: 101 - 115. 
Bijker, W.E. 1987. 'The social construction of bakelite: toward a theory of invention'. In: Bijker, W.E, Hughes, T.P. and Pinch, T. (eds.) 1987. The Social Construction of Technological Systems. New Directions in the Sociology and History of Technology. Cambridge: MIT Press, pp. $159-187$.

Bijker, W.E. 1990. 'Do not despair: there is life after constructivism'. Paper presented at the conference on 'Technological Choices: American and European Experiences', April 12 - 14 1990, Indiana University, Bloomington, IN, USA. Kennis en Methode 14: 324 - 345.

Bijker, W.E. 1992a. The Social Construction of Technology. Eijsden: PhD-thesis.

Bijker, W.E. 1992b. 'The social construction of fluorescent lighting, or how an artifact was invented in its diffusion stage'. In: Bijker, W.E. and Law, J. (eds.) Shaping Technology / Building Society. Studies in Sociotechnical Change. Cambridge, Ma.: The MIT Press, pp. 75 - 102.

Bijker, W.E. 1994. On Bikes, Bakelite and Bulbs. Towards a Theory of Technical Change. Cambridge, Ma.: The MIT Press.

Bijker, W.E., Hughes, T.P. and Pinch, T. (eds.) 1987. The Social Construction of Technological Systems. New Directions in the Sociology and History of Technology. Cambridge: MIT Press.

Bijker, W.E. and Law, J. 1992. 'General introduction'. In: Bijker, W.E. and Law, J. (eds.) 1992. Shaping Technology / Building Society. Studies in Sociotechnical Change. Cambridge, Ma.: The MIT Press, pp. 1 - 14.

Bijker, W.E. and Law, J. (eds.) 1992. Shaping Technology / Building Sociery. Studies in Sociotechnical Change. Cambridge, Ma.: The MIT Press.

Bijl, N.P.Y.M. de. 1985. 'Wet op de lijkbezorging'. Tijdschrift voor Gezondheidsrecht 1985: 207 217.

Billingham, R.E., Brent, L., and Medawar, P.B. 1953. “Actively Acquired Tolerance' of foreign cells'. Nature 172: 603.

Bjørk, V.O., Engström, C.-G. 1955. 'The treatment of ventilatory insufficiency after pulmonary resection with tracheostomy and prolonged artificial ventilation'. The Journal of Thoracic Suryery 30: $356-367$.

Bjørneboe, M. 1953a. 'Polioepidemien i København, etteråret 1952'. Tidskrift for Sygepléjı 53: 97 104.

Bjørneboe, M. 1953b. 'Studenten og polinpatienten'. Ugeskriff for Lager 115: 469 - 471.

Bjørneboe, M., Ibsen, B., Astrup, P., Everberg, G., Harvald, B., Søttrup, T., Hess Thaysen, E., Thorshauge, C. 1955. 'Active ventilation in treatment of respiratory acidosis'. The Lancet 1955: 901 - 903.

Bjørneboe, M., Ibsen, B., Johnsen, S. 1953a. 'Et tilfælde af tetanus behandlet med curarisering, tracheotomi og overtryksventilation med kvælstofforilte og ilt'. Ugeskrift for Lager 115: 1535 1537.

Bliss, M. 1982. The Discovery of Insulin. Chicago: The University of Chicago Press.

Bloor, D. 1973. 'Wittgenstein and Mannheim on the sociology of mathematics'. Studies in the History and Philosophy of Science 4: 1973 - 1991.

Bloor, D. 1976/1991. Knowledge and Social Imagery. 2nd edition. Chicago: The University of Chicago Press.

Blum, F. 1901. 'Ueber Nebennierendiabetes'. Deutsches Archiv fur klinische Medizin 71: 146 - 167. Blumentahl, F. 1898. 'Ueber Organsafttherapie bei Diabetes mellitus'. Zeitschrift fur diatetische und physikalische Therapie 1: 250 - 258.

BMJ. 1893. 'Animal Extracts as Therapeutic Agents'. British Medical Journal 1893: 1279.

BMJ. 1968. Leading Article. 'South Arican Heart Transplant'. British Medical Journal 1968: 132 133.

Borell, M. 1976a. 'Organotherapy, British physiology and discovery of internal secretions'. Journal of the History of Biology 9: 236 - 268.

Borell, M. 1976b. 'Brown-Séquard's organotherapy and its appearance in America at the end of the nineteenth century'. Bulletin of the History of Medicine 50: $309-320$.

Bower, A.G., Bennett, V.R., Dillon, J.B. 1950. 'Investigations on the care and treatment of poliomyelitis patients'. Annals of Western Medicine and Surgery 4: 561 - 582, 687 - 716.

Brain, R. 1958. 'The physiological basis of consciousness. A critical review'. Brain 81: $426-455$. 
Brandt Rehberg, P. 1974. 'August Krogh'. Dansk medicinhistorisk Arbog 1974.

Brante, T. and Hallberg, M. 1989. 'Kontroversen om dödsbegreppet'. Vest 10-11: 4 - 29.

Brante, T. and Hallberg, M. 1991. 'Brain or heart? The controversy over the concept of death'. Social Studies of Science 21: $389-413$.

Braunstein, P., Korein, J., Kricheff, I.I. and Liberman, A. 1978. 'Evaluation of the critical deficit of cerebral circulation using radioactive tracers (bolus technique)'. In: Korein, J. (ed.) 1978. Bruin Death: Internelated Medical and Social Issues. New York: The New York Academy of Sciences. Annals of the New York Academy of Sciences 315, pp. 143 - 167.

Bremer, F. 1935a. 'Cerveau isole et physiologie du sommeil'. Comtes redus des séances de la Société de Biologie et de ses Filiales 118: 1235 - 1241.

Bremer, F. 1935b. 'Quelques propriétés de l'activité électrique du cortex cérébral isolé'. Comtes redus des séances de la Société de Biologie et de ses Filiales 118: 1241 - 1244.

Bremer, F. 1938. 'L'activité électrique de l'écorce cérébrale et le probleme physiologique du sommeil'. Bollettino della Sacieta Italiana di Biologia Sperimentale 13: 271 - 290.

Briggs, A. 1961. 'Cholera and Society in the 19th Century'. Past and Present 19: 76 - 96.

Brodal, A. 1957. The reticular formation of the brain. Anatomical aspects and functional correlations. Edinburgh: Olliver \& Boyd, Ltd.

Brody, H. 1981. Ethical decisions in medicine. 2nd ed. Boston: Little Brown.

Brown-Sequard, C.-E. 1893. 'On a new therapeutic method consisting in the use of organic liquids extracted from glands and other organs'. The British Medical Journal 1893: 1145 - 1147, 1212 1214.

Bücheler, E., Käufer, C and Penin, H. 1973. 'Transfemorale zerebrale Katheterangiographie beim Hirntod'. In: Krösl, W., Scherzer, E. (Her.) 1973. Die Bestimmung des Todeszeitpunktes. Wien: Wilhelm Maudrich Verlag. pp. 155 - 161.

Burnet, F.M. 1959, The clonal selection theory of acquired immunity. Nashville: Vanderbilt University Press.

Busnardo, A.C., DiDio, L.J.A., Tidrick, R.T. and Thomford, N.R. 1983. 'History of the pancreas'. American Journal of Surgery 146: 539 - 550.

Butterfield, H. 1978 (1931). Whig Interpretation of History. New York: AMS Press (reprinted from the 1931 edition, London: G. Bell and Sons).

Cairns, H. 1952. 'Disturbances of consciousness with lesions of the brain-stem and diencephalon'. Brain 75: $109-146$.

Callon, M. 1986a. 'Some elements of a sociology of translation: domestication of the scallops and the fishermen of St Brieuc Bay'. In: Law, J. (ed.) Power, Action and Belief. A New Sociology of Knowledge? London: Routledge \& Kegan Paul, pp. 196 - 233.

Callon, M. 1986h. 'The sociology of an actor-network: the case of the electric vehicle'. In: Callon, M., Law, J. and Rip, A. (eds.) 1986. Mapping the Dynamics of Science and Technology. Sociology of Scionce in the Real World. London: The MacMillan Press Ltd., 19 - 34.

Callon, M. 1987. 'Society in the making: the study of technology as a tool for sociological analysis'. In: Bijker, W.E., Hughes, T.P. and Pinch, T. (eds.) 1987. The Social Construction of Technological Systems. New Dirrctions in the Sociology and History of Technology. Cambridge: MIT Press. pp. $83-103$.

Callon, M.. Law. J. and Rip, A. (eds.) 1986. Mapping the Dynamics of Science and Technology. Sociology of Science in the Real World. London; The MacMillan Press Ltd.

Calne, R.Y. 1960. 'The rejection of renal homografts inhibition in dogs by 6-mercaptuopurine'. Lance't 1960: 417.

Carlson, A.J. and Drennan, F.M. 1911. 'The control of pancreatic diabetes in pregnancy by the passage of the internal secretion of the pancreas of the fetus to the blood of the mother'.American Journal of Phsiology 28: 391 - 395.

Catz, E.W. 1960. 'Reanimatie-plicht'. Medisch Contact 15: 323 - 324

Cecil, R.L. 1909. 'A study of the pathological anatomy of the pancreas in ninety cases of diabetes mellitus'. The Journal of Experimensal Medicine 11: $266-290$. 
Cherington, M., Stears, J. and Hodges, J. 1976. 'Locked-in syndrome caused by a tumor'. Neurology 26: $180-181$.

Christensen, A., Lyttkens, H. (eds.) 1985. NEJ till hjarnrelaterade dodskriterier. Stockholm.

Christie, A.B., Esplen, J.R. 1953. 'Poliomyelitis in Denmark'. The Lancet 1953: 492 - 493.

Clemmesen, S., Ibsen, B., Jensen, B., Krogsgaard, M., Werner, G. 1963. 'Air-conditioning and human comfort'. Danish Medical Bulletin 10: 217 - 224.

Clowes, G.H.A. 1948. 'The Banting Memorial Address'. Proceedings of the American Diabetes Association 7: 49 - 60.

Cohen, M., Nagel, T. and Scanlon, T. (eds.) 1981. Medicine and Moral Philosophy. Princeton: Princeton University Press.

Coleman, W. and Holmes, F.L. (eds.) 1988. The Imestigative Enterprise Experimental Physiology in Ninereenth-Century Medicine. Berkeley: University of Californis Press.

Collins, G.H., Bravo-Shugarman, M.B. and Terasaki, P.I. 1969. 'Kidney preservation for transplantation. Initial perfusion and 30 hours ice storage'. Lancet 1969: 1219-1222.

Collins, H.M. 1975. 'The seven sexes: a study in the sociology of a phenomenon, or the replication of experiments in physics'. Sociology 9: 205 - 224.

Collins, H.M. 1981. 'Stages in the Empirical Programme of Relativism'. Social Studies of Science $11: 3-10$.

Collins, H.M. 1983. 'The sociology of scientific knowledge: studies of contemporary science'. Annual Review of Sociology 9: 265 - 285.

Collins, H.M. 1985. Changing Order. Replication and Induction in Scientific Practice. London: Sage Publications Ltd.

Collins, H.M. 1990. Artificial Experts. Social Knowledge and Intelligent Machines. Cambridge, Ma.: The MIT Press.

Collip, J.B. 1923. 'The original method as used for the isolation of insulin in semipure form for the treatment of the first clinical cases'. The Journal of Biological Chemistry 55: xl - xll.

Coulter, J. 1979. The social construction of mind. Studies in ethnomethodology and linguistic philosophy. London: The Macmillan Press Ltd.

Cremers, H.Th.P. 1973. 'Justitie en orgaantransplantaties'. Algemeen Politieblad van het Koninkrijk der Nederlanden 1973: 51 - 52.

Cremers, H.Th.P. 1989. 'Orgaantransplantatie en euthanasie. De taak van de gemeentelijke lijkschouwer', Medisch Contact 36: 1149.

Critchley, M., O'Leary, J.L., Jennett, B. (eds.) 1972. Scientific Foundarions of Neurology. London: William Heinemann Medical Books Ltd.

Cunningham, A. and Williams, P. (eds.) 1992. The laboratory revolution in medicine. Cambridge: Cambridge University Press.

Daston, L. 1992. 'Objectivity and the escape from perspective'. Social Studic's of Science' 22: 597 618.

Dausset, J. 1954. 'Leuco-agglutinins IV. Leuco-agglutinins and bloodtranstusion'. Vox Sanguinis 4: $190-198$.

Dell, P. 1963. 'Reticular homeostasis and critical reactivity'. In: Moruzzi, G. Fessard, A. and Jasper, H.H. (eds.) 1963. Brain mechanisms. Amsterdam: Elsevier Publishing Cu., pp. $82-114$.

Dempster, W.J., Melrose, D.G. and Bentall, H.H. 1968. 'Scientific, technical, and ethical considerations in cardiac transplantation'. British Medical Journal 1968: 177 - 178.

Derde Nota van Wijziging. 1993. Regelen omtrent het ter beschikking stellen v'an organen (Het op de orgaandonatie). 1993. Tweede Kamer der Staten Generaal, vergaderjaar 1993-1994, 22 3.58, nr. 14. 's-Gravenhage: Sdu Uitgeverij.

Drachman, A.G. 1943. Min rejse gennem livet. En gammel skibslages erindringer. In: Dumreicher, C., Olrik, H.G., Topswe-Jensen, H. (red.) 1943. Svundne tider. Erindringer og hrev'. Il. Bind. Kobenhavn: H. Hagerup.

Drennan, F.M. 1911. 'The presence of the internal secretion of the pancreas in the blood'. American Journal of Physiolngy 28: 396 - 402. 
Drinker, P., McKhann, C.F. 1929. 'The use of a new apparatus for the prolonged administration of artificial respiration. I. A fatal case of poliomyelitis'. Journal of the American Medical Association 92: $1658-1660$.

Drinker. P.A., McKhann, C.F. 1986. 'The iron lung. First practical means of respiratory support'. Journal of the American Medical Association 255: 1476 - 1480

Eijsvogel, V.P. 1967. 'Donorselectie en orgaantransplantatie'. Nederlands Tijdschrift voor Geneeskunde 111: 11661168.

Eiselberg, A. von. 1892. 'Ueber erfolgreiche Einheilung der Katzenschilddrüse in die Bauchdecke und Auftreten von Tetanie nach deren Exstirpation'. Wiener klinischen Wochenschrift 5: 81 - 85.

Elam, J.O., Brown, E.S. and Elder, J.D. 1954. 'Artificial respiration by mouth-to-mask method. A study of the respiratory gas exchange of paralyzed patients ventilated by operator's expired air'. The New England Journal of Medicine 250: 749 - 754.

Elam, J.O., Greene, D.G., Brown, E.S. and Clements, J.A. 1958. 'Oxygen and carbon dioxide exchange and energy cost of expired air resuscitation'. Journal of the American Medical Association 167: $328-334$.

Elam, J.O., Greene, D.G, Schneider, M.A., Ruben, B.H.M., Gordon, A.S., Hustead, R.F., Benson, D.W., Clements, J.A. and Ruben, A. 1960. 'Head-tilt method of oral resuscitation'. Journal of the American Medical Association 172: 812 - 815.

Engeltriet, C.P., Heersche, N.M., Eijsvogel, V.P. and Loghem, J.J. van. 1966. 'Demonstration of leucocyte iso-antigens on skin fibroblasts by means of the cytotoxic antibody test'. Wx Sanguinis 11: $625-630$.

Engelhardt, D. von (ed.). 1989. Diabetes. Its Medical and Cultural History. Heidelberg: Springer Verlag.

Engell. H.C., Ihsen, B. 1952. 'Continuous carbon dioxide measurement in the respiratory air during anaesthesia in thoracic operations'. Acta Chirurgica Scandinavica 104: 313 - 328.

Engström, C.-G. 1950. 'Om $\mathrm{CO}_{2}$-retentionens betydelse vid respirationsinsufficiencs'. Svenska lakartidningen 47: 3007 - 3011.

Engström, C.-G. 1950. 'Om lägedränage vid bulbär poliomyelit'. Sivenska Lakartidningen 47: 1457 1461 .

Engström, C.-G. 1963. 'The clinical application of prolonged controlled ventilation. With special reference to a method developed by the author'. Acta Anaesthesiologica Scandinavica Supplementum XIII.

Engström, C.-G., Svanborg, N.A. 1950. 'Kasuistik ïver fall av akut poliomyelit med respirationspares'. Sivenska Lakartidningen 47: 3011 - 3017.

Enthoven, L. 1988. Het recht op leven en dood. Deventer: Kluwer.

Evans, R.J. 1987. Deuth in Hamburg. Society and Politics in the Cholera Years 1830 - 1910. Oxford: Clarendon Press.

Faber, V., Neukirch, F., Snorrason, E. 1979. Epidemihospitalet i Kobenhavn 1879 - 1979. Kobenhavn: Rigshospitalet, afd. M.

Falkenberg, W. 1891. 'Zur Extirpation der Schilddrüse'. Verhandlungen des Congresses für innere Medizin 1891: $502-511$.

Fassaert, L.D.M. 1987. 'Hersendood en urgaandonatie'. Medisch Contact 42: 561 - 563.

Feasby, W.R. 1958. 'The discovery of insulin'. Journal of the History of Medicine and allicd Sciences 1958: $68-84$.

Feldberg, W.S. 1970. 'Henry Hallett Dale', In: Biographical Memoirs of Fellows of the Royal Society 16: 77 - 174. London: The Royal Society.

Forschbach, J. 1908a. 'Parabiose und Pankreasdiabetes'. Deutsche Medizinische Wbchenschrift 34: $910-911$.

Forschbach, J. 1908b. 'Zur Pathogenese des Pankreasdiabetes'. Archiv fur experimentelle Parhologie und Pharmakologie 60: 131 - 153.

Forschbach, J. 1909. 'Versuche zur Behandlung des Diabetes mellitus mit dem Zuelzerschen Pankreashormon'. Deutsche medizinische Hbchenschrift 35: 2053 - 2055. 
Frederiks, J.A.M. 1969. 'Consciousness'. In: Vinken, P.J., Bruyn, G.W. 1969. Disorders of higher nerwous activiry. Amsterdam: North-Holland Publishing Company.

Frenckner, P., Anderson., and Crafoord, C. 1940. 'A new and practical method of producing rhytmical ventilation during positive pressure anesthesia. Acta Oto-laryngica 28: 95.

Frerichs, Fr. Th. von. 1884. Über den Diabetes. Berlin: Verlag won August Hirschwald.

Frølund Thomsen, V. 1953. 'Kort redegørelse for den midlertidige studenterhjælp på Blegdamshospitalet'. Ugeskrift for Loger 115: 468 - 469.

Fujimura, J.H. 1992. "Crafting science: standardized packages, boundary objects, and "translation"'. In: Pickering, A. (ed.) 1992. Science as Practice and Culture. Chicago and London: The University of Chicago Press, pp. $168-211$.

Fürbringer, P. 1894. 'Ueber die moderne Behandlung mit Gewebsflüssigkeiten'. Deutsche medizinische Wbchenschrift 20: 293 - 296.

Galloway, T.C. 1943. 'Tracheotomy in bulbar poliomyelitis'. Journal of the American Medical Association 123: 1096 - 1097.

Gervais, K.G. 1986. Redefining Death. New Haven: Yale University Press.

Gewijzigd Voorstel van Wet. 1994. Regelen omtrent het ter beschikking stellen van organen (Wet op de orgaandonatie). 1994. Tweede Kamer der Staten Generaal, vergaderjaar 1993-1994, 22 358, nr. 15. 's-Gravenhage: Sdu Uitgeverij.

Gezondheidsraad. 1974. Advies inzake hersendoodscriteria. Leidschendam: Ministerie van Volksgezondheid en Milieuhygiēne.

Gezondheidsraad. 1975. De medisch-ethische en de juridische aspecten van de orgaantransplantatie. 's-Gravenhage: Staatsuitgeverij.

Gezondheidsraad. 1983. Advies inzake hersendoodscriteria. 's-Gravenhage: Staatsuitgeverij.

Gezondheidsraad. 1987. Advies inzake Algmene Transplantatieproblemutiek. 's-Gravenhage:

Staatsuitgeverij.

Goldscheider, A. 1894. 'Zur Gewebssafttheorie'. Deutsche medizinische Wbchenschrift 20: 318 - 321 and $376-378$.

Gordh, T., Gordh, U. 1952. 'Erfarenheter från behandlingen av bulbospinal poliomyelit vid Blegdamshospitalet, Köpenhamn, 1952’. Svenska Lakartidningen 49: 3103 - 3110.

Gordon, A.S., Frye, C.W., Gittelson, L, Sadove, M.S. and Beattie, E.J. 1958. 'Mouth-to-mouth versus manual artificial respiration for children and adults'. Journal of the American Medical Association 167: 320 - 328.

Gorter, E., Graaff, W.E. 1947. Klinische Diagnostiek. 6e druk. Deel I. Leiden: Stenfert Kroese.

Gould, S.J. 1989. Wonderful Life. The Burgess Shale and the Nature of History. London: Penguin Books.

Graaff, R. de, Jansen, E.N.H., Rasker, J.J., Wilts, G., Biemans, R.E.M., Wieken, L.R. van der, Bruijn, J.H. de. 1984. 'Diagnostiek hersendood 1984. Handelwijze bij vermoeden van hersendood'. Medisch Contact 1984: 591 - 592.

Green, M. and Wikler, D. 1981. 'Brain death and personal identity'. In: Cohen, M., Nagel, T. and Scanlon, T. (eds.) 1981. Medicine and Moral Philosophy. Princeton: Princeton University Press, pp. $49-77$.

Greep, J.M. (ed.) 1970. Orgaantransplantatie. Leiden: Stafleu's Wetenschappelijke Uitgeversmaatschappij N.V. .

Greep, J.M. and Brummelkamp, W.H. 1970. 'De geschiedenis van de orgaantransplantatie'. In: Greep, J.M. (ed.) Orgaantransplantatie. Leiden: Stafleu’s Wetenschappelijke Uitgeversmaatschappij N.V., pp. 17 - 38.

Gusfield, J.R. 1981. The Culture of Public Problems: Drinking-Driving and the Symbolic Order. Chicago: The University of Chicago Press.

Guyton, A.C. and Satterfield, J. 1951. 'Factors concerned in electrical defibrillation of the heart, particularly through the unopened chest'. American Journal of Physiology 167: 81 - 87.

Haglund, G. 1952. 'Anestesiologiska intryck från den danska poliomyelitepidemien'. Sienska Lakartidningen 49: 3098 - 3103. 
Hale White, W. 1893. 'On the treatment of diabetes mellitus by feeding on raw pancreas and the subcutaneous injection of liquor pancreaticus'. The British Medical Journal 1893: 452 - 453.

Hansen, J. 1953. 'Den økonomiske baggrund for poliobekæmpelsen'. Ugeskrift for Lager 115: 471 473.

Harre, R. (ed.) 1986. The Social Construction of Emotions. Oxford: Basil Blackwell Led.

Hass, W.K. and Hawkins, R.A. 1978. 'Bilateral reticular formation lesions causing coma: their effects on regional cerebral blood flow, glucose utilization and oxidative metabolism'. In: Korein, j. (ed.) 1978. Brain Death: Interrelated Medical and Social Issues. New York: The New York Academy of Sciences. Annals of the New York Academy of Sciences 315.

Have, H. ten. 1984. 'Ziekte als wijsgerig probleem'. Wijsgerig perspectief op maatschappij en wetenschap 25: 5 - 12.

Have, H. ten. 1985. 'Ethiek en techniek in de gezondheidszorg'. Wijsgerig Perspectief 26: 18 - 23.

Have, H. ten, Kimsma, G. 1987. Geneeskunde tussen droom en drama. Voortplanting, ethiek en woruitgang. Kampen: Kok Agora.

Hédon, M.E. 1890. 'Note sur la production du diabète sucré après l'extirpation du pancréas'. Comptes rendus des séances de la Société de Biologie 42: 571 - 573.

Hédon, M.E. 1891. 'Extirpation du pancréas, diabète sucré expérimental'. Archives de medicine expérimentale et d'anatomie pathologique 1: 44 - 67.

Hédon, M.E. 1909. 'Transfusion carotidienne croiseé entre chiens diabétique et chiens normaux'. Comptes rendus des séances de la Société de Biologie 67: 792 - 795.

Heiberg, K.A. 1910. Bugspytkirtelens normale og patologiske anatomi. Hvorunder forholdene ved en del tilfolde af sykkersyge. Kobenhavn: Andr. Fred. Høst \& Søn.

Henriksen, E. 1954. 'Om tetanus. Med særlig omtale af en ny behandlingsmethode'. Tidsskrift for Sygepleje 54: 387 - 389 .

Hernstein Smith, B. 1988. Contingencies of Value. Alternative Perspectives for Critical Theory. Cambridge: Harvard University Press.

Hertel, K. 1980. The store Kobenhavnske epidemier. Farsø: Klaus Hertel.

Hesser, C.M. 1949. 'Central and chemoreflex components in the respiratory activity during acid-base displacements in the blood'. Acta Physiologica Scandinavica 18: supplementum 64.

Hesser, C.M. 1949. 'A Device for measuring and recording automatically and continuously the carbon dioxide in the alveolar air'. Acta Physiologica Scandinavica 18: summplementum 64: 3 14.

Hilberman, M. 1975. 'The evolution of intensive care units'. Critical Care Medicine 3: 159 - 165.

Hooker, D.R., Kouwenhoven, W.B. and Langworthy, O.R. 1933. 'The effect of alternating electric currents on the heart'. American Journal of Physiology 103: 444 - 454

Horstmann, P. 1956. 'Polio i 3000 ar. En gammel sygdom i nyt lys'. Vor Viden 1955/56: 330 - 336.

Houssay, B.A. 1952. 'The discovery of pancreatic diabetes: the role of Oscar Minkowski'. Diabetes 1952: $112-116$.

Houwart, E.S. 1991. De Hygienisten. Artsen, staat \& wolksgezondheid in Nederland 1840 - 1890 . Groningen: Historische Uitgeverij Groningen.

Hughes, T.P. 1983. Networks of Power: Electrification in Western Society, 1880 - 1930. Baltimore: Johns Hopkins University Press.

Hughes, T.P. 1986. 'The seamless web: technology, science, etcetera, etcetera'. Social Studies of Science 16: $281-292$.

Hughes, T.P. 1987. 'The evolution of large technological systems'. In: Bijker, W.E., Hughes, T.P. and Pinch, T. (eds.) 1987. The Social Construction of Technological Systems. New Directions in the Sociology and History of Technology. Cambridge: MIT Press, pp. $51-82$.

Ibsen, B. 1953. 'Retningslinier for behandling af respirationskomplikationer ved poliomyelitis'. Ugeskrift for Laeger 115: 1203 - 1205.

Ibsen, B. 1956. 'The anaesthetist and positive pressure breathing'. In: Lassen, H.C.A.(ed.) 1956. Management of life-threatening poliomyelitis. Copenhagen, 1952 - 1956. With a surney of autopsy findings in 115 cases. Edinburgh and London: E. \& S. Livingstone Ltd.: $14-22$. 
Ibsen, B. 1962. 'Some principles in amplifier technique in the physiology of respiration and circulation'. Acta Anaesthesiologica Scandinavica 1962: supplementum X.

Ibsen, B. 1969a. Intensiv Shocktherapi. København: Nyt Nordisk Forlag.

Ibsen, B. 1969b. Causeri om alvorligt emne. København: Nyt Nordisk Forlag.

Ibsen, B. 1975. 'From Anaesthesia to Anaesthesiology. Personal experiences in Copenhagen during the past 25 years'. Acta Anaesthesiologica Scandinavica Supplementum 61: 6-68.

Ibsen, B., Dahl Kvittingen, T. 1958. 'Arbejdet på en anæsthesiologisk observationsafdeling'. Nordisk Medicin 60: $1349-1355$.

Ibsen, B., Jensen, B. 1971. 'Virkningen af dekstran $40 \mathrm{og}$ klorpromazin på perifer hudtemperatur hos normalpersoner'. Ugeskrift for Laeger 133: 1924.

Ibsen, B., Neukirch, F. 1953. Rapport om wor studierejse til U.S.A. efieräret 1953 til Landsforeningen mod Bernelammelse, Hellerup.

Ibsen, B., Neukirch, F. 1954. 'Respiratory Centers in U.S.A. Impressions from a trip Sept.-Oct. 1953'. Proceedings of the Third Congress of the Scandinavian Society of Anesthesiologists 1954: $58-62$.

Ingvar, D.H. 1973. 'Bestimmung des Sistierens der Gehirnzirkulation bei Hirntod'. In: Krösl, W., Scherzer, E. (Her.) 1973. Die Bestimmung des Todeszeitpunktes. Wien: Wilhelm Maudrich Verlag, pp. $195-198$.

Ingvar, D.H., Sourander, P. 1970. 'Destruction of the reticular core of the brain stern'. A pathoanatomical follow-up of a case of coma of three years duration'. Archives of Neurology 23: 1 - 8.

International Committee for the Study of Infantile Paralysis. 1932. Polionyelitis. Baltimore: The Williams \& Wilkins Company.

Jakobsen, E., Skovsted, P., Thorshauge, C. (eds.) 1978. 'Papers Dedicated to Ole Secher on the Occasion of the 25th Anniversary of his Department and his 60th Birthday'. Acta Anaesthesiologica Scandinavica Supplementum 67. Copenhagen: Munksgaard

Jasper, H.H., Proctor, L.D., Knighton, R.S., Noshay, W.C. and Costello, R.T. 1958. Reticular Formation of the Brain. Henry Ford Hospital International Symposium. Boston and Toronto: Little, Brown and Company.

Jennett, B. and Plum, F. 1972. 'Persistent vegetative state after brain damage. A syndrome in search of a name'. The Lancet 1972: 734 - 737.

Jensen, U.J. 1984a. Moralsk answar og menneskesyn. Om holdninger is social-og sundhedssektoren. København: Munksgaard.

Jensen, U.J. 1984b. 'A critique of essentialism in medicine'. In: Nordenfelt, L. and Lindahl, B.I.B. (eds.) 1984. Health, Disease, and Causal Explanations in Medicine. Dordrecht: D. Reidel Publishing Company, pp. $63-73$.

Jensen, U.J. 1987. Practice and Progress. A Theory for the Modern Health-care System. Oxford: Blackwell Scientific Publications.

Jørgensen, E.O. 1973. 'Spinal man after brain death'. Acta Neurochirurgica 28: 259 - 273.

Jørgensen, P.B., Jørgensen, E.O. and Rosenklint, A. 1973. 'Braindeath pathogenesis and diagnosis'. Acta Neurologica Scandinavica 49: 355 - 367.

Jouvet, M. 1969. 'Coma and other disorders of consciousness'. In: Vinken, P.J., Bruyn, G.W. 1969.

Disorders of higher nerwous activity. Amsterdam: North-Holland Publishing Company.

Kirk, J.E. 1942. Acidosens klinik og behandling. Kebenhavn: Munksgaard.

Kitzinger, C. 1987. The Social Construction of Lesbianism. London: Sage Publications.

Kjøbenhavns Magistrat. 1904. Blegdamshospizalet $i$ Kjobenhavn 1879 - 1904 . Kjøbenhavn.

Kleiner, I. 1919a. 'The action of intravenous injections of pancreas emulsions in experimental diabetes'. Journal of Biological Chemistry 40: 153 - 170.

Kleiner, I. 1919b. 'The disappearance of dextrose from the blood after intravenous injection'. The Journal of Experimental Medicine 23: 507 - 533.

Kleiner, I. 1959. 'Hypoglycemic agents - past and present'. Clinical Chemistry 5: 79 - 99.

Kleiner, I. and Meltzer, S.J. 1915a. "The influence of depancreatization upon the state of glycemia following the intravenous injections of dextrose in dogs". Proceedings of the Society for Experimental Biology and Medicine 12: 58 - 59 
Kleiner, I. and Meltzer, S.J. 1915b. 'Retention in the circulation of dextrose in normal and depancreatized animals, and the effect of an intravenous injection of an emulsion of pancreas upon this retention'. Proceedings of the National Academy of Science 1915: 338 - 341

KNMG. 1959. Medische ethiek en gedragsleer. Derde druk. Amsterdam: Koninklijke Nederlandsche Maastchappij tot Bevordering der Geneeskunst.

Knorr-Cetina, K.D. and Mulkay, M. 1983. 'Introduction: emerging principles in social studies of science'. In: Knorr-Cetina, K.D. and Mulkay, M. (eds.) 1983. Science Observed. Perspectives on the Social Study of Science. London: Sage Publications, pp. 1 - 17.

Knowlton, F.P. and Starling. E.H. 1912. 'On the nature of pancreatic diabetes. (Preliminary communication.) Proceedings of the Royal Sociery of London 1912: 218 - 223.

Knowlton, F.P. and Starling. E.H. 1912. 'Über den Zuckerverbrauch im normalen und in diabetischen Herzen.' Zentralblatt fur Physiologie 26: 169 - 173.

Kolff, W.J. and Berk, H.Th.J. 1944. 'The artificial kidney: a dialyzer with great area'. Acta medica Scandinavica 117: $121-134$.

Kolff, W.J., Berk, H.Th.J., Welle, M. ter, Ley, A.J.W. van der, Dijk, C.C. van, Noordwijk, J. van. 1943. 'De kunstmatige nier: een dialysator met een groot oppervlak. Geneeskundige Gids 21: 409 421.

Koopman, J. 1926. 'Bijdrage tot de geschiedenis der ontwikkeling van de endocrinologie. I. Ontwikkeling der opotherapie'. Nederlands Tijdschrift voor Geneeskunde 1926: 2359 - 2365.

Kooyman, F.C. 1959. "Reanimatie", aanwinst, probleem en opgave'. Medisch Consact 14: 441 - 44. Kooyman, F.C. 1960. '"Reanimatie"'. Medisch Contact 15: 186 - 187.

Korein, J. 1978. 'The problem of brain death: development and history'. In: Korein, J. (ed.) 1978. Bruin Death: Interrelated Medical and Social Issues. New York: The New York Academy of Sciences. Annals of the New' York Academy of Sciences 315: $19-38$.

Korein, J. (ed.) 1978. Brain Death: Interrelated Medical and Social Issues. New York: The New York Academy of Sciences. Annals of the New York Academy of Sciences 315.

Kouwenhoven, W.B. and Harold, J. 1951. 'A simple electrical apparatus for the clinical treatment of ventricular fibrillation'. Surgery' 30: 781 - 786.

Kouwenhoven, W.B., Hooker, D.R. and Langworthy O.R. 1932. "The current flowing through the heart under conditions of electric shock'. American Journal of Physiology 100: 344 - 350.

Kouwenhoven, W.B., Jude, R.J. and Knickerbocker, G.G. 1960. 'Closed-chest cardiac massage'. Journal of the American Medical Association 173: 1064 - 1067.

Kouwenhoven, W.B. and Milnor, W.R. 1954. 'Treatment of ventricular fibrillation using a capacitor discharge'. Journal of Applied Physiology 7: 253 - 257.

Kouwenhoven, W.B., Milnor, W.R., Knickerbocker, G.G. and Chesnut, W.R. 1957. 'Closed chest defibrillation of the heart'. Surgery 42: $550-561$.

Kramer, W. 1962. 'Van reanimatie tot deanimatie'. Nederlands Tijdschrift woor Geneeskunde 106: $637-640$.

Kramer, W. 1963. 'From reanimation to deanimation'. Acta Neurologica Scandinavica 39: 139 - 153.

Kramer, W. 1964. 'Progressive posttraumatic encephalopathy during reanimation'. Acta Neurologica Scandinavica 40: 249 - 258.

Kramer, W. 1966. 'Oorzaken woor het mislukken van reanimatie bij neurologische patiënten'. Nederlands Tijdschrift woor Geneeskunde 110: 1633 - 1635.

Kramer, W. 1970. 'Acute lethal intracranial hypertension. Clinical and experimental observations'. Psychiatria, Ncurologia, Neurochirurgia 73: 243 - 255.

Kramer, W. 1973. 'Neuropathologische Befunde nach intravitalem Hirntod'. In: Krösl, W., Scherzer, E. (Her.) 1973. Die Bestimmung des Todeszeitpunktes. Wien: Wilhelm Maudrich Verlag, pp. 223 $231 .$.

Kramer, W., Tuynman, J.A. 1967. 'Acute intracranial hypertension. An experimental investigation'. Brain Research 6: 686 - 705.

Kretschmer, E. 1940. 'Das apallische syndrom'. Zeitschrift für die gesamte Neurologie und Psychiatrie 169: 576 - 579. 
Kricheff, I.l., Pinto, R.S., George, A.E., Braunstein, P. and Korein, J. 1978. 'Angiographic tindings in brain death'. In: Korein, J. (ed.) 1978. Brain Death: Internelated Medical and Social lssues. New York: The New York Academy of Sciences. Annals of the New York Acudemy of Sciences 315: 168 $-183$.

Kriek, H.R. 1966. 'Farmacologische immunosuppressie bij orgaantransplantaties'. Nederlands Tijdschrift wor Geneeskunde 110: 728 - 735.

Krogh, A. 1910a. 'The forces governing the gas exchange in the lungs'. The British Medical Journal 1910: $1342-1343$.

Krogh, A. 1910b. 'Nogle bemærkninger om overtryksrespiration'. Hospitalstidende 26: 787 - 791.

Krogh, A. 1910c. 'Om overtryksrespiration og kulsyreudvaskning'. Hospitalstidende 29: 870 - 872.

Krogh, A. 1910d. 'Om kulsyre som regulator i organismen og aarsagerne til kirurgisk shock. En oversigt over nogle nyere undersøgelser'. Hospitalstidende 16: 441 - 449.

Krogh, A. 1924. Insulin. En opdagelse og dens betydning. Kabenhavn: G.E.C. Gads Forlag.

Krogh, A. 1932. 'En respirator efter Philip Drinker's princip'. Hospitalstidende 175: 629-632.

Krösl, W., Scherzer, E. (Her.) 1973. Die Bestimmung des Todeszeitpunktes. Wien: Wilhelm Maudrich Verlag.

Kuhn, T.S. 1962. The Structure of Scientific Revolutions. University of Chicago Press.

Kunneman, H. 1986. De waarheidstrechter. Een communicatietheoretisch perspecticf op wetenschap en samenleving. Meppel: Boom.

La Porte, T.R. (ed.) 1991. Social Responses to Large Technical Systems. Control or Anticipation. Dordrecht: Kluwer Academic Publishers.

Laguesse, M.E. 1893. 'Sur la formation des îlots de Langerhans dans le pancréas'. Comptes rendus de la Société de Biologie 5: 819 - 820.

Lamb, D. 1985. Death, brain death and ethics. London: Croom Helm.

Lange, S.A. de. 1964. 'Medische en ethische problemen rondom de reanimatie'. Midisi'h (imtact 19: $117-120$.

Langemeijer, G.E. 1960. “"Reanimatie"'. Medisch Contact 15: 188 - 191.

Langerhans, P. 1869. Beitrage zur mikroskopischen Anatomie der Bauchspeichseldrase. Berlin.

Lassen, H.C.A. 1939. 'Poliomyelitis i København 1937 med specielt henblik på epidemiologiske forhold or paresernes lokalisation'. Ugeskrift for La'ger 101: 80 - 87.

Lassen, H.C.A. 1952a. 'Om behandling af bulbær poliomyelitis - med særligt henblik på urgencebehandelngen af akut respirationsinsufficiens. En helt foreløbig meddelelse'. Ugeskrift for Lager 114: $1415-1417$

Lassen, H.C.A. 1952b. 'Behandling af akut bulbær poliomyelitis med respirationsinsufficiens'. Ugeskrift for Lager 114: 1496 - 1497.

Lassen, H.C.A. 1953. 'A preliminary report on the 1952 epidemic of poliomyelitis in Copenhagen. With special reference to the treatment of acute respiratory insufficiency'. The Lancet 1953: 37 41.

Lassen, H.C.A. 1954. 'Blegdamshospitalets 75 aars jubilæum'. Medicinsk Forum 7: 257 - 264.

Lassen, H.C.A. 1955. 'The management of respiratory and bulbar paralysis in poliomyelitis'. In: Word Health Organization. 1955. Poliomyelitis. Geneva: World Health Organization.

Lassen, H.C.A.(ed.) 1956. Management of life-threatening poliomyelitis. Copenhagen, 1952 - 1956. With a survey of auropsy findings in 115 cases. Edinburgh and London: E. \& S. Livingstone Ltd.

Lassen, H.C.A. 1959. 'Epidemihospitalet gennem tiderne'. Tidsskrift for Sygepleje 59: 166 - 171.

Lassen, H.C.A., Bjørneboe, M, Ibsen, B., Neukirch, F. 1954. 'Treatment of tetanus with curarisation, general anæstesia, and intratracheal positive-pressure ventilation'. The Lancet 1954: $1040-1044$.

Lassen, N.A. et al. 1978. 'Brain function and blood flow'. Scientific American 239: 62 - 71.

Lassen, N.A., Ingvar, D.H. 1963. 'Regional cerebral blood flow measurement in man'. Archives of Neurology 9: $615-622$.

Latour, B. 1983. 'Give me a laboratory and I will raise the world'. In: Knorr-Cetina, K.D. and Mulkay, M. (eds.) 1983. Science Obsenved. Perspectives on the Social Study of Science. London: Sage Publications, pp. $141-170$. 
Latour, B. 1987. Science in Action. How to follow scientists and engineers through society. Milton Keynes: Open University Press.

Latour, B. 1988. The Pasteurization of France. Cambridge: Harvard University Press.

Latour, B. 1990. 'Drawing things together'. In: Lynch, M. and Woolgar, S. (eds.) 1990. Representation in Scientific Practice. Cambridge, Ma.: The MIT Press, pp. 19 - 68.

Latour, B. and Woolgar, S. 1986. Laboratory Life. The Construction of Scientific Facts. Princeton: Princeton University Press. First published in 1979 by Sage Publications, Inc.

Lawson. H. and Appignanesi, L. (eds.) 1989. Dismantling Truth. Reality in the Post-Modern World. London: Weidenfeld and Nicolson.

League of Nations Health Organization. 1926. The Biological Standardisation of Insulin. Geneva.

Leigh Star, S. and Griesemer, J.R. 1989. 'Institutional Ecology, 'Translations' and Boundary Objects: Amateurs and Professionals in Berkeley's Museum of Vertebrate Zoology, 1907-39'. Social Studies of Science 19: $387-420$.

Leschke, E. 1910. 'Ueber die Wirkung des Pankreasexstraktes auf pankreasdiabetische und normale Tiere'. Archiv fur Anatomie (Physioligische Abteilung) 24: 401 - 436.

Lock, M. and Gordon, D.R. (eds.) 1988. Biomedicine Examined. Dordrecht: Kluwer Academic Publishers.

Lorentz, C. 1987. De constructie van het verleden. Een inleiding in de theorie van de geschiedenis. Meppel: Boom.

Lundberg, N. 1960. 'Continuous recording of ventricular fluid pressure'. Acta Psychiatrica Scandinavica 36: Supplement 149.

Lynch, M. and Woolgar, S. (eds.) 1990. Representation in Scientific Practice. Cambridge, Ma.: The MIT Press.

Lyne, J. 1990. 'Bio-rhetorics. Moralizing the life sciences'. In: Simons, H.W. 1990. The Rhetorical

Turn. Invention and Persuasion in the Conduct of Inquiry. Chicago: The University of Chicago Press, pp. 35 - 57.

MacKenzie, D. 1990. Inventing Accuracy. A Historical Sociology of Nuclear Missile Guidance. Cambridge, Ma.: The MIT Press.

MacKenzie, D. and Wajcman, J. (eds.) 1985. The Social Shaping of Technology. Milton Keynes: Open University Press.

Mackenzie, H.W.G. 1893. 'The Treatment of diabetes mellitus by means of pancreatic juice'. The British Medical Journal 1893: 63 - 64

Macleod, J.J.R. 1913. Diabetes: Its Pathological Physiology. London: Edward Arnold. New York: Longmans, Green \& Co.

Macleod, J.J.R. 1924. 'Insulin'. Physiological Reviews 4: 21 - 68.

Macleod, J.J.R. 1978. 'History of the researches leading to the discovery of insulin. With an introduction by Lloyd G. Stevenson'. Bulletin of the History of Medicine 52: 295-312.

Magnus, Herdis won. 1950. 'En svøbe skal besejres. Kampen mot børnelammelsen'. Vor Viden: E. Vort Legeme 1: 71 - 75.

Magnus, O. 1965. 'Het coma, neuro-fysiologische gezichtspunten'. Nederlands Tijdschrift voor Geneeskunde 109: 1941 - 1943.

Magoun, H.W. 1954. 'The ascending reticular system and wakefulness'. In: Adrian, E.D., Bremer, F. and Jasper, H.H. (eds.) 1954. Brain mechanisms and consciousness. Oxford: Blackwell Scientific Publications, pp. 1 - 15 .

Magoun, H.W. 1963. The Waking Brain. 2nd Edition. Springfield, Ill.: Charles C. Thomas Publisher. Mansell-Jones, R. 1893. 'Pancreatic juices in diabetes'. British Medical Journal 1893: 50.

Marshall, J., Spalding, J.M.K. 1953. 'Humidification in positive-pressure respiration for bulbospinal paralysis'. The Lancet 1953: 1022 - 1024.

Mauthner, L. 1890. 'Pathologie und Physiologie des Schlafes'. Wiener klinische Wbchenschrift 3: 445 $-446$.

Mayntz, R. and Hughes, T.P. (eds.) 1988. The Development of Large Technical Systems. Frankfurt am Main: Campus Verlag.

MC. 1974. 'Hersendoodcriteria bij orgaantransplantatie'. Medisch Contact 29: 343 
McMichael, J. 1968. 'Heart transplant discussion on records'. British Medical Journal 1968: 566.

Medawar, P.B. 1946. 'Immunity to homologous grafted skin. II. The relations between the antigens of blood and skin'. British Journal of experimental Pathology 27: $15-24$.

Medicinske Studenterrådet. 1952. En orientering om poliomyelitis. Isar med henblik pd epidemien 1952. Udarbejdet af de medicinske studenterrdd ved Kebenhavns Universitet (pd basis af referat fra et mode om poliomyelitis pd med. anatomisk institut den 5. november 1952. København: N. Olaf Moller.

Medvei, V.C. 1982. A History of Endocrinology. Lancaster: MTP Press Limited.

Mellinghoff, K.H. 1971. Georg Ludwig Zuelzers Beitrag zur Insulinforschung. Düsseldorf: Michael Triltsch Verlag.

Memorie van Toelichting. 1991. Regelen omtrent het ter beschikking stellen van organen (Wet op de. orgaandonatie). 1991. Tweede Kamer der Staten Generaal, vergaderjaar 1991-1992, 22 358, nr. 3. 's-Gravenhage: Sdu Uitgeverij.

Mering, J. von, and Minkowski, O. 1889. 'Diabetes mellitus nach Pankreasexstirpation'. Archiv für experimentelle Pathologie und Pharmakologie 26: 371 - 387.

Milhaud, A., Riboulot, M. and Gayet, H. 1978. 'Disconneting tests and oxygen uptake in the diagnosis of total brain death'. In: Korein, J. (ed.) 1978. Brain Death: Interrelated Medical and Social Issues. New York: The New York Academy of Sciences. Annals of the New York Academy of Sciences 315, pp. 24I - 251.

Minkowski, O. 1884. 'Ueber das Vorkommen von Oxybuttersäure im Harn bei Diahetes Mellitus'. Archiv fur experimentelle Pathologie und Pharmakologie 18: 35 - 48.

Minkowski, O. 1890. 'Diabetes mellitus und Pankreasaffection'. Berliner klinische Wbchenschrift 1890: $167-169$.

Minkowski, O. 1892. 'Weitere Mittheilungen über den Diabetes mellitus nach Exstirpation des Pankreas'. Berliner klinische Wochenschrift 1892: 90 - 94.

Minkowski, O. 1893. 'Untersuchungen über den Diabetes mellitus nach Extirpation des Pankreas'. Archiv fur experimentelle Pathologie und Pharmakologie 31: 85 - 189.

Minkowksi, O. 1929. 'Die Lehre vom Pankreas-Diabetes in ihrer geschichtlichen Entwicklung'. Munchner Medizinische Wochenschrift 76: 311 - 315.

Mollaret, P., Goulon, M. 1959. 'Le coma dépassé (mémoire préliminaire)'. Revue Neurologique 101: $3-15$.

Mollaret, P., Bertrand, 1., Mollaret, H. 1959. 'Coma dépassé et nécroses nerveuses centrales massives'. Revue Neurologique 101: 116 - 139.

Møller, K.O. 1958. 'Anæstesiologiens udvikling i Danmark fra 1915 -delvist beskrevet pá grundlag af egne oplevelser'. Medicinsk Forum 11: 97 - 118.

Møllgaard, H. 1910a. 'Teknisk redegørelse for et nyt apparat til overtryksrespiration', Hospitalstidende 21: 569 - 580.

Møllgaard, H. 1910b. 'Om overtryksrespiration og kulsyreudvaskning'. Hospitalstidende 28: 842 846.

Morrison, P., Morrison, P. and the Office of Charles \& Ray Eames. 1982. Powers of Ten. About the Relative Size of Thins in the universe. New York: W.H. Freeman and Company.

Moruzzi, G. 1954. 'The physiological properties of the brain stem reticular system'. In: Adrian, E.D., Bremer, F. and Jasper, H.H. (eds.) 1954. Brain mechanisms and consciousness. Oxford: Blackwell Scientific Publications, pp. $21-48$.

Moruzzi, G. 1972. 'The Sleep-Waking Cycle'. Reviews of Physiology 64:1-165.

Moruzzi, G., Fessard, A. and Jasper, H.H. (eds.) 1963. Brain mechanisms. Amsterdam: Elsevier Publishing $\mathrm{C} 0$.

Moruzzi, G., Magoun, H.W. 1949. 'Brain stem reticular formation and activation of the EEG. Electroencephalography and Clinical Neurophysiology 1: 455 - 473.

Moseley, J.I., Molinari, G.F. and Walker, A.E. 1976. 'Respirator brain. Report of a survey and review of current concepts'. Archives of Pathological and Laboratory Medicine 100: $61-64$.

Mulder, J.D., Voorthuisen, A.E. van (red.). 1985. Leerboek radiodiagnostiek. Utrecht: Bohn, Scheltema en Holkema. 
Murlin, J.R. and Kramer, B. 1913. 'The influence of pancreatic and duodenal extracts on the glycosuria and the repiratory metabolism of depancreatized dogs'. Proceedings of the Sociery for Experimental Biology and Medicine 10: 171 - 173.

Murnaghan, J.H. and Talalay, P. 1992. 'H.H. Dales's account of the standardization of insulin'. Bulletin for the History of Medicine 66: 440 - 450 .

Murray, G.R. 1891. 'Note on the treatment of myxoedema by hypodermic injections of an extract of the thyroid gland of a sheep'. British Medical Journal 2: 796 - 797.

Murray, I. 1969. 'The search for insulin'. Scottish Medical Journal 14: 286 - 293.

Mushin, W. 1980. Automatic Ventilation of the Lungs. Chapter 8: 'Historical Background to Automatic Ventilation'. Third edition. Oxford: Blackwell Scientific Publications.

Myers, V.C. and Baily C.B. 1916. 'The Lewis and Benedict method for the estimation of blood sugar. With some observations in disease'. Journal of Biological Chemistry 24: $147-161$.

Naunyn, B. 1925. Erinnerungen, Gedanken und Meinungen. München: J.F. Bergmann Verlag.

Nauta. J. 1959. 'De organisatie voor de behandeling van hartstilstand in het Academisch Ziekenhuis te Leiden'. Nederlands Tijdschrift voor Geneeskunde 103: 931 - 932.

Neukirch, F. 1954. 'Über die Behandlung der lebensbedrohender Poliomyelitis'. Helvetica Paediatrica Acta 9: 381 - 402.

Nielsen, A. 1936. 'Et tilfalde af morfinforgiftning behandlet i Krogh's respirator'. Ugeskrift for Lager 98: 396 - 397.

Nielsen, E.M. 1946. 'Om respiratorhehandling af respirations-pareser ved poliomyelitis anterior acuta. Ugeskrift for Loeger 108: 1341 - 1348.

Noorden, C. von. 1893. Lehrbuch der Pathologie des Stoffwechsells. Berlin: Verlag von August Hirsehwald.

Nordenfelt, L. and Lindahl, B.I.B. (eds.) 1984. Health, Disease, and Causal Explanations in Medicine. Dordrecht: D. Reidel Publishing Company.

Nouhuys, F. van. 1960. 'Kunstmatige ademhaling'. Nederlands Tijdschrifi woor Geneeskunde 104: $1419-1425$.

Opie, E.L. 1900d. 'On the relation of chronic interstitial pancreatitis to the islands of Langerhans and to diabetes mellitus'. The Journal of Experimental Medicine 1900: 397 - 428.

Opie, E.L. 1900b. 'The relation of diabetes mellitus to lesions of the pancreas. Hyaline degeneration of the islands of Langerhans'. The Journal of Experimental Medicine 1900: 527 - 540.

Pallis, C. 1983. ABC of Brain Stem Death. Articles from the British Medical Journal. London: British Medical Journal.

Pallis, C.A. 1953. 'Poliomyelitis in Denmark. Impressions from Blegdam Hospital, Copenhagen' The Lancet 1953: 726 - 728.

Papaspyros, N.S. 1964. The History of Diabetes Mellitus. Stuttgart: Georg Thieme Verlag.

Parkinson, D. 1985. 'Letter to Lars Granholm, Department of Neurosurgery, Karolinska Hospital, Stockholm, Sweden'. In: Christensen, A., Lyttkens, H. (eds.) 1985. NEJ till hjarnrelaterade dodskriterier. Stockholm, p. 90.

Pasveer, B. Shadows of Knowledge. Making a representing practice in medicine: $X$-ray pictures and pulmonary tuberculosis, 1895-1930. Amsterdam: PhD-thesis.

Pernick, M.S. 1988. 'Back from the grave: recurring controversies over defining and diagnosing death in history'. In: Zaner, R.M. 1988. Death: Beyond Whole-Brain Criteria. Dordrecht: Kluwer Academic Publishers, pp. 17 - 74.

Pflüger, E. 1905. Das Glykogen und seine Beziehungen zur Zuckerkrankheit. Zweite Auflage. Bonn: Verlag von Marting Hager.

Pflüger, E. 1877. 'Die teleologische Mechanik der lebendigen Natur'. Archiv für die gesamte Physiologie des Menschen und der Thiere 15: 57 - 103.

Pickering. A. (ed.) 1992. Science as practice and culture. Chicago and London: University of Chicago Press.

Pinch, T.J., and Bijker, W.E. 1984. 'The social construction of facts and artefacts: Or how the sociology of science and the sociology of technology might benefit each other'. Social Studies of Science 14: 399-341. 
Pinch, T.J., and Bijker, W.E. 1987. 'The social construction of fact and artifacts: Or how the sociology of science and the sociology of technology might benefit each other'. In: Bijker, W.E., Hughes, T.P., and Pinch, T.J. (eds.) 1987. The social construction of technological systems. New directions in the sociology and history of technology. Cambridge, Ma.: The MIT Press, pp. 17-50. Pirsig, R.M. 1991. Lila. An inquiry into morals. London: Black Swan.

Plum, F. 1972. 'Organic disturbances of consciousness'. In: Critchley, M., O’Leary, J.L., Jennett, B. (eds.) 1972. Scientific Foundations of Neurology. London: William Heinemann Medical Books Ltd., pp. 193 - 200.

Plum, F., Posner, J.B. 1980. The diagnosis of stupor and coma. 3rd edition. Philadelphia: F.A. Davis Company.

Pope Pius XII. 1958. 'The prolongation of life'. The Pope Speaks 4: 393 - 398. Reprinted in: Reiser, S.J., Dyck, A.J., Curran, W.J. 1977. Ethics in medicine. Historical perspectives and contemporary concerns. Cambridge: The MIT Press, pp. 501 - 504.

Poulsen, J.E. 1982. Features of the History of Diabetology. Copenhagen: Munksgaard.

Pratt, J.H. 1954. 'A reappraisal of researches leading to the discovery of insulin'. Journal of the History of Medicine 1954: 281 - 289.

Prick, J.J. 1969. 'De medische ethiek met betrekking tot de nieuwste ontwikkelingen in de geneeskunde'. Medisch Contact 24: 83 - 85.

Puccetti, R. 1988. 'Does anyone survive neocortical death?' In: Zaner, R.M. 1988. Death: Beyond Whole-Brain Criteria. Dordrecht: Kluwer Academic Publishers, pp. 75 - 90.

Putten, W.G.H.M. van der. 1992. Wet op de lijkbezorging. Wet van 7 maart 1991, Stb. 130, houdende nieuwe bepalingen inzake de lijkbezorging (Wet op de lijkbezorging), zoals deze laatselijk is gewijzigd bij de wet van 22 mei 1991, Stb. 394. Zwolle: W.E.J. Tjeenk Willink.

Rang, J.F., Rozendal, R. 1971. 'Medisch-juridische problemen rondom de orgaanextirpatie'. Medisch Contact 26: 495 - 500 .

Reale, E. 1890. 'Ueber Ursprung und Behandlung des Diabetes mellitus'. Verhandlungen des internationalen medicinischen Congresses 1890, Bd. II, Abtheil V: $97-100$.

Reiser, S.J. 1985. 'Critical care in an historical context'. In: Moskop, J.C. and Kopelman, L. 1985. Ethics and Critical Care Medicine. Dordrecht: D. Reidel Publishing Company, pp. 215 - 224.

Reiser, S.J. 1978. Medicine and the Reign of Technology. Cambridge: Cambridge University Press.

Rennie, J. and Fraser, Th. 1907. 'The islets of Langerhans in relation to diabetes'. Biochemical Journal 2: 7 - 19.

Richards, D.W. 1966. 'The effect of pancreas extract on depancreatized dogs: Ernest L. Scott's Thesis of 1911'. Perspectives in Biology and Medicine 10: 84 - 95.

Roberts, F. 1922. 'Insulin'. The British Medical Journal 1922: 1193 - 1194.

Rolleston, H.D. 1936. The Endocrine Organs in Health and Disease. With a historical revitw. London: Oxford University Press.

Rood, J.J. van, Eernisse, J.G. and Leeuwen, A. van. 1958. 'Leucocyte antibudies in sera from pregnant women'. Nature 181: 1735.

Rood, J.J. van, Leeuwen, A. van., Frederiks, E., Bosch, L.J. 1964. 'Relationship of leucocyte groups to tissue transplantation compatibility'. Annals of the New York Academy of Science 120: 285

Rood, J.J. van, Leeuwen, A. van., Schippers, A., Frederiks, E., Balner, H. and Eernisse, J.G. 1965. 'Leucocyte groups, the normal lymphocyte transfer test and homograft sensitivity'. In: Histocompatibility Testing 1965. Copenhagen: Munksgaard.

Rorbæk Madsen, E. 1975. At skabe er at leve. Struer: Bang \& Olufsen.

Rosenberg, C.E. 1992. 'Cholera in nineteenth-century Europe: A tool for social and economic analysis'. In: Rosenberg, C.E. 1992. Explaining Epidemics and Other Studies in the Histony of Medicine. Cambridge: Cambridge University Press, pp. $109-121$.

Rudwick, M.J.S. 1976. 'The emergence of a visual language for geological science $1760-1840$ '. History of Science 14: 149 - 195.

Rudwick, M.J.S. 1985. The Meaning of Fossils. Episodes in the History of Palaeontology. 2nd edition. Chicago: The University of Chicago Press. 
Russell, W.R., Schuster, E. 1953. 'Respiration pump for poliomyelitis'. The Lancet 1953: 707 - 709.

Safar, P. 1961. "Ventilatory efficacy of mouth-to-mouth artificial respiration. Airway obstruction during manual and mouth-to-mouth artificial respiration'. Journal of the American Medical Association 167: 335 - 341 .

Safar, P., Escarraga, L.A. and Elam, J.O. 1958. 'A comparison of the mouth-to-mouth and mouth-toairway methods with the chestpressure armlift methods'. New England Journal of Medicine 258: $671-677$.

Sahlin, B. 1931. 'En ny respiratortyp'. Hygienisk Revy 20: 129 - 131.

Sandmeyer, W. 1895. 'Ueber die Folgen der partiellen Pankreasexstirpation beim Hund'. Zeitschrift fur Biologie 31: 12 - 85.

Sauerbruch, F. and Heyde, M. 1908. 'Ueber Parabiose künstlich vereinigter Warmblüter'. Münchner Medizinische Wochenschrift 55: 153 - 156.

Schäfer, E.A. 1895. 'On internal secretion'. The British Medical Journal 1895: 341 - 348.

Scherzer, E. and Pendl, G. 'Die terminale Angiographie des Zerebrums'. In: Krösl, W., Scherzer, E. (Her.) 1973. Die Bestimmung des Todeszeitpunktes. Wien: Wilhelm Maudrich Verlag, pp. 137 154.

Schlich, T. 1993a. 'Making mistakes in science: Eduard Pflüger, his scientific and professional concept of physiology, and his unsuccessful theory of diabetes (1903-1910)'. Studies in the History and Philosophy of Science 24: $411-441$.

Schlich, T. 1993b. 'Vom physiologischen Experiment zur Therapie: Die Pankreastransplantation'. Medizinhistorisches Journal 28: 29 - 54.

Schmidt, M. 1934. '4 tilfælde af respirationslammelse behandlede i Krogh's respirator'. Ugeskrift for Lager 96: $406-410$.

Schöne-Seifert, B. 1993. 'Der "Erlanger Fall" im Rückblick: eine medizinethische Lektion?' Ethik in der Medizin 5: 13 - 23.

Schulze, W. 1900. 'Die Bedeutung der Langerhans'schen Inseln im Pancreas'. Archiv fur mikroskopische Anatomie 56: 491 - 509.

Schuster, E., Fischer-Williams, M. 1953. 'A rocking-bed for poliomyelitis'. The Lancet 1953: 1074 1076.

Schwarz, M. and Jansma, R. (eds.) 1988. De technologische cultuur. Amsterdam: De Balie.

Schwarz, R, Eisner, A. and Dameshek, W. 1959. 'The effect of 6-mercaptopurine on primary and secondary immune responses'. Journal of Clinical Imestiagations 38: $1394-1403$.

Schweizerische Akademie der medizinischen Wissenschaften. 1969. 'Richtlijnen voor definitie en diagnose van de dood". Medisch Contact 24: 600 - 601.

Scott, E.L. 1911. The Effect of Pancreas Extract on Depancreatized Dogs. A dissertation submitted to the faculty of the Ogden Graduate School of Science in candidacy for the degree of Master of Science Department of Physiology. Thesis No. T-10553. Published and reprinted in: Richards. D.W. 1966. "The effect of pancreas extract on depancreatized dogs: Ernest L. Scott's Thesis of 1911'. Perspectives in Biology and Medicine 10: 84 - 95.

Scott, E.L. 1912. 'On the influence of intravenous injections of an extract of the pancreas on experimental pancreatic diabetes'. Americun Journal of Plinsiology' 29: 306 - 310.

Scott, E.L. 1913. 'The relation of pancreatic extract to the sugar of the blood'. Proceedings of the Society for Experimental Biology and Medicine 10: 101 - 103.

Scott, E.L. 1923. 'Priority in discovery of a substance derived from the pancreas, active in carbohydrate metabolism'. Journal of the American Medical Association 81: 1303 - 1304.

Secher, O. 1989. 'The polio epidemic in Copenhagen 1952'. In: Atkinson, R.S. and Boulton, T.B. (eds.) 1989. The History of Anaesthesia. London and New York: Royal Society of Medicine Services and the Pathenon Publishing Group, pp. 425 - 432.

Severinghaus, J.W., Astrup, P.B. 1987. History of Blood Gas Analysis. International Anesthesiology Clinics 25, 4. Boston: Little Brown and Company.

Shapin, S. 1982. 'History of science and its sociological reconstructions'. History of Science 20: 157 211. 
Siggaard Andersen, O. 1960. 'A graphic representation of changes of the acid-base status'. Scandinavian Journal of Clinical and Laboratory Investigations 12: 311 - 314.

Siggaard Andersen, O., Engel, K. 1960. 'A new acid-base nomogram. An improved method for the calculation of the relevant blood acid-base data'. Scandinavian Journal of Clinical and Laboratory Investigations 12: 177 - 186.

Siggaard Andersen, O., Engel, K., Jørgensen, K., Astrup, P. 1960. 'A micro-method for determination of $\mathrm{pH}$, carbon dioxide tension, base excess and standard bicarbonate in capillary blood'. Scandinavian Journal of Clinical and Laboratory Investigations 12: 172 - 176.

Simons, H.W. 1990. The Rhetorical Turn. Invention and Persuasion in the Conduct of Inquiry. Chicago: The University of Chicago Press.

Sismondo, S. 1993. 'Some social constructions'. Social Studies of Science 23: 515 - 553.

Sluyters, B. 1970. 'Juridische aspecten'. In: Greep, J.M. (ed.) 1970. Orgaantransplantatie. Leiden: Stafleu's Wetenschappelijke Uitgeversmaatschappij N.V., pp. 361 - 394.

Sluyters, B. 1972. 'Transplantatie - de wetgever grijpt in'. Medisch Contact 27: 695 - 698.

Socialdepartementet. 1984. Dodsbegreppet. Huvudbetankande. Utredningen om dodsbegreppet. Stockholm: Socialdepartementet, SOU 1984:79.

Sporken, P. 1968. 'Vraag naar irreversibel moment van de dood krijgt opnieuw dringend karakter'. Medisch Contact 23: 445 - 446.

Sporken, P. 1969. Vorlopige diagnose. Inleiding tot een medische ethiek. Utrecht: Uitgeverij Ambo n.v.

Ssobolew, L.W. 1900. 'Ueber die Structur der Bauchspeichseldrüse unter gewissen pathologischen Bedingungen'. Zentralblatt fur allgemeine Pathologie und pathologische Anatomie 11: $202-203$.

Ssobolew, L.W. 1902. 'Zur normalen und pathologischen Morphologie der inneren Secretion der Bauchspeichseldrüse. Die Bedeutung der Langerhans'schen Inseln'. Virchow's Archiv fur pathologische Anatomie 168: 91 - 128.

Stanford, M. 1986. The Nature of Historical Knowledge. New York: Basil Blackwell Lid.

Stermerding, D. 1991. Plants, Animals And Formulae. Natural history in the light of Latour's Science in Action and Fourcault's The Order of Things. Enschede: Universiteit Twente.

Stevenson, L. 1947. Sir Frederick Banting. 2nd Edition. Toronto.

Stewart, G.A. 1974. 'Historical review of the analytical control of insulin'. Analyst 99: 913 - 928.

Stig Christensen, M., Sund Kristensen, H., Lyders Hansen, E. 1975. 'Artificial hyperventilation during 21 years in three cases of complete respiratory paralysis'. Acta Medica Scandinavica 198: $409-413$.

Strøm, J.(ed.) 1956. 'The poliomyelitis epidemic in Stockholm 1953. Epidemiological, clinical and laboratory investigations'. Acta Medica Scandinavica 154: Supplementum 316.

Summers, R.W., Curtis, S.J., Hartford, C.E. and Rubush, J.L. 1970. 'Acute hepatic coma treated by cross circulation with irreversibly comatose donor'. Journal of the American Medical Association 214: $2297-2301$.

Tausk, M. 1978. Organon. De geschiedenis van een bijzondere Nederlandse onderneming. Nijmegen: Dekker \& Van der Vegt.

Thiroloix, M.J. 1892. 'Note sur la physiologie du pancréas. De la dissociation expérimentale des sécrétions externe et interne de la glande - rôle dans le diabète'. Archives de physiologie normale et pathologique 1892: 716 - 720.

Till-d'Aulnis de Bourouill v., H.A.H. 1970. Medisch-juridische aspecten van het einde van het menselijk lewen. Deventer: Kluwer.

Till-d'Aulnis de Bourouill v., H.A.H. 1974. 'Dondscriteria. Achtergronden van de verwarring en een poging tot verheldering'. Tijdschrift wor Privaatrecht 1974: 495 - 542.

Till-d'Aulnis de Bourouill v, H.A.H. 1975. 'How dead can you be?' Medicine, Science and the Law 15: $133-147$.

Till-d'Aulnis de Bourouill v., H.A.H. 1976. 'Legal aspects of the definition and diagnosis of death'. In: Vinken, P.J. and Bruyn, G.W. (eds.) 1976. Injuries of the Brain and Skull. Part II. Handbook of Clinical Neurology. Volume 24. Amsterdam: North Holland Publishing Company, pp. 787 - 828. 
Till-d'Aulnis de Bourouill, v., H.A.H. 1983. 'De dood is voor allen gelijk'. Medisch Contact 43: $1363-1364$.

Till-d'Aulnis de Bourouill, v., H.A.H. 1984. 'Donorbescherming bij postmortale orgaanverwijdering'. Tijdschrift voor Gezondheidsrecht 1984: 21 - 26.

Till-d'Aulnis de Bourouill, v., H.A.H. 1986. 'Dood of niet dood, wie zal het zeggen?'. Advocatenblad 66: 529 - 533.

Till-d'Aulnis de Bourouill, v., H.A.H. 1989. 'Verwarring over de diagnose hersendood'. Medisch Contact 44: 1649 - 1653.

Veatch, R.M. 1976. Death, Dying, and the Biological Rewolution. Our Last Quest for Responsibility. New Haven: Yale University Press.

Veatch, R.M. 1978. 'The definition of death: ethical, philosophical, and policy confusion'. In: Korein, J. (ed.) 1978. Brain Death: Interrelated Medical and Social Issues. New York: The New York Academy of Sciences. Annals of the New York Academy of Sciences 315: 307 - 317.

Veatch, R.M. 1988. 'Whole brain, neocortical, and higher brain related concepts'. In: Zaner, R.M. 1988. Death: Beyond Whole-Brain Criteria. Dordrecht: Kluwer Academic Publishers, pp. 171 186.

Vink, M. 1967. 'Medisch-ethische problemen rond de niertransplantatie'. Nederlands Tijdschrift voor Geneeskunde 111: $1248-1252$.

Vinken, P.J., Bruyn, G.W. 1969. Disorders of higher nerwous activity. Amsterdam: North-Holland Publishing Company.

Wackers, G. 1988. 'Building networks: a constructivist perspective on changes in medicine and health care'. In: Jensen, U.J. and Mooney, G. (eds.) 1988. Changing Values in Medical and Health Care Decision Making. Chichester: John Wiley \& Sons, pp. $61-80$.

Wackers, G. 1992. 'The chronogeography of persuasion: normative prospects in constructivist science studies'. Social Epistemology 6: 299 - 313.

Wackers, G. 1993. 'Hersendood in meervoud: representatie in retorische context'. Kennis en Methode 27: 78 - 108.

Wackers, G. 1994a. 'Innovation in artificial respiration: how the 'iron lung' became a museum piece'. In: Lawrence, G. (ed.) 1994. Technologies of modern medicine. Proceedings of a seminar held at the Science Museum, London, March 1993. London: Science Museum, pp. 40 - 57.

Wackers, G. 1994b. 'Modern anaesthesiological principles for bulbar polio: manual IPPR in the 1952 polio-epidemic in Copenhagen'. Acta Anaesthesiologica Scandinavica 38: 420 - 431.

Waine Andersen, E. 1958. 'Tetanus treated in 1950 with decamethonium and positive-pressure ventilation'. Danish Medical Bulletin 5: 276 - 279.

Walford, R.L., Martin, C., Troup, G.M. and Goodwin, W.E. 1965. 'Skin graft survival time in human donor-recipient pairs selected on the basis of Jymphocyte typing'. In: Histocompatibility' Testing 1965. Copenhagen: Munksgaard.

Walker, A.E. 1985. Cerebral Death. 3rd edition. Baltimore-Munich: Urban \& Schwarzenberg.

Walshe, F.M.R. 1972. 'The neurophysiological approach to the problem of consciousness'. In: Critchley, M., O'Leary, J.L., Jennett, B. (eds.) 1972. Scientific Foundations of Neurology. London: William Heinemann Medical Books Ltd., pp. $181-189$

Walton, D.N. 1983. Ethics of Withdrawal of Life-Support Systems. Westport/London: Greenwood Press.

Werkö, L. 1947. 'The influence of positive pressure breathing on the circulation in man'. Actu Medica Scandinavica 128: Supplementum 193.

Wertheimer, P., Jouvet, M., Descotes, J. 1959. 'A propos du diagnostic de la mort du système nerveux. Dans les comas avec arrêt respiratoire traités par respiration artificielle'. La Presse Medicale 67: 87 - 88.

White, R.J., Wolin, L.R., Massoput Jr., L.C., Taslitz, N. and Verdura, J. 1971. 'Primate cephalic transplatation: neurogenic separation, vascular association'. Transplantation Proceedings 3: 602 604.

Wiggers, C.J. 1936. 'Cardiac massage followed by countershock in revival of mammalian ventricles from fibrillation due to coronary occlusion'. The American Journal of Physiology 116: 161 - 162. 
Wiggers, C.J. 1940. 'The physiologic basis for cardiac resuscitation from ventricular fibrillation method of serial defibrillation'. American Heart Journal 20: 413 - 422.

Williams, J.R. 1922. 'A clinical study of the effects of insulin in severe diabetes.' Journal of Metabolic Research 1922: 729 - 751.

Woelderen, C.L. van, 1969. 'Ontwikkelingen in het grensgebied van leven en dood'. Medisch Contact 24: $81-83$.

Wolstenholme, G.E.W. and O'Connor, C.M. (eds.) 1958. Ciba Foundation Symposium on the Neurological Basis of Behaviour. London: J. \& A. Churchill Ltd.

Wood, N. 1893. 'The treatment of diabetes by pancreatic extracts'. The British Medical Journal 1893: 64 .

Woolgar, S. 1988a. Science the very idea. Chichester: Ellis Horwood Limited, and London: Tavistock Publications Limited.

Woolgar, S. (ed.) 1988b. Knowledge and Reflexivity. New Frontiers in the Sociology of Knowledge. London: Sage Publications.

Woolgar, S. 1989. 'The Ideology of Representation and the Role of the Agent'. In: Lawson. H. and Appignanesi, L. (eds.) 1989. Dismantling Truth. Reality in the Post-Modern World. London: Weidenfeld and Nicolson, p. 131 - 144.

Woude, van der, A., Damstra, C.F., Vries, de, A.J.F. 1956. 'De behandeling van patiënten met een insufficiente ademhaling. Uit het Ademcentrum van de Groningse Universiteitskliniek'. Nederlands Tijdschrifi voor Geneeskunde 100: $2134-2145$.

Wrenshall, G.A., Hetenyi, G.H., Feasby, W.R. 1962. The story of insulin: forty years of success against diabetes. London: The Bodley Head.

Wulff, F. 1934. 'Om anvendelse af respirator ved poliomyelitis anterior acuta'. Ugeskrift for lager 96: $814-817$.

Wulff, H.R., Andur Pedersen, S., Rosenberg, R. 1986. Philosophy of Medicine. An Introduction. Oxford: Blackwell Scientific Publications.

Young, F.G. 1970. 'The evolution of ideas about animal hormones'. In: Needham, J. (ed.) 1970. The Chemistry of Life. Eight Lectures on the History of Biochemistry. London: Cambridge University Press, pp. $125-155$.

Zaner, R.M. 1988. Death: Beyond Whole-Brain Criteria. Dordrecht: Kluwer Academic Publishers.

Zimmermann, O.C. 1989. 'The first description of the symptoms of experimental pancreatic diabetes by the Swiss Johann Conrad Brunner (1653 - 1727)'. In: Engelhardt, D. won (ed.). 1989. Diabetes. Its Medical and Cultural History. Heidelberg: Springer Verlag: 209 - 228.

Zoll, P.M., Linenthal, A.J., Gibson, W., Paul, M.H. and Norman, L.R. 1956. 'Termination of ventricular fibrillation in man by externally applied electric countershock'. The New England Journal of Medicine 254: 727 - 732.

Zuelzer, G. 1901. 'Zur Frage des Nebennierendiabetes'. Berliner klinische Wbchenschriff 38: 1209 1210.

Zuelzer, G. 1907a. 'Experimentelle Untersuchungen über den Diabetes'. Berliner klinische Wochenschrift 44: $474-475$.

Zuelzer, G. 1907b. 'Untersuchungen über den experimentellen Diabetes'. Verhandlungen des Congresses fur innere Medicin 24: 258 - 263.

Zuelzer, G. 1908. 'Ueber Versuche einer specifischen Fermenttherapie des Diabetes'. Zeitschrift fur experimentelle Pathologie und Therapie 5: 307-318.

Zuelzer, G., Dohrn, M., Marxer, A. 1908. 'Neuere Untersuchungen über den experimentellen Diabetes'. Deutsche Medizinische Wbchenschrift 34: 1380 -1385. 


\section{Index}

academic medical ethics 12, 271 accompanying injuries in experimental diabetes 61,63

actor network theory 17-8

adrenal diabetes $53,83-4,86$

adrenal-pancreatic antagonism 84,86 Allen 78, 103

allowing to die 177

angiography 207-8, 211, 232-3, 256

anti-vivisectionist movement 94, 259

apallic syndrome $216-7,220$

arctic grail 20

Aretaeus of Capadokia on diabetes 30 arousal 194

artificial liver 212

artificialness of the life support, relevance of 190,200

ascending reticular activating system

(ARAS) 194-5, 205, 216, 255, 260

Astrup, P. 135-6, 160-162

Banting 92-104, 106-109

Barnard's heart transplantation 184

Barron 75, 92

base excess 162

basic tenets of constructivist approach 14,18

Bernard. C. 53-4, 56, 61-2, 246-248, 258-9

Best $92 \mathrm{ff}$.

biological assay $76,86,102,115,265$, 269

biological concept of disease 32-3

biological standardization 52,116 , 259

Bjørneboe 134-5, 142-44
Blegdamshospital $127 \mathrm{ff}$.

blood gas analysis 158, 161, 162

boundary formation 224

boundary object 230, 233, 266, 267

Bower's results with bulbar polio 140

brain as a whole vs whole brain 193 , 194,200

brain as integrator, regulator and coordinator 193

brain death $192 \mathrm{ff}$.

brain stem death, defition of $192 \mathrm{ff}$.

British Medical Research Council and insulin 111-3

Brunner's pancreatectomies 57

bulbar poliomyelitis $134 \mathrm{ff}$.

burden of proof in diagnosing death 204, 256

Carbovisor, Brinkman's 145-147, 149, $152,160,251,266$

cardiac transplantation 184-186

Carlson and Drennan on experimental diabetes and pregnancy 81

cellular level function and neocortical death 218

cerebral angiography $207-8,211,232-$ 3

cerebral perfusion pressure 208-9, 211

certainty

justification of 27,46

production and maintenance 27

source of 249,265

cholera epidemic in Copenhagen 127, 129

circumstances of production of inscriptions 245,248 
closure $16-7,24,222-3,235,268-9$

Collip 101-10, 114

coma artificiale prolongatum 174

coma dépassé 171

comparative testing of insulin potency

115

concepts of disease 13,22, 27, 29

conclusive proof of death 206

Connaught Anti-Toxin Laboratories

108,116

constructivist concept of disease 13

constructivist science and technology

studies 13-15, 17-19

Cooley's anemia 28

criteria of existence $51,76,90,98$

critical topos and brain death 189-90,

$193,202,216,230$

crystalline insulin 116

cuirass respirator 136,157

curare 143-4

Dale, H. 111-113, 116-7

brain damage and death

de facto 178, 222

de jure 178, 222

deanimation 174-5

definitions of death 187

diabetes mellitus $9,22,30,50,52-66$, $68,72,74-76,79,82-9,107,117-8$, $148,246,248-9,257,262-3,268$

diagnosing a dead brainstem 194-198

dietary regime $9,77,108,257$

direct cardiac massage 170

discovery of insulin $92 \mathrm{ff}$.

disposal of the non-apneic corpse 220

dissociated death 175

distribution of ontological status and

definitions of death 189

ductless glands 68,70

Duhem-Quine thesis 15

Dutch Health Council 225, 230-233, $235,267,270$

egalitarian fallacy 19

Eli Lilly \& Co 92, 104 ff.

empirical program of relativism

(EPOR) 16-7

empty wakefulness and PVS 216

Engell and carbovisor 145-6

Engström's respirator 154-5, 157

epistemic relativism 19 essentialism 22, 27, 30-33

evoked potentials and brain death 231 , 233

evolutionary concept of disease 33

experimental diabetes $58,60-1,76,84$, 246-248

experimental pathologists and diabetes 265

Forschbach 79-81, 88-90, 261,

Galen on diabetes 52

Hagedorn 113, 115

heart-beating donors 228,230

heart-lung criteria of death 172

Heine-Medin disease 130

Henderson-Hasselbalch's equation 146, 159

higher brain functions $190,215-218$

Hoffman-La Roche 91-2

horizon of relevance $223,235,267$, 271

hypoglycemic reaction 105,107

Ibsen, B. 134-137 ff., 251-253, 257 -

$8,262,266$

immutable mobiles 41-2

impartiality 15

in-house protocols $233-4,270$

infantile paralysis $30-1,126,130$

innate awareness and whole brain death 204

inscriptions $39-43,45-47,61,72,90$, $148,177,189,205,245-248,252$,

$256,257,260,262$

inside-outside boundary $259,270-1$

instruments of perception 37

insulin $9,10,19,22,46,47,50 \mathrm{ff}$., $245,250-253,258-261,265-269,272$

integrated functioning and total death $181,190-1$

internal secretion $53,65,69-72,74-79$, $81-2,84-87,89-93,96$ ff. , 118, 246$7,250,256-258,265-7$

International Distributing Company for

Danish Insulin Leo 113

international standard for insulin 117

interpretation and translation 46

interpretative flexibility 16-7

intracranial circulation and whole brain death 205-207, 209, 211, 256

intransitive reality 32 
intravital autolysis 175

inverting reality 34

iron lung $23,133,135,139,146-7$,

157

irreversible coma 202, 211-2, 220.

229-30

judgmental relativism 19

knowledge-making, approaches to 14

Kommunehospital 129, 163, 165

Kramer on dead brains 173-177

Krogh 113, 115-6, 141, 158, 259-60

Kussmaul 53

Laguesse 50, 69, 77, 99, 265

Langerhans' islands $68-74,77,118$, 190, 265

large scale production of insulin 96 , $109 \mathrm{ff}$.

large technological systems 17

Lassen 130-142, 149

latching $265-7$

latitude for disagreement 270

Law on the Disposal of the Dead

226-228, 234, 267

Leschke on non-existence of insulin

78-9

longevity experiments 99

Lovens Kemiske Fabrik 113

lower brain functions 215-6

Macleod 22, 50, 78, 81-2, 92 ff., 25

manual positive pressure ventilation 138

measuring $\mathrm{pH}$ in blood 160

mechanical students 155

medical imaging 22, 27, 46

Medicinske Studenterrådet 150, 152

Mering, Von 54-60, 63-65

metabolic alkalosis 137, 144, 159-161, 252

metaphor of scientific discovery 35

Millikan's Oximeter 147

minimum qualification of a person 204

Minkowski, O. 22, 50 ff., 246-250.

$252,256-258,262,265,268$

mouse units of insulin 115

mouth-to-mouth breathing 171

mute inscriptions $40 \mathrm{ff}$., $90,189,245$ -

6

myxoedema, treatment of 75 necessary exclusions and brain stem death 195-6

neocortical death, definition of $214 \mathrm{ff}$.

Neukirch, F. 135, 142, 144

neurogenic theory of diabetes mellitus

22,62

new function for pancreas 65

non-apneic corpse 220

non-existence of internal secretion 78 -

9,82

non-heart-beating cadaver donors 228

Nordisk Insulinfund 113

Nordisk Insulinlaboratorium 113

normal death 204, 207

ontological agnosticism 34

ontological clarification 218,255

opotherapy 75,78

organism as a whole vs whole

organism 180,190, 194

organotherapy 75,79

Pallis 194-202

parabiosis $79-81$

partial brain death 192

partial dissection 225-227, 267

partial pancreatectomies 63-4

partial transplantation and extirpation of pancreas 66

partitioning of controversy 224

patents and licenses for insulin 85 ,

$109-112,116$

patient compliance 251

penalty exemption grounds 228

perceptive immediacy $148-9,152-3$, 253

persistent vegetative state 190-2, 195, 201, 216-7, 221, 255

Pflüger, E. 56, 61-63, 76, 82, 86, 246-248, 256, 258

pharmacological immunomodulation

185

Phloridzindiabetes 53

piqûre diabétique $53,61,63,247,249$

point of no return and brain damage

$171,179-80,192$

Poliomyelitis 10, 23, 30, 43-4, $126 \mathrm{ff}$., 204, 251, 256-8, 260-3, 267

positive pressure respirators 153 
Positron Emission Tomography and neocortical death 217

postmortal autolysis 175

powers of ten 20

praeter legem development 183, 224, 225

disconnection test for apnoe 198

presumption of death 206-7

private space $223,235,252,259,271$

programs of action $244 \mathrm{ff}$.

assignment of roles 249

discursive 254, 263, 269

diagnostic 253,255

distribution of status 249

implementation of $264 \mathrm{ff}$..

therapeutic $82,118,251,253,256$ -

$7,261,269$

rabbit doses of insulin 114

Radiometer A/S 160-1

range of possible actions 233

reanimation 171-174, 183

reduction of indeterminacy 264

Rennie and Fraser 77-8

residual sentience 204

respiratory acidosis $137,141,144$,

$146,160-161,252$

responsibility for the weak 261, 264

restoration of glycogen formation 250

rhetoric 187

rhetorics of mortality rate reduction

152

right to an undisturbed deathbed 186

routinization 269

Schäfer 69-70, 75, 265

Schering $85,87,88,90$

Schulze 69, 70, 72, 74, 92, 265

SCOT 16-7

seamless web 17

secretin-stimulation of pancreas 97-9

social construction of technology 16

sociology of science 14

sociology of scientific knowledge 14

speaking for the weak $258 \mathrm{ff}$.

splitting and inversion 37,40

spokesperson $43-45,148,227,246$, 261

Ssobolew 69-74, 92

stabilization 264, 268-9

standard bicarbonate 162 standard image of scientific progress

28

standardizing roles 270

State Serum Institute 116

strategic research sited 19

sugar center $62,63,222,248,256$

supercellular functions and neocortical death 218

symmetry 15-7

systems approach $17-18$

teleological mechanicism 62

terminal bulbar polio 135

tetanus neonatorum $142-3$

thalassemia $28-9$

theater of competence $148-9,164$

theater of truth 148, 253

Till, Van on whole brain death $202 \mathrm{ff}$.

Toronto's Insulin Committee 110-3

total death, definition of $190 \mathrm{ff}$.

total pancreatectomy $54 \mathrm{ff}$.

transgenic pigs 272

transitive knowledge 32

trials of strength 18,272

utility of heart-beating corpse 184

vital mimicry 193

Whig history 35-6

whole brain death, definition of $202 \mathrm{ff}$.

xeno-transplantation 272

Zuelzer 82-92 


\section{Curriculum vitae}

Between 1972 - 1975 Ger Wackers (1956) was trained as a (medical-microbiological) laboratory technician at the Zuidlimburgse Laboratoriumschool Sittard (ZLS). Subsequently he worked as a laboratory technician at the (bacteriological) hospital laboratory of De Weverziekenhuis in Heerlen from 1975 - 1976. From 1976 - 1978 he worked at the hospital laboratory of Narvik Sykehus in Norway. From $1978-1984 \mathrm{Ger}$ Wackers studied medicine at the medical faculty of the Rijksuniversiteit Limburg (RL) in Maastricht. From 1986 - 1991 he held an appointment at the Department of Health Ethics and Philosophy of the Faculty of Health Sciences of the RL, where he started the research for this book. Since 1992 Ger Wackers holds an appointment at the Department of Philosophy of the Faculty of Cultural Sciences of the RL. 


\section{CONSTRLUCTMST MEDICINE}

This book is about modern medicine its knowledge, its technologica tool kit and is moral and social order it is the omnipresence of technology in modern medicine that sets modern medicine off against traditional forms of mediaine. Technology is taken to account for the success of an interventionist medical practice. However it is atso technology that is held responsible for the disruption of trad. tronal value systems, for abjectionable moral and social consequences. Any attempt to understand modern medicine should take into account the role that science and technology played in its generation in other words, an understanding of medicine requires an understinding of science and tectinalogy.

Drawing on recent work in constructivist science and technology stiudies for the analysis: the empincatic core of the book consisis of three case studies. The first is on the prediscovery work on insulin between 1889 en $1921: 32$ years of research yielded contlictung experimental evidence and fatled attempis to capture the internat secretion of the pancreas The second case study analyzes the events dufing the 1952. high mortality. polio-eprdemic in Copenhagens Blegdamshospltal. A free liance working anestinetists application of manuat positive pressure ventilation rectuced the mortality rate of pultar polio knom 90 to 1594 within a couple of weeks the third crete study deals with the controvensy concturning the definition and ontena of human (brain) death Many of the controversiai iscues have

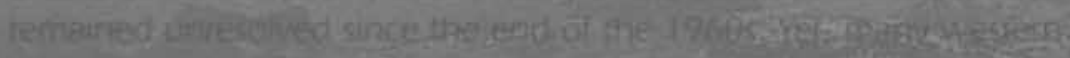

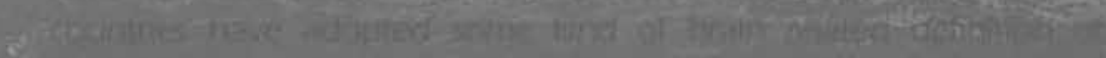

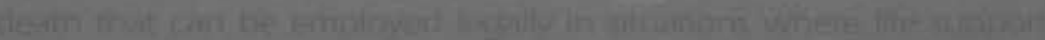

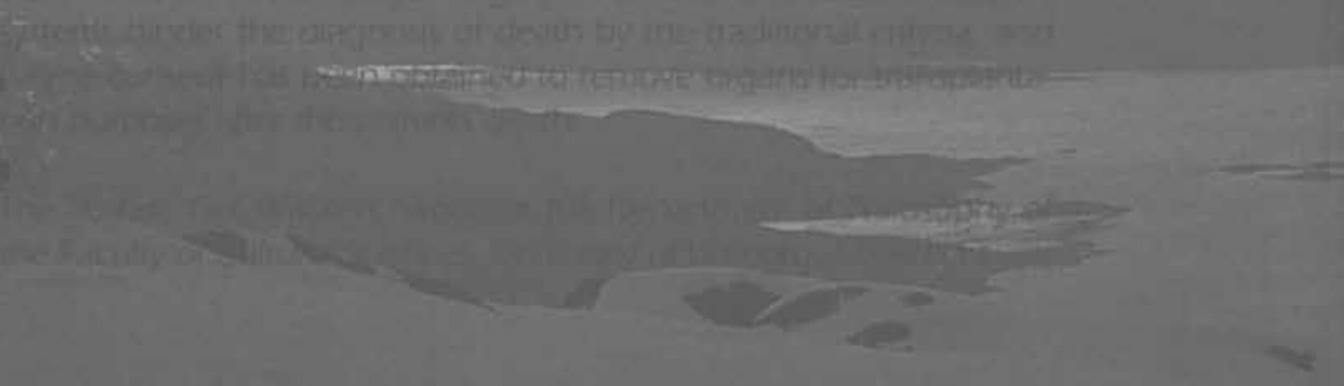

